BNL-80238-2008

\title{
World Biofuels Study
}

\section{Scenario Analysis of Global Biofuel Markets}

\author{
Thomas Alfstad
}

\author{
Prepared for \\ U.S. Department of Energy
}

\section{Energy Sciences and Technology Department}

Brookhaven National Laboratory

P.O. Box 5000

Upton, NY 11973-5000

www.bnl.gov

Notice: This manuscript has been authored by employees of Brookhaven Science Associates, LLC under Contract No. DE-AC02-98CH10886 with the U.S. Department of Energy. The publisher by accepting the manuscript for publication acknowledges that the United States Government retains a non-exclusive, paid-up, irrevocable, world-wide license to publish or reproduce the published form of this manuscript, or allow others to do so, for United States Government purposes. 


\section{DISCLAIMER}

This report was prepared as an account of work sponsored by an agency of the United States Government. Neither the United States Government nor any agency thereof, nor any of their employees, nor any of their contractors, subcontractors, or their employees, makes any warranty, express or implied, or assumes any legal liability or responsibility for the accuracy, completeness, or any third party's use or the results of such use of any information, apparatus, product, or process disclosed, or represents that its use would not infringe privately owned rights. Reference herein to any specific commercial product, process, or service by trade name, trademark, manufacturer, or otherwise, does not necessarily constitute or imply its endorsement, recommendation, or favoring by the United States Government or any agency thereof or its contractors or subcontractors. The views and opinions of authors expressed herein do not necessarily state or reflect those of the United States Government or any agency thereof. 


\section{TABLE OF CONTENTS}

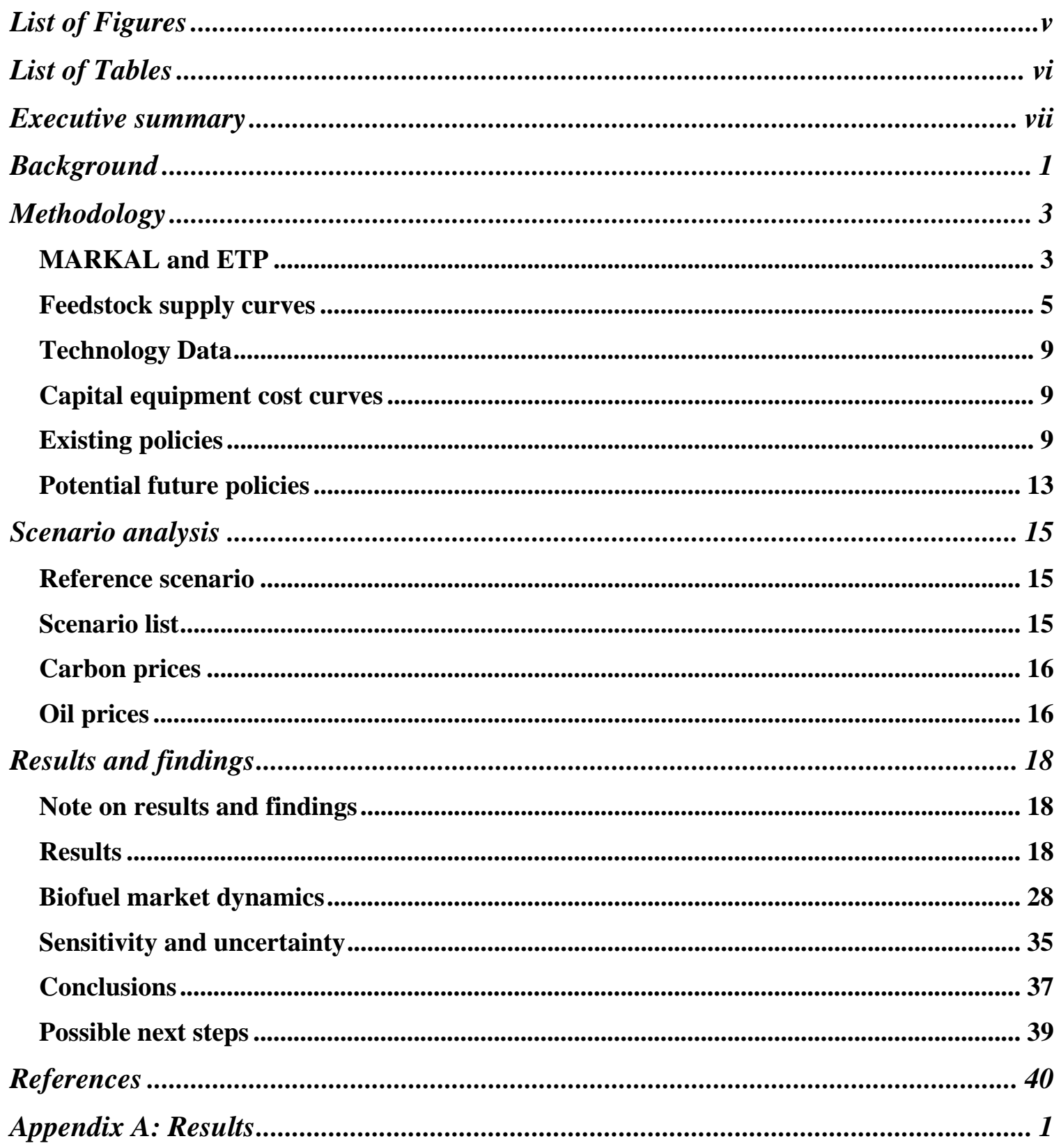


World Biofuels Study 


\section{LIST OF FIGURES}

Figure 1: World biofuels supply by type ........................................................................ viii

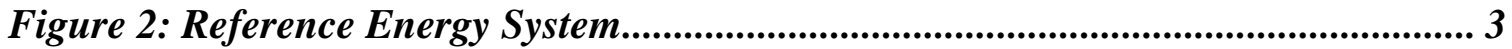

Figure 3: Projection of sugarcane availability in Brazil [8] ........................................ 6

Figure 4: Brazil sugar cane supply curve for 2017 [8] ............................................. 7

Figure 5: RFS structure with 2022 mandated volumes (Billion gallons) .................... 11

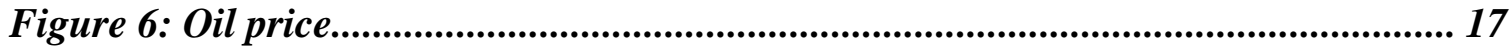

Figure 7: World biofuels supply by type for reference technology cases ....................... 19

Figure 8: World biofuels supply by type for delayed technology cases ........................... 20

Figure 9: Brazil sugarcane supply curve 2030 .............................................................. 22

Figure 10: World biofuel supply by producing region for the reference technology

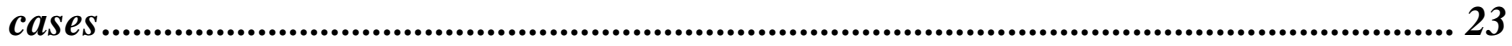

Figure 11: World biofuel supply by producing region for the delayed technology cases

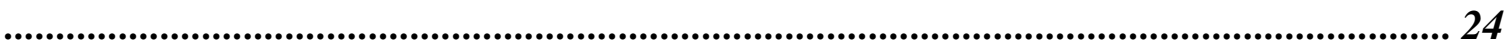

Figure 12: World biofuel demand by region for the reference technology cases ......... 25

Figure 13: World biofuel demand by region for the delayed technology cases ............ 26

Figure 14: US biofuel supply for the reference technology cases ................................ 27

Figure 15: US biofuel supply for the delayed technology cases .................................. 28

Figure 16: E85 sales in the reference case ................................................................... 30

Figure 17: Biofuels sales with E10 vs. E20 ............................................................... 32

Figure 18: Oil price impact on biofuel imports ............................................................... 33 


\section{LIST OF TABLES}

Table 1: Feedstock supply curves developed for this study [8] ..................................... 7

Table 2: Summary of aggregate feedstock projections for countries studied [8] ............ 8

Table 3: Renewable Fuel Standard in billions of gallons per year [3] ......................... 11

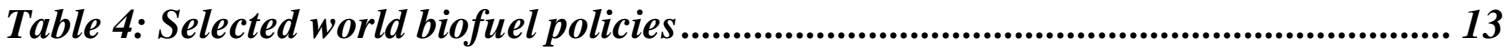

Table 5: List of scenarios.................................................................................................... 16

Table 6: Share of world (non-US) production of crop feedstocks represented by the

assessed countries [8].......................................................................................................... 21

Table 7: Change in lifecycle GHG emissions per mile traveled by replacing gasoline or

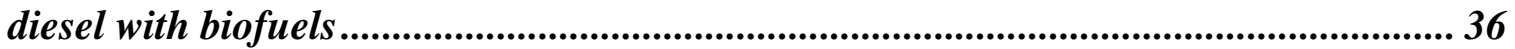




\section{EXECUTIVE SUMMARY}

This report forms part of a project entitled "World Biofuels Study." The objective is to study world biofuel markets and to examine the possible contribution that biofuel imports could make to help meet the Renewable Fuel Standard (RFS) of the Energy Independence and Security Act of 2007 (EISA).

The study was sponsored by the Biomass Program of the Assistant Secretary for Energy Efficiency and Renewable Energy (EERE), U.S. Department of Energy. It is a collaborative effort among the Office of Policy and International Affairs (PI), Department of Energy and Oak Ridge National Laboratory (ORNL), National Renewable Energy Laboratory (NREL) and Brookhaven National Laboratory (BNL).

The project consisted of three main components:

- Assessment of the resource potential for biofuel feedstocks such as sugarcane, grains, soybean, palm oil and lignocellulosic crops and development of supply curves (ORNL).

- Assessment of the cost and performance of biofuel production technologies (NREL).

- Scenario-based analysis of world biofuel markets using the ETP global energy model with data developed in the first parts of the study (BNL).

This report covers the modeling and analysis part of the project conducted by BNL in cooperation with PI.

The Energy Technology Perspectives (ETP) energy system model was used as the analytical tool for this study. ETP is a 15 region global model designed using the MARKAL framework. MARKAL-based models are partial equilibrium models that incorporate a description of the physical energy system and provide a bottom-up approach to study the entire energy system. ETP was updated for this study with biomass resource data and biofuel production technology cost and performance data developed by ORNL and NREL under Tasks 1 and 2 of this project.

Many countries around the world are embarking on ambitious biofuel policies through renewable fuel standards and economic incentives. As a result, the global biofuel demand is expected to grow very rapidly over the next two decades, provided policymakers stay the course with their policy goals.

This project relied on a scenario-based analysis to study global biofuel markets. Scenarios were designed to evaluate the impact of different policy proposals and market conditions. World biofuel supply for selected scenarios is shown in Figure 1. The reference case total biofuel production increases from 12 billion gallons of ethanol equivalent in 2005 to 54 billion gallons in 2020 and 83 billion gallons in 2030. The scenarios analyzed show volumes ranging from 46 to 64 billion gallons in 2020, and from 
about 72 to about 100 billion gallons in 2030. The highest production worldwide occurs in the scenario with high feedstock availability combined with high oil prices and more rapid improvements in cellulosic biofuel conversion technologies. The lowest global production is found in the scenario with low feedstock availability, low oil prices and slower technology progress.

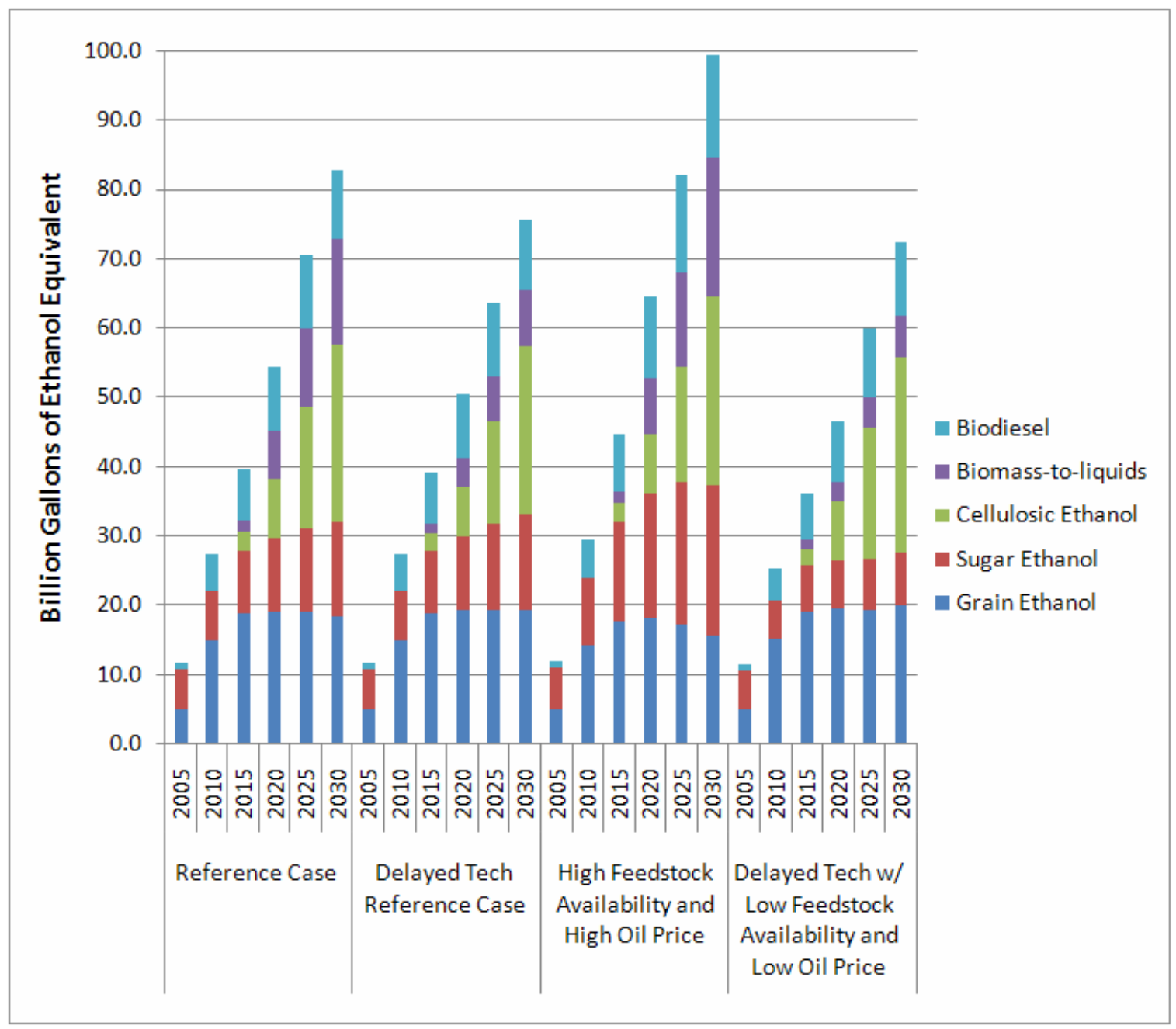

Figure 1: World biofuels supply by type

Initially, the majority of biofuels are produced from food crops. In the longer run growth rates for grain and sugar ethanol slow down. This is mainly due to limits of feedstock availability, but also because the U.S. RFS does not mandate higher volumes of these fuels. Cellulosic biofuels quickly gain significant market share after they are introduced on a commercial scale in 2012. In the reference case cellulosic biofuels have a market share of 28\% in 2020 and this grows to almost 50\% by 2030 .

The Renewable Fuel Standard in EISA is an ambitious policy that aims to raise biofuels supply in the U.S. to 36 billion gallons, with sub-targets for various types of biofuels. The challenge to the industry is vast and the scenarios presented here indicate that it may be 
difficult to reach the mandated biofuels volumes according to the schedule set out in the bill. The shortfall ranges from 0 to 5 billion gallons in 2020 depending on the scenario.

The law creates a market place where ethanol is no longer a single commodity, but can be separated into several subsets, whose value is dependent on the feedstock from which it was produced. The ethanol itself will probably trade at one price, but the associated credits will achieve different prices in the market place and thus change the total value of the ethanol to the producers.

Developing the cellulosic resource base, building biofuel production facilities and constructing the ethanol distribution infrastructure quickly enough to meet the goals of the legislation is the main obstacle to fulfilling the RFS, rather than the underlying economics of biofuels production. The overall volume requirement of the fuel standard makes it impossible to meet by blending $10 \%$ ethanol by volume into gasoline alone. In order to distribute and sell the necessary volumes of biofuels suppliers will have to market E85, biomass-to-liquids (BTL) fuels and biodiesel as well.

Because the main constraint to cellulosic biofuel production is infrastructure development rather than the underlying economics, additional incentives such as growers' payments or an extension of the ethanol blenders' tax credit does little to increase overall biofuels supply. The blenders' tax credit would be paid to marketers for volumes already mandated by law, and as policy tool would be inefficient to encourage biofuel supply. If increasing biofuel supply is the main policy goal, a targeted subsidy or "learning investment" for cellulosic biofuels would have a larger impact and also be less expensive to implement.

In markets without biofuel mandates, the price of biofuels is determined by the price premium it can achieve over gasoline or diesel due to the subsidy regimes. Higher oil prices will therefore lead to a stronger price signal for biofuel production and consequently the high oil price scenarios show higher worldwide demand for biofuels. In markets with mandates however, demand volumes are fixed through policy and changes in price signals do not do much to raise or lower demand.

These observations are true for the U.S. The U.S. biofuel demand is not very responsive to changes in the oil price, because the buy-out from the cellulosic biofuels mandate adjusts to oil price and there are no relief-valve mechanisms for the other mandated volumes. This means that higher oil prices tend to lead to domestic production substituting for imports, because the oil price hike raises biofuel demand and thereby stronger competition in international markets.

A carbon price has a similar effect to that of a higher oil price. The carbon price can in fact be seen as a price premium on fossil fuels. A carbon policy will thus promote the production and use of biofuels worldwide. However, while higher oil prices are neutral as far as feedstock and conversion technologies are concerned, a carbon price will favor cellulosic and sugar-based biofuels production over grain-based production, because the 


\section{World Biofuels Study}

latter leads to higher carbon emission per gallon. A carbon policy will thus tend to increase the share of cellulosic and sugar ethanol at the expense of grain-derived ethanol. 


\section{BACKGROUND}

In his state of the union address, President George W. Bush introduced the 20-in-10 initiative, which aims to reduce the consumption of gasoline by 20 percent over the next ten years [1]. Following this introduction, the administration sent the following two legislative proposals to Congress [2]:

- "Increasing The Supply Of Renewable And Other Alternative Fuels By Setting A Mandatory Fuels Standard To Require The Equivalent of 35 Billion Gallons Of Renewable And Other Alternative Fuels In 2017 - Nearly Five Times The 2012 Target Now In Law. In 2017, this will displace 15 percent of projected annual gasoline use.”

- "Reforming and Modernizing Corporate Average Fuel Economy (CAFE) Standards for Cars and Extending the Current Light Truck Rule. In 2017, this will reduce projected annual gasoline use by up to 8.5 billion gallons, a further 5 percent reduction that, in combination with increasing the supply of renewable and other alternative fuels, will bring the total reduction in projected annual gasoline use to 20 percent.”

These proposals were the precursors to the "Energy Independence and Security Act of 2007" [3], signed into law on December $19^{\text {th }} 2007$. The bill differs from the 20-in-10 initiative in several aspects. The timing and targets have been changed, and the requirement is now 36 billion gallons by 2022 and there are also sub targets for advanced renewable fuels, cellulosic biofuels and biodiesel.

This report forms part of a project sponsored by the U.S Department of Energy (DOE) entitled: "World biofuels study". The objective is to study world biofuel markets and examine the possible contribution that imported biofuels could make to help meet the new renewable fuel standard.

The project is a collaborative effort between the Biomass Program and the Office of Policy and International Affairs (PI) at the Department of Energy and Oak Ridge National Laboratory (ORNL), National Renewable Energy Laboratory (NREL) and Brookhaven National Laboratory (BNL).

The project consisted of three main components:

- Assessment of the resource potential for biofuel feedstocks such as sugarcane, grains, soybean, palm oil and lignocellulosic crops and development of supply curves (ORNL).

- Assessment of the cost and performance of biofuel production technologies (NREL)

- Scenario-based analysis of world biofuel markets using the ETP global energy model with data developed in the first parts of the study (BNL). 


\section{World Biofuels Study}

This report covers the modeling and analysis part of the project conducted by BNL in cooperation with PI. 


\section{METHODOLOGY}

\subsection{MARKAL and ETP}

The Energy Technology Perspectives (ETP) model is the analytic tool employed for this exercise. ETP is a 15 region global model designed using the MARKet ALlocation (MARKAL) framework. MARKAL-based models are partial equilibrium models that incorporate a description of the physical energy system [4, 5]. The components of the energy system are linked together in a flow network where the technologies form the nodes which are interlinked by energy carriers. This is illustrated in Figure 2. By representing individual technologies, MARKAL provides a bottom-up approach to study the energy system. The whole energy system, from resource extraction to service demand, is included, which allows for full "well-to-wheel" comparison of technology options.

\section{RESOURCES PROCESSES GENERATION ENERGY SERVCES}

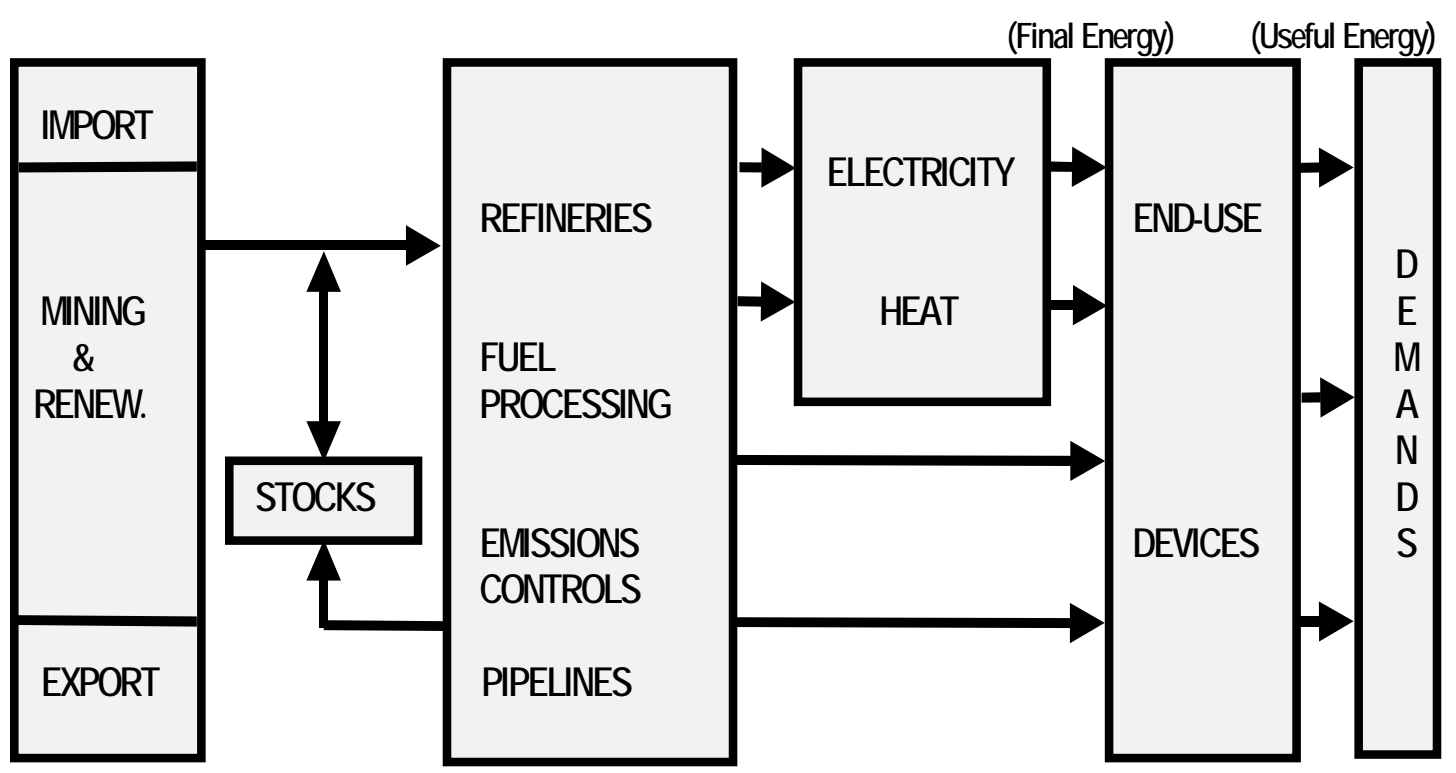

Figure 2: Reference Energy System

MARKAL models are generally solved as a cost minimization problem where future states of the energy system are determined by identifying the most cost-effective pattern of resource use and technology deployment over time. The MARKAL objective is thus to minimize the total cost of the system, discounted over the planning horizon. Each year, the total cost includes the following elements: 
- Annualized investments in technologies;

- Fixed and variable annual Operation and Maintenance (O\&M) costs of technologies;

- Cost of exogenous energy and material imports and domestic resource production (e.g., mining);

- Revenue from exogenous energy and material exports;

- Fuel and material delivery costs;

- Welfare loss resulting from reduced end-use demands.

- Taxes and subsidies associated with energy sources, technologies, and emissions.

MARKAL models are demand driven, which means that, for any feasible solution, exogenously specified energy service demands are met. The model then determines the least cost configuration of capital stock and utilization rates that will meet these demands over the full projection period. This is done while obeying a set of user-defined constraints, such as natural resource availability, technology and capital availability, environmental limitations and other constraints.

The model is dynamic, meaning that the capital stock in any period is equal to the capital stock in the preceding period plus/minus any additions or retirements. The model thus keeps track of capital stock, and the solution in one period is directly linked to the solution for other periods. Optimization is inter-temporal, which means that the optimization is performed for all periods concurrently, implicitly giving decision-makers foresight.

ETP was originally developed at the International Energy Agency for their Energy Technology Perspectives 2006 publication [6]. It consists of 15 separate regions:

- Africa

- Australia and New Zealand

- Canada

- Central and South America

- China

- Eastern Europe

- Former Soviet Union

- India

- Japan

- Mexico

- Middle East

- Other Asia

- South Korea

- United States

- Western Europe

Each of the regions listed above has a unique set of demands for all major energy services as well as energy-intensive materials such as metals, ammonia, cement, pulp and paper, 
etc. Demands can be met either through internal production or through trade with other regions.

The ETP database contains representations of hundreds of different technologies covering all stages of the energy system from extraction of primary energy to end use devices. This includes information on capital stock already in place, as well as new technologies available now, or thought to be at a future date. The general approach is that all technologies can be deployed in all world regions. However to reflect "real-world" limitations, some the following additional characterizations have been made:

- Region and sector-specific constraints;

- Region and sector-specific discount rates;

- Region-specific investment costs, fixed and variable costs;

- Region-specific supply curves for fossil fuels and renewables

- Region-specific length of seasons;

- Region-specific starting years.

The ETP model resource and technology representation was expanded in key areas for this project. A new set of biomass supply curves where added for selected feedstocks and countries (see section "2.2 Feedstock Supply Curves” below) in place of the existing representation. Because a number of the most important potential biofuel-producing countries are located in Central and South America, new biomass supply curves were developed for individual countries in this region. These curves replace the aggregated regional representation that was used previously. This added level of resolution allows more detailed study of biofuel supply in the region and the option to study trade zones.

Technology descriptions were updated and expanded based on the analysis performed by NREL for this study [7], as described in section "2.3 Technology Data" below. The model representation of international trade was also updated to reflect the new biofuel infrastructure description and tariff considerations.

\subsection{Feedstock Supply Curves}

Supply curves describe the relationship between the price and the supplied quantity of a good or service. Through interaction with the rest of the energy system they can be used to predict the volume of feedstock that will be available for conversion to biofuels at a given price. Both volumes and prices therefore are determined endogenously in the model.

Feedstock supply curves were developed by Oak Ridge National Laboratory [8] for selected countries. A set of screening criteria were used to identify the countries that had the greatest potential to supply world markets with biofuels. As a result of the screening process, seven countries and one region were selected as areas for study. 
The ORNL study analyzed current trends in feedstock production and cost at the state (or province) level in each of the selected countries. Future supply potential was projected based on historical growth rates in yield and area harvested by state over the past seven years. The methodology assumes that recent growth trends for yield and harvested area at a state level will continue into the future within a set of defined parameters. In addition to reference case projections, which are derived from past growth rates, the ORNL report also includes high and low feedstock availability curves based on historical variance in year-on-year growth. This allowed sensitivity analysis of feedstock availability.

Figure 3 shows the projection of total potential sugarcane production in Brazil. The portion of that potential utilized in a given scenario depends on the market clearing price of the feedstock as illustrated by the supply curve shown in Figure 4.

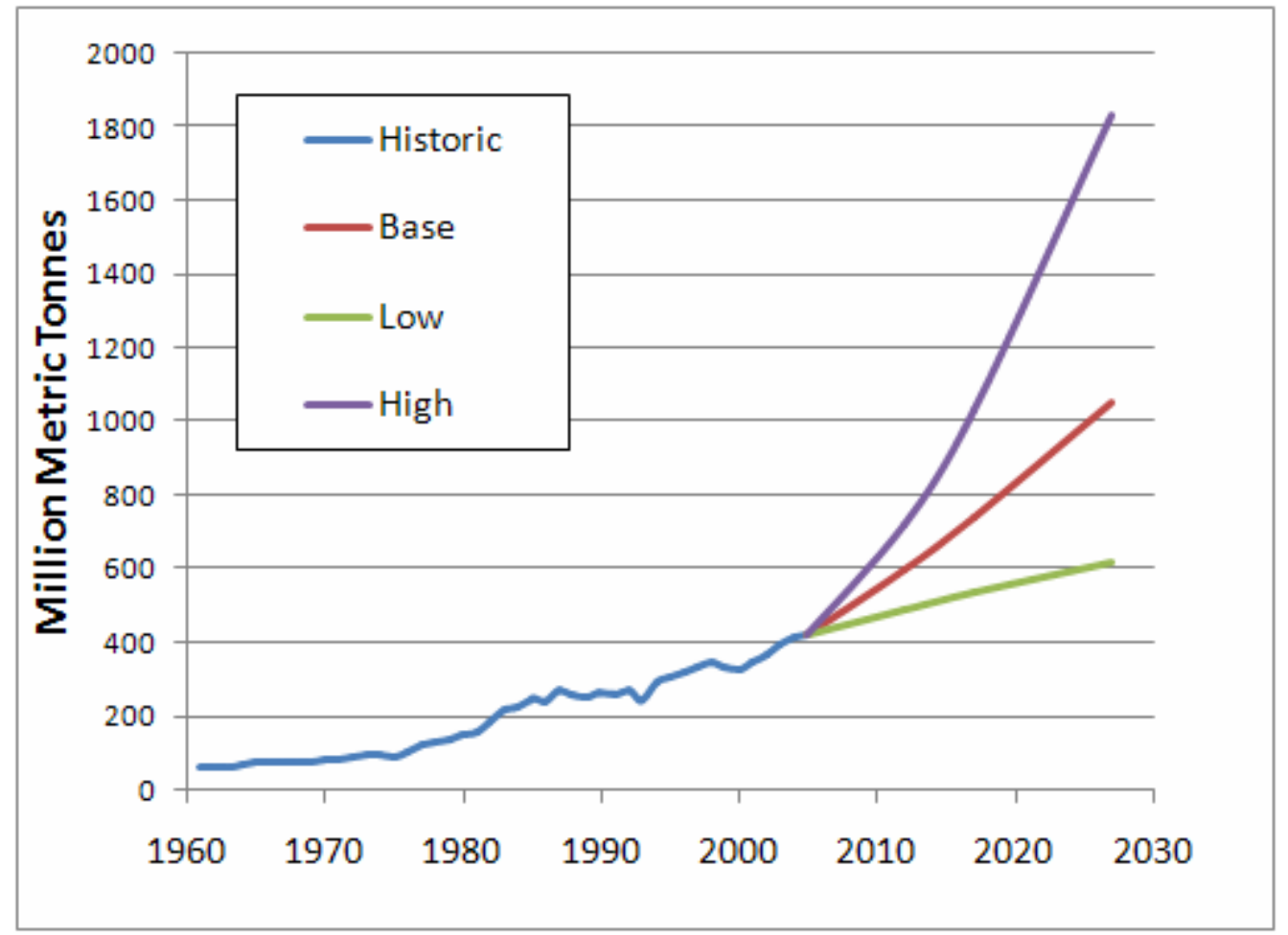

Figure 3: Projection of sugarcane availability in Brazil [8] 


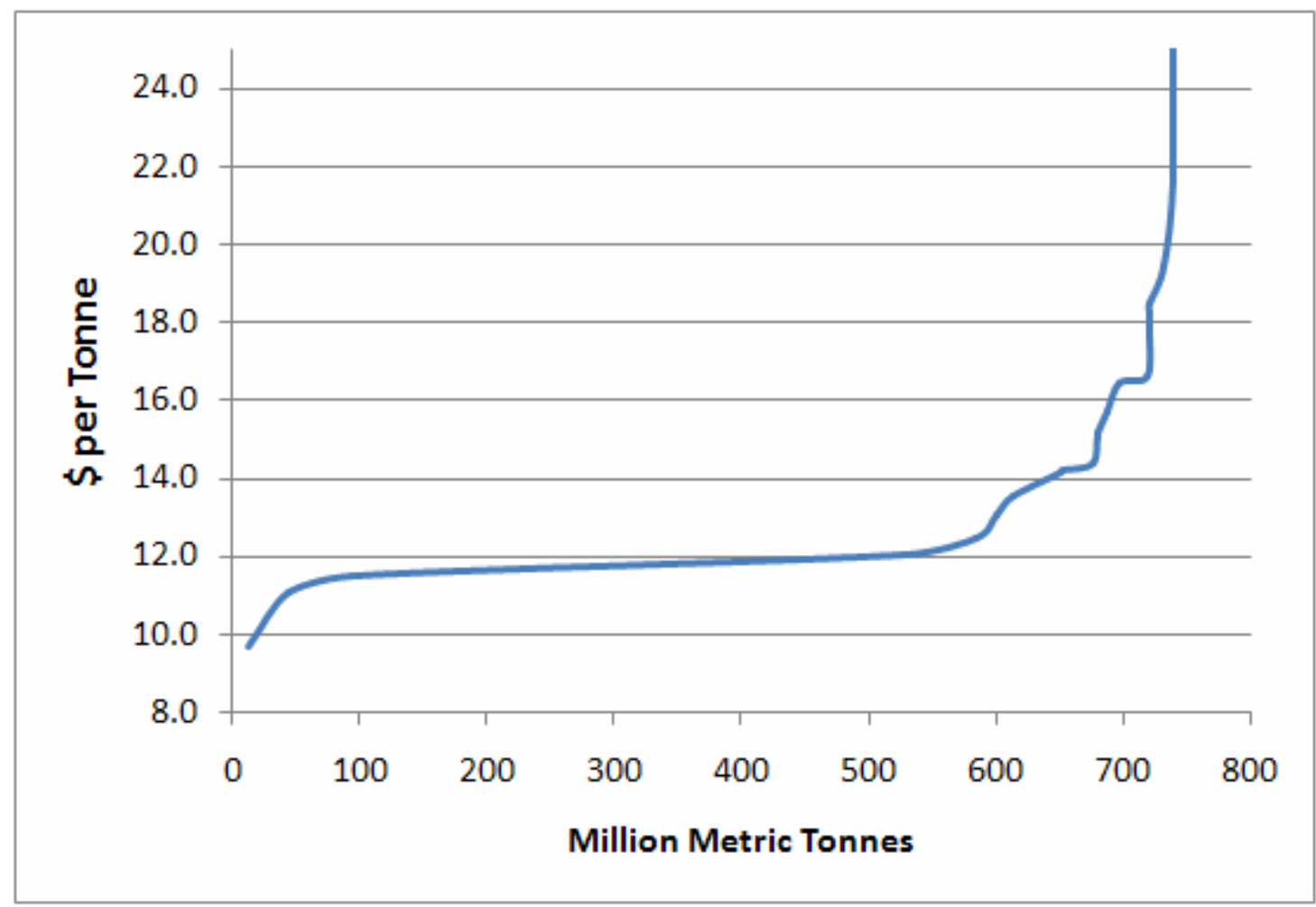

Figure 4: Brazil sugar cane supply curve for 2017 [8]

Similar curves where developed for each of the study countries and each of the applicable feedstocks. Table 1 shows the crops and countries covered in the ORNL analysis.

Table 1: Feedstock supply curves developed for this study [8]

\begin{tabular}{|c|c|c|c|c|c|c|c|c|}
\hline \multirow[b]{2}{*}{ Priority Countries } & \multicolumn{5}{|c|}{$\begin{array}{l}\text { Feedstocks for full supply curves } \\
2012,2017,2027\end{array}$} & \multicolumn{3}{|c|}{$\begin{array}{l}\text { Feedstocks for estimates } \\
2017-2027\end{array}$} \\
\hline & $\begin{array}{l}\text { sugar } \\
\text { cane }\end{array}$ & $\begin{array}{l}\text { palm } \\
\text { oil }\end{array}$ & soybean & corn & wheat & bagasse & $\begin{array}{l}\text { ag } \\
\text { residues }\end{array}$ & Othel \\
\hline Brazil & $x$ & & $x$ & $x$ & & $x$ & $\mathrm{x}$ & $x$ \\
\hline $\begin{array}{l}\mathrm{CBI} \text { (Caribbean } \\
\text { Basin/C.America) }\end{array}$ & $x$ & $x$ & & & & $x$ & & $x$ \\
\hline Colombia & $x$ & $x$ & & & & $x$ & & $x$ \\
\hline Canada & & & & $x$ & $x$ & & $x$ & $x$ \\
\hline Mexico & $\mathrm{x}$ & & & $\mathrm{X}$ & & $x$ & $\mathrm{x}$ & $\mathrm{X}$ \\
\hline Argentina & $x$ & & $x$ & $x$ & $x$ & & $x$ & $x$ \\
\hline India & $x$ & & & & & $x$ & & $x$ \\
\hline China & $x$ & & $x$ & $\mathrm{x}$ & $x$ & $x$ & $x$ & $x$ \\
\hline
\end{tabular}

Neither the ORNL study nor the ETP model directly incorporated the interaction with food markets in the analysis. Food demands were projected online and subtracted from the overall feedstock curves based on historical allocation and expert judgment. A summary of total world feedstock availability for the study countries as well as the percent available for export and biofuel production is shown in Table 2. 
Table 2: Summary of aggregate feedstock projections for countries studied [8]

\begin{tabular}{|c|c|c|c|c|c|c|}
\hline \multirow{2}{*}{ Feedstock/Year } & \multicolumn{2}{|c|}{ Baseline } & \multicolumn{2}{|c|}{ Low } & \multicolumn{2}{|c|}{ High } \\
\hline & $\begin{array}{l}\text { Total } \\
\text { supply }\end{array}$ & $\begin{array}{c}\% \\
\text { availabl } \\
\end{array}$ & $\begin{array}{c}\text { Total supply } \\
\text { mmt }\end{array}$ & $\begin{array}{c}\% \\
\text { available } \\
\end{array}$ & $\begin{array}{c}\text { Total supply } \\
\text { mmt }\end{array}$ & $\begin{array}{c}\% \\
\text { available } \\
\end{array}$ \\
\hline \multicolumn{7}{|c|}{ Feedstock Crop Supply } \\
\hline \multicolumn{7}{|l|}{ Sugarcane } \\
\hline 2012 & 1,225 & $47 \%$ & 1,003 & $42 \%$ & 1,508 & $57 \%$ \\
\hline 2017 & 1,457 & $48 \%$ & 1,066 & $40 \%$ & 2,030 & $63 \%$ \\
\hline 2027 & 1,932 & $51 \%$ & 1,174 & $38 \%$ & 3,353 & $72 \%$ \\
\hline \multicolumn{7}{|l|}{ Corn } \\
\hline 2012 & 280 & $7 \%$ & 241 & $2 \%$ & 328 & $20 \%$ \\
\hline 2017 & 325 & $7 \%$ & 257 & $2 \%$ & 428 & $29 \%$ \\
\hline 2027 & 427 & $7 \%$ & 290 & $1 \%$ & 692 & $42 \%$ \\
\hline \multicolumn{7}{|l|}{ Soybeans } \\
\hline 2012 & 155 & $65 \%$ & 130 & $62 \%$ & 188 & $71 \%$ \\
\hline 2017 & 202 & $66 \%$ & 147 & $58 \%$ & 288 & $76 \%$ \\
\hline 2027 & 314 & $67 \%$ & 178 & $49 \%$ & 652 & $84 \%$ \\
\hline \multicolumn{7}{|l|}{ Wheat } \\
\hline 2012 & 153 & $18 \%$ & 136 & $17 \%$ & 183 & $32 \%$ \\
\hline 2017 & 160 & $19 \%$ & 136 & $16 \%$ & 208 & $38 \%$ \\
\hline 2027 & 176 & $19 \%$ & 137 & $15 \%$ & 268 & $47 \%$ \\
\hline \multicolumn{7}{|l|}{ Palm Oil } \\
\hline 2012 & 2 & $41 \%$ & 2 & $18 \%$ & 3 & $53 \%$ \\
\hline 2017 & 3 & $41 \%$ & 2 & $9 \%$ & 5 & $59 \%$ \\
\hline 2027 & 7 & $40 \%$ & 2 & $0 \%$ & 12 & $66 \%$ \\
\hline \multicolumn{7}{|l|}{ Total Crops } \\
\hline 2017 & 2,144 & & 1,606 & & 2,955 & \\
\hline 2027 & 2,850 & & 1,778 & & 4,965 & \\
\hline \multicolumn{7}{|l|}{ Cellulosic Supply } \\
\hline \multicolumn{7}{|l|}{ Crop Residues $^{1}$} \\
\hline 2017 & 246 & & 182 & & 344 & \\
\hline 2027 & 326 & & 201 & & 569 & \\
\hline \multicolumn{7}{|l|}{ Other Residues $^{2}$} \\
\hline 2017 & 242 & & 242 & & 242 & \\
\hline 2027 & 294 & & 294 & & 294 & \\
\hline \multicolumn{7}{|l|}{ Total Cellulosic } \\
\hline 2017 & 488 & & 424 & & 586 & \\
\hline 2027 & 621 & & 495 & & 863 & \\
\hline
\end{tabular}

\footnotetext{
${ }^{1}$ Recoverable crop residues are derived from the crop feedstock production projected in each case and country for crops listed above. Crop residues include a percentage of bagasse (the most prominent crop residue available), corn stover, wheat straw and palm oil processing wastes. See Methodology in Annex 3. ${ }^{2}$ Other residues include estimates based on forestry residues, fuelwood supplies and perennial harvests.
} 


\subsection{Technology Data}

Technology data for biomass conversion technologies was provided by NREL in their contribution to this study [7] and used as input for ETP. Country-specific cost data was developed for a range of biofuel technologies. Data was incorporated for corn and wheat dry mills, sugar cane mills and cellulosic ethanol bio-chemical conversion plants for different plant sizes. Soy and palm oil diesel conversion technologies were also included.

The assumption for this study is that technology progress will transfer among regions, so that technology developed in one region will be available to others. However, in a report published by the UN [9], it was noted that advanced biofuel technologies tend to be developed for industrialized country applications and will be capital-intensive, laborminimizing and designed for large scale production facilities to achieve better economics of scale. For developing countries to make best use of these technologies they will have to adapt them to local crops and to their competitive advantages. This might involve substituting abundant and cheap labor for scarce capital. Differences in climate and soil conditions might also require the adaptation of technology to local crops and conditions. For this study it has been assumed that these issues are resolved and that all regions can effectively deploy these technologies.

\subsection{Capital Equipment Cost Curves}

Another addition to the ETP model is that cost curves for capital equipment have been introduced. If the capital stock of a given technology is to expand faster than a predefined "normal" rate, a price premium has to be paid for that capital stock. This represents the added cost of outbidding competitors for labor, materials and contractors. A given technology will thus take market share from competing technologies more rapidly if it has a greater cost advantage.

These cost curves have been introduced for the various biofuel production technologies and are particularly important for the market penetration of cellulosic ethanol.

\subsection{Existing Policies}

The central policies covered in this study are the provisions enacted under the Energy Independence and Security Act (EISA) of 2007 [3]. EISA is designed to improve energy efficiency and increase the supply of renewable energy. The main provisions enacted into law can be summarized as follows:

- Renewable Fuel Standard (RFS): EISA mandates the use of additional renewable fuels by modifying the existing fuel standard. The standard now requires the sale of 36 billion gallons of renewable fuels per year by 2022 . 
- Corporate Average Fuel Economy (CAFE): The law sets a fuel efficiency target of 35 miles per gallon for the combined light duty vehicle fleet by the 2020 model year.

- Appliance Energy Efficiency Standards: The bill sets energy efficiency standards for a range of commercial and household appliances, including refrigerators, freezers and lighting.

- Repeal of Oil and Gas Tax Incentives: EISA repeals two tax subsidies. The revenues from these taxes are intended to cover the cost of implementing the CAFE standards.

While the CAFE provisions have been included in the analysis, the focus in this study is on the RFS and biofuel supply and demand. All scenarios are therefore designed to address this subject.

Under the RFS provision in EISA the existing standard under the Clean Air Act is revised and expanded. The main revision is the introduction of four different categories of renewable fuel. They are as follows:

- Renewable Fuels: fuel that is produced from renewable biomass ${ }^{3}$. Any qualifying new production plant would also have to demonstrate lifecycle greenhouse gas emissions that are at least $20 \%$ lower than the baseline lifecycle greenhouse gas emissions for gasoline.

- Advanced Renewable Fuels: renewable fuels other than fuels derived from corn and other starch that has lifecycle greenhouse gas emissions that are at least 50\% lower than the baseline lifecycle greenhouse gas emissions for gasoline.

- Cellulosic Biofuels: renewable fuels derived from any cellulose, hemi-cellulose, or lignin that is derived from renewable biomass and that has lifecycle greenhouse gas emissions that are at least 60 percent less than the baseline lifecycle greenhouse gas emissions for gasoline.

- Biomass-based Diesel: renewable fuel that is biodiesel as defined in the Energy Policy Act of 1992 and that has lifecycle greenhouse gas emissions that are at least 50 percent less than the baseline lifecycle greenhouse gas emissions for gasoline.

The "renewable fuels" classification encompasses all fuels mandated under the law (e.g. all biomass-based diesel also qualifies as renewable fuel). All other categories are thus subsets of this classification. Furthermore, cellulosic biofuels and biomass-based diesel are both subsets of the advanced renewable fuels classification. This is illustrated in Figure 5, which shows the RFS structure and the mandated volumes for 2022.

\footnotetext{
${ }^{3}$ Renewable biomass is crops and crop residue harvested from agricultural land cleared or cultivated at any time prior to the enactment of the law. It also includes animal wastes, mill wastes, recycled cooking oil and algae.
} 
Renewable Fuels (36)

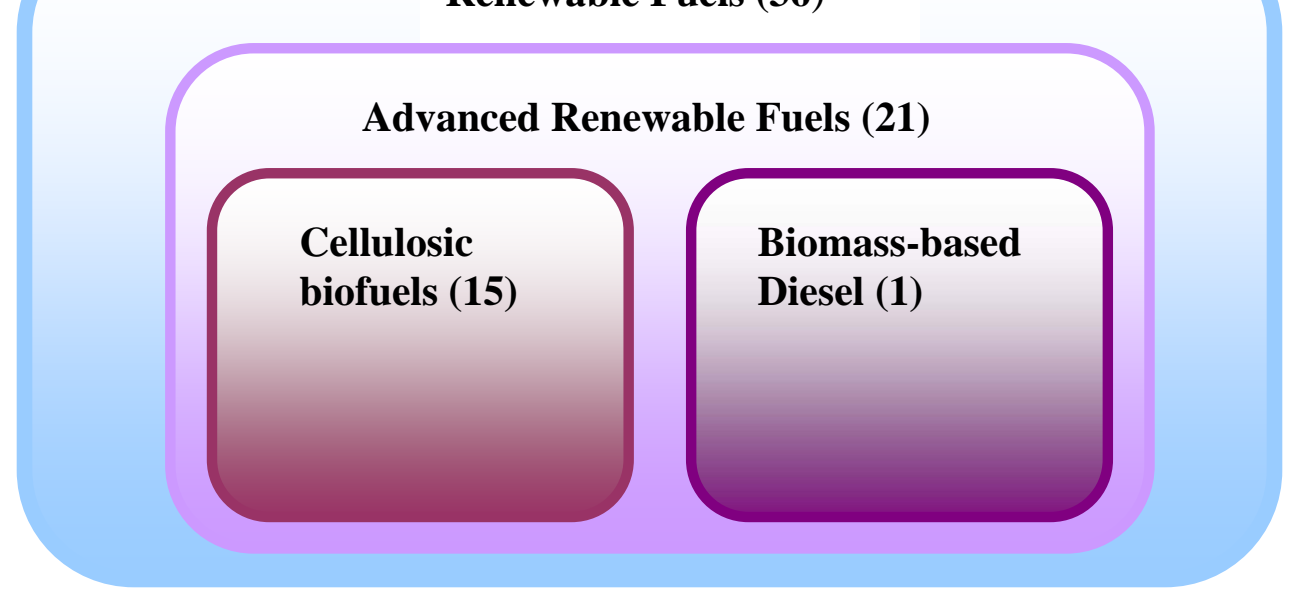

Figure 5: RFS structure with 2022 mandated volumes (Billion gallons)

The standard includes individual mandated volumes for all of the different categories. These volumes increase over time and follow the schedules shown in Table 3. From 2015 onwards all increases in the overall mandated volumes are from advanced renewable fuels. The maximum volume of grain ethanol for which distributors can receive credits is thus 15 billion gallons annually. The law does not actually prevent production of corn ethanol above this volume, but the economic incentives to produce are significantly reduced if no additional credits are available.

Table 3: Renewable Fuel Standard in billions of gallons per year [3]

\begin{tabular}{|l|c|c|c|c|}
\hline Year & $\begin{array}{c}\text { Renewable } \\
\text { Fuel }\end{array}$ & $\begin{array}{c}\text { Advanced } \\
\text { Renewable } \\
\text { Fuel }\end{array}$ & $\begin{array}{c}\text { Cellulosic } \\
\text { Biofuels }\end{array}$ & $\begin{array}{c}\text { Biomass Based } \\
\text { Diesel }\end{array}$ \\
\hline $\mathbf{2 0 0 8}$ & 9.00 & & & \\
\hline $\mathbf{2 0 0 9}$ & 11.10 & 0.60 & 0.10 & 0.65 \\
\hline $\mathbf{2 0 1 0}$ & 12.95 & 0.95 & 0.25 & 0.80 \\
\hline $\mathbf{2 0 1 1}$ & 13.95 & 1.35 & 0.50 & 1.00 \\
\hline $\mathbf{2 0 1 2}$ & 15.20 & 2.00 & 1.00 & 1.00 \\
\hline $\mathbf{2 0 1 3}$ & 16.55 & 2.75 & 1.75 & 1.00 \\
\hline $\mathbf{2 0 1 4}$ & 18.15 & 3.75 & 3.00 & 1.00 \\
\hline $\mathbf{2 0 1 5}$ & 20.50 & 5.50 & 4.25 & 1.00 \\
\hline $\mathbf{2 0 1 6}$ & 22.25 & 7.25 & 5.50 & 1.00 \\
\hline $\mathbf{2 0 1 7}$ & 24.00 & 9.00 & 7.00 & 1.00 \\
\hline $\mathbf{2 0 1 8}$ & 26.00 & 11.00 & 8.50 & 1.00 \\
\hline $\mathbf{2 0 1 9}$ & 28.00 & 13.00 & 10.50 & 1.00 \\
\hline $\mathbf{2 0 2 0}$ & 30.00 & 15.00 & 13.50 & 1.00 \\
\hline $\mathbf{2 0 2 1}$ & 33.00 & 18.00 & 16.00 & 1.00 \\
\hline $\mathbf{2 0 2 2}$ & 36.00 & 21.00 & & \\
\hline
\end{tabular}


In general, compliance is required from "refineries, blenders, distributors, and importers" for each of the volumes. The final design of the regulation is the responsibility of the Environmental Protection Agency (EPA) and the exact details of implementation and enforcement are unclear pending the rulemaking due at the end of 2008. Furthermore, the EPA can issue waivers under certain circumstances, and may also sell waivers for the cellulosic biofuel mandate. The waiver price adjusts to gasoline prices and will be the maximum of $\$ 0.25$ or $\$ 3.00$ less the wholesale price of gasoline (i.e. if the gasoline price is $\$ 2.50$ the waiver price will be $\$ 0.50$, but if the price of gasoline is higher than $\$ 2.75$ the waiver price remains at \$0.25). Until the EPA has completed its rulemaking it remains unclear how the sale of these waivers will be conducted and under what circumstances they will be allowed. For this study we have assumed that these waivers will be available for sale in the case of a shortfall of biofuels. This then acts as a "relief valve", which limits the potential cost of the mandate in a changing economic environment. The waiver price thus represents the price premium that cellulosic biofuels can achieve over gasoline (or diesel in the case of BTL distillates) and is thus the price signal for biofuels suppliers.

It is also worth noting that, as defined in EISA, distillates produced from a FischerTropsch biomass-to-liquids process would qualify as cellulosic biofuels, not as biomassbased diesel. Only fatty acid methyl esters (FAME) would be considered biomass-based diesel.

Two existing policies that were addressed in this study are the 51 cents per gallon tax credit that currently is given for blending ethanol into gasoline and the 54 cents per gallon import tariff that is charged to producers outside the NAFTA trade zone. These two polices are considered in tandem as the main purpose of the tariff is to "cancel out" the subsidy going to foreign producers. The policies are currently due to expire in 2009 and 2010 respectively. This study examines scenarios with alternative expiration dates for these policies ${ }^{4}$.

Also, there is currently a $\$ 1$ per gallon tax credit for producers of biodiesel. This study assumes that this policy is extended.

Since this is a study of global biofuel markets, policies that are in place in other countries are also of great importance. These policies are often in the form of tariffs and exemption from fuel taxes. A list of policies considered in this study is given in Table 4.

\footnotetext{
${ }^{4}$ A new farm bill is currently being debated in Congress and one of the issues involved is the extension of these two policies, although the blenders' tax credit might be reduced to 46 cents per gallon.
} 
Table 4: Selected world biofuel policies

\begin{tabular}{|c|c|c|c|c|c|}
\hline $\begin{array}{l}\text { Countryl } \\
\text { region }\end{array}$ & $\begin{array}{l}\text { Gasoline } \\
\text { tax } \\
\text { [\$/gal] }\end{array}$ & $\begin{array}{l}2010 \text { Biofuel } \\
\text { Tax } \\
\text { exemption }\end{array}$ & $\begin{array}{c}\text { Ethanol } \\
\text { tariffs }\end{array}$ & Other modeled & $\begin{array}{l}\text { Other not- } \\
\text { modeled in } \\
\text { current } \\
\text { study }\end{array}$ \\
\hline Australia & 1.40 & $100 \%$ & $90 \mathrm{c} / \mathrm{gal}$ & & \\
\hline Canada & 0.25 & $100 \%$ & $20 \mathrm{c} / \mathrm{gal}$ & & $\begin{array}{l}5 \% \text { market } \\
\text { share by } 2010\end{array}$ \\
\hline China & 0.15 & $100 \%$ & 0 & & $\begin{array}{l}\text { 15\% Market } \\
\text { share } 2015\end{array}$ \\
\hline $\begin{array}{l}\text { Central and } \\
\text { South } \\
\text { America }\end{array}$ & 0.70 & $50 \%$ & $27 \mathrm{c} / \mathrm{gal}$ & $\begin{array}{l}\text { Subsidy for hydrous } \\
\text { ethanol and flex-fuel } \\
\text { vehicles. Brazil blending } \\
\text { requirement of } 20-25 \%\end{array}$ & \\
\hline Europe & 2.80 & $90 \%$ & $90 \mathrm{c} / \mathrm{gal}$ & $\begin{array}{l}5.5 \% \text { market share in } \\
2010,10 \% \text { market } \\
\text { share in } 2020\end{array}$ & \\
\hline India & 1.90 & $0 \%$ & $200 \%$ & & $\begin{array}{l}5 \% \text { market } \\
\text { share by } 2015\end{array}$ \\
\hline Japan & 1.85 & $90 \%$ & $17 \%$ & $\begin{array}{l}500 \text { mill liter gasoline } \\
\text { equivalent by } 2010\end{array}$ & \\
\hline $\begin{array}{l}\text { South } \\
\text { Korea }\end{array}$ & 3.02 & $90 \%$ & 0 & & \\
\hline USA & 0.42 & $51 \mathrm{c} / \mathrm{gal}$ & $54 \mathrm{c} / \mathrm{gal}$ & $\begin{array}{l}36 \text { billion gallons of } \\
\text { alternative fuels by } \\
2022\end{array}$ & \\
\hline
\end{tabular}

\subsection{Potential Future Policies}

A potential additional subsidy for cellulosic biofuel production was also considered because cellulosic ethanol has three important advantages over ethanol derived from grains. First, feedstocks for this form of production do not have an alternative use as food or feed. Increased production would therefore not impact food markets directly, although competition for land, labor and capital could yield indirect impacts. Second, the resource base is potentially enormous; and third, lower water and fertilizer requirements and an overall reduction in carbon emissions means that it is seen as more environmentally friendly. During the initial commercialization phase however, this technology option will most likely need additional subsidies. The technology data developed by NREL is for an "nth of a kind" plant. To bring the cellulosic biofuels technologies to the level of commercialization implied by these numbers, some form of learning investment is most likely required. Investment could come from both public and private sources. The subsidy could be in the form of production tax credits, co-funding or other support or subsidy scheme that improves the economics of cellulosic biofuel production for the investor. A production tax credit for cellulosic biofuels is being considered as part of the farm bill [10] although it is set to expire 2012. The policy considered here could be an extension of this tax credit or a different subsidy of similar magnitude. 
Since ethanol distribution infrastructure limitations and the ability to deliver sufficient volumes of ethanol to consumers is one of the main obstacles to reaching the RFS mandate, a policy scenario where E20 (gasoline blended with 20\% ethanol by volume) is certified was considered. This would alleviate some of the infrastructure concerns, but the viability of this policy is dependent on resolving issues related to the impact higher blends of ethanol will have on engines and fuel systems. Currently most car manufacturers' will only warrantee their gasoline engines if they are fuelled with ethanol blends of $10 \%$ or less.

Another potential subsidy considered in this study is a growers' payment for U.S. farmers cultivating renewable cellulosic biomass. A \$20 per dry tonne payment would be offered to farmers starting in 2010 and expires in 2022. It is not adjusted for inflation. 


\section{SCENARIO ANALYSIS}

\subsection{Reference Scenario}

The reference scenario forms the base against which other scenarios are compared. It is calibrated to the 2007 WEO [11] with the addition of EISA provisions and the updated reference case supply curves as developed by ORNL [8]. In this case the blenders' tax credit and ethanol import tariffs both expire in 2010.

\subsection{Scenario List}

A list of scenarios is given in Table 5. Each of the scenarios was run with two sets of technology assumptions. First, with the assumption corresponding to the learning investment described in the policy section above and then with technology assumptions that correspond to a scenario where these is no learning investment. There are 14 scenarios in the list, bringing the total number of cases to 28.

For the remainder of this report the reference case with learning investment will serve as the primary comparison for other scenarios and will be referred to as "reference case" for simplicity. Likewise the reference case without learning investment will be called "delayed technology reference". Scenarios building on these will follow the same naming convention (e.g. "Delayed technology with high oil price”). 
Table 5: List of scenarios

\begin{tabular}{|c|c|c|c|c|c|}
\hline Scenario & $\begin{array}{c}\text { Ethanol } \\
\text { blenders' } \\
\text { tax } \\
\text { credit }\end{array}$ & $\begin{array}{c}\text { Ethanol } \\
\text { import } \\
\text { tariff }\end{array}$ & $\begin{array}{l}\text { Feedstock } \\
\text { availability }\end{array}$ & Oil price & Other \\
\hline Reference case & - & - & Reference & Reference & \\
\hline $\begin{array}{l}\text { Credit and Tariff } \\
\text { Extension }\end{array}$ & Extended & Extended & Reference & Reference & \\
\hline Credit Extension & Extended & - & Reference & Reference & \\
\hline E20 & - & - & Reference & Reference & E20 certified \\
\hline $\begin{array}{l}\text { \$20 per Tonne } \\
\text { Growers } \\
\text { Payment } \\
\end{array}$ & - & - & Reference & Reference & $\begin{array}{l}\text { \$20 per dry tonne } \\
\text { of biomass } \\
\text { feedstock }\end{array}$ \\
\hline $\begin{array}{l}\text { \$50 per tonne of } \\
\text { carbon dioxide }\end{array}$ & - & - & Reference & Reference & $\begin{array}{l}\text { Price of } \$ 50 \text { per } \\
\text { tonne of } \mathrm{CO}_{2}\end{array}$ \\
\hline $\begin{array}{l}\text { 70/30 fuel /food } \\
\text { split }\end{array}$ & & & & & $\begin{array}{l}70 \% \text { of sugarcane } \\
\text { in Brazil avail. } \\
\text { for biofuels }\end{array}$ \\
\hline High Oil Price & - & - & Reference & High & \\
\hline Low Oil Price & & - & Reference & Low & \\
\hline $\begin{array}{l}\text { Extra High Oil } \\
\text { Price }\end{array}$ & - & - & Reference & Extra High & \\
\hline $\begin{array}{l}\text { High Feedstock } \\
\text { Availability }\end{array}$ & - & - & High & Reference & \\
\hline $\begin{array}{l}\text { Low Feedstock } \\
\text { Availability }\end{array}$ & - & - & Low & Reference & \\
\hline $\begin{array}{l}\text { High Feedstock } \\
\text { Availability and } \\
\text { High Oil Price }\end{array}$ & - & - & High & High & \\
\hline $\begin{array}{l}\text { Low Feedstock } \\
\text { Availability and } \\
\text { Low Oil Price }\end{array}$ & - & - & Low & Low & \\
\hline
\end{tabular}

\subsection{Carbon Prices}

In the carbon scenarios, a carbon price of $\$ 50$ per metric tonne of $\mathrm{CO}_{2}$ is gradually phased in. It starts at \$12.5 per tonne in 2015 and is increased by $\$ 2.5$ per tonne annually until it reaches $\$ 50$ per tonne in 2030. The carbon price applies to all sectors of the economy and to all regions. It is adjusted for inflation.

\subsection{Oil Prices}

Oil prices are determined endogenously in the model and are thus an outcome for a given model run and not an input assumption. Many factors influence the oil price including supply curves, demand, end-use efficiency and fuel switching. Another important 
variable is OPEC rent-seeking, which can be manipulated in the model. This study used this rent-seeking as the market driver for oil prices. A high oil price scenario is thus a case where OPEC follows a more aggressive policy and restricts supply to world markets by demanding higher economic rent on each barrel it produces. Low oil prices conversely occur when OPEC reduces their rent-seeking and produces more crude oil for world markets.

In this study high oil prices indicate an additional \$18 per barrel economic rent sought by OPEC producers, while in the low price case it is \$18 per barrel lower. It is worth noting that this does not mean that oil prices will be exactly $\$ 18$ per barrel higher and lower respectively for these two scenarios, since non-OPEC producers will respond to price changes. There is also an extra high oil price case where OPEC rent-seeking is raised by $\$ 43$ per barrel. Oil price outcomes are shown in Figure 6.

The oil prices referred to in Figure 6 and in the rest of this report are the average U.S. imported prices of crude oil, also referred to as refiner's acquisition costs. Average import prices are generally significantly lower than the oil prices reported in the press. Reported oil prices are usually spot or future prices (i.e. WTI Cushing spot or NYMEX future price). These refer to reference crudes (light sweet crude oil), which are of higher quality than the average traded crude and thus receive a higher price. The latest estimates of imported contract prices available from the EIA at the time of writing (4/25/08) reports contract crude prices at $\$ 109$ per barrel [12]. This is $\$ 10$ below the WTI Cushing spot price for the same day. In its 2008 Annual Energy Outlook (AEO 2008) the EIA projects this gap to range between $\$ 8$ and $\$ 12$ per barrel over the next 20 years [13].

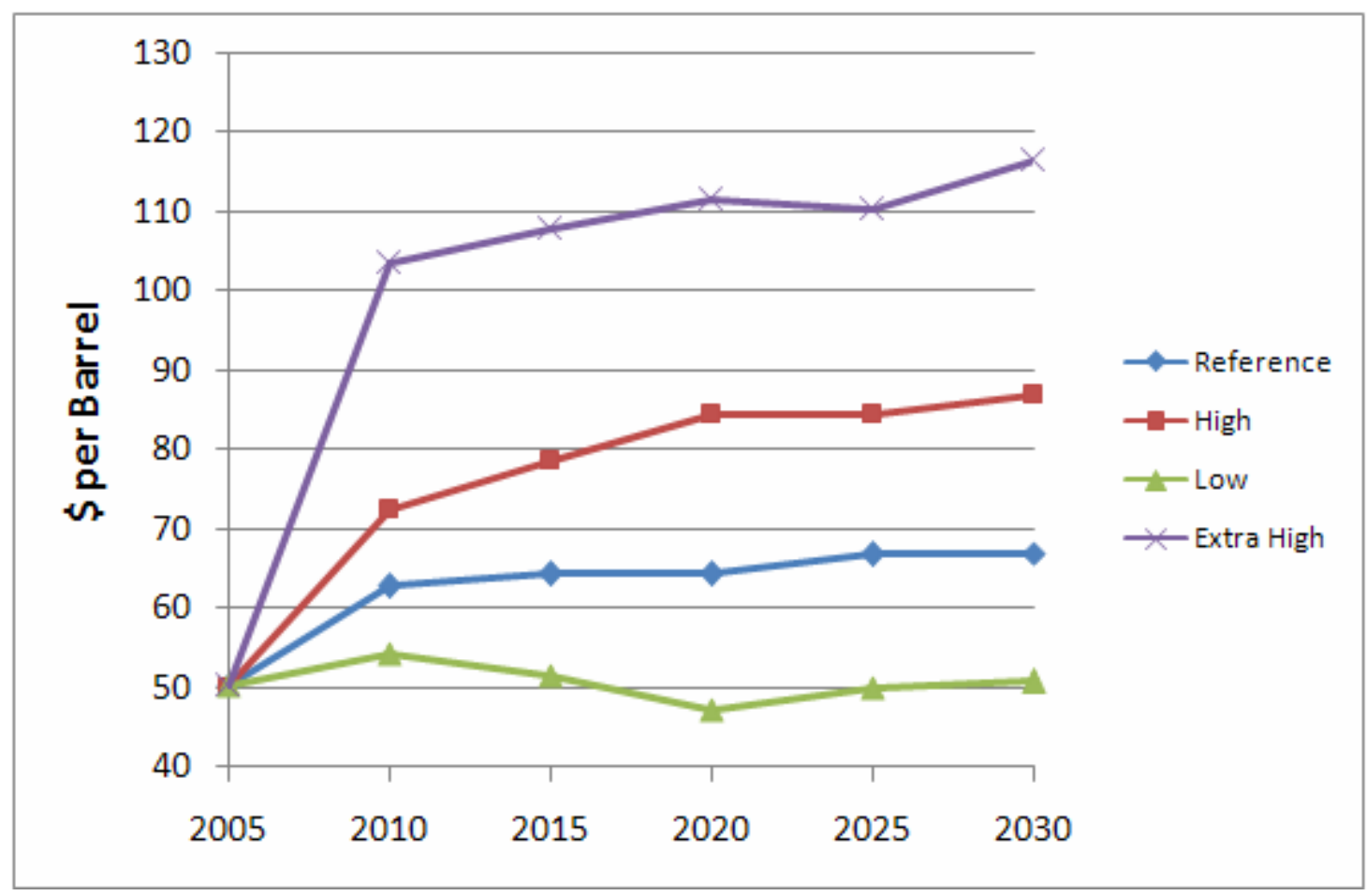

Figure 6: Oil price 


\section{RESULTS AND FINDINGS}

\subsection{Note On Results And Findings}

Results from this study should not be read as forecasts. There are too many uncertainties and unknowns to make accurate predictions about future production and traded volumes of biofuels. This has been an exploratory scenario analysis that is meant to inform the biofuels policy debate. It is thus intended to address the dynamics of the biofuel markets and the relative impact of policies and market uncertainties rather than forecast future biofuel supply. Numbers should not be viewed in isolation, but in the context of the study and underlying assumptions. For instance, an adjustment of supply curves would change produced volumes, but the overall dynamics of the markets and the relative impact of the various policies should not change. In this section the focus is therefore mainly on these issues rather than on absolute numbers. That said; every effort has been made to ensure that the reported volumes are as reasonable as possible. Furthermore, the overall spread of outcomes from the analysis probably gives a fair estimate of the range of import volumes that are feasible in the medium term. This caveat is meant to emphasize the inherent uncertainty in this type of forward-looking exercise and encourage the reader not to attach too great importance to individual values, but rather view them in context of the overall range of results.

\subsection{Results}

This section covers the results of the scenario analysis. A total of 28 scenarios were analyzed. To limit the page count and ensure readability, only selected data have been included here. Data tables for each of the scenarios can be found in Appendix A for readers who wish to access the full results.

World biofuel supply for selected scenarios is shown in Figure 7 and Figure 8. The reference case total biofuel production increases from 12 billion gallons of ethanol equivalent in 2005 to 54 billion gallons 2020 and 83 billion gallons in 2030. The scenarios analyzed showed volumes ranging from 46 to 64 billion gallons in 2020 and from about 72 to about 100 billion gallons in 2030. The highest production worldwide occurs in the scenario with high feedstock availability combined with high oil prices and more rapid improvements in cellulosic biofuel conversion technologies. The lowest global production is found in the scenario with low feedstock availability, low oil prices and slower technology progress. 


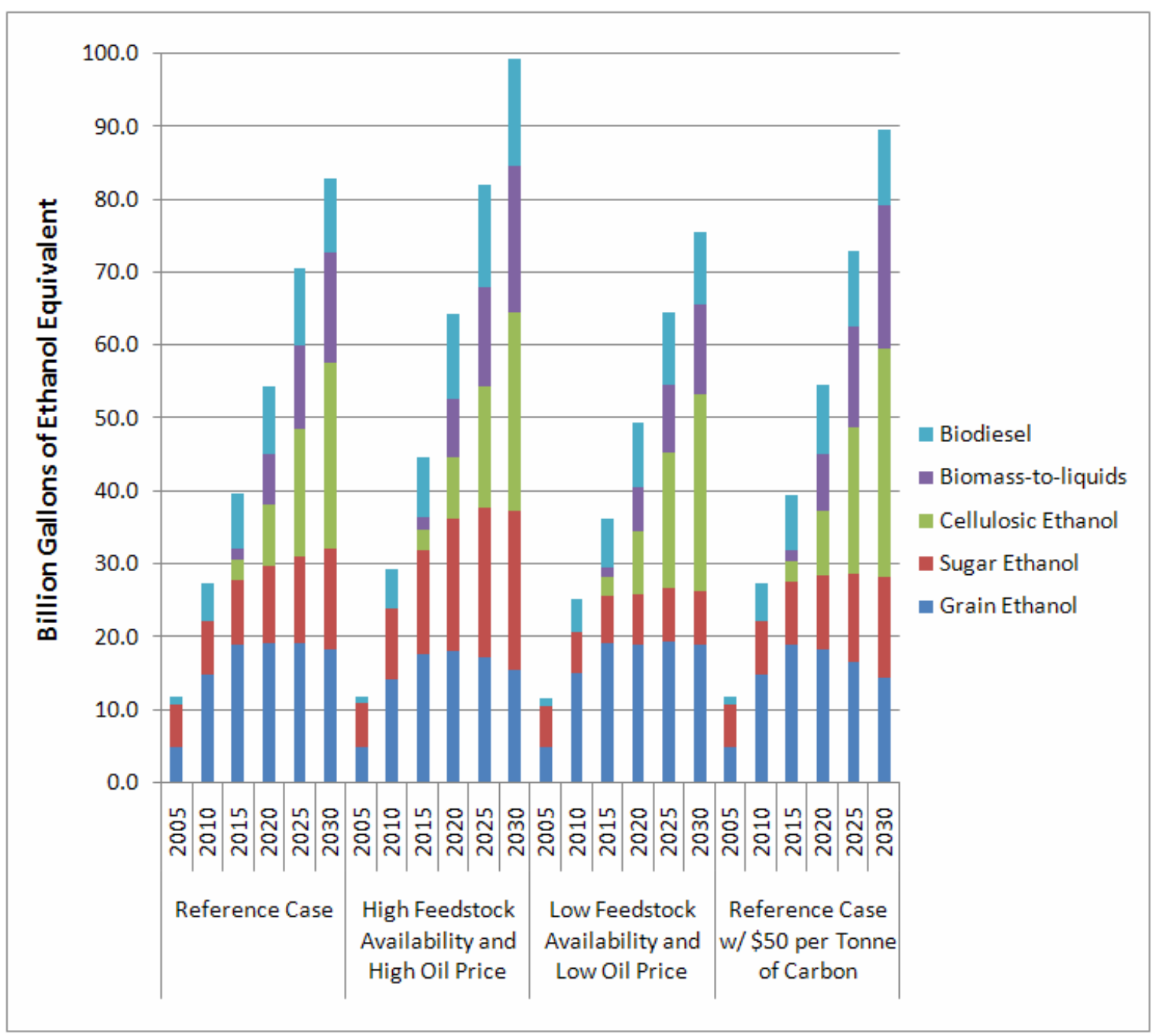

Figure 7: World biofuels supply by type for reference technology cases

Initially, the majority of biofuels are produced from food crops. In the longer run, growth rates for grain and sugar ethanol slow down. This is mainly due to limits on feedstock availability, but also because the U.S. RFS does not mandate higher volumes for these fuels. Cellulosic biofuels quickly gain significant market share after they are introduced on a commercial scale in 2012. In the reference case cellulosic biofuels have a market share of $28 \%$ in 2020 and this grows to almost 50\% by 2030 . 


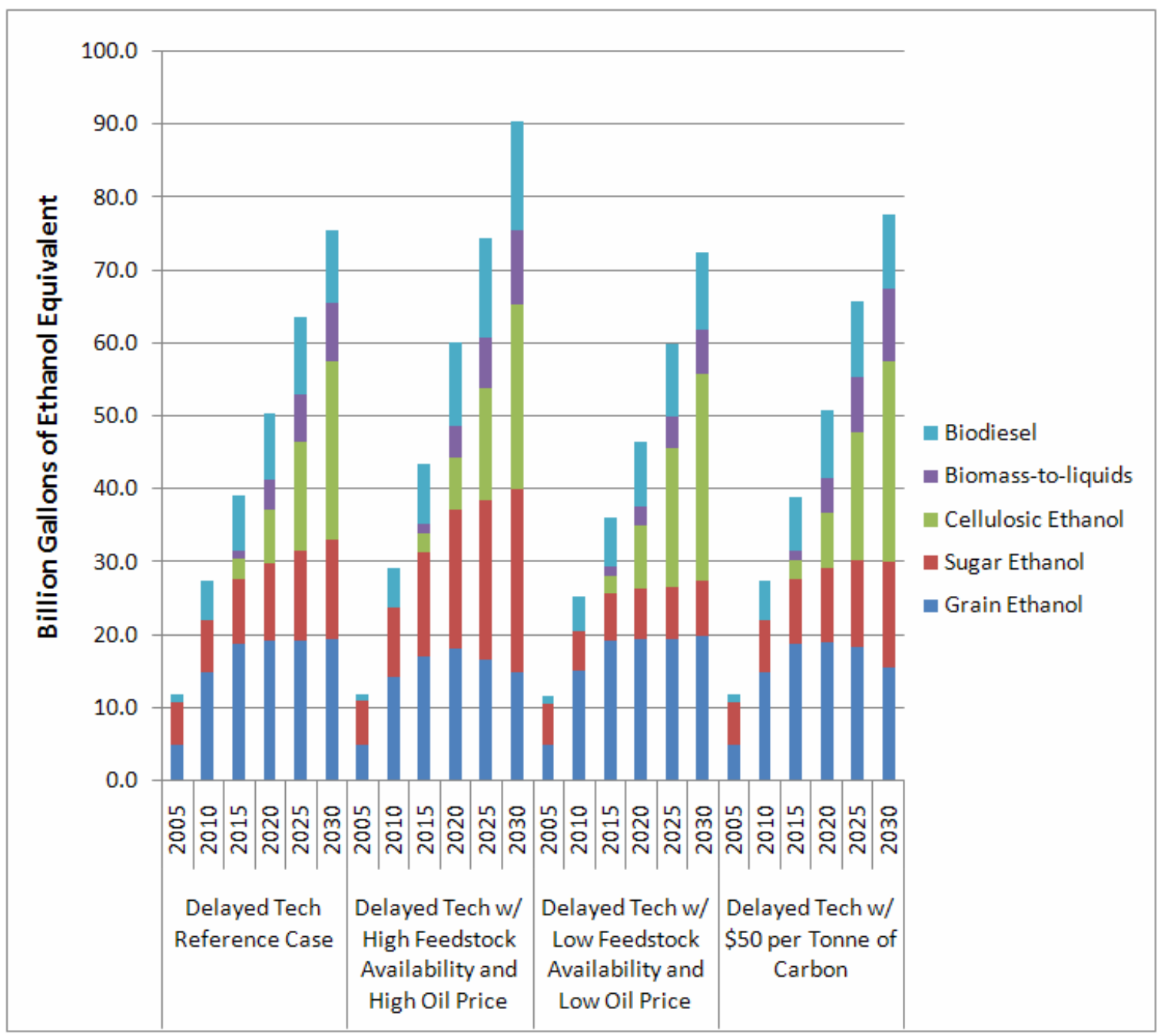

Figure 8: World biofuels supply by type for delayed technology cases

The feedstock availability mainly impacts sugar ethanol and biodiesel production, as can be seen by comparing the high and low feedstock cases to the reference case. This is because grain ethanol is not competitive outside the U.S. and cellulosic expansion is not constrained by the overall resource availability under any scenario. ${ }^{5}$ Again it is worth noting that the scenarios exploring sensitivity to feedstock availability only adjust the supply curves for the countries covered in the feedstock assessment part of this study. Worldwide sugar ethanol production in the reference case is about 11 billion gallons in 2020, while in the high feedstock growth case this rises to 18 billion gallons and in the low growth case it is as low as 7 billion gallons. The majority of sugar cane ethanol is produced in Brazil, which maintains a market share of more than $80 \%$ in all years for all scenarios. There is some feedback to grain ethanol production, as it is displaced when more cheap sugar ethanol is supplied to world markets.

\footnotetext{
${ }^{5}$ Here feedstock availability refers to the physical presence of biomass resources in the region. Availability at conversion plants depends on the ability to harvest, collect and transport the feedstocks and this is treated as an infrastructure constraint in this study.
} 
Cellulosic biofuel production is more dependent on technology cost and limits to infrastructure roll-out than feedstock availability, as can be seen by comparing Figure 7 and Figure 8. Cellulosic ethanol production remains virtually unchanged by the shifts to high and low feedstock availability. It is in fact slightly higher for the low feedstock cases because of reduced competition from sugar ethanol. However, by comparing with the corresponding cases for delayed technology assumptions instead, a clear change can be seen. World biofuel production is more than 35\% higher with reference case technology assumptions as compared to the delayed technology case. This is an indication that feedstock availability is not the constraining factor for cellulosic ethanol production, but rather infrastructure constraints and competition from cheaper sources of biofuel.

Another reason for the lack of response to changes in feedstock supply is the fact that a large share of the overall cellulosic biofuel potential is in countries not covered by the feedstock analysis part of this study. Thus, the cumulative global shift in supply curves is smaller between scenarios for cellulosic feedstocks than for sugar cane or oil seeds, where a much larger share of total supply is from the studied countries (see Table 6). This is partly because cellulosic feedstocks are more evenly distributed geographically and because the country screening process mainly focused on potential for first generation biofuels (see Section $2.2 \quad$ Feedstock Supply Curves).

Table 6: Share of world (non-U.S.) production of crop feedstocks represented by the assessed countries [8]

\begin{tabular}{|l|l|c|c|}
\hline Feedstock & Countries Assessed in Present Study & $\begin{array}{c}\text { 2006 Output } \\
\text { (mmt) }\end{array}$ & Share* \\
\hline Sugarcane & $\begin{array}{l}\text { Argentina, Brazil, China, Colombia, India, } \\
\text { Mexico, CBI }\end{array}$ & 999 & $73 \%$ \\
\hline Soybeans & Argentina, Brazil, China & 108 & $81 \%$ \\
\hline Corn & Argentina, Brazil, China, Canada Mexico & 234 & $55 \%$ \\
\hline Wheat & Argentina, Canada, China & 146 & $27 \%$ \\
\hline Palm Oil & Colombia, CBI & 1.3 & $3 \%$ \\
\hline
\end{tabular}

The introduction of carbon prices raises overall biofuel production. Total biofuel supply is 7 billion gallons higher in 2030 after the introduction of a carbon price for the reference technology set. The increase in cellulosic biofuel supply is higher at 11 billion gallons, while grain ethanol production drops about 4 billion gallons. There is also a small increase in sugar ethanol production.

This result is perhaps counterintuitive since the resulting price signal from a carbon value is about the same for sugar ethanol and cellulosic biofuels, and because grain ethanol should still receive a positive price signal. The reason for the low response in sugar ethanol production has to do with the shape of the supply curves. Sugar is the cheapest source of ethanol and supply is mainly constrained by feedstock availability. 
Figure 9 shows the sugar cane supply curve for Brazil in 2030 in million metric tonnes produced at a given price per tonne. The carbon price results in a significant increase in the feedstock market price from $\$ 15.7$ to $\$ 18.1$ per tonne for the reference case. However, since most of the ethanol is economic at prices below $\$ 15.7$ we are at the inelastic part of the supply curve where response to price signals is relatively small. As a result the increase in production is quite limited from 1,020 to 1,067 million tonnes. The same argument explains why other price signals, such as higher oil prices, also have a limited impact on sugar ethanol production and why an outward shift in the supply curve (the high feedstock growth case) has a much larger impact.

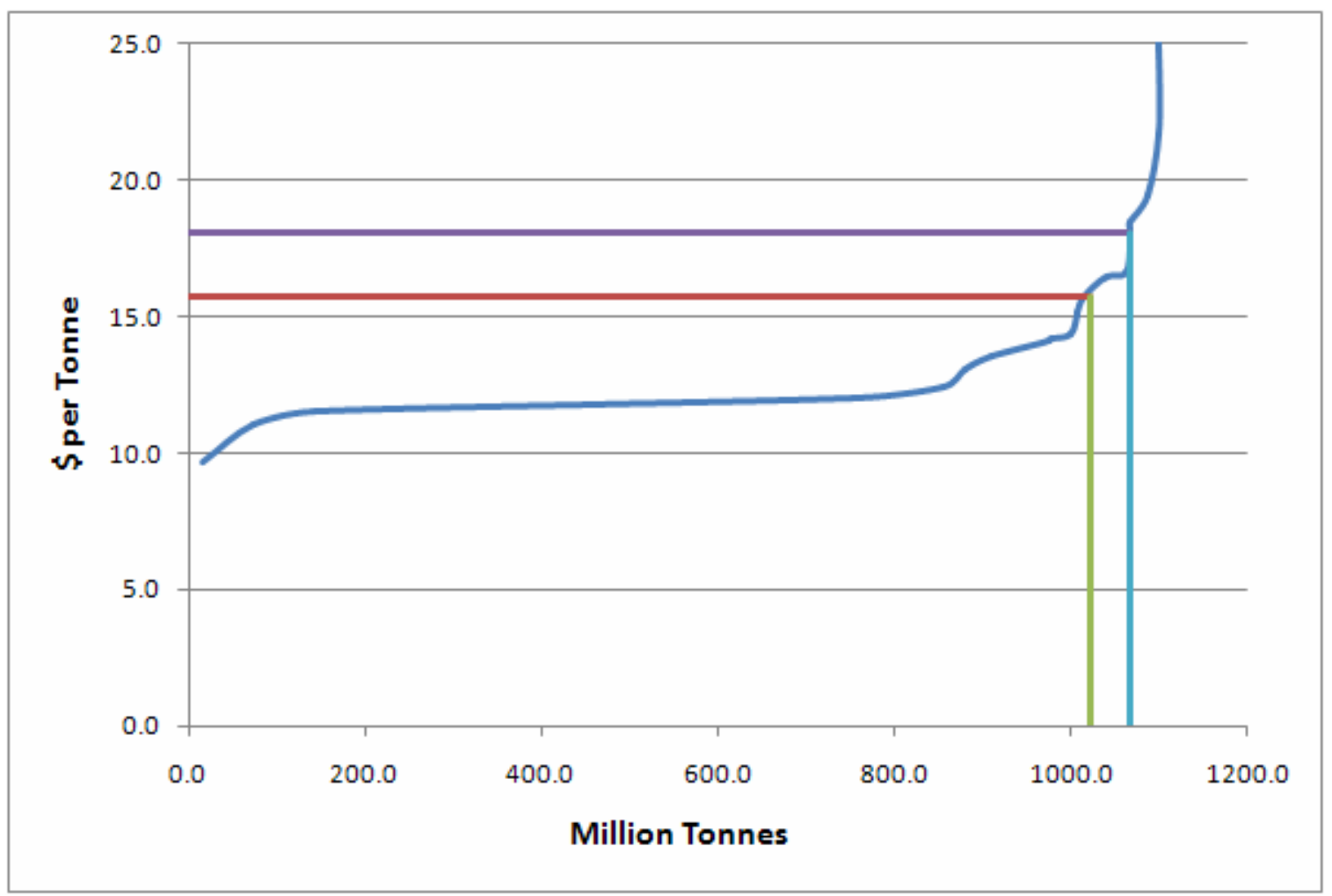

Figure 9: Effect of carbon price on Brazilian sugarcane supply in 2030

The reason why grain ethanol production is going down in spite of stronger price signals has to do with the limits on overall ethanol sales. U.S. and Europe, the biggest markets, are driven by mandates. The carbon price is not sufficient to encourage demand beyond the mandated levels and there is thus competition in a market of a fixed size. Since the economics of cellulosic biofuels relative to grain ethanol improves, the former takes market share from the latter.

In the no learning investment cases overall biofuel production is lower. For the reference case assumptions it is down 4 billion gallons in 2020 and more than 7 billion gallons in 2030 compared to the scenario with learning investment. The drop is entirely in cellulosic biofuels, and is partially offset by a small increase in production of other biofuels. 
World biofuels production is dominated by North (primarily U.S.) and South (primarily Brazil) America, with significant contribution from Europe and Asia, as can be seen in Figure 10 and Figure 11. In the reference case, the U.S. share of total biofuel production grows to over $45 \%$ in 2015 , but then drops gradually to about $35 \%$ in 2030 . In the high feedstock case the share drops to $25 \%$ in the same year. Delayed technology development does not significantly alter the market shares for the different regions.

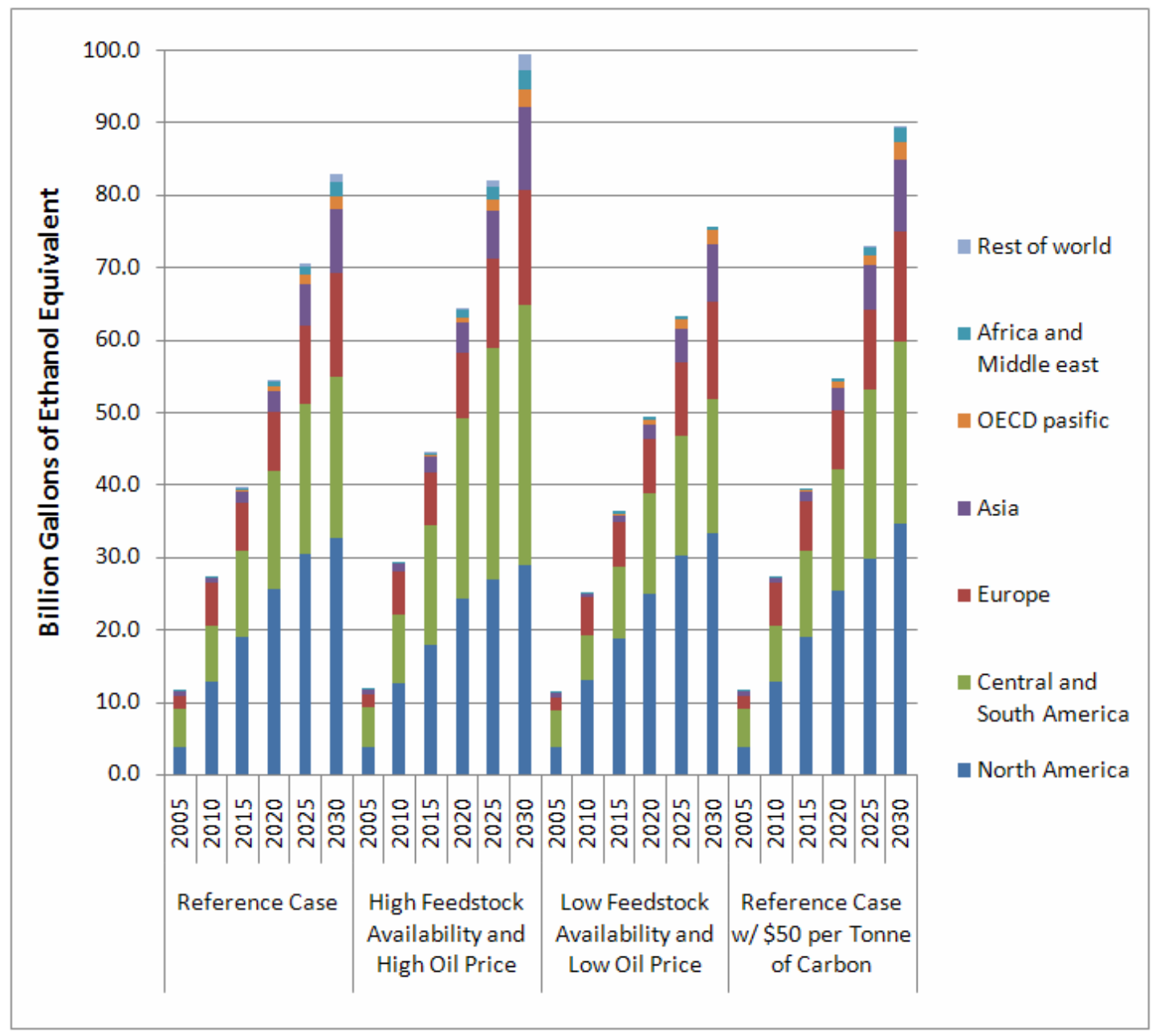

Figure 10: World biofuel supply by producing region for the reference technology cases 


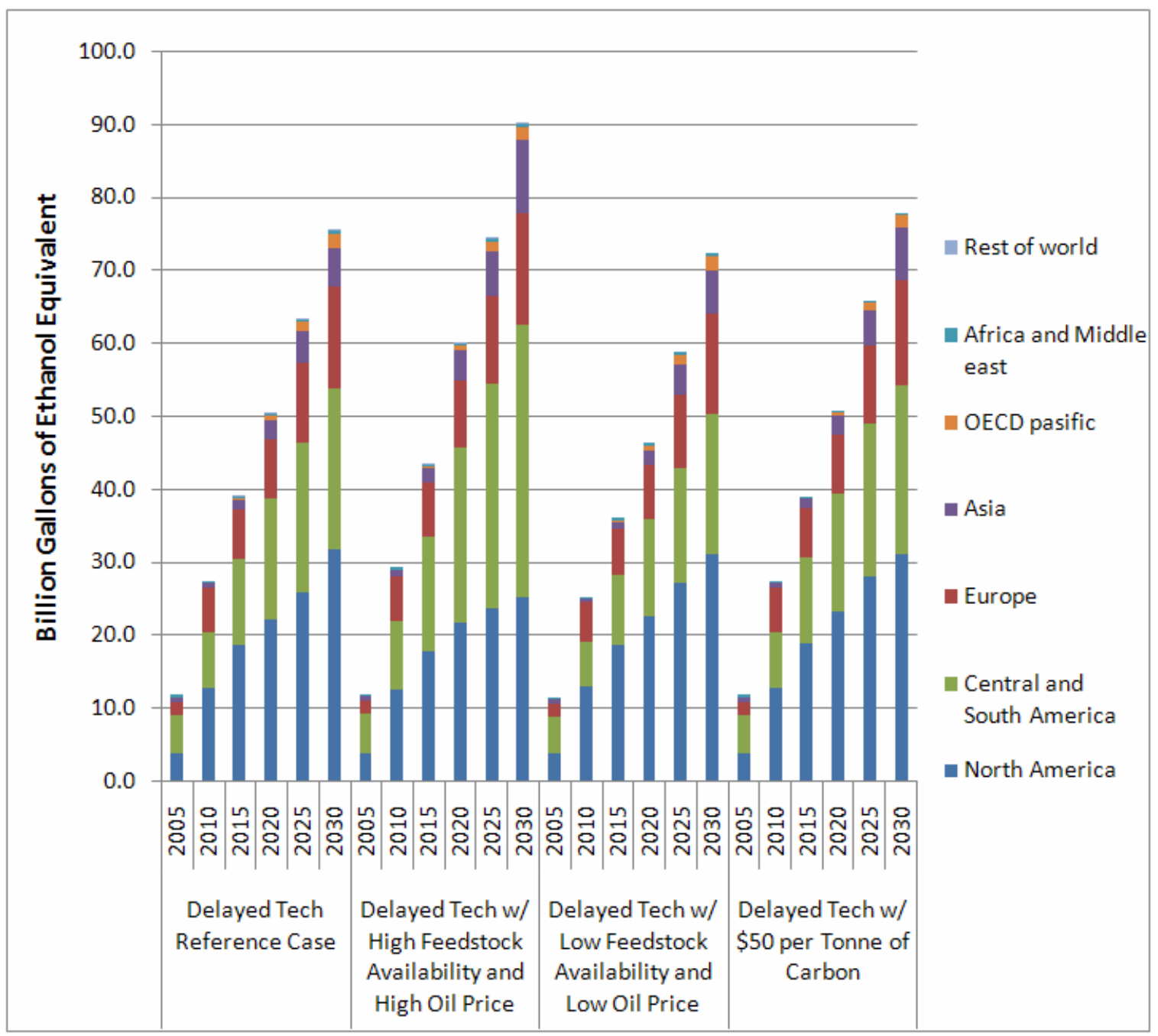

Figure 11: World biofuel supply by producing region for the delayed technology cases 


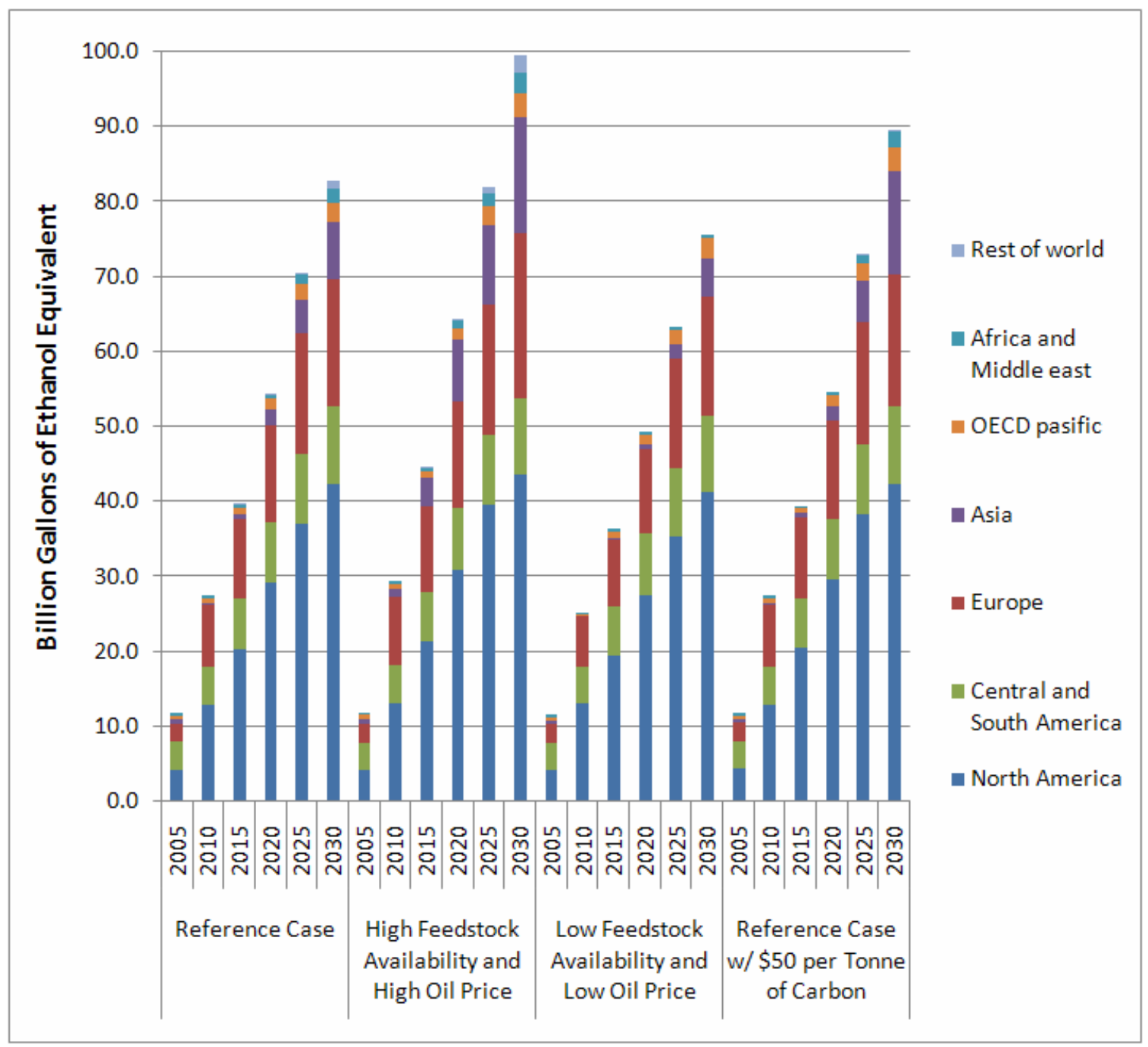

Figure 12: World biofuel demand by region for the reference technology cases

Figure 13 show biofuel demand by region. The U.S. is the biggest market for biofuels and it attracts around $50 \%$ of total supply for all years in the reference case. Europe and Brazil also attract large quantities of biofuels to satisfy their fuel standards. These three markets all have biofuel mandates, so overall demand changes little between scenarios, although there are some differences in U.S. demand since waivers can be purchased. The majority of the variation between scenarios is thus occurring in regions that have incentives, but not mandates, such as Asia and Central and South America (other than Brazil).

By comparing the supply side charts (Figure 10 and Figure 11) with the demand side it is possible to develop a view of trade flows. A total of 10 billion gallons of biofuels is traded among regions in 2020 and this grows to 15 billion gallons by 2030. For the high feedstock growth case, these trade flows are significantly higher at 15 and 26 billion gallons, respectively. 
Most of the traded ethanol originates in Central and South America, which exports 8 billion gallons of biofuels in 2020 and 12 billion gallons in 2030. The U.S. and Europe are the major importers.

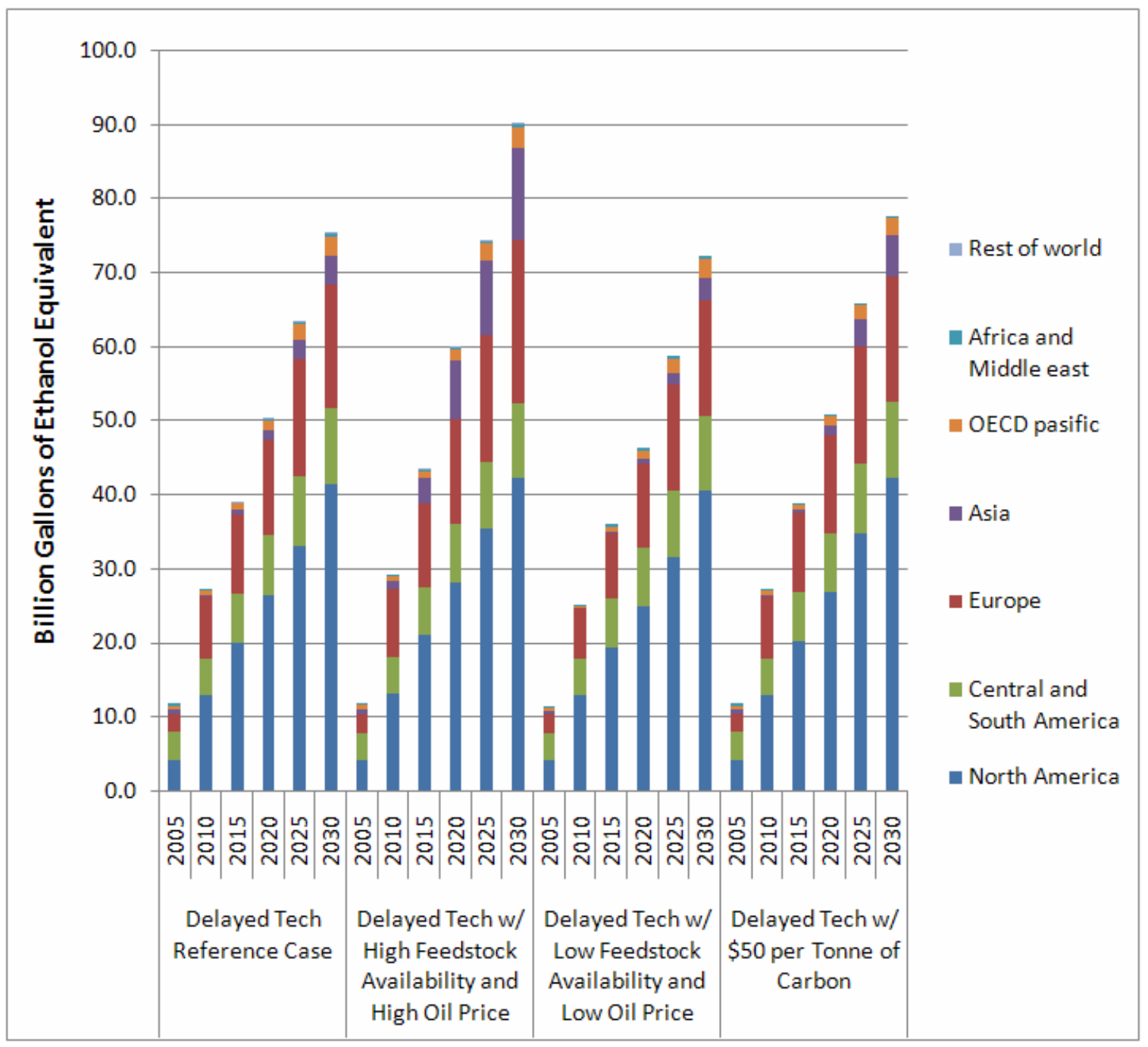

Figure 13: World biofuel demand by region for the delayed technology cases

The delayed technology assumptions do not have a major impact on global trade flows because the exporting nations tend to drop domestic consumption in order to keep regions with mandates supplied. Since fuel supply is scarcer, prices go up, lowering demand in regions without mandates, while demand in regions with mandates stay roughly the same.

Figure 14 and Figure 15 show biofuel supply to the U.S. This supply is not sufficient to meet the cellulosic biofuel mandates in the early years of the RFS. As a result waivers are purchased to cover the shortfall. Total biofuel supply in the reference case is 27.8 billion gallons in 2020 of ethanol equivalent of which about 4.3 billion gallons are imported. A little less than 20 billion gallons of this is ethanol, while the remainder is BTL fuels 
(6.1 billion gallons) and biodiesel (1.9 billion gallons). Since the total RFS requirement for this year is 30 Billion gallons the waiver requirement is 2.2 billion gallons.

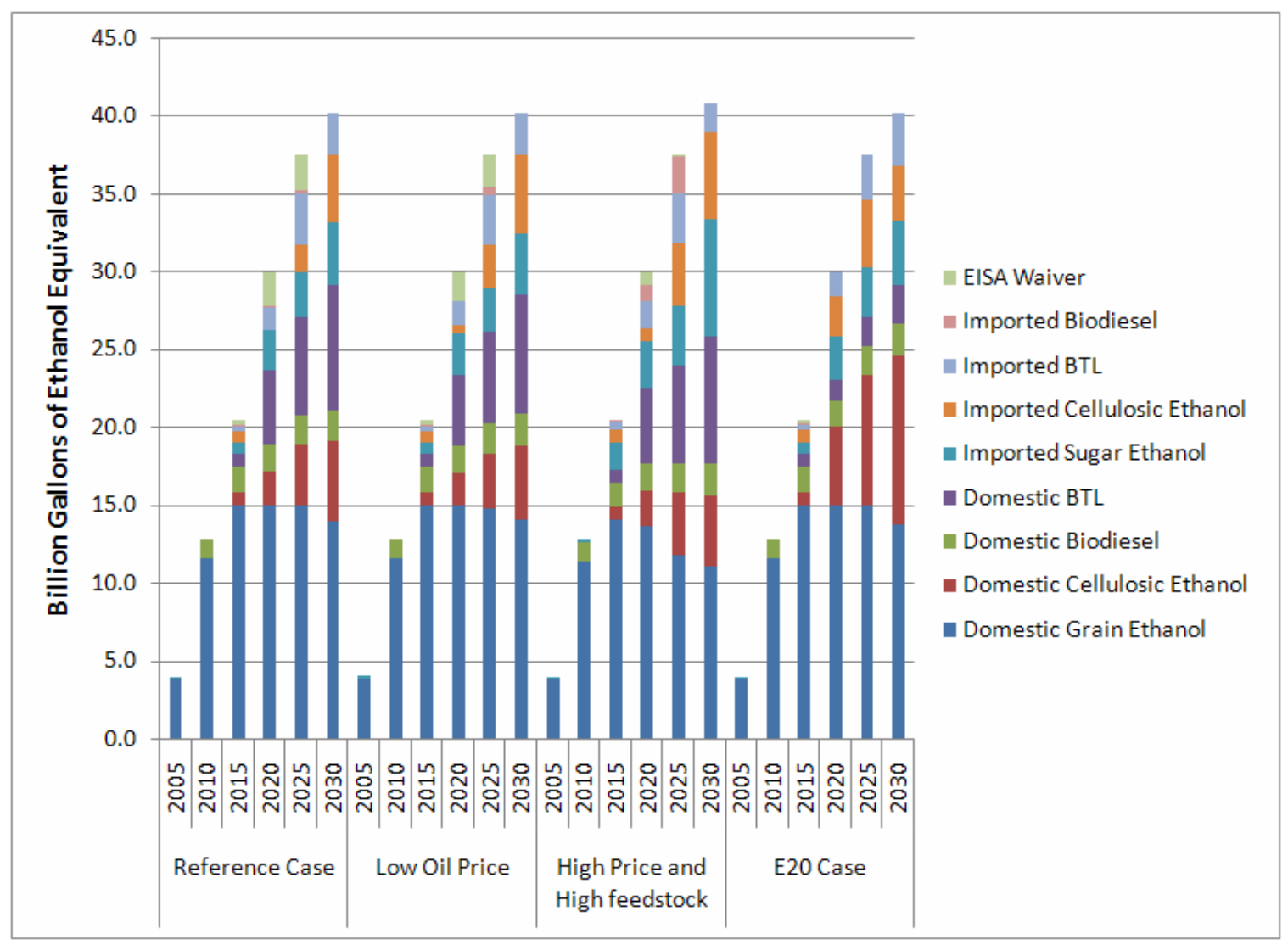

Figure 14: US biofuel supply for the reference technology cases

Allowing E20 greatly increases the share of ethanol and displaces BTL. In the reference case ethanol distribution is restricted by infrastructure constraints and the inability to deliver sufficient volumes of ethanol as E85. This brings BTL into the market to fill the RFS gap. Distributing and selling ethanol as E20 alleviates these restrictions allowing more ethanol to penetrate the market. In fact, the E20 case is the only scenario where the mandate is met in 2020 and as a consequence some BTL is displaced. This is discussed further in the "biofuel market dynamics" section below.

In scenarios where there is abundant sugar ethanol available in the world market, significant volumes of U.S. domestic grain ethanol production are displaced. In these cases the advanced renewable fuels mandate is exceeded and the additional sugar ethanol demand is not driven by the RFS but by price. Extension of the ethanol blenders' tax credit or other domestic subsidy would protect domestic producers from this foreign competition, but come at a significant cost to the treasury.

The biodiesel requirement of the RFS is met in all scenarios in all time periods and supply also increases beyond the mandated volumes to help meet the overall advanced 
biofuels target. This happens in scenarios where sugar ethanol is in short supply and higher prices justify larger imports of biodiesel.

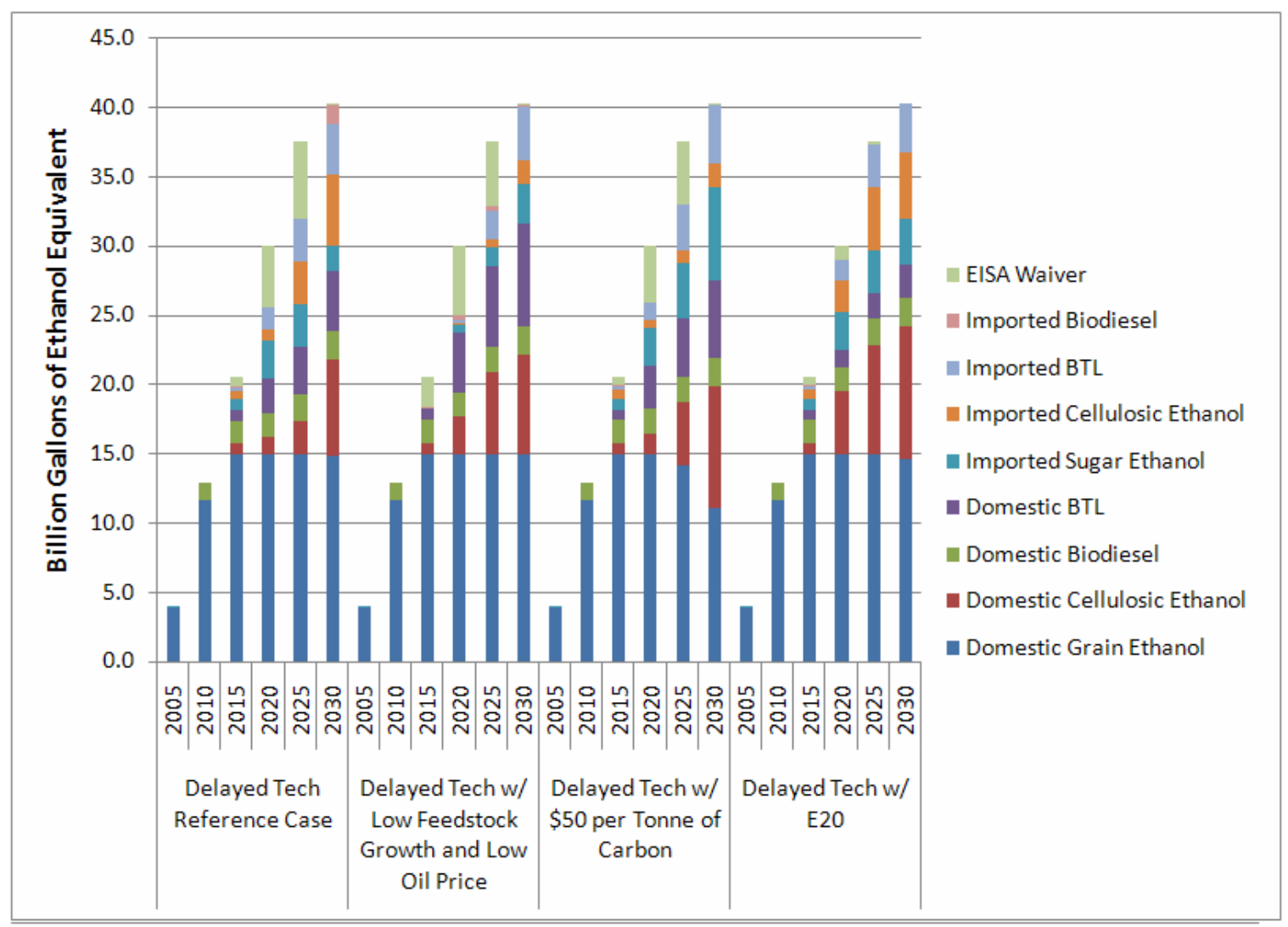

Figure 15: US biofuel supply for the delayed technology cases

The delayed technology cases show a significant reduction in cellulosic biofuel supply, with the majority of the drop in domestic BTL production. In the reference case local production of BTL fuels drop from 4.7 to 2.5 billion gallons, while imports drop only marginally. Additional waivers are needed to cover the reduction in overall supply and the requirement under the delayed tech reference is 4.4 billion gallons.

\subsection{Biofuel Market Dynamics}

The market adoption of biofuels worldwide is driven by a combination of mandates and economic incentives. If a mandate is in place the price will rise until supply is sufficient to meet it. Assuming that the targets set are achievable, there is no volumetric risk in this case, but there can be considerable risks associated with the cost of compliance. This cost risk can be mitigated through some form of relief-valve mechanism, but this would reintroduce volume risk. In a market where biofuels demand is driven by economic incentives (e.g., tax breaks, direct subsidies) the price of these fuels are determined by the price premium they can realize over gasoline or diesel due to the subsidy regimes. The cost risks are therefore reduced, but there is significant volume risk. Another way of 
describing this would be to say that under a mandate regime, prices adjust to volumes, while under a subsidy regime, volumes adjust to prices. In a region where both types of incentives are present the volumes will be determined by the mandates, while the main impact of subsidies will be to reduce prices for the consumer or increase profits for producers.

In a global market place, where some regions have mandates while others only have subsidies, these dynamics affect how biofuels are allocated among markets. Regions with mandates are willing to take the price risks and therefore tend to carve out a fixed share for themselves, regardless of what happens elsewhere ${ }^{6}$. These markets would therefore tend to be served "first". The regions that rely purely on policy induced price incentives will compete for whatever is available after all mandates have been met. Producers will sell their product in the market where they realize the highest net-back. The ethanol will thus tend to flow to the markets where price signals are the strongest, net of transport costs and tariffs. As long as there are unmet mandates this will limit the amount of ethanol going to countries without these fixed volumetric targets.

The EISA RFS creates a market place where ethanol is no longer a single commodity, but can be separated into several subsets whose value is dependent on the feedstock from which it was produced. The ethanol itself will probably trade at one price, but the associated credits will achieve different prices in the market place and thus change the total value of the ethanol. There are essentially four different types of ethanol under this regulation; ethanol that qualifies as renewable fuel (e.g., grain ethanol), ethanol that qualifies as advanced renewable fuel (e.g., sugar ethanol), ethanol that qualifies as cellulosic biofuels (e.g,. cellulosic ethanol) and ethanol that doesn't qualify for any of the credits. It is thus perfectly possible, and in fact highly probable, that the market value (price plus credit value) for each of the types of ethanol will be different. Credit prices are generally determined by the market and will reach the level required to ensure sufficient supply, but for cellulosic ethanol (and other qualifying cellulosic biofuels) the credit value is equal to the waiver price as long as the mandate remains unmet.

The general dynamics of the biofuel markets as represented in this modeling exercise can best be understood by separating it into two stages; before the U.S. mandate is met, and after the mandate is met. Before the mandate is met all production is complementary in the sense that increased production in one part of the world does not come at the expense of production in another. There is little competition among ethanol producers, and ethanol prices are set by the price premium it can realize over gasoline due to the subsidy regimes. Any producer able to deliver ethanol to the market at this price will therefore do so.

After the mandate in the U.S. has been met however, biofuel markets become a lot more competitive and prices fall. At this stage (sometime between 2020 and 2030 in most scenarios), most of the subsidies in the rest of the world has been phased out. Producers

\footnotetext{
${ }^{6}$ This is how the ETP model behaves; strictly enforcing mandates. In the real world lawmakers and regulators would likely issue waiver, adjust the regulations or otherwise accommodate markets if the mandates are deemed to have unacceptable adverse impacts.
} 
are in more direct competition, and increased production in one region leads to decreased production elsewhere. This can for instance be seen by comparing the high feedstock case to the reference case and see how domestic production changes little in the early years, but significant amounts are displaced by cheap imports in 2025 and 2030.

Another way to view this situation is to see the early years as a "sellers market" with high prices, where importers, unable to meet domestic biofuel targets, are competing for the fuel available on the open market. In the latter years the market is more balanced, prices fall and producers have to compete for market share.

Meeting the biofuel targets not only requires the production of sufficient volumes of fuel, but also that the infrastructure to bring this fuel to customers is in place, and that these consumers actually purchase it. The biofuels mandate in 2022 is 36 billion gallons of ethanol, roughly equivalent to 24 billion gallons of gasoline. In 2022 our projected gasoline demand is 139 billion gallons, so it is not feasible to meet the mandate by selling E10 alone. To sell more ethanol, retailers will be forced to market it as E85 (E85 sales in the reference case are shown in Figure 16). However, sale of E85 requires a dedicated infrastructure.

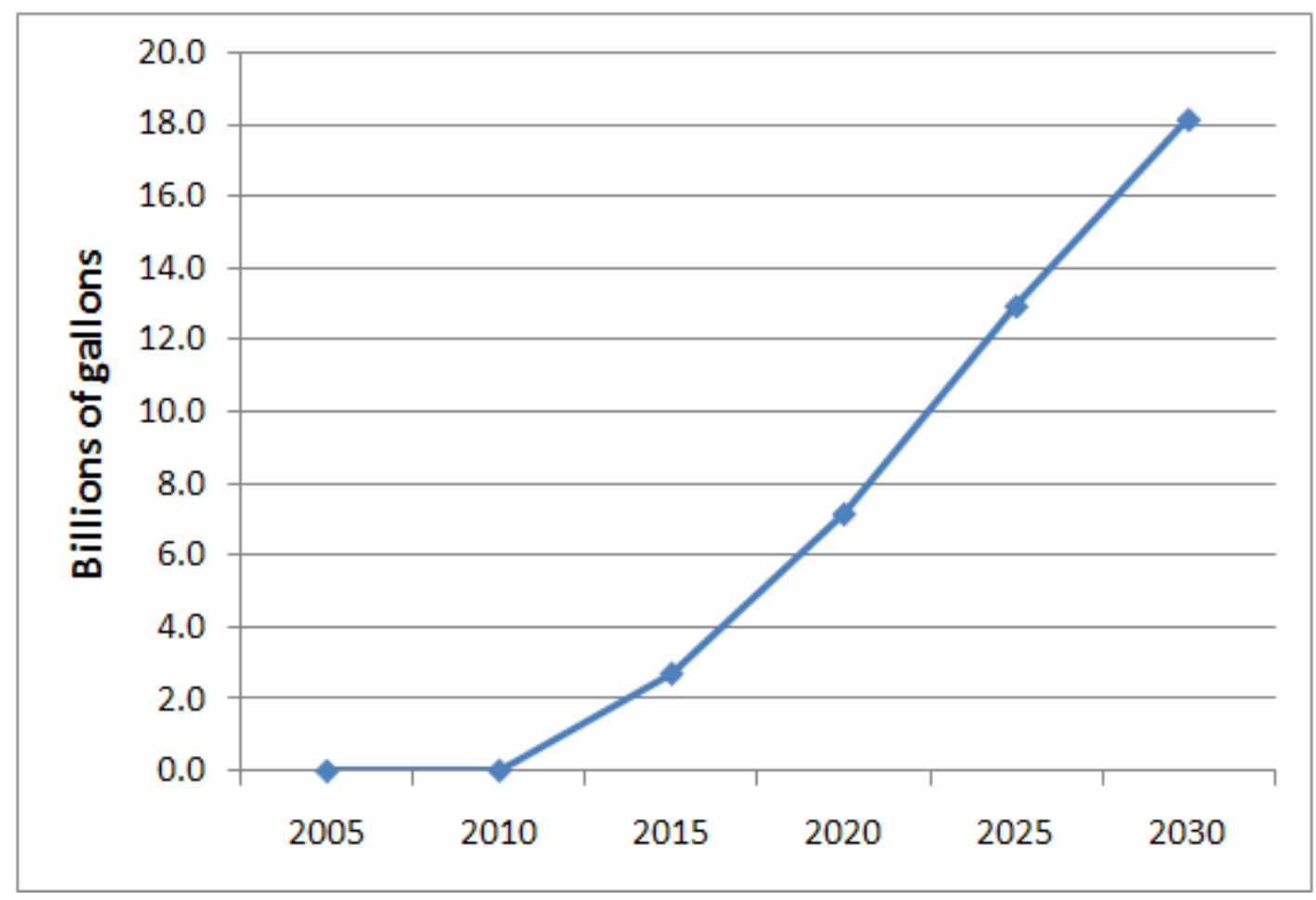

Figure 16: E85 sales in the reference case

Since the responsibility for meeting the mandate under the legislation lies with the "refineries, blenders, distributors, and importers," it falls on these entities to ensure that consumers actually purchase the fuel. This poses both infrastructure and marketing challenges. In order to sell the fuel to customers, there needs to be an underlying infrastructure to deliver it to them at fuelling stations. Not only is an expansion of the 
distribution and fuelling station infrastructure needed to deliver sufficient volumes of E85 to customers, but there also must be enough flex-fuel vehicles on the road to use it. Approximately 1200 out of almost to 170,000 fuelling stations in the U.S. sell E85 currently [14]. There are about 6.5 million flex-fuel vehicles on the road [15], but few of these actually run on E85.

Another point is that consumers not only have to drive a flex-fuel vehicle and be filling up at a station where E85 is available; they also need to be willing to buy the fuel. E85 currently trades at a premium to gasoline when adjusted for the loss in fuel efficiency due to the lower energy content for a given volume of fuel. Assuming that the bulk of consumers will buy the fuel that lets them travel at the lowest cost, this means that some form of additional incentive needs to be in place to encourage the adoption of E85. This is likely to require a cross subsidy. The ETP model optimizes across the value chain and thus assumes that the constraint (RFS) placed upon the distributors is efficiently translated into prices at the pump. The problems associated with how to structure the incentives has thus been assumed away.

Instead of supplying E85, marketers can meet the mandates by distributing FischerTropsch liquids derived from renewable biomass. These BTL fuels can be distributed in together with petroleum-based fuels in the existing infrastructure and can be used by the current fleet of light duty vehicles and trucks. Consequently, BTL fuels do not suffer from the same infrastructure constraints as E85. Based on the technology assumptions used for this study, cellulosic ethanol production is cheaper per unit of energy than Fischer-Tropsch diesel for a given feedstock price. The suppliers (as represented in the model) will therefore prefer to meet the mandates with ethanol supply as long as it can be distributed at reasonably low costs. As the sales of E85 increase, the cost of distribution goes up as the ability to expand the infrastructure and sell E85 is limited. Above a certain volume of ethanol sales, the production cost advantage is negated by the distribution cost escalation, and additional biofuel supply will then tend to be in the form of BTL distillates.

This effect can be seen by comparing the reference case, the delayed technology case and the credit extension case to the E20 case (see Figure 17). There is little variation in overall ethanol sales in the three cases with E10 as the maximum blend. Additional incentives, in the form of more rapid technology development or blenders' tax credit, have little impact. Since there are no substitutes for grain and sugar ethanol for the renewable and advanced renewable biofuel targets, the ethanol distribution constraints function as a cap on the amount of cellulosic ethanol that can be sold. Additional incentives for cellulosic biofuels will thus tend to lead to increased BTL supply. By allowing E20 to be sold to consumers, the infrastructure constraints are significantly reduced and more ethanol can be supplied to markets. This not only leads to an increase in cellulosic ethanol, but also a decrease in BTL fuels. 
Reference Case

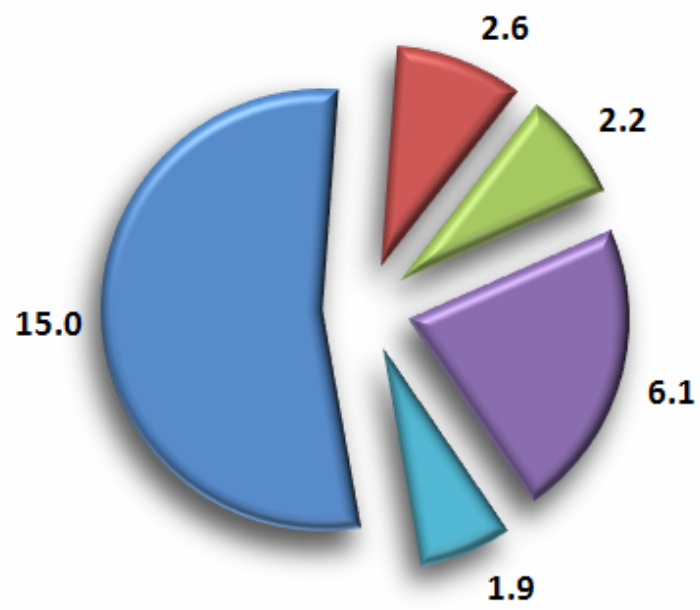

E20 Case

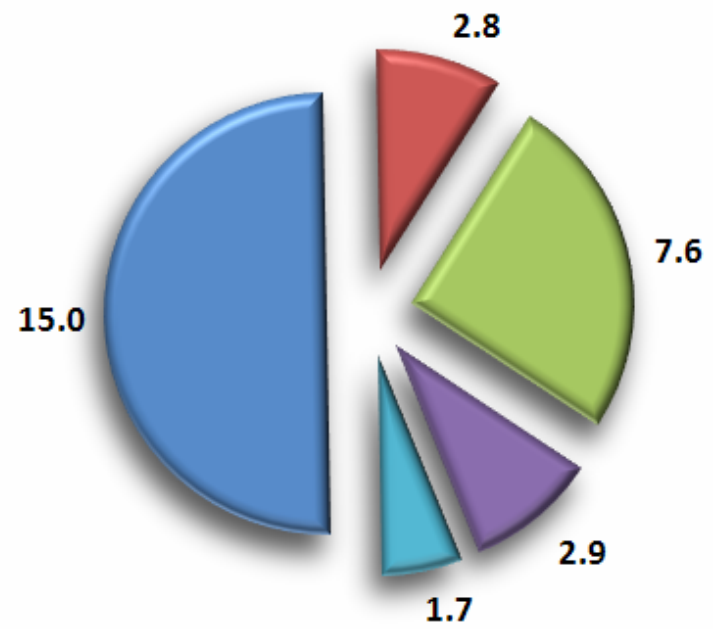

1.7
Delayed Tech Reference Case

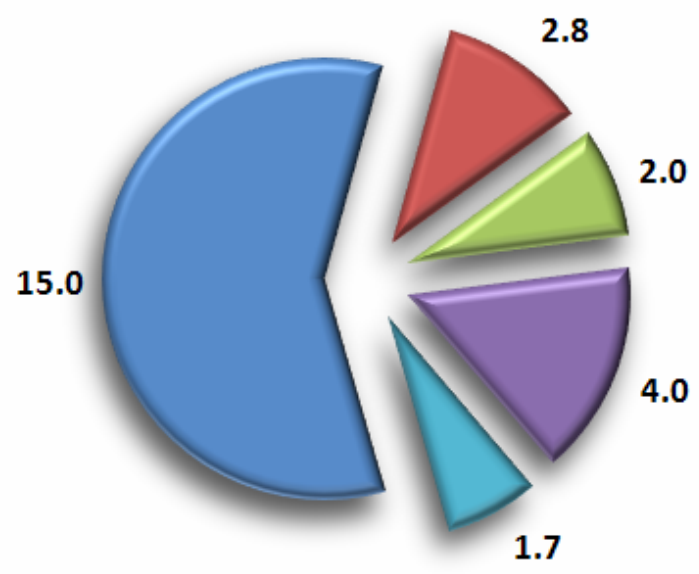

\section{Reference w/ Credit and} Tariff Extension

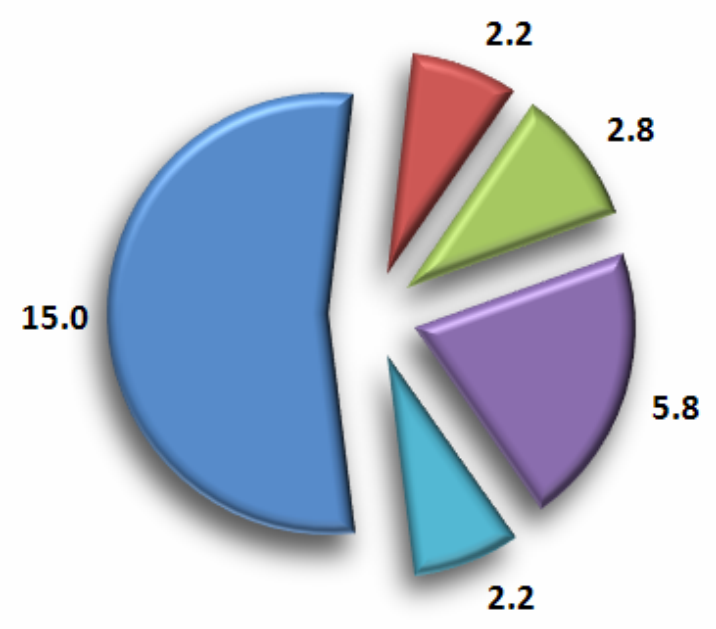

BTL $\mathbf{B}$ Biodiesel

Figure 17: Biofuels sales with E10 vs. E20

Because the buy-out from the cellulosic biofuels mandate adjusts to oil price and there are no relief-valve mechanisms for the other mandated volumes, U.S. biofuel demand is not very responsive changes in the oil price. The law removes the risk of falling oil prices to suppliers since the credit price will adjust to keep price incentives at levels that are thought to be sufficient to ensure supply. Conversely, credit prices are reduced in the event of rising oil prices to make sure that the industry is not taxed needlessly in an environment where price incentives for biofuel supply are already adequate. The impact of this is that price signal for US renewable fuel suppliers does not change much from the baseline to the high and low oil price scenarios. 
While biofuel demand in the U.S. is relatively independent of oil prices, this is not the case in countries that use price incentives rather than mandates. In these regions biofuel prices are determined by premium the subsidy regime allows them to achieve over petroleum fuels. Lower oil prices therefore mean lower biofuel prices, which leads to reduced incentives to supply these markets. With lower prices and less biofuel going to international markets the cost of importing biofuels into the U.S. drops and volumes go up. Conversely, higher oil prices mean higher demand internationally, more competition for supply and therefore higher prices and lower volumes imported into the U.S. This dynamic has two significant impacts. The first is the perhaps counterintuitive outcome that increasing oil prices lead to reduced biofuel demand in the U.S. This effect is marginal, but might have been more pronounced if it wasn't for the constraints on ethanol sales described earlier. The second is that it changes the balance of imports and domestic production.

Figure 18 shows that higher oil prices lead to reduced imports and thereby higher domestic production. The reverse would be true for falling oil prices. This relationship is consistent with the price impacts described above. This pattern changes if wholesale gasoline prices rise above $\$ 2.75$, at which point the credit price decouples from the gasoline price and is held constant at $\$ 0.25$. Oil prices above this level will increase biofuel demand in the US.

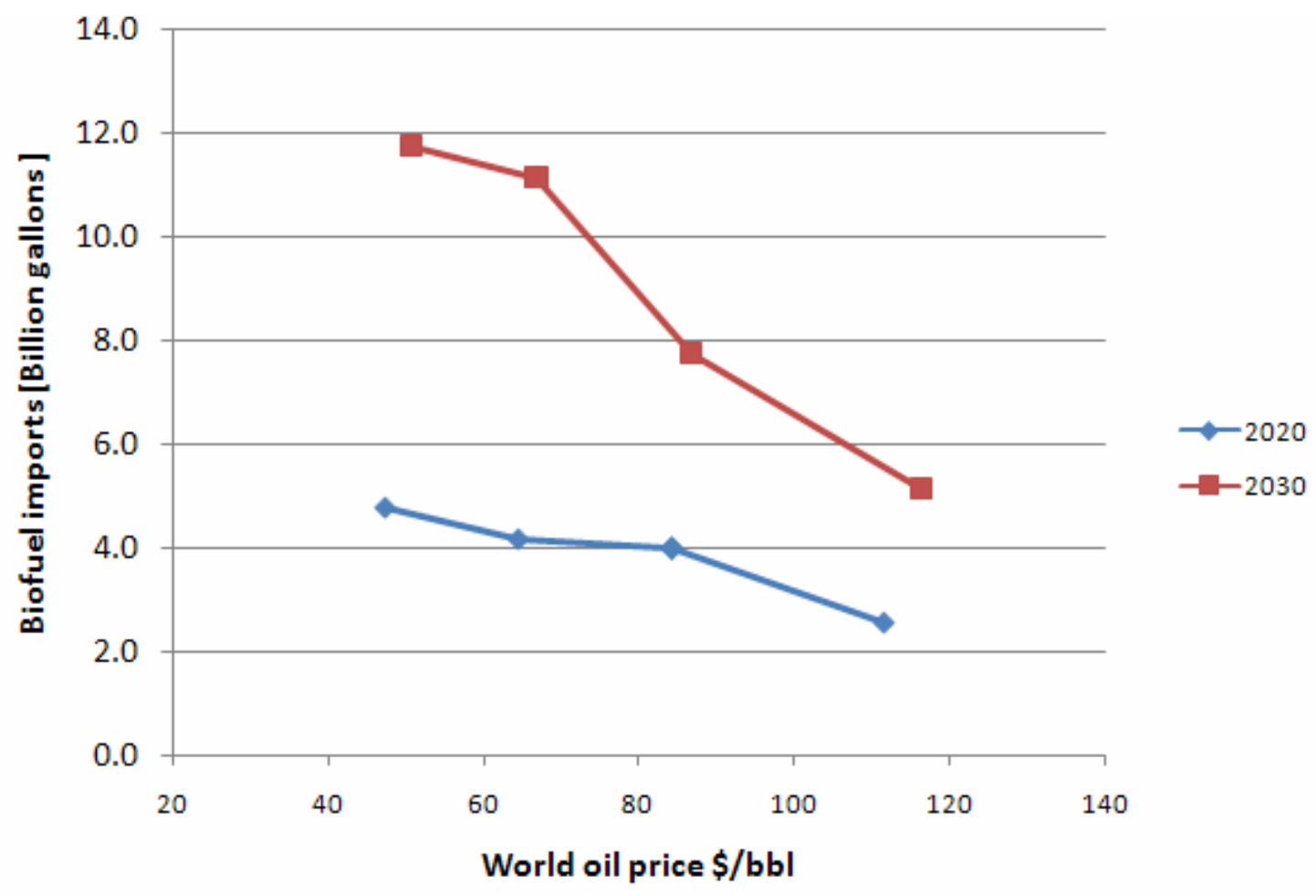

Figure 18: Oil price impact on biofuel imports 
Critical to these outcomes is the assumption that producers believe that U.S. lawmakers will stick to this arrangement even if oil prices stay low for an extended period of time. The growth rates in biofuel production seen in these scenarios require strong confidence among investors that biofuel markets will expand and remain strong. Any doubt in the willingness of the U.S. and other governments to stay the course with their biofuel policies will likely lead to under-investment.

The RFS in itself is such a strong policy that additional incentives such as growers' payments or an extension of the ethanol blenders' tax credit does little to increase overall biofuels supply. The ability to develop the cellulosic resource base, build cellulosic biofuel production facilities, and construction of ethanol distribution infrastructure fast enough is the main constraint rather than the underlying economics. The exact limitation on how quickly the infrastructure can be rolled out is uncertain, and this is an issue that needs attention. The main impact of the credit extension is thus to bring in more domestic production at the expense of imports. Towards the end of the period, when growth rates are lower and infrastructure rollout is less of a concern, it also tends to bring in more cellulosic ethanol in place of BTL fuels. Furthermore, as more subsidies are introduced in the same scenario diminishing returns to the cost of the policy will be seen.

The relative strength of the carbon value policy as a price signal compared to other policies change over time. The blenders' tax credit and growers payment are both nominal values (i.e. they stay at a given dollar price and are not adjusted for inflation) and therefore decrease in real terms over time. However, the carbon price increases over time and is also assumed to be in real dollars (i.e., inflation adjusted). This means that this policy strengthens over time.

Countries in the NAFTA trade-zone such as Mexico and the Caribbean nations currently have preferential access to U.S. markets through import waivers. Producers in these countries can realize higher net-backs to the U.S than other exporting regions do for a given domestic ethanol price. The favored market for ethanol from these countries is therefore the U.S. and they tend to be the "first" exporters serving U.S. importers. An interesting consequence of this is that extending the current tariff and blenders' tax credit policies will lead to an increase in U.S. imports from these countries. This is because the blenders' tax credit will serve as an additional incentive to producers, while the tariff normally intended to cancel out the benefits of the tax credit to foreign producers does not apply to imports from NAFTA member countries.

India and China are both potentially huge markets for biofuels, but neither import much in most of the scenarios presented here. In the case of India this is mainly due to the very high import tariffs charged for imports of fuel ethanol into the country. Currently they are importing ethanol as a chemical feedstock, but we have assumed that this can be supplied by local producers when the internal infrastructure expands, which it will have to do if current ethanol blending targets are to be met. India thus produces significant amounts of ethanol for domestic consumption, but does not import any. China does not currently have any strong economic incentives for ethanol imports. Fuel tax exemptions do not provide strong encouragement because the taxes themselves are so low. Like India, China 
relies mainly on domestic production in these scenarios. Any change in policy to help meet their ambitious targets might alter this.

\subsection{Sensitivity and Uncertainty}

The model results are relatively sensitive to some of the key assumptions. One of the most important ones is the cost at which ethanol can be produced from cellulosic feedstocks. At the conversion costs reported in the NREL study [7], cellulosic ethanol is highly competitive and penetrates the market very rapidly. If this low production cost cannot be achieved, or if it can only be achieved at a later point in time, this will have dramatic effects on overall biofuel production. This uncertainty is captured in the delayed technology scenarios. There is also great uncertainty in the rate at which cellulosic ethanol can gain market share even if these low production costs are achieved. The available historical data is insufficient to determine appropriate market penetration rates for this technology at different ethanol prices.

Assumptions regarding the biofuels policies in Europe are also important, especially in early years. Europe attracts a large share of imports in early years, because of high subsidies. The assumption here is that when the E.U. goal under current policy $(10 \%$ by 2020 ) is met, the subsidy is no longer available and any additional ethanol is available for the rest of the world. This assumes that Europeans are willing to pay these subsidies even if a large share of their supply comes from abroad. It also assumes that infrastructure to accommodate these imports is put in place as well as clear customs regulation. If these conditions are not met, considerable amounts of additional biofuels ( 1 to 2 billion gallons) could be available to U.S. markets.

Another note on the European subsidy regimes is that they are generally in the form of tax exemptions. If biofuels achieve substantial market shares (which they do in all scenarios) this leads to a considerable loss of revenue. To make up for this loss, the tax exemptions either need to be phased out as they are currently in the model (although not necessarily at the same rate), or European governments will have to find an alternative source of revenue to pay for roads. There is a lot of uncertainty regarding these subsidy provisions as they generally expire after a few years and there is no great clarity as to what will replaced them. A faster phase-out than what has been assumed here is a distinct possibility which could significantly impact trade flows.

Another issue regarding the representation of Western Europe in the model is that it is modeled as a single region. While the region has a range of various fuel taxes and exemption levels it is represented as a single market with one average fuel tax and one tax exemption level in the ETP model. This means that the model exhibits some "knifeedge” behavior, in which U.S. importers are either competitive against the all European importers or not. In reality they could be competitive against some of the European markets, but not others. 
Carbon emissions related to biofuel feedstock production is also a contentious issue. Emissions from land use changes and from diesel combustion in farm equipment, water pumping and production of fertilizer all erode the carbon benefits of biofuels. Different methodologies for estimating life cycle emissions produce different results. Most appear to arrive at estimates that show modest to significant emission benefits [16-18]. The net carbon loss of land conversion needs to be considered and is one of the greatest sources of debate and uncertainty. Soils and plant biomass are the two largest biologically active stores of terrestrial carbon and hold about 2.7 times more carbon that the atmosphere. If land is cleared to allow for cultivation of food or energy crops, the carbon contained in the standing biomass and some of the carbon stored in the soil will be released to the atmosphere. A "carbon debt" is thus incurred when native ecosystems are converted to cropland. This carbon debt is the difference between the amount of carbon stored in standing biomass and soil before land clearing and that of the crop grown in its place. The actual carbon debt is thus highly dependent on the type of ecosystem that is being cleared and the crop that replaces it.

One study [19] produced estimates for this pay-back. The actual pay-back will vary between scenarios, but even under their most optimistic assumptions it was over 30 years for corn, and under their base assumptions it was well over 100 years.

Another study on the issue [20] points out that the pay-back is highly dependent on what type of land is being converted and the type of crop is grown in its place. Their results indicate that clearing tropical or peatland rainforest to grow palmoil or soybean incur large carbon debts with paybacks of several hundred years, while using abandoned or marginal cropland to grow prairie grasses incurs little to no carbon debt.

While EISA and European legislators have tried to address these issues by requiring that only previously cultivated lands qualify for the fuel mandates, this will only deal with direct impacts. Under EISA the producer has to demonstrate that the feedstock comes from land that was cleared or under cultivation before the enactment of the law, but this does not necessarily cover secondary impacts. If pastureland is converted to sugarcane or energy cropland these crops might qualify as "renewable biomass" under the act even if this leads to conversion of forestland to pasture to accommodate the displaced livestock. How secondary impacts will be captured depends on the final rulemaking by the EPA. The emission factors used for this study, from widely referenced studies, are shown in Table 7.

Table 7: Change in lifecycle GHG emissions per mile traveled by replacing gasoline or diesel with biofuels

\begin{tabular}{|l|c|}
\hline Feedstock & Change from gasoline or diesel \\
\hline Sugar & $-81 \%[18]$ \\
\hline Maize & $-25 \%[21]$ \\
\hline Cellulose & $-73 \%[21]$ \\
\hline Wheat & $-47 \%[16]$ \\
\hline Soy bean & $-78 \%[22]$ \\
\hline
\end{tabular}


Reducing or removing tariffs and other barriers to international trade of biofuels would yield several benefits. Most importantly, it will allow the most cost-effective producers to expand production and more easily market their products abroad. This should reduce the overall cost of supplying biofuels to markets. It should also limit the political pressure to maintain subsidies provided these also benefit imported biofuels. A well established international trade network would also lessen the impact of bad harvests. If supply falls short in one year, countries with mandates can shop for biofuels in international markets. Risks are thus shared among a larger number of participants.

\subsection{Conclusions}

Many countries around the world are embarking on ambitious biofuel policies through renewable fuel standards and economic incentives. As a result, the global biofuel demand is expected to grow very rapidly over the next two decades, provided policymakers stay the course with their policy goals. In the reference case presented here, total biofuel production increases more than fivefold from 11 billion gallons in 2005 to 58 billion gallons in 2030. The infrastructure challenges are daunting, will require considerable investment, and will test the innovation systems in countries with nascent biofuel industries. The ability to transfer technology and trade in biofuels is essential to meeting the future demands.

Sugar-based ethanol is the cheapest source of biofuels and its production is mainly constrained by the availability of feedstock, which can be seen by the significant increases in production for scenarios where feedstock availability is high. Grain-based ethanol is hampered by high feedstock prices and competition with food markets, which leads to declining volumes in the long term. Cellulosic biofuels hold great promise if the necessary technology advances are made and these fuels can be produced at competitive prices. The potential for global cellulosic biomass production is sufficient to ensure that the resource base is not a constraining factor in the medium term, although the ability to bring the biomass to markets might limit the access to these resources.

The results from the ETP global model show biofuel production dominated by the U.S., Brazil and Europe. Combined they supply more than $90 \%$ of all biofuels in early years, although this decreases over time to about $80 \%$ in 2030. The majority of biofuel production is in the form of ethanol throughout the period. Sugar-based ethanol slowly looses market share as resource limitation prevents growth at the average rate of the industry.

Biofuel demand is dominated by the same regions, but the South American countries are surplus producers, and supply most of the internationally traded biofuels. In the reference case, the region exports 8 billion gallons of biofuels in 2020 and 12 billion gallons in 2030. The bulk of these volumes are sold to the U.S. and Europe, who are the largest importers. 
The ESIA RFS is an ambitious policy that will raise biofuels supply in the U.S. to 36 billion gallons. The challenge to the industry is vast and the scenarios presented here indicate that it may be difficult to reach the goal according to the schedule set out in the bill. Developing the cellulosic resource base, building cellulosic biofuel production facilities and constructing the ethanol distribution infrastructure fast enough is the main obstacle to meeting the RFS. In the scenarios presented here, the shortfalls range from 0 to 5 billion gallons in 2020 .

The law creates a marketplace where ethanol is no longer a single commodity, but can be separated into several subsets, whose value is dependent on the feedstock from which it was produced. The ethanol itself will probably trade at one price, but the associated credits will achieve different prices in the market place and thus change the total value of the ethanol to the producer.

Because the main constraint to cellulosic biofuel production is infrastructure development rather than the underlying economics, additional incentives such as growers' payments or an extension of the ethanol blenders' tax credit does little to increase overall biofuels supply. The blenders' tax credit would be paid to marketers for volumes already mandated by law and as policy tool would be inefficient to encourage biofuel supply. If increasing biofuel supply is the main policy goal, a targeted subsidy or "learning investment" for cellulosic biofuels would have a larger impact and also be cheaper to implement.

In markets without biofuel mandates, the price of biofuels is determined by the price premium it can achieve over gasoline or diesel due to the subsidy regimes. Higher oil prices will therefore lead to a stronger price signal for biofuel production and consequently the high oil price scenarios show higher worldwide demand for biofuels. In markets with mandates however, demand volumes are fixed through policy and changes in price signals do not do much to raise or lower demand.

This is also true in the U.S. The U.S. biofuel demand is not very responsive to changes in the oil price, because the buy-out from the cellulosic biofuels mandate adjusts to oil price and there are no relief-valve mechanisms for the other mandated volumes. This means that higher oil prices tend to lead to domestic production substituting for imports, because the oil price hike raises biofuel demand and thereby stronger competition in international markets.

A carbon price has a similar effect to that of a higher oil price. The carbon price can in fact be seen as a price premium on fossil fuels. A carbon policy will thus promote the production and use of biofuels worldwide. However, while higher oil prices are neutral as far as feedstock and conversion technologies are concerned, a carbon price will favor cellulosic and sugar-based biofuels production over grain-based production, which has higher carbon emission per gallon. A carbon policy will thus tend to increase the share of cellulosic and sugar ethanol at the expense of grain-derived ethanol. 


\subsection{Possible Next Steps}

There are several ways in which the current study can be expanded and enhanced in subsequent work. The competition between food and fuel markets for the crops was not fully integrated into the analysis, since neither the feedstock assessment nor the ETP energy model directly incorporate competing uses. Presently food demand is not explicitly modeled and the share of product used for food has been subtracted from the supply curves based on historical trends and expert judgment. The remainder, which is currently being exported or already going to biofuels, is considered available for biofuel production. See [8] for more details on this methodology. An approach that better captures market feedbacks and the food vs. fuel tradeoff would augment the analysis

Competition for agricultural land has only been addressed indirectly through the data input development. If it was fully integrated into the analysis the ETP model would better capture the market impacts of shifts in agricultural production from one crop to another. These dynamics could be incorporated either through a more elaborate methodology for developing the input data or by including a land use model in ETP. Both of these would both be major undertakings and most likely very time consuming and resource intensive. Availability of good underlying data would probably be a major obstacle to both approaches as well.

Detailed feedstock assessments could be developed for more countries and regions. Currently there is a lack of interaction with potential biofuel producers, and this probably leads to an underestimate of the potential for global production and international trade of biofuels.

Greenhouse gas emissions and climate policy are becoming increasingly important issues and there is a vocal debate over the real GHG emissions impact of biofuel use. Emissions related to land use changes will vary greatly between regions and crops. For this study, standardized average emission factors for feedstock production based on the available literature were used, and these regional differences were therefore not reflected. More detailed region-specific emission factors, developed under a consistent methodology, can be incorporated into the ETP model and used for future analysis of carbon policies.

Currently the link between oil prices and the cost of feedstock is not directly captured. A rise in oil prices will increase the cost of crop production. By separating the fuel requirements and related cost from other production costs the oil price feedback can be integrated into the ETP model.

With more time and resources it would also be possible to more closely examine infrastructure constraints, as well as political, institutional and regulatory barriers to the production, transport, import and export of biofuels. 


\section{REFERENCES}

1. $\quad$ President George W. Bush, State of the Union Address. 2007: Washington DC.

2. White House, T. Twenty In Ten: Strengthening America's Energy Security. 2007 http://www.whitehouse.gov/stateoftheunion/2007/initiatives/energy.html.

3. US Congress, Energy Security and Independence Act of 2007, P.L. 110-140, H.R. 6, 2007.

4. $\quad$ Fishbone L, A.H.E.R., A linear programming model for energy systems analysis: technical description of the BNL version. Energy Res 1981. 5: p. 369-79

5. Hamilton LD, G.G., Lee JC, Manne AS, Marcuse W, Morris SC, Wene C-O, MARKAL-MACRO: An overview. 1992, Brookhaven National Laboratory: Upton, NY, USA.

6. IEA, Energy Technology Perspectives. 2006, IEA: Paris, France.

7. NREL, World Biofuels Assessment, Worldwide Biomass Potential: Technology Characterizations. 2007, NREL: Golden, Colorado.

8. ORNL, Biofuel feedstock assessment for selected countries. 2007, Oak Ridge National Laboratory.

9. Larson, E.D., Biofuel production technologies: status, prospects and implications for trade and development. 2008, United Nations Conference on Trade and Development.

10. US Congress, Food and Energy Security Act of 2007, H.R. 2419, 2007.

11. IEA, 2007 World Energy Outlook. 2007, International Energy Agency: Paris, France.

12. EIA. This week in Petroleum. 2008 [cited 6 May 2008].

13. EIA, Annual Energy Outlook 2008. 2008, Energy Information Administration: Washington DC.

14. National Ethanol Vehicle Coalition. E85 Fuel stations. 2008 [cited 8 May 2008]; Available from: http://e85vehicles.com/e85-stations.htm.

15. EIA, Annual Energy Outlook 2007. 2007, Energy Information Administration: Washington DC. p. 136.

16. Armstrong, A.P., J. Baro, J. Dartoy, A.P. Groves, J. Nikkonen, D.J. Rickeard, N.D. Thompson, J-F Larivé, Energy and Greenhouse Gas Balance of Biofuels for Europe-An Update. 2002, CONCAWE: Brussels.

17. Wang, M., C. Saricks, D. Santini, Effects of Fuel Ethanol Use on Fuel-Cycle Energy and Greenhouse Gas Emissions. 1999, Center for Transportation Research, Argonne National Laboratory.

18. Macedo, I.d.C., Manoel Regis Lima Verde Lea, and João Eduardo Azevedo Ramos da Silva, Assessment of Greenhouse Gas Emissions in the Production and Use of Fuel Ethanol in Brazil. 2004.

19. Searchinger, T., Ralph Heimlich, R. A. Houghton, Fengxia Dong, Amani Elobeid, Jacinto Fabiosa, Simla Tokgoz, Dermot Hayes, Tun-Hsiang Yu, Use of U.S. Croplands for Biofuels Increases Greenhouse Gases Through Emissions from Land Use Change. Science, 2008.

20. Fargione, J., Jason Hill, David Tilman, Stephen Polasky, Peter Hawthorne, Land Clearing and the Biofuel Carbon Debt. Science, 2008. 
21. Wang, M., GREET model (v1.5a) (calculations made by IEA for reference case using the downloadable model, in consultation with Author). 2001.

22. Sheehan, J., Camobreco, Vince ,James Duffield, Michael Graboski, Housein Shapouri, An Overview of Biodiesel and Petroleum Diesel Life Cycles. 1998, National Renewable Energy Laboratory: Golden, Colorado. 


\section{Appendix A: Results}

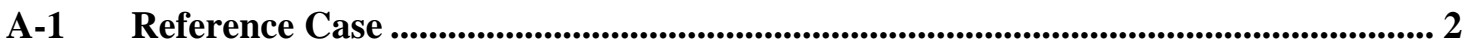

A-2 Reference Case with Credit and Tariff Extension ................................................ 12

A-3 Reference Case with Credit Extension ................................................................... 22

A-4 Reference Case with 70/30 Fuel/Feedstock Split ................................................... 32

A-5 Reference Case with High Feedstock Availability ................................................. 42

A-6 Reference Case with Low Feedstock Availability ..................................................... 52

A-7 Reference Case with High Oil Price ........................................................................... 62

A-8 Reference Case with Low Oil Price ................................................................................ 72

A-9 Reference Case with High Oil Price and High Feedstock Availability .................. 82

A-10 Reference Case with Low Oil Price and Low Feedstock Availability .................... 92

A-11 Reference Case with E20 .................................................................................................... 102

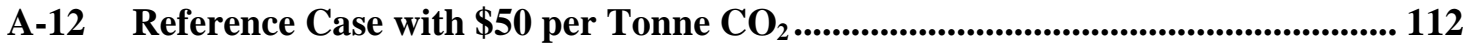

A-13 Reference Case with \$20 per Tonne Growers' Payment ......................................... 122

A-14 Delayed Technology Reference Case ........................................................................ 132

A-15 Delayed Technology Reference Case with Credit and Tariff Extension .............. 142

A-16 Delayed Technology Reference Case with Credit Extension ................................. 152

A-17 Delayed Technology Reference Case with 70/30 Fuel/Food split.......................... 162

A-18 Delayed Technology Reference Case with High Feedstock Availability .............. 172

A-19 Delayed Technology Reference Case with Low Feedstock Availability............... 182

A-20 Delayed Technology Reference Case with High Oil Price........................................ 192

A-21 Delayed Technology Reference Case with Low Oil Price......................................... 202

A-22 Delayed Technology Reference Case with High Oil Price and High Feedstock Availability............................................................................................................................ 212

A-23 Delayed Technology Reference Case with Low Oil Price and Low Feedstock Availability........................................................................................................................ 222

A-24 Delayed Technology Reference Case with E20........................................................... 232

A-25 Delayed Technology Reference Case with \$50 per Tonne $\mathrm{CO}_{2}$.............................. 242

A-26 Delayed Technology Reference Case with \$20 per Tonne Growers’ Payment .. 252 
World Biofuels Study

\section{A-1 Reference Case}

Table A-1: Reference case ethanol production [Million gallons]

\begin{tabular}{lrrrrrr} 
& $\mathbf{2 0 0 5}$ & $\mathbf{2 0 1 0}$ & $\mathbf{2 0 1 5}$ & $\mathbf{2 0 2 0}$ & $\mathbf{2 0 2 5}$ & $\mathbf{2 0 3 0}$ \\
Africa & 300 & 330 & 364 & 402 & 455 & 502 \\
Argentina & 0 & 178 & 810 & 910 & 940 & 952 \\
Australia & 0 & 0 & 71 & 267 & 709 & 1,174 \\
Brazil & 4,866 & 5,977 & 7,624 & 9,752 & 11,934 & 13,669 \\
Canada & 0 & 0 & 259 & 779 & 1,115 & 1,115 \\
Caribbean basin & 210 & 328 & 573 & 683 & 707 & 707 \\
China & 344 & 384 & 634 & 1,041 & 1,612 & 2,917 \\
Colombia & 196 & 215 & 411 & 782 & 1,501 & 2,195 \\
Eastern Europe & 110 & 226 & 434 & 780 & 1,037 & 1,149 \\
Former Soviet Union & 0 & 0 & 39 & 119 & 364 & 1,109 \\
India & 313 & 279 & 541 & 992 & 1,893 & 2,771 \\
Japan & 0 & 0 & 95 & 369 & 612 & 647 \\
Middle East & 0 & 0 & 0 & 0 & 0 & 0 \\
Mexico & 0 & 0 & 199 & 601 & 1,408 & 1,546 \\
Other Asia & 0 & 0 & 0 & 0 & 0 & 0 \\
South Korea & 0 & 0 & 36 & 36 & 36 & 36 \\
United States & 3,850 & 11,612 & 15,802 & 17,202 & 18,915 & 19,073 \\
Western Europe & 600 & 2,568 & 2,755 & 3,398 & 5,291 & 8,013 \\
\hline Total & $\mathbf{1 0 , 7 8 9}$ & $\mathbf{2 2 , 0 9 7}$ & $\mathbf{3 0 , 6 4 7}$ & $\mathbf{3 8 , 1 1 1}$ & $\mathbf{4 8 , 5 2 9}$ & $\mathbf{5 7 , 5 7 4}$ \\
\hline \hline
\end{tabular}


Table A-2: Reference case ethanol consumption [Million gallons]

\begin{tabular}{lrrrrrr} 
& $\mathbf{2 0 0 5}$ & $\mathbf{2 0 1 0}$ & $\mathbf{2 0 1 5}$ & $\mathbf{2 0 2 0}$ & $\mathbf{2 0 2 5}$ & $\mathbf{2 0 3 0}$ \\
Africa & 300 & 330 & 364 & 402 & 455 & 502 \\
Australia & 0 & 0 & 71 & 267 & 709 & 1,174 \\
Canada & 145 & 0 & 233 & 984 & 1,004 & 1,020 \\
China & 297 & 0 & 0 & 667 & 1,612 & 3,289 \\
Central and South America & 3,788 & 4,853 & 6,156 & 7,153 & 7,959 & 8,855 \\
Eastern Europe & 699 & 226 & 872 & 953 & 1,037 & 1,149 \\
Former Soviet Union & 0 & 0 & 39 & 119 & 364 & 1,109 \\
India & 313 & 279 & 541 & 992 & 1,893 & 2,399 \\
Japan & 263 & 288 & 373 & 719 & 1,008 & 1,100 \\
Middle East & 0 & 0 & 0 & 0 & 0 & 0 \\
Mexico & 0 & 0 & 30 & 396 & 865 & 1,053 \\
Other Asia & 0 & 0 & 0 & 0 & 0 & 0 \\
South Korea & 264 & 335 & 400 & 414 & 413 & 413 \\
United States & 3,984 & 11,612 & 17,287 & 19,797 & 23,562 & 27,500 \\
Western Europe & 736 & 4,174 & 4,281 & 5,249 & 7,650 & 8,013 \\
\hline Total & $\mathbf{1 0 , 7 8 9}$ & $\mathbf{2 2 , 0 9 7}$ & $\mathbf{3 0 , 6 4 7}$ & $\mathbf{3 8 , 1 1 1}$ & $\mathbf{4 8 , 5 2 9}$ & $\mathbf{5 7 , 5 7 4}$ \\
\hline \hline
\end{tabular}

Table A-3: Reference case ethanol net trade [Million gallons]

\begin{tabular}{lrrrrrr} 
& $\mathbf{2 0 0 5}$ & $\mathbf{2 0 1 0}$ & $\mathbf{2 0 1 5}$ & $\mathbf{2 0 2 0}$ & $\mathbf{2 0 2 5}$ & $\mathbf{2 0 3 0}$ \\
Africa & 0 & 0 & 0 & 0 & 0 & 0 \\
Australia & 0 & 0 & 0 & 0 & 0 & 0 \\
Canada & -145 & 0 & 26 & -205 & 112 & 96 \\
China & 47 & 384 & 634 & 373 & 0 & -372 \\
Central and South America & 1,484 & 1,845 & 3,261 & 4,974 & 7,124 & 8,668 \\
Eastern Europe & -589 & 0 & -438 & -173 & 0 & 0 \\
Former Soviet Union & 0 & 0 & 0 & 0 & 0 & 0 \\
India & 0 & 0 & 0 & 0 & 0 & 372 \\
Japan & -263 & -288 & -278 & -350 & -395 & -453 \\
Middle East & 0 & 0 & 0 & 0 & 0 & 0 \\
Mexico & 0 & 0 & 170 & 205 & 543 & 493 \\
Other Asia & 0 & 0 & 0 & 0 & 0 & 0 \\
South Korea & -264 & -335 & -364 & -378 & -377 & -377 \\
United States & -134 & 0 & $-1,485$ & $-2,594$ & $-4,647$ & $-8,427$ \\
Western Europe & -136 & $-1,607$ & $-1,526$ & $-1,851$ & $-2,359$ & 0 \\
\hline Total & $\mathbf{0}$ & $\mathbf{0}$ & $\mathbf{0}$ & $\mathbf{0}$ & $\mathbf{0}$ & $\mathbf{0}$ \\
\hline \hline
\end{tabular}


Table A-4: Reference case grain ethanol production [Million gallons]

\begin{tabular}{lrrrrrr} 
& $\mathbf{2 0 0 5}$ & $\mathbf{2 0 1 0}$ & $\mathbf{2 0 1 5}$ & $\mathbf{2 0 2 0}$ & $\mathbf{2 0 2 5}$ & $\mathbf{2 0 3 0}$ \\
Africa & 0 & 0 & 0 & 0 & 0 & 0 \\
Argentina & 0 & 178 & 604 & 604 & 604 & 604 \\
Australia & 0 & 0 & 0 & 0 & 0 & 0 \\
Brazil & 0 & 0 & 0 & 0 & 0 & 0 \\
Canada & 0 & 0 & 0 & 0 & 0 & 0 \\
Caribbean basin & 0 & 0 & 0 & 0 & 0 & 0 \\
China & 344 & 344 & 344 & 325 & 0 & 0 \\
Colombia & 0 & 0 & 0 & 0 & 0 & 0 \\
Eastern Europe & 110 & 226 & 348 & 477 & 367 & 251 \\
Former Soviet Union & 0 & 0 & 0 & 0 & 0 & 0 \\
India & 0 & 0 & 0 & 0 & 0 & 0 \\
Japan & 0 & 0 & 0 & 0 & 0 & 0 \\
Middle East & 0 & 0 & 0 & 0 & 0 & 0 \\
Mexico & 0 & 0 & 0 & 0 & 0 & 0 \\
Other Asia & 0 & 0 & 0 & 0 & 0 & 0 \\
South Korea & 0 & 0 & 0 & 0 & 0 & 0 \\
United States & 3,850 & 11,612 & 15,000 & 15,000 & 15,000 & 13,910 \\
Western Europe & 600 & 2,568 & 2,589 & 2,705 & 3,178 & 3,604 \\
\hline Total & $\mathbf{4 , 9 0 4}$ & $\mathbf{1 4 , 9 2 8}$ & $\mathbf{1 8 , 8 8 5}$ & $\mathbf{1 9 , 1 1 2}$ & $\mathbf{1 9 , 1 4 9}$ & $\mathbf{1 8 , 3 7 0}$ \\
\hline \hline
\end{tabular}

Table A-5: Reference case sugar ethanol production [Million gallons]

\begin{tabular}{lrrrrrr} 
& $\mathbf{2 0 0 5}$ & $\mathbf{2 0 1 0}$ & $\mathbf{2 0 1 5}$ & $\mathbf{2 0 2 0}$ & $\mathbf{2 0 2 5}$ & $\mathbf{2 0 3 0}$ \\
Africa & 300 & 330 & 364 & 402 & 444 & 490 \\
Argentina & 0 & 0 & 0 & 0 & 0 & 0 \\
Australia & 0 & 0 & 0 & 0 & 0 & 0 \\
Brazil & 4,866 & 5,977 & 7,329 & 8,862 & 10,057 & 11,793 \\
Canada & 0 & 0 & 0 & 0 & 0 & 0 \\
Caribbean basin & 210 & 328 & 573 & 683 & 707 & 707 \\
China & 0 & 40 & 48 & 48 & 48 & 48 \\
Colombia & 196 & 215 & 241 & 270 & 302 & 302 \\
Eastern Europe & 0 & 0 & 0 & 0 & 0 & 0 \\
Former Soviet Union & 0 & 0 & 0 & 0 & 0 & 0 \\
India & 313 & 279 & 327 & 347 & 381 & 348 \\
Japan & 0 & 0 & 0 & 0 & 0 & 0 \\
Middle East & 0 & 0 & 0 & 0 & 0 & 0 \\
Mexico & 0 & 0 & 0 & 0 & 0 & 0 \\
Other Asia & 0 & 0 & 0 & 0 & 0 & 0 \\
South Korea & 0 & 0 & 0 & 0 & 0 & 0 \\
United States & 0 & 0 & 0 & 0 & 0 & 0 \\
Western Europe & 0 & 0 & 0 & 0 & 0 & 0 \\
\hline Total & $\mathbf{5 , 8 8 4}$ & $\mathbf{7 , 1 6 9}$ & $\mathbf{8 , 8 8 2}$ & $\mathbf{1 0 , 6 1 2}$ & $\mathbf{1 1 , 9 4 0}$ & $\mathbf{1 3 , 6 8 8}$ \\
\hline \hline
\end{tabular}




\section{Table A-6: Reference case cellulosic ethanol production [Million gallons]}

\begin{tabular}{lrrrrrr} 
& $\mathbf{2 0 0 5}$ & $\mathbf{2 0 1 0}$ & $\mathbf{2 0 1 5}$ & $\mathbf{2 0 2 0}$ & $\mathbf{2 0 2 5}$ & $\mathbf{2 0 3 0}$ \\
Africa & 0 & 0 & 0 & 0 & 11 & 11 \\
Argentina & 0 & 0 & 206 & 306 & 335 & 347 \\
Australia & 0 & 0 & 71 & 267 & 709 & 1,174 \\
Brazil & 0 & 0 & 296 & 890 & 1,877 & 1,877 \\
Canada & 0 & 0 & 259 & 779 & 1,115 & 1,115 \\
Caribbean basin & 0 & 0 & 0 & 0 & 0 & 0 \\
China & 0 & 0 & 243 & 667 & 1,564 & 2,869 \\
Colombia & 0 & 0 & 170 & 512 & 1,199 & 1,893 \\
Eastern Europe & 0 & 0 & 85 & 303 & 670 & 897 \\
Former Soviet Union & 0 & 0 & 39 & 119 & 364 & 1,109 \\
India & 0 & 0 & 214 & 645 & 1,512 & 2,424 \\
Japan & 0 & 0 & 95 & 369 & 612 & 647 \\
Middle East & 0 & 0 & 0 & 0 & 0 & 0 \\
Mexico & 0 & 0 & 199 & 601 & 1,408 & 1,546 \\
Other Asia & 0 & 0 & 0 & 0 & 0 & 0 \\
South Korea & 0 & 0 & 36 & 36 & 36 & 36 \\
United States & 0 & 0 & 802 & 2,202 & 3,915 & 5,162 \\
Western Europe & 0 & 0 & 166 & 693 & 2,113 & 4,410 \\
\hline Total & $\mathbf{0}$ & $\mathbf{0}$ & $\mathbf{2 , 8 8 0}$ & $\mathbf{8 , 3 8 8}$ & $\mathbf{1 7 , 4 4 0}$ & $\mathbf{2 5 , 5 1 7}$ \\
\hline \hline
\end{tabular}

Table A-7: Reference case grain ethanol consumption [Million gallons]

\begin{tabular}{lrrrrrr} 
& $\mathbf{2 0 0 5}$ & $\mathbf{2 0 1 0}$ & $\mathbf{2 0 1 5}$ & $\mathbf{2 0 2 0}$ & $\mathbf{2 0 2 5}$ & $\mathbf{2 0 3 0}$ \\
Africa & 0 & 0 & 0 & 0 & 0 & 0 \\
Australia & 0 & 0 & 0 & 0 & 0 & 0 \\
Canada & 0 & 0 & 0 & 0 & 0 & 0 \\
China & 297 & 0 & 0 & 325 & 0 & 0 \\
Central and South America & 0 & 36 & 122 & 118 & 98 & 100 \\
Eastern Europe & 110 & 226 & 348 & 477 & 367 & 251 \\
Former Soviet Union & 0 & 0 & 0 & 0 & 0 & 0 \\
India & 0 & 0 & 0 & 0 & 0 & 0 \\
Japan & 0 & 192 & 230 & 350 & 284 & 357 \\
Middle East & 0 & 0 & 0 & 0 & 0 & 0 \\
Mexico & 0 & 0 & 0 & 0 & 0 & 0 \\
Other Asia & 0 & 0 & 0 & 0 & 0 & 0 \\
South Korea & 47 & 295 & 122 & 5 & 222 & 148 \\
United States & 3,850 & 11,612 & 15,000 & 15,000 & 15,000 & 13,910 \\
Western Europe & 600 & 2,568 & 3,064 & 2,836 & 3,178 & 3,604 \\
\hline Total & $\mathbf{4 , 9 0 4}$ & $\mathbf{1 4 , 9 2 8}$ & $\mathbf{1 8 , 8 8 5}$ & $\mathbf{1 9 , 1 1 2}$ & $\mathbf{1 9 , 1 4 9}$ & $\mathbf{1 8 , 3 7 0}$ \\
\hline \hline
\end{tabular}


Table A-8: Reference case sugar ethanol consumption [Million gallons]

\begin{tabular}{lrrrrrr} 
& $\mathbf{2 0 0 5}$ & $\mathbf{2 0 1 0}$ & $\mathbf{2 0 1 5}$ & $\mathbf{2 0 2 0}$ & $\mathbf{2 0 2 5}$ & $\mathbf{2 0 3 0}$ \\
Africa & 300 & 330 & 364 & 402 & 444 & 490 \\
Australia & 0 & 0 & 0 & 0 & 0 & 0 \\
Canada & 145 & 0 & 0 & 0 & 0 & 0 \\
China & 0 & 0 & 0 & 48 & 48 & 48 \\
Central and South America & 3,788 & 4,818 & 6,035 & 7,014 & 7,860 & 8,728 \\
Eastern Europe & 589 & 0 & 412 & 173 & 0 & 0 \\
Former Soviet Union & 0 & 0 & 0 & 0 & 0 & 0 \\
India & 313 & 279 & 327 & 347 & 381 & 348 \\
Japan & 263 & 96 & 48 & 0 & 0 & 0 \\
Middle East & 0 & 0 & 0 & 0 & 0 & 0 \\
Mexico & 0 & 0 & 0 & 0 & 0 & 0 \\
Other Asia & 0 & 0 & 0 & 0 & 0 & 0 \\
South Korea & 217 & 40 & 0 & 0 & 0 & 0 \\
United States & 134 & 0 & 788 & 2,594 & 2,920 & 4,074 \\
Western Europe & 136 & 1,607 & 908 & 33 & 287 & 0 \\
\hline Total & $\mathbf{5 , 8 8 4}$ & $\mathbf{7 , 1 6 9}$ & $\mathbf{8 , 8 8 2}$ & $\mathbf{1 0 , 6 1 2}$ & $\mathbf{1 1 , 9 4 0}$ & $\mathbf{1 3 , 6 8 8}$ \\
\hline \hline
\end{tabular}

Table A-9: Reference case cellulosic ethanol consumption [Million gallons]

\begin{tabular}{lrrrrrr} 
& $\mathbf{2 0 0 5}$ & $\mathbf{2 0 1 0}$ & $\mathbf{2 0 1 5}$ & $\mathbf{2 0 2 0}$ & $\mathbf{2 0 2 5}$ & $\mathbf{2 0 3 0}$ \\
Africa & 0 & 0 & 0 & 0 & 11 & 11 \\
Australia & 0 & 0 & 71 & 267 & 709 & 1,174 \\
Canada & 0 & 0 & 233 & 984 & 1,004 & 1,020 \\
China & 0 & 0 & 0 & 294 & 1,564 & 3,241 \\
Central and South America & 0 & 0 & 0 & 21 & 0 & 27 \\
Eastern Europe & 0 & 0 & 111 & 303 & 670 & 897 \\
Former Soviet Union & 0 & 0 & 39 & 119 & 364 & 1,109 \\
India & 0 & 0 & 214 & 645 & 1,512 & 2,051 \\
Japan & 0 & 0 & 95 & 369 & 724 & 743 \\
Middle East & 0 & 0 & 0 & 0 & 0 & 0 \\
Mexico & 0 & 0 & 30 & 396 & 865 & 1,053 \\
Other Asia & 0 & 0 & 0 & 0 & 0 & 0 \\
South Korea & 0 & 0 & 279 & 409 & 191 & 265 \\
United States & 0 & 0 & 1,500 & 2,202 & 5,642 & 9,516 \\
Western Europe & 0 & 0 & 309 & 2,379 & 4,185 & 4,410 \\
\hline Total & $\mathbf{0}$ & $\mathbf{0}$ & $\mathbf{2 , 8 8 0}$ & $\mathbf{8 , 3 8 8}$ & $\mathbf{1 7 , 4 4 0}$ & $\mathbf{2 5 , 5 1 7}$ \\
\hline \hline
\end{tabular}




\section{Table A-10: Reference case grain ethanol net trade [Million gallons]}

\begin{tabular}{lrrrrrr} 
& $\mathbf{2 0 0 5}$ & $\mathbf{2 0 1 0}$ & $\mathbf{2 0 1 5}$ & $\mathbf{2 0 2 0}$ & $\mathbf{2 0 2 5}$ & $\mathbf{2 0 3 0}$ \\
Africa & 0 & 0 & 0 & 0 & 0 & 0 \\
Australia & 0 & 0 & 0 & 0 & 0 & 0 \\
Canada & 0 & 0 & 0 & 0 & 0 & 0 \\
China & 47 & 344 & 344 & 0 & 0 & 0 \\
Central and South America & 0 & 143 & 483 & 486 & 506 & 505 \\
Eastern Europe & 0 & 0 & 0 & 0 & 0 & 0 \\
Former Soviet Union & 0 & 0 & 0 & 0 & 0 & 0 \\
India & 0 & 0 & 0 & 0 & 0 & 0 \\
Japan & 0 & -192 & -230 & -350 & -284 & -357 \\
Middle East & 0 & 0 & 0 & 0 & 0 & 0 \\
Mexico & 0 & 0 & 0 & 0 & 0 & 0 \\
Other Asia & 0 & 0 & 0 & 0 & 0 & 0 \\
South Korea & -47 & -295 & -122 & -5 & -222 & -148 \\
United States & 0 & 0 & 0 & 0 & 0 & 0 \\
Western Europe & 0 & 0 & -476 & -131 & 0 & 0 \\
\hline Total & $\mathbf{0}$ & $\mathbf{0}$ & $\mathbf{0}$ & $\mathbf{0}$ & $\mathbf{0}$ & $\mathbf{0}$ \\
\hline \hline
\end{tabular}

Table A-11: Reference case sugar ethanol net trade [Million gallons]

\begin{tabular}{lrrrrrr} 
& $\mathbf{2 0 0 5}$ & $\mathbf{2 0 1 0}$ & $\mathbf{2 0 1 5}$ & $\mathbf{2 0 2 0}$ & $\mathbf{2 0 2 5}$ & $\mathbf{2 0 3 0}$ \\
Africa & 0 & 0 & 0 & 0 & 0 & 0 \\
Australia & 0 & 0 & 0 & 0 & 0 & 0 \\
Canada & -145 & 0 & 0 & 0 & 0 & 0 \\
China & 0 & 40 & 48 & 0 & 0 & 0 \\
Central and South America & 1,484 & 1,702 & 2,108 & 2,801 & 3,207 & 4,074 \\
Eastern Europe & -589 & 0 & -412 & -173 & 0 & 0 \\
Former Soviet Union & 0 & 0 & 0 & 0 & 0 & 0 \\
India & 0 & 0 & 0 & 0 & 0 & 0 \\
Japan & -263 & -96 & -48 & 0 & 0 & 0 \\
Middle East & 0 & 0 & 0 & 0 & 0 & 0 \\
Mexico & 0 & 0 & 0 & 0 & 0 & 0 \\
Other Asia & 0 & 0 & 0 & 0 & 0 & 0 \\
South Korea & -217 & -40 & 0 & 0 & 0 & 0 \\
United States & -134 & 0 & -788 & $-2,594$ & $-2,920$ & $-4,074$ \\
Western Europe & -136 & $-1,607$ & -908 & -33 & -287 & 0 \\
\hline Total & $\mathbf{0}$ & $\mathbf{0}$ & $\mathbf{0}$ & $\mathbf{0}$ & $\mathbf{0}$ & $\mathbf{0}$ \\
\hline \hline
\end{tabular}




\section{Table A-12: Reference case cellulosic ethanol net trade [Million gallons]}

\begin{tabular}{lrrrrrr} 
& $\mathbf{2 0 0 5}$ & $\mathbf{2 0 1 0}$ & $\mathbf{2 0 1 5}$ & $\mathbf{2 0 2 0}$ & $\mathbf{2 0 2 5}$ & $\mathbf{2 0 3 0}$ \\
Africa & 0 & 0 & 0 & 0 & 0 & 0 \\
Australia & 0 & 0 & 0 & 0 & 0 & 0 \\
Canada & 0 & 0 & 26 & -205 & 112 & 96 \\
China & 0 & 0 & 243 & 373 & 0 & -372 \\
Central and South America & 0 & 0 & 671 & 1,687 & 3,411 & 4,090 \\
Eastern Europe & 0 & 0 & -26 & 0 & 0 & 0 \\
Former Soviet Union & 0 & 0 & 0 & 0 & 0 & 0 \\
India & 0 & 0 & 0 & 0 & 0 & 372 \\
Japan & 0 & 0 & 0 & 0 & -112 & -96 \\
Middle East & 0 & 0 & 0 & 0 & 0 & 0 \\
Mexico & 0 & 0 & 170 & 205 & 543 & 493 \\
Other Asia & 0 & 0 & 0 & 0 & 0 & 0 \\
South Korea & 0 & 0 & -243 & -373 & -155 & -229 \\
United States & 0 & 0 & -698 & 0 & $-1,727$ & $-4,353$ \\
Western Europe & 0 & 0 & -143 & $-1,687$ & $-2,072$ & 0 \\
\hline Total & $\mathbf{0}$ & $\mathbf{0}$ & $\mathbf{0}$ & $\mathbf{0}$ & $\mathbf{0}$ & $\mathbf{0}$ \\
\hline \hline
\end{tabular}

Table A-13: Reference case biomass-to-liquids production [Million gallons ethanol equivalent]

\begin{tabular}{lllrrrr} 
Africa & 0 & 0 & 68 & 263 & 653 & 1,177 \\
Argentina & 0 & 0 & 74 & 110 & 121 & 125 \\
Australia & 0 & 0 & 0 & 0 & 0 & 0 \\
Brazil & 0 & 0 & 106 & 320 & 675 & 675 \\
Canada & 0 & 0 & 93 & 280 & 401 & 401 \\
Caribbean basin & 0 & 0 & 0 & 0 & 0 & 0 \\
China & 0 & 0 & 57 & 240 & 563 & 563 \\
Colombia & 0 & 0 & 61 & 184 & 431 & 681 \\
Eastern Europe & 0 & 0 & 0 & 0 & 93 & 274 \\
Former Soviet Union & 0 & 0 & 0 & 0 & 0 & 0 \\
India & 0 & 0 & 77 & 232 & 544 & 544 \\
Japan & 0 & 0 & 0 & 0 & 0 & 0 \\
Middle East & 0 & 0 & 0 & 0 & 0 & 125 \\
Mexico & 0 & 0 & 72 & 216 & 506 & 556 \\
Other Asia & 0 & 0 & 89 & 448 & 1,090 & 1,942 \\
South Korea & 0 & 0 & 37 & 77 & 77 & 77 \\
United States & 0 & 0 & 822 & 4,698 & 6,246 & 8,019 \\
Western Europe & 0 & 0 & 0 & 0 & 0 & 0 \\
\hline Total & $\mathbf{0}$ & $\mathbf{0}$ & $\mathbf{1 , 5 5 6}$ & $\mathbf{7 , 0 6 9}$ & $\mathbf{1 1 , 4 0 0}$ & $\mathbf{1 5 , 1 5 8}$ \\
\hline \hline
\end{tabular}


Table A-14: Reference case biomass-to-liquids consumption [Million gallons ethanol equivalent]

\begin{tabular}{lrrrrrr} 
& $\mathbf{2 0 0 5}$ & $\mathbf{2 0 1 0}$ & $\mathbf{2 0 1 5}$ & $\mathbf{2 0 2 0}$ & $\mathbf{2 0 2 5}$ & $\mathbf{2 0 3 0}$ \\
Africa & 0 & 0 & 68 & 263 & 653 & 1,177 \\
Australia & 0 & 0 & 0 & 0 & 0 & 0 \\
Canada & 0 & 0 & 0 & 0 & 0 & 0 \\
China & 0 & 0 & 0 & 0 & 0 & 0 \\
Central and South America & 0 & 0 & 0 & 0 & 0 & 0 \\
Eastern Europe & 0 & 0 & 0 & 0 & 93 & 274 \\
Former Soviet Union & 0 & 0 & 0 & 0 & 0 & 0 \\
India & 0 & 0 & 0 & 0 & 0 & 0 \\
Japan & 0 & 0 & 0 & 0 & 0 & 0 \\
Middle East & 0 & 0 & 0 & 0 & 0 & 125 \\
Mexico & 0 & 0 & 0 & 0 & 0 & 0 \\
Other Asia & 0 & 0 & 89 & 449 & 1,090 & 1,942 \\
South Korea & 0 & 0 & 0 & 0 & 0 & 0 \\
United States & 0 & 0 & 1,111 & 6,132 & 9,564 & 10,720 \\
Western Europe & 0 & 0 & 289 & 226 & 0 & 920 \\
\hline Total & $\mathbf{0}$ & $\mathbf{0}$ & $\mathbf{1 , 5 5 6}$ & $\mathbf{7 , 0 6 9}$ & $\mathbf{1 1 , 4 0 0}$ & $\mathbf{1 5 , 1 5 8}$ \\
\hline \hline
\end{tabular}

Table A-15: Reference case biomass-to-liquids net trade [Million gallons ethanol equivalent]

\begin{tabular}{lrrrrrr} 
& $\mathbf{2 0 0 5}$ & $\mathbf{2 0 1 0}$ & $\mathbf{2 0 1 5}$ & $\mathbf{2 0 2 0}$ & $\mathbf{2 0 2 5}$ & $\mathbf{2 0 3 0}$ \\
Africa & 0 & 0 & 0 & 0 & 0 & 0 \\
Australia & 0 & 0 & 0 & 0 & 0 & 0 \\
Canada & 0 & 0 & 93 & 280 & 401 & 401 \\
China & 0 & 0 & 57 & 240 & 563 & 563 \\
Central and South America & 0 & 0 & 241 & 614 & 1,227 & 1,481 \\
Eastern Europe & 0 & 0 & 0 & 0 & 0 & 0 \\
Former Soviet Union & 0 & 0 & 0 & 0 & 0 & 0 \\
India & 0 & 0 & 77 & 232 & 544 & 544 \\
Japan & 0 & 0 & 0 & 0 & 0 & 0 \\
Middle East & 0 & 0 & 0 & 0 & 0 & 0 \\
Mexico & 0 & 0 & 72 & 216 & 506 & 556 \\
Other Asia & 0 & 0 & 0 & 0 & 0 & 0 \\
South Korea & 0 & 0 & 37 & 77 & 77 & 77 \\
United States & 0 & 0 & -289 & $-1,434$ & $-3,318$ & $-2,701$ \\
Western Europe & 0 & 0 & -289 & -226 & 0 & -920 \\
\hline Total & $\mathbf{0}$ & $\mathbf{0}$ & $\mathbf{0}$ & $\mathbf{0}$ & $\mathbf{0}$ & $\mathbf{0}$ \\
\hline \hline
\end{tabular}


Table A-16: Reference case biodiesel production [Million gallons ethanol equivalent]

\begin{tabular}{lrrrrrr} 
& $\mathbf{2 0 0 5}$ & $\mathbf{2 0 1 0}$ & $\mathbf{2 0 1 5}$ & $\mathbf{2 0 2 0}$ & $\mathbf{2 0 2 5}$ & $\mathbf{2 0 3 0}$ \\
Africa & 0 & 0 & 0 & 0 & 0 & 0 \\
Argentina & 0 & 333 & 776 & 819 & 827 & 239 \\
Australia & 0 & 0 & 0 & 0 & 0 & 0 \\
Brazil & 0 & 333 & 877 & 1,642 & 1,966 & 1,355 \\
Canada & 0 & 0 & 0 & 0 & 0 & 0 \\
Caribbean basin & 0 & 95 & 310 & 675 & 802 & 898 \\
China & 0 & 0 & 0 & 0 & 0 & 0 \\
Colombia & 0 & 119 & 298 & 474 & 650 & 782 \\
Eastern Europe & 0 & 0 & 0 & 0 & 0 & 0 \\
Former Soviet Union & 0 & 0 & 0 & 0 & 0 & 0 \\
India & 0 & 0 & 0 & 0 & 0 & 0 \\
Japan & 0 & 0 & 0 & 0 & 0 & 0 \\
Middle East & 0 & 0 & 0 & 0 & 0 & 0 \\
Mexico & 0 & 0 & 0 & 0 & 0 & 0 \\
Other Asia & 0 & 0 & 0 & 0 & 0 & 0 \\
South Korea & 0 & 0 & 0 & 0 & 0 & 0 \\
United States & 0 & 1,238 & 1,640 & 1,747 & 1,907 & 2,016 \\
Western Europe & 998 & 3,178 & 3,578 & 3,930 & 4,468 & 4,788 \\
\hline Total & $\mathbf{9 9 8}$ & $\mathbf{5 , 2 9 4}$ & $\mathbf{7 , 4 7 9}$ & $\mathbf{9 , 2 8 7}$ & $\mathbf{1 0 , 6 2 1}$ & $\mathbf{1 0 , 0 7 7}$ \\
\hline \hline
\end{tabular}

Table A-17: Reference case biodiesel consumption

[Million gallons ethanol equivalent]

\begin{tabular}{lrrrrrr} 
& $\mathbf{2 0 0 5}$ & $\mathbf{2 0 1 0}$ & $\mathbf{2 0 1 5}$ & $\mathbf{2 0 2 0}$ & $\mathbf{2 0 2 5}$ & $\mathbf{2 0 3 0}$ \\
Africa & 0 & 0 & 0 & 0 & 0 & 0 \\
Australia & 0 & 0 & 0 & 0 & 0 & 0 \\
Canada & 0 & 0 & 0 & 0 & 0 & 0 \\
China & 0 & 0 & 0 & 0 & 0 & 0 \\
Central and South America & 0 & 158 & 472 & 984 & 1,306 & 1,490 \\
Eastern Europe & 0 & 0 & 0 & 0 & 0 & 0 \\
Former Soviet Union & 0 & 0 & 0 & 0 & 0 & 0 \\
India & 0 & 0 & 0 & 0 & 0 & 0 \\
Japan & 0 & 0 & 0 & 0 & 0 & 0 \\
Middle East & 0 & 0 & 0 & 0 & 0 & 0 \\
Mexico & 0 & 0 & 0 & 0 & 0 & 0 \\
Other Asia & 0 & 0 & 0 & 0 & 0 & 0 \\
South Korea & 0 & 0 & 0 & 0 & 0 & 0 \\
United States & 0 & 1,238 & 1,713 & 1,885 & 2,080 & 2,016 \\
Western Europe & 998 & 3,898 & 5,294 & 6,419 & 7,235 & 6,572 \\
\hline Total & $\mathbf{9 9 8}$ & $\mathbf{5 , 2 9 4}$ & $\mathbf{7 , 4 7 9}$ & $\mathbf{9 , 2 8 7}$ & $\mathbf{1 0 , 6 2 1}$ & $\mathbf{1 0 , 0 7 7}$ \\
\hline \hline
\end{tabular}


World Biofuels Study

Table A-18: Reference case biodiesel net trade [Million gallons ethanol equivalent]

\begin{tabular}{lrrrrrr} 
& $\mathbf{2 0 0 5}$ & $\mathbf{2 0 1 0}$ & $\mathbf{2 0 1 5}$ & $\mathbf{2 0 2 0}$ & $\mathbf{2 0 2 5}$ & $\mathbf{2 0 3 0}$ \\
Africa & 0 & 0 & 0 & 0 & 0 & 0 \\
Australia & 0 & 0 & 0 & 0 & 0 & 0 \\
Canada & 0 & 0 & 0 & 0 & 0 & 0 \\
China & 0 & 0 & 0 & 0 & 0 & 0 \\
Central and South America & 0 & 720 & 1,788 & 2,626 & 2,940 & 1,784 \\
Eastern Europe & 0 & 0 & 0 & 0 & 0 & 0 \\
Former Soviet Union & 0 & 0 & 0 & 0 & 0 & 0 \\
India & 0 & 0 & 0 & 0 & 0 & 0 \\
Japan & 0 & 0 & 0 & 0 & 0 & 0 \\
Middle East & 0 & 0 & 0 & 0 & 0 & 0 \\
Mexico & 0 & 0 & 0 & 0 & 0 & 0 \\
Other Asia & 0 & 0 & 0 & 0 & 0 & 0 \\
South Korea & 0 & 0 & 0 & 0 & 0 & 0 \\
United States & 0 & 0 & -73 & -138 & -173 & 0 \\
Western Europe & 0 & -720 & $-1,716$ & $-2,488$ & $-2,767$ & $-1,784$ \\
\hline Total & $\mathbf{0}$ & $\mathbf{0}$ & $\mathbf{0}$ & $\mathbf{0}$ & $\mathbf{0}$ & $\mathbf{0}$ \\
\hline \hline
\end{tabular}




\section{A-2 Reference Case with Credit and Tariff Extension}

Table A-19: Reference case with credit and tariff extension ethanol production [Million gallons]

\begin{tabular}{l} 
Africa \\
Argentina \\
Australia \\
Brazil \\
Canada \\
Caribbean basin \\
China \\
Colombia \\
Eastern Europe \\
Former Soviet Union \\
India \\
Japan \\
Middle East \\
Mexico \\
Other Asia \\
South Korea \\
United States \\
Western Europe \\
Total \\
\hline \hline
\end{tabular}

\begin{tabular}{rrrrrr}
$\mathbf{2 0 0 5}$ & $\mathbf{2 0 1 0}$ & $\mathbf{2 0 1 5}$ & $\mathbf{2 0 2 0}$ & $\mathbf{2 0 2 5}$ & $\mathbf{2 0 3 0}$ \\
300 & 330 & 364 & 402 & 455 & 502 \\
0 & 178 & 745 & 841 & 904 & 347 \\
0 & 0 & 71 & 267 & 709 & 1,174 \\
4,866 & 5,977 & 7,534 & 9,597 & 11,603 & 13,338 \\
0 & 0 & 259 & 779 & 1,022 & 1,022 \\
210 & 328 & 735 & 735 & 779 & 890 \\
344 & 384 & 634 & 1,041 & 1,612 & 2,917 \\
196 & 215 & 401 & 750 & 1,469 & 2,163 \\
110 & 226 & 434 & 780 & 1,037 & 1,149 \\
0 & 0 & 39 & 119 & 364 & 1,109 \\
313 & 279 & 541 & 992 & 1,893 & 2,771 \\
0 & 0 & 95 & 369 & 612 & 647 \\
0 & 0 & 0 & 0 & 0 & 0 \\
0 & 0 & 224 & 601 & 1,408 & 1,546 \\
0 & 0 & 0 & 0 & 0 & 0 \\
0 & 0 & 35 & 35 & 35 & 35 \\
3,850 & 11,668 & 15,932 & 17,513 & 20,235 & 24,804 \\
600 & 2,568 & 2,755 & 3,398 & 5,291 & 8,013 \\
\hline $\mathbf{1 0 , 7 8 9}$ & $\mathbf{2 2 , 1 5 4}$ & $\mathbf{3 0 , 7 9 8}$ & $\mathbf{3 8 , 2 1 6}$ & $\mathbf{4 9 , 4 2 7}$ & $\mathbf{6 2 , 4 2 6}$ \\
\hline & & & & &
\end{tabular}


Table A-20: Reference case with credit and tariff extension ethanol consumption [Million gallons]

\begin{tabular}{lrrrrrr} 
& $\mathbf{2 0 0 5}$ & $\mathbf{2 0 1 0}$ & $\mathbf{2 0 1 5}$ & $\mathbf{2 0 2 0}$ & $\mathbf{2 0 2 5}$ & $\mathbf{2 0 3 0}$ \\
Africa & 300 & 330 & 364 & 402 & 455 & 502 \\
Australia & 0 & 0 & 71 & 267 & 709 & 1,174 \\
Canada & 145 & 0 & 233 & 779 & 1,004 & 1,020 \\
China & 121 & 0 & 0 & 959 & 2,896 & 5,063 \\
Central and South America & 3,788 & 4,853 & 6,065 & 7,339 & 7,954 & 8,799 \\
Eastern Europe & 699 & 226 & 872 & 953 & 1,037 & 1,149 \\
Former Soviet Union & 0 & 0 & 39 & 119 & 364 & 1,109 \\
India & 313 & 279 & 541 & 992 & 1,893 & 2,399 \\
Japan & 263 & 288 & 410 & 719 & 1,008 & 1,100 \\
Middle East & 0 & 0 & 0 & 0 & 0 & 0 \\
Mexico & 0 & 0 & 34 & 78 & 183 & 201 \\
Other Asia & 0 & 0 & 0 & 0 & 0 & 1,654 \\
South Korea & 264 & 335 & 400 & 414 & 413 & 413 \\
United States & 4,161 & 11,668 & 17,461 & 19,947 & 23,862 & 28,775 \\
Western Europe & 736 & 4,174 & 4,307 & 5,249 & 7,649 & 9,071 \\
\hline Total & $\mathbf{1 0 , 7 8 9}$ & $\mathbf{2 2 , 1 5 4}$ & $\mathbf{3 0 , 7 9 8}$ & $\mathbf{3 8 , 2 1 6}$ & $\mathbf{4 9 , 4 2 7}$ & $\mathbf{6 2 , 4 2 6}$ \\
\hline \hline
\end{tabular}

Table A-21: Reference case with credit and tariff extension ethanol net trade [Million gallons]

\begin{tabular}{lrrrrrr} 
& $\mathbf{2 0 0 5}$ & $\mathbf{2 0 1 0}$ & $\mathbf{2 0 1 5}$ & $\mathbf{2 0 2 0}$ & $\mathbf{2 0 2 5}$ & $\mathbf{2 0 3 0}$ \\
Africa & 0 & 0 & 0 & 0 & 0 & 0 \\
Australia & 0 & 0 & 0 & 0 & 0 & 0 \\
Canada & -145 & 0 & 26 & 0 & 18 & 2 \\
China & 224 & 384 & 634 & 82 & $-1,284$ & $-2,146$ \\
Central and South America & 1,484 & 1,845 & 3,350 & 4,583 & 6,801 & 7,939 \\
Eastern Europe & -589 & 0 & -438 & -173 & 0 & 0 \\
Former Soviet Union & 0 & 0 & 0 & 0 & 0 & 0 \\
India & 0 & 0 & 0 & 0 & 0 & 372 \\
Japan & -263 & -288 & -315 & -350 & -395 & -453 \\
Middle East & 0 & 0 & 0 & 0 & 0 & 0 \\
Mexico & 0 & 0 & 190 & 523 & 1,225 & 1,345 \\
Other Asia & 0 & 0 & 0 & 0 & 0 & $-1,654$ \\
South Korea & -264 & -335 & -365 & -379 & -378 & -378 \\
United States & -311 & 0 & $-1,529$ & $-2,433$ & $-3,628$ & $-3,970$ \\
Western Europe & -136 & $-1,607$ & $-1,552$ & $-1,851$ & $-2,358$ & $-1,058$ \\
\hline Total & $\mathbf{0}$ & $\mathbf{0}$ & $\mathbf{0}$ & $\mathbf{0}$ & $\mathbf{0}$ & $\mathbf{0}$ \\
\hline \hline
\end{tabular}


Table A-22: Reference case with credit and tariff extension grain ethanol production [Million gallons]

\begin{tabular}{lrrrrrr} 
& $\mathbf{2 0 0 5}$ & $\mathbf{2 0 1 0}$ & $\mathbf{2 0 1 5}$ & $\mathbf{2 0 2 0}$ & $\mathbf{2 0 2 5}$ & $\mathbf{2 0 3 0}$ \\
Africa & 0 & 0 & 0 & 0 & 0 & 0 \\
Argentina & 0 & 178 & 569 & 569 & 569 & 0 \\
Australia & 0 & 0 & 0 & 0 & 0 & 0 \\
Brazil & 0 & 0 & 0 & 0 & 0 & 0 \\
Canada & 0 & 0 & 0 & 0 & 0 & 0 \\
Caribbean basin & 0 & 0 & 0 & 0 & 0 & 0 \\
China & 344 & 344 & 344 & 325 & 0 & 0 \\
Colombia & 0 & 0 & 0 & 0 & 0 & 0 \\
Eastern Europe & 110 & 226 & 348 & 477 & 367 & 251 \\
Former Soviet Union & 0 & 0 & 0 & 0 & 0 & 0 \\
India & 0 & 0 & 0 & 0 & 0 & 0 \\
Japan & 0 & 0 & 0 & 0 & 0 & 0 \\
Middle East & 0 & 0 & 0 & 0 & 0 & 0 \\
Mexico & 0 & 0 & 0 & 0 & 0 & 0 \\
Other Asia & 0 & 0 & 0 & 0 & 0 & 0 \\
South Korea & 0 & 0 & 0 & 0 & 0 & 0 \\
United States & 3,850 & 11,668 & 15,000 & 15,000 & 15,000 & 15,000 \\
Western Europe & 600 & 2,568 & 2,589 & 2,705 & 3,178 & 3,604 \\
\hline Total & $\mathbf{4 , 9 0 4}$ & $\mathbf{1 4 , 9 8 4}$ & $\mathbf{1 8 , 8 5 0}$ & $\mathbf{1 9 , 0 7 7}$ & $\mathbf{1 9 , 1 1 4}$ & $\mathbf{1 8 , 8 5 5}$ \\
\hline \hline
\end{tabular}

Table A-23: Reference case with credit and tariff extension sugar ethanol production [Million gallons]

\begin{tabular}{lrrrrrr} 
& $\mathbf{2 0 0 5}$ & $\mathbf{2 0 1 0}$ & $\mathbf{2 0 1 5}$ & $\mathbf{2 0 2 0}$ & $\mathbf{2 0 2 5}$ & $\mathbf{2 0 3 0}$ \\
Africa & 300 & 330 & 364 & 402 & 444 & 490 \\
Argentina & 0 & 0 & 0 & 0 & 0 & 0 \\
Australia & 0 & 0 & 0 & 0 & 0 & 0 \\
Brazil & 4,866 & 5,977 & 7,233 & 8,862 & 10,057 & 11,793 \\
Canada & 0 & 0 & 0 & 0 & 0 & 0 \\
Caribbean basin & 210 & 328 & 735 & 735 & 779 & 890 \\
China & 0 & 40 & 48 & 48 & 48 & 48 \\
Colombia & 196 & 215 & 241 & 270 & 270 & 270 \\
Eastern Europe & 0 & 0 & 0 & 0 & 0 & 0 \\
Former Soviet Union & 0 & 0 & 0 & 0 & 0 & 0 \\
India & 313 & 279 & 327 & 347 & 381 & 348 \\
Japan & 0 & 0 & 0 & 0 & 0 & 0 \\
Middle East & 0 & 0 & 0 & 0 & 0 & 0 \\
Mexico & 0 & 0 & 0 & 0 & 0 & 0 \\
Other Asia & 0 & 0 & 0 & 0 & 0 & 0 \\
South Korea & 0 & 0 & 0 & 0 & 0 & 0 \\
United States & 0 & 0 & 0 & 0 & 0 & 0 \\
Western Europe & 0 & 0 & 0 & 0 & 0 & 0 \\
\hline Total & $\mathbf{5 , 8 8 4}$ & $\mathbf{7 , 1 6 9}$ & $\mathbf{8 , 9 4 8}$ & $\mathbf{1 0 , 6 6 3}$ & $\mathbf{1 1 , 9 8 0}$ & $\mathbf{1 3 , 8 3 8}$ \\
\hline \hline
\end{tabular}


Table A-24: Reference case with credit and tariff extension cellulosic ethanol production [Million gallons]

\begin{tabular}{lrrrrrr} 
& $\mathbf{2 0 0 5}$ & $\mathbf{2 0 1 0}$ & $\mathbf{2 0 1 5}$ & $\mathbf{2 0 2 0}$ & $\mathbf{2 0 2 5}$ & $\mathbf{2 0 3 0}$ \\
Africa & 0 & 0 & 0 & 0 & 11 & 11 \\
Argentina & 0 & 0 & 176 & 272 & 335 & 347 \\
Australia & 0 & 0 & 71 & 267 & 709 & 1,174 \\
Brazil & 0 & 0 & 302 & 735 & 1,546 & 1,546 \\
Canada & 0 & 0 & 259 & 779 & 1,022 & 1,022 \\
Caribbean basin & 0 & 0 & 0 & 0 & 0 & 0 \\
China & 0 & 0 & 243 & 667 & 1,564 & 2,869 \\
Colombia & 0 & 0 & 160 & 480 & 1,199 & 1,893 \\
Eastern Europe & 0 & 0 & 85 & 303 & 670 & 897 \\
Former Soviet Union & 0 & 0 & 39 & 119 & 364 & 1,109 \\
India & 0 & 0 & 214 & 645 & 1,512 & 2,424 \\
Japan & 0 & 0 & 95 & 369 & 612 & 647 \\
Middle East & 0 & 0 & 0 & 0 & 0 & 0 \\
Mexico & 0 & 0 & 224 & 601 & 1,408 & 1,546 \\
Other Asia & 0 & 0 & 0 & 0 & 0 & 0 \\
South Korea & 0 & 0 & 35 & 35 & 35 & 35 \\
United States & 0 & 0 & 932 & 2,513 & 5,235 & 9,804 \\
Western Europe & 0 & 0 & 166 & 693 & 2,113 & 4,410 \\
\hline Total & $\mathbf{0}$ & $\mathbf{0}$ & $\mathbf{3 , 0 0 0}$ & $\mathbf{8 , 4 7 7}$ & $\mathbf{1 8 , 3 3 4}$ & $\mathbf{2 9 , 7 3 3}$ \\
\hline \hline
\end{tabular}

Table A-25: Reference case with credit and tariff extension grain ethanol consumption [Million gallons]

\begin{tabular}{lrrrrrr} 
& $\mathbf{2 0 0 5}$ & $\mathbf{2 0 1 0}$ & $\mathbf{2 0 1 5}$ & $\mathbf{2 0 2 0}$ & $\mathbf{2 0 2 5}$ & $\mathbf{2 0 3 0}$ \\
Africa & 0 & 0 & 0 & 0 & 0 & 0 \\
Australia & 0 & 0 & 0 & 0 & 0 & 0 \\
Canada & 0 & 0 & 0 & 0 & 0 & 0 \\
China & 121 & 0 & 0 & 325 & 79 & 0 \\
Central and South America & 0 & 36 & 116 & 114 & 95 & 0 \\
Eastern Europe & 110 & 226 & 348 & 477 & 367 & 251 \\
Former Soviet Union & 0 & 0 & 0 & 0 & 0 & 0 \\
India & 0 & 0 & 0 & 0 & 0 & 0 \\
Japan & 0 & 192 & 267 & 350 & 395 & 0 \\
Middle East & 0 & 0 & 0 & 0 & 0 & 0 \\
Mexico & 0 & 0 & 0 & 0 & 0 & 0 \\
Other Asia & 0 & 0 & 0 & 0 & 0 & 0 \\
South Korea & 224 & 295 & 123 & 105 & 0 & 0 \\
United States & 3,850 & 11,668 & 15,000 & 15,000 & 15,000 & 15,000 \\
Western Europe & 600 & 2,568 & 2,995 & 2,705 & 3,178 & 3,604 \\
\hline Total & $\mathbf{4 , 9 0 4}$ & $\mathbf{1 4 , 9 8 4}$ & $\mathbf{1 8 , 8 5 0}$ & $\mathbf{1 9 , 0 7 7}$ & $\mathbf{1 9 , 1 1 4}$ & $\mathbf{1 8 , 8 5 5}$ \\
\hline \hline
\end{tabular}


Table A-26: Reference case with credit and tariff extension sugar ethanol consumption [Million gallons]

\begin{tabular}{lrrrrrr} 
& $\mathbf{2 0 0 5}$ & $\mathbf{2 0 1 0}$ & $\mathbf{2 0 1 5}$ & $\mathbf{2 0 2 0}$ & $\mathbf{2 0 2 5}$ & $\mathbf{2 0 3 0}$ \\
Africa & 300 & 330 & 364 & 402 & 444 & 490 \\
Australia & 0 & 0 & 0 & 0 & 0 & 0 \\
Canada & 145 & 0 & 0 & 0 & 0 & 0 \\
China & 0 & 0 & 0 & 48 & 1,236 & 1,819 \\
Central and South America & 3,788 & 4,818 & 5,949 & 7,017 & 7,516 & 8,033 \\
Eastern Europe & 589 & 0 & 412 & 173 & 0 & 0 \\
Former Soviet Union & 0 & 0 & 0 & 0 & 0 & 0 \\
India & 313 & 279 & 327 & 347 & 381 & 348 \\
Japan & 263 & 96 & 48 & 0 & 0 & 453 \\
Middle East & 0 & 0 & 0 & 0 & 0 & 0 \\
Mexico & 0 & 0 & 0 & 0 & 0 & 0 \\
Other Asia & 0 & 0 & 0 & 0 & 0 & 0 \\
South Korea & 41 & 40 & 0 & 0 & 0 & 70 \\
United States & 311 & 0 & 788 & 2,320 & 2,403 & 2,626 \\
Western Europe & 136 & 1,607 & 1,060 & 357 & 0 & 0 \\
\hline Total & $\mathbf{5 , 8 8 4}$ & $\mathbf{7 , 1 6 9}$ & $\mathbf{8 , 9 4 8}$ & $\mathbf{1 0 , 6 6 3}$ & $\mathbf{1 1 , 9 8 0}$ & $\mathbf{1 3 , 8 3 8}$ \\
\hline \hline
\end{tabular}

Table A-27: Reference case with credit and tariff extension cellulosic ethanol consumption [Million gallons]

\begin{tabular}{lrrrrrr} 
& $\mathbf{2 0 0 5}$ & $\mathbf{2 0 1 0}$ & $\mathbf{2 0 1 5}$ & $\mathbf{2 0 2 0}$ & $\mathbf{2 0 2 5}$ & $\mathbf{2 0 3 0}$ \\
Africa & 0 & 0 & 0 & 0 & 11 & 11 \\
Australia & 0 & 0 & 71 & 267 & 709 & 1,174 \\
Canada & 0 & 0 & 233 & 779 & 1,004 & 1,020 \\
China & 0 & 0 & 0 & 586 & 1,582 & 3,244 \\
Central and South America & 0 & 0 & 0 & 209 & 343 & 766 \\
Eastern Europe & 0 & 0 & 111 & 303 & 670 & 897 \\
Former Soviet Union & 0 & 0 & 39 & 119 & 364 & 1,109 \\
India & 0 & 0 & 214 & 645 & 1,512 & 2,051 \\
Japan & 0 & 0 & 95 & 369 & 612 & 647 \\
Middle East & 0 & 0 & 0 & 0 & 0 & 0 \\
Mexico & 0 & 0 & 34 & 78 & 183 & 201 \\
Other Asia & 0 & 0 & 0 & 0 & 0 & 1,654 \\
South Korea & 0 & 0 & 277 & 309 & 413 & 343 \\
United States & 0 & 0 & 1,674 & 2,627 & 6,459 & 11,149 \\
Western Europe & 0 & 0 & 252 & 2,187 & 4,472 & 5,467 \\
\hline Total & $\mathbf{0}$ & $\mathbf{0}$ & $\mathbf{3 , 0 0 0}$ & $\mathbf{8 , 4 7 7}$ & $\mathbf{1 8 , 3 3 4}$ & $\mathbf{2 9 , 7 3 3}$ \\
\hline \hline
\end{tabular}


Table A-28: Reference case with credit and tariff extension grain ethanol net trade [Million gallons]

\begin{tabular}{lrrrrrr} 
& $\mathbf{2 0 0 5}$ & $\mathbf{2 0 1 0}$ & $\mathbf{2 0 1 5}$ & $\mathbf{2 0 2 0}$ & $\mathbf{2 0 2 5}$ & $\mathbf{2 0 3 0}$ \\
Africa & 0 & 0 & 0 & 0 & 0 & 0 \\
Australia & 0 & 0 & 0 & 0 & 0 & 0 \\
Canada & 0 & 0 & 0 & 0 & 0 & 0 \\
China & 224 & 344 & 344 & 0 & -79 & 0 \\
Central and South America & 0 & 143 & 453 & 455 & 474 & 0 \\
Eastern Europe & 0 & 0 & 0 & 0 & 0 & 0 \\
Former Soviet Union & 0 & 0 & 0 & 0 & 0 & 0 \\
India & 0 & 0 & 0 & 0 & 0 & 0 \\
Japan & 0 & -192 & -267 & -350 & -395 & 0 \\
Middle East & 0 & 0 & 0 & 0 & 0 & 0 \\
Mexico & 0 & 0 & 0 & 0 & 0 & 0 \\
Other Asia & 0 & 0 & 0 & 0 & 0 & 0 \\
South Korea & -224 & -295 & -123 & -105 & 0 & 0 \\
United States & 0 & 0 & 0 & 0 & 0 & 0 \\
Western Europe & 0 & 0 & -407 & 0 & 0 & 0 \\
\hline Total & $\mathbf{0}$ & $\mathbf{0}$ & $\mathbf{0}$ & $\mathbf{0}$ & $\mathbf{0}$ & $\mathbf{0}$ \\
\hline \hline
\end{tabular}

Table A-29: Reference case with credit and tariff extension sugar ethanol net trade [Million gallons]

\begin{tabular}{lrrrrrr} 
Africa & 0 & 0 & 0 & 0 & 0 & 0 \\
Australia & 0 & 0 & 0 & 0 & 0 & 0 \\
Canada & -145 & 0 & 0 & 0 & 0 & 0 \\
China & 0 & 40 & 48 & 0 & $-1,188$ & $-1,771$ \\
Central and South America & 1,484 & 1,702 & 2,260 & 2,850 & 3,591 & 4,920 \\
Eastern Europe & -589 & 0 & -412 & -173 & 0 & 0 \\
Former Soviet Union & 0 & 0 & 0 & 0 & 0 & 0 \\
India & 0 & 0 & 0 & 0 & 0 & 0 \\
Japan & -263 & -96 & -48 & 0 & 0 & -453 \\
Middle East & 0 & 0 & 0 & 0 & 0 & 0 \\
Mexico & 0 & 0 & 0 & 0 & 0 & 0 \\
Other Asia & 0 & 0 & 0 & 0 & 0 & 0 \\
South Korea & -41 & -40 & 0 & 0 & 0 & -70 \\
United States & -311 & 0 & -788 & $-2,320$ & $-2,403$ & $-2,626$ \\
Western Europe & -136 & $-1,607$ & $-1,060$ & -357 & 0 & 0 \\
\hline Total & $\mathbf{0}$ & $\mathbf{0}$ & $\mathbf{0}$ & $\mathbf{0}$ & $\mathbf{0}$ & $\mathbf{0}$ \\
\hline \hline
\end{tabular}


Table A-30: Reference case with credit and tariff extension cellulosic ethanol net trade [Million gallons]

\begin{tabular}{lrrrrrr} 
& $\mathbf{2 0 0 5}$ & $\mathbf{2 0 1 0}$ & $\mathbf{2 0 1 5}$ & $\mathbf{2 0 2 0}$ & $\mathbf{2 0 2 5}$ & $\mathbf{2 0 3 0}$ \\
Africa & 0 & 0 & 0 & 0 & 0 & 0 \\
Australia & 0 & 0 & 0 & 0 & 0 & 0 \\
Canada & 0 & 0 & 26 & 0 & 18 & 2 \\
China & 0 & 0 & 243 & 82 & -18 & -374 \\
Central and South America & 0 & 0 & 637 & 1,278 & 2,737 & 3,020 \\
Eastern Europe & 0 & 0 & -26 & 0 & 0 & 0 \\
Former Soviet Union & 0 & 0 & 0 & 0 & 0 & 0 \\
India & 0 & 0 & 0 & 0 & 0 & 372 \\
Japan & 0 & 0 & 0 & 0 & 0 & 0 \\
Middle East & 0 & 0 & 0 & 0 & 0 & 0 \\
Mexico & 0 & 0 & 190 & 523 & 1,225 & 1,345 \\
Other Asia & 0 & 0 & 0 & 0 & 0 & $-1,654$ \\
South Korea & 0 & 0 & -243 & -275 & -378 & -308 \\
United States & 0 & 0 & -742 & -114 & $-1,225$ & $-1,345$ \\
Western Europe & 0 & 0 & -86 & $-1,494$ & $-2,358$ & $-1,058$ \\
\hline Total & $\mathbf{0}$ & $\mathbf{0}$ & $\mathbf{0}$ & $\mathbf{0}$ & $\mathbf{0}$ & $\mathbf{0}$ \\
\hline \hline
\end{tabular}

Table A-31: Reference case with credit and tariff extension biomass-to-liquids production [Million gallons ethanol equivalent]

\begin{tabular}{lrrrrrr} 
& $\mathbf{2 0 0 5}$ & $\mathbf{2 0 1 0}$ & $\mathbf{2 0 1 5}$ & $\mathbf{2 0 2 0}$ & $\mathbf{2 0 2 5}$ & $\mathbf{2 0 3 0}$ \\
Africa & 0 & 0 & 68 & 263 & 653 & 1,177 \\
Argentina & 0 & 0 & 74 & 110 & 121 & 125 \\
Australia & 0 & 0 & 0 & 0 & 0 & 0 \\
Brazil & 0 & 0 & 106 & 320 & 556 & 556 \\
Canada & 0 & 0 & 93 & 280 & 368 & 368 \\
Caribbean basin & 0 & 0 & 0 & 0 & 0 & 0 \\
China & 0 & 0 & 57 & 240 & 563 & 563 \\
Colombia & 0 & 0 & 61 & 184 & 431 & 681 \\
Eastern Europe & 0 & 0 & 0 & 0 & 93 & 274 \\
Former Soviet Union & 0 & 0 & 0 & 0 & 0 & 0 \\
India & 0 & 0 & 77 & 232 & 544 & 544 \\
Japan & 0 & 0 & 0 & 0 & 0 & 0 \\
Middle East & 0 & 0 & 0 & 0 & 0 & 125 \\
Mexico & 0 & 0 & 45 & 216 & 506 & 556 \\
Other Asia & 0 & 0 & 89 & 448 & 1,090 & 1,942 \\
South Korea & 0 & 0 & 36 & 74 & 74 & 74 \\
United States & 0 & 0 & 792 & 4,683 & 6,280 & 5,980 \\
Western Europe & 0 & 0 & 0 & 0 & 0 & 0 \\
\hline Total & $\mathbf{0}$ & $\mathbf{0}$ & $\mathbf{1 , 4 9 8}$ & $\mathbf{7 , 0 5 0}$ & $\mathbf{1 1 , 2 7 7}$ & $\mathbf{1 2 , 9 6 3}$ \\
\hline \hline
\end{tabular}


Table A-32: Reference case with credit and tariff extension biomass-to-liquids consumption [Million gallons ethanol equivalent]

\begin{tabular}{lrrrrrr} 
& $\mathbf{2 0 0 5}$ & $\mathbf{2 0 1 0}$ & $\mathbf{2 0 1 5}$ & $\mathbf{2 0 2 0}$ & $\mathbf{2 0 2 5}$ & $\mathbf{2 0 3 0}$ \\
Africa & 0 & 0 & 68 & 263 & 653 & 1,177 \\
Australia & 0 & 0 & 0 & 0 & 0 & 0 \\
Canada & 0 & 0 & 0 & 0 & 0 & 0 \\
China & 0 & 0 & 0 & 0 & 0 & 0 \\
Central and South America & 0 & 0 & 0 & 0 & 0 & 0 \\
Eastern Europe & 0 & 0 & 0 & 0 & 93 & 274 \\
Former Soviet Union & 0 & 0 & 0 & 0 & 0 & 0 \\
India & 0 & 0 & 0 & 0 & 0 & 0 \\
Japan & 0 & 0 & 0 & 0 & 0 & 0 \\
Middle East & 0 & 0 & 0 & 0 & 0 & 125 \\
Mexico & 0 & 0 & 0 & 0 & 0 & 0 \\
Other Asia & 0 & 0 & 89 & 449 & 1,090 & 1,942 \\
South Korea & 0 & 0 & 0 & 0 & 0 & 0 \\
United States & 0 & 0 & 1,067 & 5,796 & 9,442 & 9,445 \\
Western Europe & 0 & 0 & 275 & 542 & 0 & 0 \\
\hline Total & $\mathbf{0}$ & $\mathbf{0}$ & $\mathbf{1 , 4 9 8}$ & $\mathbf{7 , 0 5 0}$ & $\mathbf{1 1 , 2 7 7}$ & $\mathbf{1 2 , 9 6 3}$ \\
\hline \hline
\end{tabular}

Table A-33: Reference case with credit and tariff extension biomass-to-liquids net trade [Million gallons ethanol equivalent]

\begin{tabular}{lrrrrrr} 
& $\mathbf{2 0 0 5}$ & $\mathbf{2 0 1 0}$ & $\mathbf{2 0 1 5}$ & $\mathbf{2 0 2 0}$ & $\mathbf{2 0 2 5}$ & $\mathbf{2 0 3 0}$ \\
Africa & 0 & 0 & 0 & 0 & 0 & 0 \\
Australia & 0 & 0 & 0 & 0 & 0 & 0 \\
Canada & 0 & 0 & 93 & 280 & 368 & 368 \\
China & 0 & 0 & 57 & 240 & 563 & 563 \\
Central and South America & 0 & 0 & 241 & 614 & 1,108 & 1,362 \\
Eastern Europe & 0 & 0 & 0 & 0 & 0 & 0 \\
Former Soviet Union & 0 & 0 & 0 & 0 & 0 & 0 \\
India & 0 & 0 & 77 & 232 & 544 & 544 \\
Japan & 0 & 0 & 0 & 0 & 0 & 0 \\
Middle East & 0 & 0 & 0 & 0 & 0 & 0 \\
Mexico & 0 & 0 & 45 & 216 & 506 & 556 \\
Other Asia & 0 & 0 & 0 & 0 & 0 & 0 \\
South Korea & 0 & 0 & 36 & 74 & 74 & 74 \\
United States & 0 & 0 & -275 & $-1,114$ & $-3,162$ & $-3,466$ \\
Western Europe & 0 & 0 & -275 & -542 & 0 & 0 \\
\hline Total & $\mathbf{0}$ & $\mathbf{0}$ & $\mathbf{0}$ & $\mathbf{0}$ & $\mathbf{0}$ & $\mathbf{0}$ \\
\hline \hline
\end{tabular}


Table A-34: Reference case with credit and tariff extension biodiesel production [Million gallons ethanol equivalent]

\begin{tabular}{lrrrrrr} 
& $\mathbf{2 0 0 5}$ & $\mathbf{2 0 1 0}$ & $\mathbf{2 0 1 5}$ & $\mathbf{2 0 2 0}$ & $\mathbf{2 0 2 5}$ & $\mathbf{2 0 3 0}$ \\
Africa & 0 & 0 & 0 & 0 & 0 & 0 \\
Argentina & 0 & 333 & 776 & 819 & 827 & 94 \\
Australia & 0 & 0 & 0 & 0 & 0 & 0 \\
Brazil & 0 & 333 & 835 & 1,600 & 2,449 & 1,362 \\
Canada & 0 & 0 & 0 & 0 & 0 & 0 \\
Caribbean basin & 0 & 95 & 310 & 675 & 802 & 898 \\
China & 0 & 0 & 0 & 0 & 0 & 0 \\
Colombia & 0 & 119 & 298 & 474 & 650 & 782 \\
Eastern Europe & 0 & 0 & 0 & 0 & 0 & 0 \\
Former Soviet Union & 0 & 0 & 0 & 0 & 0 & 0 \\
India & 0 & 0 & 0 & 0 & 0 & 0 \\
Japan & 0 & 0 & 0 & 0 & 0 & 0 \\
Middle East & 0 & 0 & 0 & 0 & 0 & 0 \\
Mexico & 0 & 0 & 0 & 0 & 0 & 0 \\
Other Asia & 0 & 0 & 0 & 0 & 0 & 0 \\
South Korea & 0 & 0 & 0 & 0 & 0 & 0 \\
United States & 0 & 1,182 & 1,640 & 1,747 & 1,907 & 2,016 \\
Western Europe & 998 & 3,178 & 3,578 & 3,930 & 4,468 & 4,788 \\
\hline Total & $\mathbf{9 9 8}$ & $\mathbf{5 , 2 3 8}$ & $\mathbf{7 , 4 3 7}$ & $\mathbf{9 , 2 4 5}$ & $\mathbf{1 1 , 1 0 3}$ & $\mathbf{9 , 9 4 0}$ \\
\hline \hline
\end{tabular}

Table A-35: Reference case with credit and tariff extension biodiesel consumption [Million gallons ethanol equivalent]

\begin{tabular}{lrrrrrr} 
& $\mathbf{2 0 0 5}$ & $\mathbf{2 0 1 0}$ & $\mathbf{2 0 1 5}$ & $\mathbf{2 0 2 0}$ & $\mathbf{2 0 2 5}$ & $\mathbf{2 0 3 0}$ \\
Africa & 0 & 0 & 0 & 0 & 0 & 0 \\
Australia & 0 & 0 & 0 & 0 & 0 & 0 \\
Canada & 0 & 0 & 0 & 0 & 0 & 0 \\
China & 0 & 0 & 0 & 0 & 0 & 0 \\
Central and South America & 0 & 158 & 472 & 984 & 1,306 & 1,490 \\
Eastern Europe & 0 & 0 & 0 & 0 & 0 & 0 \\
Former Soviet Union & 0 & 0 & 0 & 0 & 0 & 0 \\
India & 0 & 0 & 0 & 0 & 0 & 0 \\
Japan & 0 & 0 & 0 & 0 & 0 & 0 \\
Middle East & 0 & 0 & 0 & 0 & 0 & 0 \\
Mexico & 0 & 0 & 0 & 0 & 0 & 0 \\
Other Asia & 0 & 0 & 0 & 0 & 0 & 0 \\
South Korea & 0 & 0 & 0 & 0 & 0 & 0 \\
United States & 0 & 1,182 & 1,713 & 2,159 & 2,562 & 2,016 \\
Western Europe & 998 & 3,898 & 5,252 & 6,102 & 7,235 & 6,434 \\
\hline Total & $\mathbf{9 9 8}$ & $\mathbf{5 , 2 3 8}$ & $\mathbf{7 , 4 3 7}$ & $\mathbf{9 , 2 4 5}$ & $\mathbf{1 1 , 1 0 3}$ & $\mathbf{9 , 9 4 0}$ \\
\hline \hline
\end{tabular}


Table A-36: Reference case with credit and tariff extension biodiesel net trade [Million gallons ethanol equivalent]

\begin{tabular}{l} 
Africa \\
Australia \\
Canada \\
China \\
Central and South America \\
Eastern Europe \\
Former Soviet Union \\
India \\
Japan \\
Middle East \\
Mexico \\
Other Asia \\
South Korea \\
United States \\
Western Europe \\
\hline Total
\end{tabular}

\begin{abstract}
2005
\end{abstract}

0

0
0

0

0

0

0

0

0
0

0

0

0

0

0

0

Total

\begin{tabular}{rrrrr}
$\mathbf{2 0 1 0}$ & $\mathbf{2 0 1 5}$ & $\mathbf{2 0 2 0}$ & $\mathbf{2 0 2 5}$ & $\mathbf{2 0 3 0}$ \\
0 & 0 & 0 & 0 & 0 \\
0 & 0 & 0 & 0 & 0 \\
0 & 0 & 0 & 0 & 0 \\
0 & 0 & 0 & 0 & 0 \\
720 & 1,746 & 2,584 & 3,422 & 1,646 \\
0 & 0 & 0 & 0 & 0 \\
0 & 0 & 0 & 0 & 0 \\
0 & 0 & 0 & 0 & 0 \\
0 & 0 & 0 & 0 & 0 \\
0 & 0 & 0 & 0 & 0 \\
0 & 0 & 0 & 0 & 0 \\
0 & 0 & 0 & 0 & 0 \\
0 & 0 & 0 & 0 & 0 \\
0 & -73 & -412 & -655 & 0 \\
-720 & $-1,674$ & $-2,172$ & $-2,767$ & $-1,646$ \\
\hline $\mathbf{0}$ & $\mathbf{0}$ & $\mathbf{0}$ & $\mathbf{0}$ & $\mathbf{0}$ \\
\hline
\end{tabular}




\section{A-3 Reference Case with Credit Extension}

\section{Table A-37: Reference case with credit extension ethanol production [Million} gallons]

\begin{tabular}{lrrrrrr} 
& $\mathbf{2 0 0 5}$ & $\mathbf{2 0 1 0}$ & $\mathbf{2 0 1 5}$ & $\mathbf{2 0 2 0}$ & $\mathbf{2 0 2 5}$ & $\mathbf{2 0 3 0}$ \\
Africa & 300 & 330 & 364 & 402 & 455 & 502 \\
Argentina & 0 & 178 & 810 & 910 & 940 & 822 \\
Australia & 0 & 0 & 71 & 267 & 709 & 1,174 \\
Brazil & 4,866 & 5,977 & 7,624 & 9,752 & 11,934 & 13,669 \\
Canada & 0 & 0 & 259 & 779 & 1,115 & 1,115 \\
Caribbean basin & 210 & 328 & 671 & 683 & 775 & 775 \\
China & 344 & 384 & 634 & 1,041 & 1,612 & 2,917 \\
Colombia & 196 & 215 & 411 & 782 & 1,501 & 2,195 \\
Eastern Europe & 110 & 226 & 434 & 780 & 1,037 & 1,149 \\
Former Soviet Union & 0 & 0 & 39 & 119 & 364 & 1,109 \\
India & 313 & 279 & 541 & 992 & 1,893 & 2,771 \\
Japan & 0 & 0 & 95 & 369 & 612 & 647 \\
Middle East & 0 & 0 & 0 & 0 & 0 & 0 \\
Mexico & 0 & 0 & 199 & 601 & 1,408 & 1,546 \\
Other Asia & 0 & 0 & 0 & 0 & 0 & 0 \\
South Korea & 0 & 0 & 35 & 35 & 35 & 35 \\
United States & 3,850 & 11,668 & 15,822 & 17,443 & 19,318 & 20,552 \\
Western Europe & 600 & 2,568 & 2,755 & 3,398 & 5,291 & 8,013 \\
\hline Total & $\mathbf{1 0 , 7 8 9}$ & $\mathbf{2 2 , 1 5 4}$ & $\mathbf{3 0 , 7 6 5}$ & $\mathbf{3 8 , 3 5 0}$ & $\mathbf{4 8 , 9 9 8}$ & $\mathbf{5 8 , 9 9 0}$ \\
\hline \hline
\end{tabular}




\section{Table A-38: Reference case with credit extension ethanol consumption}

\section{[Million gallons]}

\begin{tabular}{lrrrrrr} 
& $\mathbf{2 0 0 5}$ & $\mathbf{2 0 1 0}$ & $\mathbf{2 0 1 5}$ & $\mathbf{2 0 2 0}$ & $\mathbf{2 0 2 5}$ & $\mathbf{2 0 3 0}$ \\
Africa & 300 & 330 & 364 & 402 & 455 & 502 \\
Australia & 0 & 0 & 71 & 267 & 709 & 1,174 \\
Canada & 145 & 0 & 233 & 808 & 1,004 & 1,020 \\
China & 121 & 0 & 0 & 528 & 1,612 & 3,289 \\
Central and South America & 3,788 & 4,853 & 6,163 & 7,153 & 7,962 & 8,845 \\
Eastern Europe & 699 & 226 & 872 & 953 & 1,037 & 1,149 \\
Former Soviet Union & 0 & 0 & 39 & 119 & 364 & 1,109 \\
India & 313 & 279 & 541 & 992 & 1,893 & 2,399 \\
Japan & 263 & 288 & 373 & 719 & 1,008 & 1,100 \\
Middle East & 0 & 0 & 0 & 0 & 0 & 0 \\
Mexico & 0 & 0 & 30 & 249 & 1,031 & 1,053 \\
Other Asia & 0 & 0 & 0 & 0 & 0 & 0 \\
South Korea & 264 & 335 & 400 & 414 & 413 & 413 \\
United States & 4,161 & 11,668 & 17,383 & 19,985 & 23,897 & 28,924 \\
Western Europe & 736 & 4,174 & 4,276 & 5,249 & 7,649 & 8,013 \\
\hline Total & $\mathbf{1 0 , 7 8 9}$ & $\mathbf{2 2 , 1 5 4}$ & $\mathbf{3 0 , 7 4 4}$ & $\mathbf{3 7 , 8 3 8}$ & $\mathbf{4 9 , 0 3 3}$ & $\mathbf{6 1 , 0 2 0}$ \\
\hline \hline
\end{tabular}

Table A-39: Reference case with credit extension ethanol net trade [Million gallons]

Africa

Australia

Canada

China

Central and South America

Eastern Europe

Former Soviet Union

India

Japan

Middle East

Mexico

Other Asia

South Korea

United States

Western Europe

Total

\begin{tabular}{rrrrrr}
$\mathbf{2 0 0 5}$ & $\mathbf{2 0 1 0}$ & $\mathbf{2 0 1 5}$ & $\mathbf{2 0 2 0}$ & $\mathbf{2 0 2 5}$ & $\mathbf{2 0 3 0}$ \\
0 & 0 & 0 & 0 & 0 & 0 \\
0 & 0 & 0 & 0 & 0 & 0 \\
-145 & 0 & 26 & -29 & 112 & 96 \\
224 & 384 & 634 & 512 & 0 & -372 \\
1,484 & 1,845 & 3,354 & 4,974 & 7,187 & 8,615 \\
-589 & 0 & -438 & -173 & 0 & 0 \\
0 & 0 & 0 & 0 & 0 & 0 \\
0 & 0 & 0 & 0 & 0 & 372 \\
-263 & -288 & -278 & -350 & -395 & -453 \\
0 & 0 & 0 & 0 & 0 & 0 \\
0 & 0 & 170 & 351 & 377 & 493 \\
0 & 0 & 0 & 0 & 0 & 0 \\
-264 & -335 & -365 & -379 & -378 & -378 \\
-311 & 0 & $-1,581$ & $-3,054$ & $-4,544$ & $-8,373$ \\
-136 & $-1,607$ & $-1,521$ & $-1,851$ & $-2,358$ & 0 \\
\hline $\mathbf{0}$ & $\mathbf{0}$ & $\mathbf{0}$ & $\mathbf{0}$ & $\mathbf{0}$ & $\mathbf{0}$ \\
\hline
\end{tabular}


Table A-40: Reference case with credit extension grain ethanol production [Million gallons]

\begin{tabular}{lrrrrrr} 
& $\mathbf{2 0 0 5}$ & $\mathbf{2 0 1 0}$ & $\mathbf{2 0 1 5}$ & $\mathbf{2 0 2 0}$ & $\mathbf{2 0 2 5}$ & $\mathbf{2 0 3 0}$ \\
Africa & 0 & 0 & 0 & 0 & 0 & 0 \\
Argentina & 0 & 178 & 604 & 604 & 604 & 475 \\
Australia & 0 & 0 & 0 & 0 & 0 & 0 \\
Brazil & 0 & 0 & 0 & 0 & 0 & 0 \\
Canada & 0 & 0 & 0 & 0 & 0 & 0 \\
Caribbean basin & 0 & 0 & 0 & 0 & 0 & 0 \\
China & 344 & 344 & 344 & 325 & 0 & 0 \\
Colombia & 0 & 0 & 0 & 0 & 0 & 0 \\
Eastern Europe & 110 & 226 & 348 & 477 & 367 & 251 \\
Former Soviet Union & 0 & 0 & 0 & 0 & 0 & 0 \\
India & 0 & 0 & 0 & 0 & 0 & 0 \\
Japan & 0 & 0 & 0 & 0 & 0 & 0 \\
Middle East & 0 & 0 & 0 & 0 & 0 & 0 \\
Mexico & 0 & 0 & 0 & 0 & 0 & 0 \\
Other Asia & 0 & 0 & 0 & 0 & 0 & 0 \\
South Korea & 0 & 0 & 0 & 0 & 0 & 0 \\
United States & 3,850 & 11,668 & 15,000 & 15,000 & 15,000 & 13,716 \\
Western Europe & 600 & 2,568 & 2,589 & 2,705 & 3,178 & 3,604 \\
\hline Total & $\mathbf{4 , 9 0 4}$ & $\mathbf{1 4 , 9 8 4}$ & $\mathbf{1 8 , 8 8 6}$ & $\mathbf{1 9 , 1 1 2}$ & $\mathbf{1 9 , 1 4 9}$ & $\mathbf{1 8 , 0 4 6}$ \\
\hline \hline
\end{tabular}

Table A-41: Reference case with credit extension sugar ethanol production [Million gallons]

\begin{tabular}{lrrrrrr} 
& $\mathbf{2 0 0 5}$ & $\mathbf{2 0 1 0}$ & $\mathbf{2 0 1 5}$ & $\mathbf{2 0 2 0}$ & $\mathbf{2 0 2 5}$ & $\mathbf{2 0 3 0}$ \\
Africa & 300 & 330 & 364 & 402 & 444 & 490 \\
Argentina & 0 & 0 & 0 & 0 & 0 & 0 \\
Australia & 0 & 0 & 0 & 0 & 0 & 0 \\
Brazil & 4,866 & 5,977 & 7,329 & 8,862 & 10,057 & 11,793 \\
Canada & 0 & 0 & 0 & 0 & 0 & 0 \\
Caribbean basin & 210 & 328 & 671 & 683 & 775 & 775 \\
China & 0 & 40 & 48 & 48 & 48 & 48 \\
Colombia & 196 & 215 & 241 & 270 & 302 & 302 \\
Eastern Europe & 0 & 0 & 0 & 0 & 0 & 0 \\
Former Soviet Union & 0 & 0 & 0 & 0 & 0 & 0 \\
India & 313 & 279 & 327 & 347 & 381 & 348 \\
Japan & 0 & 0 & 0 & 0 & 0 & 0 \\
Middle East & 0 & 0 & 0 & 0 & 0 & 0 \\
Mexico & 0 & 0 & 0 & 0 & 0 & 0 \\
Other Asia & 0 & 0 & 0 & 0 & 0 & 0 \\
South Korea & 0 & 0 & 0 & 0 & 0 & 0 \\
United States & 0 & 0 & 0 & 0 & 0 & 0 \\
Western Europe & 0 & 0 & 0 & 0 & 0 & 0 \\
\hline Total & $\mathbf{5 , 8 8 4}$ & $\mathbf{7 , 1 6 9}$ & $\mathbf{8 , 9 8 0}$ & $\mathbf{1 0 , 6 1 2}$ & $\mathbf{1 2 , 0 0 7}$ & $\mathbf{1 3 , 7 5 5}$ \\
\hline \hline
\end{tabular}


Table A-42: Reference case with credit extension cellulosic ethanol production [Million gallons]

\begin{tabular}{lrrrrrr} 
& $\mathbf{2 0 0 5}$ & $\mathbf{2 0 1 0}$ & $\mathbf{2 0 1 5}$ & $\mathbf{2 0 2 0}$ & $\mathbf{2 0 2 5}$ & $\mathbf{2 0 3 0}$ \\
Africa & 0 & 0 & 0 & 0 & 11 & 11 \\
Argentina & 0 & 0 & 206 & 306 & 335 & 347 \\
Australia & 0 & 0 & 71 & 267 & 709 & 1,174 \\
Brazil & 0 & 0 & 296 & 890 & 1,877 & 1,876 \\
Canada & 0 & 0 & 259 & 779 & 1,115 & 1,115 \\
Caribbean basin & 0 & 0 & 0 & 0 & 0 & 0 \\
China & 0 & 0 & 243 & 667 & 1,564 & 2,869 \\
Colombia & 0 & 0 & 170 & 512 & 1,199 & 1,893 \\
Eastern Europe & 0 & 0 & 85 & 303 & 670 & 897 \\
Former Soviet Union & 0 & 0 & 39 & 119 & 364 & 1,109 \\
India & 0 & 0 & 214 & 645 & 1,512 & 2,424 \\
Japan & 0 & 0 & 95 & 369 & 612 & 647 \\
Middle East & 0 & 0 & 0 & 0 & 0 & 0 \\
Mexico & 0 & 0 & 199 & 601 & 1,408 & 1,546 \\
Other Asia & 0 & 0 & 0 & 0 & 0 & 0 \\
South Korea & 0 & 0 & 35 & 35 & 35 & 35 \\
United States & 0 & 0 & 802 & 1,893 & 4,318 & 6,835 \\
Western Europe & 0 & 0 & 166 & 693 & 2,113 & 4,410 \\
\hline Total & $\mathbf{0}$ & $\mathbf{0}$ & $\mathbf{2 , 8 7 9}$ & $\mathbf{8 , 0 7 7}$ & $\mathbf{1 7 , 8 4 2}$ & $\mathbf{2 7 , 1 8 8}$ \\
\hline \hline
\end{tabular}

Table A-43: Reference case with credit extension grain ethanol consumption [Million gallons]

\begin{tabular}{lrrrrrr} 
& $\mathbf{2 0 0 5}$ & $\mathbf{2 0 1 0}$ & $\mathbf{2 0 1 5}$ & $\mathbf{2 0 2 0}$ & $\mathbf{2 0 2 5}$ & $\mathbf{2 0 3 0}$ \\
Africa & 0 & 0 & 0 & 0 & 0 & 0 \\
Australia & 0 & 0 & 0 & 0 & 0 & 0 \\
Canada & 0 & 0 & 0 & 0 & 0 & 0 \\
China & 121 & 0 & 0 & 325 & 0 & 0 \\
Central and South America & 0 & 36 & 122 & 118 & 98 & 87 \\
Eastern Europe & 110 & 226 & 348 & 477 & 367 & 251 \\
Former Soviet Union & 0 & 0 & 0 & 0 & 0 & 0 \\
India & 0 & 0 & 0 & 0 & 0 & 0 \\
Japan & 0 & 192 & 230 & 0 & 284 & 357 \\
Middle East & 0 & 0 & 0 & 0 & 0 & 0 \\
Mexico & 0 & 0 & 0 & 0 & 0 & 0 \\
Other Asia & 0 & 0 & 0 & 0 & 0 & 0 \\
South Korea & 224 & 295 & 123 & 217 & 222 & 31 \\
United States & 3,850 & 11,668 & 15,000 & 15,000 & 15,000 & 13,716 \\
Western Europe & 600 & 2,568 & 3,063 & 2,974 & 3,178 & 3,604 \\
\hline Total & $\mathbf{4 , 9 0 4}$ & $\mathbf{1 4 , 9 8 4}$ & $\mathbf{1 8 , 8 8 6}$ & $\mathbf{1 9 , 1 1 2}$ & $\mathbf{1 9 , 1 4 9}$ & $\mathbf{1 8 , 0 4 6}$ \\
\hline \hline
\end{tabular}


Table A-44: Reference case with credit extension sugar ethanol consumption [Million gallons]

\begin{tabular}{lrrrrrr} 
& $\mathbf{2 0 0 5}$ & $\mathbf{2 0 1 0}$ & $\mathbf{2 0 1 5}$ & $\mathbf{2 0 2 0}$ & $\mathbf{2 0 2 5}$ & $\mathbf{2 0 3 0}$ \\
Africa & 300 & 330 & 364 & 402 & 444 & 490 \\
Australia & 0 & 0 & 0 & 0 & 0 & 0 \\
Canada & 145 & 0 & 0 & 0 & 0 & 0 \\
China & 0 & 0 & 0 & 48 & 48 & 48 \\
Central and South America & 3,788 & 4,818 & 6,041 & 6,910 & 7,864 & 8,602 \\
Eastern Europe & 589 & 0 & 412 & 173 & 0 & 0 \\
Former Soviet Union & 0 & 0 & 0 & 0 & 0 & 0 \\
India & 313 & 279 & 327 & 347 & 381 & 348 \\
Japan & 263 & 96 & 48 & 0 & 0 & 0 \\
Middle East & 0 & 0 & 0 & 0 & 0 & 0 \\
Mexico & 0 & 0 & 0 & 0 & 0 & 0 \\
Other Asia & 0 & 0 & 0 & 0 & 0 & 0 \\
South Korea & 41 & 40 & 0 & 0 & 0 & 0 \\
United States & 311 & 0 & 788 & 2,732 & 2,920 & 4,268 \\
Western Europe & 136 & 1,607 & 1,000 & 0 & 350 & 0 \\
\hline Total & $\mathbf{5 , 8 8 4}$ & $\mathbf{7 , 1 6 9}$ & $\mathbf{8 , 9 8 0}$ & $\mathbf{1 0 , 6 1 2}$ & $\mathbf{1 2 , 0 0 7}$ & $\mathbf{1 3 , 7 5 5}$ \\
\hline \hline
\end{tabular}

Table A-45: Reference case with credit extension cellulosic ethanol consumption [Million gallons]

\begin{tabular}{lrrrrrr} 
& $\mathbf{2 0 0 5}$ & $\mathbf{2 0 1 0}$ & $\mathbf{2 0 1 5}$ & $\mathbf{2 0 2 0}$ & $\mathbf{2 0 2 5}$ & $\mathbf{2 0 3 0}$ \\
Africa & 0 & 0 & 0 & 0 & 11 & 11 \\
Australia & 0 & 0 & 71 & 267 & 709 & 1,174 \\
Canada & 0 & 0 & 233 & 808 & 1,004 & 1,020 \\
China & 0 & 0 & 0 & 155 & 1,564 & 3,241 \\
Central and South America & 0 & 0 & 0 & 125 & 0 & 157 \\
Eastern Europe & 0 & 0 & 111 & 303 & 670 & 897 \\
Former Soviet Union & 0 & 0 & 39 & 119 & 364 & 1,109 \\
India & 0 & 0 & 214 & 645 & 1,512 & 2,051 \\
Japan & 0 & 0 & 95 & 719 & 724 & 743 \\
Middle East & 0 & 0 & 0 & 0 & 0 & 0 \\
Mexico & 0 & 0 & 30 & 249 & 1,031 & 1,053 \\
Other Asia & 0 & 0 & 0 & 0 & 0 & 0 \\
South Korea & 0 & 0 & 277 & 197 & 191 & 382 \\
United States & 0 & 0 & 1,595 & 2,215 & 5,942 & 10,940 \\
Western Europe & 0 & 0 & 213 & 2,275 & 4,121 & 4,410 \\
\hline Total & $\mathbf{0}$ & $\mathbf{0}$ & $\mathbf{2 , 8 7 9}$ & $\mathbf{8 , 0 7 7}$ & $\mathbf{1 7 , 8 4 2}$ & $\mathbf{2 7 , 1 8 8}$ \\
\hline \hline
\end{tabular}


Table A-46: Reference case with credit extension grain ethanol net trade [Million gallons]

\begin{tabular}{lrrrrrr} 
& $\mathbf{2 0 0 5}$ & $\mathbf{2 0 1 0}$ & $\mathbf{2 0 1 5}$ & $\mathbf{2 0 2 0}$ & $\mathbf{2 0 2 5}$ & $\mathbf{2 0 3 0}$ \\
Africa & 0 & 0 & 0 & 0 & 0 & 0 \\
Australia & 0 & 0 & 0 & 0 & 0 & 0 \\
Canada & 0 & 0 & 0 & 0 & 0 & 0 \\
China & 224 & 344 & 344 & 0 & 0 & 0 \\
Central and South America & 0 & 143 & 483 & 486 & 506 & 388 \\
Eastern Europe & 0 & 0 & 0 & 0 & 0 & 0 \\
Former Soviet Union & 0 & 0 & 0 & 0 & 0 & 0 \\
India & 0 & 0 & 0 & 0 & 0 & 0 \\
Japan & 0 & -192 & -230 & 0 & -284 & -357 \\
Middle East & 0 & 0 & 0 & 0 & 0 & 0 \\
Mexico & 0 & 0 & 0 & 0 & 0 & 0 \\
Other Asia & 0 & 0 & 0 & 0 & 0 & 0 \\
South Korea & -224 & -295 & -123 & -217 & -222 & -31 \\
United States & 0 & 0 & 0 & 0 & 0 & 0 \\
Western Europe & 0 & 0 & -474 & -269 & 0 & 0 \\
\hline Total & $\mathbf{0}$ & $\mathbf{0}$ & $\mathbf{0}$ & $\mathbf{0}$ & $\mathbf{0}$ & $\mathbf{0}$ \\
\hline \hline
\end{tabular}

Table A-47: Reference case with credit extension sugar ethanol net trade [Million gallons]

\begin{tabular}{lrrrrrr} 
Africa & 0 & 0 & 0 & 0 & 0 & 0 \\
Australia & 0 & 0 & 0 & 0 & 0 & 0 \\
Canada & -145 & 0 & 0 & 0 & 0 & 0 \\
China & 0 & 40 & 48 & 0 & 0 & 0 \\
Central and South America & 1,484 & 1,702 & 2,200 & 2,905 & 3,270 & 4,268 \\
Eastern Europe & -589 & 0 & -412 & -173 & 0 & 0 \\
Former Soviet Union & 0 & 0 & 0 & 0 & 0 & 0 \\
India & 0 & 0 & 0 & 0 & 0 & 0 \\
Japan & -263 & -96 & -48 & 0 & 0 & 0 \\
Middle East & 0 & 0 & 0 & 0 & 0 & 0 \\
Mexico & 0 & 0 & 0 & 0 & 0 & 0 \\
Other Asia & 0 & 0 & 0 & 0 & 0 & 0 \\
South Korea & -41 & -40 & 0 & 0 & 0 & 0 \\
United States & -311 & 0 & -788 & $-2,732$ & $-2,920$ & $-4,268$ \\
Western Europe & -136 & $-1,607$ & $-1,000$ & 0 & -350 & 0 \\
\hline Total & $\mathbf{0}$ & $\mathbf{0}$ & $\mathbf{0}$ & $\mathbf{0}$ & $\mathbf{0}$ & $\mathbf{0}$ \\
\hline \hline
\end{tabular}


Table A-48: Reference case with credit extension cellulosic ethanol net trade [Million gallons]

\begin{tabular}{lrrrrrr} 
& $\mathbf{2 0 0 5}$ & $\mathbf{2 0 1 0}$ & $\mathbf{2 0 1 5}$ & $\mathbf{2 0 2 0}$ & $\mathbf{2 0 2 5}$ & $\mathbf{2 0 3 0}$ \\
Africa & 0 & 0 & 0 & 0 & 0 & 0 \\
Australia & 0 & 0 & 0 & 0 & 0 & 0 \\
Canada & 0 & 0 & 26 & -29 & 112 & 96 \\
China & 0 & 0 & 243 & 512 & 0 & -372 \\
Central and South America & 0 & 0 & 671 & 1,583 & 3,411 & 3,959 \\
Eastern Europe & 0 & 0 & -26 & 0 & 0 & 0 \\
Former Soviet Union & 0 & 0 & 0 & 0 & 0 & 0 \\
India & 0 & 0 & 0 & 0 & 0 & 372 \\
Japan & 0 & 0 & 0 & -350 & -112 & -96 \\
Middle East & 0 & 0 & 0 & 0 & 0 & 0 \\
Mexico & 0 & 0 & 170 & 351 & 377 & 493 \\
Other Asia & 0 & 0 & 0 & 0 & 0 & 0 \\
South Korea & 0 & 0 & -243 & -162 & -156 & -347 \\
United States & 0 & 0 & -793 & -322 & $-1,624$ & $-4,105$ \\
Western Europe & 0 & 0 & -47 & $-1,583$ & $-2,008$ & 0 \\
\hline Total & $\mathbf{0}$ & $\mathbf{0}$ & $\mathbf{0}$ & $\mathbf{0}$ & $\mathbf{0}$ & $\mathbf{0}$ \\
\hline \hline
\end{tabular}

Table A-49: Reference case with credit extension biomass-to-liquids production [Million gallons ethanol equivalent]

\begin{tabular}{lrrrrrr} 
& $\mathbf{2 0 0 5}$ & $\mathbf{2 0 1 0}$ & $\mathbf{2 0 1 5}$ & $\mathbf{2 0 2 0}$ & $\mathbf{2 0 2 5}$ & $\mathbf{2 0 3 0}$ \\
Africa & 0 & 0 & 68 & 263 & 653 & 1,177 \\
Argentina & 0 & 0 & 74 & 110 & 121 & 125 \\
Australia & 0 & 0 & 0 & 0 & 0 & 0 \\
Brazil & 0 & 0 & 106 & 320 & 675 & 675 \\
Canada & 0 & 0 & 93 & 280 & 401 & 401 \\
Caribbean basin & 0 & 0 & 0 & 0 & 0 & 0 \\
China & 0 & 0 & 57 & 240 & 563 & 563 \\
Colombia & 0 & 0 & 61 & 184 & 431 & 681 \\
Eastern Europe & 0 & 0 & 0 & 0 & 93 & 274 \\
Former Soviet Union & 0 & 0 & 0 & 0 & 0 & 0 \\
India & 0 & 0 & 77 & 232 & 544 & 544 \\
Japan & 0 & 0 & 0 & 0 & 0 & 0 \\
Middle East & 0 & 0 & 0 & 0 & 0 & 125 \\
Mexico & 0 & 0 & 72 & 216 & 506 & 556 \\
Other Asia & 0 & 0 & 89 & 448 & 1,090 & 1,942 \\
South Korea & 0 & 0 & 36 & 74 & 74 & 74 \\
United States & 0 & 0 & 822 & 4,038 & 5,403 & 6,735 \\
Western Europe & 0 & 0 & 0 & 0 & 0 & 0 \\
\hline Total & $\mathbf{0}$ & $\mathbf{0}$ & $\mathbf{1 , 5 5 5}$ & $\mathbf{6 , 4 0 5}$ & $\mathbf{1 0 , 5 5 4}$ & $\mathbf{1 3 , 8 7 1}$ \\
\hline \hline
\end{tabular}


Table A-50: Reference case with credit extension biomass-to-liquids consumption [Million gallons ethanol equivalent]

\begin{tabular}{lrrrrrr} 
& $\mathbf{2 0 0 5}$ & $\mathbf{2 0 1 0}$ & $\mathbf{2 0 1 5}$ & $\mathbf{2 0 2 0}$ & $\mathbf{2 0 2 5}$ & $\mathbf{2 0 3 0}$ \\
Africa & 0 & 0 & 68 & 263 & 653 & 1,177 \\
Australia & 0 & 0 & 0 & 0 & 0 & 0 \\
Canada & 0 & 0 & 0 & 0 & 0 & 0 \\
China & 0 & 0 & 0 & 0 & 0 & 0 \\
Central and South America & 0 & 0 & 0 & 0 & 0 & 0 \\
Eastern Europe & 0 & 0 & 0 & 0 & 93 & 274 \\
Former Soviet Union & 0 & 0 & 0 & 0 & 0 & 0 \\
India & 0 & 0 & 0 & 0 & 0 & 0 \\
Japan & 0 & 0 & 0 & 0 & 0 & 0 \\
Middle East & 0 & 0 & 0 & 0 & 0 & 125 \\
Mexico & 0 & 0 & 0 & 0 & 0 & 0 \\
Other Asia & 0 & 0 & 89 & 449 & 1,090 & 1,942 \\
South Korea & 0 & 0 & 0 & 0 & 0 & 0 \\
United States & 0 & 0 & 1,100 & 5,606 & 8,718 & 9,295 \\
Western Europe & 0 & 0 & 298 & 88 & 0 & 1,058 \\
\hline Total & $\mathbf{0}$ & $\mathbf{0}$ & $\mathbf{1 , 5 5 5}$ & $\mathbf{6 , 4 0 5}$ & $\mathbf{1 0 , 5 5 3}$ & $\mathbf{1 3 , 8 7 1}$ \\
\hline \hline
\end{tabular}

Table A-51: Reference case with credit extension biomass-to-liquids net trade [Million gallons ethanol equivalent]

\begin{tabular}{lrrrrrr} 
& $\mathbf{2 0 0 5}$ & $\mathbf{2 0 1 0}$ & $\mathbf{2 0 1 5}$ & $\mathbf{2 0 2 0}$ & $\mathbf{2 0 2 5}$ & $\mathbf{2 0 3 0}$ \\
Africa & 0 & 0 & 0 & 0 & 0 & 0 \\
Australia & 0 & 0 & 0 & 0 & 0 & 0 \\
Canada & 0 & 0 & 93 & 280 & 401 & 401 \\
China & 0 & 0 & 57 & 240 & 563 & 563 \\
Central and South America & 0 & 0 & 241 & 614 & 1,227 & 1,481 \\
Eastern Europe & 0 & 0 & 0 & 0 & 0 & 0 \\
Former Soviet Union & 0 & 0 & 0 & 0 & 0 & 0 \\
India & 0 & 0 & 77 & 232 & 544 & 544 \\
Japan & 0 & 0 & 0 & 0 & 0 & 0 \\
Middle East & 0 & 0 & 0 & 0 & 0 & 0 \\
Mexico & 0 & 0 & 72 & 216 & 506 & 556 \\
Other Asia & 0 & 0 & 0 & 0 & 0 & 0 \\
South Korea & 0 & 0 & 36 & 74 & 74 & 74 \\
United States & 0 & 0 & -278 & $-1,568$ & $-3,315$ & $-2,560$ \\
Western Europe & 0 & 0 & -298 & -88 & 0 & $-1,058$ \\
\hline Total & $\mathbf{0}$ & $\mathbf{0}$ & $\mathbf{0}$ & $\mathbf{0}$ & $\mathbf{0}$ & $\mathbf{0}$ \\
\hline \hline
\end{tabular}


Table A-52: Reference case with credit extension biodiesel production [Million gallons ethanol equivalent]

\begin{tabular}{lrrrrrr} 
& $\mathbf{2 0 0 5}$ & $\mathbf{2 0 1 0}$ & $\mathbf{2 0 1 5}$ & $\mathbf{2 0 2 0}$ & $\mathbf{2 0 2 5}$ & $\mathbf{2 0 3 0}$ \\
Africa & 0 & 0 & 0 & 0 & 0 & 0 \\
Argentina & 0 & 333 & 776 & 819 & 827 & 94 \\
Australia & 0 & 0 & 0 & 0 & 0 & 0 \\
Brazil & 0 & 333 & 877 & 1,642 & 1,966 & 1,362 \\
Canada & 0 & 0 & 0 & 0 & 0 & 0 \\
Caribbean basin & 0 & 95 & 310 & 675 & 802 & 898 \\
China & 0 & 0 & 0 & 0 & 0 & 0 \\
Colombia & 0 & 119 & 298 & 474 & 650 & 782 \\
Eastern Europe & 0 & 0 & 0 & 0 & 0 & 0 \\
Former Soviet Union & 0 & 0 & 0 & 0 & 0 & 0 \\
India & 0 & 0 & 0 & 0 & 0 & 0 \\
Japan & 0 & 0 & 0 & 0 & 0 & 0 \\
Middle East & 0 & 0 & 0 & 0 & 0 & 0 \\
Mexico & 0 & 0 & 0 & 0 & 0 & 0 \\
Other Asia & 0 & 0 & 0 & 0 & 0 & 0 \\
South Korea & 0 & 0 & 0 & 0 & 0 & 0 \\
United States & 0 & 1,182 & 1,640 & 1,747 & 1,907 & 2,016 \\
Western Europe & 998 & 3,178 & 3,578 & 3,930 & 4,468 & 4,788 \\
\hline Total & $\mathbf{9 9 8}$ & $\mathbf{5 , 2 3 8}$ & $\mathbf{7 , 4 7 9}$ & $\mathbf{9 , 2 8 7}$ & $\mathbf{1 0 , 6 2 1}$ & $\mathbf{9 , 9 4 0}$ \\
\hline \hline
\end{tabular}

Table A-53: Reference case with credit extension biodiesel consumption [Million gallons ethanol equivalent]

\begin{tabular}{lrrrrrr} 
& $\mathbf{2 0 0 5}$ & $\mathbf{2 0 1 0}$ & $\mathbf{2 0 1 5}$ & $\mathbf{2 0 2 0}$ & $\mathbf{2 0 2 5}$ & $\mathbf{2 0 3 0}$ \\
Africa & 0 & 0 & 0 & 0 & 0 & 0 \\
Australia & 0 & 0 & 0 & 0 & 0 & 0 \\
Canada & 0 & 0 & 0 & 0 & 0 & 0 \\
China & 0 & 0 & 0 & 0 & 0 & 0 \\
Central and South America & 0 & 158 & 472 & 984 & 1,306 & 1,490 \\
Eastern Europe & 0 & 0 & 0 & 0 & 0 & 0 \\
Former Soviet Union & 0 & 0 & 0 & 0 & 0 & 0 \\
India & 0 & 0 & 0 & 0 & 0 & 0 \\
Japan & 0 & 0 & 0 & 0 & 0 & 0 \\
Middle East & 0 & 0 & 0 & 0 & 0 & 0 \\
Mexico & 0 & 0 & 0 & 0 & 0 & 0 \\
Other Asia & 0 & 0 & 0 & 0 & 0 & 0 \\
South Korea & 0 & 0 & 0 & 0 & 0 & 0 \\
United States & 0 & 1,182 & 1,713 & 1,747 & 2,080 & 2,016 \\
Western Europe & 998 & 3,898 & 5,294 & 6,556 & 7,235 & 6,434 \\
\hline Total & $\mathbf{9 9 8}$ & $\mathbf{5 , 2 3 8}$ & $\mathbf{7 , 4 7 9}$ & $\mathbf{9 , 2 8 7}$ & $\mathbf{1 0 , 6 2 1}$ & $\mathbf{9 , 9 4 0}$ \\
\hline \hline
\end{tabular}


Table A-54: Reference case with credit extension biodiesel net trade [Million gallons ethanol equivalent]

\begin{tabular}{lrrrrrr} 
& $\mathbf{2 0 0 5}$ & $\mathbf{2 0 1 0}$ & $\mathbf{2 0 1 5}$ & $\mathbf{2 0 2 0}$ & $\mathbf{2 0 2 5}$ & $\mathbf{2 0 3 0}$ \\
Africa & 0 & 0 & 0 & 0 & 0 & 0 \\
Australia & 0 & 0 & 0 & 0 & 0 & 0 \\
Canada & 0 & 0 & 0 & 0 & 0 & 0 \\
China & 0 & 0 & 0 & 0 & 0 & 0 \\
Central and South America & 0 & 720 & 1,788 & 2,626 & 2,940 & 1,646 \\
Eastern Europe & 0 & 0 & 0 & 0 & 0 & 0 \\
Former Soviet Union & 0 & 0 & 0 & 0 & 0 & 0 \\
India & 0 & 0 & 0 & 0 & 0 & 0 \\
Japan & 0 & 0 & 0 & 0 & 0 & 0 \\
Middle East & 0 & 0 & 0 & 0 & 0 & 0 \\
Mexico & 0 & 0 & 0 & 0 & 0 & 0 \\
Other Asia & 0 & 0 & 0 & 0 & 0 & 0 \\
South Korea & 0 & 0 & 0 & 0 & 0 & 0 \\
United States & 0 & 0 & -73 & 0 & -173 & 0 \\
Western Europe & 0 & -720 & $-1,716$ & $-2,626$ & $-2,767$ & $-1,646$ \\
\hline Total & $\mathbf{0}$ & $\mathbf{0}$ & $\mathbf{0}$ & $\mathbf{0}$ & $\mathbf{0}$ & $\mathbf{0}$ \\
\hline \hline
\end{tabular}




\section{A-4 Reference Case with 70/30 Fuel/Feedstock Split}

Table A-55: Reference Case with 70/30 Fuel/Feedstock split ethanol production [Million gallons]

\begin{tabular}{lrrrrrr} 
& $\mathbf{2 0 0 5}$ & $\mathbf{2 0 1 0}$ & $\mathbf{2 0 1 5}$ & $\mathbf{2 0 2 0}$ & $\mathbf{2 0 2 5}$ & $\mathbf{2 0 3 0}$ \\
Africa & 300 & 330 & 364 & 402 & 455 & 502 \\
Argentina & 0 & 178 & 727 & 875 & 904 & 916 \\
Australia & 0 & 0 & 71 & 267 & 709 & 1,174 \\
Brazil & 5,225 & 6,956 & 8,845 & 10,655 & 12,619 & 14,462 \\
Canada & 0 & 0 & 259 & 779 & 1,115 & 1,115 \\
Caribbean basin & 180 & 299 & 573 & 597 & 597 & 676 \\
China & 344 & 384 & 634 & 1,041 & 1,612 & 2,917 \\
Colombia & 196 & 215 & 411 & 782 & 1,501 & 2,195 \\
Eastern Europe & 110 & 226 & 434 & 780 & 1,037 & 1,149 \\
Former Soviet Union & 0 & 0 & 39 & 119 & 364 & 1,109 \\
India & 313 & 279 & 541 & 992 & 1,893 & 2,771 \\
Japan & 0 & 0 & 95 & 369 & 612 & 647 \\
Middle East & 0 & 0 & 0 & 0 & 0 & 0 \\
Mexico & 0 & 0 & 199 & 601 & 1,408 & 1,546 \\
Other Asia & 0 & 0 & 0 & 0 & 0 & 0 \\
South Korea & 0 & 0 & 35 & 35 & 35 & 35 \\
United States & 3,850 & 11,423 & 14,849 & 17,174 & 18,714 & 18,445 \\
Western Europe & 600 & 2,465 & 2,755 & 3,398 & 5,291 & 8,013 \\
\hline Total & $\mathbf{1 1 , 1 1 8}$ & $\mathbf{2 2 , 7 5 6}$ & $\mathbf{3 0 , 8 3 1}$ & $\mathbf{3 8 , 8 6 2}$ & $\mathbf{4 8 , 8 6 6}$ & $\mathbf{5 7 , 6 7 1}$ \\
\hline \hline
\end{tabular}


Table A-56: Reference Case with 70/30 Fuel/Feedstock split ethanol consumption [Million gallons]

\begin{tabular}{lrrrrrr} 
& $\mathbf{2 0 0 5}$ & $\mathbf{2 0 1 0}$ & $\mathbf{2 0 1 5}$ & $\mathbf{2 0 2 0}$ & $\mathbf{2 0 2 5}$ & $\mathbf{2 0 3 0}$ \\
Africa & 300 & 330 & 364 & 402 & 455 & 502 \\
Australia & 0 & 0 & 71 & 267 & 709 & 1,174 \\
Canada & 119 & 0 & 233 & 984 & 1,004 & 1,020 \\
China & 442 & 0 & 0 & 1,041 & 1,612 & 3,289 \\
Central and South America & 3,785 & 4,851 & 6,144 & 7,144 & 7,948 & 8,848 \\
Eastern Europe & 699 & 783 & 872 & 953 & 1,037 & 1,149 \\
Former Soviet Union & 0 & 0 & 39 & 119 & 364 & 1,109 \\
India & 313 & 279 & 541 & 992 & 1,893 & 2,399 \\
Japan & 300 & 325 & 410 & 751 & 1,039 & 1,100 \\
Middle East & 0 & 0 & 0 & 0 & 0 & 0 \\
Mexico & 0 & 0 & 30 & 601 & 1,031 & 1,053 \\
Other Asia & 0 & 0 & 0 & 0 & 0 & 0 \\
South Korea & 264 & 335 & 400 & 414 & 413 & 413 \\
United States & 4,161 & 11,612 & 17,383 & 19,947 & 23,712 & 27,468 \\
Western Europe & 736 & 4,241 & 4,344 & 5,249 & 7,650 & 8,149 \\
\hline Total & $\mathbf{1 1 , 1 1 8}$ & $\mathbf{2 2 , 7 5 6}$ & $\mathbf{3 0 , 8 3 1}$ & $\mathbf{3 8 , 8 6 2}$ & $\mathbf{4 8 , 8 6 6}$ & $\mathbf{5 7 , 6 7 1}$ \\
\hline \hline
\end{tabular}

Table A-57: Reference Case with 70/30 Fuel/Feedstock split ethanol net trade [Million gallons]

\begin{tabular}{lrrrrrr} 
Africa & 0 & 0 & 0 & 0 & 0 & 0 \\
Australia & 0 & 0 & 0 & 0 & 0 & 0 \\
Canada & -119 & 0 & 26 & -205 & 112 & 96 \\
China & -98 & 384 & 634 & 0 & 0 & -372 \\
Central and South America & 1,816 & 2,797 & 4,412 & 5,763 & 7,673 & 9,401 \\
Eastern Europe & -589 & -557 & -438 & -173 & 0 & 0 \\
Former Soviet Union & 0 & 0 & 0 & 0 & 0 & 0 \\
India & 0 & 0 & 0 & 0 & 0 & 372 \\
Japan & -300 & -325 & -315 & -382 & -427 & -453 \\
Middle East & 0 & 0 & 0 & 0 & 0 & 0 \\
Mexico & 0 & 0 & 170 & 0 & 377 & 493 \\
Other Asia & 0 & 0 & 0 & 0 & 0 & 0 \\
South Korea & -264 & -335 & -365 & -379 & -378 & -378 \\
United States & -311 & -189 & $-2,534$ & $-2,773$ & $-4,999$ & $-9,023$ \\
Western Europe & -136 & $-1,776$ & $-1,589$ & $-1,851$ & $-2,359$ & -135 \\
\hline Total & $\mathbf{0}$ & $\mathbf{0}$ & $\mathbf{0}$ & $\mathbf{0}$ & $\mathbf{0}$ & $\mathbf{0}$ \\
\hline \hline
\end{tabular}


Table A-58: Reference Case with 70/30 Fuel/Feedstock split grain ethanol production [Million gallons]

\begin{tabular}{lrrrrrr} 
& $\mathbf{2 0 0 5}$ & $\mathbf{2 0 1 0}$ & $\mathbf{2 0 1 5}$ & $\mathbf{2 0 2 0}$ & $\mathbf{2 0 2 5}$ & $\mathbf{2 0 3 0}$ \\
Africa & 0 & 0 & 0 & 0 & 0 & 0 \\
Argentina & 0 & 178 & 569 & 569 & 569 & 569 \\
Australia & 0 & 0 & 0 & 0 & 0 & 0 \\
Brazil & 0 & 0 & 0 & 0 & 0 & 0 \\
Canada & 0 & 0 & 0 & 0 & 0 & 0 \\
Caribbean basin & 0 & 0 & 0 & 0 & 0 & 0 \\
China & 344 & 344 & 344 & 325 & 0 & 0 \\
Colombia & 0 & 0 & 0 & 0 & 0 & 0 \\
Eastern Europe & 110 & 226 & 348 & 477 & 367 & 251 \\
Former Soviet Union & 0 & 0 & 0 & 0 & 0 & 0 \\
India & 0 & 0 & 0 & 0 & 0 & 0 \\
Japan & 0 & 0 & 0 & 0 & 0 & 0 \\
Middle East & 0 & 0 & 0 & 0 & 0 & 0 \\
Mexico & 0 & 0 & 0 & 0 & 0 & 0 \\
Other Asia & 0 & 0 & 0 & 0 & 0 & 0 \\
South Korea & 0 & 0 & 0 & 0 & 0 & 0 \\
United States & 3,850 & 11,423 & 14,047 & 15,000 & 14,815 & 13,281 \\
Western Europe & 600 & 2,465 & 2,589 & 2,705 & 3,178 & 3,604 \\
\hline Total & $\mathbf{4 , 9 0 4}$ & $\mathbf{1 4 , 6 3 6}$ & $\mathbf{1 7 , 8 9 7}$ & $\mathbf{1 9 , 0 7 7}$ & $\mathbf{1 8 , 9 2 9}$ & $\mathbf{1 7 , 7 0 5}$ \\
\hline \hline
\end{tabular}

Table A-59: Reference Case with 70/30 Fuel/Feedstock split sugar ethanol production [Million gallons]

\begin{tabular}{lrrrrrr} 
& $\mathbf{2 0 0 5}$ & $\mathbf{2 0 1 0}$ & $\mathbf{2 0 1 5}$ & $\mathbf{2 0 2 0}$ & $\mathbf{2 0 2 5}$ & $\mathbf{2 0 3 0}$ \\
Africa & 300 & 330 & 364 & 402 & 444 & 490 \\
Argentina & 0 & 0 & 0 & 0 & 0 & 0 \\
Australia & 0 & 0 & 0 & 0 & 0 & 0 \\
Brazil & 5,225 & 6,956 & 8,550 & 9,765 & 10,745 & 12,588 \\
Canada & 0 & 0 & 0 & 0 & 0 & 0 \\
Caribbean basin & 180 & 299 & 573 & 597 & 597 & 676 \\
China & 0 & 40 & 48 & 48 & 48 & 48 \\
Colombia & 196 & 215 & 241 & 270 & 302 & 302 \\
Eastern Europe & 0 & 0 & 0 & 0 & 0 & 0 \\
Former Soviet Union & 0 & 0 & 0 & 0 & 0 & 0 \\
India & 313 & 279 & 327 & 347 & 381 & 348 \\
Japan & 0 & 0 & 0 & 0 & 0 & 0 \\
Middle East & 0 & 0 & 0 & 0 & 0 & 0 \\
Mexico & 0 & 0 & 0 & 0 & 0 & 0 \\
Other Asia & 0 & 0 & 0 & 0 & 0 & 0 \\
South Korea & 0 & 0 & 0 & 0 & 0 & 0 \\
United States & 0 & 0 & 0 & 0 & 0 & 0 \\
Western Europe & 0 & 0 & 0 & 0 & 0 & 0 \\
\hline Total & $\mathbf{6 , 2 1 4}$ & $\mathbf{8 , 1 1 9}$ & $\mathbf{1 0 , 1 0 3}$ & $\mathbf{1 1 , 4 2 8}$ & $\mathbf{1 2 , 5 1 7}$ & $\mathbf{1 4 , 4 5 1}$ \\
\hline \hline
\end{tabular}


Table A-60: Reference Case with 70/30 Fuel/Feedstock split cellulosic ethanol production [Million gallons]

\begin{tabular}{lrrrrrr} 
& $\mathbf{2 0 0 5}$ & $\mathbf{2 0 1 0}$ & $\mathbf{2 0 1 5}$ & $\mathbf{2 0 2 0}$ & $\mathbf{2 0 2 5}$ & $\mathbf{2 0 3 0}$ \\
Africa & 0 & 0 & 0 & 0 & 11 & 11 \\
Argentina & 0 & 0 & 158 & 306 & 335 & 347 \\
Australia & 0 & 0 & 71 & 267 & 709 & 1,174 \\
Brazil & 0 & 0 & 296 & 890 & 1,875 & 1,875 \\
Canada & 0 & 0 & 259 & 779 & 1,115 & 1,115 \\
Caribbean basin & 0 & 0 & 0 & 0 & 0 & 0 \\
China & 0 & 0 & 243 & 667 & 1,564 & 2,869 \\
Colombia & 0 & 0 & 170 & 512 & 1,199 & 1,893 \\
Eastern Europe & 0 & 0 & 85 & 303 & 670 & 897 \\
Former Soviet Union & 0 & 0 & 39 & 119 & 364 & 1,109 \\
India & 0 & 0 & 214 & 645 & 1,512 & 2,424 \\
Japan & 0 & 0 & 95 & 369 & 612 & 647 \\
Middle East & 0 & 0 & 0 & 0 & 0 & 0 \\
Mexico & 0 & 0 & 199 & 601 & 1,408 & 1,546 \\
Other Asia & 0 & 0 & 0 & 0 & 0 & 0 \\
South Korea & 0 & 0 & 35 & 35 & 35 & 35 \\
United States & 0 & 0 & 802 & 2,174 & 3,899 & 5,164 \\
Western Europe & 0 & 0 & 166 & 693 & 2,113 & 4,410 \\
\hline Total & $\mathbf{0}$ & $\mathbf{0}$ & $\mathbf{2 , 8 3 2}$ & $\mathbf{8 , 3 5 8}$ & $\mathbf{1 7 , 4 2 0}$ & $\mathbf{2 5 , 5 1 5}$ \\
\hline \hline
\end{tabular}

\section{Table A-61: Reference Case with 70/30 Fuel/Feedstock split grain ethanol consumption [Million gallons]}

\begin{tabular}{lrrrrrr} 
& $\mathbf{2 0 0 5}$ & $\mathbf{2 0 1 0}$ & $\mathbf{2 0 1 5}$ & $\mathbf{2 0 2 0}$ & $\mathbf{2 0 2 5}$ & $\mathbf{2 0 3 0}$ \\
Africa & 0 & 0 & 0 & 0 & 0 & 0 \\
Australia & 0 & 0 & 0 & 0 & 0 & 0 \\
Canada & 0 & 0 & 0 & 0 & 0 & 0 \\
China & 344 & 0 & 0 & 325 & 0 & 0 \\
Central and South America & 0 & 36 & 109 & 114 & 93 & 95 \\
Eastern Europe & 110 & 226 & 348 & 477 & 367 & 251 \\
Former Soviet Union & 0 & 0 & 0 & 0 & 0 & 0 \\
India & 0 & 0 & 0 & 0 & 0 & 0 \\
Japan & 0 & 192 & 267 & 382 & 315 & 357 \\
Middle East & 0 & 0 & 0 & 0 & 0 & 0 \\
Mexico & 0 & 0 & 0 & 0 & 0 & 0 \\
Other Asia & 0 & 0 & 0 & 0 & 0 & 0 \\
South Korea & 0 & 295 & 123 & 74 & 160 & 117 \\
United States & 3,850 & 11,423 & 14,047 & 15,000 & 14,815 & 13,281 \\
Western Europe & 600 & 2,465 & 3,002 & 2,705 & 3,178 & 3,604 \\
\hline Total & $\mathbf{4 , 9 0 4}$ & $\mathbf{1 4 , 6 3 6}$ & $\mathbf{1 7 , 8 9 7}$ & $\mathbf{1 9 , 0 7 7}$ & $\mathbf{1 8 , 9 2 9}$ & $\mathbf{1 7 , 7 0 5}$ \\
\hline \hline
\end{tabular}




\section{Table A-62: Reference Case with 70/30 Fuel/Feedstock split sugar ethanol consumption [Million gallons]}

\begin{tabular}{lrrrrrr} 
& $\mathbf{2 0 0 5}$ & $\mathbf{2 0 1 0}$ & $\mathbf{2 0 1 5}$ & $\mathbf{2 0 2 0}$ & $\mathbf{2 0 2 5}$ & $\mathbf{2 0 3 0}$ \\
Africa & 300 & 330 & 364 & 402 & 444 & 490 \\
Australia & 0 & 0 & 0 & 0 & 0 & 0 \\
Canada & 119 & 0 & 0 & 205 & 0 & 0 \\
China & 98 & 0 & 0 & 48 & 48 & 48 \\
Central and South America & 3,785 & 4,815 & 6,035 & 7,010 & 7,855 & 8,727 \\
Eastern Europe & 589 & 557 & 412 & 173 & 0 & 0 \\
Former Soviet Union & 0 & 0 & 0 & 0 & 0 & 0 \\
India & 313 & 279 & 327 & 347 & 381 & 348 \\
Japan & 300 & 133 & 48 & 0 & 0 & 0 \\
Middle East & 0 & 0 & 0 & 0 & 0 & 0 \\
Mexico & 0 & 0 & 0 & 0 & 0 & 0 \\
Other Asia & 0 & 0 & 0 & 0 & 0 & 0 \\
South Korea & 264 & 40 & 0 & 0 & 0 & 0 \\
United States & 311 & 189 & 1,741 & 2,753 & 3,105 & 4,704 \\
Western Europe & 136 & 1,776 & 1,175 & 490 & 684 & 135 \\
\hline Total & $\mathbf{6 , 2 1 4}$ & $\mathbf{8 , 1 1 9}$ & $\mathbf{1 0 , 1 0 3}$ & $\mathbf{1 1 , 4 2 8}$ & $\mathbf{1 2 , 5 1 7}$ & $\mathbf{1 4 , 4 5 1}$ \\
\hline \hline
\end{tabular}

Table A-63: Reference Case with 70/30 Fuel/Feedstock split cellulosic ethanol consumption [Million gallons]

\begin{tabular}{lllrrrr} 
Africa & 0 & 0 & 0 & 0 & 11 & 11 \\
Australia & 0 & 0 & 71 & 267 & 709 & 1,174 \\
Canada & 0 & 0 & 233 & 779 & 1,004 & 1,020 \\
China & 0 & 0 & 0 & 667 & 1,564 & 3,241 \\
Central and South America & 0 & 0 & 0 & 21 & 0 & 27 \\
Eastern Europe & 0 & 0 & 111 & 303 & 670 & 897 \\
Former Soviet Union & 0 & 0 & 39 & 119 & 364 & 1,109 \\
India & 0 & 0 & 214 & 645 & 1,512 & 2,051 \\
Japan & 0 & 0 & 95 & 369 & 724 & 743 \\
Middle East & 0 & 0 & 0 & 0 & 0 & 0 \\
Mexico & 0 & 0 & 30 & 601 & 1,031 & 1,053 \\
Other Asia & 0 & 0 & 0 & 0 & 0 & 0 \\
South Korea & 0 & 0 & 277 & 341 & 253 & 296 \\
United States & 0 & 0 & 1,595 & 2,194 & 5,792 & 9,483 \\
Western Europe & 0 & 0 & 166 & 2,053 & 3,788 & 4,410 \\
\hline Total & $\mathbf{0}$ & $\mathbf{0}$ & $\mathbf{2 , 8 3 2}$ & $\mathbf{8 , 3 5 8}$ & $\mathbf{1 7 , 4 2 0}$ & $\mathbf{2 5 , 5 1 5}$ \\
\hline \hline
\end{tabular}


Table A-64: Reference Case with 70/30 Fuel/Feedstock split grain ethanol net trade [Million gallons]

\begin{tabular}{lrrrrrr} 
& $\mathbf{2 0 0 5}$ & $\mathbf{2 0 1 0}$ & $\mathbf{2 0 1 5}$ & $\mathbf{2 0 2 0}$ & $\mathbf{2 0 2 5}$ & $\mathbf{2 0 3 0}$ \\
Africa & 0 & 0 & 0 & 0 & 0 & 0 \\
Australia & 0 & 0 & 0 & 0 & 0 & 0 \\
Canada & 0 & 0 & 0 & 0 & 0 & 0 \\
China & 0 & 344 & 344 & 0 & 0 & 0 \\
Central and South America & 0 & 143 & 460 & 455 & 476 & 474 \\
Eastern Europe & 0 & 0 & 0 & 0 & 0 & 0 \\
Former Soviet Union & 0 & 0 & 0 & 0 & 0 & 0 \\
India & 0 & 0 & 0 & 0 & 0 & 0 \\
Japan & 0 & -192 & -267 & -382 & -315 & -357 \\
Middle East & 0 & 0 & 0 & 0 & 0 & 0 \\
Mexico & 0 & 0 & 0 & 0 & 0 & 0 \\
Other Asia & 0 & 0 & 0 & 0 & 0 & 0 \\
South Korea & 0 & -295 & -123 & -74 & -160 & -117 \\
United States & 0 & 0 & 0 & 0 & 0 & 0 \\
Western Europe & 0 & 0 & -414 & 0 & 0 & 0 \\
\hline Total & $\mathbf{0}$ & $\mathbf{0}$ & $\mathbf{0}$ & $\mathbf{0}$ & $\mathbf{0}$ & $\mathbf{0}$ \\
\hline \hline
\end{tabular}

Table A-65: Reference Case with 70/30 Fuel/Feedstock split sugar ethanol net trade [Million gallons]

\begin{tabular}{lrrrrrr} 
& $\mathbf{2 0 0 5}$ & $\mathbf{2 0 1 0}$ & $\mathbf{2 0 1 5}$ & $\mathbf{2 0 2 0}$ & $\mathbf{2 0 2 5}$ & $\mathbf{2 0 3 0}$ \\
Africa & 0 & 0 & 0 & 0 & 0 & 0 \\
Australia & 0 & 0 & 0 & 0 & 0 & 0 \\
Canada & -119 & 0 & 0 & -205 & 0 & 0 \\
China & -98 & 40 & 48 & 0 & 0 & 0 \\
Central and South America & 1,816 & 2,655 & 3,329 & 3,622 & 3,789 & 4,839 \\
Eastern Europe & -589 & -557 & -412 & -173 & 0 & 0 \\
Former Soviet Union & 0 & 0 & 0 & 0 & 0 & 0 \\
India & 0 & 0 & 0 & 0 & 0 & 0 \\
Japan & -300 & -133 & -48 & 0 & 0 & 0 \\
Middle East & 0 & 0 & 0 & 0 & 0 & 0 \\
Mexico & 0 & 0 & 0 & 0 & 0 & 0 \\
Other Asia & 0 & 0 & 0 & 0 & 0 & 0 \\
South Korea & -264 & -40 & 0 & 0 & 0 & 0 \\
United States & -311 & -189 & $-1,741$ & $-2,753$ & $-3,105$ & $-4,704$ \\
Western Europe & -136 & $-1,776$ & $-1,175$ & -490 & -684 & -135 \\
\hline Total & $\mathbf{0}$ & $\mathbf{0}$ & $\mathbf{0}$ & $\mathbf{0}$ & $\mathbf{0}$ & $\mathbf{0}$ \\
\hline \hline
\end{tabular}


Table A-66: Reference Case with 70/30 Fuel/Feedstock split cellulosic ethanol net trade [Million gallons]

\begin{tabular}{lrrrrrr} 
& $\mathbf{2 0 0 5}$ & $\mathbf{2 0 1 0}$ & $\mathbf{2 0 1 5}$ & $\mathbf{2 0 2 0}$ & $\mathbf{2 0 2 5}$ & $\mathbf{2 0 3 0}$ \\
Africa & 0 & 0 & 0 & 0 & 0 & 0 \\
Australia & 0 & 0 & 0 & 0 & 0 & 0 \\
Canada & 0 & 0 & 26 & 0 & 112 & 96 \\
China & 0 & 0 & 243 & 0 & 0 & -372 \\
Central and South America & 0 & 0 & 624 & 1,687 & 3,409 & 4,088 \\
Eastern Europe & 0 & 0 & -26 & 0 & 0 & 0 \\
Former Soviet Union & 0 & 0 & 0 & 0 & 0 & 0 \\
India & 0 & 0 & 0 & 0 & 0 & 372 \\
Japan & 0 & 0 & 0 & 0 & -112 & -96 \\
Middle East & 0 & 0 & 0 & 0 & 0 & 0 \\
Mexico & 0 & 0 & 170 & 0 & 377 & 493 \\
Other Asia & 0 & 0 & 0 & 0 & 0 & 0 \\
South Korea & 0 & 0 & -243 & -306 & -218 & -261 \\
United States & 0 & 0 & -793 & -20 & $-1,893$ & $-4,319$ \\
Western Europe & 0 & 0 & 0 & $-1,361$ & $-1,675$ & 0 \\
\hline Total & $\mathbf{0}$ & $\mathbf{0}$ & $\mathbf{0}$ & $\mathbf{0}$ & $\mathbf{0}$ & $\mathbf{0}$ \\
\hline \hline
\end{tabular}

Table A-67: Reference Case with 70/30 Fuel/Feedstock split biomass-to-liquids production [Million gallons ethanol equivalent]

\begin{tabular}{lrrrrrr} 
& $\mathbf{2 0 0 5}$ & $\mathbf{2 0 1 0}$ & $\mathbf{2 0 1 5}$ & $\mathbf{2 0 2 0}$ & $\mathbf{2 0 2 5}$ & $\mathbf{2 0 3 0}$ \\
Africa & 0 & 0 & 68 & 263 & 653 & 1,177 \\
Argentina & 0 & 0 & 57 & 110 & 121 & 125 \\
Australia & 0 & 0 & 0 & 0 & 0 & 0 \\
Brazil & 0 & 0 & 106 & 320 & 674 & 674 \\
Canada & 0 & 0 & 93 & 280 & 401 & 401 \\
Caribbean basin & 0 & 0 & 0 & 0 & 0 & 0 \\
China & 0 & 0 & 57 & 240 & 563 & 563 \\
Colombia & 0 & 0 & 61 & 184 & 431 & 681 \\
Eastern Europe & 0 & 0 & 0 & 0 & 93 & 274 \\
Former Soviet Union & 0 & 0 & 0 & 0 & 0 & 0 \\
India & 0 & 0 & 77 & 232 & 544 & 544 \\
Japan & 0 & 0 & 0 & 0 & 0 & 0 \\
Middle East & 0 & 0 & 0 & 0 & 0 & 125 \\
Mexico & 0 & 0 & 72 & 216 & 506 & 556 \\
Other Asia & 0 & 0 & 89 & 448 & 1,090 & 1,942 \\
South Korea & 0 & 0 & 36 & 74 & 74 & 74 \\
United States & 0 & 0 & 822 & 4,637 & 6,169 & 7,919 \\
Western Europe & 0 & 0 & 0 & 0 & 0 & 0 \\
\hline Total & $\mathbf{0}$ & $\mathbf{0}$ & $\mathbf{1 , 5 3 8}$ & $\mathbf{7 , 0 0 5}$ & $\mathbf{1 1 , 3 1 8}$ & $\mathbf{1 5 , 0 5 5}$ \\
\hline \hline
\end{tabular}


Table A-68: Reference Case with 70/30 Fuel/Feedstock split biomass-to-liquids consumption [Million gallons ethanol equivalent]

\begin{tabular}{lrrrrrr} 
& $\mathbf{2 0 0 5}$ & $\mathbf{2 0 1 0}$ & $\mathbf{2 0 1 5}$ & $\mathbf{2 0 2 0}$ & $\mathbf{2 0 2 5}$ & $\mathbf{2 0 3 0}$ \\
Africa & 0 & 0 & 68 & 263 & 653 & 1,177 \\
Australia & 0 & 0 & 0 & 0 & 0 & 0 \\
Canada & 0 & 0 & 0 & 0 & 0 & 0 \\
China & 0 & 0 & 0 & 0 & 0 & 0 \\
Central and South America & 0 & 0 & 0 & 0 & 0 & 0 \\
Eastern Europe & 0 & 0 & 0 & 0 & 93 & 274 \\
Former Soviet Union & 0 & 0 & 0 & 0 & 0 & 0 \\
India & 0 & 0 & 0 & 0 & 0 & 0 \\
Japan & 0 & 0 & 0 & 0 & 0 & 0 \\
Middle East & 0 & 0 & 0 & 0 & 0 & 125 \\
Mexico & 0 & 0 & 0 & 0 & 0 & 0 \\
Other Asia & 0 & 0 & 89 & 449 & 1,090 & 1,942 \\
South Korea & 0 & 0 & 0 & 0 & 0 & 0 \\
United States & 0 & 0 & 1,167 & 6,164 & 9,483 & 10,752 \\
Western Europe & 0 & 0 & 214 & 130 & 0 & 785 \\
\hline Total & $\mathbf{0}$ & $\mathbf{0}$ & $\mathbf{1 , 5 3 8}$ & $\mathbf{7 , 0 0 5}$ & $\mathbf{1 1 , 3 1 8}$ & $\mathbf{1 5 , 0 5 5}$ \\
\hline \hline
\end{tabular}

Table A-69: Reference Case with 70/30 Fuel/Feedstock split biomass-to-liquids net trade [Million gallons ethanol equivalent]

\begin{tabular}{lrrrrrr} 
& $\mathbf{2 0 0 5}$ & $\mathbf{2 0 1 0}$ & $\mathbf{2 0 1 5}$ & $\mathbf{2 0 2 0}$ & $\mathbf{2 0 2 5}$ & $\mathbf{2 0 3 0}$ \\
Africa & 0 & 0 & 0 & 0 & 0 & 0 \\
Australia & 0 & 0 & 0 & 0 & 0 & 0 \\
Canada & 0 & 0 & 93 & 280 & 401 & 401 \\
China & 0 & 0 & 57 & 240 & 563 & 563 \\
Central and South America & 0 & 0 & 224 & 614 & 1,226 & 1,480 \\
Eastern Europe & 0 & 0 & 0 & 0 & 0 & 0 \\
Former Soviet Union & 0 & 0 & 0 & 0 & 0 & 0 \\
India & 0 & 0 & 77 & 232 & 544 & 544 \\
Japan & 0 & 0 & 0 & 0 & 0 & 0 \\
Middle East & 0 & 0 & 0 & 0 & 0 & 0 \\
Mexico & 0 & 0 & 72 & 216 & 506 & 556 \\
Other Asia & 0 & 0 & 0 & 0 & 0 & 0 \\
South Korea & 0 & 0 & 36 & 74 & 74 & 74 \\
United States & 0 & 0 & -345 & $-1,526$ & $-3,314$ & $-2,833$ \\
Western Europe & 0 & 0 & -214 & -130 & 0 & -785 \\
\hline Total & $\mathbf{0}$ & $\mathbf{0}$ & $\mathbf{0}$ & $\mathbf{0}$ & $\mathbf{0}$ & $\mathbf{0}$ \\
\hline \hline
\end{tabular}


Table A-70: Reference Case with 70/30 Fuel/Feedstock split biodiesel production [Million gallons ethanol equivalent]

\begin{tabular}{lrrrrrr} 
& $\mathbf{2 0 0 5}$ & $\mathbf{2 0 1 0}$ & $\mathbf{2 0 1 5}$ & $\mathbf{2 0 2 0}$ & $\mathbf{2 0 2 5}$ & $\mathbf{2 0 3 0}$ \\
Africa & 0 & 0 & 0 & 0 & 0 & 0 \\
Argentina & 0 & 333 & 776 & 819 & 827 & 239 \\
Australia & 0 & 0 & 0 & 0 & 0 & 0 \\
Brazil & 0 & 333 & 835 & 1,600 & 1,966 & 1,355 \\
Canada & 0 & 0 & 0 & 0 & 0 & 0 \\
Caribbean basin & 0 & 95 & 310 & 675 & 802 & 898 \\
China & 0 & 0 & 0 & 0 & 0 & 0 \\
Colombia & 0 & 119 & 298 & 474 & 650 & 782 \\
Eastern Europe & 0 & 0 & 0 & 0 & 0 & 0 \\
Former Soviet Union & 0 & 0 & 0 & 0 & 0 & 0 \\
India & 0 & 0 & 0 & 0 & 0 & 0 \\
Japan & 0 & 0 & 0 & 0 & 0 & 0 \\
Middle East & 0 & 0 & 0 & 0 & 0 & 0 \\
Mexico & 0 & 0 & 0 & 0 & 0 & 0 \\
Other Asia & 0 & 0 & 0 & 0 & 0 & 0 \\
South Korea & 0 & 0 & 0 & 0 & 0 & 0 \\
United States & 0 & 1,238 & 1,640 & 1,747 & 1,907 & 2,016 \\
Western Europe & 998 & 3,178 & 3,578 & 3,930 & 4,468 & 4,788 \\
\hline Total & $\mathbf{9 9 8}$ & $\mathbf{5 , 2 9 4}$ & $\mathbf{7 , 4 3 7}$ & $\mathbf{9 , 2 4 5}$ & $\mathbf{1 0 , 6 2 1}$ & $\mathbf{1 0 , 0 7 7}$ \\
\hline \hline
\end{tabular}

Table A-71: Reference Case with 70/30 Fuel/Feedstock split biodiesel consumption [Million gallons ethanol equivalent]

\begin{tabular}{lrrrrrr} 
& $\mathbf{2 0 0 5}$ & $\mathbf{2 0 1 0}$ & $\mathbf{2 0 1 5}$ & $\mathbf{2 0 2 0}$ & $\mathbf{2 0 2 5}$ & $\mathbf{2 0 3 0}$ \\
Africa & 0 & 0 & 0 & 0 & 0 & 0 \\
Australia & 0 & 0 & 0 & 0 & 0 & 0 \\
Canada & 0 & 0 & 0 & 0 & 0 & 0 \\
China & 0 & 0 & 0 & 0 & 0 & 0 \\
Central and South America & 0 & 158 & 472 & 984 & 1,306 & 1,490 \\
Eastern Europe & 0 & 0 & 0 & 0 & 0 & 0 \\
Former Soviet Union & 0 & 0 & 0 & 0 & 0 & 0 \\
India & 0 & 0 & 0 & 0 & 0 & 0 \\
Japan & 0 & 0 & 0 & 0 & 0 & 0 \\
Middle East & 0 & 0 & 0 & 0 & 0 & 0 \\
Mexico & 0 & 0 & 0 & 0 & 0 & 0 \\
Other Asia & 0 & 0 & 0 & 0 & 0 & 0 \\
South Korea & 0 & 0 & 0 & 0 & 0 & 0 \\
United States & 0 & 1,238 & 1,713 & 1,747 & 2,080 & 2,016 \\
Western Europe & 998 & 3,898 & 5,252 & 6,514 & 7,235 & 6,572 \\
\hline Total & $\mathbf{9 9 8}$ & $\mathbf{5 , 2 9 4}$ & $\mathbf{7 , 4 3 7}$ & $\mathbf{9 , 2 4 5}$ & $\mathbf{1 0 , 6 2 1}$ & $\mathbf{1 0 , 0 7 7}$ \\
\hline \hline
\end{tabular}


Table A-72: Reference Case with 70/30 Fuel/Feedstock split biodiesel net trade [Million gallons ethanol equivalent]

\begin{tabular}{lrrrrrr} 
& $\mathbf{2 0 0 5}$ & $\mathbf{2 0 1 0}$ & $\mathbf{2 0 1 5}$ & $\mathbf{2 0 2 0}$ & $\mathbf{2 0 2 5}$ & $\mathbf{2 0 3 0}$ \\
Africa & 0 & 0 & 0 & 0 & 0 & 0 \\
Australia & 0 & 0 & 0 & 0 & 0 & 0 \\
Canada & 0 & 0 & 0 & 0 & 0 & 0 \\
China & 0 & 0 & 0 & 0 & 0 & 0 \\
Central and South America & 0 & 720 & 1,746 & 2,584 & 2,940 & 1,784 \\
Eastern Europe & 0 & 0 & 0 & 0 & 0 & 0 \\
Former Soviet Union & 0 & 0 & 0 & 0 & 0 & 0 \\
India & 0 & 0 & 0 & 0 & 0 & 0 \\
Japan & 0 & 0 & 0 & 0 & 0 & 0 \\
Middle East & 0 & 0 & 0 & 0 & 0 & 0 \\
Mexico & 0 & 0 & 0 & 0 & 0 & 0 \\
Other Asia & 0 & 0 & 0 & 0 & 0 & 0 \\
South Korea & 0 & 0 & 0 & 0 & 0 & 0 \\
United States & 0 & 0 & -73 & 0 & -173 & 0 \\
Western Europe & 0 & -720 & $-1,674$ & $-2,584$ & $-2,767$ & $-1,784$ \\
\hline Total & $\mathbf{0}$ & $\mathbf{0}$ & $\mathbf{0}$ & $\mathbf{0}$ & $\mathbf{0}$ & $\mathbf{0}$ \\
\hline \hline
\end{tabular}




\section{A-5 Reference Case with High Feedstock Availability}

Table A-73: Reference Case with high feedstock availability ethanol production [Million gallons]

\begin{tabular}{lrrrrrr} 
& $\mathbf{2 0 0 5}$ & $\mathbf{2 0 1 0}$ & $\mathbf{2 0 1 5}$ & $\mathbf{2 0 2 0}$ & $\mathbf{2 0 2 5}$ & $\mathbf{2 0 3 0}$ \\
Africa & 300 & 330 & 364 & 402 & 455 & 502 \\
Argentina & 0 & 170 & 703 & 1,512 & 2,041 & 1,941 \\
Australia & 0 & 0 & 71 & 267 & 709 & 1,174 \\
Brazil & 4,866 & 7,863 & 11,399 & 16,323 & 20,461 & 22,213 \\
Canada & 0 & 0 & 259 & 779 & 1,139 & 1,139 \\
Caribbean basin & 210 & 280 & 682 & 682 & 682 & 682 \\
China & 344 & 0 & 222 & 993 & 1,564 & 3,969 \\
Colombia & 196 & 0 & 170 & 512 & 887 & 887 \\
Eastern Europe & 110 & 226 & 428 & 774 & 1,037 & 1,149 \\
Former Soviet Union & 0 & 0 & 39 & 119 & 364 & 1,109 \\
India & 313 & 833 & 1,300 & 1,728 & 2,200 & 2,822 \\
Japan & 0 & 0 & 95 & 369 & 612 & 647 \\
Middle East & 0 & 0 & 0 & 0 & 0 & 0 \\
Mexico & 0 & 0 & 199 & 601 & 1,053 & 1,053 \\
Other Asia & 0 & 0 & 0 & 0 & 0 & 0 \\
South Korea & 0 & 0 & 31 & 31 & 31 & 31 \\
United States & 3,850 & 11,413 & 14,840 & 15,598 & 15,574 & 13,655 \\
Western Europe & 600 & 2,465 & 2,755 & 3,235 & 5,205 & 7,923 \\
\hline Total & $\mathbf{1 0 , 7 8 9}$ & $\mathbf{2 3 , 5 8 0}$ & $\mathbf{3 3 , 5 5 7}$ & $\mathbf{4 3 , 9 2 4}$ & $\mathbf{5 4 , 0 1 4}$ & $\mathbf{6 0 , 3 9 4}$ \\
\hline \hline
\end{tabular}


Table A-74: Reference Case with high feedstock availability ethanol consumption [Million gallons]

\begin{tabular}{lrrrrrr} 
& $\mathbf{2 0 0 5}$ & $\mathbf{2 0 1 0}$ & $\mathbf{2 0 1 5}$ & $\mathbf{2 0 2 0}$ & $\mathbf{2 0 2 5}$ & $\mathbf{2 0 3 0}$ \\
Africa & 300 & 330 & 364 & 402 & 455 & 502 \\
Australia & 0 & 0 & 71 & 267 & 709 & 1,174 \\
Canada & 145 & 259 & 898 & 984 & 1,025 & 1,054 \\
China & 297 & 0 & 1,296 & 3,397 & 4,131 & 5,062 \\
Central and South America & 3,788 & 4,805 & 6,111 & 7,191 & 7,974 & 9,509 \\
Eastern Europe & 699 & 783 & 872 & 953 & 1,037 & 1,149 \\
Former Soviet Union & 0 & 0 & 39 & 119 & 364 & 1,109 \\
India & 313 & 833 & 1,300 & 1,642 & 1,980 & 2,399 \\
Japan & 263 & 325 & 448 & 757 & 1,045 & 1,138 \\
Middle East & 0 & 0 & 0 & 0 & 0 & 0 \\
Mexico & 0 & 0 & 30 & 601 & 601 & 1,053 \\
Other Asia & 0 & 0 & 0 & 2,166 & 3,005 & 3,472 \\
South Korea & 264 & 335 & 400 & 414 & 413 & 413 \\
United States & 3,984 & 11,668 & 17,377 & 19,947 & 23,712 & 24,438 \\
Western Europe & 736 & $\mathbf{4 , 2 4 1}$ & 4,350 & 5,086 & 7,563 & 7,923 \\
\hline Total & $\mathbf{1 0 , 7 8 9}$ & $\mathbf{2 3 , 5 8 0}$ & $\mathbf{3 3 , 5 5 7}$ & $\mathbf{4 3 , 9 2 4}$ & $\mathbf{5 4 , 0 1 4}$ & $\mathbf{6 0 , 3 9 4}$ \\
\hline \hline
\end{tabular}

Table A-75: Reference Case with high feedstock availability ethanol net trade [Million gallons]

\begin{tabular}{lrrrrrr} 
Africa & 0 & 0 & 0 & 0 & 0 & 0 \\
Australia & 0 & 0 & 0 & 0 & 0 & 0 \\
Canada & -145 & -259 & -640 & -205 & 114 & 85 \\
China & 47 & 0 & $-1,075$ & $-2,404$ & $-2,567$ & $-1,093$ \\
Central and South America & 1,484 & 3,508 & 6,843 & 11,838 & 16,097 & 15,712 \\
Eastern Europe & -589 & -557 & -444 & -179 & 0 & 0 \\
Former Soviet Union & 0 & 0 & 0 & 0 & 0 & 0 \\
India & 0 & 0 & 0 & 86 & 220 & 423 \\
Japan & -263 & -325 & -353 & -388 & -433 & -490 \\
Middle East & 0 & 0 & 0 & 0 & 0 & 0 \\
Mexico & 0 & 0 & 170 & 0 & 452 & 0 \\
Other Asia & 0 & 0 & 0 & $-2,166$ & $-3,005$ & $-3,472$ \\
South Korea & -264 & -335 & -369 & -383 & -382 & -382 \\
United States & -134 & -256 & $-2,538$ & $-4,349$ & $-8,138$ & $-10,783$ \\
Western Europe & -136 & $-1,776$ & $-1,595$ & $-1,851$ & $-2,358$ & 0 \\
\hline Total & $\mathbf{0}$ & $\mathbf{0}$ & $\mathbf{0}$ & $\mathbf{0}$ & $\mathbf{0}$ & $\mathbf{0}$ \\
\hline \hline
\end{tabular}


Table A-76: Reference Case with high feedstock availability grain ethanol production [Million gallons]

\begin{tabular}{lrrrrrr} 
& $\mathbf{2 0 0 5}$ & $\mathbf{2 0 1 0}$ & $\mathbf{2 0 1 5}$ & $\mathbf{2 0 2 0}$ & $\mathbf{2 0 2 5}$ & $\mathbf{2 0 3 0}$ \\
Africa & 0 & 0 & 0 & 0 & 0 & 0 \\
Argentina & 0 & 170 & 525 & 1,074 & 1,495 & 1,395 \\
Australia & 0 & 0 & 0 & 0 & 0 & 0 \\
Brazil & 0 & 0 & 0 & 0 & 0 & 0 \\
Canada & 0 & 0 & 0 & 0 & 0 & 0 \\
Caribbean basin & 0 & 0 & 0 & 0 & 0 & 0 \\
China & 344 & 0 & 0 & 325 & 0 & 0 \\
Colombia & 0 & 0 & 0 & 0 & 0 & 0 \\
Eastern Europe & 110 & 226 & 348 & 477 & 367 & 251 \\
Former Soviet Union & 0 & 0 & 0 & 0 & 0 & 0 \\
India & 0 & 0 & 0 & 0 & 0 & 0 \\
Japan & 0 & 0 & 0 & 0 & 0 & 0 \\
Middle East & 0 & 0 & 0 & 0 & 0 & 0 \\
Mexico & 0 & 0 & 0 & 0 & 0 & 0 \\
Other Asia & 0 & 0 & 0 & 0 & 0 & 0 \\
South Korea & 0 & 0 & 0 & 0 & 0 & 0 \\
United States & 3,850 & 11,413 & 14,038 & 13,613 & 11,781 & 9,863 \\
Western Europe & 600 & 2,465 & 2,589 & 2,542 & 3,178 & 3,604 \\
\hline Total & $\mathbf{4 , 9 0 4}$ & $\mathbf{1 4 , 2 7 3}$ & $\mathbf{1 7 , 5 0 0}$ & $\mathbf{1 8 , 0 3 1}$ & $\mathbf{1 6 , 8 2 1}$ & $\mathbf{1 5 , 1 1 2}$ \\
\hline \hline
\end{tabular}

Table A-77: Reference Case with high feedstock availability sugar ethanol production [Million gallons]

\begin{tabular}{lrrrrrr} 
& $\mathbf{2 0 0 5}$ & $\mathbf{2 0 1 0}$ & $\mathbf{2 0 1 5}$ & $\mathbf{2 0 2 0}$ & $\mathbf{2 0 2 5}$ & $\mathbf{2 0 3 0}$ \\
Africa & 300 & 330 & 364 & 402 & 444 & 490 \\
Argentina & 0 & 0 & 0 & 0 & 0 & 0 \\
Australia & 0 & 0 & 0 & 0 & 0 & 0 \\
Brazil & 4,866 & 7,863 & 11,103 & 15,434 & 18,376 & 18,507 \\
Canada & 0 & 0 & 0 & 0 & 0 & 0 \\
Caribbean basin & 210 & 280 & 682 & 682 & 682 & 682 \\
China & 0 & 0 & 0 & 0 & 0 & 0 \\
Colombia & 196 & 0 & 0 & 0 & 0 & 0 \\
Eastern Europe & 0 & 0 & 0 & 0 & 0 & 0 \\
Former Soviet Union & 0 & 0 & 0 & 0 & 0 & 0 \\
India & 313 & 833 & 1,086 & 1,083 & 688 & 78 \\
Japan & 0 & 0 & 0 & 0 & 0 & 0 \\
Middle East & 0 & 0 & 0 & 0 & 0 & 0 \\
Mexico & 0 & 0 & 0 & 0 & 0 & 0 \\
Other Asia & 0 & 0 & 0 & 0 & 0 & 0 \\
South Korea & 0 & 0 & 0 & 0 & 0 & 0 \\
United States & 0 & 0 & 0 & 0 & 0 & 0 \\
Western Europe & 0 & 0 & 0 & 0 & 0 & 0 \\
\hline Total & $\mathbf{5 , 8 8 4}$ & $\mathbf{9 , 3 0 6}$ & $\mathbf{1 3 , 2 3 6}$ & $\mathbf{1 7 , 6 0 1}$ & $\mathbf{2 0 , 1 9 0}$ & $\mathbf{1 9 , 7 5 7}$ \\
\hline \hline
\end{tabular}


Table A-78: Reference Case with high feedstock availability cellulosic ethanol production [Million gallons]

\begin{tabular}{lrrrrrr} 
& $\mathbf{2 0 0 5}$ & $\mathbf{2 0 1 0}$ & $\mathbf{2 0 1 5}$ & $\mathbf{2 0 2 0}$ & $\mathbf{2 0 2 5}$ & $\mathbf{2 0 3 0}$ \\
Africa & 0 & 0 & 0 & 0 & 11 & 11 \\
Argentina & 0 & 0 & 178 & 438 & 547 & 547 \\
Australia & 0 & 0 & 71 & 267 & 709 & 1,174 \\
Brazil & 0 & 0 & 296 & 890 & 2,085 & 3,706 \\
Canada & 0 & 0 & 259 & 779 & 1,139 & 1,139 \\
Caribbean basin & 0 & 0 & 0 & 0 & 0 & 0 \\
China & 0 & 0 & 222 & 667 & 1,564 & 3,969 \\
Colombia & 0 & 0 & 170 & 512 & 887 & 887 \\
Eastern Europe & 0 & 0 & 80 & 297 & 670 & 897 \\
Former Soviet Union & 0 & 0 & 39 & 119 & 364 & 1,109 \\
India & 0 & 0 & 214 & 645 & 1,512 & 2,744 \\
Japan & 0 & 0 & 95 & 369 & 612 & 647 \\
Middle East & 0 & 0 & 0 & 0 & 0 & 0 \\
Mexico & 0 & 0 & 199 & 601 & 1,053 & 1,053 \\
Other Asia & 0 & 0 & 0 & 0 & 0 & 0 \\
South Korea & 0 & 0 & 31 & 31 & 31 & 31 \\
United States & 0 & 0 & 802 & 1,986 & 3,793 & 3,793 \\
Western Europe & 0 & 0 & 166 & 693 & 2,027 & 4,320 \\
\hline Total & $\mathbf{0}$ & $\mathbf{0}$ & $\mathbf{2 , 8 2 1}$ & $\mathbf{8 , 2 9 2}$ & $\mathbf{1 7 , 0 0 3}$ & $\mathbf{2 6 , 0 2 6}$ \\
\hline \hline
\end{tabular}
Table A-79: Reference Case with high feedstock availability grain ethanol
consumption [Million gallons]

\begin{tabular}{lrrrrrr} 
& $\mathbf{2 0 0 5}$ & $\mathbf{2 0 1 0}$ & $\mathbf{2 0 1 5}$ & $\mathbf{2 0 2 0}$ & $\mathbf{2 0 2 5}$ & $\mathbf{2 0 3 0}$ \\
Africa & 0 & 0 & 0 & 0 & 0 & 0 \\
Australia & 0 & 0 & 0 & 0 & 0 & 0 \\
Canada & 0 & 0 & 0 & 0 & 0 & 0 \\
China & 344 & 0 & 51 & 325 & 0 & 0 \\
Central and South America & 0 & 34 & 106 & 142 & 208 & 0 \\
Eastern Europe & 110 & 226 & 348 & 477 & 367 & 251 \\
Former Soviet Union & 0 & 0 & 0 & 0 & 0 & 0 \\
India & 0 & 0 & 0 & 0 & 0 & 0 \\
Japan & 0 & 0 & 0 & 0 & 0 & 0 \\
Middle East & 0 & 0 & 0 & 0 & 0 & 0 \\
Mexico & 0 & 0 & 0 & 0 & 0 & 0 \\
Other Asia & 0 & 0 & 0 & 932 & 905 & 0 \\
South Korea & 0 & 136 & 369 & 0 & 382 & 0 \\
United States & 3,850 & 11,413 & 14,038 & 13,613 & 11,781 & 9,863 \\
Western Europe & 600 & 2,465 & 2,589 & 2,542 & 3,178 & 3,604 \\
\hline Total & $\mathbf{4 , 9 0 4}$ & $\mathbf{1 4 , 2 7 3}$ & $\mathbf{1 7 , 5 0 0}$ & $\mathbf{1 8 , 0 3 1}$ & $\mathbf{1 6 , 8 2 1}$ & $\mathbf{1 3 , 7 1 8}$ \\
\hline \hline
\end{tabular}


Table A-80: Reference Case with high feedstock availability sugar ethanol consumption [Million gallons]

\begin{tabular}{lrrrrrr} 
& $\mathbf{2 0 0 5}$ & $\mathbf{2 0 1 0}$ & $\mathbf{2 0 1 5}$ & $\mathbf{2 0 2 0}$ & $\mathbf{2 0 2 5}$ & $\mathbf{2 0 3 0}$ \\
Africa & 300 & 330 & 364 & 402 & 444 & 490 \\
Australia & 0 & 0 & 0 & 0 & 0 & 0 \\
Canada & 145 & 259 & 640 & 205 & 0 & 0 \\
China & 0 & 0 & 1,024 & 2,318 & 2,233 & 585 \\
Central and South America & 3,788 & 4,771 & 5,980 & 6,917 & 7,634 & 8,425 \\
Eastern Europe & 589 & 557 & 444 & 179 & 0 & 0 \\
Former Soviet Union & 0 & 0 & 0 & 0 & 0 & 0 \\
India & 313 & 833 & 1,086 & 1,083 & 688 & 78 \\
Japan & 263 & 325 & 353 & 388 & 433 & 490 \\
Middle East & 0 & 0 & 0 & 0 & 0 & 0 \\
Mexico & 0 & 0 & 0 & 0 & 464 & 0 \\
Other Asia & 0 & 0 & 0 & 1,234 & 2,100 & 3,472 \\
South Korea & 217 & 199 & 0 & 0 & 0 & 359 \\
United States & 134 & 256 & 1,750 & 3,137 & 3,837 & 5,858 \\
Western Europe & 136 & 1,776 & 1,595 & 1,738 & 2,358 & 0 \\
\hline Total & $\mathbf{5 , 8 8 4}$ & $\mathbf{9 , 3 0 6}$ & $\mathbf{1 3 , 2 3 6}$ & $\mathbf{1 7 , 6 0 1}$ & $\mathbf{2 0 , 1 9 0}$ & $\mathbf{1 9 , 7 5 7}$ \\
\hline \hline
\end{tabular}

Table A-81: Reference Case with high feedstock availability cellulosic ethanol consumption [Million gallons]

\begin{tabular}{lrrrrrr} 
& $\mathbf{2 0 0 5}$ & $\mathbf{2 0 1 0}$ & $\mathbf{2 0 1 5}$ & $\mathbf{2 0 2 0}$ & $\mathbf{2 0 2 5}$ & $\mathbf{2 0 3 0}$ \\
Africa & 0 & 0 & 0 & 0 & 11 & 11 \\
Australia & 0 & 0 & 71 & 267 & 709 & 1,174 \\
Canada & 0 & 0 & 259 & 779 & 1,025 & 1,054 \\
China & 0 & 0 & 222 & 754 & 1,898 & 4,477 \\
Central and South America & 0 & 0 & 26 & 132 & 133 & 191 \\
Eastern Europe & 0 & 0 & 80 & 297 & 670 & 897 \\
Former Soviet Union & 0 & 0 & 39 & 119 & 364 & 1,109 \\
India & 0 & 0 & 214 & 559 & 1,292 & 2,321 \\
Japan & 0 & 0 & 95 & 369 & 612 & 647 \\
Middle East & 0 & 0 & 0 & 0 & 0 & 0 \\
Mexico & 0 & 0 & 30 & 601 & 137 & 1,053 \\
Other Asia & 0 & 0 & 0 & 0 & 0 & 0 \\
South Korea & 0 & 0 & 31 & 414 & 31 & 54 \\
United States & 0 & 0 & 1,590 & 3,197 & 8,094 & 8,717 \\
Western Europe & 0 & 0 & 166 & 806 & 2,027 & 4,320 \\
\hline Total & $\mathbf{0}$ & $\mathbf{0}$ & $\mathbf{2 , 8 2 1}$ & $\mathbf{8 , 2 9 2}$ & $\mathbf{1 7 , 0 0 3}$ & $\mathbf{2 6 , 0 2 6}$ \\
\hline \hline
\end{tabular}


Table A-82: Reference Case with high feedstock availability grain ethanol net trade [Million gallons]

\begin{tabular}{lrrrrrr} 
& $\mathbf{2 0 0 5}$ & $\mathbf{2 0 1 0}$ & $\mathbf{2 0 1 5}$ & $\mathbf{2 0 2 0}$ & $\mathbf{2 0 2 5}$ & $\mathbf{2 0 3 0}$ \\
Africa & 0 & 0 & 0 & 0 & 0 & 0 \\
Australia & 0 & 0 & 0 & 0 & 0 & 0 \\
Canada & 0 & 0 & 0 & 0 & 0 & 0 \\
China & 0 & 0 & -51 & 0 & 0 & 0 \\
Central and South America & 0 & 136 & 420 & 932 & 1,287 & 0 \\
Eastern Europe & 0 & 0 & 0 & 0 & 0 & 0 \\
Former Soviet Union & 0 & 0 & 0 & 0 & 0 & 0 \\
India & 0 & 0 & 0 & 0 & 0 & 0 \\
Japan & 0 & 0 & 0 & 0 & 0 & 0 \\
Middle East & 0 & 0 & 0 & 0 & 0 & 0 \\
Mexico & 0 & 0 & 0 & 0 & 0 & 0 \\
Other Asia & 0 & 0 & 0 & -932 & -905 & 0 \\
South Korea & 0 & -136 & -369 & 0 & -382 & 0 \\
United States & 0 & 0 & 0 & 0 & 0 & 0 \\
Western Europe & 0 & 0 & 0 & 0 & 0 & 0 \\
\hline Total & $\mathbf{0}$ & $\mathbf{0}$ & $\mathbf{0}$ & $\mathbf{0}$ & $\mathbf{0}$ & $\mathbf{0}$ \\
\hline \hline
\end{tabular}

Table A-83: Reference Case with high feedstock availability sugar ethanol net trade [Million gallons]

\begin{tabular}{lrrrrrr} 
Africa & 0 & 0 & 0 & 0 & 0 & 0 \\
Australia & 0 & 0 & 0 & 0 & 0 & 0 \\
Canada & -145 & -259 & -640 & -205 & 0 & 0 \\
China & 0 & 0 & $-1,024$ & $-2,318$ & $-2,233$ & -585 \\
Central and South America & 1,484 & 3,372 & 5,806 & 9,199 & 11,424 & 10,764 \\
Eastern Europe & -589 & -557 & -444 & -179 & 0 & 0 \\
Former Soviet Union & 0 & 0 & 0 & 0 & 0 & 0 \\
India & 0 & 0 & 0 & 0 & 0 & 0 \\
Japan & -263 & -325 & -353 & -388 & -433 & -490 \\
Middle East & 0 & 0 & 0 & 0 & 0 & 0 \\
Mexico & 0 & 0 & 0 & 0 & -464 & 0 \\
Other Asia & 0 & 0 & 0 & $-1,234$ & $-2,100$ & $-3,472$ \\
South Korea & -217 & -199 & 0 & 0 & 0 & -359 \\
United States & -134 & -256 & $-1,750$ & $-3,137$ & $-3,837$ & $-5,858$ \\
Western Europe & -136 & $-1,776$ & $-1,595$ & $-1,738$ & $-2,358$ & 0 \\
\hline Total & $\mathbf{0}$ & $\mathbf{0}$ & $\mathbf{0}$ & $\mathbf{0}$ & $\mathbf{0}$ & $\mathbf{0}$ \\
\hline \hline
\end{tabular}


Table A-84: Reference Case with high feedstock availability cellulosic ethanol net trade [Million gallons]

\begin{tabular}{lrrrrrr} 
& $\mathbf{2 0 0 5}$ & $\mathbf{2 0 1 0}$ & $\mathbf{2 0 1 5}$ & $\mathbf{2 0 2 0}$ & $\mathbf{2 0 2 5}$ & $\mathbf{2 0 3 0}$ \\
Africa & 0 & 0 & 0 & 0 & 0 & 0 \\
Australia & 0 & 0 & 0 & 0 & 0 & 0 \\
Canada & 0 & 0 & 0 & 0 & 114 & 85 \\
China & 0 & 0 & 0 & -86 & -334 & -509 \\
Central and South America & 0 & 0 & 618 & 1,708 & 3,386 & 4,948 \\
Eastern Europe & 0 & 0 & 0 & 0 & 0 & 0 \\
Former Soviet Union & 0 & 0 & 0 & 0 & 0 & 0 \\
India & 0 & 0 & 0 & 86 & 220 & 423 \\
Japan & 0 & 0 & 0 & 0 & 0 & 0 \\
Middle East & 0 & 0 & 0 & 0 & 0 & 0 \\
Mexico & 0 & 0 & 170 & 0 & 916 & 0 \\
Other Asia & 0 & 0 & 0 & 0 & 0 & 0 \\
South Korea & 0 & 0 & 0 & -383 & 0 & -23 \\
United States & 0 & 0 & -788 & $-1,212$ & $-4,301$ & $-4,925$ \\
Western Europe & 0 & 0 & 0 & -113 & 0 & 0 \\
\hline Total & $\mathbf{0}$ & $\mathbf{0}$ & $\mathbf{0}$ & $\mathbf{0}$ & $\mathbf{0}$ & $\mathbf{0}$ \\
\hline \hline
\end{tabular}

Table A-85: Reference Case with high feedstock availability biomass-to-liquids production [Million gallons ethanol equivalent]

\begin{tabular}{lrrrrrr} 
& $\mathbf{2 0 0 5}$ & $\mathbf{2 0 1 0}$ & $\mathbf{2 0 1 5}$ & $\mathbf{2 0 2 0}$ & $\mathbf{2 0 2 5}$ & $\mathbf{2 0 3 0}$ \\
Africa & 0 & 0 & 68 & 263 & 653 & 1,177 \\
Argentina & 0 & 0 & 64 & 158 & 197 & 197 \\
Australia & 0 & 0 & 0 & 0 & 0 & 0 \\
Brazil & 0 & 0 & 106 & 320 & 750 & 1,333 \\
Canada & 0 & 0 & 93 & 280 & 410 & 410 \\
Caribbean basin & 0 & 0 & 0 & 0 & 0 & 0 \\
China & 0 & 0 & 80 & 240 & 563 & 563 \\
Colombia & 0 & 0 & 61 & 184 & 319 & 319 \\
Eastern Europe & 0 & 0 & 0 & 0 & 85 & 262 \\
Former Soviet Union & 0 & 0 & 0 & 0 & 0 & 0 \\
India & 0 & 0 & 77 & 232 & 544 & 987 \\
Japan & 0 & 0 & 0 & 0 & 0 & 0 \\
Middle East & 0 & 0 & 0 & 0 & 0 & 125 \\
Mexico & 0 & 0 & 72 & 216 & 379 & 379 \\
Other Asia & 0 & 0 & 89 & 448 & 1,090 & 1,942 \\
South Korea & 0 & 0 & 36 & 66 & 66 & 66 \\
United States & 0 & 0 & 822 & 4,236 & 5,656 & 7,265 \\
Western Europe & 0 & 0 & 0 & 0 & 0 & 0 \\
\hline Total & $\mathbf{0}$ & $\mathbf{0}$ & $\mathbf{1 , 5 6 7}$ & $\mathbf{6 , 6 4 3}$ & $\mathbf{1 0 , 7 1 0}$ & $\mathbf{1 5 , 0 2 4}$ \\
\hline \hline
\end{tabular}


Table A-86: Reference Case with high feedstock availability biomass-to-liquids consumption [Million gallons ethanol equivalent]

\begin{tabular}{lrrrrrr} 
& $\mathbf{2 0 0 5}$ & $\mathbf{2 0 1 0}$ & $\mathbf{2 0 1 5}$ & $\mathbf{2 0 2 0}$ & $\mathbf{2 0 2 5}$ & $\mathbf{2 0 3 0}$ \\
Africa & 0 & 0 & 68 & 263 & 653 & 1,177 \\
Australia & 0 & 0 & 0 & 0 & 0 & 0 \\
Canada & 0 & 0 & 0 & 0 & 0 & 0 \\
China & 0 & 0 & 0 & 0 & 0 & 0 \\
Central and South America & 0 & 0 & 0 & 0 & 0 & 0 \\
Eastern Europe & 0 & 0 & 0 & 0 & 85 & 262 \\
Former Soviet Union & 0 & 0 & 0 & 0 & 0 & 0 \\
India & 0 & 0 & 0 & 0 & 0 & 0 \\
Japan & 0 & 0 & 0 & 0 & 0 & 0 \\
Middle East & 0 & 0 & 0 & 0 & 0 & 125 \\
Mexico & 0 & 0 & 0 & 0 & 0 & 0 \\
Other Asia & 0 & 0 & 89 & 449 & 1,090 & 1,942 \\
South Korea & 0 & 0 & 0 & 0 & 0 & 0 \\
United States & 0 & 0 & 1,410 & 5,932 & 8,883 & 11,518 \\
Western Europe & 0 & 0 & 0 & 0 & 0 & 0 \\
\hline Total & $\mathbf{0}$ & $\mathbf{0}$ & $\mathbf{1 , 5 6 7}$ & $\mathbf{6 , 6 4 3}$ & $\mathbf{1 0 , 7 1 0}$ & $\mathbf{1 5 , 0 2 4}$ \\
\hline \hline
\end{tabular}

Table A-87: Reference Case with high feedstock availability biomass-to-liquids net trade [Million gallons ethanol equivalent]

\begin{tabular}{lrrrrrr} 
& $\mathbf{2 0 0 5}$ & $\mathbf{2 0 1 0}$ & $\mathbf{2 0 1 5}$ & $\mathbf{2 0 2 0}$ & $\mathbf{2 0 2 5}$ & $\mathbf{2 0 3 0}$ \\
Africa & 0 & 0 & 0 & 0 & 0 & 0 \\
Australia & 0 & 0 & 0 & 0 & 0 & 0 \\
Canada & 0 & 0 & 93 & 280 & 410 & 410 \\
China & 0 & 0 & 80 & 240 & 563 & 563 \\
Central and South America & 0 & 0 & 232 & 662 & 1,266 & 1,849 \\
Eastern Europe & 0 & 0 & 0 & 0 & 0 & 0 \\
Former Soviet Union & 0 & 0 & 0 & 0 & 0 & 0 \\
India & 0 & 0 & 77 & 232 & 544 & 987 \\
Japan & 0 & 0 & 0 & 0 & 0 & 0 \\
Middle East & 0 & 0 & 0 & 0 & 0 & 0 \\
Mexico & 0 & 0 & 72 & 216 & 379 & 379 \\
Other Asia & 0 & 0 & 0 & 0 & 0 & 0 \\
South Korea & 0 & 0 & 36 & 66 & 66 & 66 \\
United States & 0 & 0 & -589 & $-1,696$ & $-3,227$ & $-4,253$ \\
Western Europe & 0 & 0 & 0 & 0 & 0 & 0 \\
\hline Total & $\mathbf{0}$ & $\mathbf{0}$ & $\mathbf{0}$ & $\mathbf{0}$ & $\mathbf{0}$ & $\mathbf{0}$ \\
\hline \hline
\end{tabular}


Table A-88: Reference Case with high feedstock availability biodiesel production [Million gallons ethanol equivalent]

\begin{tabular}{lrrrrrr} 
& $\mathbf{2 0 0 5}$ & $\mathbf{2 0 1 0}$ & $\mathbf{2 0 1 5}$ & $\mathbf{2 0 2 0}$ & $\mathbf{2 0 2 5}$ & $\mathbf{2 0 3 0}$ \\
Africa & 0 & 0 & 0 & 0 & 0 & 0 \\
Argentina & 0 & 333 & 877 & 1,706 & 1,853 & 0 \\
Australia & 0 & 0 & 0 & 0 & 0 & 0 \\
Brazil & 0 & 333 & 877 & 1,579 & 1,579 & 1,115 \\
Canada & 0 & 0 & 0 & 0 & 0 & 0 \\
Caribbean basin & 0 & 95 & 310 & 742 & 1,611 & 3,359 \\
China & 0 & 0 & 0 & 0 & 0 & 0 \\
Colombia & 0 & 119 & 358 & 838 & 1,648 & 2,074 \\
Eastern Europe & 0 & 0 & 0 & 0 & 0 & 0 \\
Former Soviet Union & 0 & 0 & 0 & 0 & 0 & 0 \\
India & 0 & 0 & 0 & 0 & 0 & 0 \\
Japan & 0 & 0 & 0 & 0 & 0 & 0 \\
Middle East & 0 & 0 & 0 & 0 & 0 & 0 \\
Mexico & 0 & 0 & 0 & 0 & 0 & 0 \\
Other Asia & 0 & 0 & 0 & 0 & 0 & 0 \\
South Korea & 0 & 0 & 0 & 0 & 0 & 0 \\
United States & 0 & 1,182 & 1,640 & 1,747 & 1,850 & 2,016 \\
Western Europe & 998 & 3,178 & 3,578 & 3,930 & 4,468 & 4,788 \\
\hline Total & $\mathbf{9 9 8}$ & $\mathbf{5 , 2 3 8}$ & $\mathbf{7 , 6 3 9}$ & $\mathbf{1 0 , 5 4 2}$ & $\mathbf{1 3 , 0 0 9}$ & $\mathbf{1 3 , 3 5 1}$ \\
\hline \hline
\end{tabular}

Table A-89: Reference Case with high feedstock availability biodiesel consumption [Million gallons ethanol equivalent]

\begin{tabular}{lrrrrrr} 
& $\mathbf{2 0 0 5}$ & $\mathbf{2 0 1 0}$ & $\mathbf{2 0 1 5}$ & $\mathbf{2 0 2 0}$ & $\mathbf{2 0 2 5}$ & $\mathbf{2 0 3 0}$ \\
Africa & 0 & 0 & 0 & 0 & 0 & 0 \\
Australia & 0 & 0 & 0 & 0 & 0 & 0 \\
Canada & 0 & 0 & 0 & 0 & 0 & 0 \\
China & 0 & 0 & 0 & 0 & 0 & 0 \\
Central and South America & 0 & 158 & 472 & 984 & 1,306 & 1,490 \\
Eastern Europe & 0 & 0 & 0 & 0 & 0 & 0 \\
Former Soviet Union & 0 & 0 & 0 & 0 & 0 & 0 \\
India & 0 & 0 & 0 & 0 & 0 & 0 \\
Japan & 0 & 0 & 0 & 0 & 0 & 0 \\
Middle East & 0 & 0 & 0 & 0 & 0 & 0 \\
Mexico & 0 & 0 & 0 & 0 & 0 & 0 \\
Other Asia & 0 & 0 & 0 & 0 & 0 & 0 \\
South Korea & 0 & 0 & 0 & 0 & 0 & 0 \\
United States & 0 & 1,182 & 1,713 & 2,751 & 4,382 & 4,279 \\
Western Europe & 998 & 3,898 & 5,454 & 6,807 & 7,321 & 7,582 \\
\hline Total & $\mathbf{9 9 8}$ & $\mathbf{5 , 2 3 8}$ & $\mathbf{7 , 6 3 9}$ & $\mathbf{1 0 , 5 4 2}$ & $\mathbf{1 3 , 0 0 9}$ & $\mathbf{1 3 , 3 5 1}$ \\
\hline \hline
\end{tabular}


Table A-90: Reference Case with high feedstock availability biodiesel net trade [Million gallons ethanol equivalent]

Africa

Australia

Canada

China

Central and South America

Eastern Europe

Former Soviet Union

India

Japan

Middle East

Mexico

Other Asia

South Korea

United States

Western Europe

Total

\begin{tabular}{rrrrrr}
$\mathbf{2 0 0 5}$ & $\mathbf{2 0 1 0}$ & $\mathbf{2 0 1 5}$ & $\mathbf{2 0 2 0}$ & $\mathbf{2 0 2 5}$ & $\mathbf{2 0 3 0}$ \\
0 & 0 & 0 & 0 & 0 & 0 \\
0 & 0 & 0 & 0 & 0 & 0 \\
0 & 0 & 0 & 0 & 0 & 0 \\
0 & 0 & 0 & 0 & 0 & 0 \\
0 & 720 & 1,948 & 3,881 & 5,385 & 5,057 \\
0 & 0 & 0 & 0 & 0 & 0 \\
0 & 0 & 0 & 0 & 0 & 0 \\
0 & 0 & 0 & 0 & 0 & 0 \\
0 & 0 & 0 & 0 & 0 & 0 \\
0 & 0 & 0 & 0 & 0 & 0 \\
0 & 0 & 0 & 0 & 0 & 0 \\
0 & 0 & 0 & 0 & 0 & 0 \\
0 & 0 & 0 & 0 & 0 & 0 \\
0 & 0 & -73 & $-1,004$ & $-2,532$ & $-2,263$ \\
0 & -720 & $-1,876$ & $-2,877$ & $-2,853$ & $-2,794$ \\
\hline $\mathbf{0}$ & $\mathbf{0}$ & $\mathbf{0}$ & $\mathbf{0}$ & $\mathbf{0}$ & $\mathbf{0}$ \\
\hline
\end{tabular}




\section{A-6 Reference Case with Low Feedstock Availability}

Table A-91: Reference case with low feedstock availability ethanol production [Million gallons]

\begin{tabular}{lrrrrrr} 
& $\mathbf{2 0 0 5}$ & $\mathbf{2 0 1 0}$ & $\mathbf{2 0 1 5}$ & $\mathbf{2 0 2 0}$ & $\mathbf{2 0 2 5}$ & $\mathbf{2 0 3 0}$ \\
Africa & 300 & 330 & 364 & 402 & 455 & 502 \\
Argentina & 0 & 178 & 865 & 1,634 & 1,640 & 1,640 \\
Australia & 0 & 0 & 71 & 267 & 709 & 1,174 \\
Brazil & 4,866 & 4,894 & 6,282 & 7,362 & 8,942 & 11,721 \\
Canada & 0 & 143 & 401 & 921 & 1,136 & 1,136 \\
Caribbean basin & 162 & 80 & 34 & 0 & 0 & 0 \\
China & 344 & 366 & 608 & 723 & 1,564 & 3,969 \\
Colombia & 0 & 196 & 366 & 512 & 539 & 539 \\
Eastern Europe & 110 & 226 & 434 & 798 & 1,037 & 1,149 \\
Former Soviet Union & 0 & 0 & 37 & 114 & 347 & 1,059 \\
India & 313 & 0 & 214 & 645 & 1,512 & 2,816 \\
Japan & 0 & 0 & 95 & 369 & 612 & 647 \\
Middle East & 0 & 0 & 0 & 0 & 0 & 0 \\
Mexico & 0 & 0 & 0 & 0 & 0 & 0 \\
Other Asia & 0 & 0 & 0 & 0 & 0 & 0 \\
South Korea & 0 & 0 & 42 & 42 & 42 & 42 \\
United States & 3,850 & 11,612 & 15,802 & 17,967 & 21,334 & 22,930 \\
Western Europe & 600 & 2,570 & 2,755 & 3,398 & 5,291 & 8,013 \\
\hline Total & $\mathbf{1 0 , 5 4 4}$ & $\mathbf{2 0 , 5 9 4}$ & $\mathbf{2 8 , 3 7 1}$ & $\mathbf{3 5 , 1 5 2}$ & $\mathbf{4 5 , 1 6 0}$ & $\mathbf{5 7 , 3 3 5}$ \\
\hline \hline
\end{tabular}


Table A-92: Reference case with low feedstock availability ethanol consumption [Million gallons]

\begin{tabular}{lrrrrrr} 
& $\mathbf{2 0 0 5}$ & $\mathbf{2 0 1 0}$ & $\mathbf{2 0 1 5}$ & $\mathbf{2 0 2 0}$ & $\mathbf{2 0 2 5}$ & $\mathbf{2 0 3 0}$ \\
Africa & 300 & 330 & 364 & 402 & 455 & 502 \\
Australia & 0 & 0 & 71 & 267 & 709 & 1,174 \\
Canada & 101 & 128 & 361 & 829 & 1,022 & 1,022 \\
China & 126 & 0 & 0 & 0 & 797 & 4,345 \\
Central and South America & 3,764 & 4,831 & 6,124 & 7,172 & 7,850 & 8,641 \\
Eastern Europe & 699 & 226 & 434 & 927 & 1,037 & 1,149 \\
Former Soviet Union & 0 & 0 & 37 & 114 & 347 & 1,059 \\
India & 313 & 0 & 214 & 613 & 1,361 & 2,399 \\
Japan & 257 & 132 & 345 & 719 & 1,008 & 1,100 \\
Middle East & 0 & 0 & 0 & 0 & 0 & 0 \\
Mexico & 0 & 0 & 0 & 0 & 0 & 0 \\
Other Asia & 0 & 0 & 0 & 0 & 0 & 0 \\
South Korea & 264 & 0 & 400 & 414 & 413 & 413 \\
United States & 3,984 & 11,612 & 15,802 & 18,446 & 22,512 & 27,519 \\
Western Europe & 736 & 3,335 & 4,218 & 5,249 & 7,650 & 8,013 \\
\hline Total & $\mathbf{1 0 , 5 4 4}$ & $\mathbf{2 0 , 5 9 4}$ & $\mathbf{2 8 , 3 7 1}$ & $\mathbf{3 5 , 1 5 2}$ & $\mathbf{4 5 , 1 6 0}$ & $\mathbf{5 7 , 3 3 5}$ \\
\hline \hline
\end{tabular}

Table A-93: Reference case with low feedstock availability ethanol net trade [Million gallons]

\begin{tabular}{lrrrrrr} 
Africa & 0 & 0 & 0 & 0 & 0 & 0 \\
Australia & 0 & 0 & 0 & 0 & 0 & 0 \\
Canada & -101 & 14 & 40 & 92 & 114 & 114 \\
China & 218 & 366 & 608 & 723 & 767 & -376 \\
Central and South America & 1,264 & 517 & 1,423 & 2,335 & 3,271 & 5,259 \\
Eastern Europe & -589 & 0 & 0 & -129 & 0 & 0 \\
Former Soviet Union & 0 & 0 & 0 & 0 & 0 & 0 \\
India & 0 & 0 & 0 & 32 & 151 & 417 \\
Japan & -257 & -132 & -250 & -350 & -395 & -453 \\
Middle East & 0 & 0 & 0 & 0 & 0 & 0 \\
Mexico & 0 & 0 & 0 & 0 & 0 & 0 \\
Other Asia & 0 & 0 & 0 & 0 & 0 & 0 \\
South Korea & -264 & 0 & -358 & -372 & -371 & -371 \\
United States & -134 & 0 & 0 & -479 & $-1,178$ & $-4,589$ \\
Western Europe & -136 & -765 & $-1,463$ & $-1,851$ & $-2,359$ & 0 \\
\hline Total & $\mathbf{0}$ & $\mathbf{0}$ & $\mathbf{0}$ & $\mathbf{0}$ & $\mathbf{0}$ & $\mathbf{0}$ \\
\hline \hline
\end{tabular}


Table A-94: Reference case with low feedstock availability grain ethanol production [Million gallons]

\begin{tabular}{lrrrrrr} 
& $\mathbf{2 0 0 5}$ & $\mathbf{2 0 1 0}$ & $\mathbf{2 0 1 5}$ & $\mathbf{2 0 2 0}$ & $\mathbf{2 0 2 5}$ & $\mathbf{2 0 3 0}$ \\
Africa & 0 & 0 & 0 & 0 & 0 & 0 \\
Argentina & 0 & 178 & 640 & 833 & 839 & 839 \\
Australia & 0 & 0 & 0 & 0 & 0 & 0 \\
Brazil & 0 & 0 & 0 & 0 & 0 & 0 \\
Canada & 0 & 143 & 143 & 143 & 0 & 0 \\
Caribbean basin & 0 & 0 & 0 & 0 & 0 & 0 \\
China & 344 & 366 & 366 & 55 & 0 & 0 \\
Colombia & 0 & 0 & 0 & 0 & 0 & 0 \\
Eastern Europe & 110 & 226 & 348 & 477 & 367 & 251 \\
Former Soviet Union & 0 & 0 & 0 & 0 & 0 & 0 \\
India & 0 & 0 & 0 & 0 & 0 & 0 \\
Japan & 0 & 0 & 0 & 0 & 0 & 0 \\
Middle East & 0 & 0 & 0 & 0 & 0 & 0 \\
Mexico & 0 & 0 & 0 & 0 & 0 & 0 \\
Other Asia & 0 & 0 & 0 & 0 & 0 & 0 \\
South Korea & 0 & 0 & 0 & 0 & 0 & 0 \\
United States & 3,850 & 11,612 & 15,000 & 15,000 & 15,000 & 15,000 \\
Western Europe & 600 & 2,570 & 2,589 & 2,705 & 3,178 & 3,604 \\
\hline Total & $\mathbf{4 , 9 0 4}$ & $\mathbf{1 5 , 0 9 4}$ & $\mathbf{1 9 , 0 8 5}$ & $\mathbf{1 9 , 2 1 3}$ & $\mathbf{1 9 , 3 8 4}$ & $\mathbf{1 9 , 6 9 4}$ \\
\hline \hline
\end{tabular}

Table A-95: Reference case with low feedstock availability sugar ethanol production [Million gallons]

\begin{tabular}{lrrrrrr} 
& $\mathbf{2 0 0 5}$ & $\mathbf{2 0 1 0}$ & $\mathbf{2 0 1 5}$ & $\mathbf{2 0 2 0}$ & $\mathbf{2 0 2 5}$ & $\mathbf{2 0 3 0}$ \\
Africa & 300 & 330 & 364 & 402 & 444 & 490 \\
Argentina & 0 & 0 & 0 & 0 & 0 & 0 \\
Australia & 0 & 0 & 0 & 0 & 0 & 0 \\
Brazil & 4,866 & 4,894 & 5,978 & 6,473 & 6,857 & 6,970 \\
Canada & 0 & 0 & 0 & 0 & 0 & 0 \\
Caribbean basin & 162 & 80 & 34 & 0 & 0 & 0 \\
China & 0 & 0 & 0 & 0 & 0 & 0 \\
Colombia & 0 & 196 & 196 & 0 & 0 & 0 \\
Eastern Europe & 0 & 0 & 0 & 0 & 0 & 0 \\
Former Soviet Union & 0 & 0 & 0 & 0 & 0 & 0 \\
India & 313 & 0 & 0 & 0 & 0 & 0 \\
Japan & 0 & 0 & 0 & 0 & 0 & 0 \\
Middle East & 0 & 0 & 0 & 0 & 0 & 0 \\
Mexico & 0 & 0 & 0 & 0 & 0 & 0 \\
Other Asia & 0 & 0 & 0 & 0 & 0 & 0 \\
South Korea & 0 & 0 & 0 & 0 & 0 & 0 \\
United States & 0 & 0 & 0 & 0 & 0 & 0 \\
Western Europe & 0 & 0 & 0 & 0 & 0 & 0 \\
\hline Total & $\mathbf{5 , 6 4 0}$ & $\mathbf{5 , 5 0 0}$ & $\mathbf{6 , 5 7 2}$ & $\mathbf{6 , 8 7 5}$ & $\mathbf{7 , 3 0 1}$ & $\mathbf{7 , 4 6 0}$ \\
\hline \hline
\end{tabular}


Table A-96: Reference case with low feedstock availability cellulosic ethanol production [Million gallons]

\begin{tabular}{lrrrrrr} 
& $\mathbf{2 0 0 5}$ & $\mathbf{2 0 1 0}$ & $\mathbf{2 0 1 5}$ & $\mathbf{2 0 2 0}$ & $\mathbf{2 0 2 5}$ & $\mathbf{2 0 3 0}$ \\
Africa & 0 & 0 & 0 & 0 & 11 & 11 \\
Argentina & 0 & 0 & 225 & 801 & 801 & 801 \\
Australia & 0 & 0 & 71 & 267 & 709 & 1,174 \\
Brazil & 0 & 0 & 304 & 890 & 2,085 & 4,751 \\
Canada & 0 & 0 & 259 & 779 & 1,136 & 1,136 \\
Caribbean basin & 0 & 0 & 0 & 0 & 0 & 0 \\
China & 0 & 0 & 243 & 667 & 1,564 & 3,969 \\
Colombia & 0 & 0 & 170 & 512 & 539 & 539 \\
Eastern Europe & 0 & 0 & 85 & 320 & 670 & 897 \\
Former Soviet Union & 0 & 0 & 37 & 114 & 347 & 1,059 \\
India & 0 & 0 & 214 & 645 & 1,512 & 2,816 \\
Japan & 0 & 0 & 95 & 369 & 612 & 647 \\
Middle East & 0 & 0 & 0 & 0 & 0 & 0 \\
Mexico & 0 & 0 & 0 & 0 & 0 & 0 \\
Other Asia & 0 & 0 & 0 & 0 & 0 & 0 \\
South Korea & 0 & 0 & 42 & 42 & 42 & 42 \\
United States & 0 & 0 & 802 & 2,967 & 6,334 & 7,930 \\
Western Europe & 0 & 0 & 166 & 693 & 2,113 & 4,410 \\
\hline Total & $\mathbf{0}$ & $\mathbf{0}$ & $\mathbf{2 , 7 1 3}$ & $\mathbf{9 , 0 6 4}$ & $\mathbf{1 8 , 4 7 6}$ & $\mathbf{3 0 , 1 8 1}$ \\
\hline \hline
\end{tabular}

Table A-97: Reference case with low feedstock availability grain ethanol consumption [Million gallons]

\begin{tabular}{lrrrrrr} 
& $\mathbf{2 0 0 5}$ & $\mathbf{2 0 1 0}$ & $\mathbf{2 0 1 5}$ & $\mathbf{2 0 2 0}$ & $\mathbf{2 0 2 5}$ & $\mathbf{2 0 3 0}$ \\
Africa & 0 & 0 & 0 & 0 & 0 & 0 \\
Australia & 0 & 0 & 0 & 0 & 0 & 0 \\
Canada & 0 & 128 & 143 & 143 & 0 & 0 \\
China & 126 & 0 & 0 & 0 & 0 & 0 \\
Central and South America & 0 & 36 & 130 & 212 & 169 & 169 \\
Eastern Europe & 110 & 226 & 348 & 515 & 367 & 251 \\
Former Soviet Union & 0 & 0 & 0 & 0 & 0 & 0 \\
India & 0 & 0 & 0 & 0 & 0 & 0 \\
Japan & 0 & 132 & 250 & 55 & 0 & 299 \\
Middle East & 0 & 0 & 0 & 0 & 0 & 0 \\
Mexico & 0 & 0 & 0 & 0 & 0 & 0 \\
Other Asia & 0 & 0 & 0 & 0 & 0 & 0 \\
South Korea & 218 & 0 & 116 & 0 & 0 & 371 \\
United States & 3,850 & 11,612 & 15,000 & 15,000 & 15,000 & 15,000 \\
Western Europe & 600 & 2,960 & 3,099 & 3,288 & 3,848 & 3,604 \\
\hline Total & $\mathbf{4 , 9 0 4}$ & $\mathbf{1 5 , 0 9 4}$ & $\mathbf{1 9 , 0 8 5}$ & $\mathbf{1 9 , 2 1 3}$ & $\mathbf{1 9 , 3 8 4}$ & $\mathbf{1 9 , 6 9 4}$ \\
\hline \hline
\end{tabular}


Table A-98: Reference case with low feedstock availability sugar ethanol consumption [Million gallons]

\begin{tabular}{lrrrrrr} 
& $\mathbf{2 0 0 5}$ & $\mathbf{2 0 1 0}$ & $\mathbf{2 0 1 5}$ & $\mathbf{2 0 2 0}$ & $\mathbf{2 0 2 5}$ & $\mathbf{2 0 3 0}$ \\
Africa & 300 & 330 & 364 & 402 & 444 & 490 \\
Australia & 0 & 0 & 0 & 0 & 0 & 0 \\
Canada & 101 & 0 & 0 & 0 & 0 & 0 \\
China & 0 & 0 & 0 & 0 & 0 & 0 \\
Central and South America & 3,764 & 4,795 & 5,995 & 5,993 & 5,679 & 4,074 \\
Eastern Europe & 589 & 0 & 0 & 0 & 0 & 0 \\
Former Soviet Union & 0 & 0 & 0 & 0 & 0 & 0 \\
India & 313 & 0 & 0 & 0 & 0 & 0 \\
Japan & 257 & 0 & 0 & 0 & 0 & 0 \\
Middle East & 0 & 0 & 0 & 0 & 0 & 0 \\
Mexico & 0 & 0 & 0 & 0 & 0 & 0 \\
Other Asia & 0 & 0 & 0 & 0 & 0 & 0 \\
South Korea & 46 & 0 & 0 & 0 & 0 & 0 \\
United States & 134 & 0 & 0 & 479 & 1,178 & 2,896 \\
Western Europe & 136 & 375 & 213 & 0 & 0 & 0 \\
\hline Total & $\mathbf{5 , 6 4 0}$ & $\mathbf{5 , 5 0 0}$ & $\mathbf{6 , 5 7 2}$ & $\mathbf{6 , 8 7 5}$ & $\mathbf{7 , 3 0 1}$ & $\mathbf{7 , 4 6 0}$ \\
\hline \hline
\end{tabular}

Table A-99: Reference case with low feedstock availability cellulosic ethanol consumption [Million gallons]

\begin{tabular}{lrrrrrr} 
& $\mathbf{2 0 0 5}$ & $\mathbf{2 0 1 0}$ & $\mathbf{2 0 1 5}$ & $\mathbf{2 0 2 0}$ & $\mathbf{2 0 2 5}$ & $\mathbf{2 0 3 0}$ \\
Africa & 0 & 0 & 0 & 0 & 11 & 11 \\
Australia & 0 & 0 & 71 & 267 & 709 & 1,174 \\
Canada & 0 & 0 & 219 & 687 & 1,022 & 1,022 \\
China & 0 & 0 & 0 & 0 & 797 & 4,345 \\
Central and South America & 0 & 0 & 0 & 967 & 2,002 & 4,398 \\
Eastern Europe & 0 & 0 & 85 & 413 & 670 & 897 \\
Former Soviet Union & 0 & 0 & 37 & 114 & 347 & 1,059 \\
India & 0 & 0 & 214 & 613 & 1,361 & 2,399 \\
Japan & 0 & 0 & 95 & 664 & 1,008 & 801 \\
Middle East & 0 & 0 & 0 & 0 & 0 & 0 \\
Mexico & 0 & 0 & 0 & 0 & 0 & 0 \\
Other Asia & 0 & 0 & 0 & 0 & 0 & 0 \\
South Korea & 0 & 0 & 284 & 414 & 413 & 42 \\
United States & 0 & 0 & 802 & 2,967 & 6,334 & 9,623 \\
Western Europe & 0 & 0 & 906 & 1,961 & 3,802 & 4,410 \\
\hline Total & $\mathbf{0}$ & $\mathbf{0}$ & $\mathbf{2 , 7 1 3}$ & $\mathbf{9 , 0 6 4}$ & $\mathbf{1 8 , 4 7 6}$ & $\mathbf{3 0 , 1 8 1}$ \\
\hline \hline
\end{tabular}


Table A-100: Reference case with low feedstock availability grain ethanol net trade [Million gallons]

\begin{tabular}{lrrrrrr} 
& $\mathbf{2 0 0 5}$ & $\mathbf{2 0 1 0}$ & $\mathbf{2 0 1 5}$ & $\mathbf{2 0 2 0}$ & $\mathbf{2 0 2 5}$ & $\mathbf{2 0 3 0}$ \\
Africa & 0 & 0 & 0 & 0 & 0 & 0 \\
Australia & 0 & 0 & 0 & 0 & 0 & 0 \\
Canada & 0 & 14 & 0 & 0 & 0 & 0 \\
China & 218 & 366 & 366 & 55 & 0 & 0 \\
Central and South America & 0 & 143 & 510 & 620 & 670 & 670 \\
Eastern Europe & 0 & 0 & 0 & -37 & 0 & 0 \\
Former Soviet Union & 0 & 0 & 0 & 0 & 0 & 0 \\
India & 0 & 0 & 0 & 0 & 0 & 0 \\
Japan & 0 & -132 & -250 & -55 & 0 & -299 \\
Middle East & 0 & 0 & 0 & 0 & 0 & 0 \\
Mexico & 0 & 0 & 0 & 0 & 0 & 0 \\
Other Asia & 0 & 0 & 0 & 0 & 0 & 0 \\
South Korea & -218 & 0 & -116 & 0 & 0 & -371 \\
United States & 0 & 0 & 0 & 0 & 0 & 0 \\
Western Europe & 0 & -390 & -510 & -583 & -670 & 0 \\
\hline Total & $\mathbf{0}$ & $\mathbf{0}$ & $\mathbf{0}$ & $\mathbf{0}$ & $\mathbf{0}$ & $\mathbf{0}$ \\
\hline \hline
\end{tabular}

Table A-81: Reference case with low feedstock availability sugar ethanol net trade [Million gallons]

\begin{tabular}{|c|c|c|c|c|c|c|}
\hline Africa & 0 & 0 & 0 & 0 & 0 & 0 \\
\hline Australia & 0 & 0 & 0 & 0 & 0 & 0 \\
\hline Canada & -101 & 0 & 0 & 0 & 0 & 0 \\
\hline China & 0 & 0 & 0 & 0 & 0 & 0 \\
\hline Central and South America & 1,264 & 375 & 213 & 479 & 1,178 & 2,896 \\
\hline Eastern Europe & -589 & 0 & 0 & 0 & 0 & 0 \\
\hline Former Soviet Union & 0 & 0 & 0 & 0 & 0 & 0 \\
\hline India & 0 & 0 & 0 & 0 & 0 & 0 \\
\hline Japan & -257 & 0 & 0 & 0 & 0 & 0 \\
\hline Middle East & 0 & 0 & 0 & 0 & 0 & 0 \\
\hline Mexico & 0 & 0 & 0 & 0 & 0 & 0 \\
\hline Other Asia & 0 & 0 & 0 & 0 & 0 & 0 \\
\hline South Korea & -46 & 0 & 0 & 0 & 0 & 0 \\
\hline United States & -134 & 0 & 0 & -479 & $-1,178$ & $-2,896$ \\
\hline Western Europe & -136 & -375 & -213 & 0 & 0 & 0 \\
\hline Total & $\mathbf{0}$ & $\mathbf{0}$ & $\mathbf{0}$ & $\mathbf{0}$ & $\mathbf{0}$ & $\mathbf{0}$ \\
\hline
\end{tabular}


Table A-92: Reference case with low feedstock availability cellulosic ethanol net trade [Million gallons]

\begin{tabular}{lrrrrrr} 
& $\mathbf{2 0 0 5}$ & $\mathbf{2 0 1 0}$ & $\mathbf{2 0 1 5}$ & $\mathbf{2 0 2 0}$ & $\mathbf{2 0 2 5}$ & $\mathbf{2 0 3 0}$ \\
Africa & 0 & 0 & 0 & 0 & 0 & 0 \\
Australia & 0 & 0 & 0 & 0 & 0 & 0 \\
Canada & 0 & 0 & 40 & 92 & 114 & 114 \\
China & 0 & 0 & 243 & 667 & 767 & -376 \\
Central and South America & 0 & 0 & 699 & 1,236 & 1,424 & 1,694 \\
Eastern Europe & 0 & 0 & 0 & -92 & 0 & 0 \\
Former Soviet Union & 0 & 0 & 0 & 0 & 0 & 0 \\
India & 0 & 0 & 0 & 32 & 151 & 417 \\
Japan & 0 & 0 & 0 & -295 & -395 & -154 \\
Middle East & 0 & 0 & 0 & 0 & 0 & 0 \\
Mexico & 0 & 0 & 0 & 0 & 0 & 0 \\
Other Asia & 0 & 0 & 0 & 0 & 0 & 0 \\
South Korea & 0 & 0 & -243 & -372 & -371 & 0 \\
United States & 0 & 0 & 0 & 0 & 0 & $-1,694$ \\
Western Europe & 0 & 0 & -740 & $-1,268$ & $-1,689$ & 0 \\
\hline Total & $\mathbf{0}$ & $\mathbf{0}$ & $\mathbf{0}$ & $\mathbf{0}$ & $\mathbf{0}$ & $\mathbf{0}$ \\
\hline \hline
\end{tabular}

Table A-103: Reference case with low feedstock availability biomass-to-liquids production [Million gallons ethanol equivalent]

\begin{tabular}{lrrrrrr} 
& $\mathbf{2 0 0 5}$ & $\mathbf{2 0 1 0}$ & $\mathbf{2 0 1 5}$ & $\mathbf{2 0 2 0}$ & $\mathbf{2 0 2 5}$ & $\mathbf{2 0 3 0}$ \\
Africa & 0 & 0 & 68 & 263 & 653 & 1,177 \\
Argentina & 0 & 0 & 81 & 288 & 288 & 288 \\
Australia & 0 & 0 & 0 & 0 & 0 & 0 \\
Brazil & 0 & 0 & 97 & 320 & 750 & 1,333 \\
Canada & 0 & 0 & 93 & 280 & 409 & 409 \\
Caribbean basin & 0 & 0 & 0 & 0 & 0 & 0 \\
China & 0 & 0 & 57 & 240 & 563 & 563 \\
Colombia & 0 & 0 & 61 & 184 & 194 & 194 \\
Eastern Europe & 0 & 0 & 0 & 0 & 116 & 311 \\
Former Soviet Union & 0 & 0 & 0 & 0 & 0 & 0 \\
India & 0 & 0 & 77 & 232 & 544 & 1,001 \\
Japan & 0 & 0 & 0 & 0 & 0 & 0 \\
Middle East & 0 & 0 & 0 & 0 & 0 & 125 \\
Mexico & 0 & 0 & 0 & 0 & 0 & 0 \\
Other Asia & 0 & 0 & 89 & 448 & 1,090 & 1,942 \\
South Korea & 0 & 0 & 43 & 89 & 89 & 89 \\
United States & 0 & 0 & 822 & 3,911 & 5,242 & 6,736 \\
Western Europe & 0 & 0 & 0 & 0 & 0 & 0 \\
\hline Total & $\mathbf{0}$ & $\mathbf{0}$ & $\mathbf{1 , 4 8 8}$ & $\mathbf{6 , 2 5 5}$ & $\mathbf{9 , 9 3 6}$ & $\mathbf{1 4 , 1 6 7}$ \\
\hline \hline
\end{tabular}


Table A-104: Reference case with low feedstock availability biomass-to-liquids consumption [Million gallons ethanol equivalent]

\begin{tabular}{lrrrrrr} 
& $\mathbf{2 0 0 5}$ & $\mathbf{2 0 1 0}$ & $\mathbf{2 0 1 5}$ & $\mathbf{2 0 2 0}$ & $\mathbf{2 0 2 5}$ & $\mathbf{2 0 3 0}$ \\
Africa & 0 & 0 & 68 & 263 & 653 & 1,177 \\
Australia & 0 & 0 & 0 & 0 & 0 & 0 \\
Canada & 0 & 0 & 0 & 0 & 0 & 0 \\
China & 0 & 0 & 0 & 0 & 0 & 0 \\
Central and South America & 0 & 0 & 0 & 0 & 0 & 0 \\
Eastern Europe & 0 & 0 & 0 & 0 & 116 & 311 \\
Former Soviet Union & 0 & 0 & 0 & 0 & 0 & 0 \\
India & 0 & 0 & 0 & 0 & 0 & 0 \\
Japan & 0 & 0 & 0 & 0 & 0 & 0 \\
Middle East & 0 & 0 & 0 & 0 & 0 & 125 \\
Mexico & 0 & 0 & 0 & 0 & 0 & 0 \\
Other Asia & 0 & 0 & 89 & 449 & 1,090 & 1,942 \\
South Korea & 0 & 0 & 0 & 0 & 0 & 0 \\
United States & 0 & 0 & 895 & 4,215 & 7,259 & 10,612 \\
Western Europe & 0 & 0 & 436 & 1,329 & 819 & 0 \\
\hline Total & $\mathbf{0}$ & $\mathbf{0}$ & $\mathbf{1 , 4 8 8}$ & $\mathbf{6 , 2 5 5}$ & $\mathbf{9 , 9 3 6}$ & $\mathbf{1 4 , 1 6 7}$ \\
\hline \hline
\end{tabular}

Table A-105: Reference case with low feedstock availability biomass-to-liquids net trade [Million gallons ethanol equivalent]

\begin{tabular}{lrrrrrr} 
& $\mathbf{2 0 0 5}$ & $\mathbf{2 0 1 0}$ & $\mathbf{2 0 1 5}$ & $\mathbf{2 0 2 0}$ & $\mathbf{2 0 2 5}$ & $\mathbf{2 0 3 0}$ \\
Africa & 0 & 0 & 0 & 0 & 0 & 0 \\
Australia & 0 & 0 & 0 & 0 & 0 & 0 \\
Canada & 0 & 0 & 93 & 280 & 409 & 409 \\
China & 0 & 0 & 57 & 240 & 563 & 563 \\
Central and South America & 0 & 0 & 239 & 792 & 1,232 & 1,815 \\
Eastern Europe & 0 & 0 & 0 & 0 & 0 & 0 \\
Former Soviet Union & 0 & 0 & 0 & 0 & 0 & 0 \\
India & 0 & 0 & 77 & 232 & 544 & 1,001 \\
Japan & 0 & 0 & 0 & 0 & 0 & 0 \\
Middle East & 0 & 0 & 0 & 0 & 0 & 0 \\
Mexico & 0 & 0 & 0 & 0 & 0 & 0 \\
Other Asia & 0 & 0 & 0 & 0 & 0 & 0 \\
South Korea & 0 & 0 & 43 & 89 & 89 & 89 \\
United States & 0 & 0 & -73 & -304 & $-2,017$ & $-3,876$ \\
Western Europe & 0 & 0 & -436 & $-1,329$ & -819 & 0 \\
\hline Total & $\mathbf{0}$ & $\mathbf{0}$ & $\mathbf{0}$ & $\mathbf{0}$ & $\mathbf{0}$ & $\mathbf{0}$ \\
\hline \hline
\end{tabular}


Table A-106: Reference case with low feedstock availability biodiesel production [Million gallons ethanol equivalent]

\begin{tabular}{lrrrrrr} 
& $\mathbf{2 0 0 5}$ & $\mathbf{2 0 1 0}$ & $\mathbf{2 0 1 5}$ & $\mathbf{2 0 2 0}$ & $\mathbf{2 0 2 5}$ & $\mathbf{2 0 3 0}$ \\
Africa & 0 & 0 & 0 & 0 & 0 & 0 \\
Argentina & 0 & 333 & 877 & 1,491 & 1,472 & 1,155 \\
Australia & 0 & 0 & 0 & 0 & 0 & 0 \\
Brazil & 0 & 333 & 835 & 1,473 & 1,627 & 1,330 \\
Canada & 0 & 0 & 0 & 0 & 0 & 0 \\
Caribbean basin & 0 & 95 & 310 & 742 & 1,145 & 1,220 \\
China & 0 & 0 & 0 & 0 & 0 & 0 \\
Colombia & 0 & 0 & 0 & 0 & 0 & 0 \\
Eastern Europe & 0 & 0 & 0 & 0 & 0 & 0 \\
Former Soviet Union & 0 & 0 & 0 & 0 & 0 & 0 \\
India & 0 & 0 & 0 & 0 & 0 & 0 \\
Japan & 0 & 0 & 0 & 0 & 0 & 0 \\
Middle East & 0 & 0 & 0 & 0 & 0 & 0 \\
Mexico & 0 & 0 & 0 & 0 & 0 & 0 \\
Other Asia & 0 & 0 & 0 & 0 & 0 & 0 \\
South Korea & 0 & 0 & 0 & 0 & 0 & 0 \\
United States & 0 & 1,238 & 1,640 & 1,803 & 1,907 & 2,072 \\
Western Europe & 998 & 2,910 & 3,578 & 3,930 & 4,468 & 4,788 \\
\hline Total & $\mathbf{9 9 8}$ & $\mathbf{4 , 9 0 8}$ & $\mathbf{7 , 2 3 9}$ & $\mathbf{9 , 4 4 0}$ & $\mathbf{1 0 , 6 1 9}$ & $\mathbf{1 0 , 5 6 4}$ \\
\hline \hline
\end{tabular}

Table A-107: Reference case with low feedstock availability biodiesel consumption [Million gallons ethanol equivalent]

\begin{tabular}{lrrrrrr} 
& $\mathbf{2 0 0 5}$ & $\mathbf{2 0 1 0}$ & $\mathbf{2 0 1 5}$ & $\mathbf{2 0 2 0}$ & $\mathbf{2 0 2 5}$ & $\mathbf{2 0 3 0}$ \\
Africa & 0 & 0 & 0 & 0 & 0 & 0 \\
Australia & 0 & 0 & 0 & 0 & 0 & 0 \\
Canada & 0 & 0 & 0 & 0 & 0 & 0 \\
China & 0 & 0 & 0 & 0 & 0 & 0 \\
Central and South America & 0 & 126 & 438 & 936 & 1,262 & 1,448 \\
Eastern Europe & 0 & 0 & 0 & 0 & 0 & 0 \\
Former Soviet Union & 0 & 0 & 0 & 0 & 0 & 0 \\
India & 0 & 0 & 0 & 0 & 0 & 0 \\
Japan & 0 & 0 & 0 & 0 & 0 & 0 \\
Middle East & 0 & 0 & 0 & 0 & 0 & 0 \\
Mexico & 0 & 0 & 0 & 0 & 0 & 0 \\
Other Asia & 0 & 0 & 0 & 0 & 0 & 0 \\
South Korea & 0 & 0 & 0 & 0 & 0 & 0 \\
United States & 0 & 1,238 & 2,494 & 4,000 & 3,787 & 2,104 \\
Western Europe & 998 & 3,544 & 4,307 & 4,504 & 5,570 & 7,012 \\
\hline Total & $\mathbf{9 9 8}$ & $\mathbf{4 , 9 0 8}$ & $\mathbf{7 , 2 3 9}$ & $\mathbf{9 , 4 4 0}$ & $\mathbf{1 0 , 6 1 9}$ & $\mathbf{1 0 , 5 6 4}$ \\
\hline \hline
\end{tabular}


Table A-108: Reference case with low feedstock availability biodiesel net trade [Million gallons ethanol equivalent]

\begin{tabular}{l} 
Africa \\
Australia \\
Canada \\
China \\
Central and South America \\
Eastern Europe \\
Former Soviet Union \\
India \\
Japan \\
Middle East \\
Mexico \\
Other Asia \\
South Korea \\
United States \\
Western Europe \\
\hline Total
\end{tabular}

Africa

Australia

Canada

Central and South America

Total

\begin{tabular}{rrrrrr}
$\mathbf{2 0 0 5}$ & $\mathbf{2 0 1 0}$ & $\mathbf{2 0 1 5}$ & $\mathbf{2 0 2 0}$ & $\mathbf{2 0 2 5}$ & $\mathbf{2 0 3 0}$ \\
0 & 0 & 0 & 0 & 0 & 0 \\
0 & 0 & 0 & 0 & 0 & 0 \\
0 & 0 & 0 & 0 & 0 & 0 \\
0 & 0 & 0 & 0 & 0 & 0 \\
0 & 634 & 1,583 & 2,770 & 2,982 & 2,256 \\
0 & 0 & 0 & 0 & 0 & 0 \\
0 & 0 & 0 & 0 & 0 & 0 \\
0 & 0 & 0 & 0 & 0 & 0 \\
0 & 0 & 0 & 0 & 0 & 0 \\
0 & 0 & 0 & 0 & 0 & 0 \\
0 & 0 & 0 & 0 & 0 & 0 \\
0 & 0 & 0 & 0 & 0 & 0 \\
0 & 0 & 0 & 0 & 0 & 0 \\
0 & 0 & -854 & $-2,197$ & $-1,881$ & -32 \\
0 & -634 & -729 & -574 & $-1,101$ & $-2,224$ \\
\hline $\mathbf{0}$ & $\mathbf{0}$ & $\mathbf{0}$ & $\mathbf{0}$ & $\mathbf{0}$ & $\mathbf{0}$ \\
\hline
\end{tabular}




\section{A-7 Reference Case with High Oil Price}

Table A-109: Reference case with high oil price ethanol production [Million gallons]

\begin{tabular}{lrrrrrr} 
& $\mathbf{2 0 0 5}$ & $\mathbf{2 0 1 0}$ & $\mathbf{2 0 1 5}$ & $\mathbf{2 0 2 0}$ & $\mathbf{2 0 2 5}$ & $\mathbf{2 0 3 0}$ \\
Africa & 300 & 330 & 364 & 402 & 455 & 502 \\
Argentina & 0 & 178 & 810 & 1,914 & 1,915 & 1,915 \\
Australia & 0 & 0 & 71 & 246 & 583 & 1,172 \\
Brazil & 4,866 & 5,977 & 7,624 & 9,752 & 12,299 & 13,686 \\
Canada & 0 & 0 & 259 & 779 & 1,053 & 1,057 \\
Caribbean basin & 210 & 360 & 573 & 683 & 779 & 890 \\
China & 344 & 384 & 687 & 1,171 & 2,371 & 3,561 \\
Colombia & 196 & 215 & 411 & 782 & 1,501 & 2,231 \\
Eastern Europe & 110 & 226 & 428 & 712 & 1,037 & 1,055 \\
Former Soviet Union & 0 & 0 & 39 & 120 & 367 & 1,120 \\
India & 313 & 279 & 541 & 992 & 1,893 & 2,822 \\
Japan & 0 & 0 & 95 & 369 & 609 & 727 \\
Middle East & 0 & 0 & 0 & 0 & 0 & 0 \\
Mexico & 0 & 0 & 199 & 601 & 1,408 & 1,546 \\
Other Asia & 0 & 0 & 0 & 0 & 0 & 0 \\
South Korea & 0 & 0 & 42 & 42 & 42 & 42 \\
United States & 3,850 & 11,612 & 15,802 & 17,258 & 20,065 & 22,205 \\
Western Europe & 600 & 2,570 & 2,755 & 3,235 & 5,291 & 8,013 \\
\hline Total & $\mathbf{1 0 , 7 8 9}$ & $\mathbf{2 2 , 1 3 1}$ & $\mathbf{3 0 , 7 0 1}$ & $\mathbf{3 9 , 0 5 7}$ & $\mathbf{5 1 , 6 7 0}$ & $\mathbf{6 2 , 5 4 1}$ \\
\hline \hline
\end{tabular}


Table A-110: Reference case with high oil price ethanol consumption [Million gallons]

\begin{tabular}{lrrrrrr} 
& $\mathbf{2 0 0 5}$ & $\mathbf{2 0 1 0}$ & $\mathbf{2 0 1 5}$ & $\mathbf{2 0 2 0}$ & $\mathbf{2 0 2 5}$ & $\mathbf{2 0 3 0}$ \\
Africa & 300 & 330 & 364 & 402 & 455 & 502 \\
Australia & 0 & 0 & 71 & 246 & 583 & 1,172 \\
Canada & 145 & 0 & 259 & 895 & 948 & 951 \\
China & 265 & 0 & 0 & 1,511 & 4,132 & 5,063 \\
Central and South America & 3,788 & 4,856 & 6,156 & 7,284 & 8,060 & 8,966 \\
Eastern Europe & 699 & 226 & 872 & 953 & 1,037 & 1,055 \\
Former Soviet Union & 0 & 0 & 39 & 120 & 367 & 1,120 \\
India & 313 & 279 & 541 & 992 & 1,893 & 2,399 \\
Japan & 295 & 320 & 424 & 757 & 1,042 & 1,194 \\
Middle East & 0 & 0 & 0 & 0 & 0 & 0 \\
Mexico & 0 & 0 & 30 & 601 & 1,030 & 1,052 \\
Other Asia & 0 & 0 & 0 & 0 & 497 & 3,472 \\
South Korea & 264 & 335 & 400 & 414 & 413 & 413 \\
United States & 3,984 & 11,612 & 17,268 & 19,797 & 23,562 & 27,170 \\
Western Europe & 736 & 4,173 & 4,276 & 5,086 & 7,650 & 8,013 \\
\hline Total & $\mathbf{1 0 , 7 8 9}$ & $\mathbf{2 2 , 1 3 1}$ & $\mathbf{3 0 , 7 0 1}$ & $\mathbf{3 9 , 0 5 7}$ & $\mathbf{5 1 , 6 7 0}$ & $\mathbf{6 2 , 5 4 1}$ \\
\hline \hline
\end{tabular}

Table A-111: Reference case with high oil price ethanol net trade [Million gallons]

Africa

\begin{tabular}{rrrrrr}
$\mathbf{2 0 0 5}$ & $\mathbf{2 0 1 0}$ & $\mathbf{2 0 1 5}$ & $\mathbf{2 0 2 0}$ & $\mathbf{2 0 2 5}$ & $\mathbf{2 0 3 0}$ \\
0 & 0 & 0 & 0 & 0 & 0 \\
0 & 0 & 0 & 0 & 0 & 0 \\
-145 & 0 & 0 & -116 & 105 & 106 \\
80 & 384 & 687 & -340 & $-1,761$ & $-1,502$ \\
1,484 & 1,874 & 3,262 & 5,847 & 8,434 & 9,755 \\
-589 & 0 & -444 & -241 & 0 & 0 \\
0 & 0 & 0 & 0 & 0 & 0 \\
0 & 0 & 0 & 0 & 0 & 423 \\
-295 & -320 & -329 & -388 & -433 & -467 \\
0 & 0 & 0 & 0 & 0 & 0 \\
0 & 0 & 170 & 0 & 378 & 493 \\
0 & 0 & 0 & 0 & -497 & $-3,472$ \\
-264 & -335 & -358 & -372 & -371 & -371 \\
-134 & 0 & $-1,466$ & $-2,538$ & $-3,497$ & $-4,965$ \\
-136 & $-1,604$ & $-1,521$ & $-1,851$ & $-2,359$ & 0 \\
$\mathbf{0}$ & $\mathbf{0}$ & $\mathbf{0}$ & $\mathbf{0}$ & $\mathbf{0}$ & $\mathbf{0}$ \\
\hline
\end{tabular}




\section{World Biofuels Study}

Table A-112: Reference case with high oil price grain ethanol production [Million gallons]

\begin{tabular}{lrrrrrr} 
& $\mathbf{2 0 0 5}$ & $\mathbf{2 0 1 0}$ & $\mathbf{2 0 1 5}$ & $\mathbf{2 0 2 0}$ & $\mathbf{2 0 2 5}$ & $\mathbf{2 0 3 0}$ \\
Africa & 0 & 0 & 0 & 0 & 0 & 0 \\
Argentina & 0 & 178 & 604 & 1,153 & 1,153 & 1,153 \\
Australia & 0 & 0 & 0 & 0 & 0 & 0 \\
Brazil & 0 & 0 & 0 & 0 & 0 & 0 \\
Canada & 0 & 0 & 0 & 0 & 0 & 0 \\
Caribbean basin & 0 & 0 & 0 & 0 & 0 & 0 \\
China & 344 & 344 & 344 & 344 & 344 & 223 \\
Colombia & 0 & 0 & 0 & 0 & 0 & 0 \\
Eastern Europe & 110 & 226 & 348 & 477 & 567 & 451 \\
Former Soviet Union & 0 & 0 & 0 & 0 & 0 & 0 \\
India & 0 & 0 & 0 & 0 & 0 & 0 \\
Japan & 0 & 0 & 0 & 0 & 0 & 0 \\
Middle East & 0 & 0 & 0 & 0 & 0 & 0 \\
Mexico & 0 & 0 & 0 & 0 & 0 & 0 \\
Other Asia & 0 & 0 & 0 & 0 & 0 & 0 \\
South Korea & 0 & 0 & 0 & 0 & 0 & 0 \\
United States & 3,850 & 11,612 & 15,000 & 15,000 & 15,000 & 15,000 \\
Western Europe & 600 & 2,570 & 2,589 & 2,542 & 3,178 & 3,604 \\
\hline Total & $\mathbf{4 , 9 0 4}$ & $\mathbf{1 4 , 9 3 0}$ & $\mathbf{1 8 , 8 8 6}$ & $\mathbf{1 9 , 5 1 7}$ & $\mathbf{2 0 , 2 4 2}$ & $\mathbf{2 0 , 4 3 1}$ \\
\hline \hline
\end{tabular}

Table A-113: Reference case with high oil price sugar ethanol production [Million gallons]

\begin{tabular}{lrrrrrr} 
& $\mathbf{2 0 0 5}$ & $\mathbf{2 0 1 0}$ & $\mathbf{2 0 1 5}$ & $\mathbf{2 0 2 0}$ & $\mathbf{2 0 2 5}$ & $\mathbf{2 0 3 0}$ \\
Africa & 300 & 330 & 364 & 402 & 444 & 490 \\
Argentina & 0 & 0 & 0 & 0 & 0 & 0 \\
Australia & 0 & 0 & 0 & 0 & 0 & 0 \\
Brazil & 4,866 & 5,977 & 7,329 & 8,862 & 10,406 & 11,793 \\
Canada & 0 & 0 & 0 & 0 & 0 & 0 \\
Caribbean basin & 210 & 360 & 573 & 683 & 779 & 890 \\
China & 0 & 40 & 48 & 53 & 58 & 64 \\
Colombia & 196 & 215 & 241 & 270 & 302 & 338 \\
Eastern Europe & 0 & 0 & 0 & 0 & 0 & 0 \\
Former Soviet Union & 0 & 0 & 0 & 0 & 0 & 0 \\
India & 313 & 279 & 327 & 347 & 381 & 348 \\
Japan & 0 & 0 & 0 & 0 & 0 & 0 \\
Middle East & 0 & 0 & 0 & 0 & 0 & 0 \\
Mexico & 0 & 0 & 0 & 0 & 0 & 0 \\
Other Asia & 0 & 0 & 0 & 0 & 0 & 0 \\
South Korea & 0 & 0 & 0 & 0 & 0 & 0 \\
United States & 0 & 0 & 0 & 0 & 0 & 0 \\
Western Europe & 0 & 0 & 0 & 0 & 0 & 0 \\
\hline Total & $\mathbf{5 , 8 8 4}$ & $\mathbf{7 , 2 0 1}$ & $\mathbf{8 , 8 8 2}$ & $\mathbf{1 0 , 6 1 6}$ & $\mathbf{1 2 , 3 7 1}$ & $\mathbf{1 3 , 9 2 3}$ \\
\hline \hline
\end{tabular}


Table A-114: Reference case with high oil price cellulosic ethanol production [Million gallons]

\begin{tabular}{lrrrrrr} 
& $\mathbf{2 0 0 5}$ & $\mathbf{2 0 1 0}$ & $\mathbf{2 0 1 5}$ & $\mathbf{2 0 2 0}$ & $\mathbf{2 0 2 5}$ & $\mathbf{2 0 3 0}$ \\
Africa & 0 & 0 & 0 & 0 & 11 & 11 \\
Argentina & 0 & 0 & 206 & 762 & 762 & 762 \\
Australia & 0 & 0 & 71 & 246 & 583 & 1,172 \\
Brazil & 0 & 0 & 296 & 890 & 1,893 & 1,893 \\
Canada & 0 & 0 & 259 & 779 & 1,053 & 1,057 \\
Caribbean basin & 0 & 0 & 0 & 0 & 0 & 0 \\
China & 0 & 0 & 296 & 774 & 1,970 & 3,275 \\
Colombia & 0 & 0 & 170 & 512 & 1,199 & 1,893 \\
Eastern Europe & 0 & 0 & 80 & 235 & 470 & 604 \\
Former Soviet Union & 0 & 0 & 39 & 120 & 367 & 1,120 \\
India & 0 & 0 & 214 & 645 & 1,512 & 2,475 \\
Japan & 0 & 0 & 95 & 369 & 609 & 727 \\
Middle East & 0 & 0 & 0 & 0 & 0 & 0 \\
Mexico & 0 & 0 & 199 & 601 & 1,408 & 1,546 \\
Other Asia & 0 & 0 & 0 & 0 & 0 & 0 \\
South Korea & 0 & 0 & 42 & 42 & 42 & 42 \\
United States & 0 & 0 & 802 & 2,258 & 5,065 & 7,205 \\
Western Europe & 0 & 0 & 166 & 693 & 2,113 & 4,410 \\
\hline Total & $\mathbf{0}$ & $\mathbf{0}$ & $\mathbf{2 , 9 3 4}$ & $\mathbf{8 , 9 2 4}$ & $\mathbf{1 9 , 0 5 6}$ & $\mathbf{2 8 , 1 8 8}$ \\
\hline \hline
\end{tabular}

Table A-115: Reference case with high oil price grain ethanol consumption [Million gallons]

\begin{tabular}{lrrrrrr} 
& $\mathbf{2 0 0 5}$ & $\mathbf{2 0 1 0}$ & $\mathbf{2 0 1 5}$ & $\mathbf{2 0 2 0}$ & $\mathbf{2 0 2 5}$ & $\mathbf{2 0 3 0}$ \\
Africa & 0 & 0 & 0 & 0 & 0 & 0 \\
Australia & 0 & 0 & 0 & 0 & 0 & 0 \\
Canada & 0 & 0 & 0 & 0 & 0 & 0 \\
China & 265 & 0 & 0 & 488 & 868 & 223 \\
Central and South America & 0 & 36 & 122 & 249 & 196 & 196 \\
Eastern Europe & 110 & 226 & 348 & 477 & 567 & 451 \\
Former Soviet Union & 0 & 0 & 0 & 0 & 0 & 0 \\
India & 0 & 0 & 0 & 0 & 0 & 0 \\
Japan & 0 & 192 & 281 & 388 & 433 & 467 \\
Middle East & 0 & 0 & 0 & 0 & 0 & 0 \\
Mexico & 0 & 0 & 0 & 0 & 0 & 0 \\
Other Asia & 0 & 0 & 0 & 0 & 0 & 491 \\
South Korea & 80 & 295 & 63 & 372 & 0 & 0 \\
United States & 3,850 & 11,612 & 15,000 & 15,000 & 15,000 & 15,000 \\
Western Europe & 600 & 2,570 & 3,072 & 2,542 & 3,178 & 3,604 \\
\hline Total & $\mathbf{4 , 9 0 4}$ & $\mathbf{1 4 , 9 3 0}$ & $\mathbf{1 8 , 8 8 6}$ & $\mathbf{1 9 , 5 1 7}$ & $\mathbf{2 0 , 2 4 2}$ & $\mathbf{2 0 , 4 3 1}$ \\
\hline \hline
\end{tabular}


Table A-116: Reference case with high oil price sugar ethanol consumption [Million gallons]

\begin{tabular}{lrrrrrr} 
& $\mathbf{2 0 0 5}$ & $\mathbf{2 0 1 0}$ & $\mathbf{2 0 1 5}$ & $\mathbf{2 0 2 0}$ & $\mathbf{2 0 2 5}$ & $\mathbf{2 0 3 0}$ \\
Africa & 300 & 330 & 364 & 402 & 444 & 490 \\
Australia & 0 & 0 & 0 & 0 & 0 & 0 \\
Canada & 145 & 0 & 0 & 116 & 0 & 0 \\
China & 0 & 0 & 0 & 53 & 799 & 1,037 \\
Central and South America & 3,788 & 4,820 & 6,035 & 7,008 & 7,653 & 8,770 \\
Eastern Europe & 589 & 0 & 444 & 153 & 0 & 0 \\
Former Soviet Union & 0 & 0 & 0 & 0 & 0 & 0 \\
India & 313 & 279 & 327 & 347 & 381 & 348 \\
Japan & 295 & 128 & 48 & 0 & 0 & 0 \\
Middle East & 0 & 0 & 0 & 0 & 0 & 0 \\
Mexico & 0 & 0 & 0 & 0 & 0 & 0 \\
Other Asia & 0 & 0 & 0 & 0 & 0 & 346 \\
South Korea & 185 & 40 & 0 & 0 & 0 & 4 \\
United States & 134 & 0 & 788 & 2,538 & 3,094 & 2,928 \\
Western Europe & 136 & 1,604 & 876 & 0 & 0 & 0 \\
\hline Total & $\mathbf{5 , 8 8 4}$ & $\mathbf{7 , 2 0 1}$ & $\mathbf{8 , 8 8 2}$ & $\mathbf{1 0 , 6 1 6}$ & $\mathbf{1 2 , 3 7 1}$ & $\mathbf{1 3 , 9 2 3}$ \\
\hline \hline
\end{tabular}

Table A-117: Reference case with high oil price cellulosic ethanol consumption [Million gallons]

\begin{tabular}{lrrrrrr} 
& $\mathbf{2 0 0 5}$ & $\mathbf{2 0 1 0}$ & $\mathbf{2 0 1 5}$ & $\mathbf{2 0 2 0}$ & $\mathbf{2 0 2 5}$ & $\mathbf{2 0 3 0}$ \\
Africa & 0 & 0 & 0 & 0 & 11 & 11 \\
Australia & 0 & 0 & 71 & 246 & 583 & 1,172 \\
Canada & 0 & 0 & 259 & 779 & 948 & 951 \\
China & 0 & 0 & 0 & 971 & 2,465 & 3,804 \\
Central and South America & 0 & 0 & 0 & 27 & 211 & 0 \\
Eastern Europe & 0 & 0 & 80 & 323 & 470 & 604 \\
Former Soviet Union & 0 & 0 & 39 & 120 & 367 & 1,120 \\
India & 0 & 0 & 214 & 645 & 1,512 & 2,051 \\
Japan & 0 & 0 & 95 & 369 & 609 & 727 \\
Middle East & 0 & 0 & 0 & 0 & 0 & 0 \\
Mexico & 0 & 0 & 30 & 601 & 1,030 & 1,052 \\
Other Asia & 0 & 0 & 0 & 0 & 497 & 2,636 \\
South Korea & 0 & 0 & 337 & 42 & 413 & 409 \\
United States & 0 & 0 & 1,480 & 2,258 & 5,469 & 9,242 \\
Western Europe & 0 & 0 & 328 & 2,544 & 4,472 & 4,410 \\
\hline Total & $\mathbf{0}$ & $\mathbf{0}$ & $\mathbf{2 , 9 3 4}$ & $\mathbf{8 , 9 2 4}$ & $\mathbf{1 9 , 0 5 6}$ & $\mathbf{2 8 , 1 8 8}$ \\
\hline \hline
\end{tabular}


Table A-118: Reference case with high oil price grain ethanol net trade [Million gallons]

\begin{tabular}{lrrrrrr} 
& $\mathbf{2 0 0 5}$ & $\mathbf{2 0 1 0}$ & $\mathbf{2 0 1 5}$ & $\mathbf{2 0 2 0}$ & $\mathbf{2 0 2 5}$ & $\mathbf{2 0 3 0}$ \\
Africa & 0 & 0 & 0 & 0 & 0 & 0 \\
Australia & 0 & 0 & 0 & 0 & 0 & 0 \\
Canada & 0 & 0 & 0 & 0 & 0 & 0 \\
China & 80 & 344 & 344 & -144 & -524 & 0 \\
Central and South America & 0 & 143 & 483 & 904 & 957 & 957 \\
Eastern Europe & 0 & 0 & 0 & 0 & 0 & 0 \\
Former Soviet Union & 0 & 0 & 0 & 0 & 0 & 0 \\
India & 0 & 0 & 0 & 0 & 0 & 0 \\
Japan & 0 & -192 & -281 & -388 & -433 & -467 \\
Middle East & 0 & 0 & 0 & 0 & 0 & 0 \\
Mexico & 0 & 0 & 0 & 0 & 0 & 0 \\
Other Asia & 0 & 0 & 0 & 0 & 0 & -491 \\
South Korea & -80 & -295 & -63 & -372 & 0 & 0 \\
United States & 0 & 0 & 0 & 0 & 0 & 0 \\
Western Europe & 0 & 0 & -483 & 0 & 0 & 0 \\
\hline Total & $\mathbf{0}$ & $\mathbf{0}$ & $\mathbf{0}$ & $\mathbf{0}$ & $\mathbf{0}$ & $\mathbf{0}$ \\
\hline \hline
\end{tabular}

Table A-119: Reference case with high oil price sugar ethanol net trade [Million gallons]

\begin{tabular}{lrrrrrr} 
Africa & 0 & 0 & 0 & 0 & 0 & 0 \\
Australia & 0 & 0 & 0 & 0 & 0 & 0 \\
Canada & -145 & 0 & 0 & -116 & 0 & 0 \\
China & 0 & 40 & 48 & 0 & -741 & -973 \\
Central and South America & 1,484 & 1,732 & 2,108 & 2,807 & 3,835 & 4,251 \\
Eastern Europe & -589 & 0 & -444 & -153 & 0 & 0 \\
Former Soviet Union & 0 & 0 & 0 & 0 & 0 & 0 \\
India & 0 & 0 & 0 & 0 & 0 & 0 \\
Japan & -295 & -128 & -48 & 0 & 0 & 0 \\
Middle East & 0 & 0 & 0 & 0 & 0 & 0 \\
Mexico & 0 & 0 & 0 & 0 & 0 & 0 \\
Other Asia & 0 & 0 & 0 & 0 & 0 & -346 \\
South Korea & -185 & -40 & 0 & 0 & 0 & -4 \\
United States & -134 & 0 & -788 & $-2,538$ & $-3,094$ & $-2,928$ \\
Western Europe & -136 & $-1,604$ & -876 & 0 & 0 & 0 \\
\hline Total & $\mathbf{0}$ & $\mathbf{0}$ & $\mathbf{0}$ & $\mathbf{0}$ & $\mathbf{0}$ & $\mathbf{0}$ \\
\hline \hline
\end{tabular}




\section{World Biofuels Study}

Table A-120: Reference case with high oil price cellulosic ethanol net trade [Million gallons]

\begin{tabular}{lrrrrrr} 
& $\mathbf{2 0 0 5}$ & $\mathbf{2 0 1 0}$ & $\mathbf{2 0 1 5}$ & $\mathbf{2 0 2 0}$ & $\mathbf{2 0 2 5}$ & $\mathbf{2 0 3 0}$ \\
Africa & 0 & 0 & 0 & 0 & 0 & 0 \\
Australia & 0 & 0 & 0 & 0 & 0 & 0 \\
Canada & 0 & 0 & 0 & 0 & 105 & 106 \\
China & 0 & 0 & 296 & -197 & -495 & -529 \\
Central and South America & 0 & 0 & 671 & 2,136 & 3,643 & 4,547 \\
Eastern Europe & 0 & 0 & 0 & -89 & 0 & 0 \\
Former Soviet Union & 0 & 0 & 0 & 0 & 0 & 0 \\
India & 0 & 0 & 0 & 0 & 0 & 423 \\
Japan & 0 & 0 & 0 & 0 & 0 & 0 \\
Middle East & 0 & 0 & 0 & 0 & 0 & 0 \\
Mexico & 0 & 0 & 170 & 0 & 378 & 493 \\
Other Asia & 0 & 0 & 0 & 0 & -497 & $-2,636$ \\
South Korea & 0 & 0 & -296 & 0 & -371 & -367 \\
United States & 0 & 0 & -678 & 0 & -403 & $-2,037$ \\
Western Europe & 0 & 0 & -162 & $-1,851$ & $-2,359$ & 0 \\
\hline Total & $\mathbf{0}$ & $\mathbf{0}$ & $\mathbf{0}$ & $\mathbf{0}$ & $\mathbf{0}$ & $\mathbf{0}$ \\
\hline \hline
\end{tabular}

Table A-121: Reference case with high oil price biomass-to-liquids production [Million gallons ethanol equivalent]

\begin{tabular}{lrrrrrr} 
& $\mathbf{2 0 0 5}$ & $\mathbf{2 0 1 0}$ & $\mathbf{2 0 1 5}$ & $\mathbf{2 0 2 0}$ & $\mathbf{2 0 2 5}$ & $\mathbf{2 0 3 0}$ \\
Africa & 0 & 0 & 82 & 415 & 921 & 1,584 \\
Argentina & 0 & 0 & 74 & 274 & 274 & 274 \\
Australia & 0 & 0 & 0 & 46 & 217 & 474 \\
Brazil & 0 & 0 & 106 & 320 & 681 & 681 \\
Canada & 0 & 0 & 93 & 280 & 379 & 379 \\
Caribbean basin & 0 & 0 & 0 & 0 & 0 & 0 \\
China & 0 & 0 & 0 & 125 & 125 & 125 \\
Colombia & 0 & 0 & 61 & 184 & 431 & 681 \\
Eastern Europe & 0 & 0 & 0 & 125 & 376 & 731 \\
Former Soviet Union & 0 & 0 & 44 & 213 & 553 & 1,016 \\
India & 0 & 0 & 77 & 232 & 544 & 890 \\
Japan & 0 & 0 & 0 & 0 & 0 & 0 \\
Middle East & 0 & 0 & 0 & 111 & 348 & 685 \\
Mexico & 0 & 0 & 72 & 216 & 506 & 556 \\
Other Asia & 0 & 0 & 96 & 554 & 1,511 & 2,682 \\
South Korea & 0 & 0 & 43 & 89 & 89 & 89 \\
United States & 0 & 0 & 822 & 4,818 & 6,399 & 8,213 \\
Western Europe & 0 & 0 & 0 & 0 & 0 & 0 \\
\hline Total & $\mathbf{0}$ & $\mathbf{0}$ & $\mathbf{1 , 5 6 9}$ & $\mathbf{8 , 0 0 1}$ & $\mathbf{1 3 , 3 5 3}$ & $\mathbf{1 9 , 0 6 0}$ \\
\hline \hline
\end{tabular}


Table A-122: Reference case with high oil price biomass-to-liquids consumption [Million gallons ethanol equivalent]

\begin{tabular}{lrrrrrr} 
& $\mathbf{2 0 0 5}$ & $\mathbf{2 0 1 0}$ & $\mathbf{2 0 1 5}$ & $\mathbf{2 0 2 0}$ & $\mathbf{2 0 2 5}$ & $\mathbf{2 0 3 0}$ \\
Africa & 0 & 0 & 82 & 415 & 921 & 1,585 \\
Australia & 0 & 0 & 0 & 46 & 217 & 474 \\
Canada & 0 & 0 & 0 & 0 & 0 & 0 \\
China & 0 & 0 & 0 & 0 & 0 & 0 \\
Central and South America & 0 & 0 & 0 & 0 & 0 & 0 \\
Eastern Europe & 0 & 0 & 0 & 125 & 377 & 731 \\
Former Soviet Union & 0 & 0 & 44 & 213 & 554 & 1,016 \\
India & 0 & 0 & 0 & 0 & 0 & 0 \\
Japan & 0 & 0 & 0 & 0 & 0 & 0 \\
Middle East & 0 & 0 & 0 & 111 & 348 & 685 \\
Mexico & 0 & 0 & 0 & 0 & 0 & 0 \\
Other Asia & 0 & 0 & 96 & 554 & 1,511 & 2,683 \\
South Korea & 0 & 0 & 0 & 0 & 0 & 0 \\
United States & 0 & 0 & 1,069 & 6,058 & 9,428 & 10,993 \\
Western Europe & 0 & 0 & 279 & 480 & 0 & 894 \\
\hline Total & $\mathbf{0}$ & $\mathbf{0}$ & $\mathbf{1 , 5 6 9}$ & $\mathbf{8 , 0 0 1}$ & $\mathbf{1 3 , 3 5 4}$ & $\mathbf{1 9 , 0 6 1}$ \\
\hline \hline
\end{tabular}

Table A-123: Reference case with high oil price biomass-to-liquids net trade [Million gallons ethanol equivalent]

\begin{tabular}{lllrrrr} 
Africa & 0 & 0 & 0 & 0 & 0 & 0 \\
Australia & 0 & 0 & 0 & 0 & 0 & 0 \\
Canada & 0 & 0 & 93 & 280 & 379 & 379 \\
China & 0 & 0 & 0 & 125 & 125 & 125 \\
Central and South America & 0 & 0 & 241 & 778 & 1,386 & 1,635 \\
Eastern Europe & 0 & 0 & 0 & 0 & 0 & 0 \\
Former Soviet Union & 0 & 0 & 0 & 0 & 0 & 0 \\
India & 0 & 0 & 77 & 232 & 544 & 890 \\
Japan & 0 & 0 & 0 & 0 & 0 & 0 \\
Middle East & 0 & 0 & 0 & 0 & 0 & 0 \\
Mexico & 0 & 0 & 72 & 216 & 506 & 556 \\
Other Asia & 0 & 0 & 0 & 0 & 0 & 0 \\
South Korea & 0 & 0 & 43 & 89 & 89 & 89 \\
United States & 0 & 0 & -247 & $-1,240$ & $-3,029$ & $-2,780$ \\
Western Europe & 0 & 0 & -279 & -480 & 0 & -894 \\
\hline Total & $\mathbf{0}$ & $\mathbf{0}$ & $\mathbf{0}$ & $\mathbf{0}$ & $\mathbf{0}$ & $\mathbf{- 1}$ \\
\hline \hline
\end{tabular}


Table A-124: Reference case with high oil price biodiesel production [Million gallons ethanol equivalent]

\begin{tabular}{lrrrrrr} 
& $\mathbf{2 0 0 5}$ & $\mathbf{2 0 1 0}$ & $\mathbf{2 0 1 5}$ & $\mathbf{2 0 2 0}$ & $\mathbf{2 0 2 5}$ & $\mathbf{2 0 3 0}$ \\
Africa & 0 & 0 & 0 & 0 & 0 & 0 \\
Argentina & 0 & 333 & 776 & 819 & 827 & 239 \\
Australia & 0 & 0 & 0 & 0 & 0 & 0 \\
Brazil & 0 & 333 & 877 & 1,579 & 1,768 & 1,351 \\
Canada & 0 & 0 & 0 & 0 & 0 & 0 \\
Caribbean basin & 0 & 95 & 310 & 675 & 802 & 898 \\
China & 0 & 0 & 0 & 0 & 0 & 0 \\
Colombia & 0 & 119 & 298 & 474 & 650 & 782 \\
Eastern Europe & 0 & 0 & 0 & 0 & 0 & 0 \\
Former Soviet Union & 0 & 0 & 0 & 0 & 0 & 0 \\
India & 0 & 0 & 0 & 0 & 0 & 0 \\
Japan & 0 & 0 & 0 & 0 & 0 & 0 \\
Middle East & 0 & 0 & 0 & 0 & 0 & 0 \\
Mexico & 0 & 0 & 0 & 0 & 0 & 0 \\
Other Asia & 0 & 0 & 0 & 0 & 0 & 0 \\
South Korea & 0 & 0 & 0 & 0 & 0 & 0 \\
United States & 0 & 1,238 & 1,640 & 1,747 & 1,907 & 2,072 \\
Western Europe & 998 & 3,445 & 4,172 & 5,110 & 5,532 & 5,997 \\
\hline Total & $\mathbf{9 9 8}$ & $\mathbf{5 , 5 6 1}$ & $\mathbf{8 , 0 7 2}$ & $\mathbf{1 0 , 4 0 3}$ & $\mathbf{1 1 , 4 8 5}$ & $\mathbf{1 1 , 3 3 9}$ \\
\hline \hline
\end{tabular}

Table A-125: Reference case with high oil price biodiesel consumption [Million gallons ethanol equivalent]

\begin{tabular}{lrrrrrr} 
& $\mathbf{2 0 0 5}$ & $\mathbf{2 0 1 0}$ & $\mathbf{2 0 1 5}$ & $\mathbf{2 0 2 0}$ & $\mathbf{2 0 2 5}$ & $\mathbf{2 0 3 0}$ \\
Africa & 0 & 0 & 0 & 0 & 0 & 0 \\
Australia & 0 & 0 & 0 & 0 & 0 & 0 \\
Canada & 0 & 0 & 0 & 0 & 0 & 0 \\
China & 0 & 0 & 0 & 0 & 0 & 0 \\
Central and South America & 0 & 158 & 458 & 969 & 1,300 & 1,486 \\
Eastern Europe & 0 & 0 & 0 & 0 & 0 & 0 \\
Former Soviet Union & 0 & 0 & 0 & 0 & 0 & 0 \\
India & 0 & 0 & 0 & 0 & 0 & 0 \\
Japan & 0 & 0 & 0 & 0 & 0 & 0 \\
Middle East & 0 & 0 & 0 & 0 & 0 & 0 \\
Mexico & 0 & 0 & 0 & 0 & 0 & 0 \\
Other Asia & 0 & 0 & 0 & 0 & 0 & 0 \\
South Korea & 0 & 0 & 0 & 0 & 0 & 0 \\
United States & 0 & 1,238 & 1,713 & 1,941 & 1,907 & 2,072 \\
Western Europe & 998 & 4,165 & 5,902 & 7,494 & 8,278 & 7,781 \\
\hline Total & $\mathbf{9 9 8}$ & $\mathbf{5 , 5 6 1}$ & $\mathbf{8 , 0 7 2}$ & $\mathbf{1 0 , 4 0 3}$ & $\mathbf{1 1 , 4 8 5}$ & $\mathbf{1 1 , 3 3 9}$ \\
\hline \hline
\end{tabular}




\section{Table A-126: Reference case with high oil price biodiesel net trade [Million gallons ethanol equivalent]}

Africa
Australia
Canada
China
Central and South America
Eastern Europe
Former Soviet Union
India
Japan
Middle East
Mexico
Other Asia
South Korea
United States
Western Europe
Total

\begin{tabular}{rrrrrr}
$\mathbf{2 0 0 5}$ & $\mathbf{2 0 1 0}$ & $\mathbf{2 0 1 5}$ & $\mathbf{2 0 2 0}$ & $\mathbf{2 0 2 5}$ & $\mathbf{2 0 3 0}$ \\
0 & 0 & 0 & 0 & 0 & 0 \\
0 & 0 & 0 & 0 & 0 & 0 \\
0 & 0 & 0 & 0 & 0 & 0 \\
0 & 0 & 0 & 0 & 0 & 0 \\
0 & 720 & 1,803 & 2,577 & 2,747 & 1,784 \\
0 & 0 & 0 & 0 & 0 & 0 \\
0 & 0 & 0 & 0 & 0 & 0 \\
0 & 0 & 0 & 0 & 0 & 0 \\
0 & 0 & 0 & 0 & 0 & 0 \\
0 & 0 & 0 & 0 & 0 & 0 \\
0 & 0 & 0 & 0 & 0 & 0 \\
0 & 0 & 0 & 0 & 0 & 0 \\
0 & 0 & 0 & 0 & 0 & 0 \\
0 & 0 & -73 & -194 & 0 & 0 \\
0 & -720 & $-1,730$ & $-2,384$ & $-2,747$ & $-1,784$ \\
\hline $\mathbf{0}$ & $\mathbf{0}$ & $\mathbf{0}$ & $\mathbf{0}$ & $\mathbf{0}$ & $\mathbf{0}$ \\
\hline
\end{tabular}




\section{A-8 Reference Case with Low Oil Price}

Table A-127: Reference case with low oil price ethanol production [Million gallons]

\begin{tabular}{|c|c|c|c|c|c|c|}
\hline Africa & 300 & 330 & 364 & 402 & 455 & 490 \\
\hline Argentina & 0 & 178 & 810 & 910 & 940 & 532 \\
\hline Australia & 0 & 0 & 66 & 247 & 616 & 1,172 \\
\hline Brazil & 4,866 & 5,977 & 7,624 & 9,539 & 11,603 & 13,338 \\
\hline Canada & 0 & 0 & 259 & 779 & 1,069 & 1,069 \\
\hline Caribbean basin & 210 & 328 & 573 & 597 & 597 & 597 \\
\hline China & 344 & 384 & 634 & 993 & 1,564 & 1,564 \\
\hline Colombia & 196 & 215 & 411 & 782 & 1,469 & 2,163 \\
\hline Eastern Europe & 110 & 226 & 434 & 780 & 1,037 & 1,149 \\
\hline Former Soviet Union & 0 & 0 & 0 & 0 & 0 & 0 \\
\hline India & 313 & 275 & 534 & 974 & 1,850 & 2,527 \\
\hline Japan & 0 & 0 & 95 & 348 & 612 & 644 \\
\hline Middle East & 0 & 0 & 0 & 0 & 0 & 0 \\
\hline Mexico & 0 & 0 & 199 & 601 & 1,408 & 1,546 \\
\hline Other Asia & 0 & 0 & 0 & 0 & 0 & 0 \\
\hline South Korea & 0 & 0 & 35 & 35 & 25 & 35 \\
\hline United States & 3,850 & 11,612 & 15,802 & 17,094 & 18,336 & 18,836 \\
\hline Western Europe & 600 & 2,549 & 2,755 & 3,398 & 5,291 & 8,013 \\
\hline Total & 10,788 & 22,074 & 30,595 & 37,477 & 46,871 & 53,673 \\
\hline
\end{tabular}


Table A-128: Reference case with low oil price ethanol consumption [Million gallons]

\begin{tabular}{lrrrrrr} 
& $\mathbf{2 0 0 5}$ & $\mathbf{2 0 1 0}$ & $\mathbf{2 0 1 5}$ & $\mathbf{2 0 2 0}$ & $\mathbf{2 0 2 5}$ & $\mathbf{2 0 3 0}$ \\
Africa & 300 & 330 & 364 & 402 & 455 & 490 \\
Australia & 0 & 0 & 66 & 247 & 616 & 1,172 \\
Canada & 145 & 0 & 233 & 701 & 1,069 & 1,061 \\
China & 263 & 0 & 0 & 263 & 780 & 771 \\
Central and South America & 3,788 & 4,853 & 6,156 & 7,149 & 7,948 & 8,803 \\
Eastern Europe & 699 & 226 & 872 & 953 & 1,037 & 1,149 \\
Former Soviet Union & 0 & 0 & 0 & 0 & 0 & 0 \\
India & 313 & 275 & 534 & 974 & 1,850 & 2,399 \\
Japan & 263 & 250 & 364 & 698 & 1,008 & 1,060 \\
Middle East & 0 & 0 & 0 & 0 & 0 & 0 \\
Mexico & 0 & 0 & 30 & 78 & 183 & 201 \\
Other Asia & 0 & 0 & 0 & 0 & 0 & 0 \\
South Korea & 264 & 335 & 400 & 414 & 413 & 413 \\
United States & 4,018 & 11,612 & 17,232 & 20,349 & 23,862 & 27,880 \\
Western Europe & 736 & 4,193 & 4,344 & 5,249 & 7,649 & 8,276 \\
\hline Total & $\mathbf{1 0 , 7 8 8}$ & $\mathbf{2 2 , 0 7 4}$ & $\mathbf{3 0 , 5 9 5}$ & $\mathbf{3 7 , 4 7 7}$ & $\mathbf{4 6 , 8 7 1}$ & $\mathbf{5 3 , 6 7 3}$ \\
\hline \hline
\end{tabular}

Table A-129: Reference case with low oil price ethanol net trade [Million gallons]

Africa

\begin{tabular}{rrrrrr}
$\mathbf{2 0 0 5}$ & $\mathbf{2 0 1 0}$ & $\mathbf{2 0 1 5}$ & $\mathbf{2 0 2 0}$ & $\mathbf{2 0 2 5}$ & $\mathbf{2 0 3 0}$ \\
0 & 0 & 0 & 0 & 0 & 0 \\
0 & 0 & 0 & 0 & 0 & 0 \\
-145 & 0 & 26 & 78 & 0 & 8 \\
81 & 384 & 634 & 730 & 784 & 794 \\
1,484 & 1,845 & 3,262 & 4,678 & 6,660 & 7,827 \\
-589 & 0 & -438 & -173 & 0 & 0 \\
0 & 0 & 0 & 0 & 0 & 0 \\
0 & 0 & 0 & 0 & 0 & 128 \\
-263 & -250 & -269 & -350 & -395 & -415 \\
0 & 0 & 0 & 0 & 0 & 0 \\
0 & 0 & 170 & 523 & 1,225 & 1,345 \\
0 & 0 & 0 & 0 & 0 & 0 \\
-264 & -335 & -365 & -379 & -388 & -378 \\
-168 & 0 & $-1,430$ & $-3,255$ & $-5,526$ & $-9,045$ \\
-136 & $-1,644$ & $-1,589$ & $-1,851$ & $-2,358$ & -263 \\
\hline $\mathbf{0}$ & $\mathbf{0}$ & $\mathbf{0}$ & $\mathbf{0}$ & $\mathbf{0}$ & $\mathbf{0}$ \\
\hline
\end{tabular}


Table A-130: Reference case with low oil price grain ethanol production [Million gallons]

\begin{tabular}{lrrrrrr} 
& $\mathbf{2 0 0 5}$ & $\mathbf{2 0 1 0}$ & $\mathbf{2 0 1 5}$ & $\mathbf{2 0 2 0}$ & $\mathbf{2 0 2 5}$ & $\mathbf{2 0 3 0}$ \\
Africa & 0 & 0 & 0 & 0 & 0 & 0 \\
Argentina & 0 & 178 & 604 & 604 & 604 & 185 \\
Australia & 0 & 0 & 0 & 0 & 0 & 0 \\
Brazil & 0 & 0 & 0 & 0 & 0 & 0 \\
Canada & 0 & 0 & 0 & 0 & 0 & 0 \\
Caribbean basin & 0 & 0 & 0 & 0 & 0 & 0 \\
China & 344 & 344 & 344 & 325 & 0 & 0 \\
Colombia & 0 & 0 & 0 & 0 & 0 & 0 \\
Eastern Europe & 110 & 226 & 348 & 477 & 367 & 251 \\
Former Soviet Union & 0 & 0 & 0 & 0 & 0 & 0 \\
India & 0 & 0 & 0 & 0 & 0 & 0 \\
Japan & 0 & 0 & 0 & 0 & 0 & 0 \\
Middle East & 0 & 0 & 0 & 0 & 0 & 0 \\
Mexico & 0 & 0 & 0 & 0 & 0 & 0 \\
Other Asia & 0 & 0 & 0 & 0 & 0 & 0 \\
South Korea & 0 & 0 & 0 & 0 & 0 & 0 \\
United States & 3,850 & 11,612 & 15,000 & 15,000 & 14,815 & 14,015 \\
Western Europe & 600 & 2,549 & 2,589 & 2,705 & 3,178 & 3,604 \\
\hline Total & $\mathbf{4 , 9 0 4}$ & $\mathbf{1 4 , 9 0 9}$ & $\mathbf{1 8 , 8 8 6}$ & $\mathbf{1 9 , 1 1 2}$ & $\mathbf{1 8 , 9 6 4}$ & $\mathbf{1 8 , 0 5 5}$ \\
\hline \hline
\end{tabular}

Table A-131: Reference case with low oil price sugar ethanol production [Million gallons]

\begin{tabular}{lrrrrrr} 
& $\mathbf{2 0 0 5}$ & $\mathbf{2 0 1 0}$ & $\mathbf{2 0 1 5}$ & $\mathbf{2 0 2 0}$ & $\mathbf{2 0 2 5}$ & $\mathbf{2 0 3 0}$ \\
Africa & 300 & 330 & 364 & 402 & 444 & 490 \\
Argentina & 0 & 0 & 0 & 0 & 0 & 0 \\
Australia & 0 & 0 & 0 & 0 & 0 & 0 \\
Brazil & 4,866 & 5,977 & 7,329 & 8,649 & 10,057 & 11,793 \\
Canada & 0 & 0 & 0 & 0 & 0 & 0 \\
Caribbean basin & 210 & 328 & 573 & 597 & 597 & 597 \\
China & 0 & 40 & 48 & 0 & 0 & 0 \\
Colombia & 196 & 215 & 241 & 270 & 270 & 270 \\
Eastern Europe & 0 & 0 & 0 & 0 & 0 & 0 \\
Former Soviet Union & 0 & 0 & 0 & 0 & 0 & 0 \\
India & 313 & 275 & 320 & 329 & 338 & 332 \\
Japan & 0 & 0 & 0 & 0 & 0 & 0 \\
Middle East & 0 & 0 & 0 & 0 & 0 & 0 \\
Mexico & 0 & 0 & 0 & 0 & 0 & 0 \\
Other Asia & 0 & 0 & 0 & 0 & 0 & 0 \\
South Korea & 0 & 0 & 0 & 0 & 0 & 0 \\
United States & 0 & 0 & 0 & 0 & 0 & 0 \\
Western Europe & 0 & 0 & 0 & 0 & 0 & 0 \\
\hline Total & $\mathbf{5 , 8 8 4}$ & $\mathbf{7 , 1 6 5}$ & $\mathbf{8 , 8 7 4}$ & $\mathbf{1 0 , 2 4 6}$ & $\mathbf{1 1 , 7 0 6}$ & $\mathbf{1 3 , 4 8 1}$ \\
\hline \hline
\end{tabular}


Table A-132: Reference case with low oil price cellulosic ethanol production [Million gallons]

\begin{tabular}{lrrrrrr} 
& $\mathbf{2 0 0 5}$ & $\mathbf{2 0 1 0}$ & $\mathbf{2 0 1 5}$ & $\mathbf{2 0 2 0}$ & $\mathbf{2 0 2 5}$ & $\mathbf{2 0 3 0}$ \\
Africa & 0 & 0 & 0 & 0 & 11 & 0 \\
Argentina & 0 & 0 & 206 & 306 & 335 & 347 \\
Australia & 0 & 0 & 66 & 247 & 616 & 1,172 \\
Brazil & 0 & 0 & 296 & 890 & 1,546 & 1,546 \\
Canada & 0 & 0 & 259 & 779 & 1,069 & 1,069 \\
Caribbean basin & 0 & 0 & 0 & 0 & 0 & 0 \\
China & 0 & 0 & 243 & 667 & 1,564 & 1,564 \\
Colombia & 0 & 0 & 170 & 512 & 1,199 & 1,893 \\
Eastern Europe & 0 & 0 & 85 & 303 & 670 & 897 \\
Former Soviet Union & 0 & 0 & 0 & 0 & 0 & 0 \\
India & 0 & 0 & 214 & 645 & 1,512 & 2,196 \\
Japan & 0 & 0 & 95 & 348 & 612 & 644 \\
Middle East & 0 & 0 & 0 & 0 & 0 & 0 \\
Mexico & 0 & 0 & 199 & 601 & 1,408 & 1,546 \\
Other Asia & 0 & 0 & 0 & 0 & 0 & 0 \\
South Korea & 0 & 0 & 35 & 35 & 25 & 35 \\
United States & 0 & 0 & 802 & 2,094 & 3,521 & 4,820 \\
Western Europe & 0 & 0 & 166 & 693 & 2,113 & 4,410 \\
\hline Total & $\mathbf{0}$ & $\mathbf{0}$ & $\mathbf{2 , 8 3 5}$ & $\mathbf{8 , 1 1 8}$ & $\mathbf{1 6 , 2 0 1}$ & $\mathbf{2 2 , 1 3 7}$ \\
\hline \hline
\end{tabular}

Table A-133: Reference case with low oil price grain ethanol consumption [Million gallons]

\begin{tabular}{lrrrrrr} 
& $\mathbf{2 0 0 5}$ & $\mathbf{2 0 1 0}$ & $\mathbf{2 0 1 5}$ & $\mathbf{2 0 2 0}$ & $\mathbf{2 0 2 5}$ & $\mathbf{2 0 3 0}$ \\
Africa & 0 & 0 & 0 & 0 & 0 & 0 \\
Australia & 0 & 0 & 0 & 0 & 0 & 0 \\
Canada & 0 & 0 & 0 & 0 & 0 & 0 \\
China & 263 & 0 & 0 & 263 & 0 & 0 \\
Central and South America & 0 & 36 & 122 & 118 & 98 & 58 \\
Eastern Europe & 110 & 226 & 348 & 477 & 367 & 251 \\
Former Soviet Union & 0 & 0 & 0 & 0 & 0 & 0 \\
India & 0 & 0 & 0 & 0 & 0 & 0 \\
Japan & 0 & 192 & 221 & 62 & 0 & 0 \\
Middle East & 0 & 0 & 0 & 0 & 0 & 0 \\
Mexico & 0 & 0 & 0 & 0 & 0 & 0 \\
Other Asia & 0 & 0 & 0 & 0 & 0 & 0 \\
South Korea & 81 & 295 & 123 & 0 & 0 & 0 \\
United States & 3,850 & 11,612 & 15,000 & 15,000 & 14,815 & 14,015 \\
Western Europe & 600 & 2,549 & 3,072 & 3,191 & 3,684 & 3,731 \\
\hline Total & $\mathbf{4 , 9 0 4}$ & $\mathbf{1 4 , 9 0 9}$ & $\mathbf{1 8 , 8 8 6}$ & $\mathbf{1 9 , 1 1 2}$ & $\mathbf{1 8 , 9 6 4}$ & $\mathbf{1 8 , 0 5 5}$ \\
\hline \hline
\end{tabular}


Table A-134: Reference case with low oil price sugar ethanol consumption [Million gallons]

\begin{tabular}{lrrrrrr} 
& $\mathbf{2 0 0 5}$ & $\mathbf{2 0 1 0}$ & $\mathbf{2 0 1 5}$ & $\mathbf{2 0 2 0}$ & $\mathbf{2 0 2 5}$ & $\mathbf{2 0 3 0}$ \\
Africa & 300 & 330 & 364 & 402 & 444 & 490 \\
Australia & 0 & 0 & 0 & 0 & 0 & 0 \\
Canada & 145 & 0 & 0 & 0 & 0 & 0 \\
China & 0 & 0 & 0 & 0 & 0 & 0 \\
Central and South America & 3,788 & 4,818 & 6,035 & 6,631 & 7,850 & 8,690 \\
Eastern Europe & 589 & 0 & 412 & 96 & 0 & 0 \\
Former Soviet Union & 0 & 0 & 0 & 0 & 0 & 0 \\
India & 313 & 275 & 320 & 329 & 338 & 332 \\
Japan & 263 & 58 & 48 & 0 & 0 & 0 \\
Middle East & 0 & 0 & 0 & 0 & 0 & 0 \\
Mexico & 0 & 0 & 0 & 0 & 0 & 0 \\
Other Asia & 0 & 0 & 0 & 0 & 0 & 0 \\
South Korea & 183 & 40 & 0 & 0 & 0 & 0 \\
United States & 168 & 0 & 788 & 2,732 & 2,756 & 3,969 \\
Western Europe & 136 & 1,644 & 908 & 57 & 318 & 0 \\
\hline Total & $\mathbf{5 , 8 8 4}$ & $\mathbf{7 , 1 6 5}$ & $\mathbf{8 , 8 7 4}$ & $\mathbf{1 0 , 2 4 6}$ & $\mathbf{1 1 , 7 0 6}$ & $\mathbf{1 3 , 4 8 1}$ \\
\hline \hline
\end{tabular}

Table A-135: Reference case with low oil price cellulosic ethanol consumption [Million gallons]

\begin{tabular}{lllrrrr} 
Africa & 0 & 0 & 0 & 0 & 11 & 0 \\
Australia & 0 & 0 & 66 & 247 & 616 & 1,172 \\
Canada & 0 & 0 & 233 & 701 & 1,069 & 1,061 \\
China & 0 & 0 & 0 & 0 & 780 & 771 \\
Central and South America & 0 & 0 & 0 & 400 & 0 & 55 \\
Eastern Europe & 0 & 0 & 111 & 380 & 670 & 897 \\
Former Soviet Union & 0 & 0 & 0 & 0 & 0 & 0 \\
India & 0 & 0 & 214 & 645 & 1,512 & 2,067 \\
Japan & 0 & 0 & 95 & 636 & 1,008 & 1,060 \\
Middle East & 0 & 0 & 0 & 0 & 0 & 0 \\
Mexico & 0 & 0 & 30 & 78 & 183 & 201 \\
Other Asia & 0 & 0 & 0 & 0 & 0 & 0 \\
South Korea & 0 & 0 & 277 & 414 & 413 & 413 \\
United States & 0 & 0 & 1,444 & 2,617 & 6,291 & 9,896 \\
Western Europe & 0 & 0 & 365 & 2,000 & 3,648 & 4,546 \\
\hline Total & $\mathbf{0}$ & $\mathbf{0}$ & $\mathbf{2 , 8 3 5}$ & $\mathbf{8 , 1 1 8}$ & $\mathbf{1 6 , 2 0 1}$ & $\mathbf{2 2 , 1 3 7}$ \\
\hline \hline
\end{tabular}


Table A-136: Reference case with low oil price grain ethanol net trade [Million gallons]

\begin{tabular}{lrrrrrr} 
& $\mathbf{2 0 0 5}$ & $\mathbf{2 0 1 0}$ & $\mathbf{2 0 1 5}$ & $\mathbf{2 0 2 0}$ & $\mathbf{2 0 2 5}$ & $\mathbf{2 0 3 0}$ \\
Africa & 0 & 0 & 0 & 0 & 0 & 0 \\
Australia & 0 & 0 & 0 & 0 & 0 & 0 \\
Canada & 0 & 0 & 0 & 0 & 0 & 0 \\
China & 81 & 344 & 344 & 62 & 0 & 0 \\
Central and South America & 0 & 143 & 483 & 486 & 506 & 127 \\
Eastern Europe & 0 & 0 & 0 & 0 & 0 & 0 \\
Former Soviet Union & 0 & 0 & 0 & 0 & 0 & 0 \\
India & 0 & 0 & 0 & 0 & 0 & 0 \\
Japan & 0 & -192 & -221 & -62 & 0 & 0 \\
Middle East & 0 & 0 & 0 & 0 & 0 & 0 \\
Mexico & 0 & 0 & 0 & 0 & 0 & 0 \\
Other Asia & 0 & 0 & 0 & 0 & 0 & 0 \\
South Korea & -81 & -295 & -123 & 0 & 0 & 0 \\
United States & 0 & 0 & 0 & 0 & 0 & 0 \\
Western Europe & 0 & 0 & -483 & -486 & -506 & -127 \\
\hline Total & $\mathbf{0}$ & $\mathbf{0}$ & $\mathbf{0}$ & $\mathbf{0}$ & $\mathbf{0}$ & $\mathbf{0}$ \\
\hline \hline
\end{tabular}

Table A-137: Reference case with low oil price sugar ethanol net trade [Million gallons]

\begin{tabular}{lrrrrrr} 
Africa & 0 & 0 & 0 & 0 & 0 & 0 \\
Australia & 0 & 0 & 0 & 0 & 0 & 0 \\
Canada & -145 & 0 & 0 & 0 & 0 & 0 \\
China & 0 & 40 & 48 & 0 & 0 & 0 \\
Central and South America & 1,484 & 1,702 & 2,108 & 2,885 & 3,074 & 3,969 \\
Eastern Europe & -589 & 0 & -412 & -96 & 0 & 0 \\
Former Soviet Union & 0 & 0 & 0 & 0 & 0 & 0 \\
India & 0 & 0 & 0 & 0 & 0 & 0 \\
Japan & -263 & -58 & -48 & 0 & 0 & 0 \\
Middle East & 0 & 0 & 0 & 0 & 0 & 0 \\
Mexico & 0 & 0 & 0 & 0 & 0 & 0 \\
Other Asia & 0 & 0 & 0 & 0 & 0 & 0 \\
South Korea & -183 & -40 & 0 & 0 & 0 & 0 \\
United States & -168 & 0 & -788 & $-2,732$ & $-2,756$ & $-3,969$ \\
Western Europe & -136 & $-1,644$ & -908 & -57 & -318 & 0 \\
\hline Total & $\mathbf{0}$ & $\mathbf{0}$ & $\mathbf{0}$ & $\mathbf{0}$ & $\mathbf{0}$ & $\mathbf{0}$ \\
\hline \hline
\end{tabular}


Table A-138: Reference case with low oil price cellulosic ethanol net trade [Million gallons]

\begin{tabular}{lrrrrrr} 
& $\mathbf{2 0 0 5}$ & $\mathbf{2 0 1 0}$ & $\mathbf{2 0 1 5}$ & $\mathbf{2 0 2 0}$ & $\mathbf{2 0 2 5}$ & $\mathbf{2 0 3 0}$ \\
Africa & 0 & 0 & 0 & 0 & 0 & 0 \\
Australia & 0 & 0 & 0 & 0 & 0 & 0 \\
Canada & 0 & 0 & 26 & 78 & 0 & 8 \\
China & 0 & 0 & 243 & 667 & 784 & 794 \\
Central and South America & 0 & 0 & 671 & 1,308 & 3,080 & 3,731 \\
Eastern Europe & 0 & 0 & -26 & -78 & 0 & 0 \\
Former Soviet Union & 0 & 0 & 0 & 0 & 0 & 0 \\
India & 0 & 0 & 0 & 0 & 0 & 128 \\
Japan & 0 & 0 & 0 & -288 & -395 & -415 \\
Middle East & 0 & 0 & 0 & 0 & 0 & 0 \\
Mexico & 0 & 0 & 170 & 523 & 1,225 & 1,345 \\
Other Asia & 0 & 0 & 0 & 0 & 0 & 0 \\
South Korea & 0 & 0 & -243 & -379 & -388 & -378 \\
United States & 0 & 0 & -642 & -523 & $-2,770$ & $-5,076$ \\
Western Europe & 0 & 0 & -199 & $-1,308$ & $-1,535$ & -136 \\
\hline Total & $\mathbf{0}$ & $\mathbf{0}$ & $\mathbf{0}$ & $\mathbf{0}$ & $\mathbf{0}$ & $\mathbf{0}$ \\
\hline \hline
\end{tabular}

Table A-139: Reference case with low oil price biomass-to-liquids production [Million gallons ethanol equivalent]

\begin{tabular}{lrrrrrr} 
& $\mathbf{2 0 0 5}$ & $\mathbf{2 0 1 0}$ & $\mathbf{2 0 1 5}$ & $\mathbf{2 0 2 0}$ & $\mathbf{2 0 2 5}$ & $\mathbf{2 0 3 0}$ \\
Africa & 0 & 0 & 0 & 0 & 0 & 0 \\
Argentina & 0 & 0 & 74 & 110 & 121 & 125 \\
Australia & 0 & 0 & 0 & 0 & 0 & 0 \\
Brazil & 0 & 0 & 106 & 320 & 556 & 556 \\
Canada & 0 & 0 & 93 & 280 & 384 & 384 \\
Caribbean basin & 0 & 0 & 0 & 0 & 0 & 0 \\
China & 0 & 0 & 57 & 240 & 563 & 563 \\
Colombia & 0 & 0 & 61 & 184 & 431 & 681 \\
Eastern Europe & 0 & 0 & 0 & 0 & 0 & 0 \\
Former Soviet Union & 0 & 0 & 0 & 0 & 0 & 0 \\
India & 0 & 0 & 77 & 232 & 544 & 790 \\
Japan & 0 & 0 & 0 & 0 & 0 & 0 \\
Middle East & 0 & 0 & 0 & 0 & 0 & 0 \\
Mexico & 0 & 0 & 72 & 216 & 506 & 556 \\
Other Asia & 0 & 0 & 0 & 188 & 565 & 1,097 \\
South Korea & 0 & 0 & 36 & 74 & 74 & 74 \\
United States & 0 & 0 & 822 & 4,468 & 5,952 & 7,643 \\
Western Europe & 0 & 0 & 0 & 0 & 0 & 0 \\
\hline Total & $\mathbf{0}$ & $\mathbf{0}$ & $\mathbf{1 , 3 9 8}$ & $\mathbf{6 , 3 1 2}$ & $\mathbf{9 , 6 9 6}$ & $\mathbf{1 2 , 4 6 8}$ \\
\hline \hline
\end{tabular}


Table A-140: Reference case with low oil price biomass-to-liquids consumption [Million gallons ethanol equivalent]

\begin{tabular}{lrrrrrr} 
& $\mathbf{2 0 0 5}$ & $\mathbf{2 0 1 0}$ & $\mathbf{2 0 1 5}$ & $\mathbf{2 0 2 0}$ & $\mathbf{2 0 2 5}$ & $\mathbf{2 0 3 0}$ \\
Africa & 0 & 0 & 0 & 0 & 0 & 0 \\
Australia & 0 & 0 & 0 & 0 & 0 & 0 \\
Canada & 0 & 0 & 0 & 0 & 0 & 0 \\
China & 0 & 0 & 0 & 0 & 0 & 0 \\
Central and South America & 0 & 0 & 0 & 0 & 0 & 0 \\
Eastern Europe & 0 & 0 & 0 & 0 & 0 & 0 \\
Former Soviet Union & 0 & 0 & 0 & 0 & 0 & 0 \\
India & 0 & 0 & 0 & 0 & 0 & 0 \\
Japan & 0 & 0 & 0 & 0 & 0 & 0 \\
Middle East & 0 & 0 & 0 & 0 & 0 & 0 \\
Mexico & 0 & 0 & 0 & 0 & 0 & 0 \\
Other Asia & 0 & 0 & 0 & 188 & 565 & 1,097 \\
South Korea & 0 & 0 & 0 & 0 & 0 & 0 \\
United States & 0 & 0 & 1,172 & 5,987 & 9,131 & 10,340 \\
Western Europe & 0 & 0 & 226 & 138 & 0 & 1,032 \\
\hline Total & $\mathbf{0}$ & $\mathbf{0}$ & $\mathbf{1 , 3 9 8}$ & $\mathbf{6 , 3 1 2}$ & $\mathbf{9 , 6 9 6}$ & $\mathbf{1 2 , 4 6 8}$ \\
\hline \hline
\end{tabular}

Table A-141: Reference case with low oil price biomass-to-liquids net trade [Million gallons ethanol equivalent]

\begin{tabular}{lrrrrrr} 
& $\mathbf{2 0 0 5}$ & $\mathbf{2 0 1 0}$ & $\mathbf{2 0 1 5}$ & $\mathbf{2 0 2 0}$ & $\mathbf{2 0 2 5}$ & $\mathbf{2 0 3 0}$ \\
Africa & 0 & 0 & 0 & 0 & 0 & 0 \\
Australia & 0 & 0 & 0 & 0 & 0 & 0 \\
Canada & 0 & 0 & 93 & 280 & 384 & 384 \\
China & 0 & 0 & 57 & 240 & 563 & 563 \\
Central and South America & 0 & 0 & 241 & 614 & 1,108 & 1,362 \\
Eastern Europe & 0 & 0 & 0 & 0 & 0 & 0 \\
Former Soviet Union & 0 & 0 & 0 & 0 & 0 & 0 \\
India & 0 & 0 & 77 & 232 & 544 & 790 \\
Japan & 0 & 0 & 0 & 0 & 0 & 0 \\
Middle East & 0 & 0 & 0 & 0 & 0 & 0 \\
Mexico & 0 & 0 & 72 & 216 & 506 & 556 \\
Other Asia & 0 & 0 & 0 & 0 & 0 & 0 \\
South Korea & 0 & 0 & 36 & 74 & 74 & 74 \\
United States & 0 & 0 & -350 & $-1,519$ & $-3,179$ & $-2,697$ \\
Western Europe & 0 & 0 & -226 & -138 & 0 & $-1,032$ \\
\hline Total & $\mathbf{0}$ & $\mathbf{0}$ & $\mathbf{0}$ & $\mathbf{0}$ & $\mathbf{0}$ & $\mathbf{0}$ \\
\hline \hline
\end{tabular}


Table A-142: Reference case with low oil price biodiesel production [Million gallons ethanol equivalent]

\begin{tabular}{lrrrrrr} 
& $\mathbf{2 0 0 5}$ & $\mathbf{2 0 1 0}$ & $\mathbf{2 0 1 5}$ & $\mathbf{2 0 2 0}$ & $\mathbf{2 0 2 5}$ & $\mathbf{2 0 3 0}$ \\
Africa & 0 & 0 & 0 & 0 & 0 & 0 \\
Argentina & 0 & 333 & 776 & 819 & 827 & 239 \\
Australia & 0 & 0 & 0 & 0 & 0 & 0 \\
Brazil & 0 & 333 & 835 & 1,600 & 2,469 & 1,359 \\
Canada & 0 & 0 & 0 & 0 & 0 & 0 \\
Caribbean basin & 0 & 95 & 310 & 675 & 802 & 898 \\
China & 0 & 0 & 0 & 0 & 0 & 0 \\
Colombia & 0 & 119 & 298 & 474 & 650 & 782 \\
Eastern Europe & 0 & 0 & 0 & 0 & 0 & 0 \\
Former Soviet Union & 0 & 0 & 0 & 0 & 0 & 0 \\
India & 0 & 0 & 0 & 0 & 0 & 0 \\
Japan & 0 & 0 & 0 & 0 & 0 & 0 \\
Middle East & 0 & 0 & 0 & 0 & 0 & 0 \\
Mexico & 0 & 0 & 0 & 0 & 0 & 0 \\
Other Asia & 0 & 0 & 0 & 0 & 0 & 0 \\
South Korea & 0 & 0 & 0 & 0 & 0 & 0 \\
United States & 0 & 1,238 & 1,640 & 1,747 & 1,907 & 2,016 \\
Western Europe & 998 & 2,643 & 3,281 & 3,307 & 3,760 & 4,114 \\
\hline Total & $\mathbf{9 9 8}$ & $\mathbf{4 , 7 6 0}$ & $\mathbf{7 , 1 4 0}$ & $\mathbf{8 , 6 2 2}$ & $\mathbf{1 0 , 4 1 5}$ & $\mathbf{9 , 4 0 8}$ \\
\hline \hline
\end{tabular}

Table A-143: Reference case with low oil price biodiesel consumption

[Million gallons ethanol equivalent]

\begin{tabular}{lrrrrrr} 
& $\mathbf{2 0 0 5}$ & $\mathbf{2 0 1 0}$ & $\mathbf{2 0 1 5}$ & $\mathbf{2 0 2 0}$ & $\mathbf{2 0 2 5}$ & $\mathbf{2 0 3 0}$ \\
Africa & 0 & 0 & 0 & 0 & 0 & 0 \\
Australia & 0 & 0 & 0 & 0 & 0 & 0 \\
Canada & 0 & 0 & 0 & 0 & 0 & 0 \\
China & 0 & 0 & 0 & 0 & 0 & 0 \\
Central and South America & 0 & 158 & 477 & 985 & 1,309 & 1,494 \\
Eastern Europe & 0 & 0 & 0 & 0 & 0 & 0 \\
Former Soviet Union & 0 & 0 & 0 & 0 & 0 & 0 \\
India & 0 & 0 & 0 & 0 & 0 & 0 \\
Japan & 0 & 0 & 0 & 0 & 0 & 0 \\
Middle East & 0 & 0 & 0 & 0 & 0 & 0 \\
Mexico & 0 & 0 & 0 & 0 & 0 & 0 \\
Other Asia & 0 & 0 & 0 & 0 & 0 & 0 \\
South Korea & 0 & 0 & 0 & 0 & 0 & 0 \\
United States & 0 & 1,238 & 1,713 & 1,747 & 2,429 & 2,016 \\
Western Europe & 998 & 3,364 & 4,951 & 5,890 & 6,677 & 5,898 \\
\hline Total & $\mathbf{9 9 8}$ & $\mathbf{4 , 7 6 0}$ & $\mathbf{7 , 1 4 0}$ & $\mathbf{8 , 6 2 2}$ & $\mathbf{1 0 , 4 1 5}$ & $\mathbf{9 , 4 0 8}$ \\
\hline \hline
\end{tabular}




\section{Table A-144: Reference case with low oil price biodiesel net trade}

[Million gallons ethanol equivalent]

\begin{tabular}{l} 
Africa \\
Australia \\
Canada \\
China \\
Central and South America \\
Eastern Europe \\
Former Soviet Union \\
India \\
Japan \\
Middle East \\
Mexico \\
Other Asia \\
South Korea \\
United States \\
Western Europe \\
\hline
\end{tabular}

2005

\begin{tabular}{rrrrrr}
$\mathbf{2 0 0 5}$ & $\mathbf{2 0 1 0}$ & $\mathbf{2 0 1 5}$ & $\mathbf{2 0 2 0}$ & $\mathbf{2 0 2 5}$ & $\mathbf{2 0 3 0}$ \\
0 & 0 & 0 & 0 & 0 & 0 \\
0 & 0 & 0 & 0 & 0 & 0 \\
0 & 0 & 0 & 0 & 0 & 0 \\
0 & 0 & 0 & 0 & 0 & 0 \\
0 & 720 & 1,742 & 2,583 & 3,440 & 1,784 \\
0 & 0 & 0 & 0 & 0 & 0 \\
0 & 0 & 0 & 0 & 0 & 0 \\
0 & 0 & 0 & 0 & 0 & 0 \\
0 & 0 & 0 & 0 & 0 & 0 \\
0 & 0 & 0 & 0 & 0 & 0 \\
0 & 0 & 0 & 0 & 0 & 0 \\
0 & 0 & 0 & 0 & 0 & 0 \\
0 & 0 & 0 & 0 & 0 & 0 \\
0 & 0 & -73 & 0 & -523 & 0 \\
0 & -720 & $-1,670$ & $-2,583$ & $-2,917$ & $-1,784$ \\
\hline $\mathbf{0}$ & $\mathbf{0}$ & $\mathbf{0}$ & $\mathbf{0}$ & $\mathbf{0}$ & $\mathbf{0}$ \\
\hline
\end{tabular}




\section{A-9 Reference Case with High Oil Price and High Feedstock Availability}

Table A-145: Reference case with high oil price and high feedstock availability ethanol production [Million gallons]

\begin{tabular}{lrrrrrr} 
& $\mathbf{2 0 0 5}$ & $\mathbf{2 0 1 0}$ & $\mathbf{2 0 1 5}$ & $\mathbf{2 0 2 0}$ & $\mathbf{2 0 2 5}$ & $\mathbf{2 0 3 0}$ \\
Africa & 300 & 330 & 364 & 402 & 455 & 502 \\
Argentina & 0 & 178 & 788 & 1,591 & 2,010 & 781 \\
Australia & 0 & 0 & 71 & 246 & 583 & 1,172 \\
Brazil & 5,225 & 8,034 & 12,103 & 16,323 & 20,293 & 24,835 \\
Canada & 0 & 0 & 259 & 779 & 1,054 & 1,067 \\
Caribbean basin & 164 & 282 & 687 & 795 & 795 & 795 \\
China & 344 & 119 & 587 & 1,350 & 1,922 & 3,937 \\
Colombia & 0 & 0 & 170 & 512 & 887 & 960 \\
Eastern Europe & 110 & 226 & 399 & 632 & 1,037 & 1,055 \\
Former Soviet Union & 0 & 0 & 39 & 120 & 367 & 1,120 \\
India & 313 & 842 & 1,300 & 1,728 & 2,200 & 2,822 \\
Japan & 0 & 0 & 95 & 369 & 609 & 727 \\
Middle East & 0 & 0 & 0 & 0 & 0 & 0 \\
Mexico & 0 & 0 & 199 & 601 & 1,088 & 1,088 \\
Other Asia & 0 & 0 & 0 & 0 & 0 & 0 \\
South Korea & 0 & 0 & 35 & 35 & 35 & 35 \\
United States & 3,850 & 11,413 & 14,840 & 15,918 & 15,775 & 15,653 \\
Western Europe & 600 & 2,465 & 2,755 & 3,235 & 5,205 & 7,923 \\
\hline Total & $\mathbf{1 0 , 9 0 5}$ & $\mathbf{2 3 , 8 8 9}$ & $\mathbf{3 4 , 6 9 0}$ & $\mathbf{4 4 , 6 3 6}$ & $\mathbf{5 4 , 3 1 6}$ & $\mathbf{6 4 , 4 7 1}$ \\
\hline \hline
\end{tabular}


Table A-146: Reference case with high oil price and high feedstock availability ethanol consumption [Million gallons]

\begin{tabular}{lrrrrrr} 
& $\mathbf{2 0 0 5}$ & $\mathbf{2 0 1 0}$ & $\mathbf{2 0 1 5}$ & $\mathbf{2 0 2 0}$ & $\mathbf{2 0 2 5}$ & $\mathbf{2 0 3 0}$ \\
Africa & 300 & 330 & 364 & 402 & 455 & 502 \\
Australia & 0 & 0 & 71 & 333 & 803 & 1,174 \\
Canada & 103 & 261 & 851 & 895 & 949 & 960 \\
China & 442 & 297 & 2,465 & 3,397 & 4,132 & 5,063 \\
Central and South America & 3,764 & 4,807 & 6,124 & 7,207 & 7,977 & 8,656 \\
Eastern Europe & 699 & 783 & 872 & 953 & 1,037 & 1,055 \\
Former Soviet Union & 0 & 0 & 39 & 120 & 367 & 1,120 \\
India & 313 & 842 & 1,300 & 1,642 & 1,980 & 2,399 \\
Japan & 300 & 325 & 448 & 757 & 1,042 & 1,217 \\
Middle East & 0 & 0 & 0 & 0 & 0 & 0 \\
Mexico & 0 & 0 & 30 & 998 & 1,030 & 1,052 \\
Other Asia & 0 & 0 & 0 & 2,637 & 3,005 & 3,472 \\
South Korea & 264 & 335 & 400 & 414 & 413 & 413 \\
United States & 3,984 & 11,668 & 17,383 & 19,797 & 23,562 & 28,684 \\
Western Europe & 736 & $\mathbf{4 , 2 4 1}$ & 4,343 & 5,086 & 7,563 & 8,703 \\
\hline Total & $\mathbf{1 0 , 9 0 5}$ & $\mathbf{2 3 , 8 8 9}$ & $\mathbf{3 4 , 6 9 0}$ & $\mathbf{4 4 , 6 3 6}$ & $\mathbf{5 4 , 3 1 6}$ & $\mathbf{6 4 , 4 7 1}$ \\
\hline \hline
\end{tabular}

Table A-147: Reference case with high oil price and high feedstock availability ethanol net trade [Million gallons]

\begin{tabular}{lrrrrrr} 
Africa & 0 & 0 & 0 & 0 & 0 & 0 \\
Australia & 0 & 0 & 0 & -86 & -220 & -3 \\
Canada & -103 & -261 & -592 & -116 & 105 & 107 \\
China & -98 & -178 & $-1,879$ & $-2,046$ & $-2,211$ & $-1,126$ \\
Central and South America & 1,624 & 3,688 & 7,624 & 12,014 & 16,008 & 18,715 \\
Eastern Europe & -589 & -557 & -473 & -321 & 0 & 0 \\
Former Soviet Union & 0 & 0 & 0 & 0 & 0 & 0 \\
India & 0 & 0 & 0 & 86 & 220 & 423 \\
Japan & -300 & -325 & -353 & -388 & -433 & -490 \\
Middle East & 0 & 0 & 0 & 0 & 0 & 0 \\
Mexico & 0 & 0 & 170 & -398 & 59 & 36 \\
Other Asia & 0 & 0 & 0 & $-2,637$ & $-3,005$ & $-3,472$ \\
South Korea & -264 & -335 & -365 & -379 & -378 & -378 \\
United States & -134 & -256 & $-2,543$ & $-3,879$ & $-7,788$ & $-13,032$ \\
Western Europe & -136 & $-1,776$ & $-1,589$ & $-1,851$ & $-2,358$ & -780 \\
\hline Total & $\mathbf{0}$ & $\mathbf{0}$ & $\mathbf{0}$ & $\mathbf{0}$ & $\mathbf{0}$ & $\mathbf{0}$ \\
\hline \hline
\end{tabular}


Table A-148: Reference case with high oil price and high feedstock availability grain ethanol production [Million gallons]

\begin{tabular}{lrrrrrr} 
& $\mathbf{2 0 0 5}$ & $\mathbf{2 0 1 0}$ & $\mathbf{2 0 1 5}$ & $\mathbf{2 0 2 0}$ & $\mathbf{2 0 2 5}$ & $\mathbf{2 0 3 0}$ \\
Africa & 0 & 0 & 0 & 0 & 0 & 0 \\
Argentina & 0 & 178 & 604 & 1,153 & 1,495 & 234 \\
Australia & 0 & 0 & 0 & 0 & 0 & 0 \\
Brazil & 0 & 0 & 0 & 0 & 0 & 0 \\
Canada & 0 & 0 & 0 & 0 & 0 & 0 \\
Caribbean basin & 0 & 0 & 0 & 0 & 0 & 0 \\
China & 344 & 0 & 0 & 325 & 0 & 0 \\
Colombia & 0 & 0 & 0 & 0 & 0 & 0 \\
Eastern Europe & 110 & 226 & 348 & 477 & 716 & 600 \\
Former Soviet Union & 0 & 0 & 0 & 0 & 0 & 0 \\
India & 0 & 0 & 0 & 0 & 0 & 0 \\
Japan & 0 & 0 & 0 & 0 & 0 & 0 \\
Middle East & 0 & 0 & 0 & 0 & 0 & 0 \\
Mexico & 0 & 0 & 0 & 0 & 0 & 0 \\
Other Asia & 0 & 0 & 0 & 0 & 0 & 0 \\
South Korea & 0 & 0 & 0 & 0 & 0 & 0 \\
United States & 3,850 & 11,413 & 14,038 & 13,660 & 11,828 & 11,111 \\
Western Europe & 600 & 2,465 & 2,589 & 2,542 & 3,178 & 3,604 \\
\hline Total & $\mathbf{4 , 9 0 4}$ & $\mathbf{1 4 , 2 8 2}$ & $\mathbf{1 7 , 5 7 9}$ & $\mathbf{1 8 , 1 5 7}$ & $\mathbf{1 7 , 2 1 7}$ & $\mathbf{1 5 , 5 4 9}$ \\
\hline \hline
\end{tabular}

Table A-149: Reference case with high oil price and high feedstock availability sugar ethanol production [Million gallons]

\begin{tabular}{lrrrrrr} 
& $\mathbf{2 0 0 5}$ & $\mathbf{2 0 1 0}$ & $\mathbf{2 0 1 5}$ & $\mathbf{2 0 2 0}$ & $\mathbf{2 0 2 5}$ & $\mathbf{2 0 3 0}$ \\
Africa & 300 & 330 & 364 & 402 & 444 & 490 \\
Argentina & 0 & 0 & 0 & 0 & 0 & 0 \\
Australia & 0 & 0 & 0 & 0 & 0 & 0 \\
Brazil & 5,225 & 8,034 & 11,807 & 15,433 & 18,208 & 20,112 \\
Canada & 0 & 0 & 0 & 0 & 0 & 0 \\
Caribbean basin & 164 & 282 & 687 & 795 & 795 & 795 \\
China & 0 & 119 & 358 & 358 & 358 & 358 \\
Colombia & 0 & 0 & 0 & 0 & 0 & 0 \\
Eastern Europe & 0 & 0 & 0 & 0 & 0 & 0 \\
Former Soviet Union & 0 & 0 & 0 & 0 & 0 & 0 \\
India & 313 & 842 & 1,086 & 1,083 & 688 & 40 \\
Japan & 0 & 0 & 0 & 0 & 0 & 0 \\
Middle East & 0 & 0 & 0 & 0 & 0 & 0 \\
Mexico & 0 & 0 & 0 & 0 & 0 & 0 \\
Other Asia & 0 & 0 & 0 & 0 & 0 & 0 \\
South Korea & 0 & 0 & 0 & 0 & 0 & 0 \\
United States & 0 & 0 & 0 & 0 & 0 & 0 \\
Western Europe & 0 & 0 & 0 & 0 & 0 & 0 \\
\hline Total & $\mathbf{6 , 0 0 1}$ & $\mathbf{9 , 6 0 8}$ & $\mathbf{1 4 , 3 0 2}$ & $\mathbf{1 8 , 0 7 2}$ & $\mathbf{2 0 , 4 9 3}$ & $\mathbf{2 1 , 7 9 5}$ \\
\hline \hline
\end{tabular}


Table A-150: Reference case with high oil price and high feedstock availability cellulosic ethanol production [Million gallons]

\begin{tabular}{lrrrrrr} 
& $\mathbf{2 0 0 5}$ & $\mathbf{2 0 1 0}$ & $\mathbf{2 0 1 5}$ & $\mathbf{2 0 2 0}$ & $\mathbf{2 0 2 5}$ & $\mathbf{2 0 3 0}$ \\
Africa & 0 & 0 & 0 & 0 & 11 & 11 \\
Argentina & 0 & 0 & 184 & 438 & 516 & 547 \\
Australia & 0 & 0 & 71 & 246 & 583 & 1,172 \\
Brazil & 0 & 0 & 296 & 890 & 2,085 & 4,723 \\
Canada & 0 & 0 & 259 & 779 & 1,054 & 1,067 \\
Caribbean basin & 0 & 0 & 0 & 0 & 0 & 0 \\
China & 0 & 0 & 229 & 667 & 1,564 & 3,580 \\
Colombia & 0 & 0 & 170 & 512 & 887 & 960 \\
Eastern Europe & 0 & 0 & 51 & 155 & 321 & 455 \\
Former Soviet Union & 0 & 0 & 39 & 120 & 367 & 1,120 \\
India & 0 & 0 & 214 & 645 & 1,512 & 2,782 \\
Japan & 0 & 0 & 95 & 369 & 609 & 727 \\
Middle East & 0 & 0 & 0 & 0 & 0 & 0 \\
Mexico & 0 & 0 & 199 & 601 & 1,088 & 1,088 \\
Other Asia & 0 & 0 & 0 & 0 & 0 & 0 \\
South Korea & 0 & 0 & 35 & 35 & 35 & 35 \\
United States & 0 & 0 & 802 & 2,258 & 3,946 & 4,541 \\
Western Europe & 0 & 0 & 166 & 693 & 2,027 & 4,320 \\
\hline Total & $\mathbf{0}$ & $\mathbf{0}$ & $\mathbf{2 , 8 0 9}$ & $\mathbf{8 , 4 0 7}$ & $\mathbf{1 6 , 6 0 6}$ & $\mathbf{2 7 , 1 2 7}$ \\
\hline \hline
\end{tabular}

Table A-151: Reference case with high oil price and high feedstock availability grain ethanol consumption [Million gallons]

\begin{tabular}{lrrrrrr} 
& $\mathbf{2 0 0 5}$ & $\mathbf{2 0 1 0}$ & $\mathbf{2 0 1 5}$ & $\mathbf{2 0 2 0}$ & $\mathbf{2 0 2 5}$ & $\mathbf{2 0 3 0}$ \\
Africa & 0 & 0 & 0 & 0 & 0 & 0 \\
Australia & 0 & 0 & 0 & 0 & 0 & 0 \\
Canada & 0 & 0 & 0 & 0 & 0 & 0 \\
China & 344 & 0 & 121 & 325 & 0 & 0 \\
Central and South America & 0 & 36 & 118 & 148 & 205 & 82 \\
Eastern Europe & 110 & 226 & 348 & 477 & 716 & 600 \\
Former Soviet Union & 0 & 0 & 0 & 0 & 0 & 0 \\
India & 0 & 0 & 0 & 0 & 0 & 0 \\
Japan & 0 & 0 & 0 & 0 & 0 & 153 \\
Middle East & 0 & 0 & 0 & 0 & 0 & 0 \\
Mexico & 0 & 0 & 0 & 0 & 0 & 0 \\
Other Asia & 0 & 0 & 0 & 1,005 & 912 & 0 \\
South Korea & 0 & 143 & 365 & 0 & 378 & 0 \\
United States & 3,850 & 11,413 & 14,038 & 13,660 & 11,828 & 11,111 \\
Western Europe & 600 & 2,465 & 2,589 & 2,542 & 3,178 & 3,604 \\
\hline Total & $\mathbf{4 , 9 0 4}$ & $\mathbf{1 4 , 2 8 2}$ & $\mathbf{1 7 , 5 7 9}$ & $\mathbf{1 8 , 1 5 7}$ & $\mathbf{1 7 , 2 1 7}$ & $\mathbf{1 5 , 5 4 9}$ \\
\hline \hline
\end{tabular}


Table A-152: Reference case with high oil price and high feedstock availability sugar ethanol consumption [Million gallons]

\begin{tabular}{lrrrrrr} 
& $\mathbf{2 0 0 5}$ & $\mathbf{2 0 1 0}$ & $\mathbf{2 0 1 5}$ & $\mathbf{2 0 2 0}$ & $\mathbf{2 0 2 5}$ & $\mathbf{2 0 3 0}$ \\
Africa & 300 & 330 & 364 & 402 & 444 & 490 \\
Australia & 0 & 0 & 0 & 0 & 0 & 3 \\
Canada & 103 & 261 & 592 & 116 & 0 & 0 \\
China & 98 & 297 & 2,115 & 2,404 & 2,463 & 788 \\
Central and South America & 3,764 & 4,771 & 5,980 & 6,923 & 7,639 & 8,430 \\
Eastern Europe & 589 & 557 & 473 & 321 & 0 & 0 \\
Former Soviet Union & 0 & 0 & 0 & 0 & 0 & 0 \\
India & 313 & 842 & 1,086 & 1,083 & 688 & 37 \\
Japan & 300 & 325 & 353 & 388 & 433 & 338 \\
Middle East & 0 & 0 & 0 & 0 & 0 & 0 \\
Mexico & 0 & 0 & 0 & 398 & 570 & 0 \\
Other Asia & 0 & 0 & 0 & 1,632 & 2,093 & 3,472 \\
South Korea & 264 & 193 & 0 & 0 & 0 & 0 \\
United States & 134 & 256 & 1,750 & 3,068 & 3,805 & 7,456 \\
Western Europe & 136 & 1,776 & 1,589 & 1,337 & 2,358 & 780 \\
\hline Total & $\mathbf{6 , 0 0 1}$ & $\mathbf{9 , 6 0 8}$ & $\mathbf{1 4 , 3 0 2}$ & $\mathbf{1 8 , 0 7 2}$ & $\mathbf{2 0 , 4 9 3}$ & $\mathbf{2 1 , 7 9 5}$ \\
\hline \hline
\end{tabular}

Table A-153: Reference case with high oil price and high feedstock availability cellulosic ethanol consumption [Million gallons]

\begin{tabular}{lrrrrrr} 
& $\mathbf{2 0 0 5}$ & $\mathbf{2 0 1 0}$ & $\mathbf{2 0 1 5}$ & $\mathbf{2 0 2 0}$ & $\mathbf{2 0 2 5}$ & $\mathbf{2 0 3 0}$ \\
Africa & 0 & 0 & 0 & 0 & 11 & 11 \\
Australia & 0 & 0 & 71 & 333 & 803 & 1,172 \\
Canada & 0 & 0 & 259 & 779 & 949 & 960 \\
China & 0 & 0 & 229 & 667 & 1,669 & 4,276 \\
Central and South America & 0 & 0 & 26 & 136 & 133 & 144 \\
Eastern Europe & 0 & 0 & 51 & 155 & 321 & 455 \\
Former Soviet Union & 0 & 0 & 39 & 120 & 367 & 1,120 \\
India & 0 & 0 & 214 & 559 & 1,292 & 2,362 \\
Japan & 0 & 0 & 95 & 369 & 609 & 727 \\
Middle East & 0 & 0 & 0 & 0 & 0 & 0 \\
Mexico & 0 & 0 & 30 & 601 & 460 & 1,052 \\
Other Asia & 0 & 0 & 0 & 0 & 0 & 0 \\
South Korea & 0 & 0 & 35 & 414 & 35 & 413 \\
United States & 0 & 0 & 1,595 & 3,069 & 7,929 & 10,117 \\
Western Europe & 0 & 0 & 166 & 1,207 & 2,027 & 4,320 \\
\hline Total & $\mathbf{0}$ & $\mathbf{0}$ & $\mathbf{2 , 8 0 9}$ & $\mathbf{8 , 4 0 7}$ & $\mathbf{1 6 , 6 0 6}$ & $\mathbf{2 7 , 1 2 7}$ \\
\hline \hline
\end{tabular}


Table A-154: Reference case with high oil price and high feedstock availability grain ethanol net trade [Million gallons]

\begin{tabular}{lrrrrrr} 
& $\mathbf{2 0 0 5}$ & $\mathbf{2 0 1 0}$ & $\mathbf{2 0 1 5}$ & $\mathbf{2 0 2 0}$ & $\mathbf{2 0 2 5}$ & $\mathbf{2 0 3 0}$ \\
Africa & 0 & 0 & 0 & 0 & 0 & 0 \\
Australia & 0 & 0 & 0 & 0 & 0 & 0 \\
Canada & 0 & 0 & 0 & 0 & 0 & 0 \\
China & 0 & 0 & -121 & 0 & 0 & 0 \\
Central and South America & 0 & 143 & 486 & 1,005 & 1,290 & 153 \\
Eastern Europe & 0 & 0 & 0 & 0 & 0 & 0 \\
Former Soviet Union & 0 & 0 & 0 & 0 & 0 & 0 \\
India & 0 & 0 & 0 & 0 & 0 & 0 \\
Japan & 0 & 0 & 0 & 0 & 0 & -153 \\
Middle East & 0 & 0 & 0 & 0 & 0 & 0 \\
Mexico & 0 & 0 & 0 & 0 & 0 & 0 \\
Other Asia & 0 & 0 & 0 & $-1,005$ & -912 & 0 \\
South Korea & 0 & -143 & -365 & 0 & -378 & 0 \\
United States & 0 & 0 & 0 & 0 & 0 & 0 \\
Western Europe & 0 & 0 & 0 & 0 & 0 & 0 \\
\hline Total & $\mathbf{0}$ & $\mathbf{0}$ & $\mathbf{0}$ & $\mathbf{0}$ & $\mathbf{0}$ & $\mathbf{0}$ \\
\hline \hline
\end{tabular}

Table A-155: Reference case with high oil price and high feedstock availability sugar ethanol net trade [Million gallons]

\begin{tabular}{lrrrrrr} 
Africa & 0 & 0 & 0 & 0 & 0 & 0 \\
Australia & 0 & 0 & 0 & 0 & 0 & -3 \\
Canada & -103 & -261 & -592 & -116 & 0 & 0 \\
China & -98 & -178 & $-1,758$ & $-2,046$ & $-2,105$ & -430 \\
Central and South America & 1,624 & 3,545 & 6,514 & 9,306 & 11,364 & 12,476 \\
Eastern Europe & -589 & -557 & -473 & -321 & 0 & 0 \\
Former Soviet Union & 0 & 0 & 0 & 0 & 0 & 0 \\
India & 0 & 0 & 0 & 0 & 0 & 3 \\
Japan & -300 & -325 & -353 & -388 & -433 & -338 \\
Middle East & 0 & 0 & 0 & 0 & 0 & 0 \\
Mexico & 0 & 0 & 0 & -398 & -570 & 0 \\
Other Asia & 0 & 0 & 0 & $-1,632$ & $-2,093$ & $-3,472$ \\
South Korea & -264 & -193 & 0 & 0 & 0 & 0 \\
United States & -134 & -256 & $-1,750$ & $-3,068$ & $-3,805$ & $-7,456$ \\
Western Europe & -136 & $-1,776$ & $-1,589$ & $-1,337$ & $-2,358$ & -780 \\
\hline Total & $\mathbf{0}$ & $\mathbf{0}$ & $\mathbf{0}$ & $\mathbf{0}$ & $\mathbf{0}$ & $\mathbf{0}$ \\
\hline \hline
\end{tabular}


Table A-156: Reference case with high oil price and high feedstock availability cellulosic ethanol net trade [Million gallons]

\begin{tabular}{lrrrrrr} 
& $\mathbf{2 0 0 5}$ & $\mathbf{2 0 1 0}$ & $\mathbf{2 0 1 5}$ & $\mathbf{2 0 2 0}$ & $\mathbf{2 0 2 5}$ & $\mathbf{2 0 3 0}$ \\
Africa & 0 & 0 & 0 & 0 & 0 & 0 \\
Australia & 0 & 0 & 0 & -86 & -220 & 0 \\
Canada & 0 & 0 & 0 & 0 & 105 & 107 \\
China & 0 & 0 & 0 & 0 & -105 & -696 \\
Central and South America & 0 & 0 & 624 & 1,704 & 3,355 & 6,086 \\
Eastern Europe & 0 & 0 & 0 & 0 & 0 & 0 \\
Former Soviet Union & 0 & 0 & 0 & 0 & 0 & 0 \\
India & 0 & 0 & 0 & 86 & 220 & 421 \\
Japan & 0 & 0 & 0 & 0 & 0 & 0 \\
Middle East & 0 & 0 & 0 & 0 & 0 & 0 \\
Mexico & 0 & 0 & 170 & 0 & 628 & 36 \\
Other Asia & 0 & 0 & 0 & 0 & 0 & 0 \\
South Korea & 0 & 0 & 0 & -379 & 0 & -378 \\
United States & 0 & 0 & -793 & -811 & $-3,983$ & $-5,576$ \\
Western Europe & 0 & 0 & 0 & -514 & 0 & 0 \\
\hline Total & $\mathbf{0}$ & $\mathbf{0}$ & $\mathbf{0}$ & $\mathbf{0}$ & $\mathbf{0}$ & $\mathbf{0}$ \\
\hline \hline
\end{tabular}

Table A-157: Reference case with high oil price and high feedstock availability biomass-to-liquids production [Million gallons ethanol equivalent]

\begin{tabular}{lrrrrrr} 
& $\mathbf{2 0 0 5}$ & $\mathbf{2 0 1 0}$ & $\mathbf{2 0 1 5}$ & $\mathbf{2 0 2 0}$ & $\mathbf{2 0 2 5}$ & $\mathbf{2 0 3 0}$ \\
Africa & 0 & 0 & 82 & 415 & 921 & 1,584 \\
Argentina & 0 & 0 & 66 & 158 & 186 & 197 \\
Australia & 0 & 0 & 0 & 46 & 217 & 474 \\
Brazil & 0 & 0 & 106 & 320 & 750 & 1,363 \\
Canada & 0 & 0 & 93 & 280 & 379 & 379 \\
Caribbean basin & 0 & 0 & 0 & 0 & 0 & 0 \\
China & 0 & 0 & 72 & 240 & 563 & 982 \\
Colombia & 0 & 0 & 61 & 184 & 319 & 346 \\
Eastern Europe & 0 & 0 & 38 & 204 & 536 & 988 \\
Former Soviet Union & 0 & 0 & 44 & 213 & 553 & 1,016 \\
India & 0 & 0 & 77 & 232 & 544 & 1,001 \\
Japan & 0 & 0 & 0 & 0 & 0 & 0 \\
Middle East & 0 & 0 & 0 & 111 & 348 & 685 \\
Mexico & 0 & 0 & 72 & 216 & 391 & 391 \\
Other Asia & 0 & 0 & 96 & 554 & 1,511 & 2,519 \\
South Korea & 0 & 0 & 36 & 74 & 74 & 74 \\
United States & 0 & 0 & 822 & 4,818 & 6,399 & 8,213 \\
Western Europe & 0 & 0 & 0 & 0 & 0 & 0 \\
\hline Total & $\mathbf{0}$ & $\mathbf{0}$ & $\mathbf{1 , 6 6 4}$ & $\mathbf{8 , 0 6 4}$ & $\mathbf{1 3 , 6 8 9}$ & $\mathbf{2 0 , 2 1 3}$ \\
\hline \hline
\end{tabular}


Table A-158: Reference case with high oil price and high feedstock availability biomass-to-liquids consumption [Million gallons ethanol equivalent]

\begin{tabular}{lrrrrrr} 
& $\mathbf{2 0 0 5}$ & $\mathbf{2 0 1 0}$ & $\mathbf{2 0 1 5}$ & $\mathbf{2 0 2 0}$ & $\mathbf{2 0 2 5}$ & $\mathbf{2 0 3 0}$ \\
Africa & 0 & 0 & 82 & 415 & 921 & 1,585 \\
Australia & 0 & 0 & 0 & 46 & 217 & 474 \\
Canada & 0 & 0 & 0 & 0 & 0 & 379 \\
China & 0 & 0 & 0 & 0 & 0 & 982 \\
Central and South America & 0 & 0 & 0 & 0 & 0 & 0 \\
Eastern Europe & 0 & 0 & 38 & 204 & 536 & 988 \\
Former Soviet Union & 0 & 0 & 44 & 213 & 554 & 1,016 \\
India & 0 & 0 & 0 & 0 & 0 & 1,001 \\
Japan & 0 & 0 & 0 & 0 & 0 & 0 \\
Middle East & 0 & 0 & 0 & 111 & 348 & 685 \\
Mexico & 0 & 0 & 0 & 0 & 0 & 392 \\
Other Asia & 0 & 0 & 96 & 554 & 1,511 & 2,519 \\
South Korea & 0 & 0 & 0 & 0 & 0 & 74 \\
United States & 0 & 0 & 1,405 & 6,522 & 9,604 & 10,118 \\
Western Europe & 0 & 0 & 0 & 0 & 0 & 0 \\
\hline Total & $\mathbf{0}$ & $\mathbf{0}$ & $\mathbf{1 , 6 6 4}$ & $\mathbf{8 , 0 6 5}$ & $\mathbf{1 3 , 6 8 9}$ & $\mathbf{2 0 , 2 1 3}$ \\
\hline \hline
\end{tabular}

Table A-159: Reference case with high oil price and high feedstock availability biomass-to-liquids net trade [Million gallons ethanol equivalent]

\begin{tabular}{lrrrrrr} 
& $\mathbf{2 0 0 5}$ & $\mathbf{2 0 1 0}$ & $\mathbf{2 0 1 5}$ & $\mathbf{2 0 2 0}$ & $\mathbf{2 0 2 5}$ & $\mathbf{2 0 3 0}$ \\
Africa & 0 & 0 & 0 & 0 & 0 & 0 \\
Australia & 0 & 0 & 0 & 0 & 0 & 0 \\
Canada & 0 & 0 & 93 & 280 & 379 & 0 \\
China & 0 & 0 & 72 & 240 & 563 & 0 \\
Central and South America & 0 & 0 & 234 & 662 & 1,255 & 1,905 \\
Eastern Europe & 0 & 0 & 0 & 0 & 0 & 0 \\
Former Soviet Union & 0 & 0 & 0 & 0 & 0 & 0 \\
India & 0 & 0 & 77 & 232 & 544 & 0 \\
Japan & 0 & 0 & 0 & 0 & 0 & 0 \\
Middle East & 0 & 0 & 0 & 0 & 0 & 0 \\
Mexico & 0 & 0 & 72 & 216 & 391 & 0 \\
Other Asia & 0 & 0 & 0 & 0 & 0 & 0 \\
South Korea & 0 & 0 & 36 & 74 & 74 & 0 \\
United States & 0 & 0 & -583 & $-1,704$ & $-3,205$ & $-1,905$ \\
Western Europe & 0 & 0 & 0 & 0 & 0 & 0 \\
\hline Total & $\mathbf{0}$ & $\mathbf{0}$ & $\mathbf{0}$ & $\mathbf{0}$ & $\mathbf{0}$ & $\mathbf{0}$ \\
\hline \hline
\end{tabular}


Table A-160: Reference case with high oil price and high feedstock availability biodiesel production [Million gallons ethanol equivalent]

\begin{tabular}{lrrrrrr} 
& $\mathbf{2 0 0 5}$ & $\mathbf{2 0 1 0}$ & $\mathbf{2 0 1 5}$ & $\mathbf{2 0 2 0}$ & $\mathbf{2 0 2 5}$ & $\mathbf{2 0 3 0}$ \\
Africa & 0 & 0 & 0 & 0 & 0 & 0 \\
Argentina & 0 & 333 & 877 & 1,706 & 1,815 & 198 \\
Australia & 0 & 0 & 0 & 0 & 0 & 0 \\
Brazil & 0 & 333 & 877 & 1,579 & 1,579 & 1,103 \\
Canada & 0 & 0 & 0 & 0 & 0 & 0 \\
Caribbean basin & 0 & 95 & 310 & 742 & 1,611 & 3,359 \\
China & 0 & 0 & 0 & 0 & 0 & 0 \\
Colombia & 0 & 119 & 358 & 838 & 1,648 & 2,074 \\
Eastern Europe & 0 & 0 & 0 & 0 & 0 & 0 \\
Former Soviet Union & 0 & 0 & 0 & 0 & 0 & 0 \\
India & 0 & 0 & 0 & 0 & 0 & 0 \\
Japan & 0 & 0 & 0 & 0 & 0 & 0 \\
Middle East & 0 & 0 & 0 & 0 & 0 & 0 \\
Mexico & 0 & 0 & 0 & 0 & 0 & 0 \\
Other Asia & 0 & 0 & 0 & 0 & 0 & 0 \\
South Korea & 0 & 0 & 0 & 0 & 0 & 0 \\
United States & 0 & 1,182 & 1,640 & 1,747 & 1,850 & 2,016 \\
Western Europe & 998 & 3,445 & 4,172 & 5,110 & 5,532 & 5,997 \\
\hline Total & $\mathbf{9 9 8}$ & $\mathbf{5 , 5 0 5}$ & $\mathbf{8 , 2 3 2}$ & $\mathbf{1 1 , 7 2 2}$ & $\mathbf{1 4 , 0 3 4}$ & $\mathbf{1 4 , 7 4 6}$ \\
\hline \hline
\end{tabular}

Table A-161: Reference case with high oil price and high feedstock availability biodiesel consumption [Million gallons ethanol equivalent]

\begin{tabular}{lrrrrrr} 
& $\mathbf{2 0 0 5}$ & $\mathbf{2 0 1 0}$ & $\mathbf{2 0 1 5}$ & $\mathbf{2 0 2 0}$ & $\mathbf{2 0 2 5}$ & $\mathbf{2 0 3 0}$ \\
Africa & 0 & 0 & 0 & 0 & 0 & 0 \\
Australia & 0 & 0 & 0 & 0 & 0 & 0 \\
Canada & 0 & 0 & 0 & 0 & 0 & 0 \\
China & 0 & 0 & 0 & 0 & 0 & 0 \\
Central and South America & 0 & 158 & 464 & 975 & 1,303 & 1,488 \\
Eastern Europe & 0 & 0 & 0 & 0 & 0 & 0 \\
Former Soviet Union & 0 & 0 & 0 & 0 & 0 & 0 \\
India & 0 & 0 & 0 & 0 & 0 & 0 \\
Japan & 0 & 0 & 0 & 0 & 0 & 0 \\
Middle East & 0 & 0 & 0 & 0 & 0 & 0 \\
Mexico & 0 & 0 & 0 & 0 & 0 & 0 \\
Other Asia & 0 & 0 & 0 & 0 & 0 & 0 \\
South Korea & 0 & 0 & 0 & 0 & 0 & 0 \\
United States & 0 & 1,182 & 1,713 & 2,772 & 4,367 & 2,016 \\
Western Europe & 998 & 4,165 & 6,056 & 7,974 & 8,365 & 11,242 \\
\hline Total & $\mathbf{9 9 8}$ & $\mathbf{5 , 5 0 5}$ & $\mathbf{8 , 2 3 2}$ & $\mathbf{1 1 , 7 2 2}$ & $\mathbf{1 4 , 0 3 4}$ & $\mathbf{1 4 , 7 4 6}$ \\
\hline \hline
\end{tabular}




\section{World Biofuels Study}

Table A-162: Reference case with high oil price and high feedstock availability biodiesel net trade [Million gallons ethanol equivalent]

\begin{tabular}{lrrrrrr} 
& $\mathbf{2 0 0 5}$ & $\mathbf{2 0 1 0}$ & $\mathbf{2 0 1 5}$ & $\mathbf{2 0 2 0}$ & $\mathbf{2 0 2 5}$ & $\mathbf{2 0 3 0}$ \\
Africa & 0 & 0 & 0 & 0 & 0 & 0 \\
Australia & 0 & 0 & 0 & 0 & 0 & 0 \\
Canada & 0 & 0 & 0 & 0 & 0 & 0 \\
China & 0 & 0 & 0 & 0 & 0 & 0 \\
Central and South America & 0 & 720 & 1,956 & 3,889 & 5,350 & 5,245 \\
Eastern Europe & 0 & 0 & 0 & 0 & 0 & 0 \\
Former Soviet Union & 0 & 0 & 0 & 0 & 0 & 0 \\
India & 0 & 0 & 0 & 0 & 0 & 0 \\
Japan & 0 & 0 & 0 & 0 & 0 & 0 \\
Middle East & 0 & 0 & 0 & 0 & 0 & 0 \\
Mexico & 0 & 0 & 0 & 0 & 0 & 0 \\
Other Asia & 0 & 0 & 0 & 0 & 0 & 0 \\
South Korea & 0 & 0 & 0 & 0 & 0 & 0 \\
United States & 0 & 0 & -73 & $-1,025$ & $-2,517$ & 0 \\
Western Europe & 0 & -720 & $-1,884$ & $-2,864$ & $-2,833$ & $-5,245$ \\
\hline Total & $\mathbf{0}$ & $\mathbf{0}$ & $\mathbf{0}$ & $\mathbf{0}$ & $\mathbf{0}$ & $\mathbf{0}$ \\
\hline \hline
\end{tabular}




\section{A-10 Reference Case with Low Oil Price and Low Feedstock Availability}

\section{Table A-163: Reference case with low oil price and low feedstock availability ethanol production [Million gallons]}

\begin{tabular}{lrrrrrr} 
& $\mathbf{2 0 0 5}$ & $\mathbf{2 0 1 0}$ & $\mathbf{2 0 1 5}$ & $\mathbf{2 0 2 0}$ & $\mathbf{2 0 2 5}$ & $\mathbf{2 0 3 0}$ \\
Africa & 300 & 330 & 364 & 402 & 455 & 490 \\
Argentina & 0 & 178 & 865 & 1,634 & 1,634 & 895 \\
Australia & 0 & 0 & 66 & 247 & 616 & 1,172 \\
Brazil & 4,866 & 4,894 & 6,286 & 7,371 & 8,942 & 11,721 \\
Canada & 0 & 143 & 401 & 779 & 1,199 & 1,199 \\
Caribbean basin & 162 & 80 & 34 & 0 & 0 & 0 \\
China & 344 & 366 & 608 & 667 & 1,564 & 2,532 \\
Colombia & 0 & 196 & 375 & 512 & 512 & 512 \\
Eastern Europe & 110 & 226 & 434 & 780 & 1,037 & 1,149 \\
Former Soviet Union & 0 & 0 & 0 & 0 & 0 & 0 \\
India & 313 & 0 & 214 & 645 & 1,512 & 2,816 \\
Japan & 0 & 0 & 95 & 348 & 612 & 644 \\
Middle East & 0 & 0 & 0 & 0 & 0 & 0 \\
Mexico & 0 & 0 & 0 & 0 & 0 & 0 \\
Other Asia & 0 & 0 & 0 & 0 & 0 & 0 \\
South Korea & 0 & 0 & 35 & 35 & 35 & 19 \\
United States & 3,850 & 11,612 & 15,802 & 17,643 & 20,834 & 22,159 \\
Western Europe & 600 & 2,570 & 2,755 & 3,398 & 5,291 & 8,013 \\
\hline Total & $\mathbf{1 0 , 5 4 4}$ & $\mathbf{2 0 , 5 9 4}$ & $\mathbf{2 8 , 3 3 5}$ & $\mathbf{3 4 , 4 6 0}$ & $\mathbf{4 4 , 2 4 3}$ & $\mathbf{5 3 , 3 2 0}$ \\
\hline \hline
\end{tabular}


Table A-164: Reference case with low oil price and low feedstock availability ethanol consumption [Million gallons]

\begin{tabular}{lrrrrrr} 
& $\mathbf{2 0 0 5}$ & $\mathbf{2 0 1 0}$ & $\mathbf{2 0 1 5}$ & $\mathbf{2 0 2 0}$ & $\mathbf{2 0 2 5}$ & $\mathbf{2 0 3 0}$ \\
Africa & 300 & 330 & 364 & 402 & 455 & 490 \\
Australia & 0 & 0 & 66 & 247 & 616 & 1,172 \\
Canada & 101 & 128 & 361 & 701 & 1,079 & 1,079 \\
China & 126 & 0 & 0 & 0 & 0 & 1,685 \\
Central and South America & 3,764 & 4,831 & 6,126 & 7,172 & 7,845 & 8,562 \\
Eastern Europe & 699 & 226 & 434 & 780 & 1,037 & 1,149 \\
Former Soviet Union & 0 & 0 & 0 & 0 & 0 & 0 \\
India & 313 & 0 & 214 & 613 & 1,361 & 2,399 \\
Japan & 257 & 132 & 335 & 636 & 1,008 & 1,097 \\
Middle East & 0 & 0 & 0 & 0 & 0 & 0 \\
Mexico & 0 & 0 & 0 & 0 & 0 & 0 \\
Other Asia & 0 & 0 & 0 & 0 & 0 & 0 \\
South Korea & 264 & 0 & 400 & 414 & 413 & 413 \\
United States & 3,984 & 11,612 & 15,802 & 18,246 & 22,779 & 26,725 \\
Western Europe & 736 & 3,335 & 4,233 & 5,249 & 7,649 & 8,550 \\
\hline Total & $\mathbf{1 0 , 5 4 4}$ & $\mathbf{2 0 , 5 9 4}$ & $\mathbf{2 8 , 3 3 5}$ & $\mathbf{3 4 , 4 6 0}$ & $\mathbf{4 4 , 2 4 3}$ & $\mathbf{5 3 , 3 2 0}$ \\
\hline \hline
\end{tabular}

Table A-165: Reference case with low oil price and low feedstock availability ethanol net trade [Million gallons]

\begin{tabular}{lrrrrrr} 
Africa & 0 & 0 & 0 & 0 & 0 & 0 \\
Australia & 0 & 0 & 0 & 0 & 0 & 0 \\
Canada & -101 & 14 & 40 & 78 & 120 & 120 \\
China & 218 & 366 & 608 & 667 & 1,564 & 847 \\
Central and South America & 1,264 & 517 & 1,435 & 2,344 & 3,242 & 4,566 \\
Eastern Europe & -589 & 0 & 0 & 0 & 0 & 0 \\
Former Soviet Union & 0 & 0 & 0 & 0 & 0 & 0 \\
India & 0 & 0 & 0 & 32 & 151 & 417 \\
Japan & -257 & -132 & -240 & -288 & -395 & -453 \\
Middle East & 0 & 0 & 0 & 0 & 0 & 0 \\
Mexico & 0 & 0 & 0 & 0 & 0 & 0 \\
Other Asia & 0 & 0 & 0 & 0 & 0 & 0 \\
South Korea & -264 & 0 & -365 & -379 & -378 & -394 \\
United States & -134 & 0 & 0 & -603 & $-1,945$ & $-4,566$ \\
Western Europe & -136 & -765 & $-1,478$ & $-1,851$ & $-2,358$ & -537 \\
\hline Total & $\mathbf{0}$ & $\mathbf{0}$ & $\mathbf{0}$ & $\mathbf{0}$ & $\mathbf{0}$ & $\mathbf{0}$ \\
\hline \hline
\end{tabular}


Table A-166: Reference case with low oil price and low feedstock availability grain ethanol production [Million gallons]

\begin{tabular}{lrrrrrr} 
& $\mathbf{2 0 0 5}$ & $\mathbf{2 0 1 0}$ & $\mathbf{2 0 1 5}$ & $\mathbf{2 0 2 0}$ & $\mathbf{2 0 2 5}$ & $\mathbf{2 0 3 0}$ \\
Africa & 0 & 0 & 0 & 0 & 0 & 0 \\
Argentina & 0 & 178 & 640 & 833 & 833 & 95 \\
Australia & 0 & 0 & 0 & 0 & 0 & 0 \\
Brazil & 0 & 0 & 0 & 0 & 0 & 0 \\
Canada & 0 & 143 & 143 & 0 & 0 & 0 \\
Caribbean basin & 0 & 0 & 0 & 0 & 0 & 0 \\
China & 344 & 366 & 366 & 0 & 0 & 0 \\
Colombia & 0 & 0 & 0 & 0 & 0 & 0 \\
Eastern Europe & 110 & 226 & 348 & 477 & 367 & 251 \\
Former Soviet Union & 0 & 0 & 0 & 0 & 0 & 0 \\
India & 0 & 0 & 0 & 0 & 0 & 0 \\
Japan & 0 & 0 & 0 & 0 & 0 & 0 \\
Middle East & 0 & 0 & 0 & 0 & 0 & 0 \\
Mexico & 0 & 0 & 0 & 0 & 0 & 0 \\
Other Asia & 0 & 0 & 0 & 0 & 0 & 0 \\
South Korea & 0 & 0 & 0 & 0 & 0 & 0 \\
United States & 3,850 & 11,612 & 15,000 & 15,000 & 15,000 & 15,000 \\
Western Europe & 600 & 2,570 & 2,589 & 2,705 & 3,178 & 3,604 \\
\hline Total & $\mathbf{4 , 9 0 4}$ & $\mathbf{1 5 , 0 9 4}$ & $\mathbf{1 9 , 0 8 5}$ & $\mathbf{1 9 , 0 1 5}$ & $\mathbf{1 9 , 3 7 8}$ & $\mathbf{1 8 , 9 5 0}$ \\
\hline \hline
\end{tabular}

Table A-167: Reference case with low oil price and low feedstock availability sugar ethanol production [Million gallons]

\begin{tabular}{lrrrrrr} 
& $\mathbf{2 0 0 5}$ & $\mathbf{2 0 1 0}$ & $\mathbf{2 0 1 5}$ & $\mathbf{2 0 2 0}$ & $\mathbf{2 0 2 5}$ & $\mathbf{2 0 3 0}$ \\
Africa & 300 & 330 & 364 & 402 & 444 & 490 \\
Argentina & 0 & 0 & 0 & 0 & 0 & 0 \\
Australia & 0 & 0 & 0 & 0 & 0 & 0 \\
Brazil & 4,866 & 4,894 & 5,978 & 6,473 & 6,857 & 6,970 \\
Canada & 0 & 0 & 0 & 0 & 0 & 0 \\
Caribbean basin & 162 & 80 & 34 & 0 & 0 & 0 \\
China & 0 & 0 & 0 & 0 & 0 & 0 \\
Colombia & 0 & 196 & 196 & 0 & 0 & 0 \\
Eastern Europe & 0 & 0 & 0 & 0 & 0 & 0 \\
Former Soviet Union & 0 & 0 & 0 & 0 & 0 & 0 \\
India & 313 & 0 & 0 & 0 & 0 & 0 \\
Japan & 0 & 0 & 0 & 0 & 0 & 0 \\
Middle East & 0 & 0 & 0 & 0 & 0 & 0 \\
Mexico & 0 & 0 & 0 & 0 & 0 & 0 \\
Other Asia & 0 & 0 & 0 & 0 & 0 & 0 \\
South Korea & 0 & 0 & 0 & 0 & 0 & 0 \\
United States & 0 & 0 & 0 & 0 & 0 & 0 \\
Western Europe & 0 & 0 & 0 & 0 & 0 & 0 \\
\hline Total & $\mathbf{5 , 6 4 0}$ & $\mathbf{5 , 5 0 0}$ & $\mathbf{6 , 5 7 2}$ & $\mathbf{6 , 8 7 5}$ & $\mathbf{7 , 3 0 1}$ & $\mathbf{7 , 4 6 0}$ \\
\hline \hline
\end{tabular}


Table A-168: Reference case with low oil price and low feedstock availability cellulosic ethanol production [Million gallons]

\begin{tabular}{lrrrrrr} 
& $\mathbf{2 0 0 5}$ & $\mathbf{2 0 1 0}$ & $\mathbf{2 0 1 5}$ & $\mathbf{2 0 2 0}$ & $\mathbf{2 0 2 5}$ & $\mathbf{2 0 3 0}$ \\
Africa & 0 & 0 & 0 & 0 & 11 & 0 \\
Argentina & 0 & 0 & 225 & 801 & 801 & 801 \\
Australia & 0 & 0 & 66 & 247 & 616 & 1,172 \\
Brazil & 0 & 0 & 308 & 899 & 2,085 & 4,751 \\
Canada & 0 & 0 & 259 & 779 & 1,199 & 1,199 \\
Caribbean basin & 0 & 0 & 0 & 0 & 0 & 0 \\
China & 0 & 0 & 243 & 667 & 1,564 & 2,532 \\
Colombia & 0 & 0 & 179 & 512 & 512 & 512 \\
Eastern Europe & 0 & 0 & 85 & 303 & 670 & 897 \\
Former Soviet Union & 0 & 0 & 0 & 0 & 0 & 0 \\
India & 0 & 0 & 214 & 645 & 1,512 & 2,816 \\
Japan & 0 & 0 & 95 & 348 & 612 & 644 \\
Middle East & 0 & 0 & 0 & 0 & 0 & 0 \\
Mexico & 0 & 0 & 0 & 0 & 0 & 0 \\
Other Asia & 0 & 0 & 0 & 0 & 0 & 0 \\
South Korea & 0 & 0 & 35 & 35 & 35 & 19 \\
United States & 0 & 0 & 802 & 2,643 & 5,834 & 7,159 \\
Western Europe & 0 & 0 & 166 & 693 & 2,113 & 4,410 \\
\hline Total & $\mathbf{0}$ & $\mathbf{0}$ & $\mathbf{2 , 6 7 8}$ & $\mathbf{8 , 5 7 0}$ & $\mathbf{1 7 , 5 6 5}$ & $\mathbf{2 6 , 9 1 1}$ \\
\hline \hline
\end{tabular}

Table A-169: Reference case with low oil price and low feedstock availability grain ethanol consumption [Million gallons]

\begin{tabular}{lrrrrrr} 
& $\mathbf{2 0 0 5}$ & $\mathbf{2 0 1 0}$ & $\mathbf{2 0 1 5}$ & $\mathbf{2 0 2 0}$ & $\mathbf{2 0 2 5}$ & $\mathbf{2 0 3 0}$ \\
Africa & 0 & 0 & 0 & 0 & 0 & 0 \\
Australia & 0 & 0 & 0 & 0 & 0 & 0 \\
Canada & 0 & 128 & 143 & 0 & 0 & 0 \\
China & 126 & 0 & 0 & 0 & 0 & 0 \\
Central and South America & 0 & 36 & 130 & 212 & 168 & 95 \\
Eastern Europe & 110 & 226 & 348 & 477 & 367 & 251 \\
Former Soviet Union & 0 & 0 & 0 & 0 & 0 & 0 \\
India & 0 & 0 & 0 & 0 & 0 & 0 \\
Japan & 0 & 132 & 240 & 0 & 0 & 0 \\
Middle East & 0 & 0 & 0 & 0 & 0 & 0 \\
Mexico & 0 & 0 & 0 & 0 & 0 & 0 \\
Other Asia & 0 & 0 & 0 & 0 & 0 & 0 \\
South Korea & 218 & 0 & 126 & 0 & 0 & 0 \\
United States & 3,850 & 11,612 & 15,000 & 15,000 & 15,000 & 15,000 \\
Western Europe & 600 & 2,960 & 3,099 & 3,325 & 3,842 & 3,604 \\
\hline Total & $\mathbf{4 , 9 0 4}$ & $\mathbf{1 5 , 0 9 4}$ & $\mathbf{1 9 , 0 8 5}$ & $\mathbf{1 9 , 0 1 5}$ & $\mathbf{1 9 , 3 7 8}$ & $\mathbf{1 8 , 9 5 0}$ \\
\hline \hline
\end{tabular}


Table A-170: Reference case with low oil price and low feedstock availability sugar ethanol consumption [Million gallons]

\begin{tabular}{lrrrrrr} 
& $\mathbf{2 0 0 5}$ & $\mathbf{2 0 1 0}$ & $\mathbf{2 0 1 5}$ & $\mathbf{2 0 2 0}$ & $\mathbf{2 0 2 5}$ & $\mathbf{2 0 3 0}$ \\
Africa & 300 & 330 & 364 & 402 & 444 & 490 \\
Australia & 0 & 0 & 0 & 0 & 0 & 0 \\
Canada & 101 & 0 & 0 & 0 & 0 & 0 \\
China & 0 & 0 & 0 & 0 & 0 & 0 \\
Central and South America & 3,764 & 4,795 & 5,996 & 5,985 & 5,514 & 4,196 \\
Eastern Europe & 589 & 0 & 0 & 0 & 0 & 0 \\
Former Soviet Union & 0 & 0 & 0 & 0 & 0 & 0 \\
India & 313 & 0 & 0 & 0 & 0 & 0 \\
Japan & 257 & 0 & 0 & 0 & 0 & 0 \\
Middle East & 0 & 0 & 0 & 0 & 0 & 0 \\
Mexico & 0 & 0 & 0 & 0 & 0 & 0 \\
Other Asia & 0 & 0 & 0 & 0 & 0 & 0 \\
South Korea & 46 & 0 & 0 & 0 & 0 & 0 \\
United States & 134 & 0 & 0 & 488 & 1,342 & 2,774 \\
Western Europe & 136 & 375 & 212 & 0 & 0 & 0 \\
\hline Total & $\mathbf{5 , 6 4 0}$ & $\mathbf{5 , 5 0 0}$ & $\mathbf{6 , 5 7 2}$ & $\mathbf{6 , 8 7 5}$ & $\mathbf{7 , 3 0 1}$ & $\mathbf{7 , 4 6 0}$ \\
\hline \hline
\end{tabular}

Table A-171: Reference case with low oil price and low feedstock availability cellulosic ethanol consumption [Million gallons]

\begin{tabular}{lrrrrrr} 
& $\mathbf{2 0 0 5}$ & $\mathbf{2 0 1 0}$ & $\mathbf{2 0 1 5}$ & $\mathbf{2 0 2 0}$ & $\mathbf{2 0 2 5}$ & $\mathbf{2 0 3 0}$ \\
Africa & 0 & 0 & 0 & 0 & 11 & 0 \\
Australia & 0 & 0 & 66 & 247 & 616 & 1,172 \\
Canada & 0 & 0 & 219 & 701 & 1,079 & 1,079 \\
China & 0 & 0 & 0 & 0 & 0 & 1,685 \\
Central and South America & 0 & 0 & 0 & 975 & 2,162 & 4,272 \\
Eastern Europe & 0 & 0 & 85 & 303 & 670 & 897 \\
Former Soviet Union & 0 & 0 & 0 & 0 & 0 & 0 \\
India & 0 & 0 & 214 & 613 & 1,361 & 2,399 \\
Japan & 0 & 0 & 95 & 636 & 1,008 & 1,097 \\
Middle East & 0 & 0 & 0 & 0 & 0 & 0 \\
Mexico & 0 & 0 & 0 & 0 & 0 & 0 \\
Other Asia & 0 & 0 & 0 & 0 & 0 & 0 \\
South Korea & 0 & 0 & 274 & 414 & 413 & 413 \\
United States & 0 & 0 & 802 & 2,758 & 6,437 & 8,951 \\
Western Europe & 0 & 0 & 922 & 1,923 & 3,807 & 4,946 \\
\hline Total & $\mathbf{0}$ & $\mathbf{0}$ & $\mathbf{2 , 6 7 8}$ & $\mathbf{8 , 5 7 0}$ & $\mathbf{1 7 , 5 6 5}$ & $\mathbf{2 6 , 9 1 1}$ \\
\hline \hline
\end{tabular}


Table A-172: Reference case with low oil price and low feedstock availability grain ethanol net trade [Million gallons]

\begin{tabular}{lrrrrrr} 
& $\mathbf{2 0 0 5}$ & $\mathbf{2 0 1 0}$ & $\mathbf{2 0 1 5}$ & $\mathbf{2 0 2 0}$ & $\mathbf{2 0 2 5}$ & $\mathbf{2 0 3 0}$ \\
Africa & 0 & 0 & 0 & 0 & 0 & 0 \\
Australia & 0 & 0 & 0 & 0 & 0 & 0 \\
Canada & 0 & 14 & 0 & 0 & 0 & 0 \\
China & 218 & 366 & 366 & 0 & 0 & 0 \\
Central and South America & 0 & 143 & 510 & 620 & 664 & 0 \\
Eastern Europe & 0 & 0 & 0 & 0 & 0 & 0 \\
Former Soviet Union & 0 & 0 & 0 & 0 & 0 & 0 \\
India & 0 & 0 & 0 & 0 & 0 & 0 \\
Japan & 0 & -132 & -240 & 0 & 0 & 0 \\
Middle East & 0 & 0 & 0 & 0 & 0 & 0 \\
Mexico & 0 & 0 & 0 & 0 & 0 & 0 \\
Other Asia & 0 & 0 & 0 & 0 & 0 & 0 \\
South Korea & -218 & 0 & -126 & 0 & 0 & 0 \\
United States & 0 & 0 & 0 & 0 & 0 & 0 \\
Western Europe & 0 & -390 & -510 & -620 & -664 & 0 \\
\hline Total & $\mathbf{0}$ & $\mathbf{0}$ & $\mathbf{0}$ & $\mathbf{0}$ & $\mathbf{0}$ & $\mathbf{0}$ \\
\hline \hline
\end{tabular}

Table A-173: Reference case with low oil price and low feedstock availability sugar ethanol net trade [Million gallons]

\begin{tabular}{|c|c|c|c|c|c|c|}
\hline Africa & 0 & 0 & 0 & 0 & 0 & 0 \\
\hline Australia & 0 & 0 & 0 & 0 & 0 & 0 \\
\hline Canada & -101 & 0 & 0 & 0 & 0 & 0 \\
\hline China & 0 & 0 & 0 & 0 & 0 & 0 \\
\hline Central and South America & 1,264 & 375 & 212 & 488 & 1,342 & 2,774 \\
\hline Eastern Europe & -589 & 0 & 0 & 0 & 0 & 0 \\
\hline Former Soviet Union & 0 & 0 & 0 & 0 & 0 & 0 \\
\hline India & 0 & 0 & 0 & 0 & 0 & 0 \\
\hline Japan & -257 & 0 & 0 & 0 & 0 & 0 \\
\hline Middle East & 0 & 0 & 0 & 0 & 0 & 0 \\
\hline Mexico & 0 & 0 & 0 & 0 & 0 & 0 \\
\hline Other Asia & 0 & 0 & 0 & 0 & 0 & 0 \\
\hline South Korea & -46 & 0 & 0 & 0 & 0 & 0 \\
\hline United States & -134 & 0 & 0 & -488 & $-1,342$ & $-2,774$ \\
\hline Western Europe & -136 & -375 & -212 & 0 & 0 & 0 \\
\hline Total & $\mathbf{0}$ & $\mathbf{0}$ & $\mathbf{0}$ & $\mathbf{0}$ & $\mathbf{0}$ & $\mathbf{0}$ \\
\hline
\end{tabular}


Table A-174: Reference case with low oil price and low feedstock availability cellulosic ethanol net trade [Million gallons]

\begin{tabular}{lrrrrrr} 
& $\mathbf{2 0 0 5}$ & $\mathbf{2 0 1 0}$ & $\mathbf{2 0 1 5}$ & $\mathbf{2 0 2 0}$ & $\mathbf{2 0 2 5}$ & $\mathbf{2 0 3 0}$ \\
Africa & 0 & 0 & 0 & 0 & 0 & 0 \\
Australia & 0 & 0 & 0 & 0 & 0 & 0 \\
Canada & 0 & 0 & 40 & 78 & 120 & 120 \\
China & 0 & 0 & 243 & 667 & 1,564 & 847 \\
Central and South America & 0 & 0 & 713 & 1,236 & 1,236 & 1,792 \\
Eastern Europe & 0 & 0 & 0 & 0 & 0 & 0 \\
Former Soviet Union & 0 & 0 & 0 & 0 & 0 & 0 \\
India & 0 & 0 & 0 & 32 & 151 & 417 \\
Japan & 0 & 0 & 0 & -288 & -395 & -453 \\
Middle East & 0 & 0 & 0 & 0 & 0 & 0 \\
Mexico & 0 & 0 & 0 & 0 & 0 & 0 \\
Other Asia & 0 & 0 & 0 & 0 & 0 & 0 \\
South Korea & 0 & 0 & -240 & -379 & -378 & -394 \\
United States & 0 & 0 & 0 & -115 & -603 & $-1,792$ \\
Western Europe & 0 & 0 & -756 & $-1,231$ & $-1,694$ & -537 \\
\hline Total & $\mathbf{0}$ & $\mathbf{0}$ & $\mathbf{0}$ & $\mathbf{0}$ & $\mathbf{0}$ & $\mathbf{0}$ \\
\hline \hline
\end{tabular}

Table A-175: Reference case with low oil price and low feedstock availability biomass-to-liquids production [Million gallons ethanol equivalent]

\begin{tabular}{lrrrrrr} 
& $\mathbf{2 0 0 5}$ & $\mathbf{2 0 1 0}$ & $\mathbf{2 0 1 5}$ & $\mathbf{2 0 2 0}$ & $\mathbf{2 0 2 5}$ & $\mathbf{2 0 3 0}$ \\
Africa & 0 & 0 & 0 & 0 & 0 & 0 \\
Argentina & 0 & 0 & 81 & 288 & 288 & 288 \\
Australia & 0 & 0 & 0 & 0 & 0 & 0 \\
Brazil & 0 & 0 & 92 & 311 & 750 & 1,333 \\
Canada & 0 & 0 & 93 & 280 & 431 & 431 \\
Caribbean basin & 0 & 0 & 0 & 0 & 0 & 0 \\
China & 0 & 0 & 57 & 240 & 563 & 563 \\
Colombia & 0 & 0 & 51 & 184 & 184 & 184 \\
Eastern Europe & 0 & 0 & 0 & 0 & 0 & 0 \\
Former Soviet Union & 0 & 0 & 0 & 0 & 0 & 0 \\
India & 0 & 0 & 77 & 232 & 544 & 1,001 \\
Japan & 0 & 0 & 0 & 0 & 0 & 0 \\
Middle East & 0 & 0 & 0 & 0 & 0 & 0 \\
Mexico & 0 & 0 & 0 & 0 & 0 & 0 \\
Other Asia & 0 & 0 & 0 & 184 & 558 & 1,086 \\
South Korea & 0 & 0 & 36 & 74 & 74 & 74 \\
United States & 0 & 0 & 822 & 4,325 & 5,770 & 7,411 \\
Western Europe & 0 & 0 & 0 & 0 & 0 & 0 \\
\hline Total & $\mathbf{0}$ & $\mathbf{0}$ & $\mathbf{1 , 3 0 9}$ & $\mathbf{6 , 1 1 8}$ & $\mathbf{9 , 1 6 2}$ & $\mathbf{1 2 , 3 7 0}$ \\
\hline \hline
\end{tabular}


Table A-176: Reference case with low oil price and low feedstock availability biomass-to-liquids consumption [Million gallons ethanol equivalent]

\begin{tabular}{lrrrrrr} 
& $\mathbf{2 0 0 5}$ & $\mathbf{2 0 1 0}$ & $\mathbf{2 0 1 5}$ & $\mathbf{2 0 2 0}$ & $\mathbf{2 0 2 5}$ & $\mathbf{2 0 3 0}$ \\
Africa & 0 & 0 & 0 & 0 & 0 & 0 \\
Australia & 0 & 0 & 0 & 0 & 0 & 0 \\
Canada & 0 & 0 & 0 & 0 & 0 & 0 \\
China & 0 & 0 & 0 & 0 & 0 & 0 \\
Central and South America & 0 & 0 & 0 & 0 & 0 & 0 \\
Eastern Europe & 0 & 0 & 0 & 0 & 0 & 0 \\
Former Soviet Union & 0 & 0 & 0 & 0 & 0 & 0 \\
India & 0 & 0 & 0 & 0 & 0 & 0 \\
Japan & 0 & 0 & 0 & 0 & 0 & 0 \\
Middle East & 0 & 0 & 0 & 0 & 0 & 0 \\
Mexico & 0 & 0 & 0 & 0 & 0 & 0 \\
Other Asia & 0 & 0 & 0 & 184 & 558 & 1,086 \\
South Korea & 0 & 0 & 0 & 0 & 0 & 0 \\
United States & 0 & 0 & 874 & 4,612 & 7,854 & 11,285 \\
Western Europe & 0 & 0 & 435 & 1,322 & 750 & 0 \\
\hline Total & $\mathbf{0}$ & $\mathbf{0}$ & $\mathbf{1 , 3 0 9}$ & $\mathbf{6 , 1 1 8}$ & $\mathbf{9 , 1 6 2}$ & $\mathbf{1 2 , 3 7 0}$ \\
\hline \hline
\end{tabular}

Table A-177: Reference case with low oil price and low feedstock availability biomass-to-liquids net trade [Million gallons ethanol equivalent]

\begin{tabular}{lrrrrrr} 
& $\mathbf{2 0 0 5}$ & $\mathbf{2 0 1 0}$ & $\mathbf{2 0 1 5}$ & $\mathbf{2 0 2 0}$ & $\mathbf{2 0 2 5}$ & $\mathbf{2 0 3 0}$ \\
Africa & 0 & 0 & 0 & 0 & 0 & 0 \\
Australia & 0 & 0 & 0 & 0 & 0 & 0 \\
Canada & 0 & 0 & 93 & 280 & 431 & 431 \\
China & 0 & 0 & 57 & 240 & 563 & 563 \\
Central and South America & 0 & 0 & 225 & 783 & 1,222 & 1,805 \\
Eastern Europe & 0 & 0 & 0 & 0 & 0 & 0 \\
Former Soviet Union & 0 & 0 & 0 & 0 & 0 & 0 \\
India & 0 & 0 & 77 & 232 & 544 & 1,001 \\
Japan & 0 & 0 & 0 & 0 & 0 & 0 \\
Middle East & 0 & 0 & 0 & 0 & 0 & 0 \\
Mexico & 0 & 0 & 0 & 0 & 0 & 0 \\
Other Asia & 0 & 0 & 0 & 0 & 0 & 0 \\
South Korea & 0 & 0 & 36 & 74 & 74 & 74 \\
United States & 0 & 0 & -52 & -287 & $-2,084$ & $-3,874$ \\
Western Europe & 0 & 0 & -435 & $-1,322$ & -750 & 0 \\
\hline Total & $\mathbf{0}$ & $\mathbf{0}$ & $\mathbf{0}$ & $\mathbf{0}$ & $\mathbf{0}$ & $\mathbf{0}$ \\
\hline \hline
\end{tabular}


Table A-178: Reference case with low oil price and low feedstock availability biodiesel production [Million gallons ethanol equivalent]

\begin{tabular}{lrrrrrr} 
& $\mathbf{2 0 0 5}$ & $\mathbf{2 0 1 0}$ & $\mathbf{2 0 1 5}$ & $\mathbf{2 0 2 0}$ & $\mathbf{2 0 2 5}$ & $\mathbf{2 0 3 0}$ \\
Africa & 0 & 0 & 0 & 0 & 0 & 0 \\
Argentina & 0 & 333 & 877 & 1,491 & 1,472 & 1,155 \\
Australia & 0 & 0 & 0 & 0 & 0 & 0 \\
Brazil & 0 & 333 & 835 & 1,537 & 1,769 & 1,334 \\
Canada & 0 & 0 & 0 & 0 & 0 & 0 \\
Caribbean basin & 0 & 95 & 310 & 742 & 1,145 & 1,220 \\
China & 0 & 0 & 0 & 0 & 0 & 0 \\
Colombia & 0 & 0 & 0 & 0 & 0 & 0 \\
Eastern Europe & 0 & 0 & 0 & 0 & 0 & 0 \\
Former Soviet Union & 0 & 0 & 0 & 0 & 0 & 0 \\
India & 0 & 0 & 0 & 0 & 0 & 0 \\
Japan & 0 & 0 & 0 & 0 & 0 & 0 \\
Middle East & 0 & 0 & 0 & 0 & 0 & 0 \\
Mexico & 0 & 0 & 0 & 0 & 0 & 0 \\
Other Asia & 0 & 0 & 0 & 0 & 0 & 0 \\
South Korea & 0 & 0 & 0 & 0 & 0 & 0 \\
United States & 0 & 1,238 & 1,640 & 1,803 & 1,907 & 2,072 \\
Western Europe & 998 & 2,643 & 2,984 & 3,240 & 3,684 & 4,114 \\
\hline Total & $\mathbf{9 9 8}$ & $\mathbf{4 , 6 4 1}$ & $\mathbf{6 , 6 4 6}$ & $\mathbf{8 , 8 1 3}$ & $\mathbf{9 , 9 7 6}$ & $\mathbf{9 , 8 9 5}$ \\
\hline \hline
\end{tabular}

Table A-179: Reference case with low oil price and low feedstock availability biodiesel consumption [Million gallons ethanol equivalent]

\begin{tabular}{lrrrrrr} 
& $\mathbf{2 0 0 5}$ & $\mathbf{2 0 1 0}$ & $\mathbf{2 0 1 5}$ & $\mathbf{2 0 2 0}$ & $\mathbf{2 0 2 5}$ & $\mathbf{2 0 3 0}$ \\
Africa & 0 & 0 & 0 & 0 & 0 & 0 \\
Australia & 0 & 0 & 0 & 0 & 0 & 0 \\
Canada & 0 & 0 & 0 & 0 & 0 & 0 \\
China & 0 & 0 & 0 & 0 & 0 & 0 \\
Central and South America & 0 & 126 & 443 & 937 & 1,265 & 1,453 \\
Eastern Europe & 0 & 0 & 0 & 0 & 0 & 0 \\
Former Soviet Union & 0 & 0 & 0 & 0 & 0 & 0 \\
India & 0 & 0 & 0 & 0 & 0 & 0 \\
Japan & 0 & 0 & 0 & 0 & 0 & 0 \\
Middle East & 0 & 0 & 0 & 0 & 0 & 0 \\
Mexico & 0 & 0 & 0 & 0 & 0 & 0 \\
Other Asia & 0 & 0 & 0 & 0 & 0 & 0 \\
South Korea & 0 & 0 & 0 & 0 & 0 & 0 \\
United States & 0 & 1,238 & 2,494 & 3,991 & 3,623 & 2,226 \\
Western Europe & 998 & 3,277 & 3,709 & 3,885 & 5,089 & 6,216 \\
\hline Total & $\mathbf{9 9 8}$ & $\mathbf{4 , 6 4 1}$ & $\mathbf{6 , 6 4 6}$ & $\mathbf{8 , 8 1 3}$ & $\mathbf{9 , 9 7 6}$ & $\mathbf{9 , 8 9 5}$ \\
\hline \hline
\end{tabular}




\section{World Biofuels Study}

Table A-180: Reference case with low oil price and low feedstock availability biodiesel net trade [Million gallons ethanol equivalent]

\begin{tabular}{lrrrrrr} 
& $\mathbf{2 0 0 5}$ & $\mathbf{2 0 1 0}$ & $\mathbf{2 0 1 5}$ & $\mathbf{2 0 2 0}$ & $\mathbf{2 0 2 5}$ & $\mathbf{2 0 3 0}$ \\
Africa & 0 & 0 & 0 & 0 & 0 & 0 \\
Australia & 0 & 0 & 0 & 0 & 0 & 0 \\
Canada & 0 & 0 & 0 & 0 & 0 & 0 \\
China & 0 & 0 & 0 & 0 & 0 & 0 \\
Central and South America & 0 & 634 & 1,579 & 2,833 & 3,122 & 2,256 \\
Eastern Europe & 0 & 0 & 0 & 0 & 0 & 0 \\
Former Soviet Union & 0 & 0 & 0 & 0 & 0 & 0 \\
India & 0 & 0 & 0 & 0 & 0 & 0 \\
Japan & 0 & 0 & 0 & 0 & 0 & 0 \\
Middle East & 0 & 0 & 0 & 0 & 0 & 0 \\
Mexico & 0 & 0 & 0 & 0 & 0 & 0 \\
Other Asia & 0 & 0 & 0 & 0 & 0 & 0 \\
South Korea & 0 & 0 & 0 & 0 & 0 & 0 \\
United States & 0 & 0 & -854 & $-2,188$ & $-1,716$ & -154 \\
Western Europe & 0 & -634 & -725 & -645 & $-1,405$ & $-2,102$ \\
\hline Total & $\mathbf{0}$ & $\mathbf{0}$ & $\mathbf{0}$ & $\mathbf{0}$ & $\mathbf{0}$ & $\mathbf{0}$ \\
\hline \hline
\end{tabular}




\section{A-11 Reference Case with E20}

Table A-181: Reference case with E20 ethanol production [Million gallons]

BIOREF20

\section{Ethanol production} [Million gallons]

Africa
Argentina
Australia
Brazil
Canada
Caribbean basin
China
Colombia
Eastern Europe
Former Soviet Union
India
Japan
Middle East
Mexico
Other Asia
South Korea
United States
Western Europe

Total

$\mathbf{0 , 7 8 9}$

\begin{tabular}{rrrrrr}
$\mathbf{2 0 0 5}$ & $\mathbf{2 0 1 0}$ & $\mathbf{2 0 1 5}$ & $\mathbf{2 0 2 0}$ & $\mathbf{2 0 2 5}$ & $\mathbf{2 0 3 0}$ \\
300 & 330 & 364 & 402 & 455 & 502 \\
0 & 178 & 846 & 2,074 & 2,074 & 1,002 \\
0 & 0 & 71 & 267 & 709 & 1,174 \\
4,866 & 5,977 & 7,624 & 9,926 & 12,283 & 13,669 \\
0 & 0 & 259 & 779 & 1,115 & 1,115 \\
210 & 328 & 719 & 779 & 779 & 779 \\
344 & 384 & 634 & 1,041 & 1,911 & 3,216 \\
196 & 215 & 411 & 782 & 1,501 & 2,195 \\
110 & 226 & 434 & 798 & 1,037 & 1,149 \\
0 & 0 & 39 & 119 & 364 & 1,109 \\
313 & 279 & 541 & 992 & 1,893 & 2,771 \\
0 & 0 & 95 & 369 & 612 & 647 \\
0 & 0 & 0 & 0 & 0 & 0 \\
0 & 0 & 199 & 601 & 1,408 & 1,546 \\
0 & 0 & 0 & 0 & 0 & 0 \\
0 & 0 & 35 & 35 & 35 & 35 \\
3,850 & 11,612 & 15,802 & 20,007 & 23,359 & 24,618 \\
600 & 2,568 & 2,755 & 3,357 & 5,291 & 8,013 \\
\hline $\mathbf{1 0 , 7 8 9}$ & $\mathbf{2 2 , 0 9 7}$ & $\mathbf{3 0 , 8 2 8}$ & $\mathbf{4 2 , 3 2 6}$ & $\mathbf{5 4 , 8 2 5}$ & $\mathbf{6 3 , 5 4 0}$ \\
\hline
\end{tabular}


World Biofuels Study

Table A-182: Reference case with E20 ethanol consumption [Million gallons]

\begin{tabular}{lrrrrrr} 
& $\mathbf{2 0 0 5}$ & $\mathbf{2 0 1 0}$ & $\mathbf{2 0 1 5}$ & $\mathbf{2 0 2 0}$ & $\mathbf{2 0 2 5}$ & $\mathbf{2 0 3 0}$ \\
Africa & 300 & 330 & 364 & 402 & 455 & 502 \\
Australia & 0 & 0 & 71 & 267 & 709 & 1,174 \\
Canada & 145 & 0 & 233 & 701 & 1,004 & 1,020 \\
China & 297 & 0 & 0 & 0 & 1,137 & 3,588 \\
Central and South America & 3,788 & 4,853 & 6,171 & 7,309 & 8,076 & 8,864 \\
Eastern Europe & 699 & 226 & 872 & 798 & 1,037 & 1,149 \\
Former Soviet Union & 0 & 0 & 39 & 119 & 364 & 1,109 \\
India & 313 & 279 & 541 & 942 & 1,893 & 2,399 \\
Japan & 263 & 288 & 373 & 719 & 1,008 & 1,100 \\
Middle East & 0 & 0 & 0 & 0 & 0 & 0 \\
Mexico & 0 & 0 & 30 & 78 & 183 & 1,053 \\
Other Asia & 0 & 0 & 0 & 0 & 0 & 0 \\
South Korea & 264 & 335 & 400 & 414 & 413 & 413 \\
United States & 3,984 & 11,612 & 17,430 & 25,368 & 30,898 & 32,237 \\
Western Europe & 736 & 4,174 & 4,304 & 5,208 & 7,649 & 8,933 \\
\hline Total & $\mathbf{1 0 , 7 8 9}$ & $\mathbf{2 2 , 0 9 7}$ & $\mathbf{3 0 , 8 2 8}$ & $\mathbf{4 2 , 3 2 6}$ & $\mathbf{5 4 , 8 2 5}$ & $\mathbf{6 3 , 5 4 0}$ \\
\hline \hline
\end{tabular}

\section{Table A-183: Reference case with E20 ethanol net trade [Million gallons]}

\begin{tabular}{lrrrrrr} 
& $\mathbf{2 0 0 5}$ & $\mathbf{2 0 1 0}$ & $\mathbf{2 0 1 5}$ & $\mathbf{2 0 2 0}$ & $\mathbf{2 0 2 5}$ & $\mathbf{2 0 3 0}$ \\
Africa & 0 & 0 & 0 & 0 & 0 & 0 \\
Australia & 0 & 0 & 0 & 0 & 0 & 0 \\
Canada & -145 & 0 & 26 & 78 & 112 & 96 \\
China & 47 & 384 & 634 & 1,041 & 774 & -372 \\
Central and South America & 1,484 & 1,845 & 3,429 & 6,251 & 8,561 & 8,782 \\
Eastern Europe & -589 & 0 & -438 & 0 & 0 & 0 \\
Former Soviet Union & 0 & 0 & 0 & 0 & 0 & 0 \\
India & 0 & 0 & 0 & 50 & 0 & 372 \\
Japan & -263 & -288 & -278 & -350 & -395 & -453 \\
Middle East & 0 & 0 & 0 & 0 & 0 & 0 \\
Mexico & 0 & 0 & 170 & 523 & 1,225 & 493 \\
Other Asia & 0 & 0 & 0 & 0 & 0 & 0 \\
South Korea & -264 & -335 & -365 & -379 & -378 & -378 \\
United States & -134 & 0 & $-1,628$ & $-5,361$ & $-7,539$ & $-7,620$ \\
Western Europe & -136 & $-1,607$ & $-1,549$ & $-1,851$ & $-2,358$ & -920 \\
\hline Total & $\mathbf{0}$ & $\mathbf{0}$ & $\mathbf{0}$ & $\mathbf{0}$ & $\mathbf{0}$ & $\mathbf{0}$ \\
\hline \hline
\end{tabular}


Table A-184: Reference case with E20 grain ethanol production [Million gallons]

\begin{tabular}{lrrrrrr} 
& $\mathbf{2 0 0 5}$ & $\mathbf{2 0 1 0}$ & $\mathbf{2 0 1 5}$ & $\mathbf{2 0 2 0}$ & $\mathbf{2 0 2 5}$ & $\mathbf{2 0 3 0}$ \\
Africa & 0 & 0 & 0 & 0 & 0 & 0 \\
Argentina & 0 & 178 & 640 & 1,260 & 1,260 & 188 \\
Australia & 0 & 0 & 0 & 0 & 0 & 0 \\
Brazil & 0 & 0 & 0 & 0 & 0 & 0 \\
Canada & 0 & 0 & 0 & 0 & 0 & 0 \\
Caribbean basin & 0 & 0 & 0 & 0 & 0 & 0 \\
China & 344 & 344 & 344 & 325 & 0 & 0 \\
Colombia & 0 & 0 & 0 & 0 & 0 & 0 \\
Eastern Europe & 110 & 226 & 348 & 477 & 367 & 251 \\
Former Soviet Union & 0 & 0 & 0 & 0 & 0 & 0 \\
India & 0 & 0 & 0 & 0 & 0 & 0 \\
Japan & 0 & 0 & 0 & 0 & 0 & 0 \\
Middle East & 0 & 0 & 0 & 0 & 0 & 0 \\
Mexico & 0 & 0 & 0 & 0 & 0 & 0 \\
Other Asia & 0 & 0 & 0 & 0 & 0 & 0 \\
South Korea & 0 & 0 & 0 & 0 & 0 & 0 \\
United States & 3,850 & 11,612 & 15,000 & 15,000 & 15,000 & 13,786 \\
Western Europe & 600 & 2,568 & 2,589 & 2,665 & 3,178 & 3,604 \\
\hline Total & $\mathbf{4 , 9 0 4}$ & $\mathbf{1 4 , 9 2 8}$ & $\mathbf{1 8 , 9 2 1}$ & $\mathbf{1 9 , 7 2 7}$ & $\mathbf{1 9 , 8 0 5}$ & $\mathbf{1 7 , 8 2 9}$ \\
\hline \hline
\end{tabular}

Table A-185: Reference case with E20 sugar ethanol production [Million gallons]

\begin{tabular}{lrrrrrr} 
& $\mathbf{2 0 0 5}$ & $\mathbf{2 0 1 0}$ & $\mathbf{2 0 1 5}$ & $\mathbf{2 0 2 0}$ & $\mathbf{2 0 2 5}$ & $\mathbf{2 0 3 0}$ \\
Africa & 300 & 330 & 364 & 402 & 444 & 490 \\
Argentina & 0 & 0 & 0 & 0 & 0 & 0 \\
Australia & 0 & 0 & 0 & 0 & 0 & 0 \\
Brazil & 4,866 & 5,977 & 7,329 & 9,036 & 10,406 & 11,793 \\
Canada & 0 & 0 & 0 & 0 & 0 & 0 \\
Caribbean basin & 210 & 328 & 719 & 779 & 779 & 779 \\
China & 0 & 40 & 48 & 48 & 48 & 48 \\
Colombia & 196 & 215 & 241 & 270 & 302 & 302 \\
Eastern Europe & 0 & 0 & 0 & 0 & 0 & 0 \\
Former Soviet Union & 0 & 0 & 0 & 0 & 0 & 0 \\
India & 313 & 279 & 327 & 347 & 381 & 348 \\
Japan & 0 & 0 & 0 & 0 & 0 & 0 \\
Middle East & 0 & 0 & 0 & 0 & 0 & 0 \\
Mexico & 0 & 0 & 0 & 0 & 0 & 0 \\
Other Asia & 0 & 0 & 0 & 0 & 0 & 0 \\
South Korea & 0 & 0 & 0 & 0 & 0 & 0 \\
United States & 0 & 0 & 0 & 0 & 0 & 0 \\
Western Europe & 0 & 0 & 0 & 0 & 0 & 0 \\
\hline Total & $\mathbf{5 , 8 8 4}$ & $\mathbf{7 , 1 6 9}$ & $\mathbf{9 , 0 2 8}$ & $\mathbf{1 0 , 8 8 2}$ & $\mathbf{1 2 , 3 6 1}$ & $\mathbf{1 3 , 7 6 0}$ \\
\hline \hline
\end{tabular}


Table A-186: Reference case with E20 cellulosic ethanol production [Million gallons]

\begin{tabular}{lrrrrrr} 
& $\mathbf{2 0 0 5}$ & $\mathbf{2 0 1 0}$ & $\mathbf{2 0 1 5}$ & $\mathbf{2 0 2 0}$ & $\mathbf{2 0 2 5}$ & $\mathbf{2 0 3 0}$ \\
Africa & 0 & 0 & 0 & 0 & 11 & 11 \\
Argentina & 0 & 0 & 206 & 814 & 814 & 814 \\
Australia & 0 & 0 & 71 & 267 & 709 & 1,174 \\
Brazil & 0 & 0 & 296 & 890 & 1,877 & 1,877 \\
Canada & 0 & 0 & 259 & 779 & 1,115 & 1,115 \\
Caribbean basin & 0 & 0 & 0 & 0 & 0 & 0 \\
China & 0 & 0 & 243 & 668 & 1,863 & 3,168 \\
Colombia & 0 & 0 & 170 & 512 & 1,199 & 1,893 \\
Eastern Europe & 0 & 0 & 85 & 320 & 670 & 897 \\
Former Soviet Union & 0 & 0 & 39 & 119 & 364 & 1,109 \\
India & 0 & 0 & 214 & 645 & 1,512 & 2,424 \\
Japan & 0 & 0 & 95 & 369 & 612 & 647 \\
Middle East & 0 & 0 & 0 & 0 & 0 & 0 \\
Mexico & 0 & 0 & 199 & 601 & 1,408 & 1,546 \\
Other Asia & 0 & 0 & 0 & 0 & 0 & 0 \\
South Korea & 0 & 0 & 35 & 35 & 35 & 35 \\
United States & 0 & 0 & 802 & 5,007 & 8,359 & 10,832 \\
Western Europe & 0 & 0 & 166 & 693 & 2,113 & 4,410 \\
\hline Total & $\mathbf{0}$ & $\mathbf{0}$ & $\mathbf{2 , 8 7 9}$ & $\mathbf{1 1 , 7 1 8}$ & $\mathbf{2 2 , 6 6 0}$ & $\mathbf{3 1 , 9 5 1}$ \\
\hline \hline
\end{tabular}

Table A-187: Reference case with E20 grain ethanol consumption [Million gallons]

\begin{tabular}{lrrrrrr} 
& $\mathbf{2 0 0 5}$ & $\mathbf{2 0 1 0}$ & $\mathbf{2 0 1 5}$ & $\mathbf{2 0 2 0}$ & $\mathbf{2 0 2 5}$ & $\mathbf{2 0 3 0}$ \\
Africa & 0 & 0 & 0 & 0 & 0 & 0 \\
Australia & 0 & 0 & 0 & 0 & 0 & 0 \\
Canada & 0 & 0 & 0 & 0 & 0 & 0 \\
China & 297 & 0 & 0 & 0 & 0 & 0 \\
Central and South America & 0 & 36 & 127 & 270 & 212 & 105 \\
Eastern Europe & 110 & 226 & 348 & 477 & 367 & 251 \\
Former Soviet Union & 0 & 0 & 0 & 0 & 0 & 0 \\
India & 0 & 0 & 0 & 0 & 0 & 0 \\
Japan & 0 & 192 & 230 & 303 & 0 & 83 \\
Middle East & 0 & 0 & 0 & 0 & 0 & 0 \\
Mexico & 0 & 0 & 0 & 0 & 0 & 0 \\
Other Asia & 0 & 0 & 0 & 0 & 0 & 0 \\
South Korea & 47 & 295 & 123 & 23 & 0 & 0 \\
United States & 3,850 & 11,612 & 15,000 & 15,000 & 15,000 & 13,786 \\
Western Europe & 600 & 2,568 & 3,093 & 3,655 & 4,226 & 3,604 \\
\hline Total & $\mathbf{4 , 9 0 4}$ & $\mathbf{1 4 , 9 2 8}$ & $\mathbf{1 8 , 9 2 1}$ & $\mathbf{1 9 , 7 2 7}$ & $\mathbf{1 9 , 8 0 5}$ & $\mathbf{1 7 , 8 2 9}$ \\
\hline \hline
\end{tabular}


Table A-188: Reference case with E20 sugar ethanol consumption [Million gallons]

\begin{tabular}{lrrrrrr} 
& $\mathbf{2 0 0 5}$ & $\mathbf{2 0 1 0}$ & $\mathbf{2 0 1 5}$ & $\mathbf{2 0 2 0}$ & $\mathbf{2 0 2 5}$ & $\mathbf{2 0 3 0}$ \\
Africa & 300 & 330 & 364 & 402 & 444 & 490 \\
Australia & 0 & 0 & 0 & 0 & 0 & 0 \\
Canada & 145 & 0 & 0 & 0 & 0 & 0 \\
China & 0 & 0 & 0 & 0 & 48 & 48 \\
Central and South America & 3,788 & 4,818 & 6,044 & 7,040 & 7,864 & 8,732 \\
Eastern Europe & 589 & 0 & 412 & 0 & 0 & 0 \\
Former Soviet Union & 0 & 0 & 0 & 0 & 0 & 0 \\
India & 313 & 279 & 327 & 347 & 381 & 348 \\
Japan & 263 & 96 & 48 & 48 & 0 & 0 \\
Middle East & 0 & 0 & 0 & 0 & 0 & 0 \\
Mexico & 0 & 0 & 0 & 0 & 0 & 0 \\
Other Asia & 0 & 0 & 0 & 0 & 0 & 0 \\
South Korea & 217 & 40 & 0 & 0 & 0 & 0 \\
United States & 134 & 0 & 788 & 2,753 & 3,150 & 4,143 \\
Western Europe & 136 & 1,607 & 1,045 & 293 & 474 & 0 \\
\hline Total & $\mathbf{5 , 8 8 4}$ & $\mathbf{7 , 1 6 9}$ & $\mathbf{9 , 0 2 8}$ & $\mathbf{1 0 , 8 8 2}$ & $\mathbf{1 2 , 3 6 1}$ & $\mathbf{1 3 , 7 6 0}$ \\
\hline \hline
\end{tabular}

Table A-189: Reference case with E20 cellulosic ethanol consumption [Million gallons]

\begin{tabular}{lrrrrrr} 
& $\mathbf{2 0 0 5}$ & $\mathbf{2 0 1 0}$ & $\mathbf{2 0 1 5}$ & $\mathbf{2 0 2 0}$ & $\mathbf{2 0 2 5}$ & $\mathbf{2 0 3 0}$ \\
Africa & 0 & 0 & 0 & 0 & 11 & 11 \\
Australia & 0 & 0 & 71 & 267 & 709 & 1,174 \\
Canada & 0 & 0 & 233 & 701 & 1,004 & 1,020 \\
China & 0 & 0 & 0 & 0 & 1,089 & 3,540 \\
Central and South America & 0 & 0 & 0 & 0 & 0 & 27 \\
Eastern Europe & 0 & 0 & 111 & 320 & 670 & 897 \\
Former Soviet Union & 0 & 0 & 39 & 119 & 364 & 1,109 \\
India & 0 & 0 & 214 & 596 & 1,512 & 2,051 \\
Japan & 0 & 0 & 95 & 369 & 1,008 & 1,016 \\
Middle East & 0 & 0 & 0 & 0 & 0 & 0 \\
Mexico & 0 & 0 & 30 & 78 & 183 & 1,053 \\
Other Asia & 0 & 0 & 0 & 0 & 0 & 0 \\
South Korea & 0 & 0 & 277 & 392 & 413 & 413 \\
United States & 0 & 0 & 1,643 & 7,615 & 12,748 & 14,309 \\
Western Europe & 0 & 0 & 166 & 1,261 & 2,949 & 5,330 \\
\hline Total & $\mathbf{0}$ & $\mathbf{0}$ & $\mathbf{2 , 8 7 9}$ & $\mathbf{1 1 , 7 1 8}$ & $\mathbf{2 2 , 6 6 0}$ & $\mathbf{3 1 , 9 5 1}$ \\
\hline \hline
\end{tabular}


Table A-190: Reference case with E20 grain ethanol net trade [Million gallons]

\begin{tabular}{lrrrrrr} 
& $\mathbf{2 0 0 5}$ & $\mathbf{2 0 1 0}$ & $\mathbf{2 0 1 5}$ & $\mathbf{2 0 2 0}$ & $\mathbf{2 0 2 5}$ & $\mathbf{2 0 3 0}$ \\
Africa & 0 & 0 & 0 & 0 & 0 & 0 \\
Australia & 0 & 0 & 0 & 0 & 0 & 0 \\
Canada & 0 & 0 & 0 & 0 & 0 & 0 \\
China & 47 & 344 & 344 & 325 & 0 & 0 \\
Central and South America & 0 & 143 & 513 & 990 & 1,048 & 83 \\
Eastern Europe & 0 & 0 & 0 & 0 & 0 & 0 \\
Former Soviet Union & 0 & 0 & 0 & 0 & 0 & 0 \\
India & 0 & 0 & 0 & 0 & 0 & 0 \\
Japan & 0 & -192 & -230 & -303 & 0 & -83 \\
Middle East & 0 & 0 & 0 & 0 & 0 & 0 \\
Mexico & 0 & 0 & 0 & 0 & 0 & 0 \\
Other Asia & 0 & 0 & 0 & 0 & 0 & 0 \\
South Korea & -47 & -295 & -123 & -23 & 0 & 0 \\
United States & 0 & 0 & 0 & 0 & 0 & 0 \\
Western Europe & 0 & 0 & -504 & -990 & $-1,048$ & 0 \\
\hline Total & $\mathbf{0}$ & $\mathbf{0}$ & $\mathbf{0}$ & $\mathbf{0}$ & $\mathbf{0}$ & $\mathbf{0}$ \\
\hline \hline
\end{tabular}

Table A-191: Reference case with E20 sugar ethanol net trade [Million gallons]

\begin{tabular}{lrrrrrr} 
& $\mathbf{2 0 0 5}$ & $\mathbf{2 0 1 0}$ & $\mathbf{2 0 1 5}$ & $\mathbf{2 0 2 0}$ & $\mathbf{2 0 2 5}$ & $\mathbf{2 0 3 0}$ \\
Africa & 0 & 0 & 0 & 0 & 0 & 0 \\
Australia & 0 & 0 & 0 & 0 & 0 & 0 \\
Canada & -145 & 0 & 0 & 0 & 0 & 0 \\
China & 0 & 40 & 48 & 48 & 0 & 0 \\
Central and South America & 1,484 & 1,702 & 2,245 & 3,045 & 3,624 & 4,143 \\
Eastern Europe & -589 & 0 & -412 & 0 & 0 & 0 \\
Former Soviet Union & 0 & 0 & 0 & 0 & 0 & 0 \\
India & 0 & 0 & 0 & 0 & 0 & 0 \\
Japan & -263 & -96 & -48 & -48 & 0 & 0 \\
Middle East & 0 & 0 & 0 & 0 & 0 & 0 \\
Mexico & 0 & 0 & 0 & 0 & 0 & 0 \\
Other Asia & 0 & 0 & 0 & 0 & 0 & 0 \\
South Korea & -217 & -40 & 0 & 0 & 0 & 0 \\
United States & -134 & 0 & -788 & $-2,753$ & $-3,150$ & $-4,143$ \\
Western Europe & -136 & $-1,607$ & $-1,045$ & -293 & -474 & 0 \\
\hline Total & $\mathbf{0}$ & $\mathbf{0}$ & $\mathbf{0}$ & $\mathbf{0}$ & $\mathbf{0}$ & $\mathbf{0}$ \\
\hline \hline
\end{tabular}


Table A-192: Reference case with E20 cellulosic ethanol net trade [Million gallons]

\begin{tabular}{lrrrrrr} 
& $\mathbf{2 0 0 5}$ & $\mathbf{2 0 1 0}$ & $\mathbf{2 0 1 5}$ & $\mathbf{2 0 2 0}$ & $\mathbf{2 0 2 5}$ & $\mathbf{2 0 3 0}$ \\
Africa & 0 & 0 & 0 & 0 & 0 & 0 \\
Australia & 0 & 0 & 0 & 0 & 0 & 0 \\
Canada & 0 & 0 & 26 & 78 & 112 & 96 \\
China & 0 & 0 & 243 & 668 & 774 & -372 \\
Central and South America & 0 & 0 & 671 & 2,216 & 3,890 & 4,556 \\
Eastern Europe & 0 & 0 & -26 & 0 & 0 & 0 \\
Former Soviet Union & 0 & 0 & 0 & 0 & 0 & 0 \\
India & 0 & 0 & 0 & 50 & 0 & 372 \\
Japan & 0 & 0 & 0 & 0 & -395 & -370 \\
Middle East & 0 & 0 & 0 & 0 & 0 & 0 \\
Mexico & 0 & 0 & 170 & 523 & 1,225 & 493 \\
Other Asia & 0 & 0 & 0 & 0 & 0 & 0 \\
South Korea & 0 & 0 & -243 & -357 & -378 & -378 \\
United States & 0 & 0 & -841 & $-2,608$ & $-4,389$ & $-3,477$ \\
Western Europe & 0 & 0 & 0 & -568 & -836 & -920 \\
\hline Total & $\mathbf{0}$ & $\mathbf{0}$ & $\mathbf{0}$ & $\mathbf{0}$ & $\mathbf{0}$ & $\mathbf{0}$ \\
\hline \hline
\end{tabular}

Table A-193: Reference case with E20 biomass-to-liquids production [Million gallons ethanol equivalent]

\begin{tabular}{lrrrrrr} 
& $\mathbf{2 0 0 5}$ & $\mathbf{2 0 1 0}$ & $\mathbf{2 0 1 5}$ & $\mathbf{2 0 2 0}$ & $\mathbf{2 0 2 5}$ & $\mathbf{2 0 3 0}$ \\
Africa & 0 & 0 & 68 & 263 & 653 & 1,177 \\
Argentina & 0 & 0 & 74 & 274 & 274 & 274 \\
Australia & 0 & 0 & 0 & 0 & 0 & 0 \\
Brazil & 0 & 0 & 106 & 320 & 675 & 675 \\
Canada & 0 & 0 & 93 & 280 & 401 & 401 \\
Caribbean basin & 0 & 0 & 0 & 0 & 0 & 0 \\
China & 0 & 0 & 57 & 240 & 240 & 240 \\
Colombia & 0 & 0 & 61 & 184 & 431 & 681 \\
Eastern Europe & 0 & 0 & 0 & 0 & 116 & 311 \\
Former Soviet Union & 0 & 0 & 0 & 0 & 0 & 0 \\
India & 0 & 0 & 77 & 232 & 544 & 544 \\
Japan & 0 & 0 & 0 & 0 & 0 & 0 \\
Middle East & 0 & 0 & 0 & 0 & 0 & 125 \\
Mexico & 0 & 0 & 72 & 216 & 506 & 556 \\
Other Asia & 0 & 0 & 89 & 448 & 1,090 & 1,942 \\
South Korea & 0 & 0 & 36 & 74 & 74 & 74 \\
United States & 0 & 0 & 822 & 1,299 & 1,908 & 2,481 \\
Western Europe & 0 & 0 & 0 & 0 & 0 & 0 \\
\hline Total & $\mathbf{0}$ & $\mathbf{0}$ & $\mathbf{1 , 5 5 5}$ & $\mathbf{3 , 8 3 0}$ & $\mathbf{6 , 9 1 2}$ & $\mathbf{9 , 4 8 1}$ \\
\hline \hline
\end{tabular}


Table A-194: Reference case with E20 biomass-to-liquids consumption [Million gallons ethanol equivalent]

\begin{tabular}{lrrrrrr} 
& $\mathbf{2 0 0 5}$ & $\mathbf{2 0 1 0}$ & $\mathbf{2 0 1 5}$ & $\mathbf{2 0 2 0}$ & $\mathbf{2 0 2 5}$ & $\mathbf{2 0 3 0}$ \\
Africa & 0 & 0 & 68 & 263 & 653 & 1,177 \\
Australia & 0 & 0 & 0 & 0 & 0 & 0 \\
Canada & 0 & 0 & 0 & 0 & 0 & 0 \\
China & 0 & 0 & 0 & 0 & 0 & 0 \\
Central and South America & 0 & 0 & 0 & 0 & 0 & 0 \\
Eastern Europe & 0 & 0 & 0 & 0 & 116 & 311 \\
Former Soviet Union & 0 & 0 & 0 & 0 & 0 & 0 \\
India & 0 & 0 & 0 & 0 & 0 & 0 \\
Japan & 0 & 0 & 0 & 0 & 0 & 0 \\
Middle East & 0 & 0 & 0 & 0 & 0 & 125 \\
Mexico & 0 & 0 & 0 & 0 & 0 & 0 \\
Other Asia & 0 & 0 & 89 & 449 & 1,090 & 1,942 \\
South Korea & 0 & 0 & 0 & 0 & 0 & 0 \\
United States & 0 & 0 & 1,110 & 2,885 & 4,785 & 5,926 \\
Western Europe & 0 & 0 & 288 & 235 & 268 & 0 \\
\hline Total & $\mathbf{0}$ & $\mathbf{0}$ & $\mathbf{1 , 5 5 5}$ & $\mathbf{3 , 8 3 0}$ & $\mathbf{6 , 9 1 1}$ & $\mathbf{9 , 4 8 1}$ \\
\hline \hline
\end{tabular}

Table A-195: Reference case with E20 biomass-to-liquids net trade [Million gallons ethanol equivalent]

\begin{tabular}{lrrrrrr} 
& $\mathbf{2 0 0 5}$ & $\mathbf{2 0 1 0}$ & $\mathbf{2 0 1 5}$ & $\mathbf{2 0 2 0}$ & $\mathbf{2 0 2 5}$ & $\mathbf{2 0 3 0}$ \\
Africa & 0 & 0 & 0 & 0 & 0 & 0 \\
Australia & 0 & 0 & 0 & 0 & 0 & 0 \\
Canada & 0 & 0 & 93 & 280 & 401 & 401 \\
China & 0 & 0 & 57 & 240 & 240 & 240 \\
Central and South America & 0 & 0 & 241 & 778 & 1,380 & 1,630 \\
Eastern Europe & 0 & 0 & 0 & 0 & 0 & 0 \\
Former Soviet Union & 0 & 0 & 0 & 0 & 0 & 0 \\
India & 0 & 0 & 77 & 232 & 544 & 544 \\
Japan & 0 & 0 & 0 & 0 & 0 & 0 \\
Middle East & 0 & 0 & 0 & 0 & 0 & 0 \\
Mexico & 0 & 0 & 72 & 216 & 506 & 556 \\
Other Asia & 0 & 0 & 0 & 0 & 0 & 0 \\
South Korea & 0 & 0 & 36 & 74 & 74 & 74 \\
United States & 0 & 0 & -288 & $-1,586$ & $-2,878$ & $-3,445$ \\
Western Europe & 0 & 0 & -288 & -235 & -268 & 0 \\
\hline Total & $\mathbf{0}$ & $\mathbf{0}$ & $\mathbf{0}$ & $\mathbf{0}$ & $\mathbf{0}$ & $\mathbf{0}$ \\
\hline \hline
\end{tabular}


Table A-196: Reference case with E20 biodiesel production [Million gallons ethanol equivalent]

\begin{tabular}{lrrrrrr} 
& $\mathbf{2 0 0 5}$ & $\mathbf{2 0 1 0}$ & $\mathbf{2 0 1 5}$ & $\mathbf{2 0 2 0}$ & $\mathbf{2 0 2 5}$ & $\mathbf{2 0 3 0}$ \\
Africa & 0 & 0 & 0 & 0 & 0 & 0 \\
Argentina & 0 & 333 & 776 & 819 & 827 & 239 \\
Australia & 0 & 0 & 0 & 0 & 0 & 0 \\
Brazil & 0 & 333 & 835 & 1,537 & 1,525 & 1,355 \\
Canada & 0 & 0 & 0 & 0 & 0 & 0 \\
Caribbean basin & 0 & 95 & 310 & 675 & 802 & 898 \\
China & 0 & 0 & 0 & 0 & 0 & 0 \\
Colombia & 0 & 119 & 298 & 474 & 650 & 782 \\
Eastern Europe & 0 & 0 & 0 & 0 & 0 & 0 \\
Former Soviet Union & 0 & 0 & 0 & 0 & 0 & 0 \\
India & 0 & 0 & 0 & 0 & 0 & 0 \\
Japan & 0 & 0 & 0 & 0 & 0 & 0 \\
Middle East & 0 & 0 & 0 & 0 & 0 & 0 \\
Mexico & 0 & 0 & 0 & 0 & 0 & 0 \\
Other Asia & 0 & 0 & 0 & 0 & 0 & 0 \\
South Korea & 0 & 0 & 0 & 0 & 0 & 0 \\
United States & 0 & 1,238 & 1,640 & 1,747 & 1,850 & 2,072 \\
Western Europe & 998 & 3,178 & 3,578 & 3,930 & 4,468 & 4,788 \\
\hline Total & $\mathbf{9 9 8}$ & $\mathbf{5 , 2 9 4}$ & $\mathbf{7 , 4 3 7}$ & $\mathbf{9 , 1 8 1}$ & $\mathbf{1 0 , 1 2 3}$ & $\mathbf{1 0 , 1 3 4}$ \\
\hline \hline
\end{tabular}

Table A-197: Reference case with E20 biodiesel consumption

[Million gallons ethanol equivalent]

\begin{tabular}{lrrrrrr} 
& $\mathbf{2 0 0 5}$ & $\mathbf{2 0 1 0}$ & $\mathbf{2 0 1 5}$ & $\mathbf{2 0 2 0}$ & $\mathbf{2 0 2 5}$ & $\mathbf{2 0 3 0}$ \\
Africa & 0 & 0 & 0 & 0 & 0 & 0 \\
Australia & 0 & 0 & 0 & 0 & 0 & 0 \\
Canada & 0 & 0 & 0 & 0 & 0 & 0 \\
China & 0 & 0 & 0 & 0 & 0 & 0 \\
Central and South America & 0 & 158 & 472 & 984 & 1,306 & 1,490 \\
Eastern Europe & 0 & 0 & 0 & 0 & 0 & 0 \\
Former Soviet Union & 0 & 0 & 0 & 0 & 0 & 0 \\
India & 0 & 0 & 0 & 0 & 0 & 0 \\
Japan & 0 & 0 & 0 & 0 & 0 & 0 \\
Middle East & 0 & 0 & 0 & 0 & 0 & 0 \\
Mexico & 0 & 0 & 0 & 0 & 0 & 0 \\
Other Asia & 0 & 0 & 0 & 0 & 0 & 0 \\
South Korea & 0 & 0 & 0 & 0 & 0 & 0 \\
United States & 0 & 1,238 & 1,713 & 1,747 & 1,850 & 2,072 \\
Western Europe & 998 & 3,898 & 5,252 & 6,451 & 6,967 & 6,572 \\
\hline Total & $\mathbf{9 9 8}$ & $\mathbf{5 , 2 9 4}$ & $\mathbf{7 , 4 3 7}$ & $\mathbf{9 , 1 8 1}$ & $\mathbf{1 0 , 1 2 3}$ & $\mathbf{1 0 , 1 3 4}$ \\
\hline \hline
\end{tabular}


Table A-198: Reference case with E20 biodiesel net trade [Million gallons ethanol equivalent]

\begin{tabular}{l} 
Africa \\
Australia \\
Canada \\
China \\
Central and South America \\
Eastern Europe \\
Former Soviet Union \\
India \\
Japan \\
Middle East \\
Mexico \\
Other Asia \\
South Korea \\
United States \\
Western Europe \\
\hline Total
\end{tabular}

\begin{tabular}{rrrrrr}
$\mathbf{2 0 0 5}$ & $\mathbf{2 0 1 0}$ & $\mathbf{2 0 1 5}$ & $\mathbf{2 0 2 0}$ & $\mathbf{2 0 2 5}$ & $\mathbf{2 0 3 0}$ \\
0 & 0 & 0 & 0 & 0 & 0 \\
0 & 0 & 0 & 0 & 0 & 0 \\
0 & 0 & 0 & 0 & 0 & 0 \\
0 & 0 & 0 & 0 & 0 & 0 \\
0 & 720 & 1,746 & 2,520 & 2,499 & 1,784 \\
0 & 0 & 0 & 0 & 0 & 0 \\
0 & 0 & 0 & 0 & 0 & 0 \\
0 & 0 & 0 & 0 & 0 & 0 \\
0 & 0 & 0 & 0 & 0 & 0 \\
0 & 0 & 0 & 0 & 0 & 0 \\
0 & 0 & 0 & 0 & 0 & 0 \\
0 & 0 & 0 & 0 & 0 & 0 \\
0 & 0 & 0 & 0 & 0 & 0 \\
0 & 0 & -73 & 0 & 0 & 0 \\
0 & -720 & $-1,674$ & $-2,520$ & $-2,499$ & $-1,784$ \\
\hline $\mathbf{0}$ & $\mathbf{0}$ & $\mathbf{0}$ & $\mathbf{0}$ & $\mathbf{0}$ & $\mathbf{0}$ \\
\hline
\end{tabular}




\section{A-12 Reference Case with $\$ 50$ per Tonne $\mathbf{C O}_{2}$}

\section{Table A-199: Reference case with $\$ 50$ per tonne $\mathrm{CO}_{2}$ ethanol production} [Million gallons]

\begin{tabular}{lrrrrrr} 
& $\mathbf{2 0 0 5}$ & $\mathbf{2 0 1 0}$ & $\mathbf{2 0 1 5}$ & $\mathbf{2 0 2 0}$ & $\mathbf{2 0 2 5}$ & $\mathbf{2 0 3 0}$ \\
Africa & 300 & 330 & 73 & 81 & 89 & 98 \\
Argentina & 0 & 178 & 810 & 1,366 & 2,481 & 2,481 \\
Australia & 0 & 0 & 71 & 246 & 583 & 1,172 \\
Brazil & 4,866 & 5,977 & 7,624 & 9,752 & 12,491 & 14,318 \\
Canada & 0 & 0 & 259 & 779 & 1,043 & 1,043 \\
Caribbean basin & 210 & 328 & 683 & 683 & 779 & 890 \\
China & 344 & 384 & 634 & 1,045 & 1,622 & 2,933 \\
Colombia & 196 & 215 & 411 & 782 & 1,501 & 2,231 \\
Eastern Europe & 110 & 226 & 434 & 751 & 1,037 & 1,149 \\
Former Soviet Union & 0 & 0 & 0 & 0 & 0 & 0 \\
India & 313 & 279 & 541 & 992 & 1,893 & 2,822 \\
Japan & 0 & 0 & 95 & 369 & 580 & 676 \\
Middle East & 0 & 0 & 0 & 0 & 0 & 0 \\
Mexico & 0 & 0 & 199 & 601 & 1,408 & 2,543 \\
Other Asia & 0 & 0 & 0 & 0 & 0 & 0 \\
South Korea & 0 & 0 & 30 & 30 & 30 & 30 \\
United States & 3,850 & 11,612 & 15,802 & 16,630 & 17,984 & 19,077 \\
Western Europe & 600 & 2,570 & 2,755 & 3,235 & 5,298 & 8,190 \\
\hline Total & $\mathbf{1 0 , 7 8 9}$ & $\mathbf{2 2 , 0 9 9}$ & $\mathbf{3 0 , 4 2 2}$ & $\mathbf{3 7 , 3 4 0}$ & $\mathbf{4 8 , 8 2 0}$ & $\mathbf{5 9 , 6 5 2}$ \\
\hline \hline
\end{tabular}


Table A-200: Reference case with $\$ 50$ per tonne $\mathrm{CO}_{2}$ ethanol consumption [Million gallons]

\begin{tabular}{lrrrrrr} 
& $\mathbf{2 0 0 5}$ & $\mathbf{2 0 1 0}$ & $\mathbf{2 0 1 5}$ & $\mathbf{2 0 2 0}$ & $\mathbf{2 0 2 5}$ & $\mathbf{2 0 3 0}$ \\
Africa & 300 & 330 & 73 & 81 & 89 & 98 \\
Australia & 0 & 0 & 71 & 246 & 583 & 1,172 \\
Canada & 145 & 0 & 233 & 779 & 938 & 938 \\
China & 118 & 0 & 0 & 274 & 1,726 & 5,063 \\
Central and South America & 3,788 & 4,853 & 6,163 & 7,212 & 8,116 & 9,022 \\
Eastern Europe & 699 & 226 & 872 & 953 & 1,037 & 1,149 \\
Former Soviet Union & 0 & 0 & 0 & 0 & 0 & 0 \\
India & 313 & 279 & 541 & 992 & 1,893 & 2,399 \\
Japan & 265 & 290 & 373 & 757 & 1,013 & 1,129 \\
Middle East & 0 & 0 & 0 & 0 & 0 & 0 \\
Mexico & 0 & 0 & 30 & 601 & 1,031 & 1,053 \\
Other Asia & 0 & 0 & 0 & 0 & 461 & 3,472 \\
South Korea & 264 & 335 & 400 & 414 & 413 & 413 \\
United States & $\mathbf{4} 161$ & 11,612 & 17,361 & 19,947 & 23,862 & 25,555 \\
Western Europe & 736 & 4,173 & 4,305 & 5,086 & 7,657 & 8,190 \\
\hline Total & $\mathbf{1 0 , 7 8 9}$ & $\mathbf{2 2 , 0 9 9}$ & $\mathbf{3 0 , 4 2 2}$ & $\mathbf{3 7 , 3 4 0}$ & $\mathbf{4 8 , 8 2 0}$ & $\mathbf{5 9 , 6 5 2}$ \\
\hline \hline
\end{tabular}

Table A-201: Reference case with $\$ 50$ per tonne $\mathrm{CO}_{2}$ ethanol net trade [Million gallons]

\begin{tabular}{lrrrrrr} 
Africa & 0 & 0 & 0 & 0 & 0 & 0 \\
Australia & 0 & 0 & 0 & 0 & 0 & 0 \\
Canada & -145 & 0 & 26 & 0 & 104 & 104 \\
China & 226 & 384 & 634 & 772 & -104 & $-2,130$ \\
Central and South America & 1,484 & 1,845 & 3,365 & 5,370 & 9,137 & 10,898 \\
Eastern Europe & -589 & 0 & -438 & -202 & 0 & 0 \\
Former Soviet Union & 0 & 0 & 0 & 0 & 0 & 0 \\
India & 0 & 0 & 0 & 0 & 0 & 423 \\
Japan & -265 & -290 & -278 & -388 & -433 & -453 \\
Middle East & 0 & 0 & 0 & 0 & 0 & 0 \\
Mexico & 0 & 0 & 170 & 0 & 377 & 1,490 \\
Other Asia & 0 & 0 & 0 & 0 & -461 & $-3,472$ \\
South Korea & -264 & -335 & -370 & -384 & -383 & -383 \\
United States & -311 & 0 & $-1,559$ & $-3,317$ & $-5,878$ & $-6,478$ \\
Western Europe & -136 & $-1,604$ & $-1,551$ & $-1,851$ & $-2,359$ & 0 \\
\hline Total & $\mathbf{0}$ & $\mathbf{0}$ & $\mathbf{0}$ & $\mathbf{0}$ & $\mathbf{0}$ & $\mathbf{0}$ \\
\hline \hline
\end{tabular}


Table A-202: Reference case with $\$ 50$ per tonne $\mathrm{CO}_{2}$ grain ethanol production [Million gallons]

\begin{tabular}{lrrrrrr} 
& $\mathbf{2 0 0 5}$ & $\mathbf{2 0 1 0}$ & $\mathbf{2 0 1 5}$ & $\mathbf{2 0 2 0}$ & $\mathbf{2 0 2 5}$ & $\mathbf{2 0 3 0}$ \\
Africa & 0 & 0 & 0 & 0 & 0 & 0 \\
Argentina & 0 & 178 & 604 & 604 & 604 & 604 \\
Australia & 0 & 0 & 0 & 0 & 0 & 0 \\
Brazil & 0 & 0 & 0 & 0 & 0 & 0 \\
Canada & 0 & 0 & 0 & 0 & 0 & 0 \\
Caribbean basin & 0 & 0 & 0 & 0 & 0 & 0 \\
China & 344 & 344 & 344 & 325 & 0 & 0 \\
Colombia & 0 & 0 & 0 & 0 & 0 & 0 \\
Eastern Europe & 110 & 226 & 348 & 477 & 367 & 251 \\
Former Soviet Union & 0 & 0 & 0 & 0 & 0 & 0 \\
India & 0 & 0 & 0 & 0 & 0 & 0 \\
Japan & 0 & 0 & 0 & 0 & 0 & 0 \\
Middle East & 0 & 0 & 0 & 0 & 0 & 0 \\
Mexico & 0 & 0 & 0 & 0 & 0 & 0 \\
Other Asia & 0 & 0 & 0 & 0 & 0 & 0 \\
South Korea & 0 & 0 & 0 & 0 & 0 & 0 \\
United States & 3,850 & 11,612 & 15,000 & 14,278 & 12,447 & 9,863 \\
Western Europe & 600 & 2,570 & 2,589 & 2,542 & 3,178 & 3,604 \\
\hline Total & $\mathbf{4 , 9 0 4}$ & $\mathbf{1 4 , 9 3 0}$ & $\mathbf{1 8 , 8 8 6}$ & $\mathbf{1 8 , 2 2 7}$ & $\mathbf{1 6 , 5 9 6}$ & $\mathbf{1 4 , 3 2 2}$ \\
\hline \hline
\end{tabular}

Table A-203: Reference case with $\$ 50$ per tonne $\mathrm{CO}_{2}$ sugar ethanol production [Million gallons]

\begin{tabular}{lrrrrrr} 
& $\mathbf{2 0 0 5}$ & $\mathbf{2 0 1 0}$ & $\mathbf{2 0 1 5}$ & $\mathbf{2 0 2 0}$ & $\mathbf{2 0 2 5}$ & $\mathbf{2 0 3 0}$ \\
Africa & 300 & 330 & 73 & 81 & 89 & 98 \\
Argentina & 0 & 0 & 0 & 0 & 0 & 0 \\
Australia & 0 & 0 & 0 & 0 & 0 & 0 \\
Brazil & 4,866 & 5,977 & 7,329 & 8,862 & 10,406 & 12,233 \\
Canada & 0 & 0 & 0 & 0 & 0 & 0 \\
Caribbean basin & 210 & 328 & 683 & 683 & 779 & 890 \\
China & 0 & 40 & 48 & 53 & 58 & 64 \\
Colombia & 196 & 215 & 241 & 270 & 302 & 339 \\
Eastern Europe & 0 & 0 & 0 & 0 & 0 & 0 \\
Former Soviet Union & 0 & 0 & 0 & 0 & 0 & 0 \\
India & 313 & 279 & 327 & 347 & 381 & 348 \\
Japan & 0 & 0 & 0 & 0 & 0 & 0 \\
Middle East & 0 & 0 & 0 & 0 & 0 & 0 \\
Mexico & 0 & 0 & 0 & 0 & 0 & 0 \\
Other Asia & 0 & 0 & 0 & 0 & 0 & 0 \\
South Korea & 0 & 0 & 0 & 0 & 0 & 0 \\
United States & 0 & 0 & 0 & 0 & 0 & 0 \\
Western Europe & 0 & 0 & 0 & 0 & 0 & 0 \\
\hline Total & $\mathbf{5 , 8 8 4}$ & $\mathbf{7 , 1 7 0}$ & $\mathbf{8 , 7 0 1}$ & $\mathbf{1 0 , 2 9 5}$ & $\mathbf{1 2 , 0 1 6}$ & $\mathbf{1 3 , 9 7 1}$ \\
\hline \hline
\end{tabular}


Table A-204: Reference case with $\$ 50$ per tonne $\mathrm{CO}_{2}$ cellulosic ethanol production [Million gallons]

\begin{tabular}{lrrrrrr} 
& $\mathbf{2 0 0 5}$ & $\mathbf{2 0 1 0}$ & $\mathbf{2 0 1 5}$ & $\mathbf{2 0 2 0}$ & $\mathbf{2 0 2 5}$ & $\mathbf{2 0 3 0}$ \\
Africa & 0 & 0 & 0 & 0 & 0 & 0 \\
Argentina & 0 & 0 & 206 & 762 & 1,877 & 1,877 \\
Australia & 0 & 0 & 71 & 246 & 583 & 1,172 \\
Brazil & 0 & 0 & 296 & 890 & 2,085 & 2,085 \\
Canada & 0 & 0 & 259 & 779 & 1,043 & 1,043 \\
Caribbean basin & 0 & 0 & 0 & 0 & 0 & 0 \\
China & 0 & 0 & 243 & 667 & 1,564 & 2,869 \\
Colombia & 0 & 0 & 170 & 512 & 1,199 & 1,893 \\
Eastern Europe & 0 & 0 & 85 & 274 & 670 & 897 \\
Former Soviet Union & 0 & 0 & 0 & 0 & 0 & 0 \\
India & 0 & 0 & 214 & 645 & 1,512 & 2,475 \\
Japan & 0 & 0 & 95 & 369 & 580 & 676 \\
Middle East & 0 & 0 & 0 & 0 & 0 & 0 \\
Mexico & 0 & 0 & 199 & 601 & 1,408 & 2,543 \\
Other Asia & 0 & 0 & 0 & 0 & 0 & 0 \\
South Korea & 0 & 0 & 30 & 30 & 30 & 30 \\
United States & 0 & 0 & 802 & 2,352 & 5,538 & 9,215 \\
Western Europe & 0 & 0 & 166 & 693 & 2,120 & 4,586 \\
\hline Total & $\mathbf{0}$ & $\mathbf{0}$ & $\mathbf{2 , 8 3 6}$ & $\mathbf{8 , 8 1 9}$ & $\mathbf{2 0 , 2 0 9}$ & $\mathbf{3 1 , 3 6 0}$ \\
\hline \hline
\end{tabular}

Table A-205: Reference case with $\$ 50$ per tonne $\mathrm{CO}_{2}$ grain ethanol consumption [Million gallons]

\begin{tabular}{lrrrrrr} 
& $\mathbf{2 0 0 5}$ & $\mathbf{2 0 1 0}$ & $\mathbf{2 0 1 5}$ & $\mathbf{2 0 2 0}$ & $\mathbf{2 0 2 5}$ & $\mathbf{2 0 3 0}$ \\
Africa & 0 & 0 & 0 & 0 & 0 & 0 \\
Australia & 0 & 0 & 0 & 0 & 0 & 0 \\
Canada & 0 & 0 & 0 & 0 & 0 & 0 \\
China & 118 & 0 & 0 & 0 & 0 & 152 \\
Central and South America & 0 & 36 & 122 & 178 & 222 & 0 \\
Eastern Europe & 110 & 226 & 348 & 477 & 367 & 251 \\
Former Soviet Union & 0 & 0 & 0 & 0 & 0 & 0 \\
India & 0 & 0 & 0 & 0 & 0 & 0 \\
Japan & 226 & 290 & 230 & 325 & 0 & 453 \\
Middle East & 0 & 0 & 0 & 0 & 0 & 0 \\
Mexico & 0 & 0 & 0 & 0 & 0 & 0 \\
Other Asia & 0 & 0 & 0 & 0 & 0 & 0 \\
South Korea & 0 & 196 & 127 & 0 & 383 & 0 \\
United States & 3,850 & 11,612 & 15,000 & 14,278 & 12,447 & 9,863 \\
Western Europe & 600 & 2,570 & 3,059 & 2,969 & 3,178 & 3,604 \\
\hline Total & $\mathbf{4 , 9 0 4}$ & $\mathbf{1 4 , 9 3 0}$ & $\mathbf{1 8 , 8 8 6}$ & $\mathbf{1 8 , 2 2 7}$ & $\mathbf{1 6 , 5 9 6}$ & $\mathbf{1 4 , 3 2 2}$ \\
\hline \hline
\end{tabular}


Table A-206: Reference case with $\$ 50$ per tonne $\mathrm{CO}_{2}$ sugar ethanol consumption [Million gallons]

\begin{tabular}{lrrrrrr} 
& $\mathbf{2 0 0 5}$ & $\mathbf{2 0 1 0}$ & $\mathbf{2 0 1 5}$ & $\mathbf{2 0 2 0}$ & $\mathbf{2 0 2 5}$ & $\mathbf{2 0 3 0}$ \\
Africa & 300 & 330 & 73 & 81 & 89 & 98 \\
Australia & 0 & 0 & 0 & 0 & 0 & 0 \\
Canada & 145 & 0 & 0 & 0 & 0 & 0 \\
China & 0 & 0 & 0 & 0 & 58 & 411 \\
Central and South America & 3,788 & 4,818 & 5,980 & 6,345 & 5,574 & 8,435 \\
Eastern Europe & 589 & 0 & 412 & 0 & 0 & 0 \\
Former Soviet Union & 0 & 0 & 0 & 0 & 0 & 0 \\
India & 313 & 279 & 327 & 347 & 381 & 0 \\
Japan & 39 & 0 & 48 & 53 & 0 & 0 \\
Middle East & 0 & 0 & 0 & 0 & 0 & 0 \\
Mexico & 0 & 0 & 0 & 0 & 0 & 0 \\
Other Asia & 0 & 0 & 0 & 0 & 0 & 1,741 \\
South Korea & 264 & 139 & 0 & 0 & 0 & 339 \\
United States & 311 & 0 & 788 & 3,317 & 5,612 & 2,946 \\
Western Europe & 136 & 1,604 & 1,073 & 153 & 302 & 0 \\
\hline Total & $\mathbf{5 , 8 8 4}$ & $\mathbf{7 , 1 7 0}$ & $\mathbf{8 , 7 0 1}$ & $\mathbf{1 0 , 2 9 5}$ & $\mathbf{1 2 , 0 1 6}$ & $\mathbf{1 3 , 9 7 1}$ \\
\hline \hline
\end{tabular}

Table A-207: Reference case with $\$ 50$ per tonne $\mathrm{CO}_{2}$ cellulosic ethanol consumption [Million gallons]

\begin{tabular}{lllrrrr} 
Africa & 0 & 0 & 0 & 0 & 0 & 0 \\
Australia & 0 & 0 & 71 & 246 & 583 & 1,172 \\
Canada & 0 & 0 & 233 & 779 & 938 & 938 \\
China & 0 & 0 & 0 & 274 & 1,668 & 4,501 \\
Central and South America & 0 & 0 & 62 & 689 & 2,321 & 587 \\
Eastern Europe & 0 & 0 & 111 & 476 & 670 & 897 \\
Former Soviet Union & 0 & 0 & 0 & 0 & 0 & 0 \\
India & 0 & 0 & 214 & 645 & 1,512 & 2,399 \\
Japan & 0 & 0 & 95 & 379 & 1,013 & 676 \\
Middle East & 0 & 0 & 0 & 0 & 0 & 0 \\
Mexico & 0 & 0 & 30 & 601 & 1,031 & 1,053 \\
Other Asia & 0 & 0 & 0 & 0 & 461 & 1,731 \\
South Korea & 0 & 0 & 273 & 414 & 30 & 74 \\
United States & 0 & 0 & 1,573 & 2,352 & 5,804 & 12,746 \\
Western Europe & 0 & 0 & 174 & 1,965 & 4,177 & 4,586 \\
\hline Total & $\mathbf{0}$ & $\mathbf{0}$ & $\mathbf{2 , 8 3 6}$ & $\mathbf{8 , 8 1 9}$ & $\mathbf{2 0 , 2 0 9}$ & $\mathbf{3 1 , 3 6 0}$ \\
\hline \hline
\end{tabular}


Table A-208: Reference case with $\$ 50$ per tonne $\mathrm{CO}_{2}$ grain ethanol net trade [Million gallons]

\begin{tabular}{lrrrrrr} 
& $\mathbf{2 0 0 5}$ & $\mathbf{2 0 1 0}$ & $\mathbf{2 0 1 5}$ & $\mathbf{2 0 2 0}$ & $\mathbf{2 0 2 5}$ & $\mathbf{2 0 3 0}$ \\
Africa & 0 & 0 & 0 & 0 & 0 & 0 \\
Australia & 0 & 0 & 0 & 0 & 0 & 0 \\
Canada & 0 & 0 & 0 & 0 & 0 & 0 \\
China & 226 & 344 & 344 & 325 & 0 & -152 \\
Central and South America & 0 & 143 & 483 & 427 & 383 & 604 \\
Eastern Europe & 0 & 0 & 0 & 0 & 0 & 0 \\
Former Soviet Union & 0 & 0 & 0 & 0 & 0 & 0 \\
India & 0 & 0 & 0 & 0 & 0 & 0 \\
Japan & -226 & -290 & -230 & -325 & 0 & -453 \\
Middle East & 0 & 0 & 0 & 0 & 0 & 0 \\
Mexico & 0 & 0 & 0 & 0 & 0 & 0 \\
Other Asia & 0 & 0 & 0 & 0 & 0 & 0 \\
South Korea & 0 & -196 & -127 & 0 & -383 & 0 \\
United States & 0 & 0 & 0 & 0 & 0 & 0 \\
Western Europe & 0 & 0 & -470 & -427 & 0 & 0 \\
\hline Total & $\mathbf{0}$ & $\mathbf{0}$ & $\mathbf{0}$ & $\mathbf{0}$ & $\mathbf{0}$ & $\mathbf{0}$ \\
\hline \hline
\end{tabular}

Table A-209: Reference case with $\$ 50$ per tonne $\mathrm{CO}_{2}$ sugar ethanol net trade [Million gallons]

\begin{tabular}{lrrrrrr} 
Africa & 0 & 0 & 0 & 0 & 0 & 0 \\
Australia & 0 & 0 & 0 & 0 & 0 & 0 \\
Canada & -145 & 0 & 0 & 0 & 0 & 0 \\
China & 0 & 40 & 48 & 53 & 0 & -348 \\
Central and South America & 1,484 & 1,702 & 2,273 & 3,470 & 5,914 & 5,026 \\
Eastern Europe & -589 & 0 & -412 & 0 & 0 & 0 \\
Former Soviet Union & 0 & 0 & 0 & 0 & 0 & 0 \\
India & 0 & 0 & 0 & 0 & 0 & 348 \\
Japan & -39 & 0 & -48 & -53 & 0 & 0 \\
Middle East & 0 & 0 & 0 & 0 & 0 & 0 \\
Mexico & 0 & 0 & 0 & 0 & 0 & 0 \\
Other Asia & 0 & 0 & 0 & 0 & 0 & $-1,741$ \\
South Korea & -264 & -139 & 0 & 0 & 0 & -339 \\
United States & -311 & 0 & -788 & $-3,317$ & $-5,612$ & $-2,946$ \\
Western Europe & -136 & $-1,604$ & $-1,073$ & -153 & -302 & 0 \\
\hline Total & $\mathbf{0}$ & $\mathbf{0}$ & $\mathbf{0}$ & $\mathbf{0}$ & $\mathbf{0}$ & $\mathbf{0}$ \\
\hline \hline
\end{tabular}


Table A-210: Reference case with $\$ 50$ per tonne $\mathrm{CO}_{2}$ cellulosic ethanol net trade [Million gallons]

\begin{tabular}{lrrrrrr} 
& $\mathbf{2 0 0 5}$ & $\mathbf{2 0 1 0}$ & $\mathbf{2 0 1 5}$ & $\mathbf{2 0 2 0}$ & $\mathbf{2 0 2 5}$ & $\mathbf{2 0 3 0}$ \\
Africa & 0 & 0 & 0 & 0 & 0 & 0 \\
Australia & 0 & 0 & 0 & 0 & 0 & 0 \\
Canada & 0 & 0 & 26 & 0 & 104 & 104 \\
China & 0 & 0 & 243 & 394 & -104 & $-1,631$ \\
Central and South America & 0 & 0 & 610 & 1,474 & 2,840 & 5,268 \\
Eastern Europe & 0 & 0 & -26 & -202 & 0 & 0 \\
Former Soviet Union & 0 & 0 & 0 & 0 & 0 & 0 \\
India & 0 & 0 & 0 & 0 & 0 & 76 \\
Japan & 0 & 0 & 0 & -10 & -433 & 0 \\
Middle East & 0 & 0 & 0 & 0 & 0 & 0 \\
Mexico & 0 & 0 & 170 & 0 & 377 & 1,490 \\
Other Asia & 0 & 0 & 0 & 0 & -461 & $-1,731$ \\
South Korea & 0 & 0 & -243 & -384 & 0 & -44 \\
United States & 0 & 0 & -771 & 0 & -266 & $-3,531$ \\
Western Europe & 0 & 0 & -8 & $-1,272$ & $-2,057$ & 0 \\
\hline Total & $\mathbf{0}$ & $\mathbf{0}$ & $\mathbf{0}$ & $\mathbf{0}$ & $\mathbf{0}$ & $\mathbf{0}$ \\
\hline \hline
\end{tabular}

Table A-211: Reference case with $\$ 50$ per tonne $\mathrm{CO}_{2}$ biomass-to-liquids production [Million gallons ethanol equivalent]

\begin{tabular}{lrrrrrr} 
& $\mathbf{2 0 0 5}$ & $\mathbf{2 0 1 0}$ & $\mathbf{2 0 1 5}$ & $\mathbf{2 0 2 0}$ & $\mathbf{2 0 2 5}$ & $\mathbf{2 0 3 0}$ \\
Africa & 0 & 0 & 68 & 313 & 880 & 1,403 \\
Argentina & 0 & 0 & 74 & 274 & 675 & 675 \\
Australia & 0 & 0 & 0 & 46 & 217 & 474 \\
Brazil & 0 & 0 & 106 & 320 & 750 & 750 \\
Canada & 0 & 0 & 93 & 280 & 375 & 375 \\
Caribbean basin & 0 & 0 & 0 & 0 & 0 & 0 \\
China & 0 & 0 & 57 & 240 & 563 & 563 \\
Colombia & 0 & 0 & 61 & 184 & 431 & 681 \\
Eastern Europe & 0 & 0 & 0 & 36 & 198 & 569 \\
Former Soviet Union & 0 & 0 & 0 & 0 & 23 & 163 \\
India & 0 & 0 & 77 & 232 & 544 & 890 \\
Japan & 0 & 0 & 0 & 0 & 0 & 0 \\
Middle East & 0 & 0 & 0 & 102 & 330 & 656 \\
Mexico & 0 & 0 & 72 & 216 & 506 & 915 \\
Other Asia & 0 & 0 & 96 & 554 & 1,489 & 2,773 \\
South Korea & 0 & 0 & 31 & 44 & 44 & 44 \\
United States & 0 & 0 & 822 & 5,018 & 6,654 & 8,742 \\
Western Europe & 0 & 0 & 0 & 0 & 0 & 0 \\
\hline Total & $\mathbf{0}$ & $\mathbf{0}$ & $\mathbf{1 , 5 5 7}$ & $\mathbf{7 , 8 5 9}$ & $\mathbf{1 3 , 6 7 9}$ & $\mathbf{1 9 , 6 7 3}$ \\
\hline \hline
\end{tabular}




\section{Table A-212: Reference case with $\$ 50$ per tonne $\mathrm{CO}_{2}$ biomass-to-liquids consumption [Million gallons ethanol equivalent]}

\begin{tabular}{l} 
Africa \\
Australia \\
Canada \\
China \\
Central and South America \\
Eastern Europe \\
Former Soviet Union \\
India \\
Japan \\
Middle East \\
Mexico \\
Other Asia \\
South Korea \\
United States \\
Western Europe \\
\hline Total \\
\hline \hline
\end{tabular}

\begin{tabular}{rrrrrr}
$\mathbf{2 0 0 5}$ & $\mathbf{2 0 1 0}$ & $\mathbf{2 0 1 5}$ & $\mathbf{2 0 2 0}$ & $\mathbf{2 0 2 5}$ & $\mathbf{2 0 3 0}$ \\
0 & 0 & 68 & 313 & 880 & 1,403 \\
0 & 0 & 0 & 46 & 217 & 474 \\
0 & 0 & 0 & 0 & 0 & 0 \\
0 & 0 & 0 & 0 & 0 & 0 \\
0 & 0 & 0 & 0 & 0 & 0 \\
0 & 0 & 0 & 36 & 198 & 569 \\
0 & 0 & 0 & 0 & 23 & 163 \\
0 & 0 & 0 & 0 & 0 & 0 \\
0 & 0 & 0 & 0 & 0 & 0 \\
0 & 0 & 0 & 102 & 330 & 656 \\
0 & 0 & 0 & 0 & 0 & 0 \\
0 & 0 & 96 & 554 & 1,489 & 2,773 \\
0 & 0 & 0 & 0 & 0 & 0 \\
0 & 0 & 1,108 & 6,314 & 10,542 & 12,664 \\
0 & 0 & 286 & 494 & 0 & 970 \\
\hline $\mathbf{0}$ & $\mathbf{0}$ & $\mathbf{1 , 5 5 7}$ & $\mathbf{7 , 8 5 9}$ & $\mathbf{1 3 , 6 7 9}$ & $\mathbf{1 9 , 6 7 3}$ \\
\hline
\end{tabular}

Table A-213: Reference case with $\$ 50$ per tonne $\mathrm{CO}_{2}$ biomass-to-liquids net trade [Million gallons ethanol equivalent]

\begin{tabular}{lrrrrrr} 
& $\mathbf{2 0 0 5}$ & $\mathbf{2 0 1 0}$ & $\mathbf{2 0 1 5}$ & $\mathbf{2 0 2 0}$ & $\mathbf{2 0 2 5}$ & $\mathbf{2 0 3 0}$ \\
Africa & 0 & 0 & 0 & 0 & 0 & 0 \\
Australia & 0 & 0 & 0 & 0 & 0 & 0 \\
Canada & 0 & 0 & 93 & 280 & 375 & 375 \\
China & 0 & 0 & 57 & 240 & 563 & 563 \\
Central and South America & 0 & 0 & 241 & 778 & 1,857 & 2,106 \\
Eastern Europe & 0 & 0 & 0 & 0 & 0 & 0 \\
Former Soviet Union & 0 & 0 & 0 & 0 & 0 & 0 \\
India & 0 & 0 & 77 & 232 & 544 & 890 \\
Japan & 0 & 0 & 0 & 0 & 0 & 0 \\
Middle East & 0 & 0 & 0 & 0 & 0 & 0 \\
Mexico & 0 & 0 & 72 & 216 & 506 & 915 \\
Other Asia & 0 & 0 & 0 & 0 & 0 & 0 \\
South Korea & 0 & 0 & 31 & 44 & 44 & 44 \\
United States & 0 & 0 & -286 & $-1,296$ & $-3,888$ & $-3,922$ \\
Western Europe & 0 & 0 & -286 & -494 & 0 & -970 \\
\hline Total & $\mathbf{0}$ & $\mathbf{0}$ & $\mathbf{0}$ & $\mathbf{0}$ & $\mathbf{0}$ & $\mathbf{0}$ \\
\hline \hline
\end{tabular}


Table A-214: Reference case with $\$ 50$ per tonne $\mathrm{CO}_{2}$ biodiesel production [Million gallons ethanol equivalent]

\begin{tabular}{lrrrrrr} 
& $\mathbf{2 0 0 5}$ & $\mathbf{2 0 1 0}$ & $\mathbf{2 0 1 5}$ & $\mathbf{2 0 2 0}$ & $\mathbf{2 0 2 5}$ & $\mathbf{2 0 3 0}$ \\
Africa & 0 & 0 & 0 & 0 & 0 & 0 \\
Argentina & 0 & 333 & 776 & 819 & 827 & 0 \\
Australia & 0 & 0 & 0 & 0 & 0 & 0 \\
Brazil & 0 & 333 & 835 & 1,537 & 1,785 & 1,367 \\
Canada & 0 & 0 & 0 & 0 & 0 & 0 \\
Caribbean basin & 0 & 95 & 310 & 675 & 802 & 898 \\
China & 0 & 0 & 0 & 0 & 0 & 0 \\
Colombia & 0 & 119 & 298 & 474 & 650 & 782 \\
Eastern Europe & 0 & 0 & 0 & 0 & 0 & 0 \\
Former Soviet Union & 0 & 0 & 0 & 0 & 0 & 0 \\
India & 0 & 0 & 0 & 0 & 0 & 0 \\
Japan & 0 & 0 & 0 & 0 & 0 & 0 \\
Middle East & 0 & 0 & 0 & 0 & 0 & 0 \\
Mexico & 0 & 0 & 0 & 0 & 0 & 0 \\
Other Asia & 0 & 0 & 0 & 0 & 0 & 0 \\
South Korea & 0 & 0 & 0 & 0 & 0 & 0 \\
United States & 0 & 1,238 & 1,640 & 1,747 & 1,907 & 2,016 \\
Western Europe & 998 & 3,178 & 3,578 & 4,175 & 4,566 & 5,191 \\
\hline Total & $\mathbf{9 9 8}$ & $\mathbf{5 , 2 9 4}$ & $\mathbf{7 , 4 3 7}$ & $\mathbf{9 , 4 2 6}$ & $\mathbf{1 0 , 5 3 7}$ & $\mathbf{1 0 , 2 5 3}$ \\
\hline \hline
\end{tabular}

Table A-215: Reference case with $\$ 50$ per tonne $\mathrm{CO}_{2}$ biodiesel consumption [Million gallons ethanol equivalent]

\begin{tabular}{lrrrrrr} 
& $\mathbf{2 0 0 5}$ & $\mathbf{2 0 1 0}$ & $\mathbf{2 0 1 5}$ & $\mathbf{2 0 2 0}$ & $\mathbf{2 0 2 5}$ & $\mathbf{2 0 3 0}$ \\
Africa & 0 & 0 & 0 & 0 & 0 & 0 \\
Australia & 0 & 0 & 0 & 0 & 0 & 0 \\
Canada & 0 & 0 & 0 & 0 & 0 & 0 \\
China & 0 & 0 & 0 & 0 & 0 & 0 \\
Central and South America & 0 & 158 & 472 & 984 & 1,306 & 1,490 \\
Eastern Europe & 0 & 0 & 0 & 0 & 0 & 0 \\
Former Soviet Union & 0 & 0 & 0 & 0 & 0 & 0 \\
India & 0 & 0 & 0 & 0 & 0 & 0 \\
Japan & 0 & 0 & 0 & 0 & 0 & 0 \\
Middle East & 0 & 0 & 0 & 0 & 0 & 0 \\
Mexico & 0 & 0 & 0 & 0 & 0 & 0 \\
Other Asia & 0 & 0 & 0 & 0 & 0 & 0 \\
South Korea & 0 & 0 & 0 & 0 & 0 & 0 \\
United States & 0 & 1,238 & 1,713 & 1,884 & 1,907 & 2,016 \\
Western Europe & 998 & 3,898 & 5,252 & 6,558 & 7,324 & 6,748 \\
\hline Total & $\mathbf{9 9 8}$ & $\mathbf{5 , 2 9 4}$ & $\mathbf{7 , 4 3 7}$ & $\mathbf{9 , 4 2 6}$ & $\mathbf{1 0 , 5 3 7}$ & $\mathbf{1 0 , 2 5 3}$ \\
\hline \hline
\end{tabular}




\section{Table A-216: Reference case with $\$ 50$ per tonne $\mathrm{CO}_{2}$ biodiesel net trade} [Million gallons ethanol equivalent]

\begin{tabular}{lrrrrrr} 
& $\mathbf{2 0 0 5}$ & $\mathbf{2 0 1 0}$ & $\mathbf{2 0 1 5}$ & $\mathbf{2 0 2 0}$ & $\mathbf{2 0 2 5}$ & $\mathbf{2 0 3 0}$ \\
Africa & 0 & 0 & 0 & 0 & 0 & 0 \\
Australia & 0 & 0 & 0 & 0 & 0 & 0 \\
Canada & 0 & 0 & 0 & 0 & 0 & 0 \\
China & 0 & 0 & 0 & 0 & 0 & 0 \\
Central and South America & 0 & 720 & 1,746 & 2,520 & 2,759 & 1,557 \\
Eastern Europe & 0 & 0 & 0 & 0 & 0 & 0 \\
Former Soviet Union & 0 & 0 & 0 & 0 & 0 & 0 \\
India & 0 & 0 & 0 & 0 & 0 & 0 \\
Japan & 0 & 0 & 0 & 0 & 0 & 0 \\
Middle East & 0 & 0 & 0 & 0 & 0 & 0 \\
Mexico & 0 & 0 & 0 & 0 & 0 & 0 \\
Other Asia & 0 & 0 & 0 & 0 & 0 & 0 \\
South Korea & 0 & 0 & 0 & 0 & 0 & 0 \\
United States & 0 & 0 & -73 & -137 & 0 & 0 \\
Western Europe & 0 & -720 & $-1,674$ & $-2,383$ & $-2,759$ & $-1,557$ \\
\hline Total & $\mathbf{0}$ & $\mathbf{0}$ & $\mathbf{0}$ & $\mathbf{0}$ & $\mathbf{0}$ & $\mathbf{0}$ \\
\hline \hline
\end{tabular}




\section{A-13 Reference Case with \$20 per Tonne Growers' Payment}

\section{Table A-217: Reference case with $\$ 20$ per tonne growers’ payment ethanol production [Million gallons]}

\begin{tabular}{lrrrrrr} 
& $\mathbf{2 0 0 5}$ & $\mathbf{2 0 1 0}$ & $\mathbf{2 0 1 5}$ & $\mathbf{2 0 2 0}$ & $\mathbf{2 0 2 5}$ & $\mathbf{2 0 3 0}$ \\
Africa & 300 & 330 & 364 & 402 & 455 & 502 \\
Argentina & 0 & 178 & 810 & 910 & 940 & 820 \\
Australia & 0 & 0 & 71 & 267 & 709 & 1,174 \\
Brazil & 4,866 & 5,977 & 7,624 & 9,752 & 11,934 & 13,669 \\
Canada & 0 & 0 & 259 & 779 & 1,115 & 1,115 \\
Caribbean basin & 210 & 328 & 573 & 683 & 707 & 707 \\
China & 344 & 384 & 634 & 1,041 & 1,612 & 2,917 \\
Colombia & 196 & 215 & 411 & 782 & 1,501 & 2,195 \\
Eastern Europe & 110 & 226 & 434 & 780 & 1,037 & 1,149 \\
Former Soviet Union & 0 & 0 & 39 & 119 & 364 & 1,109 \\
India & 313 & 279 & 541 & 992 & 1,893 & 2,771 \\
Japan & 0 & 0 & 95 & 369 & 612 & 647 \\
Middle East & 0 & 0 & 0 & 0 & 0 & 0 \\
Mexico & 0 & 0 & 199 & 601 & 1,408 & 1,546 \\
Other Asia & 0 & 0 & 0 & 0 & 0 & 0 \\
South Korea & 0 & 0 & 36 & 36 & 36 & 36 \\
United States & 3,850 & 11,612 & 15,802 & 17,258 & 18,946 & 19,034 \\
Western Europe & 600 & 2,568 & 2,755 & 3,398 & 5,291 & 8,013 \\
\hline Total & $\mathbf{1 0 , 7 8 9}$ & $\mathbf{2 2 , 0 9 7}$ & $\mathbf{3 0 , 6 4 7}$ & $\mathbf{3 8 , 1 6 7}$ & $\mathbf{4 8 , 5 6 0}$ & $\mathbf{5 7 , 4 0 4}$ \\
\hline \hline
\end{tabular}


Table A-218: Reference case with $\$ 20$ per tonne growers’ payment ethanol consumption [Million gallons]

\begin{tabular}{lrrrrrr} 
& $\mathbf{2 0 0 5}$ & $\mathbf{2 0 1 0}$ & $\mathbf{2 0 1 5}$ & $\mathbf{2 0 2 0}$ & $\mathbf{2 0 2 5}$ & $\mathbf{2 0 3 0}$ \\
Africa & 300 & 330 & 364 & 402 & 455 & 502 \\
Australia & 0 & 0 & 71 & 267 & 709 & 1,174 \\
Canada & 145 & 0 & 233 & 984 & 1,004 & 1,020 \\
China & 297 & 0 & 0 & 723 & 1,612 & 3,289 \\
Central and South America & 3,788 & 4,853 & 6,156 & 7,153 & 7,959 & 8,842 \\
Eastern Europe & 699 & 226 & 872 & 953 & 1,037 & 1,149 \\
Former Soviet Union & 0 & 0 & 39 & 119 & 364 & 1,109 \\
India & 313 & 279 & 541 & 992 & 1,893 & 2,399 \\
Japan & 263 & 288 & 373 & 719 & 1,008 & 1,100 \\
Middle East & 0 & 0 & 0 & 0 & 0 & 0 \\
Mexico & 0 & 0 & 30 & 396 & 896 & 1,053 \\
Other Asia & 0 & 0 & 0 & 0 & 0 & 0 \\
South Korea & 264 & 335 & 400 & 414 & 413 & 413 \\
United States & 3,984 & 11,612 & 17,287 & 19,797 & 23,562 & 27,343 \\
Western Europe & 736 & 4,174 & 4,281 & 5,249 & 7,650 & 8,013 \\
\hline Total & $\mathbf{1 0 , 7 8 9}$ & $\mathbf{2 2 , 0 9 7}$ & $\mathbf{3 0 , 6 4 7}$ & $\mathbf{3 8 , 1 6 7}$ & $\mathbf{4 8 , 5 6 0}$ & $\mathbf{5 7 , 4 0 4}$ \\
\hline \hline
\end{tabular}

Table A-219: Reference case with \$20 per tonne growers’ payment ethanol net trade [Million gallons]

\begin{tabular}{lrrrrrr} 
Africa & 0 & 0 & 0 & 0 & 0 & 0 \\
Australia & 0 & 0 & 0 & 0 & 0 & 0 \\
Canada & -145 & 0 & 26 & -205 & 112 & 96 \\
China & 47 & 384 & 634 & 318 & 0 & -372 \\
Central and South America & 1,484 & 1,845 & 3,261 & 4,974 & 7,124 & 8,550 \\
Eastern Europe & -589 & 0 & -438 & -173 & 0 & 0 \\
Former Soviet Union & 0 & 0 & 0 & 0 & 0 & 0 \\
India & 0 & 0 & 0 & 0 & 0 & 372 \\
Japan & -263 & -288 & -278 & -350 & -395 & -453 \\
Middle East & 0 & 0 & 0 & 0 & 0 & 0 \\
Mexico & 0 & 0 & 170 & 205 & 512 & 493 \\
Other Asia & 0 & 0 & 0 & 0 & 0 & 0 \\
South Korea & -264 & -335 & -364 & -378 & -377 & -377 \\
United States & -134 & 0 & $-1,485$ & $-2,538$ & $-4,616$ & $-8,309$ \\
Western Europe & -136 & $-1,607$ & $-1,526$ & $-1,851$ & $-2,359$ & 0 \\
\hline Total & $\mathbf{0}$ & $\mathbf{0}$ & $\mathbf{0}$ & $\mathbf{0}$ & $\mathbf{0}$ & $\mathbf{0}$ \\
\hline \hline
\end{tabular}


Table A-220: Reference case with \$20 per tonne growers' payment grain ethanol production [Million gallons]

\begin{tabular}{lrrrrrr} 
& $\mathbf{2 0 0 5}$ & $\mathbf{2 0 1 0}$ & $\mathbf{2 0 1 5}$ & $\mathbf{2 0 2 0}$ & $\mathbf{2 0 2 5}$ & $\mathbf{2 0 3 0}$ \\
Africa & 0 & 0 & 0 & 0 & 0 & 0 \\
Argentina & 0 & 178 & 604 & 604 & 604 & 473 \\
Australia & 0 & 0 & 0 & 0 & 0 & 0 \\
Brazil & 0 & 0 & 0 & 0 & 0 & 0 \\
Canada & 0 & 0 & 0 & 0 & 0 & 0 \\
Caribbean basin & 0 & 0 & 0 & 0 & 0 & 0 \\
China & 344 & 344 & 344 & 325 & 0 & 0 \\
Colombia & 0 & 0 & 0 & 0 & 0 & 0 \\
Eastern Europe & 110 & 226 & 348 & 477 & 367 & 251 \\
Former Soviet Union & 0 & 0 & 0 & 0 & 0 & 0 \\
India & 0 & 0 & 0 & 0 & 0 & 0 \\
Japan & 0 & 0 & 0 & 0 & 0 & 0 \\
Middle East & 0 & 0 & 0 & 0 & 0 & 0 \\
Mexico & 0 & 0 & 0 & 0 & 0 & 0 \\
Other Asia & 0 & 0 & 0 & 0 & 0 & 0 \\
South Korea & 0 & 0 & 0 & 0 & 0 & 0 \\
United States & 3,850 & 11,612 & 15,000 & 15,000 & 15,000 & 13,874 \\
Western Europe & 600 & 2,568 & 2,589 & 2,705 & 3,178 & 3,604 \\
\hline Total & $\mathbf{4 , 9 0 4}$ & $\mathbf{1 4 , 9 2 8}$ & $\mathbf{1 8 , 8 8 5}$ & $\mathbf{1 9 , 1 1 2}$ & $\mathbf{1 9 , 1 4 9}$ & $\mathbf{1 8 , 2 0 2}$ \\
\hline \hline
\end{tabular}

Table A-221: Reference case with \$20 per tonne growers’ payment sugar ethanol production [Million gallons]

\begin{tabular}{lrrrrrr} 
& $\mathbf{2 0 0 5}$ & $\mathbf{2 0 1 0}$ & $\mathbf{2 0 1 5}$ & $\mathbf{2 0 2 0}$ & $\mathbf{2 0 2 5}$ & $\mathbf{2 0 3 0}$ \\
Africa & 300 & 330 & 364 & 402 & 444 & 490 \\
Argentina & 0 & 0 & 0 & 0 & 0 & 0 \\
Australia & 0 & 0 & 0 & 0 & 0 & 0 \\
Brazil & 4,866 & 5,977 & 7,329 & 8,862 & 10,057 & 11,793 \\
Canada & 0 & 0 & 0 & 0 & 0 & 0 \\
Caribbean basin & 210 & 328 & 573 & 683 & 707 & 707 \\
China & 0 & 40 & 48 & 48 & 48 & 48 \\
Colombia & 196 & 215 & 241 & 270 & 302 & 302 \\
Eastern Europe & 0 & 0 & 0 & 0 & 0 & 0 \\
Former Soviet Union & 0 & 0 & 0 & 0 & 0 & 0 \\
India & 313 & 279 & 327 & 347 & 381 & 348 \\
Japan & 0 & 0 & 0 & 0 & 0 & 0 \\
Middle East & 0 & 0 & 0 & 0 & 0 & 0 \\
Mexico & 0 & 0 & 0 & 0 & 0 & 0 \\
Other Asia & 0 & 0 & 0 & 0 & 0 & 0 \\
South Korea & 0 & 0 & 0 & 0 & 0 & 0 \\
United States & 0 & 0 & 0 & 0 & 0 & 0 \\
Western Europe & 0 & 0 & 0 & 0 & 0 & 0 \\
\hline Total & $\mathbf{5 , 8 8 4}$ & $\mathbf{7 , 1 6 9}$ & $\mathbf{8 , 8 8 2}$ & $\mathbf{1 0 , 6 1 2}$ & $\mathbf{1 1 , 9 4 0}$ & $\mathbf{1 3 , 6 8 8}$ \\
\hline \hline
\end{tabular}


Table A-222: Reference case with \$20 per tonne growers' payment cellulosic ethanol production [Million gallons]

\begin{tabular}{lrrrrrr} 
& $\mathbf{2 0 0 5}$ & $\mathbf{2 0 1 0}$ & $\mathbf{2 0 1 5}$ & $\mathbf{2 0 2 0}$ & $\mathbf{2 0 2 5}$ & $\mathbf{2 0 3 0}$ \\
Africa & 0 & 0 & 0 & 0 & 11 & 11 \\
Argentina & 0 & 0 & 206 & 306 & 335 & 347 \\
Australia & 0 & 0 & 71 & 267 & 709 & 1,174 \\
Brazil & 0 & 0 & 296 & 890 & 1,877 & 1,876 \\
Canada & 0 & 0 & 259 & 779 & 1,115 & 1,115 \\
Caribbean basin & 0 & 0 & 0 & 0 & 0 & 0 \\
China & 0 & 0 & 243 & 668 & 1,564 & 2,869 \\
Colombia & 0 & 0 & 170 & 512 & 1,199 & 1,893 \\
Eastern Europe & 0 & 0 & 85 & 303 & 670 & 897 \\
Former Soviet Union & 0 & 0 & 39 & 119 & 364 & 1,109 \\
India & 0 & 0 & 214 & 645 & 1,512 & 2,424 \\
Japan & 0 & 0 & 95 & 369 & 612 & 647 \\
Middle East & 0 & 0 & 0 & 0 & 0 & 0 \\
Mexico & 0 & 0 & 199 & 601 & 1,408 & 1,546 \\
Other Asia & 0 & 0 & 0 & 0 & 0 & 0 \\
South Korea & 0 & 0 & 36 & 36 & 36 & 36 \\
United States & 0 & 0 & 802 & 2,258 & 3,946 & 5,160 \\
Western Europe & 0 & 0 & 166 & 693 & 2,113 & 4,410 \\
\hline Total & $\mathbf{0}$ & $\mathbf{0}$ & $\mathbf{2 , 8 8 0}$ & $\mathbf{8 , 4 4 4}$ & $\mathbf{1 7 , 4 7 1}$ & $\mathbf{2 5 , 5 1 4}$ \\
\hline \hline
\end{tabular}

Table A-223: Reference case with $\$ 20$ per tonne growers’ payment grain ethanol consumption [Million gallons]

\begin{tabular}{lrrrrrr} 
& $\mathbf{2 0 0 5}$ & $\mathbf{2 0 1 0}$ & $\mathbf{2 0 1 5}$ & $\mathbf{2 0 2 0}$ & $\mathbf{2 0 2 5}$ & $\mathbf{2 0 3 0}$ \\
Africa & 0 & 0 & 0 & 0 & 0 & 0 \\
Australia & 0 & 0 & 0 & 0 & 0 & 0 \\
Canada & 0 & 0 & 0 & 0 & 0 & 0 \\
China & 297 & 0 & 0 & 325 & 0 & 0 \\
Central and South America & 0 & 36 & 122 & 118 & 98 & 86 \\
Eastern Europe & 110 & 226 & 348 & 477 & 367 & 251 \\
Former Soviet Union & 0 & 0 & 0 & 0 & 0 & 0 \\
India & 0 & 0 & 0 & 0 & 0 & 0 \\
Japan & 0 & 192 & 230 & 350 & 284 & 357 \\
Middle East & 0 & 0 & 0 & 0 & 0 & 0 \\
Mexico & 0 & 0 & 0 & 0 & 0 & 0 \\
Other Asia & 0 & 0 & 0 & 0 & 0 & 0 \\
South Korea & 47 & 295 & 122 & 61 & 222 & 30 \\
United States & 3,850 & 11,612 & 15,000 & 15,000 & 15,000 & 13,874 \\
Western Europe & 600 & 2,568 & 3,064 & 2,780 & 3,178 & 3,604 \\
\hline Total & $\mathbf{4 , 9 0 4}$ & $\mathbf{1 4 , 9 2 8}$ & $\mathbf{1 8 , 8 8 5}$ & $\mathbf{1 9 , 1 1 2}$ & $\mathbf{1 9 , 1 4 9}$ & $\mathbf{1 8 , 2 0 2}$ \\
\hline \hline
\end{tabular}


Table A-224: Reference case with $\$ 20$ per tonne growers’ payment sugar ethanol consumption [Million gallons]

\begin{tabular}{lrrrrrr} 
& $\mathbf{2 0 0 5}$ & $\mathbf{2 0 1 0}$ & $\mathbf{2 0 1 5}$ & $\mathbf{2 0 2 0}$ & $\mathbf{2 0 2 5}$ & $\mathbf{2 0 3 0}$ \\
Africa & 300 & 330 & 364 & 402 & 444 & 490 \\
Australia & 0 & 0 & 0 & 0 & 0 & 0 \\
Canada & 145 & 0 & 0 & 0 & 0 & 0 \\
China & 0 & 0 & 0 & 48 & 48 & 48 \\
Central and South America & 3,788 & 4,818 & 6,035 & 7,014 & 7,860 & 8,692 \\
Eastern Europe & 589 & 0 & 412 & 173 & 0 & 0 \\
Former Soviet Union & 0 & 0 & 0 & 0 & 0 & 0 \\
India & 313 & 279 & 327 & 347 & 381 & 348 \\
Japan & 263 & 96 & 48 & 0 & 0 & 0 \\
Middle East & 0 & 0 & 0 & 0 & 0 & 0 \\
Mexico & 0 & 0 & 0 & 0 & 0 & 0 \\
Other Asia & 0 & 0 & 0 & 0 & 0 & 0 \\
South Korea & 217 & 40 & 0 & 0 & 0 & 0 \\
United States & 134 & 0 & 788 & 2,538 & 2,920 & 4,110 \\
Western Europe & 136 & 1,607 & 908 & 89 & 287 & 0 \\
\hline Total & $\mathbf{5 , 8 8 4}$ & $\mathbf{7 , 1 6 9}$ & $\mathbf{8 , 8 8 2}$ & $\mathbf{1 0 , 6 1 2}$ & $\mathbf{1 1 , 9 4 0}$ & $\mathbf{1 3 , 6 8 8}$ \\
\hline \hline
\end{tabular}

Table A-225: Reference case with $\$ 20$ per tonne growers’ payment cellulosic ethanol consumption [Million gallons]

\begin{tabular}{lllrrrr} 
Africa & 0 & 0 & 0 & 0 & 11 & 11 \\
Australia & 0 & 0 & 71 & 267 & 709 & 1,174 \\
Canada & 0 & 0 & 233 & 984 & 1,004 & 1,020 \\
China & 0 & 0 & 0 & 350 & 1,564 & 3,241 \\
Central and South America & 0 & 0 & 0 & 21 & 0 & 63 \\
Eastern Europe & 0 & 0 & 111 & 303 & 670 & 897 \\
Former Soviet Union & 0 & 0 & 39 & 119 & 364 & 1,109 \\
India & 0 & 0 & 214 & 645 & 1,512 & 2,051 \\
Japan & 0 & 0 & 95 & 369 & 724 & 743 \\
Middle East & 0 & 0 & 0 & 0 & 0 & 0 \\
Mexico & 0 & 0 & 30 & 396 & 896 & 1,053 \\
Other Asia & 0 & 0 & 0 & 0 & 0 & 0 \\
South Korea & 0 & 0 & 279 & 354 & 191 & 383 \\
United States & 0 & 0 & 1,500 & 2,258 & 5,642 & 9,359 \\
Western Europe & 0 & 0 & 309 & 2,379 & 4,185 & 4,410 \\
\hline Total & $\mathbf{0}$ & $\mathbf{0}$ & $\mathbf{2 , 8 8 0}$ & $\mathbf{8 , 4 4 4}$ & $\mathbf{1 7 , 4 7 1}$ & $\mathbf{2 5 , 5 1 4}$ \\
\hline \hline
\end{tabular}


Table A-226: Reference case with \$20 per tonne growers’ payment grain ethanol net trade [Million gallons]

\begin{tabular}{lrrrrrr} 
& $\mathbf{2 0 0 5}$ & $\mathbf{2 0 1 0}$ & $\mathbf{2 0 1 5}$ & $\mathbf{2 0 2 0}$ & $\mathbf{2 0 2 5}$ & $\mathbf{2 0 3 0}$ \\
Africa & 0 & 0 & 0 & 0 & 0 & 0 \\
Australia & 0 & 0 & 0 & 0 & 0 & 0 \\
Canada & 0 & 0 & 0 & 0 & 0 & 0 \\
China & 47 & 344 & 344 & 0 & 0 & 0 \\
Central and South America & 0 & 143 & 483 & 486 & 506 & 387 \\
Eastern Europe & 0 & 0 & 0 & 0 & 0 & 0 \\
Former Soviet Union & 0 & 0 & 0 & 0 & 0 & 0 \\
India & 0 & 0 & 0 & 0 & 0 & 0 \\
Japan & 0 & -192 & -230 & -350 & -284 & -357 \\
Middle East & 0 & 0 & 0 & 0 & 0 & 0 \\
Mexico & 0 & 0 & 0 & 0 & 0 & 0 \\
Other Asia & 0 & 0 & 0 & 0 & 0 & 0 \\
South Korea & -47 & -295 & -122 & -61 & -222 & -30 \\
United States & 0 & 0 & 0 & 0 & 0 & 0 \\
Western Europe & 0 & 0 & -476 & -75 & 0 & 0 \\
\hline Total & $\mathbf{0}$ & $\mathbf{0}$ & $\mathbf{0}$ & $\mathbf{0}$ & $\mathbf{0}$ & $\mathbf{0}$ \\
\hline \hline
\end{tabular}

Table A-227: Reference case with \$20 per tonne growers’ payment sugar ethanol net trade [Million gallons]

\begin{tabular}{lrrrrrr} 
Africa & 0 & 0 & 0 & 0 & 0 & 0 \\
Australia & 0 & 0 & 0 & 0 & 0 & 0 \\
Canada & -145 & 0 & 0 & 0 & 0 & 0 \\
China & 0 & 40 & 48 & 0 & 0 & 0 \\
Central and South America & 1,484 & 1,702 & 2,108 & 2,801 & 3,207 & 4,110 \\
Eastern Europe & -589 & 0 & -412 & -173 & 0 & 0 \\
Former Soviet Union & 0 & 0 & 0 & 0 & 0 & 0 \\
India & 0 & 0 & 0 & 0 & 0 & 0 \\
Japan & -263 & -96 & -48 & 0 & 0 & 0 \\
Middle East & 0 & 0 & 0 & 0 & 0 & 0 \\
Mexico & 0 & 0 & 0 & 0 & 0 & 0 \\
Other Asia & 0 & 0 & 0 & 0 & 0 & 0 \\
South Korea & -217 & -40 & 0 & 0 & 0 & 0 \\
United States & -134 & 0 & -788 & $-2,538$ & $-2,920$ & $-4,110$ \\
Western Europe & -136 & $-1,607$ & -908 & -89 & -287 & 0 \\
\hline Total & $\mathbf{0}$ & $\mathbf{0}$ & $\mathbf{0}$ & $\mathbf{0}$ & $\mathbf{0}$ & $\mathbf{0}$ \\
\hline \hline
\end{tabular}


Table A-228: Reference case with \$20 per tonne growers’ payment cellulosic ethanol net trade [Million gallons]

\begin{tabular}{lrrrrrr} 
& $\mathbf{2 0 0 5}$ & $\mathbf{2 0 1 0}$ & $\mathbf{2 0 1 5}$ & $\mathbf{2 0 2 0}$ & $\mathbf{2 0 2 5}$ & $\mathbf{2 0 3 0}$ \\
Africa & 0 & 0 & 0 & 0 & 0 & 0 \\
Australia & 0 & 0 & 0 & 0 & 0 & 0 \\
Canada & 0 & 0 & 26 & -205 & 112 & 96 \\
China & 0 & 0 & 243 & 318 & 0 & -372 \\
Central and South America & 0 & 0 & 671 & 1,687 & 3,411 & 4,054 \\
Eastern Europe & 0 & 0 & -26 & 0 & 0 & 0 \\
Former Soviet Union & 0 & 0 & 0 & 0 & 0 & 0 \\
India & 0 & 0 & 0 & 0 & 0 & 372 \\
Japan & 0 & 0 & 0 & 0 & -112 & -96 \\
Middle East & 0 & 0 & 0 & 0 & 0 & 0 \\
Mexico & 0 & 0 & 170 & 205 & 512 & 493 \\
Other Asia & 0 & 0 & 0 & 0 & 0 & 0 \\
South Korea & 0 & 0 & -243 & -318 & -155 & -347 \\
United States & 0 & 0 & -698 & 0 & $-1,696$ & $-4,199$ \\
Western Europe & 0 & 0 & -143 & $-1,687$ & $-2,072$ & 0 \\
\hline Total & $\mathbf{0}$ & $\mathbf{0}$ & $\mathbf{0}$ & $\mathbf{0}$ & $\mathbf{0}$ & $\mathbf{0}$ \\
\hline \hline
\end{tabular}

Table A-229: Reference case with \$20 per tonne growers’ payment biomass-toliquids production [Million gallons ethanol equivalent]

\begin{tabular}{lrrrrrr} 
& $\mathbf{2 0 0 5}$ & $\mathbf{2 0 1 0}$ & $\mathbf{2 0 1 5}$ & $\mathbf{2 0 2 0}$ & $\mathbf{2 0 2 5}$ & $\mathbf{2 0 3 0}$ \\
Africa & 0 & 0 & 68 & 263 & 653 & 1,177 \\
Argentina & 0 & 0 & 74 & 110 & 121 & 125 \\
Australia & 0 & 0 & 0 & 0 & 0 & 0 \\
Brazil & 0 & 0 & 106 & 320 & 675 & 675 \\
Canada & 0 & 0 & 93 & 280 & 401 & 401 \\
Caribbean basin & 0 & 0 & 0 & 0 & 0 & 0 \\
China & 0 & 0 & 57 & 240 & 563 & 563 \\
Colombia & 0 & 0 & 61 & 184 & 431 & 681 \\
Eastern Europe & 0 & 0 & 0 & 0 & 93 & 274 \\
Former Soviet Union & 0 & 0 & 0 & 0 & 0 & 0 \\
India & 0 & 0 & 77 & 232 & 544 & 544 \\
Japan & 0 & 0 & 0 & 0 & 0 & 0 \\
Middle East & 0 & 0 & 0 & 0 & 0 & 125 \\
Mexico & 0 & 0 & 72 & 216 & 506 & 556 \\
Other Asia & 0 & 0 & 89 & 448 & 1,090 & 1,942 \\
South Korea & 0 & 0 & 37 & 77 & 77 & 77 \\
United States & 0 & 0 & 822 & 4,818 & 6,399 & 8,213 \\
Western Europe & 0 & 0 & 0 & 0 & 0 & 0 \\
\hline Total & $\mathbf{0}$ & $\mathbf{0}$ & $\mathbf{1 , 5 5 6}$ & $\mathbf{7 , 1 8 8}$ & $\mathbf{1 1 , 5 5 2}$ & $\mathbf{1 5 , 3 5 2}$ \\
\hline \hline
\end{tabular}


Table A-230: Reference case with \$20 per tonne growers’ payment biomass-toliquids consumption [Million gallons ethanol equivalent]

\begin{tabular}{lrrrrrr} 
& $\mathbf{2 0 0 5}$ & $\mathbf{2 0 1 0}$ & $\mathbf{2 0 1 5}$ & $\mathbf{2 0 2 0}$ & $\mathbf{2 0 2 5}$ & $\mathbf{2 0 3 0}$ \\
Africa & 0 & 0 & 68 & 263 & 653 & 1,177 \\
Australia & 0 & 0 & 0 & 0 & 0 & 0 \\
Canada & 0 & 0 & 0 & 0 & 0 & 0 \\
China & 0 & 0 & 0 & 0 & 0 & 0 \\
Central and South America & 0 & 0 & 0 & 0 & 0 & 0 \\
Eastern Europe & 0 & 0 & 0 & 0 & 93 & 274 \\
Former Soviet Union & 0 & 0 & 0 & 0 & 0 & 0 \\
India & 0 & 0 & 0 & 0 & 0 & 0 \\
Japan & 0 & 0 & 0 & 0 & 0 & 0 \\
Middle East & 0 & 0 & 0 & 0 & 0 & 125 \\
Mexico & 0 & 0 & 0 & 0 & 0 & 0 \\
Other Asia & 0 & 0 & 89 & 449 & 1,090 & 1,942 \\
South Korea & 0 & 0 & 0 & 0 & 0 & 0 \\
United States & 0 & 0 & 1,111 & 6,195 & 9,717 & 10,877 \\
Western Europe & 0 & 0 & 289 & 282 & 0 & 957 \\
\hline Total & $\mathbf{0}$ & $\mathbf{0}$ & $\mathbf{1 , 5 5 6}$ & $\mathbf{7 , 1 8 8}$ & $\mathbf{1 1 , 5 5 2}$ & $\mathbf{1 5 , 3 5 2}$ \\
\hline \hline
\end{tabular}

Table A-231: Reference case with \$20 per tonne growers’ payment biomass-toliquids net trade [Million gallons ethanol equivalent]

\begin{tabular}{lrrrrrr} 
& $\mathbf{2 0 0 5}$ & $\mathbf{2 0 1 0}$ & $\mathbf{2 0 1 5}$ & $\mathbf{2 0 2 0}$ & $\mathbf{2 0 2 5}$ & $\mathbf{2 0 3 0}$ \\
Africa & 0 & 0 & 0 & 0 & 0 & 0 \\
Australia & 0 & 0 & 0 & 0 & 0 & 0 \\
Canada & 0 & 0 & 93 & 280 & 401 & 401 \\
China & 0 & 0 & 57 & 240 & 563 & 563 \\
Central and South America & 0 & 0 & 241 & 614 & 1,227 & 1,481 \\
Eastern Europe & 0 & 0 & 0 & 0 & 0 & 0 \\
Former Soviet Union & 0 & 0 & 0 & 0 & 0 & 0 \\
India & 0 & 0 & 77 & 232 & 544 & 544 \\
Japan & 0 & 0 & 0 & 0 & 0 & 0 \\
Middle East & 0 & 0 & 0 & 0 & 0 & 0 \\
Mexico & 0 & 0 & 72 & 216 & 506 & 556 \\
Other Asia & 0 & 0 & 0 & 0 & 0 & 0 \\
South Korea & 0 & 0 & 37 & 77 & 77 & 77 \\
United States & 0 & 0 & -289 & $-1,378$ & $-3,318$ & $-2,664$ \\
Western Europe & 0 & 0 & -289 & -282 & 0 & -957 \\
\hline Total & $\mathbf{0}$ & $\mathbf{0}$ & $\mathbf{0}$ & $\mathbf{0}$ & $\mathbf{0}$ & $\mathbf{0}$ \\
\hline \hline
\end{tabular}


Table A-232: Reference case with $\$ 20$ per tonne growers' payment biodiesel production [Million gallons ethanol equivalent]

\begin{tabular}{lrrrrrr} 
& $\mathbf{2 0 0 5}$ & $\mathbf{2 0 1 0}$ & $\mathbf{2 0 1 5}$ & $\mathbf{2 0 2 0}$ & $\mathbf{2 0 2 5}$ & $\mathbf{2 0 3 0}$ \\
Africa & 0 & 0 & 0 & 0 & 0 & 0 \\
Argentina & 0 & 333 & 776 & 819 & 827 & 200 \\
Australia & 0 & 0 & 0 & 0 & 0 & 0 \\
Brazil & 0 & 333 & 877 & 1,642 & 1,966 & 1,357 \\
Canada & 0 & 0 & 0 & 0 & 0 & 0 \\
Caribbean basin & 0 & 95 & 310 & 675 & 802 & 898 \\
China & 0 & 0 & 0 & 0 & 0 & 0 \\
Colombia & 0 & 119 & 298 & 474 & 650 & 782 \\
Eastern Europe & 0 & 0 & 0 & 0 & 0 & 0 \\
Former Soviet Union & 0 & 0 & 0 & 0 & 0 & 0 \\
India & 0 & 0 & 0 & 0 & 0 & 0 \\
Japan & 0 & 0 & 0 & 0 & 0 & 0 \\
Middle East & 0 & 0 & 0 & 0 & 0 & 0 \\
Mexico & 0 & 0 & 0 & 0 & 0 & 0 \\
Other Asia & 0 & 0 & 0 & 0 & 0 & 0 \\
South Korea & 0 & 0 & 0 & 0 & 0 & 0 \\
United States & 0 & 1,238 & 1,640 & 1,747 & 1,907 & 2,016 \\
Western Europe & 998 & 3,178 & 3,578 & 3,930 & 4,468 & 4,788 \\
\hline Total & $\mathbf{9 9 8}$ & $\mathbf{5 , 2 9 4}$ & $\mathbf{7 , 4 7 9}$ & $\mathbf{9 , 2 8 7}$ & $\mathbf{1 0 , 6 2 1}$ & $\mathbf{1 0 , 0 4 0}$ \\
\hline \hline
\end{tabular}

Table A-233: Reference case with $\$ 20$ per tonne growers’ payment biodiesel consumption [Million gallons ethanol equivalent]

\begin{tabular}{lrrrrrr} 
& $\mathbf{2 0 0 5}$ & $\mathbf{2 0 1 0}$ & $\mathbf{2 0 1 5}$ & $\mathbf{2 0 2 0}$ & $\mathbf{2 0 2 5}$ & $\mathbf{2 0 3 0}$ \\
Africa & 0 & 0 & 0 & 0 & 0 & 0 \\
Australia & 0 & 0 & 0 & 0 & 0 & 0 \\
Canada & 0 & 0 & 0 & 0 & 0 & 0 \\
China & 0 & 0 & 0 & 0 & 0 & 0 \\
Central and South America & 0 & 158 & 472 & 984 & 1,306 & 1,490 \\
Eastern Europe & 0 & 0 & 0 & 0 & 0 & 0 \\
Former Soviet Union & 0 & 0 & 0 & 0 & 0 & 0 \\
India & 0 & 0 & 0 & 0 & 0 & 0 \\
Japan & 0 & 0 & 0 & 0 & 0 & 0 \\
Middle East & 0 & 0 & 0 & 0 & 0 & 0 \\
Mexico & 0 & 0 & 0 & 0 & 0 & 0 \\
Other Asia & 0 & 0 & 0 & 0 & 0 & 0 \\
South Korea & 0 & 0 & 0 & 0 & 0 & 0 \\
United States & 0 & 1,238 & 1,713 & 1,941 & 2,080 & 2,016 \\
Western Europe & 998 & 3,898 & 5,294 & 6,363 & 7,235 & 6,535 \\
\hline Total & $\mathbf{9 9 8}$ & $\mathbf{5 , 2 9 4}$ & $\mathbf{7 , 4 7 9}$ & $\mathbf{9 , 2 8 7}$ & $\mathbf{1 0 , 6 2 1}$ & $\mathbf{1 0 , 0 4 0}$ \\
\hline \hline
\end{tabular}


Table A-234: Reference case with $\$ 20$ per tonne growers’ payment biodiesel net trade [Million gallons ethanol equivalent]

\begin{tabular}{lrrrrrr} 
& $\mathbf{2 0 0 5}$ & $\mathbf{2 0 1 0}$ & $\mathbf{2 0 1 5}$ & $\mathbf{2 0 2 0}$ & $\mathbf{2 0 2 5}$ & $\mathbf{2 0 3 0}$ \\
Africa & 0 & 0 & 0 & 0 & 0 & 0 \\
Australia & 0 & 0 & 0 & 0 & 0 & 0 \\
Canada & 0 & 0 & 0 & 0 & 0 & 0 \\
China & 0 & 0 & 0 & 0 & 0 & 0 \\
Central and South America & 0 & 720 & 1,788 & 2,626 & 2,940 & 1,747 \\
Eastern Europe & 0 & 0 & 0 & 0 & 0 & 0 \\
Former Soviet Union & 0 & 0 & 0 & 0 & 0 & 0 \\
India & 0 & 0 & 0 & 0 & 0 & 0 \\
Japan & 0 & 0 & 0 & 0 & 0 & 0 \\
Middle East & 0 & 0 & 0 & 0 & 0 & 0 \\
Mexico & 0 & 0 & 0 & 0 & 0 & 0 \\
Other Asia & 0 & 0 & 0 & 0 & 0 & 0 \\
South Korea & 0 & 0 & 0 & 0 & 0 & 0 \\
United States & 0 & 0 & -73 & -194 & -173 & 0 \\
Western Europe & 0 & -720 & $-1,716$ & $-2,432$ & $-2,767$ & $-1,747$ \\
\hline Total & $\mathbf{0}$ & $\mathbf{0}$ & $\mathbf{0}$ & $\mathbf{0}$ & $\mathbf{0}$ & $\mathbf{0}$ \\
\hline \hline
\end{tabular}




\section{A-14 Delayed Technology Reference Case}

Table A-235: Delayed technology reference case ethanol production [Million gallons]

\begin{tabular}{lrrrrrr} 
& $\mathbf{2 0 0 5}$ & $\mathbf{2 0 1 0}$ & $\mathbf{2 0 1 5}$ & $\mathbf{2 0 2 0}$ & $\mathbf{2 0 2 5}$ & $\mathbf{2 0 3 0}$ \\
Africa & 300 & 330 & 364 & 402 & 455 & 502 \\
Argentina & 0 & 178 & 664 & 1,047 & 1,077 & 1,088 \\
Australia & 0 & 0 & 66 & 247 & 625 & 1,172 \\
Brazil & 4,866 & 5,977 & 7,624 & 9,801 & 11,947 & 13,338 \\
Canada & 0 & 0 & 259 & 779 & 1,004 & 1,004 \\
Caribbean basin & 210 & 328 & 573 & 683 & 775 & 775 \\
China & 344 & 384 & 614 & 1,041 & 1,485 & 1,485 \\
Colombia & 196 & 215 & 411 & 782 & 1,501 & 2,227 \\
Eastern Europe & 110 & 226 & 434 & 780 & 1,037 & 1,149 \\
Former Soviet Union & 0 & 0 & 6 & 19 & 57 & 174 \\
India & 313 & 279 & 541 & 992 & 1,893 & 2,543 \\
Japan & 0 & 0 & 95 & 348 & 612 & 647 \\
Middle East & 0 & 0 & 0 & 0 & 0 & 0 \\
Mexico & 0 & 0 & 199 & 601 & 1,408 & 1,546 \\
Other Asia & 0 & 0 & 0 & 0 & 0 & 0 \\
South Korea & 0 & 0 & 35 & 35 & 35 & 35 \\
United States & 3,850 & 11,612 & 15,732 & 16,156 & 17,368 & 21,748 \\
Western Europe & 600 & 2,568 & 2,755 & 3,398 & 5,291 & 8,013 \\
\hline Total & $\mathbf{1 0 , 7 8 9}$ & $\mathbf{2 2 , 0 9 7}$ & $\mathbf{3 0 , 3 7 1}$ & $\mathbf{3 7 , 1 0 9}$ & $\mathbf{4 6 , 5 7 0}$ & $\mathbf{5 7 , 4 4 4}$ \\
\hline \hline
\end{tabular}


Table A-236: Delayed technology reference case ethanol consumption [Million gallons]

\begin{tabular}{lrrrrrr} 
& $\mathbf{2 0 0 5}$ & $\mathbf{2 0 1 0}$ & $\mathbf{2 0 1 5}$ & $\mathbf{2 0 2 0}$ & $\mathbf{2 0 2 5}$ & $\mathbf{2 0 3 0}$ \\
Africa & 300 & 330 & 364 & 402 & 455 & 502 \\
Australia & 0 & 0 & 66 & 247 & 625 & 1,172 \\
Canada & 145 & 0 & 233 & 779 & 1,004 & 1,020 \\
China & 297 & 0 & 0 & 311 & 712 & 1,485 \\
Central and South America & 3,788 & 4,853 & 6,134 & 7,171 & 7,971 & 8,872 \\
Eastern Europe & 699 & 226 & 872 & 953 & 1,037 & 1,149 \\
Former Soviet Union & 0 & 0 & 6 & 19 & 57 & 174 \\
India & 313 & 279 & 541 & 992 & 1,893 & 2,399 \\
Japan & 263 & 288 & 373 & 698 & 1,008 & 1,100 \\
Middle East & 0 & 0 & 0 & 0 & 0 & 0 \\
Mexico & 0 & 0 & 30 & 78 & 183 & 201 \\
Other Asia & 0 & 0 & 0 & 0 & 0 & 0 \\
South Korea & 264 & 335 & 400 & 414 & 413 & 413 \\
United States & 3,984 & 11,612 & 17,062 & 19,797 & 23,562 & 28,684 \\
Western Europe & 736 & 4,174 & 4,289 & 5,249 & 7,650 & 10,274 \\
\hline Total & $\mathbf{1 0 , 7 8 9}$ & $\mathbf{2 2 , 0 9 7}$ & $\mathbf{3 0 , 3 7 1}$ & $\mathbf{3 7 , 1 0 9}$ & $\mathbf{4 6 , 5 7 0}$ & $\mathbf{5 7 , 4 4 4}$ \\
\hline \hline
\end{tabular}

Table A-237: Delayed technology reference case ethanol net trade [Million gallons]

Africa

\begin{tabular}{rrrrrr}
$\mathbf{2 0 0 5}$ & $\mathbf{2 0 1 0}$ & $\mathbf{2 0 1 5}$ & $\mathbf{2 0 2 0}$ & $\mathbf{2 0 2 5}$ & $\mathbf{2 0 3 0}$ \\
0 & 0 & 0 & 0 & 0 & 0 \\
0 & 0 & 0 & 0 & 0 & 0 \\
-145 & 0 & 26 & 0 & 0 & -16 \\
47 & 384 & 614 & 730 & 774 & 0 \\
1,484 & 1,845 & 3,137 & 5,142 & 7,328 & 8,556 \\
-589 & 0 & -438 & -173 & 0 & 0 \\
0 & 0 & 0 & 0 & 0 & 0 \\
0 & 0 & 0 & 0 & 0 & 144 \\
-263 & -288 & -278 & -350 & -395 & -453 \\
0 & 0 & 0 & 0 & 0 & 0 \\
0 & 0 & 170 & 523 & 1,225 & 1,345 \\
0 & 0 & 0 & 0 & 0 & 0 \\
-264 & -335 & -365 & -379 & -378 & -378 \\
-134 & 0 & $-1,330$ & $-3,641$ & $-6,194$ & $-6,937$ \\
-136 & $-1,607$ & $-1,535$ & $-1,851$ & $-2,359$ & $-2,261$ \\
\hline $\mathbf{0}$ & $\mathbf{0}$ & $\mathbf{0}$ & $\mathbf{0}$ & $\mathbf{0}$ & $\mathbf{0}$ \\
\hline
\end{tabular}


Table A-238: Delayed technology reference case grain ethanol production [Million gallons]

\begin{tabular}{lrrrrrr} 
& $\mathbf{2 0 0 5}$ & $\mathbf{2 0 1 0}$ & $\mathbf{2 0 1 5}$ & $\mathbf{2 0 2 0}$ & $\mathbf{2 0 2 5}$ & $\mathbf{2 0 3 0}$ \\
Africa & 0 & 0 & 0 & 0 & 0 & 0 \\
Argentina & 0 & 178 & 604 & 741 & 741 & 741 \\
Australia & 0 & 0 & 0 & 0 & 0 & 0 \\
Brazil & 0 & 0 & 0 & 0 & 0 & 0 \\
Canada & 0 & 0 & 0 & 0 & 0 & 0 \\
Caribbean basin & 0 & 0 & 0 & 0 & 0 & 0 \\
China & 344 & 344 & 344 & 325 & 0 & 0 \\
Colombia & 0 & 0 & 0 & 0 & 0 & 0 \\
Eastern Europe & 110 & 226 & 348 & 477 & 367 & 251 \\
Former Soviet Union & 0 & 0 & 0 & 0 & 0 & 0 \\
India & 0 & 0 & 0 & 0 & 0 & 0 \\
Japan & 0 & 0 & 0 & 0 & 0 & 0 \\
Middle East & 0 & 0 & 0 & 0 & 0 & 0 \\
Mexico & 0 & 0 & 0 & 0 & 0 & 0 \\
Other Asia & 0 & 0 & 0 & 0 & 0 & 0 \\
South Korea & 0 & 0 & 0 & 0 & 0 & 0 \\
United States & 3,850 & 11,612 & 15,000 & 15,000 & 15,000 & 14,780 \\
Western Europe & 600 & 2,568 & 2,589 & 2,705 & 3,178 & 3,604 \\
\hline Total & $\mathbf{4 , 9 0 4}$ & $\mathbf{1 4 , 9 2 8}$ & $\mathbf{1 8 , 8 8 5}$ & $\mathbf{1 9 , 2 4 9}$ & $\mathbf{1 9 , 2 8 6}$ & $\mathbf{1 9 , 3 7 7}$ \\
\hline \hline
\end{tabular}

Table A-239: Delayed technology reference case sugar ethanol production [Million gallons]

\begin{tabular}{lrrrrrr} 
& $\mathbf{2 0 0 5}$ & $\mathbf{2 0 1 0}$ & $\mathbf{2 0 1 5}$ & $\mathbf{2 0 2 0}$ & $\mathbf{2 0 2 5}$ & $\mathbf{2 0 3 0}$ \\
Africa & 300 & 330 & 364 & 402 & 444 & 490 \\
Argentina & 0 & 0 & 0 & 0 & 0 & 0 \\
Australia & 0 & 0 & 0 & 0 & 0 & 0 \\
Brazil & 4,866 & 5,977 & 7,329 & 8,911 & 10,406 & 11,793 \\
Canada & 0 & 0 & 0 & 0 & 0 & 0 \\
Caribbean basin & 210 & 328 & 573 & 683 & 775 & 775 \\
China & 0 & 40 & 48 & 48 & 48 & 48 \\
Colombia & 196 & 215 & 241 & 270 & 302 & 334 \\
Eastern Europe & 0 & 0 & 0 & 0 & 0 & 0 \\
Former Soviet Union & 0 & 0 & 0 & 0 & 0 & 0 \\
India & 313 & 279 & 327 & 347 & 381 & 348 \\
Japan & 0 & 0 & 0 & 0 & 0 & 0 \\
Middle East & 0 & 0 & 0 & 0 & 0 & 0 \\
Mexico & 0 & 0 & 0 & 0 & 0 & 0 \\
Other Asia & 0 & 0 & 0 & 0 & 0 & 0 \\
South Korea & 0 & 0 & 0 & 0 & 0 & 0 \\
United States & 0 & 0 & 0 & 0 & 0 & 0 \\
Western Europe & 0 & 0 & 0 & 0 & 0 & 0 \\
\hline Total & $\mathbf{5 , 8 8 4}$ & $\mathbf{7 , 1 6 9}$ & $\mathbf{8 , 8 8 2}$ & $\mathbf{1 0 , 6 6 1}$ & $\mathbf{1 2 , 3 5 6}$ & $\mathbf{1 3 , 7 8 7}$ \\
\hline \hline
\end{tabular}


Table A-240: Delayed technology reference case cellulosic ethanol production [Million gallons]

\begin{tabular}{lrrrrrr} 
& $\mathbf{2 0 0 5}$ & $\mathbf{2 0 1 0}$ & $\mathbf{2 0 1 5}$ & $\mathbf{2 0 2 0}$ & $\mathbf{2 0 2 5}$ & $\mathbf{2 0 3 0}$ \\
Africa & 0 & 0 & 0 & 0 & 11 & 11 \\
Argentina & 0 & 0 & 59 & 306 & 335 & 347 \\
Australia & 0 & 0 & 66 & 247 & 625 & 1,172 \\
Brazil & 0 & 0 & 296 & 890 & 1,541 & 1,546 \\
Canada & 0 & 0 & 259 & 779 & 1,004 & 1,004 \\
Caribbean basin & 0 & 0 & 0 & 0 & 0 & 0 \\
China & 0 & 0 & 222 & 667 & 1,438 & 1,438 \\
Colombia & 0 & 0 & 170 & 512 & 1,199 & 1,893 \\
Eastern Europe & 0 & 0 & 85 & 303 & 670 & 897 \\
Former Soviet Union & 0 & 0 & 6 & 19 & 57 & 174 \\
India & 0 & 0 & 214 & 645 & 1,512 & 2,196 \\
Japan & 0 & 0 & 95 & 348 & 612 & 647 \\
Middle East & 0 & 0 & 0 & 0 & 0 & 0 \\
Mexico & 0 & 0 & 199 & 601 & 1,408 & 1,546 \\
Other Asia & 0 & 0 & 0 & 0 & 0 & 0 \\
South Korea & 0 & 0 & 35 & 35 & 35 & 35 \\
United States & 0 & 0 & 732 & 1,156 & 2,368 & 6,967 \\
Western Europe & 0 & 0 & 166 & 693 & 2,113 & 4,410 \\
\hline Total & $\mathbf{0}$ & $\mathbf{0}$ & $\mathbf{2 , 6 0 4}$ & $\mathbf{7 , 1 9 9}$ & $\mathbf{1 4 , 9 2 8}$ & $\mathbf{2 4 , 2 8 1}$ \\
\hline \hline
\end{tabular}

Table A-241: Delayed technology reference case grain ethanol consumption [Million gallons]

\begin{tabular}{lrrrrrr} 
& $\mathbf{2 0 0 5}$ & $\mathbf{2 0 1 0}$ & $\mathbf{2 0 1 5}$ & $\mathbf{2 0 2 0}$ & $\mathbf{2 0 2 5}$ & $\mathbf{2 0 3 0}$ \\
Africa & 0 & 0 & 0 & 0 & 0 & 0 \\
Australia & 0 & 0 & 0 & 0 & 0 & 0 \\
Canada & 0 & 0 & 0 & 0 & 0 & 0 \\
China & 297 & 0 & 0 & 263 & 0 & 0 \\
Central and South America & 0 & 36 & 100 & 136 & 108 & 109 \\
Eastern Europe & 110 & 226 & 348 & 477 & 367 & 251 \\
Former Soviet Union & 0 & 0 & 0 & 0 & 0 & 0 \\
India & 0 & 0 & 0 & 0 & 0 & 0 \\
Japan & 0 & 192 & 230 & 62 & 0 & 254 \\
Middle East & 0 & 0 & 0 & 0 & 0 & 0 \\
Mexico & 0 & 0 & 0 & 0 & 0 & 0 \\
Other Asia & 0 & 0 & 0 & 0 & 0 & 0 \\
South Korea & 47 & 295 & 144 & 0 & 0 & 378 \\
United States & 3,850 & 11,612 & 15,000 & 15,000 & 15,000 & 14,780 \\
Western Europe & 600 & 2,568 & 3,064 & 3,310 & 3,811 & 3,604 \\
\hline Total & $\mathbf{4 , 9 0 4}$ & $\mathbf{1 4 , 9 2 8}$ & $\mathbf{1 8 , 8 8 5}$ & $\mathbf{1 9 , 2 4 9}$ & $\mathbf{1 9 , 2 8 6}$ & $\mathbf{1 9 , 3 7 7}$ \\
\hline \hline
\end{tabular}


Table A-242: Delayed technology reference case sugar ethanol consumption [Million gallons]

\begin{tabular}{lrrrrrr} 
& $\mathbf{2 0 0 5}$ & $\mathbf{2 0 1 0}$ & $\mathbf{2 0 1 5}$ & $\mathbf{2 0 2 0}$ & $\mathbf{2 0 2 5}$ & $\mathbf{2 0 3 0}$ \\
Africa & 300 & 330 & 364 & 402 & 444 & 490 \\
Australia & 0 & 0 & 0 & 0 & 0 & 0 \\
Canada & 145 & 0 & 0 & 0 & 0 & 16 \\
China & 0 & 0 & 0 & 48 & 48 & 48 \\
Central and South America & 3,788 & 4,818 & 6,035 & 6,938 & 7,864 & 8,763 \\
Eastern Europe & 589 & 0 & 412 & 173 & 0 & 0 \\
Former Soviet Union & 0 & 0 & 0 & 0 & 0 & 0 \\
India & 313 & 279 & 327 & 347 & 381 & 348 \\
Japan & 263 & 96 & 48 & 0 & 0 & 54 \\
Middle East & 0 & 0 & 0 & 0 & 0 & 0 \\
Mexico & 0 & 0 & 0 & 0 & 0 & 0 \\
Other Asia & 0 & 0 & 0 & 0 & 0 & 0 \\
South Korea & 217 & 40 & 0 & 0 & 0 & 0 \\
United States & 134 & 0 & 788 & 2,753 & 3,093 & 1,807 \\
Western Europe & 136 & 1,607 & 908 & 0 & 526 & 2,261 \\
\hline Total & $\mathbf{5 , 8 8 4}$ & $\mathbf{7 , 1 6 9}$ & $\mathbf{8 , 8 8 2}$ & $\mathbf{1 0 , 6 6 1}$ & $\mathbf{1 2 , 3 5 6}$ & $\mathbf{1 3 , 7 8 7}$ \\
\hline \hline
\end{tabular}

Table A-243: Delayed technology reference case cellulosic ethanol consumption [Million gallons]

\begin{tabular}{lrrrrrr} 
& $\mathbf{2 0 0 5}$ & $\mathbf{2 0 1 0}$ & $\mathbf{2 0 1 5}$ & $\mathbf{2 0 2 0}$ & $\mathbf{2 0 2 5}$ & $\mathbf{2 0 3 0}$ \\
Africa & 0 & 0 & 0 & 0 & 11 & 11 \\
Australia & 0 & 0 & 66 & 247 & 625 & 1,172 \\
Canada & 0 & 0 & 233 & 779 & 1,004 & 1,004 \\
China & 0 & 0 & 0 & 0 & 664 & 1,438 \\
Central and South America & 0 & 0 & 0 & 96 & 0 & 0 \\
Eastern Europe & 0 & 0 & 111 & 303 & 670 & 897 \\
Former Soviet Union & 0 & 0 & 6 & 19 & 57 & 174 \\
India & 0 & 0 & 214 & 645 & 1,512 & 2,051 \\
Japan & 0 & 0 & 95 & 636 & 1,008 & 791 \\
Middle East & 0 & 0 & 0 & 0 & 0 & 0 \\
Mexico & 0 & 0 & 30 & 78 & 183 & 201 \\
Other Asia & 0 & 0 & 0 & 0 & 0 & 0 \\
South Korea & 0 & 0 & 256 & 414 & 413 & 35 \\
United States & 0 & 0 & 1,274 & 2,044 & 5,469 & 12,098 \\
Western Europe & 0 & 0 & 318 & 1,939 & 3,312 & 4,410 \\
\hline Total & $\mathbf{0}$ & $\mathbf{0}$ & $\mathbf{2 , 6 0 4}$ & $\mathbf{7 , 1 9 9}$ & $\mathbf{1 4 , 9 2 8}$ & $\mathbf{2 4 , 2 8 1}$ \\
\hline \hline
\end{tabular}


Table A-244: Delayed technology reference case grain ethanol net trade [Million gallons]

\begin{tabular}{lrrrrrr} 
& $\mathbf{2 0 0 5}$ & $\mathbf{2 0 1 0}$ & $\mathbf{2 0 1 5}$ & $\mathbf{2 0 2 0}$ & $\mathbf{2 0 2 5}$ & $\mathbf{2 0 3 0}$ \\
Africa & 0 & 0 & 0 & 0 & 0 & 0 \\
Australia & 0 & 0 & 0 & 0 & 0 & 0 \\
Canada & 0 & 0 & 0 & 0 & 0 & 0 \\
China & 47 & 344 & 344 & 62 & 0 & 0 \\
Central and South America & 0 & 143 & 505 & 605 & 634 & 632 \\
Eastern Europe & 0 & 0 & 0 & 0 & 0 & 0 \\
Former Soviet Union & 0 & 0 & 0 & 0 & 0 & 0 \\
India & 0 & 0 & 0 & 0 & 0 & 0 \\
Japan & 0 & -192 & -230 & -62 & 0 & -254 \\
Middle East & 0 & 0 & 0 & 0 & 0 & 0 \\
Mexico & 0 & 0 & 0 & 0 & 0 & 0 \\
Other Asia & 0 & 0 & 0 & 0 & 0 & 0 \\
South Korea & -47 & -295 & -144 & 0 & 0 & -378 \\
United States & 0 & 0 & 0 & 0 & 0 & 0 \\
Western Europe & 0 & 0 & -475 & -605 & -634 & 0 \\
\hline Total & $\mathbf{0}$ & $\mathbf{0}$ & $\mathbf{0}$ & $\mathbf{0}$ & $\mathbf{0}$ & $\mathbf{0}$ \\
\hline \hline
\end{tabular}

Table A-245: Delayed technology reference case sugar ethanol net trade [Million gallons]

\begin{tabular}{lrrrrrr} 
Africa & 0 & 0 & 0 & 0 & 0 & 0 \\
Australia & 0 & 0 & 0 & 0 & 0 & 0 \\
Canada & -145 & 0 & 0 & 0 & 0 & -16 \\
China & 0 & 40 & 48 & 0 & 0 & 0 \\
Central and South America & 1,484 & 1,702 & 2,108 & 2,926 & 3,619 & 4,138 \\
Eastern Europe & -589 & 0 & -412 & -173 & 0 & 0 \\
Former Soviet Union & 0 & 0 & 0 & 0 & 0 & 0 \\
India & 0 & 0 & 0 & 0 & 0 & 0 \\
Japan & -263 & -96 & -48 & 0 & 0 & -54 \\
Middle East & 0 & 0 & 0 & 0 & 0 & 0 \\
Mexico & 0 & 0 & 0 & 0 & 0 & 0 \\
Other Asia & 0 & 0 & 0 & 0 & 0 & 0 \\
South Korea & -217 & -40 & 0 & 0 & 0 & 0 \\
United States & -134 & 0 & -788 & $-2,753$ & $-3,093$ & $-1,807$ \\
Western Europe & -136 & $-1,607$ & -908 & 0 & -526 & $-2,261$ \\
\hline Total & $\mathbf{0}$ & $\mathbf{0}$ & $\mathbf{0}$ & $\mathbf{0}$ & $\mathbf{0}$ & $\mathbf{0}$ \\
\hline \hline
\end{tabular}


Table A-246: Delayed technology reference case cellulosic ethanol net trade [Million gallons]

\begin{tabular}{lrrrrrr} 
& $\mathbf{2 0 0 5}$ & $\mathbf{2 0 1 0}$ & $\mathbf{2 0 1 5}$ & $\mathbf{2 0 2 0}$ & $\mathbf{2 0 2 5}$ & $\mathbf{2 0 3 0}$ \\
Africa & 0 & 0 & 0 & 0 & 0 & 0 \\
Australia & 0 & 0 & 0 & 0 & 0 & 0 \\
Canada & 0 & 0 & 26 & 0 & 0 & 0 \\
China & 0 & 0 & 222 & 667 & 774 & 0 \\
Central and South America & 0 & 0 & 525 & 1,611 & 3,076 & 3,786 \\
Eastern Europe & 0 & 0 & -26 & 0 & 0 & 0 \\
Former Soviet Union & 0 & 0 & 0 & 0 & 0 & 0 \\
India & 0 & 0 & 0 & 0 & 0 & 144 \\
Japan & 0 & 0 & 0 & -288 & -395 & -144 \\
Middle East & 0 & 0 & 0 & 0 & 0 & 0 \\
Mexico & 0 & 0 & 170 & 523 & 1,225 & 1,345 \\
Other Asia & 0 & 0 & 0 & 0 & 0 & 0 \\
South Korea & 0 & 0 & -222 & -379 & -378 & 0 \\
United States & 0 & 0 & -543 & -888 & $-3,101$ & $-5,130$ \\
Western Europe & 0 & 0 & -152 & $-1,246$ & $-1,199$ & 0 \\
\hline Total & $\mathbf{0}$ & $\mathbf{0}$ & $\mathbf{0}$ & $\mathbf{0}$ & $\mathbf{0}$ & $\mathbf{0}$ \\
\hline \hline
\end{tabular}

Table A-247: Delayed technology reference case biomass-to-liquids production [Million gallons]

\begin{tabular}{lrrrrrr} 
& $\mathbf{2 0 0 5}$ & $\mathbf{2 0 1 0}$ & $\mathbf{2 0 1 5}$ & $\mathbf{2 0 2 0}$ & $\mathbf{2 0 2 5}$ & $\mathbf{2 0 3 0}$ \\
Africa & 0 & 0 & 0 & 0 & 0 & 0 \\
Argentina & 0 & 0 & 21 & 110 & 121 & 125 \\
Australia & 0 & 0 & 0 & 0 & 0 & 0 \\
Brazil & 0 & 0 & 106 & 320 & 554 & 556 \\
Canada & 0 & 0 & 93 & 280 & 361 & 361 \\
Caribbean basin & 0 & 0 & 0 & 0 & 0 & 0 \\
China & 0 & 0 & 80 & 240 & 517 & 517 \\
Colombia & 0 & 0 & 61 & 184 & 431 & 681 \\
Eastern Europe & 0 & 0 & 0 & 0 & 0 & 0 \\
Former Soviet Union & 0 & 0 & 0 & 0 & 0 & 0 \\
India & 0 & 0 & 77 & 232 & 544 & 790 \\
Japan & 0 & 0 & 0 & 0 & 0 & 0 \\
Middle East & 0 & 0 & 0 & 0 & 0 & 0 \\
Mexico & 0 & 0 & 72 & 216 & 506 & 556 \\
Other Asia & 0 & 0 & 0 & 0 & 0 & 0 \\
South Korea & 0 & 0 & 36 & 56 & 56 & 56 \\
United States & 0 & 0 & 750 & 2,467 & 3,398 & 4,383 \\
Western Europe & 0 & 0 & 0 & 0 & 0 & 0 \\
\hline Total & $\mathbf{0}$ & $\mathbf{0}$ & $\mathbf{1 , 2 9 6}$ & $\mathbf{4 , 1 0 5}$ & $\mathbf{6 , 4 8 8}$ & $\mathbf{8 , 0 2 4}$ \\
\hline \hline
\end{tabular}


Table A-248: Delayed technology reference case biomass-to-liquids consumption [Million gallons]

\begin{tabular}{lrrrrrr} 
& $\mathbf{2 0 0 5}$ & $\mathbf{2 0 1 0}$ & $\mathbf{2 0 1 5}$ & $\mathbf{2 0 2 0}$ & $\mathbf{2 0 2 5}$ & $\mathbf{2 0 3 0}$ \\
Africa & 0 & 0 & 0 & 0 & 0 & 0 \\
Australia & 0 & 0 & 0 & 0 & 0 & 0 \\
Canada & 0 & 0 & 0 & 0 & 0 & 0 \\
China & 0 & 0 & 0 & 0 & 0 & 0 \\
Central and South America & 0 & 0 & 0 & 0 & 0 & 0 \\
Eastern Europe & 0 & 0 & 0 & 0 & 0 & 0 \\
Former Soviet Union & 0 & 0 & 0 & 0 & 0 & 0 \\
India & 0 & 0 & 0 & 0 & 0 & 0 \\
Japan & 0 & 0 & 0 & 0 & 0 & 0 \\
Middle East & 0 & 0 & 0 & 0 & 0 & 0 \\
Mexico & 0 & 0 & 0 & 0 & 0 & 0 \\
Other Asia & 0 & 0 & 0 & 0 & 0 & 0 \\
South Korea & 0 & 0 & 0 & 0 & 0 & 0 \\
United States & 0 & 0 & 1,023 & 4,016 & 6,488 & 8,024 \\
Western Europe & 0 & 0 & 273 & 88 & 0 & 0 \\
\hline Total & $\mathbf{0}$ & $\mathbf{0}$ & $\mathbf{1 , 2 9 6}$ & $\mathbf{4 , 1 0 5}$ & $\mathbf{6 , 4 8 8}$ & $\mathbf{8 , 0 2 4}$ \\
\hline \hline
\end{tabular}

Table A-249: Delayed technology reference case biomass-to-liquids net trade [Million gallons]

\begin{tabular}{lrrrrrr} 
& $\mathbf{2 0 0 5}$ & $\mathbf{2 0 1 0}$ & $\mathbf{2 0 1 5}$ & $\mathbf{2 0 2 0}$ & $\mathbf{2 0 2 5}$ & $\mathbf{2 0 3 0}$ \\
Africa & 0 & 0 & 0 & 0 & 0 & 0 \\
Australia & 0 & 0 & 0 & 0 & 0 & 0 \\
Canada & 0 & 0 & 93 & 280 & 361 & 361 \\
China & 0 & 0 & 80 & 240 & 517 & 517 \\
Central and South America & 0 & 0 & 189 & 614 & 1,106 & 1,362 \\
Eastern Europe & 0 & 0 & 0 & 0 & 0 & 0 \\
Former Soviet Union & 0 & 0 & 0 & 0 & 0 & 0 \\
India & 0 & 0 & 77 & 232 & 544 & 790 \\
Japan & 0 & 0 & 0 & 0 & 0 & 0 \\
Middle East & 0 & 0 & 0 & 0 & 0 & 0 \\
Mexico & 0 & 0 & 72 & 216 & 506 & 556 \\
Other Asia & 0 & 0 & 0 & 0 & 0 & 0 \\
South Korea & 0 & 0 & 36 & 56 & 56 & 56 \\
United States & 0 & 0 & -273 & $-1,550$ & $-3,090$ & $-3,641$ \\
Western Europe & 0 & 0 & -273 & -88 & 0 & 0 \\
\hline Total & $\mathbf{0}$ & $\mathbf{0}$ & $\mathbf{0}$ & $\mathbf{0}$ & $\mathbf{0}$ & $\mathbf{0}$ \\
\hline \hline
\end{tabular}


Table A-250: Delayed technology reference case biodiesel production [Million gallons]

\begin{tabular}{lrrrrrr} 
& $\mathbf{2 0 0 5}$ & $\mathbf{2 0 1 0}$ & $\mathbf{2 0 1 5}$ & $\mathbf{2 0 2 0}$ & $\mathbf{2 0 2 5}$ & $\mathbf{2 0 3 0}$ \\
Africa & 0 & 0 & 0 & 0 & 0 & 0 \\
Argentina & 0 & 333 & 776 & 819 & 827 & 239 \\
Australia & 0 & 0 & 0 & 0 & 0 & 0 \\
Brazil & 0 & 333 & 877 & 1,642 & 1,793 & 1,355 \\
Canada & 0 & 0 & 0 & 0 & 0 & 0 \\
Caribbean basin & 0 & 95 & 310 & 675 & 802 & 898 \\
China & 0 & 0 & 0 & 0 & 0 & 0 \\
Colombia & 0 & 119 & 298 & 474 & 650 & 782 \\
Eastern Europe & 0 & 0 & 0 & 0 & 0 & 0 \\
Former Soviet Union & 0 & 0 & 0 & 0 & 0 & 0 \\
India & 0 & 0 & 0 & 0 & 0 & 0 \\
Japan & 0 & 0 & 0 & 0 & 0 & 0 \\
Middle East & 0 & 0 & 0 & 0 & 0 & 0 \\
Mexico & 0 & 0 & 0 & 0 & 0 & 0 \\
Other Asia & 0 & 0 & 0 & 0 & 0 & 0 \\
South Korea & 0 & 0 & 0 & 0 & 0 & 0 \\
United States & 0 & 1,238 & 1,640 & 1,747 & 1,907 & 2,072 \\
Western Europe & 998 & 3,178 & 3,578 & 3,930 & 4,468 & 4,788 \\
\hline Total & $\mathbf{9 9 8}$ & $\mathbf{5 , 2 9 4}$ & $\mathbf{7 , 4 7 9}$ & $\mathbf{9 , 2 8 7}$ & $\mathbf{1 0 , 4 4 8}$ & $\mathbf{1 0 , 1 3 4}$ \\
\hline \hline
\end{tabular}

Table A-251: Delayed technology reference case biodiesel consumption [Million gallons]

\begin{tabular}{lrrrrrr} 
& $\mathbf{2 0 0 5}$ & $\mathbf{2 0 1 0}$ & $\mathbf{2 0 1 5}$ & $\mathbf{2 0 2 0}$ & $\mathbf{2 0 2 5}$ & $\mathbf{2 0 3 0}$ \\
Africa & 0 & 0 & 0 & 0 & 0 & 0 \\
Australia & 0 & 0 & 0 & 0 & 0 & 0 \\
Canada & 0 & 0 & 0 & 0 & 0 & 0 \\
China & 0 & 0 & 0 & 0 & 0 & 0 \\
Central and South America & 0 & 158 & 472 & 984 & 1,306 & 1,490 \\
Eastern Europe & 0 & 0 & 0 & 0 & 0 & 0 \\
Former Soviet Union & 0 & 0 & 0 & 0 & 0 & 0 \\
India & 0 & 0 & 0 & 0 & 0 & 0 \\
Japan & 0 & 0 & 0 & 0 & 0 & 0 \\
Middle East & 0 & 0 & 0 & 0 & 0 & 0 \\
Mexico & 0 & 0 & 0 & 0 & 0 & 0 \\
Other Asia & 0 & 0 & 0 & 0 & 0 & 0 \\
South Korea & 0 & 0 & 0 & 0 & 0 & 0 \\
United States & 0 & 1,238 & 1,713 & 1,747 & 1,907 & 3,413 \\
Western Europe & 998 & 3,898 & 5,294 & 6,556 & 7,235 & 5,231 \\
\hline Total & $\mathbf{9 9 8}$ & $\mathbf{5 , 2 9 4}$ & $\mathbf{7 , 4 7 9}$ & $\mathbf{9 , 2 8 7}$ & $\mathbf{1 0 , 4 4 8}$ & $\mathbf{1 0 , 1 3 4}$ \\
\hline \hline
\end{tabular}




\section{Table A-252: Delayed technology reference case biodiesel net trade [Million gallons]}

\begin{tabular}{lrrrrrr} 
& $\mathbf{2 0 0 5}$ & $\mathbf{2 0 1 0}$ & $\mathbf{2 0 1 5}$ & $\mathbf{2 0 2 0}$ & $\mathbf{2 0 2 5}$ & $\mathbf{2 0 3 0}$ \\
Africa & 0 & 0 & 0 & 0 & 0 & 0 \\
Australia & 0 & 0 & 0 & 0 & 0 & 0 \\
Canada & 0 & 0 & 0 & 0 & 0 & 0 \\
China & 0 & 0 & 0 & 0 & 0 & 0 \\
Central and South America & 0 & 720 & 1,788 & 2,626 & 2,767 & 1,784 \\
Eastern Europe & 0 & 0 & 0 & 0 & 0 & 0 \\
Former Soviet Union & 0 & 0 & 0 & 0 & 0 & 0 \\
India & 0 & 0 & 0 & 0 & 0 & 0 \\
Japan & 0 & 0 & 0 & 0 & 0 & 0 \\
Middle East & 0 & 0 & 0 & 0 & 0 & 0 \\
Mexico & 0 & 0 & 0 & 0 & 0 & 0 \\
Other Asia & 0 & 0 & 0 & 0 & 0 & 0 \\
South Korea & 0 & 0 & 0 & 0 & 0 & 0 \\
United States & 0 & 0 & -73 & 0 & 0 & $-1,341$ \\
Western Europe & 0 & -720 & $-1,716$ & $-2,626$ & $-2,767$ & -443 \\
\hline Total & $\mathbf{0}$ & $\mathbf{0}$ & $\mathbf{0}$ & $\mathbf{0}$ & $\mathbf{0}$ & $\mathbf{0}$ \\
\hline \hline
\end{tabular}




\section{A-15 Delayed Technology Reference Case with Credit and Tariff Extension}

Table A-253: Delayed technology reference case with credit and tariff extension ethanol production [Million gallons]

BIOREFDC

\section{Ethanol production [Million gallons]}

\begin{tabular}{l} 
Africa \\
Argentina \\
Australia \\
Brazil \\
Canada \\
Caribbean basin \\
China \\
Colombia \\
Eastern Europe \\
Former Soviet Union \\
India \\
Japan \\
Middle East \\
Mexico \\
Other Asia \\
South Korea \\
United States \\
Western Europe \\
\hline Total \\
\hline
\end{tabular}

\begin{tabular}{rrrrrr}
$\mathbf{2 0 0 5}$ & $\mathbf{2 0 1 0}$ & $\mathbf{2 0 1 5}$ & $\mathbf{2 0 2 0}$ & $\mathbf{2 0 2 5}$ & $\mathbf{2 0 3 0}$ \\
300 & 330 & 364 & 402 & 455 & 502 \\
0 & 178 & 628 & 875 & 904 & 478 \\
0 & 0 & 66 & 247 & 622 & 1,172 \\
4,866 & 5,977 & 7,624 & 9,475 & 11,246 & 13,338 \\
0 & 0 & 259 & 779 & 1,004 & 1,004 \\
210 & 328 & 735 & 735 & 779 & 792 \\
344 & 384 & 614 & 1,041 & 1,319 & 1,319 \\
196 & 215 & 411 & 782 & 1,388 & 1,542 \\
110 & 226 & 428 & 738 & 1,037 & 1,149 \\
0 & 0 & 6 & 17 & 52 & 157 \\
313 & 279 & 541 & 992 & 1,893 & 2,543 \\
0 & 0 & 95 & 348 & 612 & 647 \\
0 & 0 & 0 & 0 & 0 & 0 \\
0 & 0 & 199 & 601 & 1,408 & 1,546 \\
0 & 0 & 0 & 0 & 0 & 0 \\
0 & 0 & 35 & 35 & 35 & 35 \\
3,850 & 11,668 & 15,767 & 16,736 & 19,667 & 24,124 \\
600 & 2,568 & 2,755 & 3,398 & 5,291 & 8,013 \\
\hline $\mathbf{1 0 , 7 8 9}$ & $\mathbf{2 2 , 1 5 4}$ & $\mathbf{3 0 , 5 2 7}$ & $\mathbf{3 7 , 1 9 8}$ & $\mathbf{4 7 , 7 1 1}$ & $\mathbf{5 8 , 3 5 9}$ \\
\hline
\end{tabular}


Table A-254: Delayed technology reference case with credit and tariff extension ethanol consumption [Million gallons]

\begin{tabular}{lrrrrrr} 
& $\mathbf{2 0 0 5}$ & $\mathbf{2 0 1 0}$ & $\mathbf{2 0 1 5}$ & $\mathbf{2 0 2 0}$ & $\mathbf{2 0 2 5}$ & $\mathbf{2 0 3 0}$ \\
Africa & 300 & 330 & 364 & 402 & 455 & 502 \\
Australia & 0 & 0 & 66 & 247 & 622 & 1,172 \\
Canada & 145 & 0 & 233 & 779 & 1,004 & 1,004 \\
China & 121 & 0 & 0 & 1,041 & 1,103 & 1,463 \\
Central and South America & 3,788 & 4,853 & 6,139 & 6,863 & 8,250 & 8,709 \\
Eastern Europe & 699 & 226 & 872 & 953 & 1,037 & 1,149 \\
Former Soviet Union & 0 & 0 & 6 & 17 & 52 & 157 \\
India & 313 & 279 & 541 & 992 & 1,893 & 2,399 \\
Japan & 263 & 288 & 410 & 730 & 1,039 & 1,100 \\
Middle East & 0 & 0 & 0 & 0 & 0 & 0 \\
Mexico & 0 & 0 & 30 & 78 & 183 & 201 \\
Other Asia & 0 & 0 & 0 & 0 & 0 & 0 \\
South Korea & 264 & 335 & 400 & 414 & 413 & 413 \\
United States & 4,161 & 11,668 & 17,153 & 19,947 & 24,012 & 30,075 \\
Western Europe & 736 & 4,174 & 4,312 & 4,737 & 7,649 & 10,017 \\
\hline Total & $\mathbf{1 0 , 7 8 9}$ & $\mathbf{2 2 , 1 5 4}$ & $\mathbf{3 0 , 5 2 7}$ & $\mathbf{3 7 , 1 9 8}$ & $\mathbf{4 7 , 7 1 1}$ & $\mathbf{5 8 , 3 5 9}$ \\
\hline \hline
\end{tabular}

Table A-255: Delayed technology reference case with credit and tariff extension ethanol net trade [Million gallons]

\begin{tabular}{lrrrrrr} 
& $\mathbf{2 0 0 5}$ & $\mathbf{2 0 1 0}$ & $\mathbf{2 0 1 5}$ & $\mathbf{2 0 2 0}$ & $\mathbf{2 0 2 5}$ & $\mathbf{2 0 3 0}$ \\
Africa & 0 & 0 & 0 & 0 & 0 & 0 \\
Australia & 0 & 0 & 0 & 0 & 0 & 0 \\
Canada & -145 & 0 & 26 & 0 & 0 & 0 \\
China & 224 & 384 & 614 & 0 & 216 & -144 \\
Central and South America & 1,484 & 1,845 & 3,259 & 5,003 & 6,068 & 7,441 \\
Eastern Europe & -589 & 0 & -444 & -215 & 0 & 0 \\
Former Soviet Union & 0 & 0 & 0 & 0 & 0 & 0 \\
India & 0 & 0 & 0 & 0 & 0 & 144 \\
Japan & -263 & -288 & -315 & -382 & -427 & -453 \\
Middle East & 0 & 0 & 0 & 0 & 0 & 0 \\
Mexico & 0 & 0 & 170 & 523 & 1,225 & 1,345 \\
Other Asia & 0 & 0 & 0 & 0 & 0 & 0 \\
South Korea & -264 & -335 & -365 & -379 & -378 & -378 \\
United States & -311 & 0 & $-1,386$ & $-3,211$ & $-4,345$ & $-5,951$ \\
Western Europe & -136 & $-1,607$ & $-1,557$ & $-1,339$ & $-2,358$ & $-2,003$ \\
\hline Total & $\mathbf{0}$ & $\mathbf{0}$ & $\mathbf{0}$ & $\mathbf{0}$ & $\mathbf{0}$ & $\mathbf{0}$ \\
\hline \hline
\end{tabular}


Table A-256: Delayed technology reference case with credit and tariff extension grain ethanol production [Million gallons]

\begin{tabular}{lrrrrrr} 
& $\mathbf{2 0 0 5}$ & $\mathbf{2 0 1 0}$ & $\mathbf{2 0 1 5}$ & $\mathbf{2 0 2 0}$ & $\mathbf{2 0 2 5}$ & $\mathbf{2 0 3 0}$ \\
Africa & 0 & 0 & 0 & 0 & 0 & 0 \\
Argentina & 0 & 178 & 569 & 569 & 569 & 142 \\
Australia & 0 & 0 & 0 & 0 & 0 & 0 \\
Brazil & 0 & 0 & 0 & 0 & 0 & 0 \\
Canada & 0 & 0 & 0 & 0 & 0 & 0 \\
Caribbean basin & 0 & 0 & 0 & 0 & 0 & 0 \\
China & 344 & 344 & 344 & 325 & 0 & 0 \\
Colombia & 0 & 0 & 0 & 0 & 0 & 0 \\
Eastern Europe & 110 & 226 & 348 & 477 & 367 & 251 \\
Former Soviet Union & 0 & 0 & 0 & 0 & 0 & 0 \\
India & 0 & 0 & 0 & 0 & 0 & 0 \\
Japan & 0 & 0 & 0 & 0 & 0 & 0 \\
Middle East & 0 & 0 & 0 & 0 & 0 & 0 \\
Mexico & 0 & 0 & 0 & 0 & 0 & 0 \\
Other Asia & 0 & 0 & 0 & 0 & 0 & 0 \\
South Korea & 0 & 0 & 0 & 0 & 0 & 0 \\
United States & 3,850 & 11,668 & 15,000 & 15,000 & 15,000 & 15,000 \\
Western Europe & 600 & 2,568 & 2,589 & 2,705 & 3,178 & 3,604 \\
\hline Total & $\mathbf{4 , 9 0 4}$ & $\mathbf{1 4 , 9 8 4}$ & $\mathbf{1 8 , 8 5 0}$ & $\mathbf{1 9 , 0 7 7}$ & $\mathbf{1 9 , 1 1 4}$ & $\mathbf{1 8 , 9 9 7}$ \\
\hline \hline
\end{tabular}

Table A-257: Delayed technology reference case with credit and tariff extension sugar ethanol production [Million gallons]

\begin{tabular}{lrrrrrr} 
& $\mathbf{2 0 0 5}$ & $\mathbf{2 0 1 0}$ & $\mathbf{2 0 1 5}$ & $\mathbf{2 0 2 0}$ & $\mathbf{2 0 2 5}$ & $\mathbf{2 0 3 0}$ \\
Africa & 300 & 330 & 364 & 402 & 444 & 490 \\
Argentina & 0 & 0 & 0 & 0 & 0 & 0 \\
Australia & 0 & 0 & 0 & 0 & 0 & 0 \\
Brazil & 4,866 & 5,977 & 7,329 & 8,585 & 9,855 & 11,793 \\
Canada & 0 & 0 & 0 & 0 & 0 & 0 \\
Caribbean basin & 210 & 328 & 735 & 735 & 779 & 792 \\
China & 0 & 40 & 48 & 48 & 48 & 48 \\
Colombia & 196 & 215 & 241 & 270 & 270 & 270 \\
Eastern Europe & 0 & 0 & 0 & 0 & 0 & 0 \\
Former Soviet Union & 0 & 0 & 0 & 0 & 0 & 0 \\
India & 313 & 279 & 327 & 347 & 381 & 348 \\
Japan & 0 & 0 & 0 & 0 & 0 & 0 \\
Middle East & 0 & 0 & 0 & 0 & 0 & 0 \\
Mexico & 0 & 0 & 0 & 0 & 0 & 0 \\
Other Asia & 0 & 0 & 0 & 0 & 0 & 0 \\
South Korea & 0 & 0 & 0 & 0 & 0 & 0 \\
United States & 0 & 0 & 0 & 0 & 0 & 0 \\
Western Europe & 0 & 0 & 0 & 0 & 0 & 0 \\
\hline Total & $\mathbf{5 , 8 8 4}$ & $\mathbf{7 , 1 6 9}$ & $\mathbf{9 , 0 4 4}$ & $\mathbf{1 0 , 3 8 7}$ & $\mathbf{1 1 , 7 7 8}$ & $\mathbf{1 3 , 7 4 1}$ \\
\hline \hline
\end{tabular}


Table A-258: Delayed technology reference case with credit and tariff extension cellulosic ethanol production [Million gallons]

\begin{tabular}{lrrrrrr} 
& $\mathbf{2 0 0 5}$ & $\mathbf{2 0 1 0}$ & $\mathbf{2 0 1 5}$ & $\mathbf{2 0 2 0}$ & $\mathbf{2 0 2 5}$ & $\mathbf{2 0 3 0}$ \\
Africa & 0 & 0 & 0 & 0 & 11 & 11 \\
Argentina & 0 & 0 & 59 & 306 & 335 & 335 \\
Australia & 0 & 0 & 66 & 247 & 622 & 1,172 \\
Brazil & 0 & 0 & 296 & 890 & 1,391 & 1,546 \\
Canada & 0 & 0 & 259 & 779 & 1,004 & 1,004 \\
Caribbean basin & 0 & 0 & 0 & 0 & 0 & 0 \\
China & 0 & 0 & 222 & 667 & 1,271 & 1,271 \\
Colombia & 0 & 0 & 170 & 512 & 1,118 & 1,272 \\
Eastern Europe & 0 & 0 & 80 & 261 & 670 & 897 \\
Former Soviet Union & 0 & 0 & 6 & 17 & 52 & 157 \\
India & 0 & 0 & 214 & 645 & 1,512 & 2,196 \\
Japan & 0 & 0 & 95 & 348 & 612 & 647 \\
Middle East & 0 & 0 & 0 & 0 & 0 & 0 \\
Mexico & 0 & 0 & 199 & 601 & 1,408 & 1,546 \\
Other Asia & 0 & 0 & 0 & 0 & 0 & 0 \\
South Korea & 0 & 0 & 35 & 35 & 35 & 35 \\
United States & 0 & 0 & 767 & 1,736 & 4,667 & 9,124 \\
Western Europe & 0 & 0 & 166 & 693 & 2,113 & 4,410 \\
\hline Total & $\mathbf{0}$ & $\mathbf{0}$ & $\mathbf{2 , 6 3 3}$ & $\mathbf{7 , 7 3 5}$ & $\mathbf{1 6 , 8 2 0}$ & $\mathbf{2 5 , 6 2 2}$ \\
\hline \hline
\end{tabular}

Table A-259: Delayed technology reference case with credit and tariff extension grain ethanol consumption [Million gallons]

\begin{tabular}{lrrrrrr} 
& $\mathbf{2 0 0 5}$ & $\mathbf{2 0 1 0}$ & $\mathbf{2 0 1 5}$ & $\mathbf{2 0 2 0}$ & $\mathbf{2 0 2 5}$ & $\mathbf{2 0 3 0}$ \\
Africa & 0 & 0 & 0 & 0 & 0 & 0 \\
Australia & 0 & 0 & 0 & 0 & 0 & 0 \\
Canada & 0 & 0 & 0 & 0 & 0 & 0 \\
China & 121 & 0 & 0 & 325 & 387 & 0 \\
Central and South America & 0 & 36 & 94 & 114 & 90 & 48 \\
Eastern Europe & 110 & 226 & 348 & 477 & 367 & 251 \\
Former Soviet Union & 0 & 0 & 0 & 0 & 0 & 0 \\
India & 0 & 0 & 0 & 0 & 0 & 0 \\
Japan & 0 & 192 & 267 & 382 & 91 & 0 \\
Middle East & 0 & 0 & 0 & 0 & 0 & 0 \\
Mexico & 0 & 0 & 0 & 0 & 0 & 0 \\
Other Asia & 0 & 0 & 0 & 0 & 0 & 0 \\
South Korea & 224 & 295 & 144 & 74 & 0 & 94 \\
United States & 3,850 & 11,668 & 15,000 & 15,000 & 15,000 & 15,000 \\
Western Europe & 600 & 2,568 & 2,996 & 2,705 & 3,178 & 3,604 \\
\hline Total & $\mathbf{4 , 9 0 4}$ & $\mathbf{1 4 , 9 8 4}$ & $\mathbf{1 8 , 8 5 0}$ & $\mathbf{1 9 , 0 7 7}$ & $\mathbf{1 9 , 1 1 4}$ & $\mathbf{1 8 , 9 9 7}$ \\
\hline \hline
\end{tabular}


Table A-260: Delayed technology reference case with credit and tariff extension sugar ethanol consumption [Million gallons]

\begin{tabular}{lrrrrrr} 
& $\mathbf{2 0 0 5}$ & $\mathbf{2 0 1 0}$ & $\mathbf{2 0 1 5}$ & $\mathbf{2 0 2 0}$ & $\mathbf{2 0 2 5}$ & $\mathbf{2 0 3 0}$ \\
Africa & 300 & 330 & 364 & 402 & 444 & 490 \\
Australia & 0 & 0 & 0 & 0 & 0 & 0 \\
Canada & 145 & 0 & 0 & 0 & 0 & 0 \\
China & 0 & 0 & 0 & 48 & 48 & 48 \\
Central and South America & 3,788 & 4,818 & 6,045 & 6,749 & 8,156 & 8,662 \\
Eastern Europe & 589 & 0 & 418 & 153 & 0 & 0 \\
Former Soviet Union & 0 & 0 & 0 & 0 & 0 & 0 \\
India & 313 & 279 & 327 & 347 & 381 & 348 \\
Japan & 263 & 96 & 48 & 0 & 0 & 453 \\
Middle East & 0 & 0 & 0 & 0 & 0 & 0 \\
Mexico & 0 & 0 & 0 & 0 & 0 & 0 \\
Other Asia & 0 & 0 & 0 & 0 & 0 & 0 \\
South Korea & 41 & 40 & 0 & 0 & 0 & 284 \\
United States & 311 & 0 & 788 & 2,689 & 2,749 & 1,454 \\
Western Europe & 136 & 1,607 & 1,054 & 0 & 0 & 2,003 \\
\hline Total & $\mathbf{5 , 8 8 4}$ & $\mathbf{7 , 1 6 9}$ & $\mathbf{9 , 0 4 4}$ & $\mathbf{1 0 , 3 8 7}$ & $\mathbf{1 1 , 7 7 8}$ & $\mathbf{1 3 , 7 4 1}$ \\
\hline \hline
\end{tabular}

Table A-261: Delayed technology reference case with credit and tariff extension cellulosic ethanol consumption [Million gallons]

\begin{tabular}{lrrrrrr} 
& $\mathbf{2 0 0 5}$ & $\mathbf{2 0 1 0}$ & $\mathbf{2 0 1 5}$ & $\mathbf{2 0 2 0}$ & $\mathbf{2 0 2 5}$ & $\mathbf{2 0 3 0}$ \\
Africa & 0 & 0 & 0 & 0 & 11 & 11 \\
Australia & 0 & 0 & 66 & 247 & 622 & 1,172 \\
Canada & 0 & 0 & 233 & 779 & 1,004 & 1,004 \\
China & 0 & 0 & 0 & 667 & 667 & 1,415 \\
Central and South America & 0 & 0 & 0 & 0 & 4 & 0 \\
Eastern Europe & 0 & 0 & 106 & 323 & 670 & 897 \\
Former Soviet Union & 0 & 0 & 6 & 17 & 52 & 157 \\
India & 0 & 0 & 214 & 645 & 1,512 & 2,051 \\
Japan & 0 & 0 & 95 & 348 & 948 & 647 \\
Middle East & 0 & 0 & 0 & 0 & 0 & 0 \\
Mexico & 0 & 0 & 30 & 78 & 183 & 201 \\
Other Asia & 0 & 0 & 0 & 0 & 0 & 0 \\
South Korea & 0 & 0 & 256 & 341 & 413 & 35 \\
United States & 0 & 0 & 1,365 & 2,258 & 6,264 & 13,622 \\
Western Europe & 0 & 0 & 262 & 2,032 & 4,472 & 4,410 \\
\hline Total & $\mathbf{0}$ & $\mathbf{0}$ & $\mathbf{2 , 6 3 3}$ & $\mathbf{7 , 7 3 5}$ & $\mathbf{1 6 , 8 2 0}$ & $\mathbf{2 5 , 6 2 2}$ \\
\hline \hline
\end{tabular}


Table A-262: Delayed technology reference case with credit and tariff extension grain ethanol net trade [Million gallons]

\begin{tabular}{lrrrrrr} 
& $\mathbf{2 0 0 5}$ & $\mathbf{2 0 1 0}$ & $\mathbf{2 0 1 5}$ & $\mathbf{2 0 2 0}$ & $\mathbf{2 0 2 5}$ & $\mathbf{2 0 3 0}$ \\
Africa & 0 & 0 & 0 & 0 & 0 & 0 \\
Australia & 0 & 0 & 0 & 0 & 0 & 0 \\
Canada & 0 & 0 & 0 & 0 & 0 & 0 \\
China & 224 & 344 & 344 & 0 & -387 & 0 \\
Central and South America & 0 & 143 & 475 & 455 & 479 & 94 \\
Eastern Europe & 0 & 0 & 0 & 0 & 0 & 0 \\
Former Soviet Union & 0 & 0 & 0 & 0 & 0 & 0 \\
India & 0 & 0 & 0 & 0 & 0 & 0 \\
Japan & 0 & -192 & -267 & -382 & -91 & 0 \\
Middle East & 0 & 0 & 0 & 0 & 0 & 0 \\
Mexico & 0 & 0 & 0 & 0 & 0 & 0 \\
Other Asia & 0 & 0 & 0 & 0 & 0 & 0 \\
South Korea & -224 & -295 & -144 & -74 & 0 & -94 \\
United States & 0 & 0 & 0 & 0 & 0 & 0 \\
Western Europe & 0 & 0 & -408 & 0 & 0 & 0 \\
\hline Total & $\mathbf{0}$ & $\mathbf{0}$ & $\mathbf{0}$ & $\mathbf{0}$ & $\mathbf{0}$ & $\mathbf{0}$ \\
\hline \hline
\end{tabular}

Table A-263: Delayed technology reference case with credit and tariff extension sugar ethanol net trade [Million gallons]

\begin{tabular}{lrrrrrr} 
& $\mathbf{2 0 0 5}$ & $\mathbf{2 0 1 0}$ & $\mathbf{2 0 1 5}$ & $\mathbf{2 0 2 0}$ & $\mathbf{2 0 2 5}$ & $\mathbf{2 0 3 0}$ \\
Africa & 0 & 0 & 0 & 0 & 0 & 0 \\
Australia & 0 & 0 & 0 & 0 & 0 & 0 \\
Canada & -145 & 0 & 0 & 0 & 0 & 0 \\
China & 0 & 40 & 48 & 0 & 0 & 0 \\
Central and South America & 1,484 & 1,702 & 2,260 & 2,841 & 2,749 & 4,193 \\
Eastern Europe & -589 & 0 & -418 & -153 & 0 & 0 \\
Former Soviet Union & 0 & 0 & 0 & 0 & 0 & 0 \\
India & 0 & 0 & 0 & 0 & 0 & 0 \\
Japan & -263 & -96 & -48 & 0 & 0 & -453 \\
Middle East & 0 & 0 & 0 & 0 & 0 & 0 \\
Mexico & 0 & 0 & 0 & 0 & 0 & 0 \\
Other Asia & 0 & 0 & 0 & 0 & 0 & 0 \\
South Korea & -41 & -40 & 0 & 0 & 0 & -284 \\
United States & -311 & 0 & -788 & $-2,689$ & $-2,749$ & $-1,454$ \\
Western Europe & -136 & $-1,607$ & $-1,054$ & 0 & 0 & $-2,003$ \\
\hline Total & $\mathbf{0}$ & $\mathbf{0}$ & $\mathbf{0}$ & $\mathbf{0}$ & $\mathbf{0}$ & $\mathbf{0}$ \\
\hline \hline
\end{tabular}


Table A-264: Delayed technology reference case with credit and tariff extension cellulosic ethanol net trade [Million gallons]

\begin{tabular}{lrrrrrr} 
& $\mathbf{2 0 0 5}$ & $\mathbf{2 0 1 0}$ & $\mathbf{2 0 1 5}$ & $\mathbf{2 0 2 0}$ & $\mathbf{2 0 2 5}$ & $\mathbf{2 0 3 0}$ \\
Africa & 0 & 0 & 0 & 0 & 0 & 0 \\
Australia & 0 & 0 & 0 & 0 & 0 & 0 \\
Canada & 0 & 0 & 26 & 0 & 0 & 0 \\
China & 0 & 0 & 222 & 0 & 603 & -144 \\
Central and South America & 0 & 0 & 525 & 1,707 & 2,841 & 3,153 \\
Eastern Europe & 0 & 0 & -26 & -62 & 0 & 0 \\
Former Soviet Union & 0 & 0 & 0 & 0 & 0 & 0 \\
India & 0 & 0 & 0 & 0 & 0 & 144 \\
Japan & 0 & 0 & 0 & 0 & -335 & 0 \\
Middle East & 0 & 0 & 0 & 0 & 0 & 0 \\
Mexico & 0 & 0 & 170 & 523 & 1,225 & 1,345 \\
Other Asia & 0 & 0 & 0 & 0 & 0 & 0 \\
South Korea & 0 & 0 & -222 & -306 & -378 & 0 \\
United States & 0 & 0 & -599 & -523 & $-1,597$ & $-4,498$ \\
Western Europe & 0 & 0 & -96 & $-1,339$ & $-2,358$ & 0 \\
\hline Total & $\mathbf{0}$ & $\mathbf{0}$ & $\mathbf{0}$ & $\mathbf{0}$ & $\mathbf{0}$ & $\mathbf{0}$ \\
\hline \hline
\end{tabular}

Table A-265: Delayed technology reference case with credit and tariff extension biomass-to-liquids production [Million gallons ethanol equivalent]

\begin{tabular}{lrrrrrr} 
& $\mathbf{2 0 0 5}$ & $\mathbf{2 0 1 0}$ & $\mathbf{2 0 1 5}$ & $\mathbf{2 0 2 0}$ & $\mathbf{2 0 2 5}$ & $\mathbf{2 0 3 0}$ \\
Africa & 0 & 0 & 0 & 0 & 0 & 0 \\
Argentina & 0 & 0 & 21 & 110 & 121 & 121 \\
Australia & 0 & 0 & 0 & 0 & 0 & 0 \\
Brazil & 0 & 0 & 106 & 320 & 554 & 556 \\
Canada & 0 & 0 & 93 & 280 & 361 & 361 \\
Caribbean basin & 0 & 0 & 0 & 0 & 0 & 0 \\
China & 0 & 0 & 80 & 240 & 457 & 457 \\
Colombia & 0 & 0 & 61 & 184 & 431 & 458 \\
Eastern Europe & 0 & 0 & 0 & 0 & 0 & 0 \\
Former Soviet Union & 0 & 0 & 0 & 0 & 0 & 0 \\
India & 0 & 0 & 77 & 232 & 544 & 790 \\
Japan & 0 & 0 & 0 & 0 & 0 & 0 \\
Middle East & 0 & 0 & 0 & 0 & 0 & 0 \\
Mexico & 0 & 0 & 72 & 216 & 506 & 556 \\
Other Asia & 0 & 0 & 0 & 0 & 0 & 0 \\
South Korea & 0 & 0 & 36 & 52 & 52 & 52 \\
United States & 0 & 0 & 786 & 3,253 & 3,349 & 3,264 \\
Western Europe & 0 & 0 & 0 & 0 & 0 & 0 \\
\hline Total & $\mathbf{0}$ & $\mathbf{0}$ & $\mathbf{1 , 3 3 2}$ & $\mathbf{4 , 8 8 7}$ & $\mathbf{6 , 3 7 5}$ & $\mathbf{6 , 6 1 4}$ \\
\hline \hline
\end{tabular}


Table A-266: Delayed technology reference case with credit and tariff extension biomass-to-liquids consumption [Million gallons ethanol equivalent]

\begin{tabular}{lrrrrrr} 
& $\mathbf{2 0 0 5}$ & $\mathbf{2 0 1 0}$ & $\mathbf{2 0 1 5}$ & $\mathbf{2 0 2 0}$ & $\mathbf{2 0 2 5}$ & $\mathbf{2 0 3 0}$ \\
Africa & 0 & 0 & 0 & 0 & 0 & 0 \\
Australia & 0 & 0 & 0 & 0 & 0 & 0 \\
Canada & 0 & 0 & 0 & 0 & 0 & 0 \\
China & 0 & 0 & 0 & 0 & 0 & 0 \\
Central and South America & 0 & 0 & 0 & 0 & 0 & 0 \\
Eastern Europe & 0 & 0 & 0 & 0 & 0 & 0 \\
Former Soviet Union & 0 & 0 & 0 & 0 & 0 & 0 \\
India & 0 & 0 & 0 & 0 & 0 & 0 \\
Japan & 0 & 0 & 0 & 0 & 0 & 0 \\
Middle East & 0 & 0 & 0 & 0 & 0 & 0 \\
Mexico & 0 & 0 & 0 & 0 & 0 & 0 \\
Other Asia & 0 & 0 & 0 & 0 & 0 & 0 \\
South Korea & 0 & 0 & 0 & 0 & 0 & 0 \\
United States & 0 & 0 & 1,059 & 3,913 & 6,375 & 6,614 \\
Western Europe & 0 & 0 & 273 & 974 & 0 & 0 \\
\hline Total & $\mathbf{0}$ & $\mathbf{0}$ & $\mathbf{1 , 3 3 2}$ & $\mathbf{4 , 8 8 7}$ & $\mathbf{6 , 3 7 5}$ & $\mathbf{6 , 6 1 4}$ \\
\hline \hline
\end{tabular}

Table A-267: Delayed technology reference case with credit and tariff extension biomass-to-liquids net trade [Million gallons ethanol equivalent]

\begin{tabular}{lrrrrrr} 
& $\mathbf{2 0 0 5}$ & $\mathbf{2 0 1 0}$ & $\mathbf{2 0 1 5}$ & $\mathbf{2 0 2 0}$ & $\mathbf{2 0 2 5}$ & $\mathbf{2 0 3 0}$ \\
Africa & 0 & 0 & 0 & 0 & 0 & 0 \\
Australia & 0 & 0 & 0 & 0 & 0 & 0 \\
Canada & 0 & 0 & 93 & 280 & 361 & 361 \\
China & 0 & 0 & 80 & 240 & 457 & 457 \\
Central and South America & 0 & 0 & 189 & 614 & 1,106 & 1,134 \\
Eastern Europe & 0 & 0 & 0 & 0 & 0 & 0 \\
Former Soviet Union & 0 & 0 & 0 & 0 & 0 & 0 \\
India & 0 & 0 & 77 & 232 & 544 & 790 \\
Japan & 0 & 0 & 0 & 0 & 0 & 0 \\
Middle East & 0 & 0 & 0 & 0 & 0 & 0 \\
Mexico & 0 & 0 & 72 & 216 & 506 & 556 \\
Other Asia & 0 & 0 & 0 & 0 & 0 & 0 \\
South Korea & 0 & 0 & 36 & 52 & 52 & 52 \\
United States & 0 & 0 & -273 & -660 & $-3,026$ & $-3,350$ \\
Western Europe & 0 & 0 & -273 & -974 & 0 & 0 \\
\hline Total & $\mathbf{0}$ & $\mathbf{0}$ & $\mathbf{0}$ & $\mathbf{0}$ & $\mathbf{0}$ & $\mathbf{0}$ \\
\hline \hline
\end{tabular}


Table A-268: Delayed technology reference case with credit and tariff extension biodiesel production [Million gallons ethanol equivalent]

\begin{tabular}{lrrrrrr} 
& $\mathbf{2 0 0 5}$ & $\mathbf{2 0 1 0}$ & $\mathbf{2 0 1 5}$ & $\mathbf{2 0 2 0}$ & $\mathbf{2 0 2 5}$ & $\mathbf{2 0 3 0}$ \\
Africa & 0 & 0 & 0 & 0 & 0 & 0 \\
Argentina & 0 & 333 & 776 & 819 & 827 & 651 \\
Australia & 0 & 0 & 0 & 0 & 0 & 0 \\
Brazil & 0 & 333 & 835 & 1,600 & 2,103 & 1,334 \\
Canada & 0 & 0 & 0 & 0 & 0 & 0 \\
Caribbean basin & 0 & 95 & 310 & 675 & 802 & 898 \\
China & 0 & 0 & 0 & 0 & 0 & 0 \\
Colombia & 0 & 119 & 298 & 474 & 650 & 782 \\
Eastern Europe & 0 & 0 & 0 & 0 & 0 & 0 \\
Former Soviet Union & 0 & 0 & 0 & 0 & 0 & 0 \\
India & 0 & 0 & 0 & 0 & 0 & 0 \\
Japan & 0 & 0 & 0 & 0 & 0 & 0 \\
Middle East & 0 & 0 & 0 & 0 & 0 & 0 \\
Mexico & 0 & 0 & 0 & 0 & 0 & 0 \\
Other Asia & 0 & 0 & 0 & 0 & 0 & 0 \\
South Korea & 0 & 0 & 0 & 0 & 0 & 0 \\
United States & 0 & 1,182 & 1,640 & 1,747 & 1,907 & 2,072 \\
Western Europe & 998 & 3,178 & 3,578 & 3,930 & 4,468 & 4,788 \\
\hline Total & $\mathbf{9 9 8}$ & $\mathbf{5 , 2 3 8}$ & $\mathbf{7 , 4 3 7}$ & $\mathbf{9 , 2 4 5}$ & $\mathbf{1 0 , 7 5 7}$ & $\mathbf{1 0 , 5 2 5}$ \\
\hline \hline
\end{tabular}

Table A-269: Delayed technology reference case with credit and tariff extension biodiesel consumption [Million gallons ethanol equivalent]

\begin{tabular}{lrrrrrr} 
& $\mathbf{2 0 0 5}$ & $\mathbf{2 0 1 0}$ & $\mathbf{2 0 1 5}$ & $\mathbf{2 0 2 0}$ & $\mathbf{2 0 2 5}$ & $\mathbf{2 0 3 0}$ \\
Africa & 0 & 0 & 0 & 0 & 0 & 0 \\
Australia & 0 & 0 & 0 & 0 & 0 & 0 \\
Canada & 0 & 0 & 0 & 0 & 0 & 0 \\
China & 0 & 0 & 0 & 0 & 0 & 0 \\
Central and South America & 0 & 158 & 472 & 984 & 1,306 & 1,490 \\
Eastern Europe & 0 & 0 & 0 & 0 & 0 & 0 \\
Former Soviet Union & 0 & 0 & 0 & 0 & 0 & 0 \\
India & 0 & 0 & 0 & 0 & 0 & 0 \\
Japan & 0 & 0 & 0 & 0 & 0 & 0 \\
Middle East & 0 & 0 & 0 & 0 & 0 & 0 \\
Mexico & 0 & 0 & 0 & 0 & 0 & 0 \\
Other Asia & 0 & 0 & 0 & 0 & 0 & 0 \\
South Korea & 0 & 0 & 0 & 0 & 0 & 0 \\
United States & 0 & 1,182 & 1,713 & 1,791 & 2,216 & 3,546 \\
Western Europe & 998 & 3,898 & 5,252 & 6,471 & 7,235 & 5,489 \\
\hline Total & $\mathbf{9 9 8}$ & $\mathbf{5 , 2 3 8}$ & $\mathbf{7 , 4 3 7}$ & $\mathbf{9 , 2 4 5}$ & $\mathbf{1 0 , 7 5 7}$ & $\mathbf{1 0 , 5 2 5}$ \\
\hline \hline
\end{tabular}


Table A-270: Delayed technology reference case with credit and tariff extension biodiesel net trade [Million gallons ethanol equivalent]

\begin{tabular}{lrrrrrr} 
& $\mathbf{2 0 0 5}$ & $\mathbf{2 0 1 0}$ & $\mathbf{2 0 1 5}$ & $\mathbf{2 0 2 0}$ & $\mathbf{2 0 2 5}$ & $\mathbf{2 0 3 0}$ \\
Africa & 0 & 0 & 0 & 0 & 0 & 0 \\
Australia & 0 & 0 & 0 & 0 & 0 & 0 \\
Canada & 0 & 0 & 0 & 0 & 0 & 0 \\
China & 0 & 0 & 0 & 0 & 0 & 0 \\
Central and South America & 0 & 720 & 1,746 & 2,584 & 3,076 & 2,175 \\
Eastern Europe & 0 & 0 & 0 & 0 & 0 & 0 \\
Former Soviet Union & 0 & 0 & 0 & 0 & 0 & 0 \\
India & 0 & 0 & 0 & 0 & 0 & 0 \\
Japan & 0 & 0 & 0 & 0 & 0 & 0 \\
Middle East & 0 & 0 & 0 & 0 & 0 & 0 \\
Mexico & 0 & 0 & 0 & 0 & 0 & 0 \\
Other Asia & 0 & 0 & 0 & 0 & 0 & 0 \\
South Korea & 0 & 0 & 0 & 0 & 0 & 0 \\
United States & 0 & 0 & -73 & -43 & -310 & $-1,474$ \\
Western Europe & 0 & -720 & $-1,674$ & $-2,541$ & $-2,767$ & -701 \\
\hline Total & $\mathbf{0}$ & $\mathbf{0}$ & $\mathbf{0}$ & $\mathbf{0}$ & $\mathbf{0}$ & $\mathbf{0}$ \\
\hline \hline
\end{tabular}




\section{A-16 Delayed Technology Reference Case with Credit Extension}

Table A-271: Delayed technology reference case with credit extension ethanol production [Million gallons]

\begin{tabular}{lrrrrrr} 
& $\mathbf{2 0 0 5}$ & $\mathbf{2 0 1 0}$ & $\mathbf{2 0 1 5}$ & $\mathbf{2 0 2 0}$ & $\mathbf{2 0 2 5}$ & $\mathbf{2 0 3 0}$ \\
Africa & 300 & 330 & 364 & 402 & 455 & 502 \\
Argentina & 0 & 178 & 699 & 946 & 975 & 987 \\
Australia & 0 & 0 & 66 & 247 & 622 & 1,172 \\
Brazil & 4,866 & 5,977 & 7,624 & 9,452 & 11,347 & 13,363 \\
Canada & 0 & 0 & 259 & 779 & 1,004 & 1,004 \\
Caribbean basin & 210 & 328 & 683 & 683 & 775 & 775 \\
China & 344 & 384 & 614 & 1,041 & 1,549 & 1,549 \\
Colombia & 196 & 215 & 411 & 782 & 1,501 & 2,231 \\
Eastern Europe & 110 & 226 & 434 & 780 & 1,037 & 1,149 \\
Former Soviet Union & 0 & 0 & 6 & 17 & 52 & 157 \\
India & 313 & 279 & 541 & 992 & 1,893 & 2,543 \\
Japan & 0 & 0 & 95 & 348 & 612 & 646 \\
Middle East & 0 & 0 & 0 & 0 & 0 & 0 \\
Mexico & 0 & 0 & 199 & 601 & 1,408 & 1,546 \\
Other Asia & 0 & 0 & 0 & 0 & 0 & 0 \\
South Korea & 0 & 0 & 35 & 35 & 35 & 35 \\
United States & 3,850 & 11,668 & 15,767 & 16,736 & 19,559 & 23,155 \\
Western Europe & 600 & 2,568 & 2,755 & 3,698 & 5,291 & 8,013 \\
\hline Total & $\mathbf{1 0 , 7 8 9}$ & $\mathbf{2 2 , 1 5 4}$ & $\mathbf{3 0 , 5 5 1}$ & $\mathbf{3 7 , 5 3 6}$ & $\mathbf{4 8 , 1 1 3}$ & $\mathbf{5 8 , 8 2 6}$ \\
\hline \hline
\end{tabular}


Table A-272: Delayed technology reference case with credit extension ethanol consumption [Million gallons]

\begin{tabular}{lrrrrrr} 
& $\mathbf{2 0 0 5}$ & $\mathbf{2 0 1 0}$ & $\mathbf{2 0 1 5}$ & $\mathbf{2 0 2 0}$ & $\mathbf{2 0 2 5}$ & $\mathbf{2 0 3 0}$ \\
Africa & 300 & 330 & 364 & 402 & 455 & 502 \\
Australia & 0 & 0 & 66 & 247 & 622 & 1,172 \\
Canada & 145 & 0 & 233 & 779 & 1,004 & 1,020 \\
China & 121 & 0 & 0 & 903 & 1,100 & 1,374 \\
Central and South America & 3,788 & 4,853 & 6,147 & 6,858 & 8,237 & 8,863 \\
Eastern Europe & 699 & 226 & 872 & 953 & 1,037 & 1,149 \\
Former Soviet Union & 0 & 0 & 6 & 17 & 52 & 157 \\
India & 313 & 279 & 541 & 992 & 1,893 & 2,399 \\
Japan & 263 & 288 & 368 & 698 & 1,007 & 1,099 \\
Middle East & 0 & 0 & 0 & 0 & 0 & 0 \\
Mexico & 0 & 0 & 30 & 78 & 183 & 201 \\
Other Asia & 0 & 0 & 0 & 0 & 0 & 0 \\
South Korea & 264 & 335 & 400 & 414 & 413 & 413 \\
United States & 4,161 & 11,668 & 17,249 & 19,947 & 24,487 & 31,676 \\
Western Europe & 736 & 4,174 & 4,276 & 5,249 & 7,624 & 8,803 \\
\hline Total & $\mathbf{1 0 , 7 8 9}$ & $\mathbf{2 2 , 1 5 4}$ & $\mathbf{3 0 , 5 5 1}$ & $\mathbf{3 7 , 5 3 6}$ & $\mathbf{4 8 , 1 1 3}$ & $\mathbf{5 8 , 8 2 6}$ \\
\hline \hline
\end{tabular}

Table A-273: Delayed technology reference case with credit extension ethanol net trade [Million gallons]

\begin{tabular}{lrrrrrr} 
Africa & 0 & 0 & 0 & 0 & 0 & 0 \\
Australia & 0 & 0 & 0 & 0 & 0 & 0 \\
Canada & -145 & 0 & 26 & 0 & 0 & -16 \\
China & 224 & 384 & 614 & 138 & 449 & 175 \\
Central and South America & 1,484 & 1,845 & 3,271 & 5,005 & 6,361 & 8,493 \\
Eastern Europe & -589 & 0 & -438 & -173 & 0 & 0 \\
Former Soviet Union & 0 & 0 & 0 & 0 & 0 & 0 \\
India & 0 & 0 & 0 & 0 & 0 & 144 \\
Japan & -263 & -288 & -273 & -350 & -395 & -453 \\
Middle East & 0 & 0 & 0 & 0 & 0 & 0 \\
Mexico & 0 & 0 & 170 & 523 & 1,225 & 1,345 \\
Other Asia & 0 & 0 & 0 & 0 & 0 & 0 \\
South Korea & -264 & -335 & -365 & -379 & -378 & -378 \\
United States & -311 & 0 & $-1,482$ & $-3,211$ & $-4,928$ & $-8,521$ \\
Western Europe & -136 & $-1,607$ & $-1,521$ & $-1,551$ & $-2,333$ & -790 \\
\hline Total & $\mathbf{0}$ & $\mathbf{0}$ & $\mathbf{0}$ & $\mathbf{0}$ & $\mathbf{0}$ & $\mathbf{0}$ \\
\hline \hline
\end{tabular}


Table A-274: Delayed technology reference case with credit extension grain ethanol production [Million gallons]

\begin{tabular}{lrrrrrr} 
& $\mathbf{2 0 0 5}$ & $\mathbf{2 0 1 0}$ & $\mathbf{2 0 1 5}$ & $\mathbf{2 0 2 0}$ & $\mathbf{2 0 2 5}$ & $\mathbf{2 0 3 0}$ \\
Africa & 0 & 0 & 0 & 0 & 0 & 0 \\
Argentina & 0 & 178 & 640 & 640 & 640 & 640 \\
Australia & 0 & 0 & 0 & 0 & 0 & 0 \\
Brazil & 0 & 0 & 0 & 0 & 0 & 0 \\
Canada & 0 & 0 & 0 & 0 & 0 & 0 \\
Caribbean basin & 0 & 0 & 0 & 0 & 0 & 0 \\
China & 344 & 344 & 344 & 325 & 0 & 0 \\
Colombia & 0 & 0 & 0 & 0 & 0 & 0 \\
Eastern Europe & 110 & 226 & 348 & 477 & 367 & 251 \\
Former Soviet Union & 0 & 0 & 0 & 0 & 0 & 0 \\
India & 0 & 0 & 0 & 0 & 0 & 0 \\
Japan & 0 & 0 & 0 & 0 & 0 & 0 \\
Middle East & 0 & 0 & 0 & 0 & 0 & 0 \\
Mexico & 0 & 0 & 0 & 0 & 0 & 0 \\
Other Asia & 0 & 0 & 0 & 0 & 0 & 0 \\
South Korea & 0 & 0 & 0 & 0 & 0 & 0 \\
United States & 3,850 & 11,668 & 15,000 & 15,000 & 15,000 & 14,868 \\
Western Europe & 600 & 2,568 & 2,589 & 3,005 & 3,178 & 3,604 \\
\hline Total & $\mathbf{4 , 9 0 4}$ & $\mathbf{1 4 , 9 8 4}$ & $\mathbf{1 8 , 9 2 1}$ & $\mathbf{1 9 , 4 4 8}$ & $\mathbf{1 9 , 1 8 5}$ & $\mathbf{1 9 , 3 6 3}$ \\
\hline \hline
\end{tabular}

Table A-275: Delayed technology reference case with credit extension sugar ethanol production [Million gallons]

\begin{tabular}{lrrrrrr} 
& $\mathbf{2 0 0 5}$ & $\mathbf{2 0 1 0}$ & $\mathbf{2 0 1 5}$ & $\mathbf{2 0 2 0}$ & $\mathbf{2 0 2 5}$ & $\mathbf{2 0 3 0}$ \\
Africa & 300 & 330 & 364 & 402 & 444 & 490 \\
Argentina & 0 & 0 & 0 & 0 & 0 & 0 \\
Australia & 0 & 0 & 0 & 0 & 0 & 0 \\
Brazil & 4,866 & 5,977 & 7,329 & 8,562 & 9,806 & 11,818 \\
Canada & 0 & 0 & 0 & 0 & 0 & 0 \\
Caribbean basin & 210 & 328 & 683 & 683 & 775 & 775 \\
China & 0 & 40 & 48 & 48 & 48 & 48 \\
Colombia & 196 & 215 & 241 & 270 & 302 & 338 \\
Eastern Europe & 0 & 0 & 0 & 0 & 0 & 0 \\
Former Soviet Union & 0 & 0 & 0 & 0 & 0 & 0 \\
India & 313 & 279 & 327 & 347 & 381 & 348 \\
Japan & 0 & 0 & 0 & 0 & 0 & 0 \\
Middle East & 0 & 0 & 0 & 0 & 0 & 0 \\
Mexico & 0 & 0 & 0 & 0 & 0 & 0 \\
Other Asia & 0 & 0 & 0 & 0 & 0 & 0 \\
South Korea & 0 & 0 & 0 & 0 & 0 & 0 \\
United States & 0 & 0 & 0 & 0 & 0 & 0 \\
Western Europe & 0 & 0 & 0 & 0 & 0 & 0 \\
\hline Total & $\mathbf{5 , 8 8 4}$ & $\mathbf{7 , 1 6 9}$ & $\mathbf{8 , 9 9 2}$ & $\mathbf{1 0 , 3 1 2}$ & $\mathbf{1 1 , 7 5 6}$ & $\mathbf{1 3 , 8 1 6}$ \\
\hline \hline
\end{tabular}


Table A-276: Delayed technology reference case with credit extension cellulosic ethanol production [Million gallons]

\begin{tabular}{lrrrrrr} 
& $\mathbf{2 0 0 5}$ & $\mathbf{2 0 1 0}$ & $\mathbf{2 0 1 5}$ & $\mathbf{2 0 2 0}$ & $\mathbf{2 0 2 5}$ & $\mathbf{2 0 3 0}$ \\
Africa & 0 & 0 & 0 & 0 & 11 & 11 \\
Argentina & 0 & 0 & 59 & 306 & 335 & 347 \\
Australia & 0 & 0 & 66 & 247 & 622 & 1,172 \\
Brazil & 0 & 0 & 296 & 890 & 1,541 & 1,546 \\
Canada & 0 & 0 & 259 & 779 & 1,004 & 1,004 \\
Caribbean basin & 0 & 0 & 0 & 0 & 0 & 0 \\
China & 0 & 0 & 222 & 667 & 1,501 & 1,501 \\
Colombia & 0 & 0 & 170 & 512 & 1,199 & 1,893 \\
Eastern Europe & 0 & 0 & 85 & 303 & 670 & 897 \\
Former Soviet Union & 0 & 0 & 6 & 17 & 52 & 157 \\
India & 0 & 0 & 214 & 645 & 1,512 & 2,196 \\
Japan & 0 & 0 & 95 & 348 & 612 & 646 \\
Middle East & 0 & 0 & 0 & 0 & 0 & 0 \\
Mexico & 0 & 0 & 199 & 601 & 1,408 & 1,546 \\
Other Asia & 0 & 0 & 0 & 0 & 0 & 0 \\
South Korea & 0 & 0 & 35 & 35 & 35 & 35 \\
United States & 0 & 0 & 767 & 1,736 & 4,559 & 8,288 \\
Western Europe & 0 & 0 & 166 & 693 & 2,113 & 4,410 \\
\hline Total & $\mathbf{0}$ & $\mathbf{0}$ & $\mathbf{2 , 6 3 8}$ & $\mathbf{7 , 7 7 7}$ & $\mathbf{1 7 , 1 7 2}$ & $\mathbf{2 5 , 6 4 7}$ \\
\hline \hline
\end{tabular}

Table A-277: Delayed technology reference case with credit extension grain ethanol consumption [Million gallons]

\begin{tabular}{lrrrrrr} 
& $\mathbf{2 0 0 5}$ & $\mathbf{2 0 1 0}$ & $\mathbf{2 0 1 5}$ & $\mathbf{2 0 2 0}$ & $\mathbf{2 0 2 5}$ & $\mathbf{2 0 3 0}$ \\
Africa & 0 & 0 & 0 & 0 & 0 & 0 \\
Australia & 0 & 0 & 0 & 0 & 0 & 0 \\
Canada & 0 & 0 & 0 & 0 & 0 & 0 \\
China & 121 & 0 & 0 & 263 & 0 & 0 \\
Central and South America & 0 & 36 & 105 & 123 & 98 & 99 \\
Eastern Europe & 110 & 226 & 348 & 477 & 367 & 251 \\
Former Soviet Union & 0 & 0 & 0 & 0 & 0 & 0 \\
India & 0 & 0 & 0 & 0 & 0 & 0 \\
Japan & 0 & 192 & 225 & 62 & 0 & 163 \\
Middle East & 0 & 0 & 0 & 0 & 0 & 0 \\
Mexico & 0 & 0 & 0 & 0 & 0 & 0 \\
Other Asia & 0 & 0 & 0 & 0 & 0 & 0 \\
South Korea & 224 & 295 & 144 & 0 & 0 & 378 \\
United States & 3,850 & 11,668 & 15,000 & 15,000 & 15,000 & 14,868 \\
Western Europe & 600 & 2,568 & 3,099 & 3,522 & 3,720 & 3,604 \\
\hline Total & $\mathbf{4 , 9 0 4}$ & $\mathbf{1 4 , 9 8 4}$ & $\mathbf{1 8 , 9 2 1}$ & $\mathbf{1 9 , 4 4 8}$ & $\mathbf{1 9 , 1 8 5}$ & $\mathbf{1 9 , 3 6 3}$ \\
\hline \hline
\end{tabular}


Table A-278: Delayed technology reference case with credit extension sugar ethanol consumption [Million gallons]

\begin{tabular}{lrrrrrr} 
& $\mathbf{2 0 0 5}$ & $\mathbf{2 0 1 0}$ & $\mathbf{2 0 1 5}$ & $\mathbf{2 0 2 0}$ & $\mathbf{2 0 2 5}$ & $\mathbf{2 0 3 0}$ \\
Africa & 300 & 330 & 364 & 402 & 444 & 490 \\
Australia & 0 & 0 & 0 & 0 & 0 & 0 \\
Canada & 145 & 0 & 0 & 0 & 0 & 16 \\
China & 0 & 0 & 0 & 48 & 48 & 48 \\
Central and South America & 3,788 & 4,818 & 6,042 & 6,589 & 7,790 & 8,764 \\
Eastern Europe & 589 & 0 & 412 & 173 & 0 & 0 \\
Former Soviet Union & 0 & 0 & 0 & 0 & 0 & 0 \\
India & 313 & 279 & 327 & 347 & 381 & 348 \\
Japan & 263 & 96 & 48 & 0 & 0 & 146 \\
Middle East & 0 & 0 & 0 & 0 & 0 & 0 \\
Mexico & 0 & 0 & 0 & 0 & 0 & 0 \\
Other Asia & 0 & 0 & 0 & 0 & 0 & 0 \\
South Korea & 41 & 40 & 0 & 0 & 0 & 0 \\
United States & 311 & 0 & 788 & 2,753 & 3,093 & 3,091 \\
Western Europe & 136 & 1,607 & 1,011 & 0 & 0 & 915 \\
\hline Total & $\mathbf{5 , 8 8 4}$ & $\mathbf{7 , 1 6 9}$ & $\mathbf{8 , 9 9 2}$ & $\mathbf{1 0 , 3 1 2}$ & $\mathbf{1 1 , 7 5 6}$ & $\mathbf{1 3 , 8 1 6}$ \\
\hline \hline
\end{tabular}

Table A-279: Delayed technology reference case with credit extension cellulosic ethanol consumption [Million gallons]

\begin{tabular}{lllrrrr} 
Africa & 0 & 0 & 0 & 0 & 11 & 11 \\
Australia & 0 & 0 & 66 & 247 & 622 & 1,172 \\
Canada & 0 & 0 & 233 & 779 & 1,004 & 1,004 \\
China & 0 & 0 & 0 & 592 & 1,052 & 1,326 \\
Central and South America & 0 & 0 & 0 & 146 & 350 & 0 \\
Eastern Europe & 0 & 0 & 111 & 303 & 670 & 897 \\
Former Soviet Union & 0 & 0 & 6 & 17 & 52 & 157 \\
India & 0 & 0 & 214 & 645 & 1,512 & 2,051 \\
Japan & 0 & 0 & 95 & 636 & 1,007 & 790 \\
Middle East & 0 & 0 & 0 & 0 & 0 & 0 \\
Mexico & 0 & 0 & 30 & 78 & 183 & 201 \\
Other Asia & 0 & 0 & 0 & 0 & 0 & 0 \\
South Korea & 0 & 0 & 256 & 414 & 413 & 35 \\
United States & 0 & 0 & 1,461 & 2,194 & 6,394 & 13,718 \\
Western Europe & 0 & 0 & 166 & 1,727 & 3,903 & 4,285 \\
\hline Total & $\mathbf{0}$ & $\mathbf{0}$ & $\mathbf{2 , 6 3 8}$ & $\mathbf{7 , 7 7 7}$ & $\mathbf{1 7 , 1 7 2}$ & $\mathbf{2 5 , 6 4 7}$ \\
\hline \hline
\end{tabular}


Table A-280: Delayed technology reference case with credit extension grain ethanol net trade [Million gallons]

\begin{tabular}{lrrrrrr} 
& $\mathbf{2 0 0 5}$ & $\mathbf{2 0 1 0}$ & $\mathbf{2 0 1 5}$ & $\mathbf{2 0 2 0}$ & $\mathbf{2 0 2 5}$ & $\mathbf{2 0 3 0}$ \\
Africa & 0 & 0 & 0 & 0 & 0 & 0 \\
Australia & 0 & 0 & 0 & 0 & 0 & 0 \\
Canada & 0 & 0 & 0 & 0 & 0 & 0 \\
China & 224 & 344 & 344 & 62 & 0 & 0 \\
Central and South America & 0 & 143 & 535 & 517 & 542 & 541 \\
Eastern Europe & 0 & 0 & 0 & 0 & 0 & 0 \\
Former Soviet Union & 0 & 0 & 0 & 0 & 0 & 0 \\
India & 0 & 0 & 0 & 0 & 0 & 0 \\
Japan & 0 & -192 & -225 & -62 & 0 & -163 \\
Middle East & 0 & 0 & 0 & 0 & 0 & 0 \\
Mexico & 0 & 0 & 0 & 0 & 0 & 0 \\
Other Asia & 0 & 0 & 0 & 0 & 0 & 0 \\
South Korea & -224 & -295 & -144 & 0 & 0 & -378 \\
United States & 0 & 0 & 0 & 0 & 0 & 0 \\
Western Europe & 0 & 0 & -510 & -517 & -542 & 0 \\
\hline Total & $\mathbf{0}$ & $\mathbf{0}$ & $\mathbf{0}$ & $\mathbf{0}$ & $\mathbf{0}$ & $\mathbf{0}$ \\
\hline \hline
\end{tabular}

Table A-281: Delayed technology reference case with credit extension sugar ethanol net trade [Million gallons]

\begin{tabular}{lrrrrrr} 
Africa & 0 & 0 & 0 & 0 & 0 & 0 \\
Australia & 0 & 0 & 0 & 0 & 0 & 0 \\
Canada & -145 & 0 & 0 & 0 & 0 & -16 \\
China & 0 & 40 & 48 & 0 & 0 & 0 \\
Central and South America & 1,484 & 1,702 & 2,211 & 2,926 & 3,093 & 4,167 \\
Eastern Europe & -589 & 0 & -412 & -173 & 0 & 0 \\
Former Soviet Union & 0 & 0 & 0 & 0 & 0 & 0 \\
India & 0 & 0 & 0 & 0 & 0 & 0 \\
Japan & -263 & -96 & -48 & 0 & 0 & -146 \\
Middle East & 0 & 0 & 0 & 0 & 0 & 0 \\
Mexico & 0 & 0 & 0 & 0 & 0 & 0 \\
Other Asia & 0 & 0 & 0 & 0 & 0 & 0 \\
South Korea & -41 & -40 & 0 & 0 & 0 & 0 \\
United States & -311 & 0 & -788 & $-2,753$ & $-3,093$ & $-3,091$ \\
Western Europe & -136 & $-1,607$ & $-1,011$ & 0 & 0 & -915 \\
\hline Total & $\mathbf{0}$ & $\mathbf{0}$ & $\mathbf{0}$ & $\mathbf{0}$ & $\mathbf{0}$ & $\mathbf{0}$ \\
\hline \hline
\end{tabular}


Table A-282: Delayed technology reference case with credit extension cellulosic ethanol net trade [Million gallons]

\begin{tabular}{lrrrrrr} 
& $\mathbf{2 0 0 5}$ & $\mathbf{2 0 1 0}$ & $\mathbf{2 0 1 5}$ & $\mathbf{2 0 2 0}$ & $\mathbf{2 0 2 5}$ & $\mathbf{2 0 3 0}$ \\
Africa & 0 & 0 & 0 & 0 & 0 & 0 \\
Australia & 0 & 0 & 0 & 0 & 0 & 0 \\
Canada & 0 & 0 & 26 & 0 & 0 & 0 \\
China & 0 & 0 & 222 & 75 & 449 & 175 \\
Central and South America & 0 & 0 & 525 & 1,561 & 2,726 & 3,786 \\
Eastern Europe & 0 & 0 & -26 & 0 & 0 & 0 \\
Former Soviet Union & 0 & 0 & 0 & 0 & 0 & 0 \\
India & 0 & 0 & 0 & 0 & 0 & 144 \\
Japan & 0 & 0 & 0 & -288 & -395 & -144 \\
Middle East & 0 & 0 & 0 & 0 & 0 & 0 \\
Mexico & 0 & 0 & 170 & 523 & 1,225 & 1,345 \\
Other Asia & 0 & 0 & 0 & 0 & 0 & 0 \\
South Korea & 0 & 0 & -222 & -379 & -378 & 0 \\
United States & 0 & 0 & -694 & -458 & $-1,835$ & $-5,430$ \\
Western Europe & 0 & 0 & 0 & $-1,034$ & $-1,790$ & 125 \\
\hline Total & $\mathbf{0}$ & $\mathbf{0}$ & $\mathbf{0}$ & $\mathbf{0}$ & $\mathbf{0}$ & $\mathbf{0}$ \\
\hline \hline
\end{tabular}

Table A-283: Delayed technology reference case with credit extension biomass-toliquids production [Million gallons ethanol equivalent]

\begin{tabular}{lrrrrrr} 
& $\mathbf{2 0 0 5}$ & $\mathbf{2 0 1 0}$ & $\mathbf{2 0 1 5}$ & $\mathbf{2 0 2 0}$ & $\mathbf{2 0 2 5}$ & $\mathbf{2 0 3 0}$ \\
Africa & 0 & 0 & 0 & 0 & 0 & 0 \\
Argentina & 0 & 0 & 21 & 110 & 121 & 125 \\
Australia & 0 & 0 & 0 & 0 & 0 & 0 \\
Brazil & 0 & 0 & 106 & 320 & 554 & 556 \\
Canada & 0 & 0 & 93 & 280 & 361 & 361 \\
Caribbean basin & 0 & 0 & 0 & 0 & 0 & 0 \\
China & 0 & 0 & 80 & 240 & 477 & 477 \\
Colombia & 0 & 0 & 61 & 184 & 431 & 681 \\
Eastern Europe & 0 & 0 & 0 & 0 & 0 & 0 \\
Former Soviet Union & 0 & 0 & 0 & 0 & 0 & 0 \\
India & 0 & 0 & 77 & 232 & 544 & 790 \\
Japan & 0 & 0 & 0 & 0 & 0 & 0 \\
Middle East & 0 & 0 & 0 & 0 & 0 & 0 \\
Mexico & 0 & 0 & 72 & 216 & 506 & 556 \\
Other Asia & 0 & 0 & 0 & 0 & 0 & 0 \\
South Korea & 0 & 0 & 36 & 52 & 52 & 52 \\
United States & 0 & 0 & 786 & 2,532 & 3,407 & 5,031 \\
Western Europe & 0 & 0 & 0 & 0 & 0 & 0 \\
\hline Total & $\mathbf{0}$ & $\mathbf{0}$ & $\mathbf{1 , 3 3 2}$ & $\mathbf{4 , 1 6 6}$ & $\mathbf{6 , 4 5 3}$ & $\mathbf{8 , 6 2 8}$ \\
\hline \hline
\end{tabular}


Table A-284: Delayed technology reference case with credit extension biomass-toliquids consumption [Million gallons ethanol equivalent]

\begin{tabular}{lrrrrrr} 
& $\mathbf{2 0 0 5}$ & $\mathbf{2 0 1 0}$ & $\mathbf{2 0 1 5}$ & $\mathbf{2 0 2 0}$ & $\mathbf{2 0 2 5}$ & $\mathbf{2 0 3 0}$ \\
Africa & 0 & 0 & 0 & 0 & 0 & 0 \\
Australia & 0 & 0 & 0 & 0 & 0 & 0 \\
Canada & 0 & 0 & 0 & 0 & 0 & 0 \\
China & 0 & 0 & 0 & 0 & 0 & 0 \\
Central and South America & 0 & 0 & 0 & 0 & 0 & 0 \\
Eastern Europe & 0 & 0 & 0 & 0 & 0 & 0 \\
Former Soviet Union & 0 & 0 & 0 & 0 & 0 & 0 \\
India & 0 & 0 & 0 & 0 & 0 & 0 \\
Japan & 0 & 0 & 0 & 0 & 0 & 0 \\
Middle East & 0 & 0 & 0 & 0 & 0 & 0 \\
Mexico & 0 & 0 & 0 & 0 & 0 & 0 \\
Other Asia & 0 & 0 & 0 & 0 & 0 & 0 \\
South Korea & 0 & 0 & 0 & 0 & 0 & 0 \\
United States & 0 & 0 & 1,034 & 4,078 & 6,453 & 6,517 \\
Western Europe & 0 & 0 & 298 & 88 & 0 & 0 \\
\hline Total & $\mathbf{0}$ & $\mathbf{0}$ & $\mathbf{1 , 3 3 2}$ & $\mathbf{4 , 1 6 6}$ & $\mathbf{6 , 4 5 3}$ & $\mathbf{6 , 5 1 7}$ \\
\hline \hline
\end{tabular}

Table A-285: Delayed technology reference case with credit extension biomass-toliquids net trade [Million gallons ethanol equivalent]

\begin{tabular}{lrrrrrr} 
& $\mathbf{2 0 0 5}$ & $\mathbf{2 0 1 0}$ & $\mathbf{2 0 1 5}$ & $\mathbf{2 0 2 0}$ & $\mathbf{2 0 2 5}$ & $\mathbf{2 0 3 0}$ \\
Africa & 0 & 0 & 0 & 0 & 0 & 0 \\
Australia & 0 & 0 & 0 & 0 & 0 & 0 \\
Canada & 0 & 0 & 93 & 280 & 361 & 361 \\
China & 0 & 0 & 80 & 240 & 477 & 477 \\
Central and South America & 0 & 0 & 189 & 614 & 1,106 & 1,362 \\
Eastern Europe & 0 & 0 & 0 & 0 & 0 & 0 \\
Former Soviet Union & 0 & 0 & 0 & 0 & 0 & 0 \\
India & 0 & 0 & 77 & 232 & 544 & 790 \\
Japan & 0 & 0 & 0 & 0 & 0 & 0 \\
Middle East & 0 & 0 & 0 & 0 & 0 & 0 \\
Mexico & 0 & 0 & 72 & 216 & 506 & 556 \\
Other Asia & 0 & 0 & 0 & 0 & 0 & 0 \\
South Korea & 0 & 0 & 36 & 52 & 52 & 52 \\
United States & 0 & 0 & -248 & $-1,546$ & $-3,046$ & $-3,597$ \\
Western Europe & 0 & 0 & -298 & -88 & 0 & 0 \\
\hline Total & $\mathbf{0}$ & $\mathbf{0}$ & $\mathbf{0}$ & $\mathbf{0}$ & $\mathbf{0}$ & $\mathbf{0}$ \\
\hline \hline
\end{tabular}


Table A-286: Delayed technology reference case with credit extension biodiesel production [Million gallons ethanol equivalent]

\begin{tabular}{lrrrrrr} 
& $\mathbf{2 0 0 5}$ & $\mathbf{2 0 1 0}$ & $\mathbf{2 0 1 5}$ & $\mathbf{2 0 2 0}$ & $\mathbf{2 0 2 5}$ & $\mathbf{2 0 3 0}$ \\
Africa & 0 & 0 & 0 & 0 & 0 & 0 \\
Argentina & 0 & 333 & 776 & 819 & 827 & 239 \\
Australia & 0 & 0 & 0 & 0 & 0 & 0 \\
Brazil & 0 & 333 & 877 & 1,642 & 1,793 & 1,355 \\
Canada & 0 & 0 & 0 & 0 & 0 & 0 \\
Caribbean basin & 0 & 95 & 310 & 675 & 802 & 898 \\
China & 0 & 0 & 0 & 0 & 0 & 0 \\
Colombia & 0 & 119 & 298 & 474 & 650 & 782 \\
Eastern Europe & 0 & 0 & 0 & 0 & 0 & 0 \\
Former Soviet Union & 0 & 0 & 0 & 0 & 0 & 0 \\
India & 0 & 0 & 0 & 0 & 0 & 0 \\
Japan & 0 & 0 & 0 & 0 & 0 & 0 \\
Middle East & 0 & 0 & 0 & 0 & 0 & 0 \\
Mexico & 0 & 0 & 0 & 0 & 0 & 0 \\
Other Asia & 0 & 0 & 0 & 0 & 0 & 0 \\
South Korea & 0 & 0 & 0 & 0 & 0 & 0 \\
United States & 0 & 1,182 & 1,640 & 1,747 & 1,907 & 2,072 \\
Western Europe & 998 & 3,178 & 3,578 & 3,930 & 4,468 & 4,788 \\
\hline Total & $\mathbf{9 9 8}$ & $\mathbf{5 , 2 3 8}$ & $\mathbf{7 , 4 7 9}$ & $\mathbf{9 , 2 8 7}$ & $\mathbf{1 0 , 4 4 8}$ & $\mathbf{1 0 , 1 3 4}$ \\
\hline \hline
\end{tabular}

Table A-287: Delayed technology reference case with credit extension biodiesel consumption [Million gallons ethanol equivalent]

\begin{tabular}{lrrrrrr} 
& $\mathbf{2 0 0 5}$ & $\mathbf{2 0 1 0}$ & $\mathbf{2 0 1 5}$ & $\mathbf{2 0 2 0}$ & $\mathbf{2 0 2 5}$ & $\mathbf{2 0 3 0}$ \\
Africa & 0 & 0 & 0 & 0 & 0 & 0 \\
Australia & 0 & 0 & 0 & 0 & 0 & 0 \\
Canada & 0 & 0 & 0 & 0 & 0 & 0 \\
China & 0 & 0 & 0 & 0 & 0 & 0 \\
Central and South America & 0 & 158 & 472 & 984 & 1,306 & 1,490 \\
Eastern Europe & 0 & 0 & 0 & 0 & 0 & 0 \\
Former Soviet Union & 0 & 0 & 0 & 0 & 0 & 0 \\
India & 0 & 0 & 0 & 0 & 0 & 0 \\
Japan & 0 & 0 & 0 & 0 & 0 & 0 \\
Middle East & 0 & 0 & 0 & 0 & 0 & 0 \\
Mexico & 0 & 0 & 0 & 0 & 0 & 0 \\
Other Asia & 0 & 0 & 0 & 0 & 0 & 0 \\
South Korea & 0 & 0 & 0 & 0 & 0 & 0 \\
United States & 0 & 1,182 & 1,713 & 1,747 & 1,907 & 2,042 \\
Western Europe & 998 & 3,898 & 5,294 & 6,556 & 7,235 & 6,602 \\
\hline Total & $\mathbf{9 9 8}$ & $\mathbf{5 , 2 3 8}$ & $\mathbf{7 , 4 7 9}$ & $\mathbf{9 , 2 8 7}$ & $\mathbf{1 0 , 4 4 8}$ & $\mathbf{1 0 , 1 3 4}$ \\
\hline \hline
\end{tabular}


Table A-288: Delayed technology reference case with credit extension biodiesel net trade [Million gallons ethanol equivalent]

\begin{tabular}{lrrrrrr} 
& $\mathbf{2 0 0 5}$ & $\mathbf{2 0 1 0}$ & $\mathbf{2 0 1 5}$ & $\mathbf{2 0 2 0}$ & $\mathbf{2 0 2 5}$ & $\mathbf{2 0 3 0}$ \\
Africa & 0 & 0 & 0 & 0 & 0 & 0 \\
Australia & 0 & 0 & 0 & 0 & 0 & 0 \\
Canada & 0 & 0 & 0 & 0 & 0 & 0 \\
China & 0 & 0 & 0 & 0 & 0 & 0 \\
Central and South America & 0 & 720 & 1,788 & 2,626 & 2,767 & 1,784 \\
Eastern Europe & 0 & 0 & 0 & 0 & 0 & 0 \\
Former Soviet Union & 0 & 0 & 0 & 0 & 0 & 0 \\
India & 0 & 0 & 0 & 0 & 0 & 0 \\
Japan & 0 & 0 & 0 & 0 & 0 & 0 \\
Middle East & 0 & 0 & 0 & 0 & 0 & 0 \\
Mexico & 0 & 0 & 0 & 0 & 0 & 0 \\
Other Asia & 0 & 0 & 0 & 0 & 0 & 0 \\
South Korea & 0 & 0 & 0 & 0 & 0 & 0 \\
United States & 0 & 0 & -73 & 0 & 0 & 31 \\
Western Europe & 0 & -720 & $-1,716$ & $-2,626$ & $-2,767$ & $-1,815$ \\
\hline Total & $\mathbf{0}$ & $\mathbf{0}$ & $\mathbf{0}$ & $\mathbf{0}$ & $\mathbf{0}$ & $\mathbf{0}$ \\
\hline \hline
\end{tabular}




\section{A-17 Delayed Technology Reference Case with 70/30 Fuel/Food split}

Table A-289: Delayed technology reference case with 70/30 food/fuel split ethanol production [Million gallons]

BIORF70D

\section{Ethanol production [Million gallons]}

\begin{tabular}{lrrrrrr} 
Africa & 300 & 330 & 364 & 402 & 455 & 502 \\
Argentina & 0 & 178 & 628 & 875 & 904 & 916 \\
Australia & 0 & 0 & 66 & 247 & 624 & 1,172 \\
Brazil & 5,225 & 6,956 & 8,845 & 10,906 & 13,274 & 14,133 \\
Canada & 0 & 0 & 259 & 779 & 1,004 & 1,004 \\
Caribbean basin & 180 & 299 & 573 & 683 & 775 & 775 \\
China & 344 & 384 & 614 & 1,041 & 1,485 & 1,485 \\
Colombia & 196 & 215 & 411 & 782 & 1,501 & 2,227 \\
Eastern Europe & 110 & 226 & 428 & 738 & 1,037 & 1,149 \\
Former Soviet Union & 0 & 0 & 6 & 19 & 57 & 174 \\
India & 313 & 279 & 541 & 992 & 1,893 & 2,543 \\
Japan & 0 & 0 & 95 & 348 & 612 & 647 \\
Middle East & 0 & 0 & 0 & 0 & 0 & 0 \\
Mexico & 0 & 0 & 199 & 601 & 1,408 & 1,546 \\
Other Asia & 0 & 0 & 0 & 0 & 0 & 0 \\
South Korea & 0 & 0 & 35 & 35 & 35 & 35 \\
United States & 3,850 & 11,423 & 14,790 & 16,127 & 16,196 & 21,110 \\
Western Europe & 600 & 2,465 & 2,755 & 3,398 & 5,291 & 8,013 \\
\hline Total & $\mathbf{1 1 , 1 1 8}$ & $\mathbf{2 2 , 7 5 6}$ & $\mathbf{3 0 , 6 0 9}$ & $\mathbf{3 7 , 9 7 1}$ & $\mathbf{4 6 , 5 5 1}$ & $\mathbf{5 7 , 4 2 8}$ \\
\hline \hline
\end{tabular}


Table A-290: Delayed technology reference case with 70/30 food/fuel split ethanol consumption [Million gallons]

\begin{tabular}{lrrrrrr} 
& $\mathbf{2 0 0 5}$ & $\mathbf{2 0 1 0}$ & $\mathbf{2 0 1 5}$ & $\mathbf{2 0 2 0}$ & $\mathbf{2 0 2 5}$ & $\mathbf{2 0 3 0}$ \\
Africa & 300 & 330 & 364 & 402 & 455 & 502 \\
Australia & 0 & 0 & 66 & 247 & 624 & 1,172 \\
Canada & 119 & 0 & 233 & 933 & 1,004 & 1,020 \\
China & 442 & 0 & 0 & 1,041 & 712 & 1,485 \\
Central and South America & 3,785 & 4,851 & 6,129 & 7,148 & 7,954 & 8,855 \\
Eastern Europe & 699 & 783 & 872 & 953 & 1,037 & 1,149 \\
Former Soviet Union & 0 & 0 & 6 & 19 & 57 & 174 \\
India & 313 & 279 & 541 & 992 & 1,893 & 2,399 \\
Japan & 300 & 325 & 410 & 698 & 1,007 & 1,100 \\
Middle East & 0 & 0 & 0 & 0 & 0 & 0 \\
Mexico & 0 & 0 & 30 & 78 & 183 & 201 \\
Other Asia & 0 & 0 & 0 & 0 & 0 & 0 \\
South Korea & 264 & 335 & 400 & 414 & 413 & 413 \\
United States & 4,161 & 11,612 & 17,213 & 19,797 & 23,562 & 28,685 \\
Western Europe & 736 & 4,241 & 4,344 & 5,249 & 7,649 & 10,276 \\
\hline Total & $\mathbf{1 1 , 1 1 8}$ & $\mathbf{2 2 , 7 5 6}$ & $\mathbf{3 0 , 6 0 9}$ & $\mathbf{3 7 , 9 7 1}$ & $\mathbf{4 6 , 5 5 1}$ & $\mathbf{5 7 , 4 2 8}$ \\
\hline \hline
\end{tabular}

Table A-291: Delayed technology reference case with 70/30 food/fuel split ethanol net trade [Million gallons]

\begin{tabular}{lrrrrrr} 
Africa & 0 & 0 & 0 & 0 & 0 & 0 \\
Australia & 0 & 0 & 0 & 0 & 0 & 0 \\
Canada & -119 & 0 & 26 & -154 & 0 & -16 \\
China & -98 & 384 & 614 & 0 & 774 & 0 \\
Central and South America & 1,816 & 2,797 & 4,328 & 6,097 & 8,500 & 9,195 \\
Eastern Europe & -589 & -557 & -444 & -215 & 0 & 0 \\
Former Soviet Union & 0 & 0 & 0 & 0 & 0 & 0 \\
India & 0 & 0 & 0 & 0 & 0 & 144 \\
Japan & -300 & -325 & -315 & -350 & -395 & -453 \\
Middle East & 0 & 0 & 0 & 0 & 0 & 0 \\
Mexico & 0 & 0 & 170 & 523 & 1,225 & 1,345 \\
Other Asia & 0 & 0 & 0 & 0 & 0 & 0 \\
South Korea & -264 & -335 & -365 & -379 & -378 & -378 \\
United States & -311 & -189 & $-2,424$ & $-3,669$ & $-7,366$ & $-7,575$ \\
Western Europe & -136 & $-1,776$ & $-1,589$ & $-1,851$ & $-2,358$ & $-2,263$ \\
\hline Total & $\mathbf{0}$ & $\mathbf{0}$ & $\mathbf{0}$ & $\mathbf{0}$ & $\mathbf{0}$ & $\mathbf{0}$ \\
\hline \hline
\end{tabular}


Table A-292: Delayed technology reference case with 70/30 food/fuel split grain ethanol production [Million gallons]

\begin{tabular}{lrrrrrr} 
& $\mathbf{2 0 0 5}$ & $\mathbf{2 0 1 0}$ & $\mathbf{2 0 1 5}$ & $\mathbf{2 0 2 0}$ & $\mathbf{2 0 2 5}$ & $\mathbf{2 0 3 0}$ \\
Africa & 0 & 0 & 0 & 0 & 0 & 0 \\
Argentina & 0 & 178 & 569 & 569 & 569 & 569 \\
Australia & 0 & 0 & 0 & 0 & 0 & 0 \\
Brazil & 0 & 0 & 0 & 0 & 0 & 0 \\
Canada & 0 & 0 & 0 & 0 & 0 & 0 \\
Caribbean basin & 0 & 0 & 0 & 0 & 0 & 0 \\
China & 344 & 344 & 344 & 325 & 0 & 0 \\
Colombia & 0 & 0 & 0 & 0 & 0 & 0 \\
Eastern Europe & 110 & 226 & 348 & 477 & 367 & 251 \\
Former Soviet Union & 0 & 0 & 0 & 0 & 0 & 0 \\
India & 0 & 0 & 0 & 0 & 0 & 0 \\
Japan & 0 & 0 & 0 & 0 & 0 & 0 \\
Middle East & 0 & 0 & 0 & 0 & 0 & 0 \\
Mexico & 0 & 0 & 0 & 0 & 0 & 0 \\
Other Asia & 0 & 0 & 0 & 0 & 0 & 0 \\
South Korea & 0 & 0 & 0 & 0 & 0 & 0 \\
United States & 3,850 & 11,423 & 14,058 & 15,000 & 15,000 & 14,141 \\
Western Europe & 600 & 2,465 & 2,589 & 2,705 & 3,178 & 3,604 \\
\hline Total & $\mathbf{4 , 9 0 4}$ & $\mathbf{1 4 , 6 3 6}$ & $\mathbf{1 7 , 9 0 8}$ & $\mathbf{1 9 , 0 7 7}$ & $\mathbf{1 9 , 1 1 4}$ & $\mathbf{1 8 , 5 6 5}$ \\
\hline \hline
\end{tabular}

Table A-293: Delayed technology reference case with 70/30 food/fuel split sugar ethanol production [Million gallons]

\begin{tabular}{lrrrrrr} 
& $\mathbf{2 0 0 5}$ & $\mathbf{2 0 1 0}$ & $\mathbf{2 0 1 5}$ & $\mathbf{2 0 2 0}$ & $\mathbf{2 0 2 5}$ & $\mathbf{2 0 3 0}$ \\
Africa & 300 & 330 & 364 & 402 & 444 & 490 \\
Argentina & 0 & 0 & 0 & 0 & 0 & 0 \\
Australia & 0 & 0 & 0 & 0 & 0 & 0 \\
Brazil & 5,225 & 6,956 & 8,550 & 10,016 & 11,733 & 12,588 \\
Canada & 0 & 0 & 0 & 0 & 0 & 0 \\
Caribbean basin & 180 & 299 & 573 & 683 & 775 & 775 \\
China & 0 & 40 & 48 & 48 & 48 & 48 \\
Colombia & 196 & 215 & 241 & 270 & 302 & 334 \\
Eastern Europe & 0 & 0 & 0 & 0 & 0 & 0 \\
Former Soviet Union & 0 & 0 & 0 & 0 & 0 & 0 \\
India & 313 & 279 & 327 & 347 & 381 & 348 \\
Japan & 0 & 0 & 0 & 0 & 0 & 0 \\
Middle East & 0 & 0 & 0 & 0 & 0 & 0 \\
Mexico & 0 & 0 & 0 & 0 & 0 & 0 \\
Other Asia & 0 & 0 & 0 & 0 & 0 & 0 \\
South Korea & 0 & 0 & 0 & 0 & 0 & 0 \\
United States & 0 & 0 & 0 & 0 & 0 & 0 \\
Western Europe & 0 & 0 & 0 & 0 & 0 & 0 \\
\hline Total & $\mathbf{6 , 2 1 4}$ & $\mathbf{8 , 1 1 9}$ & $\mathbf{1 0 , 1 0 3}$ & $\mathbf{1 1 , 7 6 6}$ & $\mathbf{1 3 , 6 8 3}$ & $\mathbf{1 4 , 5 8 2}$ \\
\hline \hline
\end{tabular}


Table A-294: Delayed technology reference case with 70/30 food/fuel split cellulosic ethanol production [Million gallons]

\begin{tabular}{lrrrrrr} 
& $\mathbf{2 0 0 5}$ & $\mathbf{2 0 1 0}$ & $\mathbf{2 0 1 5}$ & $\mathbf{2 0 2 0}$ & $\mathbf{2 0 2 5}$ & $\mathbf{2 0 3 0}$ \\
Africa & 0 & 0 & 0 & 0 & 11 & 11 \\
Argentina & 0 & 0 & 59 & 306 & 335 & 347 \\
Australia & 0 & 0 & 66 & 247 & 624 & 1,172 \\
Brazil & 0 & 0 & 296 & 890 & 1,541 & 1,546 \\
Canada & 0 & 0 & 259 & 779 & 1,004 & 1,004 \\
Caribbean basin & 0 & 0 & 0 & 0 & 0 & 0 \\
China & 0 & 0 & 222 & 667 & 1,438 & 1,438 \\
Colombia & 0 & 0 & 170 & 512 & 1,199 & 1,893 \\
Eastern Europe & 0 & 0 & 80 & 261 & 670 & 897 \\
Former Soviet Union & 0 & 0 & 6 & 19 & 57 & 174 \\
India & 0 & 0 & 214 & 645 & 1,512 & 2,196 \\
Japan & 0 & 0 & 95 & 348 & 612 & 647 \\
Middle East & 0 & 0 & 0 & 0 & 0 & 0 \\
Mexico & 0 & 0 & 199 & 601 & 1,408 & 1,546 \\
Other Asia & 0 & 0 & 0 & 0 & 0 & 0 \\
South Korea & 0 & 0 & 35 & 35 & 35 & 35 \\
United States & 0 & 0 & 732 & 1,127 & 1,196 & 6,969 \\
Western Europe & 0 & 0 & 166 & 693 & 2,113 & 4,410 \\
\hline Total & $\mathbf{0}$ & $\mathbf{0}$ & $\mathbf{2 , 5 9 8}$ & $\mathbf{7 , 1 2 8}$ & $\mathbf{1 3 , 7 5 5}$ & $\mathbf{2 4 , 2 8 2}$ \\
\hline \hline
\end{tabular}

Table A-295: Delayed technology reference case with 70/30 food/fuel split grain ethanol consumption [Million gallons]

\begin{tabular}{lrrrrrr} 
& $\mathbf{2 0 0 5}$ & $\mathbf{2 0 1 0}$ & $\mathbf{2 0 1 5}$ & $\mathbf{2 0 2 0}$ & $\mathbf{2 0 2 5}$ & $\mathbf{2 0 3 0}$ \\
Africa & 0 & 0 & 0 & 0 & 0 & 0 \\
Australia & 0 & 0 & 0 & 0 & 0 & 0 \\
Canada & 0 & 0 & 0 & 0 & 0 & 0 \\
China & 344 & 0 & 0 & 325 & 0 & 0 \\
Central and South America & 0 & 36 & 94 & 114 & 90 & 92 \\
Eastern Europe & 110 & 226 & 348 & 477 & 367 & 251 \\
Former Soviet Union & 0 & 0 & 0 & 0 & 0 & 0 \\
India & 0 & 0 & 0 & 0 & 0 & 0 \\
Japan & 0 & 192 & 267 & 350 & 0 & 99 \\
Middle East & 0 & 0 & 0 & 0 & 0 & 0 \\
Mexico & 0 & 0 & 0 & 0 & 0 & 0 \\
Other Asia & 0 & 0 & 0 & 0 & 0 & 0 \\
South Korea & 0 & 295 & 144 & 105 & 0 & 378 \\
United States & 3,850 & 11,423 & 14,058 & 15,000 & 15,000 & 14,141 \\
Western Europe & 600 & 2,465 & 2,996 & 2,705 & 3,656 & 3,604 \\
\hline Total & $\mathbf{4 , 9 0 4}$ & $\mathbf{1 4 , 6 3 6}$ & $\mathbf{1 7 , 9 0 8}$ & $\mathbf{1 9 , 0 7 7}$ & $\mathbf{1 9 , 1 1 4}$ & $\mathbf{1 8 , 5 6 5}$ \\
\hline \hline
\end{tabular}


Table A-296: Delayed technology reference case with 70/30 food/fuel split sugar ethanol consumption [Million gallons]

\begin{tabular}{lrrrrrr} 
& $\mathbf{2 0 0 5}$ & $\mathbf{2 0 1 0}$ & $\mathbf{2 0 1 5}$ & $\mathbf{2 0 2 0}$ & $\mathbf{2 0 2 5}$ & $\mathbf{2 0 3 0}$ \\
Africa & 300 & 330 & 364 & 402 & 444 & 490 \\
Australia & 0 & 0 & 0 & 0 & 0 & 0 \\
Canada & 119 & 0 & 0 & 0 & 0 & 16 \\
China & 98 & 0 & 0 & 48 & 48 & 48 \\
Central and South America & 3,785 & 4,815 & 6,035 & 7,004 & 7,864 & 8,763 \\
Eastern Europe & 589 & 557 & 418 & 184 & 0 & 0 \\
Former Soviet Union & 0 & 0 & 0 & 0 & 0 & 0 \\
India & 313 & 279 & 327 & 347 & 381 & 348 \\
Japan & 300 & 133 & 48 & 0 & 0 & 210 \\
Middle East & 0 & 0 & 0 & 0 & 0 & 0 \\
Mexico & 0 & 0 & 0 & 0 & 0 & 0 \\
Other Asia & 0 & 0 & 0 & 0 & 0 & 0 \\
South Korea & 264 & 40 & 0 & 0 & 0 & 0 \\
United States & 311 & 189 & 1,729 & 2,753 & 3,093 & 2,445 \\
Western Europe & 136 & 1,776 & 1,181 & 1,029 & 1,853 & 2,263 \\
\hline Total & $\mathbf{6 , 2 1 4}$ & $\mathbf{8 , 1 1 9}$ & $\mathbf{1 0 , 1 0 3}$ & $\mathbf{1 1 , 7 6 6}$ & $\mathbf{1 3 , 6 8 3}$ & $\mathbf{1 4 , 5 8 2}$ \\
\hline \hline
\end{tabular}

Table A-297: Delayed technology reference case with 70/30 food/fuel split cellulosic ethanol consumption [Million gallons]

\begin{tabular}{lllrrrr} 
Africa & 0 & 0 & 0 & 0 & 11 & 11 \\
Australia & 0 & 0 & 66 & 247 & 624 & 1,172 \\
Canada & 0 & 0 & 233 & 933 & 1,004 & 1,004 \\
China & 0 & 0 & 0 & 667 & 664 & 1,438 \\
Central and South America & 0 & 0 & 0 & 31 & 0 & 0 \\
Eastern Europe & 0 & 0 & 106 & 292 & 670 & 897 \\
Former Soviet Union & 0 & 0 & 6 & 19 & 57 & 174 \\
India & 0 & 0 & 214 & 645 & 1,512 & 2,051 \\
Japan & 0 & 0 & 95 & 348 & 1,007 & 791 \\
Middle East & 0 & 0 & 0 & 0 & 0 & 0 \\
Mexico & 0 & 0 & 30 & 78 & 183 & 201 \\
Other Asia & 0 & 0 & 0 & 0 & 0 & 0 \\
South Korea & 0 & 0 & 256 & 309 & 413 & 35 \\
United States & 0 & 0 & 1,426 & 2,044 & 5,469 & 12,099 \\
Western Europe & 0 & 0 & 166 & 1,515 & 2,140 & 4,410 \\
\hline Total & $\mathbf{0}$ & $\mathbf{0}$ & $\mathbf{2 , 5 9 8}$ & $\mathbf{7 , 1 2 8}$ & $\mathbf{1 3 , 7 5 5}$ & $\mathbf{2 4 , 2 8 2}$ \\
\hline \hline
\end{tabular}


Table A-298: Delayed technology reference case with 70/30 food/fuel split grain ethanol net trade [Million gallons]

\begin{tabular}{lrrrrrr} 
& $\mathbf{2 0 0 5}$ & $\mathbf{2 0 1 0}$ & $\mathbf{2 0 1 5}$ & $\mathbf{2 0 2 0}$ & $\mathbf{2 0 2 5}$ & $\mathbf{2 0 3 0}$ \\
Africa & 0 & 0 & 0 & 0 & 0 & 0 \\
Australia & 0 & 0 & 0 & 0 & 0 & 0 \\
Canada & 0 & 0 & 0 & 0 & 0 & 0 \\
China & 0 & 344 & 344 & 0 & 0 & 0 \\
Central and South America & 0 & 143 & 475 & 455 & 478 & 477 \\
Eastern Europe & 0 & 0 & 0 & 0 & 0 & 0 \\
Former Soviet Union & 0 & 0 & 0 & 0 & 0 & 0 \\
India & 0 & 0 & 0 & 0 & 0 & 0 \\
Japan & 0 & -192 & -267 & -350 & 0 & -99 \\
Middle East & 0 & 0 & 0 & 0 & 0 & 0 \\
Mexico & 0 & 0 & 0 & 0 & 0 & 0 \\
Other Asia & 0 & 0 & 0 & 0 & 0 & 0 \\
South Korea & 0 & -295 & -144 & -105 & 0 & -378 \\
United States & 0 & 0 & 0 & 0 & 0 & 0 \\
Western Europe & 0 & 0 & -408 & 0 & -478 & 0 \\
\hline Total & $\mathbf{0}$ & $\mathbf{0}$ & $\mathbf{0}$ & $\mathbf{0}$ & $\mathbf{0}$ & $\mathbf{0}$ \\
\hline \hline
\end{tabular}

Table A-299: Delayed technology reference case with 70/30 food/fuel split sugar ethanol net trade [Million gallons]

\begin{tabular}{lrrrrrr} 
Africa & 0 & 0 & 0 & 0 & 0 & 0 \\
Australia & 0 & 0 & 0 & 0 & 0 & 0 \\
Canada & -119 & 0 & 0 & 0 & 0 & -16 \\
China & -98 & 40 & 48 & 0 & 0 & 0 \\
Central and South America & 1,816 & 2,655 & 3,329 & 3,966 & 4,946 & 4,933 \\
Eastern Europe & -589 & -557 & -418 & -184 & 0 & 0 \\
Former Soviet Union & 0 & 0 & 0 & 0 & 0 & 0 \\
India & 0 & 0 & 0 & 0 & 0 & 0 \\
Japan & -300 & -133 & -48 & 0 & 0 & -210 \\
Middle East & 0 & 0 & 0 & 0 & 0 & 0 \\
Mexico & 0 & 0 & 0 & 0 & 0 & 0 \\
Other Asia & 0 & 0 & 0 & 0 & 0 & 0 \\
South Korea & -264 & -40 & 0 & 0 & 0 & 0 \\
United States & -311 & -189 & $-1,729$ & $-2,753$ & $-3,093$ & $-2,445$ \\
Western Europe & -136 & $-1,776$ & $-1,181$ & $-1,029$ & $-1,853$ & $-2,263$ \\
\hline Total & $\mathbf{0}$ & $\mathbf{0}$ & $\mathbf{0}$ & $\mathbf{0}$ & $\mathbf{0}$ & $\mathbf{0}$ \\
\hline \hline
\end{tabular}


Table A-300: Delayed technology reference case with 70/30 food/fuel split cellulosic ethanol net trade [Million gallons]

\begin{tabular}{lrrrrrr} 
& $\mathbf{2 0 0 5}$ & $\mathbf{2 0 1 0}$ & $\mathbf{2 0 1 5}$ & $\mathbf{2 0 2 0}$ & $\mathbf{2 0 2 5}$ & $\mathbf{2 0 3 0}$ \\
Africa & 0 & 0 & 0 & 0 & 0 & 0 \\
Australia & 0 & 0 & 0 & 0 & 0 & 0 \\
Canada & 0 & 0 & 26 & -154 & 0 & 0 \\
China & 0 & 0 & 222 & 0 & 774 & 0 \\
Central and South America & 0 & 0 & 525 & 1,676 & 3,075 & 3,786 \\
Eastern Europe & 0 & 0 & -26 & -31 & 0 & 0 \\
Former Soviet Union & 0 & 0 & 0 & 0 & 0 & 0 \\
India & 0 & 0 & 0 & 0 & 0 & 144 \\
Japan & 0 & 0 & 0 & 0 & -395 & -144 \\
Middle East & 0 & 0 & 0 & 0 & 0 & 0 \\
Mexico & 0 & 0 & 170 & 523 & 1,225 & 1,345 \\
Other Asia & 0 & 0 & 0 & 0 & 0 & 0 \\
South Korea & 0 & 0 & -222 & -275 & -378 & 0 \\
United States & 0 & 0 & -694 & -917 & $-4,273$ & $-5,130$ \\
Western Europe & 0 & 0 & 0 & -822 & -27 & 0 \\
\hline Total & $\mathbf{0}$ & $\mathbf{0}$ & $\mathbf{0}$ & $\mathbf{0}$ & $\mathbf{0}$ & $\mathbf{0}$ \\
\hline \hline
\end{tabular}

Table A-301: Delayed technology reference case with 70/30 food/fuel split biomassto-liquids production [Million gallons ethanol equivalent]

\begin{tabular}{lrrrrrr} 
& $\mathbf{2 0 0 5}$ & $\mathbf{2 0 1 0}$ & $\mathbf{2 0 1 5}$ & $\mathbf{2 0 2 0}$ & $\mathbf{2 0 2 5}$ & $\mathbf{2 0 3 0}$ \\
Africa & 0 & 0 & 0 & 0 & 0 & 0 \\
Argentina & 0 & 0 & 21 & 110 & 121 & 125 \\
Australia & 0 & 0 & 0 & 0 & 0 & 0 \\
Brazil & 0 & 0 & 106 & 320 & 554 & 556 \\
Canada & 0 & 0 & 93 & 280 & 361 & 361 \\
Caribbean basin & 0 & 0 & 0 & 0 & 0 & 0 \\
China & 0 & 0 & 80 & 240 & 517 & 517 \\
Colombia & 0 & 0 & 61 & 184 & 431 & 681 \\
Eastern Europe & 0 & 0 & 0 & 0 & 0 & 0 \\
Former Soviet Union & 0 & 0 & 0 & 0 & 0 & 0 \\
India & 0 & 0 & 77 & 232 & 544 & 790 \\
Japan & 0 & 0 & 0 & 0 & 0 & 0 \\
Middle East & 0 & 0 & 0 & 0 & 0 & 0 \\
Mexico & 0 & 0 & 72 & 216 & 506 & 556 \\
Other Asia & 0 & 0 & 0 & 0 & 0 & 0 \\
South Korea & 0 & 0 & 36 & 56 & 56 & 56 \\
United States & 0 & 0 & 750 & 2,405 & 3,319 & 4,283 \\
Western Europe & 0 & 0 & 0 & 0 & 0 & 0 \\
\hline Total & $\mathbf{0}$ & $\mathbf{0}$ & $\mathbf{1 , 2 9 6}$ & $\mathbf{4 , 0 4 3}$ & $\mathbf{6 , 4 1 0}$ & $\mathbf{7 , 9 2 4}$ \\
\hline \hline
\end{tabular}


Table A-302: Delayed technology reference case with 70/30 food/fuel split biomassto-liquids consumption [Million gallons ethanol equivalent]

\begin{tabular}{lrrrrrr} 
& $\mathbf{2 0 0 5}$ & $\mathbf{2 0 1 0}$ & $\mathbf{2 0 1 5}$ & $\mathbf{2 0 2 0}$ & $\mathbf{2 0 2 5}$ & $\mathbf{2 0 3 0}$ \\
Africa & 0 & 0 & 0 & 0 & 0 & 0 \\
Australia & 0 & 0 & 0 & 0 & 0 & 0 \\
Canada & 0 & 0 & 0 & 0 & 0 & 0 \\
China & 0 & 0 & 0 & 0 & 0 & 0 \\
Central and South America & 0 & 0 & 0 & 0 & 0 & 0 \\
Eastern Europe & 0 & 0 & 0 & 0 & 0 & 0 \\
Former Soviet Union & 0 & 0 & 0 & 0 & 0 & 0 \\
India & 0 & 0 & 0 & 0 & 0 & 0 \\
Japan & 0 & 0 & 0 & 0 & 0 & 0 \\
Middle East & 0 & 0 & 0 & 0 & 0 & 0 \\
Mexico & 0 & 0 & 0 & 0 & 0 & 0 \\
Other Asia & 0 & 0 & 0 & 0 & 0 & 0 \\
South Korea & 0 & 0 & 0 & 0 & 0 & 0 \\
United States & 0 & 0 & 1,082 & 3,913 & 6,409 & 7,924 \\
Western Europe & 0 & 0 & 214 & 130 & 0 & 0 \\
\hline Total & $\mathbf{0}$ & $\mathbf{0}$ & $\mathbf{1 , 2 9 6}$ & $\mathbf{4 , 0 4 3}$ & $\mathbf{6 , 4 0 9}$ & $\mathbf{7 , 9 2 4}$ \\
\hline \hline
\end{tabular}

Table A-303: Delayed technology reference case with 70/30 food/fuel split biomassto-liquids net trade [Million gallons ethanol equivalent]

\begin{tabular}{lrrrrrr} 
& $\mathbf{2 0 0 5}$ & $\mathbf{2 0 1 0}$ & $\mathbf{2 0 1 5}$ & $\mathbf{2 0 2 0}$ & $\mathbf{2 0 2 5}$ & $\mathbf{2 0 3 0}$ \\
Africa & 0 & 0 & 0 & 0 & 0 & 0 \\
Australia & 0 & 0 & 0 & 0 & 0 & 0 \\
Canada & 0 & 0 & 93 & 280 & 361 & 361 \\
China & 0 & 0 & 80 & 240 & 517 & 517 \\
Central and South America & 0 & 0 & 189 & 614 & 1,106 & 1,362 \\
Eastern Europe & 0 & 0 & 0 & 0 & 0 & 0 \\
Former Soviet Union & 0 & 0 & 0 & 0 & 0 & 0 \\
India & 0 & 0 & 77 & 232 & 544 & 790 \\
Japan & 0 & 0 & 0 & 0 & 0 & 0 \\
Middle East & 0 & 0 & 0 & 0 & 0 & 0 \\
Mexico & 0 & 0 & 72 & 216 & 506 & 556 \\
Other Asia & 0 & 0 & 0 & 0 & 0 & 0 \\
South Korea & 0 & 0 & 36 & 56 & 56 & 56 \\
United States & 0 & 0 & -332 & $-1,508$ & $-3,090$ & $-3,641$ \\
Western Europe & 0 & 0 & -214 & -130 & 0 & 0 \\
\hline Total & $\mathbf{0}$ & $\mathbf{0}$ & $\mathbf{0}$ & $\mathbf{0}$ & $\mathbf{0}$ & $\mathbf{0}$ \\
\hline \hline
\end{tabular}




\section{World Biofuels Study}

Table A-304: Delayed technology reference case with 70/30 food/fuel split biodiesel production [Million gallons ethanol equivalent]

\begin{tabular}{lrrrrrr} 
& $\mathbf{2 0 0 5}$ & $\mathbf{2 0 1 0}$ & $\mathbf{2 0 1 5}$ & $\mathbf{2 0 2 0}$ & $\mathbf{2 0 2 5}$ & $\mathbf{2 0 3 0}$ \\
Africa & 0 & 0 & 0 & 0 & 0 & 0 \\
Argentina & 0 & 333 & 776 & 819 & 827 & 239 \\
Australia & 0 & 0 & 0 & 0 & 0 & 0 \\
Brazil & 0 & 333 & 835 & 1,600 & 1,793 & 1,355 \\
Canada & 0 & 0 & 0 & 0 & 0 & 0 \\
Caribbean basin & 0 & 95 & 310 & 675 & 802 & 898 \\
China & 0 & 0 & 0 & 0 & 0 & 0 \\
Colombia & 0 & 119 & 298 & 474 & 650 & 782 \\
Eastern Europe & 0 & 0 & 0 & 0 & 0 & 0 \\
Former Soviet Union & 0 & 0 & 0 & 0 & 0 & 0 \\
India & 0 & 0 & 0 & 0 & 0 & 0 \\
Japan & 0 & 0 & 0 & 0 & 0 & 0 \\
Middle East & 0 & 0 & 0 & 0 & 0 & 0 \\
Mexico & 0 & 0 & 0 & 0 & 0 & 0 \\
Other Asia & 0 & 0 & 0 & 0 & 0 & 0 \\
South Korea & 0 & 0 & 0 & 0 & 0 & 0 \\
United States & 0 & 1,238 & 1,640 & 1,747 & 1,907 & 2,072 \\
Western Europe & 998 & 3,178 & 3,578 & 3,930 & 4,468 & 4,788 \\
\hline Total & $\mathbf{9 9 8}$ & $\mathbf{5 , 2 9 4}$ & $\mathbf{7 , 4 3 7}$ & $\mathbf{9 , 2 4 5}$ & $\mathbf{1 0 , 4 4 8}$ & $\mathbf{1 0 , 1 3 4}$ \\
\hline \hline
\end{tabular}

Table A-305: Delayed technology reference case with 70/30 food/fuel split biodiesel consumption [Million gallons ethanol equivalent]

\begin{tabular}{lrrrrrr} 
& $\mathbf{2 0 0 5}$ & $\mathbf{2 0 1 0}$ & $\mathbf{2 0 1 5}$ & $\mathbf{2 0 2 0}$ & $\mathbf{2 0 2 5}$ & $\mathbf{2 0 3 0}$ \\
Africa & 0 & 0 & 0 & 0 & 0 & 0 \\
Australia & 0 & 0 & 0 & 0 & 0 & 0 \\
Canada & 0 & 0 & 0 & 0 & 0 & 0 \\
China & 0 & 0 & 0 & 0 & 0 & 0 \\
Central and South America & 0 & 158 & 472 & 984 & 1,306 & 1,490 \\
Eastern Europe & 0 & 0 & 0 & 0 & 0 & 0 \\
Former Soviet Union & 0 & 0 & 0 & 0 & 0 & 0 \\
India & 0 & 0 & 0 & 0 & 0 & 0 \\
Japan & 0 & 0 & 0 & 0 & 0 & 0 \\
Middle East & 0 & 0 & 0 & 0 & 0 & 0 \\
Mexico & 0 & 0 & 0 & 0 & 0 & 0 \\
Other Asia & 0 & 0 & 0 & 0 & 0 & 0 \\
South Korea & 0 & 0 & 0 & 0 & 0 & 0 \\
United States & 0 & 1,238 & 1,713 & 1,747 & 1,907 & 3,415 \\
Western Europe & 998 & 3,898 & 5,252 & 6,514 & 7,235 & 5,229 \\
\hline Total & $\mathbf{9 9 8}$ & $\mathbf{5 , 2 9 4}$ & $\mathbf{7 , 4 3 7}$ & $\mathbf{9 , 2 4 5}$ & $\mathbf{1 0 , 4 4 8}$ & $\mathbf{1 0 , 1 3 4}$ \\
\hline \hline
\end{tabular}




\section{World Biofuels Study}

Table A-306: Delayed technology reference case with 70/30 food/fuel split biodiesel net trade [Million gallons ethanol equivalent]

\begin{tabular}{lrrrrrr} 
& $\mathbf{2 0 0 5}$ & $\mathbf{2 0 1 0}$ & $\mathbf{2 0 1 5}$ & $\mathbf{2 0 2 0}$ & $\mathbf{2 0 2 5}$ & $\mathbf{2 0 3 0}$ \\
Africa & 0 & 0 & 0 & 0 & 0 & 0 \\
Australia & 0 & 0 & 0 & 0 & 0 & 0 \\
Canada & 0 & 0 & 0 & 0 & 0 & 0 \\
China & 0 & 0 & 0 & 0 & 0 & 0 \\
Central and South America & 0 & 720 & 1,746 & 2,584 & 2,767 & 1,784 \\
Eastern Europe & 0 & 0 & 0 & 0 & 0 & 0 \\
Former Soviet Union & 0 & 0 & 0 & 0 & 0 & 0 \\
India & 0 & 0 & 0 & 0 & 0 & 0 \\
Japan & 0 & 0 & 0 & 0 & 0 & 0 \\
Middle East & 0 & 0 & 0 & 0 & 0 & 0 \\
Mexico & 0 & 0 & 0 & 0 & 0 & 0 \\
Other Asia & 0 & 0 & 0 & 0 & 0 & 0 \\
South Korea & 0 & 0 & 0 & 0 & 0 & 0 \\
United States & 0 & 0 & -73 & 0 & 0 & $-1,343$ \\
Western Europe & 0 & -720 & $-1,674$ & $-2,584$ & $-2,767$ & -442 \\
\hline Total & $\mathbf{0}$ & $\mathbf{0}$ & $\mathbf{0}$ & $\mathbf{0}$ & $\mathbf{0}$ & $\mathbf{0}$ \\
\hline \hline
\end{tabular}




\section{A-18 Delayed Technology Reference Case with High Feedstock Availability}

Table A-307: Delayed technology reference case with high feedstock availability ethanol production [Million gallons]

\begin{tabular}{lrrrrrr} 
& $\mathbf{2 0 0 5}$ & $\mathbf{2 0 1 0}$ & $\mathbf{2 0 1 5}$ & $\mathbf{2 0 2 0}$ & $\mathbf{2 0 2 5}$ & $\mathbf{2 0 3 0}$ \\
Africa & 300 & 330 & 364 & 402 & 455 & 502 \\
Argentina & 0 & 0 & 70 & 449 & 573 & 607 \\
Australia & 0 & 0 & 66 & 247 & 627 & 1,172 \\
Brazil & 4,866 & 7,998 & 11,399 & 16,323 & 20,592 & 23,010 \\
Canada & 0 & 0 & 259 & 779 & 1,004 & 1,020 \\
Caribbean basin & 210 & 280 & 682 & 682 & 682 & 683 \\
China & 344 & 0 & 222 & 993 & 1,564 & 3,251 \\
Colombia & 196 & 0 & 170 & 512 & 887 & 887 \\
Eastern Europe & 110 & 226 & 428 & 738 & 1,037 & 1,149 \\
Former Soviet Union & 0 & 0 & 6 & 19 & 57 & 173 \\
India & 313 & 833 & 1,300 & 1,728 & 2,200 & 2,822 \\
Japan & 0 & 0 & 95 & 348 & 612 & 647 \\
Middle East & 0 & 0 & 0 & 0 & 0 & 0 \\
Mexico & 0 & 0 & 199 & 561 & 635 & 665 \\
Other Asia & 0 & 0 & 0 & 0 & 0 & 0 \\
South Korea & 0 & 0 & 25 & 25 & 25 & 25 \\
United States & 3,850 & 11,413 & 14,769 & 14,344 & 15,501 & 16,640 \\
Western Europe & 600 & 2,465 & 2,755 & 3,235 & 5,205 & 7,923 \\
\hline Total & $\mathbf{1 0 , 7 8 9}$ & $\mathbf{2 3 , 5 4 6}$ & $\mathbf{3 2 , 8 0 9}$ & $\mathbf{4 1 , 3 8 5}$ & $\mathbf{5 1 , 6 5 6}$ & $\mathbf{6 1 , 1 7 3}$ \\
\hline \hline
\end{tabular}


Table A-308: Delayed technology reference case with high feedstock availability ethanol consumption [Million gallons]

\begin{tabular}{lrrrrrr} 
& $\mathbf{2 0 0 5}$ & $\mathbf{2 0 1 0}$ & $\mathbf{2 0 1 5}$ & $\mathbf{2 0 2 0}$ & $\mathbf{2 0 2 5}$ & $\mathbf{2 0 3 0}$ \\
Africa & 300 & 330 & 364 & 402 & 455 & 502 \\
Australia & 0 & 0 & 66 & 247 & 627 & 1,172 \\
Canada & 145 & 259 & 898 & 984 & 1,004 & 1,020 \\
China & 297 & 0 & 848 & 3,397 & 4,132 & 5,063 \\
Central and South America & 3,788 & 4,771 & 6,016 & 7,053 & 7,824 & 8,619 \\
Eastern Europe & 699 & 783 & 872 & 953 & 1,037 & 1,149 \\
Former Soviet Union & 0 & 0 & 6 & 19 & 57 & 173 \\
India & 313 & 833 & 1,300 & 1,642 & 1,980 & 2,399 \\
Japan & 263 & 325 & 448 & 736 & 1,008 & 1,100 \\
Middle East & 0 & 0 & 0 & 0 & 0 & 0 \\
Mexico & 0 & 0 & 30 & 130 & 83 & 130 \\
Other Asia & 0 & 0 & 0 & 526 & 1,912 & 2,828 \\
South Korea & 264 & 335 & 400 & 414 & 413 & 413 \\
United States & 3,984 & 11,668 & 17,188 & 19,797 & 23,562 & 28,684 \\
Western Europe & 736 & 4,241 & 4,373 & 5,086 & 7,563 & 7,923 \\
\hline Total & $\mathbf{1 0 , 7 8 9}$ & $\mathbf{2 3 , 5 4 6}$ & $\mathbf{3 2 , 8 0 9}$ & $\mathbf{4 1 , 3 8 5}$ & $\mathbf{5 1 , 6 5 6}$ & $\mathbf{6 1 , 1 7 3}$ \\
\hline \hline
\end{tabular}

Table A-309: Delayed technology reference case with high feedstock availability ethanol net trade [Million gallons]

\begin{tabular}{lrrrrrr} 
Africa & 0 & 0 & 0 & 0 & 0 & 0 \\
Australia & 0 & 0 & 0 & 0 & 0 & 0 \\
Canada & -145 & -259 & -640 & -205 & 0 & 0 \\
China & 47 & 0 & -626 & $-2,404$ & $-2,568$ & $-1,812$ \\
Central and South America & 1,484 & 3,508 & 6,305 & 10,914 & 14,910 & 16,568 \\
Eastern Europe & -589 & -557 & -444 & -215 & 0 & 0 \\
Former Soviet Union & 0 & 0 & 0 & 0 & 0 & 0 \\
India & 0 & 0 & 0 & 86 & 220 & 423 \\
Japan & -263 & -325 & -353 & -388 & -395 & -453 \\
Middle East & 0 & 0 & 0 & 0 & 0 & 0 \\
Mexico & 0 & 0 & 170 & 431 & 553 & 535 \\
Other Asia & 0 & 0 & 0 & -526 & $-1,912$ & $-2,828$ \\
South Korea & -264 & -335 & -375 & -389 & -388 & -388 \\
United States & -134 & -256 & $-2,419$ & $-5,452$ & $-8,061$ & $-12,044$ \\
Western Europe & -136 & $-1,776$ & $-1,618$ & $-1,851$ & $-2,358$ & 0 \\
\hline Total & $\mathbf{0}$ & $\mathbf{0}$ & $\mathbf{0}$ & $\mathbf{0}$ & $\mathbf{0}$ & $\mathbf{0}$ \\
\hline \hline
\end{tabular}


Table A-110: Delayed technology reference case with high feedstock availability

\begin{tabular}{lrrrrrr}
\multicolumn{7}{c}{ grain ethanol production [Million gallons] } \\
Africa & $\mathbf{2 0 0 5}$ & $\mathbf{2 0 1 0}$ & $\mathbf{2 0 1 5}$ & $\mathbf{2 0 2 0}$ & $\mathbf{2 0 2 5}$ & $\mathbf{2 0 3 0}$ \\
Argentina & 0 & 0 & 0 & 0 & 0 & 0 \\
Australia & 0 & 0 & 11 & 11 & 57 & 61 \\
Brazil & 0 & 0 & 0 & 0 & 0 & 0 \\
Canada & 0 & 0 & 0 & 0 & 0 & 0 \\
Caribbean basin & 0 & 0 & 0 & 0 & 0 & 0 \\
China & 0 & 0 & 0 & 0 & 0 & 0 \\
Colombia & 344 & 0 & 0 & 325 & 0 & 0 \\
Eastern Europe & 0 & 0 & 0 & 0 & 0 & 0 \\
Former Soviet Union & 110 & 226 & 348 & 477 & 367 & 251 \\
India & 0 & 0 & 0 & 0 & 0 & 0 \\
Japan & 0 & 0 & 0 & 0 & 0 & 0 \\
Middle East & 0 & 0 & 0 & 0 & 0 & 0 \\
Mexico & 0 & 0 & 0 & 0 & 0 & 0 \\
Other Asia & 0 & 0 & 0 & 0 & 0 & 0 \\
South Korea & 0 & 0 & 0 & 0 & 0 & 0 \\
United States & 0 & 0 & 0 & 0 & 0 & 0 \\
Western Europe & 3,850 & 11,413 & 14,038 & 13,613 & 11,781 & 9,863 \\
Total & 600 & 2,465 & 2,589 & 2,542 & 3,178 & 3,604 \\
\hline \hline
\end{tabular}

Table A-121: Delayed technology reference case with high feedstock availability sugar ethanol production [Million gallons]

\begin{tabular}{lrrrrrr} 
& $\mathbf{2 0 0 5}$ & $\mathbf{2 0 1 0}$ & $\mathbf{2 0 1 5}$ & $\mathbf{2 0 2 0}$ & $\mathbf{2 0 2 5}$ & $\mathbf{2 0 3 0}$ \\
Africa & 300 & 330 & 364 & 402 & 444 & 490 \\
Argentina & 0 & 0 & 0 & 0 & 0 & 0 \\
Australia & 0 & 0 & 0 & 0 & 0 & 0 \\
Brazil & 4,866 & 7,998 & 11,104 & 15,434 & 18,507 & 18,507 \\
Canada & 0 & 0 & 0 & 0 & 0 & 0 \\
Caribbean basin & 210 & 280 & 682 & 682 & 682 & 683 \\
China & 0 & 0 & 0 & 0 & 0 & 0 \\
Colombia & 196 & 0 & 0 & 0 & 0 & 0 \\
Eastern Europe & 0 & 0 & 0 & 0 & 0 & 0 \\
Former Soviet Union & 0 & 0 & 0 & 0 & 0 & 0 \\
India & 313 & 833 & 1,086 & 1,086 & 1,086 & 1,086 \\
Japan & 0 & 0 & 0 & 0 & 0 & 0 \\
Middle East & 0 & 0 & 0 & 0 & 0 & 0 \\
Mexico & 0 & 0 & 0 & 0 & 0 & 0 \\
Other Asia & 0 & 0 & 0 & 0 & 0 & 0 \\
South Korea & 0 & 0 & 0 & 0 & 0 & 0 \\
United States & 0 & 0 & 0 & 0 & 0 & 0 \\
Western Europe & 0 & 0 & 0 & 0 & 0 & 0 \\
\hline Total & $\mathbf{5 , 8 8 4}$ & $\mathbf{9 , 4 4 2}$ & $\mathbf{1 3 , 2 3 6}$ & $\mathbf{1 7 , 6 0 4}$ & $\mathbf{2 0 , 7 1 9}$ & $\mathbf{2 0 , 7 6 5}$ \\
\hline \hline
\end{tabular}


Table A-312: Delayed technology reference case with high feedstock availability cellulosic ethanol production [Million gallons]

\begin{tabular}{lrrrrrr} 
& $\mathbf{2 0 0 5}$ & $\mathbf{2 0 1 0}$ & $\mathbf{2 0 1 5}$ & $\mathbf{2 0 2 0}$ & $\mathbf{2 0 2 5}$ & $\mathbf{2 0 3 0}$ \\
Africa & 0 & 0 & 0 & 0 & 11 & 11 \\
Argentina & 0 & 0 & 59 & 438 & 516 & 547 \\
Australia & 0 & 0 & 66 & 247 & 627 & 1,172 \\
Brazil & 0 & 0 & 296 & 890 & 2,085 & 4,504 \\
Canada & 0 & 0 & 259 & 779 & 1,004 & 1,020 \\
Caribbean basin & 0 & 0 & 0 & 0 & 0 & 0 \\
China & 0 & 0 & 222 & 667 & 1,564 & 3,251 \\
Colombia & 0 & 0 & 170 & 512 & 887 & 887 \\
Eastern Europe & 0 & 0 & 80 & 261 & 670 & 897 \\
Former Soviet Union & 0 & 0 & 6 & 19 & 57 & 173 \\
India & 0 & 0 & 214 & 642 & 1,114 & 1,736 \\
Japan & 0 & 0 & 95 & 348 & 612 & 647 \\
Middle East & 0 & 0 & 0 & 0 & 0 & 0 \\
Mexico & 0 & 0 & 199 & 561 & 635 & 665 \\
Other Asia & 0 & 0 & 0 & 0 & 0 & 0 \\
South Korea & 0 & 0 & 25 & 25 & 25 & 25 \\
United States & 0 & 0 & 732 & 732 & 3,720 & 6,777 \\
Western Europe & 0 & 0 & 166 & 693 & 2,027 & 4,320 \\
\hline Total & $\mathbf{0}$ & $\mathbf{0}$ & $\mathbf{2 , 5 8 8}$ & $\mathbf{6 , 8 1 3}$ & $\mathbf{1 5 , 5 5 4}$ & $\mathbf{2 6 , 6 3 0}$ \\
\hline \hline
\end{tabular}

Table A-313: Delayed technology reference case with high feedstock availability grain ethanol consumption [Million gallons]

\begin{tabular}{lrrrrrr} 
& $\mathbf{2 0 0 5}$ & $\mathbf{2 0 1 0}$ & $\mathbf{2 0 1 5}$ & $\mathbf{2 0 2 0}$ & $\mathbf{2 0 2 5}$ & $\mathbf{2 0 3 0}$ \\
Africa & 0 & 0 & 0 & 0 & 0 & 0 \\
Australia & 0 & 0 & 0 & 0 & 0 & 0 \\
Canada & 0 & 0 & 0 & 0 & 0 & 0 \\
China & 344 & 0 & 0 & 325 & 0 & 0 \\
Central and South America & 0 & 0 & 11 & 11 & 57 & 61 \\
Eastern Europe & 110 & 226 & 348 & 477 & 367 & 251 \\
Former Soviet Union & 0 & 0 & 0 & 0 & 0 & 0 \\
India & 0 & 0 & 0 & 0 & 0 & 0 \\
Japan & 0 & 0 & 0 & 0 & 0 & 0 \\
Middle East & 0 & 0 & 0 & 0 & 0 & 0 \\
Mexico & 0 & 0 & 0 & 0 & 0 & 0 \\
Other Asia & 0 & 0 & 0 & 0 & 0 & 0 \\
South Korea & 0 & 0 & 0 & 0 & 0 & 0 \\
United States & 3,850 & 11,413 & 14,038 & 13,613 & 11,781 & 9,863 \\
Western Europe & 600 & 2,465 & 2,589 & 2,542 & 3,178 & 3,604 \\
\hline Total & $\mathbf{4 , 9 0 4}$ & $\mathbf{1 4 , 1 0 4}$ & $\mathbf{1 6 , 9 8 5}$ & $\mathbf{1 6 , 9 6 8}$ & $\mathbf{1 5 , 3 8 4}$ & $\mathbf{1 3 , 7 7 8}$ \\
\hline \hline
\end{tabular}


Table A-314: Delayed technology reference case with high feedstock availability sugar ethanol consumption [Million gallons]

\begin{tabular}{lrrrrrr} 
& $\mathbf{2 0 0 5}$ & $\mathbf{2 0 1 0}$ & $\mathbf{2 0 1 5}$ & $\mathbf{2 0 2 0}$ & $\mathbf{2 0 2 5}$ & $\mathbf{2 0 3 0}$ \\
Africa & 300 & 330 & 364 & 402 & 444 & 490 \\
Australia & 0 & 0 & 0 & 0 & 0 & 0 \\
Canada & 145 & 259 & 640 & 205 & 0 & 0 \\
China & 0 & 0 & 626 & 2,318 & 2,348 & 1,389 \\
Central and South America & 3,788 & 4,771 & 5,980 & 6,917 & 7,634 & 8,425 \\
Eastern Europe & 589 & 557 & 444 & 215 & 0 & 0 \\
Former Soviet Union & 0 & 0 & 0 & 0 & 0 & 0 \\
India & 313 & 833 & 1,086 & 1,086 & 1,086 & 1,086 \\
Japan & 263 & 325 & 353 & 388 & 395 & 453 \\
Middle East & 0 & 0 & 0 & 0 & 0 & 0 \\
Mexico & 0 & 0 & 0 & 0 & 0 & 44 \\
Other Asia & 0 & 0 & 0 & 526 & 1,912 & 2,828 \\
South Korea & 217 & 335 & 375 & 389 & 388 & 388 \\
United States & 134 & 256 & 1,750 & 3,306 & 4,154 & 5,662 \\
Western Europe & 136 & 1,776 & 1,618 & 1,851 & 2,358 & 0 \\
\hline Total & $\mathbf{5 , 8 8 4}$ & $\mathbf{9 , 4 4 2}$ & $\mathbf{1 3 , 2 3 6}$ & $\mathbf{1 7 , 6 0 4}$ & $\mathbf{2 0 , 7 1 9}$ & $\mathbf{2 0 , 7 6 5}$ \\
\hline \hline
\end{tabular}

Table A-315: Delayed technology reference case with high feedstock availability cellulosic ethanol consumption [Million gallons]

\begin{tabular}{lrrrrrr} 
& $\mathbf{2 0 0 5}$ & $\mathbf{2 0 1 0}$ & $\mathbf{2 0 1 5}$ & $\mathbf{2 0 2 0}$ & $\mathbf{2 0 2 5}$ & $\mathbf{2 0 3 0}$ \\
Africa & 0 & 0 & 0 & 0 & 11 & 11 \\
Australia & 0 & 0 & 66 & 247 & 627 & 1,172 \\
Canada & 0 & 0 & 259 & 779 & 1,004 & 1,020 \\
China & 0 & 0 & 222 & 754 & 1,784 & 3,674 \\
Central and South America & 0 & 0 & 26 & 125 & 133 & 133 \\
Eastern Europe & 0 & 0 & 80 & 261 & 670 & 897 \\
Former Soviet Union & 0 & 0 & 6 & 19 & 57 & 173 \\
India & 0 & 0 & 214 & 556 & 894 & 1,313 \\
Japan & 0 & 0 & 95 & 348 & 612 & 647 \\
Middle East & 0 & 0 & 0 & 0 & 0 & 0 \\
Mexico & 0 & 0 & 30 & 130 & 83 & 86 \\
Other Asia & 0 & 0 & 0 & 0 & 0 & 0 \\
South Korea & 0 & 0 & 25 & 25 & 25 & 25 \\
United States & 0 & 0 & 1,400 & 2,878 & 7,627 & 13,160 \\
Western Europe & 0 & 0 & 166 & 693 & 2,027 & 4,320 \\
\hline Total & $\mathbf{0}$ & $\mathbf{0}$ & $\mathbf{2 , 5 8 8}$ & $\mathbf{6 , 8 1 3}$ & $\mathbf{1 5 , 5 5 4}$ & $\mathbf{2 6 , 6 3 0}$ \\
\hline \hline
\end{tabular}


Table A-316: Delayed technology reference case with high feedstock availability grain ethanol net trade [Million gallons]

\begin{tabular}{lrrrrrr} 
& $\mathbf{2 0 0 5}$ & $\mathbf{2 0 1 0}$ & $\mathbf{2 0 1 5}$ & $\mathbf{2 0 2 0}$ & $\mathbf{2 0 2 5}$ & $\mathbf{2 0 3 0}$ \\
Africa & 0 & 0 & 0 & 0 & 0 & 0 \\
Australia & 0 & 0 & 0 & 0 & 0 & 0 \\
Canada & 0 & 0 & 0 & 0 & 0 & 0 \\
China & 0 & 0 & 0 & 0 & 0 & 0 \\
Central and South America & 0 & 0 & 0 & 0 & 0 & 0 \\
Eastern Europe & 0 & 0 & 0 & 0 & 0 & 0 \\
Former Soviet Union & 0 & 0 & 0 & 0 & 0 & 0 \\
India & 0 & 0 & 0 & 0 & 0 & 0 \\
Japan & 0 & 0 & 0 & 0 & 0 & 0 \\
Middle East & 0 & 0 & 0 & 0 & 0 & 0 \\
Mexico & 0 & 0 & 0 & 0 & 0 & 0 \\
Other Asia & 0 & 0 & 0 & 0 & 0 & 0 \\
South Korea & 0 & 0 & 0 & 0 & 0 & 0 \\
United States & 0 & 0 & 0 & 0 & 0 & 0 \\
Western Europe & 0 & 0 & 0 & 0 & 0 & 0 \\
\hline Total & $\mathbf{0}$ & $\mathbf{0}$ & $\mathbf{0}$ & $\mathbf{0}$ & $\mathbf{0}$ & $\mathbf{0}$ \\
\hline \hline
\end{tabular}

Table A-317: Delayed technology reference case with high feedstock availability sugar ethanol net trade [Million gallons]

\begin{tabular}{lrrrrrr} 
Africa & 0 & 0 & 0 & 0 & 0 & 0 \\
Australia & 0 & 0 & 0 & 0 & 0 & 0 \\
Canada & -145 & -259 & -640 & -205 & 0 & 0 \\
China & 0 & 0 & -626 & $-2,318$ & $-2,348$ & $-1,389$ \\
Central and South America & 1,484 & 3,508 & 5,806 & 9,199 & 11,555 & 10,764 \\
Eastern Europe & -589 & -557 & -444 & -215 & 0 & 0 \\
Former Soviet Union & 0 & 0 & 0 & 0 & 0 & 0 \\
India & 0 & 0 & 0 & 0 & 0 & 0 \\
Japan & -263 & -325 & -353 & -388 & -395 & -453 \\
Middle East & 0 & 0 & 0 & 0 & 0 & 0 \\
Mexico & 0 & 0 & 0 & 0 & 0 & -44 \\
Other Asia & 0 & 0 & 0 & -526 & $-1,912$ & $-2,828$ \\
South Korea & -217 & -335 & -375 & -389 & -388 & -388 \\
United States & -134 & -256 & $-1,750$ & $-3,306$ & $-4,154$ & $-5,662$ \\
Western Europe & -136 & $-1,776$ & $-1,618$ & $-1,851$ & $-2,358$ & 0 \\
\hline Total & $\mathbf{0}$ & $\mathbf{0}$ & $\mathbf{0}$ & $\mathbf{0}$ & $\mathbf{0}$ & $\mathbf{0}$ \\
\hline \hline
\end{tabular}


Table A-318: Delayed technology reference case with high feedstock availability cellulosic ethanol net trade [Million gallons]

\begin{tabular}{lrrrrrr} 
& $\mathbf{2 0 0 5}$ & $\mathbf{2 0 1 0}$ & $\mathbf{2 0 1 5}$ & $\mathbf{2 0 2 0}$ & $\mathbf{2 0 2 5}$ & $\mathbf{2 0 3 0}$ \\
Africa & 0 & 0 & 0 & 0 & 0 & 0 \\
Australia & 0 & 0 & 0 & 0 & 0 & 0 \\
Canada & 0 & 0 & 0 & 0 & 0 & 0 \\
China & 0 & 0 & 0 & -86 & -220 & -423 \\
Central and South America & 0 & 0 & 499 & 1,715 & 3,355 & 5,804 \\
Eastern Europe & 0 & 0 & 0 & 0 & 0 & 0 \\
Former Soviet Union & 0 & 0 & 0 & 0 & 0 & 0 \\
India & 0 & 0 & 0 & 86 & 220 & 423 \\
Japan & 0 & 0 & 0 & 0 & 0 & 0 \\
Middle East & 0 & 0 & 0 & 0 & 0 & 0 \\
Mexico & 0 & 0 & 170 & 431 & 553 & 579 \\
Other Asia & 0 & 0 & 0 & 0 & 0 & 0 \\
South Korea & 0 & 0 & 0 & 0 & 0 & 0 \\
United States & 0 & 0 & -669 & $-2,146$ & $-3,907$ & $-6,382$ \\
Western Europe & 0 & 0 & 0 & 0 & 0 & 0 \\
\hline Total & $\mathbf{0}$ & $\mathbf{0}$ & $\mathbf{0}$ & $\mathbf{0}$ & $\mathbf{0}$ & $\mathbf{0}$ \\
\hline \hline
\end{tabular}

Table A-319: Delayed technology reference case with high feedstock availability biomass-to-liquids production [Million gallons ethanol equivalent]

\begin{tabular}{lrrrrrr} 
& $\mathbf{2 0 0 5}$ & $\mathbf{2 0 1 0}$ & $\mathbf{2 0 1 5}$ & $\mathbf{2 0 2 0}$ & $\mathbf{2 0 2 5}$ & $\mathbf{2 0 3 0}$ \\
Africa & 0 & 0 & 0 & 0 & 0 & 0 \\
Argentina & 0 & 0 & 21 & 158 & 186 & 197 \\
Australia & 0 & 0 & 0 & 0 & 0 & 0 \\
Brazil & 0 & 0 & 106 & 320 & 750 & 1,333 \\
Canada & 0 & 0 & 93 & 280 & 361 & 367 \\
Caribbean basin & 0 & 0 & 0 & 0 & 0 & 0 \\
China & 0 & 0 & 80 & 240 & 563 & 1,031 \\
Colombia & 0 & 0 & 61 & 184 & 319 & 319 \\
Eastern Europe & 0 & 0 & 0 & 0 & 0 & 0 \\
Former Soviet Union & 0 & 0 & 0 & 0 & 0 & 0 \\
India & 0 & 0 & 77 & 231 & 401 & 625 \\
Japan & 0 & 0 & 0 & 0 & 0 & 0 \\
Middle East & 0 & 0 & 0 & 0 & 0 & 0 \\
Mexico & 0 & 0 & 72 & 202 & 229 & 239 \\
Other Asia & 0 & 0 & 0 & 0 & 0 & 0 \\
South Korea & 0 & 0 & 31 & 53 & 58 & 58 \\
United States & 0 & 0 & 750 & 1,561 & 2,242 & 2,908 \\
Western Europe & 0 & 0 & 0 & 0 & 0 & 0 \\
\hline Total & $\mathbf{0}$ & $\mathbf{0}$ & $\mathbf{1 , 2 9 1}$ & $\mathbf{3 , 2 2 9}$ & $\mathbf{5 , 1 0 7}$ & $\mathbf{7 , 0 7 5}$ \\
\hline \hline
\end{tabular}


Table A-320: Delayed technology reference case with high feedstock availability biomass-to-liquids consumption [Million gallons ethanol equivalent]

\begin{tabular}{lrrrrrr} 
& $\mathbf{2 0 0 5}$ & $\mathbf{2 0 1 0}$ & $\mathbf{2 0 1 5}$ & $\mathbf{2 0 2 0}$ & $\mathbf{2 0 2 5}$ & $\mathbf{2 0 3 0}$ \\
Africa & 0 & 0 & 0 & 0 & 0 & 0 \\
Australia & 0 & 0 & 0 & 0 & 0 & 0 \\
Canada & 0 & 0 & 0 & 0 & 0 & 0 \\
China & 0 & 0 & 0 & 0 & 0 & 0 \\
Central and South America & 0 & 0 & 0 & 0 & 0 & 0 \\
Eastern Europe & 0 & 0 & 0 & 0 & 0 & 0 \\
Former Soviet Union & 0 & 0 & 0 & 0 & 0 & 0 \\
India & 0 & 0 & 0 & 0 & 0 & 0 \\
Japan & 0 & 0 & 0 & 0 & 0 & 0 \\
Middle East & 0 & 0 & 0 & 0 & 0 & 0 \\
Mexico & 0 & 0 & 0 & 0 & 0 & 0 \\
Other Asia & 0 & 0 & 0 & 0 & 0 & 0 \\
South Korea & 0 & 0 & 0 & 0 & 0 & 0 \\
United States & 0 & 0 & 1,291 & 3,229 & 5,107 & 7,076 \\
Western Europe & 0 & 0 & 0 & 0 & 0 & 0 \\
\hline Total & $\mathbf{0}$ & $\mathbf{0}$ & $\mathbf{1 , 2 9 1}$ & $\mathbf{3 , 2 2 9}$ & $\mathbf{5 , 1 0 7}$ & $\mathbf{7 , 0 7 6}$ \\
\hline \hline
\end{tabular}

Table A-321: Delayed technology reference case with high feedstock availability biomass-to-liquids net trade [Million gallons ethanol equivalent]

\begin{tabular}{lrrrrrr} 
& $\mathbf{2 0 0 5}$ & $\mathbf{2 0 1 0}$ & $\mathbf{2 0 1 5}$ & $\mathbf{2 0 2 0}$ & $\mathbf{2 0 2 5}$ & $\mathbf{2 0 3 0}$ \\
Africa & 0 & 0 & 0 & 0 & 0 & 0 \\
Australia & 0 & 0 & 0 & 0 & 0 & 0 \\
Canada & 0 & 0 & 93 & 280 & 361 & 367 \\
China & 0 & 0 & 80 & 240 & 563 & 1,031 \\
Central and South America & 0 & 0 & 189 & 662 & 1,255 & 1,849 \\
Eastern Europe & 0 & 0 & 0 & 0 & 0 & 0 \\
Former Soviet Union & 0 & 0 & 0 & 0 & 0 & 0 \\
India & 0 & 0 & 77 & 231 & 401 & 625 \\
Japan & 0 & 0 & 0 & 0 & 0 & 0 \\
Middle East & 0 & 0 & 0 & 0 & 0 & 0 \\
Mexico & 0 & 0 & 72 & 202 & 229 & 239 \\
Other Asia & 0 & 0 & 0 & 0 & 0 & 0 \\
South Korea & 0 & 0 & 31 & 53 & 58 & 58 \\
United States & 0 & 0 & -542 & $-1,668$ & $-2,865$ & $-4,168$ \\
Western Europe & 0 & 0 & 0 & 0 & 0 & 0 \\
\hline Total & $\mathbf{0}$ & $\mathbf{0}$ & $\mathbf{0}$ & $\mathbf{0}$ & $\mathbf{0}$ & $\mathbf{0}$ \\
\hline \hline
\end{tabular}


Table A-322: Delayed technology reference case with high feedstock availability biodiesel production [Million gallons ethanol equivalent]

\begin{tabular}{lrrrrrr} 
& $\mathbf{2 0 0 5}$ & $\mathbf{2 0 1 0}$ & $\mathbf{2 0 1 5}$ & $\mathbf{2 0 2 0}$ & $\mathbf{2 0 2 5}$ & $\mathbf{2 0 3 0}$ \\
Africa & 0 & 0 & 0 & 0 & 0 & 0 \\
Argentina & 0 & 333 & 877 & 1,642 & 1,642 & 198 \\
Australia & 0 & 0 & 0 & 0 & 0 & 0 \\
Brazil & 0 & 333 & 835 & 1,473 & 1,473 & 1,105 \\
Canada & 0 & 0 & 0 & 0 & 0 & 0 \\
Caribbean basin & 0 & 95 & 310 & 742 & 1,611 & 3,359 \\
China & 0 & 0 & 0 & 0 & 0 & 0 \\
Colombia & 0 & 119 & 358 & 838 & 1,648 & 2,074 \\
Eastern Europe & 0 & 0 & 0 & 0 & 0 & 0 \\
Former Soviet Union & 0 & 0 & 0 & 0 & 0 & 0 \\
India & 0 & 0 & 0 & 0 & 0 & 0 \\
Japan & 0 & 0 & 0 & 0 & 0 & 0 \\
Middle East & 0 & 0 & 0 & 0 & 0 & 0 \\
Mexico & 0 & 0 & 0 & 0 & 0 & 0 \\
Other Asia & 0 & 0 & 0 & 0 & 0 & 0 \\
South Korea & 0 & 0 & 0 & 0 & 0 & 0 \\
United States & 0 & 1,182 & 1,640 & 1,747 & 1,850 & 2,024 \\
Western Europe & 998 & 3,178 & 3,578 & 3,930 & 4,468 & 4,788 \\
\hline Total & $\mathbf{9 9 8}$ & $\mathbf{5 , 2 3 8}$ & $\mathbf{7 , 5 9 7}$ & $\mathbf{1 0 , 3 7 2}$ & $\mathbf{1 2 , 6 9 2}$ & $\mathbf{1 3 , 5 4 7}$ \\
\hline \hline
\end{tabular}

Table A-323: Delayed technology reference case with high feedstock availability biodiesel consumption [Million gallons ethanol equivalent]

\begin{tabular}{lrrrrrr} 
& $\mathbf{2 0 0 5}$ & $\mathbf{2 0 1 0}$ & $\mathbf{2 0 1 5}$ & $\mathbf{2 0 2 0}$ & $\mathbf{2 0 2 5}$ & $\mathbf{2 0 3 0}$ \\
Africa & 0 & 0 & 0 & 0 & 0 & 0 \\
Australia & 0 & 0 & 0 & 0 & 0 & 0 \\
Canada & 0 & 0 & 0 & 0 & 0 & 0 \\
China & 0 & 0 & 0 & 0 & 0 & 0 \\
Central and South America & 0 & 158 & 472 & 984 & 1,306 & 1,490 \\
Eastern Europe & 0 & 0 & 0 & 0 & 0 & 0 \\
Former Soviet Union & 0 & 0 & 0 & 0 & 0 & 0 \\
India & 0 & 0 & 0 & 0 & 0 & 0 \\
Japan & 0 & 0 & 0 & 0 & 0 & 0 \\
Middle East & 0 & 0 & 0 & 0 & 0 & 0 \\
Mexico & 0 & 0 & 0 & 0 & 0 & 0 \\
Other Asia & 0 & 0 & 0 & 0 & 0 & 0 \\
South Korea & 0 & 0 & 0 & 0 & 0 & 0 \\
United States & 0 & 1,182 & 1,713 & 2,581 & 4,065 & 4,475 \\
Western Europe & 998 & 3,898 & 5,412 & 6,807 & 7,321 & 7,582 \\
\hline Total & $\mathbf{9 9 8}$ & $\mathbf{5 , 2 3 8}$ & $\mathbf{7 , 5 9 7}$ & $\mathbf{1 0 , 3 7 2}$ & $\mathbf{1 2 , 6 9 2}$ & $\mathbf{1 3 , 5 4 7}$ \\
\hline \hline
\end{tabular}


Table A-324: Delayed technology reference case with high feedstock availability biodiesel net trade [Million gallons ethanol equivalent]

\begin{tabular}{lrrrrrr} 
& $\mathbf{2 0 0 5}$ & $\mathbf{2 0 1 0}$ & $\mathbf{2 0 1 5}$ & $\mathbf{2 0 2 0}$ & $\mathbf{2 0 2 5}$ & $\mathbf{2 0 3 0}$ \\
Africa & 0 & 0 & 0 & 0 & 0 & 0 \\
Australia & 0 & 0 & 0 & 0 & 0 & 0 \\
Canada & 0 & 0 & 0 & 0 & 0 & 0 \\
China & 0 & 0 & 0 & 0 & 0 & 0 \\
Central and South America & 0 & 720 & 1,907 & 3,711 & 5,068 & 5,245 \\
Eastern Europe & 0 & 0 & 0 & 0 & 0 & 0 \\
Former Soviet Union & 0 & 0 & 0 & 0 & 0 & 0 \\
India & 0 & 0 & 0 & 0 & 0 & 0 \\
Japan & 0 & 0 & 0 & 0 & 0 & 0 \\
Middle East & 0 & 0 & 0 & 0 & 0 & 0 \\
Mexico & 0 & 0 & 0 & 0 & 0 & 0 \\
Other Asia & 0 & 0 & 0 & 0 & 0 & 0 \\
South Korea & 0 & 0 & 0 & 0 & 0 & 0 \\
United States & 0 & 0 & -73 & -834 & $-2,215$ & $-2,451$ \\
Western Europe & 0 & -720 & $-1,834$ & $-2,877$ & $-2,853$ & $-2,794$ \\
\hline Total & $\mathbf{0}$ & $\mathbf{0}$ & $\mathbf{0}$ & $\mathbf{0}$ & $\mathbf{0}$ & $\mathbf{0}$ \\
\hline \hline
\end{tabular}




\section{A-19 Delayed Technology Reference Case with Low Feedstock Availability}

\section{Table A-325: Delayed technology reference case with low feedstock availability} ethanol production [Million gallons]

\begin{tabular}{lrrrrrr} 
& $\mathbf{2 0 0 5}$ & $\mathbf{2 0 1 0}$ & $\mathbf{2 0 1 5}$ & $\mathbf{2 0 2 0}$ & $\mathbf{2 0 2 5}$ & $\mathbf{2 0 3 0}$ \\
Africa & 300 & 330 & 364 & 402 & 455 & 502 \\
Argentina & 0 & 178 & 755 & 1,141 & 1,147 & 1,147 \\
Australia & 0 & 0 & 66 & 247 & 635 & 1,172 \\
Brazil & 4,866 & 4,894 & 6,282 & 7,363 & 8,942 & 11,817 \\
Canada & 0 & 143 & 401 & 921 & 1,115 & 1,130 \\
Caribbean basin & 162 & 80 & 34 & 0 & 0 & 0 \\
China & 344 & 366 & 608 & 965 & 1,564 & 2,290 \\
Colombia & 0 & 196 & 418 & 433 & 433 & 433 \\
Eastern Europe & 110 & 226 & 434 & 780 & 1,037 & 1,149 \\
Former Soviet Union & 0 & 0 & 6 & 18 & 54 & 165 \\
India & 313 & 0 & 214 & 645 & 1,512 & 2,807 \\
Japan & 0 & 0 & 95 & 348 & 612 & 647 \\
Middle East & 0 & 0 & 0 & 0 & 0 & 0 \\
Mexico & 0 & 0 & 0 & 0 & 0 & 0 \\
Other Asia & 0 & 0 & 0 & 0 & 0 & 0 \\
South Korea & 0 & 0 & 35 & 35 & 35 & 35 \\
United States & 3,850 & 11,612 & 15,767 & 18,998 & 22,316 & 24,911 \\
Western Europe & 600 & 2,570 & 2,755 & 3,398 & 5,291 & 8,392 \\
\hline Total & $\mathbf{1 0 , 5 4 4}$ & $\mathbf{2 0 , 5 9 4}$ & $\mathbf{2 8 , 2 3 4}$ & $\mathbf{3 5 , 6 9 3}$ & $\mathbf{4 5 , 1 4 9}$ & $\mathbf{5 6 , 5 9 6}$ \\
\hline \hline
\end{tabular}


Table A-326: Delayed technology reference case with low feedstock availability ethanol consumption [Million gallons]

\begin{tabular}{lrrrrrr} 
& $\mathbf{2 0 0 5}$ & $\mathbf{2 0 1 0}$ & $\mathbf{2 0 1 5}$ & $\mathbf{2 0 2 0}$ & $\mathbf{2 0 2 5}$ & $\mathbf{2 0 3 0}$ \\
Africa & 300 & 330 & 364 & 402 & 455 & 502 \\
Australia & 0 & 0 & 66 & 247 & 635 & 1,172 \\
Canada & 101 & 128 & 361 & 829 & 1,004 & 1,020 \\
China & 126 & 0 & 0 & 0 & 790 & 1,497 \\
Central and South America & 3,764 & 4,831 & 6,116 & 7,097 & 7,780 & 8,571 \\
Eastern Europe & 699 & 226 & 434 & 780 & 1,037 & 1,149 \\
Former Soviet Union & 0 & 0 & 6 & 18 & 54 & 165 \\
India & 313 & 0 & 214 & 613 & 1,361 & 2,399 \\
Japan & 257 & 132 & 298 & 698 & 1,008 & 1,062 \\
Middle East & 0 & 0 & 0 & 0 & 0 & 0 \\
Mexico & 0 & 0 & 0 & 0 & 0 & 0 \\
Other Asia & 0 & 0 & 0 & 0 & 0 & 0 \\
South Korea & 264 & 0 & 400 & 414 & 413 & 413 \\
United States & 3,984 & 11,612 & 15,767 & 19,347 & 22,962 & 28,414 \\
Western Europe & 736 & 3,335 & 4,208 & 5,249 & 7,649 & 10,233 \\
\hline Total & $\mathbf{1 0 , 5 4 4}$ & $\mathbf{2 0 , 5 9 4}$ & $\mathbf{2 8 , 2 3 4}$ & $\mathbf{3 5 , 6 9 3}$ & $\mathbf{4 5 , 1 4 9}$ & $\mathbf{5 6 , 5 9 6}$ \\
\hline \hline
\end{tabular}

Table A-327: Delayed technology reference case with low feedstock availability ethanol net trade [Million gallons]

\begin{tabular}{lrrrrrr} 
Africa & 0 & 0 & 0 & 0 & 0 & 0 \\
Australia & 0 & 0 & 0 & 0 & 0 & 0 \\
Canada & -101 & 14 & 40 & 92 & 112 & 110 \\
China & 218 & 366 & 608 & 965 & 774 & 794 \\
Central and South America & 1,264 & 517 & 1,373 & 1,840 & 2,742 & 4,826 \\
Eastern Europe & -589 & 0 & 0 & 0 & 0 & 0 \\
Former Soviet Union & 0 & 0 & 0 & 0 & 0 & 0 \\
India & 0 & 0 & 0 & 32 & 151 & 408 \\
Japan & -257 & -132 & -203 & -350 & -395 & -415 \\
Middle East & 0 & 0 & 0 & 0 & 0 & 0 \\
Mexico & 0 & 0 & 0 & 0 & 0 & 0 \\
Other Asia & 0 & 0 & 0 & 0 & 0 & 0 \\
South Korea & -264 & 0 & -365 & -379 & -378 & -378 \\
United States & -134 & 0 & 0 & -348 & -647 & $-3,504$ \\
Western Europe & -136 & -765 & $-1,454$ & $-1,851$ & $-2,358$ & $-1,841$ \\
\hline Total & $\mathbf{0}$ & $\mathbf{0}$ & $\mathbf{0}$ & $\mathbf{0}$ & $\mathbf{0}$ & $\mathbf{0}$ \\
\hline \hline
\end{tabular}


Table A-328: Delayed technology reference case with low feedstock availability grain ethanol production [Million gallons]

\begin{tabular}{lrrrrrr} 
& $\mathbf{2 0 0 5}$ & $\mathbf{2 0 1 0}$ & $\mathbf{2 0 1 5}$ & $\mathbf{2 0 2 0}$ & $\mathbf{2 0 2 5}$ & $\mathbf{2 0 3 0}$ \\
Africa & 0 & 0 & 0 & 0 & 0 & 0 \\
Argentina & 0 & 178 & 676 & 833 & 839 & 839 \\
Australia & 0 & 0 & 0 & 0 & 0 & 0 \\
Brazil & 0 & 0 & 0 & 0 & 0 & 0 \\
Canada & 0 & 143 & 143 & 143 & 0 & 0 \\
Caribbean basin & 0 & 0 & 0 & 0 & 0 & 0 \\
China & 344 & 366 & 366 & 298 & 0 & 0 \\
Colombia & 0 & 0 & 0 & 0 & 0 & 0 \\
Eastern Europe & 110 & 226 & 348 & 477 & 367 & 251 \\
Former Soviet Union & 0 & 0 & 0 & 0 & 0 & 0 \\
India & 0 & 0 & 0 & 0 & 0 & 0 \\
Japan & 0 & 0 & 0 & 0 & 0 & 0 \\
Middle East & 0 & 0 & 0 & 0 & 0 & 0 \\
Mexico & 0 & 0 & 0 & 0 & 0 & 0 \\
Other Asia & 0 & 0 & 0 & 0 & 0 & 0 \\
South Korea & 0 & 0 & 0 & 0 & 0 & 0 \\
United States & 3,850 & 11,612 & 15,000 & 15,000 & 15,000 & 15,000 \\
Western Europe & 600 & 2,570 & 2,589 & 2,705 & 3,178 & 3,813 \\
\hline Total & $\mathbf{4 , 9 0 4}$ & $\mathbf{1 5 , 0 9 4}$ & $\mathbf{1 9 , 1 2 1}$ & $\mathbf{1 9 , 4 5 5}$ & $\mathbf{1 9 , 3 8 4}$ & $\mathbf{1 9 , 9 0 4}$ \\
\hline \hline
\end{tabular}

Table A-329: Delayed technology reference case with low feedstock availability sugar ethanol production [Million gallons]

\begin{tabular}{lrrrrrr} 
& $\mathbf{2 0 0 5}$ & $\mathbf{2 0 1 0}$ & $\mathbf{2 0 1 5}$ & $\mathbf{2 0 2 0}$ & $\mathbf{2 0 2 5}$ & $\mathbf{2 0 3 0}$ \\
Africa & 300 & 330 & 364 & 402 & 444 & 490 \\
Argentina & 0 & 0 & 0 & 0 & 0 & 0 \\
Australia & 0 & 0 & 0 & 0 & 0 & 0 \\
Brazil & 4,866 & 4,894 & 5,978 & 6,473 & 6,857 & 7,093 \\
Canada & 0 & 0 & 0 & 0 & 0 & 0 \\
Caribbean basin & 162 & 80 & 34 & 0 & 0 & 0 \\
China & 0 & 0 & 0 & 0 & 0 & 0 \\
Colombia & 0 & 196 & 248 & 0 & 0 & 0 \\
Eastern Europe & 0 & 0 & 0 & 0 & 0 & 0 \\
Former Soviet Union & 0 & 0 & 0 & 0 & 0 & 0 \\
India & 313 & 0 & 0 & 0 & 0 & 0 \\
Japan & 0 & 0 & 0 & 0 & 0 & 0 \\
Middle East & 0 & 0 & 0 & 0 & 0 & 0 \\
Mexico & 0 & 0 & 0 & 0 & 0 & 0 \\
Other Asia & 0 & 0 & 0 & 0 & 0 & 0 \\
South Korea & 0 & 0 & 0 & 0 & 0 & 0 \\
United States & 0 & 0 & 0 & 0 & 0 & 0 \\
Western Europe & 0 & 0 & 0 & 0 & 0 & 0 \\
\hline Total & $\mathbf{5 , 6 4 0}$ & $\mathbf{5 , 5 0 0}$ & $\mathbf{6 , 6 2 4}$ & $\mathbf{6 , 8 7 5}$ & $\mathbf{7 , 3 0 1}$ & $\mathbf{7 , 5 8 4}$ \\
\hline \hline
\end{tabular}


Table A-330: Delayed technology reference case with low feedstock availability cellulosic ethanol production [Million gallons]

\begin{tabular}{lrrrrrr} 
& $\mathbf{2 0 0 5}$ & $\mathbf{2 0 1 0}$ & $\mathbf{2 0 1 5}$ & $\mathbf{2 0 2 0}$ & $\mathbf{2 0 2 5}$ & $\mathbf{2 0 3 0}$ \\
Africa & 0 & 0 & 0 & 0 & 11 & 11 \\
Argentina & 0 & 0 & 79 & 308 & 308 & 308 \\
Australia & 0 & 0 & 66 & 247 & 635 & 1,172 \\
Brazil & 0 & 0 & 304 & 890 & 2,085 & 4,723 \\
Canada & 0 & 0 & 259 & 779 & 1,115 & 1,130 \\
Caribbean basin & 0 & 0 & 0 & 0 & 0 & 0 \\
China & 0 & 0 & 243 & 668 & 1,564 & 2,290 \\
Colombia & 0 & 0 & 170 & 433 & 433 & 433 \\
Eastern Europe & 0 & 0 & 85 & 303 & 670 & 897 \\
Former Soviet Union & 0 & 0 & 6 & 18 & 54 & 165 \\
India & 0 & 0 & 214 & 645 & 1,512 & 2,807 \\
Japan & 0 & 0 & 95 & 348 & 612 & 647 \\
Middle East & 0 & 0 & 0 & 0 & 0 & 0 \\
Mexico & 0 & 0 & 0 & 0 & 0 & 0 \\
Other Asia & 0 & 0 & 0 & 0 & 0 & 0 \\
South Korea & 0 & 0 & 35 & 35 & 35 & 35 \\
United States & 0 & 0 & 767 & 3,998 & 7,316 & 9,911 \\
Western Europe & 0 & 0 & 166 & 693 & 2,113 & 4,579 \\
\hline Total & $\mathbf{0}$ & $\mathbf{0}$ & $\mathbf{2 , 4 8 9}$ & $\mathbf{9 , 3 6 3}$ & $\mathbf{1 8 , 4 6 4}$ & $\mathbf{2 9 , 1 0 9}$ \\
\hline \hline
\end{tabular}

Table A-331: Delayed technology reference case with low feedstock availability grain ethanol consumption [Million gallons]

\begin{tabular}{lrrrrrr} 
& $\mathbf{2 0 0 5}$ & $\mathbf{2 0 1 0}$ & $\mathbf{2 0 1 5}$ & $\mathbf{2 0 2 0}$ & $\mathbf{2 0 2 5}$ & $\mathbf{2 0 3 0}$ \\
Africa & 0 & 0 & 0 & 0 & 0 & 0 \\
Australia & 0 & 0 & 0 & 0 & 0 & 0 \\
Canada & 0 & 128 & 143 & 143 & 0 & 0 \\
China & 126 & 0 & 0 & 0 & 0 & 0 \\
Central and South America & 0 & 36 & 113 & 148 & 115 & 115 \\
Eastern Europe & 110 & 226 & 348 & 477 & 367 & 251 \\
Former Soviet Union & 0 & 0 & 0 & 0 & 0 & 0 \\
India & 0 & 0 & 0 & 0 & 0 & 0 \\
Japan & 0 & 132 & 203 & 298 & 0 & 0 \\
Middle East & 0 & 0 & 0 & 0 & 0 & 0 \\
Mexico & 0 & 0 & 0 & 0 & 0 & 0 \\
Other Asia & 0 & 0 & 0 & 0 & 0 & 0 \\
South Korea & 218 & 0 & 163 & 0 & 0 & 0 \\
United States & 3,850 & 11,612 & 15,000 & 15,000 & 15,000 & 15,000 \\
Western Europe & 600 & 2,960 & 3,151 & 3,389 & 3,902 & 4,537 \\
\hline Total & $\mathbf{4 , 9 0 4}$ & $\mathbf{1 5 , 0 9 4}$ & $\mathbf{1 9 , 1 2 1}$ & $\mathbf{1 9 , 4 5 5}$ & $\mathbf{1 9 , 3 8 4}$ & $\mathbf{1 9 , 9 0 4}$ \\
\hline \hline
\end{tabular}


Table A-332: Delayed technology reference case with low feedstock availability sugar ethanol consumption [Million gallons]

\begin{tabular}{lrrrrrr} 
& $\mathbf{2 0 0 5}$ & $\mathbf{2 0 1 0}$ & $\mathbf{2 0 1 5}$ & $\mathbf{2 0 2 0}$ & $\mathbf{2 0 2 5}$ & $\mathbf{2 0 3 0}$ \\
Africa & 300 & 330 & 364 & 402 & 444 & 490 \\
Australia & 0 & 0 & 0 & 0 & 0 & 0 \\
Canada & 101 & 0 & 0 & 0 & 0 & 0 \\
China & 0 & 0 & 0 & 0 & 0 & 0 \\
Central and South America & 3,764 & 4,795 & 6,002 & 6,124 & 6,210 & 7,020 \\
Eastern Europe & 589 & 0 & 0 & 0 & 0 & 0 \\
Former Soviet Union & 0 & 0 & 0 & 0 & 0 & 0 \\
India & 313 & 0 & 0 & 0 & 0 & 0 \\
Japan & 257 & 0 & 0 & 0 & 0 & 0 \\
Middle East & 0 & 0 & 0 & 0 & 0 & 0 \\
Mexico & 0 & 0 & 0 & 0 & 0 & 0 \\
Other Asia & 0 & 0 & 0 & 0 & 0 & 0 \\
South Korea & 46 & 0 & 0 & 0 & 0 & 0 \\
United States & 134 & 0 & 0 & 348 & 647 & 73 \\
Western Europe & 136 & 375 & 258 & 0 & 0 & 0 \\
\hline Total & $\mathbf{5 , 6 4 0}$ & $\mathbf{5 , 5 0 0}$ & $\mathbf{6 , 6 2 4}$ & $\mathbf{6 , 8 7 5}$ & $\mathbf{7 , 3 0 1}$ & $\mathbf{7 , 5 8 4}$ \\
\hline \hline
\end{tabular}

Table A-333: Delayed technology reference case with low feedstock availability cellulosic ethanol consumption [Million gallons]

\begin{tabular}{lrrrrrr} 
& $\mathbf{2 0 0 5}$ & $\mathbf{2 0 1 0}$ & $\mathbf{2 0 1 5}$ & $\mathbf{2 0 2 0}$ & $\mathbf{2 0 2 5}$ & $\mathbf{2 0 3 0}$ \\
Africa & 0 & 0 & 0 & 0 & 11 & 11 \\
Australia & 0 & 0 & 66 & 247 & 635 & 1,172 \\
Canada & 0 & 0 & 219 & 687 & 1,004 & 1,020 \\
China & 0 & 0 & 0 & 0 & 790 & 1,497 \\
Central and South America & 0 & 0 & 0 & 824 & 1,455 & 1,436 \\
Eastern Europe & 0 & 0 & 85 & 303 & 670 & 897 \\
Former Soviet Union & 0 & 0 & 6 & 18 & 54 & 165 \\
India & 0 & 0 & 214 & 613 & 1,361 & 2,399 \\
Japan & 0 & 0 & 95 & 401 & 1,008 & 1,062 \\
Middle East & 0 & 0 & 0 & 0 & 0 & 0 \\
Mexico & 0 & 0 & 0 & 0 & 0 & 0 \\
Other Asia & 0 & 0 & 0 & 0 & 0 & 0 \\
South Korea & 0 & 0 & 237 & 414 & 413 & 413 \\
United States & 0 & 0 & 767 & 3,998 & 7,316 & 13,341 \\
Western Europe & 0 & 0 & 800 & 1,859 & 3,748 & 5,696 \\
\hline Total & $\mathbf{0}$ & $\mathbf{0}$ & $\mathbf{2 , 4 8 9}$ & $\mathbf{9 , 3 6 3}$ & $\mathbf{1 8 , 4 6 4}$ & $\mathbf{2 9 , 1 0 9}$ \\
\hline \hline
\end{tabular}


Table A-334: Delayed technology reference case with low feedstock availability grain ethanol net trade [Million gallons]

\begin{tabular}{lrrrrrr} 
& $\mathbf{2 0 0 5}$ & $\mathbf{2 0 1 0}$ & $\mathbf{2 0 1 5}$ & $\mathbf{2 0 2 0}$ & $\mathbf{2 0 2 5}$ & $\mathbf{2 0 3 0}$ \\
Africa & 0 & 0 & 0 & 0 & 0 & 0 \\
Australia & 0 & 0 & 0 & 0 & 0 & 0 \\
Canada & 0 & 14 & 0 & 0 & 0 & 0 \\
China & 218 & 366 & 366 & 298 & 0 & 0 \\
Central and South America & 0 & 143 & 562 & 684 & 724 & 724 \\
Eastern Europe & 0 & 0 & 0 & 0 & 0 & 0 \\
Former Soviet Union & 0 & 0 & 0 & 0 & 0 & 0 \\
India & 0 & 0 & 0 & 0 & 0 & 0 \\
Japan & 0 & -132 & -203 & -298 & 0 & 0 \\
Middle East & 0 & 0 & 0 & 0 & 0 & 0 \\
Mexico & 0 & 0 & 0 & 0 & 0 & 0 \\
Other Asia & 0 & 0 & 0 & 0 & 0 & 0 \\
South Korea & -218 & 0 & -163 & 0 & 0 & 0 \\
United States & 0 & 0 & 0 & 0 & 0 & 0 \\
Western Europe & 0 & -390 & -562 & -684 & -724 & -724 \\
\hline Total & $\mathbf{0}$ & $\mathbf{0}$ & $\mathbf{0}$ & $\mathbf{0}$ & $\mathbf{0}$ & $\mathbf{0}$ \\
\hline \hline
\end{tabular}

Table A-335: Delayed technology reference case with low feedstock availability sugar ethanol net trade [Million gallons]

\begin{tabular}{|c|c|c|c|c|c|c|}
\hline Africa & 0 & 0 & 0 & 0 & 0 & 0 \\
\hline Australia & 0 & 0 & 0 & 0 & 0 & 0 \\
\hline Canada & -101 & 0 & 0 & 0 & 0 & 0 \\
\hline China & 0 & 0 & 0 & 0 & 0 & 0 \\
\hline Central and South America & 1,264 & 375 & 258 & 348 & 647 & 73 \\
\hline Eastern Europe & -589 & 0 & 0 & 0 & 0 & 0 \\
\hline Former Soviet Union & 0 & 0 & 0 & 0 & 0 & 0 \\
\hline India & 0 & 0 & 0 & 0 & 0 & 0 \\
\hline Japan & -257 & 0 & 0 & 0 & 0 & 0 \\
\hline Middle East & 0 & 0 & 0 & 0 & 0 & 0 \\
\hline Mexico & 0 & 0 & 0 & 0 & 0 & 0 \\
\hline Other Asia & 0 & 0 & 0 & 0 & 0 & 0 \\
\hline South Korea & -46 & 0 & 0 & 0 & 0 & 0 \\
\hline United States & -134 & 0 & 0 & -348 & -647 & -73 \\
\hline Western Europe & -136 & -375 & -258 & 0 & 0 & 0 \\
\hline Total & 0 & 0 & 0 & 0 & $\mathbf{0}$ & $\mathbf{0}$ \\
\hline
\end{tabular}


Table A-336: Delayed technology reference case with low feedstock availability cellulosic ethanol net trade [Million gallons]

\begin{tabular}{lrrrrrr} 
& $\mathbf{2 0 0 5}$ & $\mathbf{2 0 1 0}$ & $\mathbf{2 0 1 5}$ & $\mathbf{2 0 2 0}$ & $\mathbf{2 0 2 5}$ & $\mathbf{2 0 3 0}$ \\
Africa & 0 & 0 & 0 & 0 & 0 & 0 \\
Australia & 0 & 0 & 0 & 0 & 0 & 0 \\
Canada & 0 & 0 & 40 & 92 & 112 & 110 \\
China & 0 & 0 & 243 & 668 & 774 & 794 \\
Central and South America & 0 & 0 & 553 & 807 & 1,372 & 4,029 \\
Eastern Europe & 0 & 0 & 0 & 0 & 0 & 0 \\
Former Soviet Union & 0 & 0 & 0 & 0 & 0 & 0 \\
India & 0 & 0 & 0 & 32 & 151 & 408 \\
Japan & 0 & 0 & 0 & -53 & -395 & -415 \\
Middle East & 0 & 0 & 0 & 0 & 0 & 0 \\
Mexico & 0 & 0 & 0 & 0 & 0 & 0 \\
Other Asia & 0 & 0 & 0 & 0 & 0 & 0 \\
South Korea & 0 & 0 & -202 & -379 & -378 & -378 \\
United States & 0 & 0 & 0 & 0 & 0 & $-3,430$ \\
Western Europe & 0 & 0 & -634 & $-1,167$ & $-1,635$ & $-1,117$ \\
\hline Total & $\mathbf{0}$ & $\mathbf{0}$ & $\mathbf{0}$ & $\mathbf{0}$ & $\mathbf{0}$ & $\mathbf{0}$ \\
\hline \hline
\end{tabular}

Table A-337: Delayed technology reference case with low feedstock availability biomass-to-liquids production [Million gallons ethanol equivalent]

\begin{tabular}{lrrrrrr} 
& $\mathbf{2 0 0 5}$ & $\mathbf{2 0 1 0}$ & $\mathbf{2 0 1 5}$ & $\mathbf{2 0 2 0}$ & $\mathbf{2 0 2 5}$ & $\mathbf{2 0 3 0}$ \\
Africa & 0 & 0 & 0 & 0 & 0 & 0 \\
Argentina & 0 & 0 & 29 & 111 & 111 & 111 \\
Australia & 0 & 0 & 0 & 0 & 0 & 0 \\
Brazil & 0 & 0 & 97 & 320 & 750 & 1,363 \\
Canada & 0 & 0 & 93 & 280 & 401 & 407 \\
Caribbean basin & 0 & 0 & 0 & 0 & 0 & 0 \\
China & 0 & 0 & 57 & 240 & 563 & 824 \\
Colombia & 0 & 0 & 61 & 156 & 156 & 156 \\
Eastern Europe & 0 & 0 & 0 & 0 & 0 & 0 \\
Former Soviet Union & 0 & 0 & 0 & 0 & 0 & 0 \\
India & 0 & 0 & 77 & 232 & 544 & 1,010 \\
Japan & 0 & 0 & 0 & 0 & 0 & 0 \\
Middle East & 0 & 0 & 0 & 0 & 0 & 0 \\
Mexico & 0 & 0 & 0 & 0 & 0 & 0 \\
Other Asia & 0 & 0 & 0 & 0 & 0 & 0 \\
South Korea & 0 & 0 & 36 & 52 & 52 & 52 \\
United States & 0 & 0 & 786 & 1,253 & 1,849 & 2,406 \\
Western Europe & 0 & 0 & 0 & 0 & 0 & 0 \\
\hline Total & $\mathbf{0}$ & $\mathbf{0}$ & $\mathbf{1 , 2 3 5}$ & $\mathbf{2 , 6 4 3}$ & $\mathbf{4 , 4 2 5}$ & $\mathbf{6 , 3 2 7}$ \\
\hline \hline
\end{tabular}


Table A-338: Delayed technology reference case with low feedstock availability biomass-to-liquids consumption [Million gallons ethanol equivalent]

\begin{tabular}{lrrrrrr} 
& $\mathbf{2 0 0 5}$ & $\mathbf{2 0 1 0}$ & $\mathbf{2 0 1 5}$ & $\mathbf{2 0 2 0}$ & $\mathbf{2 0 2 5}$ & $\mathbf{2 0 3 0}$ \\
Africa & 0 & 0 & 0 & 0 & 0 & 0 \\
Australia & 0 & 0 & 0 & 0 & 0 & 0 \\
Canada & 0 & 0 & 0 & 0 & 0 & 0 \\
China & 0 & 0 & 0 & 0 & 0 & 0 \\
Central and South America & 0 & 0 & 0 & 0 & 0 & 0 \\
Eastern Europe & 0 & 0 & 0 & 0 & 0 & 0 \\
Former Soviet Union & 0 & 0 & 0 & 0 & 0 & 0 \\
India & 0 & 0 & 0 & 0 & 0 & 0 \\
Japan & 0 & 0 & 0 & 0 & 0 & 0 \\
Middle East & 0 & 0 & 0 & 0 & 0 & 0 \\
Mexico & 0 & 0 & 0 & 0 & 0 & 0 \\
Other Asia & 0 & 0 & 0 & 0 & 0 & 0 \\
South Korea & 0 & 0 & 0 & 0 & 0 & 0 \\
United States & 0 & 0 & 788 & 1,253 & 3,025 & 6,328 \\
Western Europe & 0 & 0 & 447 & 1,391 & 1,400 & 0 \\
\hline Total & $\mathbf{0}$ & $\mathbf{0}$ & $\mathbf{1 , 2 3 5}$ & $\mathbf{2 , 6 4 3}$ & $\mathbf{4 , 4 2 5}$ & $\mathbf{6 , 3 2 8}$ \\
\hline \hline
\end{tabular}

Table A-339: Delayed technology reference case with low feedstock availability biomass-to-liquids net trade [Million gallons ethanol equivalent]

\begin{tabular}{lrrrrrr} 
& $\mathbf{2 0 0 5}$ & $\mathbf{2 0 1 0}$ & $\mathbf{2 0 1 5}$ & $\mathbf{2 0 2 0}$ & $\mathbf{2 0 2 5}$ & $\mathbf{2 0 3 0}$ \\
Africa & 0 & 0 & 0 & 0 & 0 & 0 \\
Australia & 0 & 0 & 0 & 0 & 0 & 0 \\
Canada & 0 & 0 & 93 & 280 & 401 & 407 \\
China & 0 & 0 & 57 & 240 & 563 & 824 \\
Central and South America & 0 & 0 & 187 & 587 & 1,017 & 1,630 \\
Eastern Europe & 0 & 0 & 0 & 0 & 0 & 0 \\
Former Soviet Union & 0 & 0 & 0 & 0 & 0 & 0 \\
India & 0 & 0 & 77 & 232 & 544 & 1,010 \\
Japan & 0 & 0 & 0 & 0 & 0 & 0 \\
Middle East & 0 & 0 & 0 & 0 & 0 & 0 \\
Mexico & 0 & 0 & 0 & 0 & 0 & 0 \\
Other Asia & 0 & 0 & 0 & 0 & 0 & 0 \\
South Korea & 0 & 0 & 36 & 52 & 52 & 52 \\
United States & 0 & 0 & -2 & 0 & $-1,176$ & $-3,921$ \\
Western Europe & 0 & 0 & -447 & $-1,391$ & $-1,400$ & 0 \\
\hline Total & $\mathbf{0}$ & $\mathbf{0}$ & $\mathbf{0}$ & $\mathbf{0}$ & $\mathbf{0}$ & $\mathbf{0}$ \\
\hline \hline
\end{tabular}


Table A-340: Delayed technology reference case with low feedstock availability biodiesel production [Million gallons ethanol equivalent]

\begin{tabular}{lrrrrrr} 
& $\mathbf{2 0 0 5}$ & $\mathbf{2 0 1 0}$ & $\mathbf{2 0 1 5}$ & $\mathbf{2 0 2 0}$ & $\mathbf{2 0 2 5}$ & $\mathbf{2 0 3 0}$ \\
Africa & 0 & 0 & 0 & 0 & 0 & 0 \\
Argentina & 0 & 333 & 877 & 1,491 & 1,472 & 1,458 \\
Australia & 0 & 0 & 0 & 0 & 0 & 0 \\
Brazil & 0 & 333 & 835 & 1,537 & 1,569 & 1,569 \\
Canada & 0 & 0 & 0 & 0 & 0 & 0 \\
Caribbean basin & 0 & 95 & 310 & 742 & 1,145 & 1,220 \\
China & 0 & 0 & 0 & 0 & 0 & 0 \\
Colombia & 0 & 0 & 0 & 0 & 0 & 0 \\
Eastern Europe & 0 & 0 & 0 & 0 & 0 & 0 \\
Former Soviet Union & 0 & 0 & 0 & 0 & 0 & 0 \\
India & 0 & 0 & 0 & 0 & 0 & 0 \\
Japan & 0 & 0 & 0 & 0 & 0 & 0 \\
Middle East & 0 & 0 & 0 & 0 & 0 & 0 \\
Mexico & 0 & 0 & 0 & 0 & 0 & 0 \\
Other Asia & 0 & 0 & 0 & 0 & 0 & 0 \\
South Korea & 0 & 0 & 0 & 0 & 0 & 0 \\
United States & 0 & 1,238 & 1,640 & 1,803 & 1,907 & 2,128 \\
Western Europe & 998 & 2,910 & 3,578 & 3,930 & 4,468 & 4,788 \\
\hline Total & $\mathbf{9 9 8}$ & $\mathbf{4 , 9 0 8}$ & $\mathbf{7 , 2 3 9}$ & $\mathbf{9 , 5 0 3}$ & $\mathbf{1 0 , 5 6 1}$ & $\mathbf{1 1 , 1 6 3}$ \\
\hline \hline
\end{tabular}

Table A-341: Delayed technology reference case with low feedstock availability biodiesel consumption [Million gallons ethanol equivalent]

\begin{tabular}{lrrrrrr} 
& $\mathbf{2 0 0 5}$ & $\mathbf{2 0 1 0}$ & $\mathbf{2 0 1 5}$ & $\mathbf{2 0 2 0}$ & $\mathbf{2 0 2 5}$ & $\mathbf{2 0 3 0}$ \\
Africa & 0 & 0 & 0 & 0 & 0 & 0 \\
Australia & 0 & 0 & 0 & 0 & 0 & 0 \\
Canada & 0 & 0 & 0 & 0 & 0 & 0 \\
China & 0 & 0 & 0 & 0 & 0 & 0 \\
Central and South America & 0 & 126 & 438 & 936 & 1,262 & 1,448 \\
Eastern Europe & 0 & 0 & 0 & 0 & 0 & 0 \\
Former Soviet Union & 0 & 0 & 0 & 0 & 0 & 0 \\
India & 0 & 0 & 0 & 0 & 0 & 0 \\
Japan & 0 & 0 & 0 & 0 & 0 & 0 \\
Middle East & 0 & 0 & 0 & 0 & 0 & 0 \\
Mexico & 0 & 0 & 0 & 0 & 0 & 0 \\
Other Asia & 0 & 0 & 0 & 0 & 0 & 0 \\
South Korea & 0 & 0 & 0 & 0 & 0 & 0 \\
United States & 0 & 1,238 & 2,494 & 4,131 & 4,319 & 4,927 \\
Western Europe & 998 & 3,544 & 4,307 & 4,437 & 4,981 & 4,788 \\
\hline Total & $\mathbf{9 9 8}$ & $\mathbf{4 , 9 0 8}$ & $\mathbf{7 , 2 3 9}$ & $\mathbf{9 , 5 0 3}$ & $\mathbf{1 0 , 5 6 1}$ & $\mathbf{1 1 , 1 6 3}$ \\
\hline \hline
\end{tabular}


Table A-342: Delayed technology reference case with low feedstock availability biodiesel net trade [Million gallons ethanol equivalent]

\begin{tabular}{lrrrrrr} 
& $\mathbf{2 0 0 5}$ & $\mathbf{2 0 1 0}$ & $\mathbf{2 0 1 5}$ & $\mathbf{2 0 2 0}$ & $\mathbf{2 0 2 5}$ & $\mathbf{2 0 3 0}$ \\
Africa & 0 & 0 & 0 & 0 & 0 & 0 \\
Australia & 0 & 0 & 0 & 0 & 0 & 0 \\
Canada & 0 & 0 & 0 & 0 & 0 & 0 \\
China & 0 & 0 & 0 & 0 & 0 & 0 \\
Central and South America & 0 & 634 & 1,583 & 2,834 & 2,924 & 2,798 \\
Eastern Europe & 0 & 0 & 0 & 0 & 0 & 0 \\
Former Soviet Union & 0 & 0 & 0 & 0 & 0 & 0 \\
India & 0 & 0 & 0 & 0 & 0 & 0 \\
Japan & 0 & 0 & 0 & 0 & 0 & 0 \\
Middle East & 0 & 0 & 0 & 0 & 0 & 0 \\
Mexico & 0 & 0 & 0 & 0 & 0 & 0 \\
Other Asia & 0 & 0 & 0 & 0 & 0 & 0 \\
South Korea & 0 & 0 & 0 & 0 & 0 & 0 \\
United States & 0 & 0 & -854 & $-2,327$ & $-2,412$ & $-2,798$ \\
Western Europe & 0 & -634 & -729 & -507 & -512 & 0 \\
\hline Total & $\mathbf{0}$ & $\mathbf{0}$ & $\mathbf{0}$ & $\mathbf{0}$ & $\mathbf{0}$ & $\mathbf{0}$ \\
\hline \hline
\end{tabular}




\section{A-20 Delayed Technology Reference Case with High Oil Price}

Table A-343: Delayed technology reference case with high oil price ethanol production [Million gallons]

\begin{tabular}{lrrrrrr} 
& $\mathbf{2 0 0 5}$ & $\mathbf{2 0 1 0}$ & $\mathbf{2 0 1 5}$ & $\mathbf{2 0 2 0}$ & $\mathbf{2 0 2 5}$ & $\mathbf{2 0 3 0}$ \\
Africa & 300 & 330 & 364 & 402 & 455 & 502 \\
Argentina & 0 & 178 & 699 & 1,549 & 1,653 & 1,665 \\
Australia & 0 & 0 & 71 & 267 & 709 & 1,174 \\
Brazil & 4,866 & 5,977 & 7,624 & 9,926 & 11,947 & 13,779 \\
Canada & 0 & 0 & 259 & 779 & 914 & 914 \\
Caribbean basin & 210 & 360 & 573 & 683 & 779 & 890 \\
China & 344 & 384 & 614 & 1,064 & 1,966 & 2,951 \\
Colombia & 196 & 215 & 411 & 782 & 1,502 & 2,231 \\
Eastern Europe & 110 & 226 & 434 & 780 & 1,037 & 1,055 \\
Former Soviet Union & 0 & 0 & 6 & 19 & 57 & 175 \\
India & 313 & 279 & 541 & 992 & 1,893 & 2,822 \\
Japan & 0 & 0 & 95 & 369 & 608 & 648 \\
Middle East & 0 & 0 & 0 & 0 & 0 & 0 \\
Mexico & 0 & 0 & 199 & 601 & 1,408 & 1,546 \\
Other Asia & 0 & 0 & 0 & 0 & 0 & 0 \\
South Korea & 0 & 0 & 35 & 35 & 35 & 35 \\
United States & 3,850 & 11,612 & 15,732 & 16,851 & 19,096 & 22,833 \\
Western Europe & 600 & 2,570 & 2,755 & 3,235 & 5,291 & 8,392 \\
\hline Total & $\mathbf{1 0 , 7 8 9}$ & $\mathbf{2 2 , 1 3 1}$ & $\mathbf{3 0 , 4 1 1}$ & $\mathbf{3 8 , 3 3 2}$ & $\mathbf{4 9 , 3 4 9}$ & $\mathbf{6 1 , 6 0 9}$ \\
\hline \hline
\end{tabular}


Table A-344: Delayed technology reference case with high oil price ethanol consumption [Million gallons]

\begin{tabular}{lrrrrrr} 
& $\mathbf{2 0 0 5}$ & $\mathbf{2 0 1 0}$ & $\mathbf{2 0 1 5}$ & $\mathbf{2 0 2 0}$ & $\mathbf{2 0 2 5}$ & $\mathbf{2 0 3 0}$ \\
Africa & 300 & 330 & 364 & 402 & 455 & 502 \\
Australia & 0 & 0 & 71 & 267 & 709 & 1,174 \\
Canada & 145 & 0 & 259 & 895 & 911 & 914 \\
China & 265 & 0 & 0 & 1,064 & 2,713 & 5,063 \\
Central and South America & 3,788 & 4,856 & 6,140 & 7,236 & 8,029 & 8,936 \\
Eastern Europe & 699 & 226 & 872 & 953 & 1,037 & 1,055 \\
Former Soviet Union & 0 & 0 & 6 & 19 & 57 & 175 \\
India & 313 & 279 & 541 & 992 & 1,893 & 2,399 \\
Japan & 295 & 320 & 410 & 757 & 1,041 & 1,101 \\
Middle East & 0 & 0 & 0 & 0 & 0 & 0 \\
Mexico & 0 & 0 & 30 & 601 & 1,030 & 1,052 \\
Other Asia & 0 & 0 & 0 & 0 & 0 & 530 \\
South Korea & 264 & 335 & 400 & 414 & 413 & 413 \\
United States & 3,984 & 11,612 & 17,042 & 19,647 & 23,412 & 28,594 \\
Western Europe & 736 & 4,173 & 4,276 & 5,086 & 7,650 & 9,702 \\
\hline Total & $\mathbf{1 0 , 7 8 9}$ & $\mathbf{2 2 , 1 3 1}$ & $\mathbf{3 0 , 4 1 1}$ & $\mathbf{3 8 , 3 3 2}$ & $\mathbf{4 9 , 3 4 9}$ & $\mathbf{6 1 , 6 0 9}$ \\
\hline \hline
\end{tabular}

Table A-345: Delayed technology reference case with high oil price ethanol net trade [Million gallons]

\begin{tabular}{lrrrrrr} 
Africa & 0 & 0 & 0 & 0 & 0 & 0 \\
Australia & 0 & 0 & 0 & 0 & 0 & 0 \\
Canada & -145 & 0 & 0 & -116 & 3 & 0 \\
China & 80 & 384 & 614 & 0 & -747 & $-2,112$ \\
Central and South America & 1,484 & 1,874 & 3,167 & 5,704 & 7,852 & 9,628 \\
Eastern Europe & -589 & 0 & -438 & -173 & 0 & 0 \\
Former Soviet Union & 0 & 0 & 0 & 0 & 0 & 0 \\
India & 0 & 0 & 0 & 0 & 0 & 423 \\
Japan & -295 & -320 & -315 & -388 & -433 & -453 \\
Middle East & 0 & 0 & 0 & 0 & 0 & 0 \\
Mexico & 0 & 0 & 170 & 0 & 378 & 493 \\
Other Asia & 0 & 0 & 0 & 0 & 0 & -530 \\
South Korea & -264 & -335 & -365 & -379 & -378 & -378 \\
United States & -134 & 0 & $-1,310$ & $-2,796$ & $-4,317$ & $-5,762$ \\
Western Europe & -136 & $-1,604$ & $-1,521$ & $-1,851$ & $-2,359$ & $-1,310$ \\
\hline Total & $\mathbf{0}$ & $\mathbf{0}$ & $\mathbf{0}$ & $\mathbf{0}$ & $\mathbf{0}$ & $\mathbf{0}$ \\
\hline \hline
\end{tabular}


Table A-346: Delayed technology reference case with high oil price grain ethanol production [Million gallons]

\begin{tabular}{lrrrrrr} 
& $\mathbf{2 0 0 5}$ & $\mathbf{2 0 1 0}$ & $\mathbf{2 0 1 5}$ & $\mathbf{2 0 2 0}$ & $\mathbf{2 0 2 5}$ & $\mathbf{2 0 3 0}$ \\
Africa & 0 & 0 & 0 & 0 & 0 & 0 \\
Argentina & 0 & 178 & 640 & 1,243 & 1,317 & 1,317 \\
Australia & 0 & 0 & 0 & 0 & 0 & 0 \\
Brazil & 0 & 0 & 0 & 0 & 0 & 0 \\
Canada & 0 & 0 & 0 & 0 & 0 & 0 \\
Caribbean basin & 0 & 0 & 0 & 0 & 0 & 0 \\
China & 344 & 344 & 344 & 344 & 344 & 344 \\
Colombia & 0 & 0 & 0 & 0 & 0 & 0 \\
Eastern Europe & 110 & 226 & 348 & 477 & 394 & 278 \\
Former Soviet Union & 0 & 0 & 0 & 0 & 0 & 0 \\
India & 0 & 0 & 0 & 0 & 0 & 0 \\
Japan & 0 & 0 & 0 & 0 & 0 & 0 \\
Middle East & 0 & 0 & 0 & 0 & 0 & 0 \\
Mexico & 0 & 0 & 0 & 0 & 0 & 0 \\
Other Asia & 0 & 0 & 0 & 0 & 0 & 0 \\
South Korea & 0 & 0 & 0 & 0 & 0 & 0 \\
United States & 3,850 & 11,612 & 15,000 & 15,000 & 15,000 & 15,000 \\
Western Europe & 600 & 2,570 & 2,589 & 2,542 & 3,178 & 3,813 \\
\hline Total & $\mathbf{4 , 9 0 4}$ & $\mathbf{1 4 , 9 3 0}$ & $\mathbf{1 8 , 9 2 1}$ & $\mathbf{1 9 , 6 0 7}$ & $\mathbf{2 0 , 2 3 3}$ & $\mathbf{2 0 , 7 5 2}$ \\
\hline \hline
\end{tabular}

Table A-347: Delayed technology reference case with high oil price sugar ethanol production [Million gallons]

\begin{tabular}{lrrrrrr} 
& $\mathbf{2 0 0 5}$ & $\mathbf{2 0 1 0}$ & $\mathbf{2 0 1 5}$ & $\mathbf{2 0 2 0}$ & $\mathbf{2 0 2 5}$ & $\mathbf{2 0 3 0}$ \\
Africa & 300 & 330 & 364 & 402 & 444 & 490 \\
Argentina & 0 & 0 & 0 & 0 & 0 & 0 \\
Australia & 0 & 0 & 0 & 0 & 0 & 0 \\
Brazil & 4,866 & 5,977 & 7,329 & 9,036 & 10,406 & 12,233 \\
Canada & 0 & 0 & 0 & 0 & 0 & 0 \\
Caribbean basin & 210 & 360 & 573 & 683 & 779 & 890 \\
China & 0 & 40 & 48 & 53 & 58 & 64 \\
Colombia & 196 & 215 & 241 & 270 & 302 & 338 \\
Eastern Europe & 0 & 0 & 0 & 0 & 0 & 0 \\
Former Soviet Union & 0 & 0 & 0 & 0 & 0 & 0 \\
India & 313 & 279 & 327 & 347 & 381 & 450 \\
Japan & 0 & 0 & 0 & 0 & 0 & 0 \\
Middle East & 0 & 0 & 0 & 0 & 0 & 0 \\
Mexico & 0 & 0 & 0 & 0 & 0 & 0 \\
Other Asia & 0 & 0 & 0 & 0 & 0 & 0 \\
South Korea & 0 & 0 & 0 & 0 & 0 & 0 \\
United States & 0 & 0 & 0 & 0 & 0 & 0 \\
Western Europe & 0 & 0 & 0 & 0 & 0 & 0 \\
\hline Total & $\mathbf{5 , 8 8 4}$ & $\mathbf{7 , 2 0 1}$ & $\mathbf{8 , 8 8 2}$ & $\mathbf{1 0 , 7 9 0}$ & $\mathbf{1 2 , 3 7 1}$ & $\mathbf{1 4 , 4 6 5}$ \\
\hline \hline
\end{tabular}


Table A-348: Delayed technology reference case with high oil price cellulosic ethanol production [Million gallons]

\begin{tabular}{lrrrrrr} 
& $\mathbf{2 0 0 5}$ & $\mathbf{2 0 1 0}$ & $\mathbf{2 0 1 5}$ & $\mathbf{2 0 2 0}$ & $\mathbf{2 0 2 5}$ & $\mathbf{2 0 3 0}$ \\
Africa & 0 & 0 & 0 & 0 & 11 & 11 \\
Argentina & 0 & 0 & 59 & 306 & 335 & 347 \\
Australia & 0 & 0 & 71 & 267 & 709 & 1,174 \\
Brazil & 0 & 0 & 296 & 890 & 1,541 & 1,546 \\
Canada & 0 & 0 & 259 & 779 & 914 & 914 \\
Caribbean basin & 0 & 0 & 0 & 0 & 0 & 0 \\
China & 0 & 0 & 222 & 667 & 1,564 & 2,543 \\
Colombia & 0 & 0 & 170 & 512 & 1,199 & 1,893 \\
Eastern Europe & 0 & 0 & 85 & 303 & 644 & 778 \\
Former Soviet Union & 0 & 0 & 6 & 19 & 57 & 175 \\
India & 0 & 0 & 214 & 645 & 1,512 & 2,373 \\
Japan & 0 & 0 & 95 & 369 & 608 & 648 \\
Middle East & 0 & 0 & 0 & 0 & 0 & 0 \\
Mexico & 0 & 0 & 199 & 601 & 1,408 & 1,546 \\
Other Asia & 0 & 0 & 0 & 0 & 0 & 0 \\
South Korea & 0 & 0 & 35 & 35 & 35 & 35 \\
United States & 0 & 0 & 732 & 1,851 & 4,096 & 7,833 \\
Western Europe & 0 & 0 & 166 & 693 & 2,113 & 4,579 \\
\hline Total & $\mathbf{0}$ & $\mathbf{0}$ & $\mathbf{2 , 6 0 9}$ & $\mathbf{7 , 9 3 4}$ & $\mathbf{1 6 , 7 4 6}$ & $\mathbf{2 6 , 3 9 2}$ \\
\hline \hline
\end{tabular}

Table A-349: Delayed technology reference case with high oil price grain ethanol consumption [Million gallons]

\begin{tabular}{lrrrrrr} 
& $\mathbf{2 0 0 5}$ & $\mathbf{2 0 1 0}$ & $\mathbf{2 0 1 5}$ & $\mathbf{2 0 2 0}$ & $\mathbf{2 0 2 5}$ & $\mathbf{2 0 3 0}$ \\
Africa & 0 & 0 & 0 & 0 & 0 & 0 \\
Australia & 0 & 0 & 0 & 0 & 0 & 0 \\
Canada & 0 & 0 & 0 & 0 & 0 & 0 \\
China & 265 & 0 & 0 & 344 & 1,020 & 587 \\
Central and South America & 0 & 36 & 105 & 201 & 165 & 167 \\
Eastern Europe & 110 & 226 & 348 & 477 & 394 & 278 \\
Former Soviet Union & 0 & 0 & 0 & 0 & 0 & 0 \\
India & 0 & 0 & 0 & 0 & 0 & 0 \\
Japan & 0 & 192 & 267 & 388 & 433 & 0 \\
Middle East & 0 & 0 & 0 & 0 & 0 & 0 \\
Mexico & 0 & 0 & 0 & 0 & 0 & 0 \\
Other Asia & 0 & 0 & 0 & 0 & 0 & 530 \\
South Korea & 80 & 295 & 144 & 379 & 43 & 378 \\
United States & 3,850 & 11,612 & 15,000 & 15,000 & 15,000 & 15,000 \\
Western Europe & 600 & 2,570 & 3,057 & 2,817 & 3,178 & 3,813 \\
\hline Total & $\mathbf{4 , 9 0 4}$ & $\mathbf{1 4 , 9 3 0}$ & $\mathbf{1 8 , 9 2 1}$ & $\mathbf{1 9 , 6 0 7}$ & $\mathbf{2 0 , 2 3 3}$ & $\mathbf{2 0 , 7 5 2}$ \\
\hline \hline
\end{tabular}


Table A-350: Delayed technology reference case with high oil price sugar ethanol consumption [Million gallons]

\begin{tabular}{lrrrrrr} 
& $\mathbf{2 0 0 5}$ & $\mathbf{2 0 1 0}$ & $\mathbf{2 0 1 5}$ & $\mathbf{2 0 2 0}$ & $\mathbf{2 0 2 5}$ & $\mathbf{2 0 3 0}$ \\
Africa & 300 & 330 & 364 & 402 & 444 & 490 \\
Australia & 0 & 0 & 0 & 0 & 0 & 0 \\
Canada & 145 & 0 & 0 & 116 & 0 & 0 \\
China & 0 & 0 & 0 & 53 & 125 & 1,509 \\
Central and South America & 3,788 & 4,820 & 6,035 & 6,947 & 7,864 & 8,770 \\
Eastern Europe & 589 & 0 & 438 & 173 & 0 & 0 \\
Former Soviet Union & 0 & 0 & 0 & 0 & 0 & 0 \\
India & 313 & 279 & 327 & 347 & 381 & 450 \\
Japan & 295 & 128 & 48 & 0 & 0 & 453 \\
Middle East & 0 & 0 & 0 & 0 & 0 & 0 \\
Mexico & 0 & 0 & 0 & 0 & 0 & 0 \\
Other Asia & 0 & 0 & 0 & 0 & 0 & 0 \\
South Korea & 185 & 40 & 0 & 0 & 0 & 0 \\
United States & 134 & 0 & 788 & 2,753 & 3,094 & 1,483 \\
Western Europe & 136 & 1,604 & 882 & 0 & 464 & 1,310 \\
\hline Total & $\mathbf{5 , 8 8 4}$ & $\mathbf{7 , 2 0 1}$ & $\mathbf{8 , 8 8 2}$ & $\mathbf{1 0 , 7 9 0}$ & $\mathbf{1 2 , 3 7 1}$ & $\mathbf{1 4 , 4 6 5}$ \\
\hline \hline
\end{tabular}

Table A-351: Delayed technology reference case with high oil price cellulosic ethanol consumption [Million gallons]

\begin{tabular}{lrrrrrr} 
& $\mathbf{2 0 0 5}$ & $\mathbf{2 0 1 0}$ & $\mathbf{2 0 1 5}$ & $\mathbf{2 0 2 0}$ & $\mathbf{2 0 2 5}$ & $\mathbf{2 0 3 0}$ \\
Africa & 0 & 0 & 0 & 0 & 11 & 11 \\
Australia & 0 & 0 & 71 & 267 & 709 & 1,174 \\
Canada & 0 & 0 & 259 & 779 & 911 & 914 \\
China & 0 & 0 & 0 & 667 & 1,567 & 2,966 \\
Central and South America & 0 & 0 & 0 & 88 & 0 & 0 \\
Eastern Europe & 0 & 0 & 85 & 303 & 644 & 778 \\
Former Soviet Union & 0 & 0 & 6 & 19 & 57 & 175 \\
India & 0 & 0 & 214 & 645 & 1,512 & 1,949 \\
Japan & 0 & 0 & 95 & 369 & 608 & 648 \\
Middle East & 0 & 0 & 0 & 0 & 0 & 0 \\
Mexico & 0 & 0 & 30 & 601 & 1,030 & 1,052 \\
Other Asia & 0 & 0 & 0 & 0 & 0 & 0 \\
South Korea & 0 & 0 & 256 & 35 & 370 & 35 \\
United States & 0 & 0 & 1,254 & 1,894 & 5,319 & 12,112 \\
Western Europe & 0 & 0 & 338 & 2,269 & 4,008 & 4,579 \\
\hline Total & $\mathbf{0}$ & $\mathbf{0}$ & $\mathbf{2 , 6 0 9}$ & $\mathbf{7 , 9 3 4}$ & $\mathbf{1 6 , 7 4 6}$ & $\mathbf{2 6 , 3 9 2}$ \\
\hline \hline
\end{tabular}


Table A-352: Delayed technology reference case with high oil price grain ethanol net trade [Million gallons]

\begin{tabular}{lrrrrrr} 
& $\mathbf{2 0 0 5}$ & $\mathbf{2 0 1 0}$ & $\mathbf{2 0 1 5}$ & $\mathbf{2 0 2 0}$ & $\mathbf{2 0 2 5}$ & $\mathbf{2 0 3 0}$ \\
Africa & 0 & 0 & 0 & 0 & 0 & 0 \\
Australia & 0 & 0 & 0 & 0 & 0 & 0 \\
Canada & 0 & 0 & 0 & 0 & 0 & 0 \\
China & 80 & 344 & 344 & 0 & -676 & -243 \\
Central and South America & 0 & 143 & 535 & 1,042 & 1,152 & 1,151 \\
Eastern Europe & 0 & 0 & 0 & 0 & 0 & 0 \\
Former Soviet Union & 0 & 0 & 0 & 0 & 0 & 0 \\
India & 0 & 0 & 0 & 0 & 0 & 0 \\
Japan & 0 & -192 & -267 & -388 & -433 & 0 \\
Middle East & 0 & 0 & 0 & 0 & 0 & 0 \\
Mexico & 0 & 0 & 0 & 0 & 0 & 0 \\
Other Asia & 0 & 0 & 0 & 0 & 0 & -530 \\
South Korea & -80 & -295 & -144 & -379 & -43 & -378 \\
United States & 0 & 0 & 0 & 0 & 0 & 0 \\
Western Europe & 0 & 0 & -468 & -275 & 0 & 0 \\
\hline Total & $\mathbf{0}$ & $\mathbf{0}$ & $\mathbf{0}$ & $\mathbf{0}$ & $\mathbf{0}$ & $\mathbf{0}$ \\
\hline \hline
\end{tabular}

Table A-353: Delayed technology reference case with high oil price sugar ethanol net trade [Million gallons]

\begin{tabular}{lrrrrrr} 
Africa & 0 & 0 & 0 & 0 & 0 & 0 \\
Australia & 0 & 0 & 0 & 0 & 0 & 0 \\
Canada & -145 & 0 & 0 & -116 & 0 & 0 \\
China & 0 & 40 & 48 & 0 & -67 & $-1,446$ \\
Central and South America & 1,484 & 1,732 & 2,108 & 3,042 & 3,624 & 4,692 \\
Eastern Europe & -589 & 0 & -438 & -173 & 0 & 0 \\
Former Soviet Union & 0 & 0 & 0 & 0 & 0 & 0 \\
India & 0 & 0 & 0 & 0 & 0 & 0 \\
Japan & -295 & -128 & -48 & 0 & 0 & -453 \\
Middle East & 0 & 0 & 0 & 0 & 0 & 0 \\
Mexico & 0 & 0 & 0 & 0 & 0 & 0 \\
Other Asia & 0 & 0 & 0 & 0 & 0 & 0 \\
South Korea & -185 & -40 & 0 & 0 & 0 & 0 \\
United States & -134 & 0 & -788 & $-2,753$ & $-3,094$ & $-1,483$ \\
Western Europe & -136 & $-1,604$ & -882 & 0 & -464 & $-1,310$ \\
\hline Total & $\mathbf{0}$ & $\mathbf{0}$ & $\mathbf{0}$ & $\mathbf{0}$ & $\mathbf{0}$ & $\mathbf{0}$ \\
\hline \hline
\end{tabular}


Table A-354: Delayed technology reference case with high oil price cellulosic ethanol net trade [Million gallons]

\begin{tabular}{lrrrrrr} 
& $\mathbf{2 0 0 5}$ & $\mathbf{2 0 1 0}$ & $\mathbf{2 0 1 5}$ & $\mathbf{2 0 2 0}$ & $\mathbf{2 0 2 5}$ & $\mathbf{2 0 3 0}$ \\
Africa & 0 & 0 & 0 & 0 & 0 & 0 \\
Australia & 0 & 0 & 0 & 0 & 0 & 0 \\
Canada & 0 & 0 & 0 & 0 & 3 & 0 \\
China & 0 & 0 & 222 & 0 & -3 & -423 \\
Central and South America & 0 & 0 & 525 & 1,619 & 3,076 & 3,786 \\
Eastern Europe & 0 & 0 & 0 & 0 & 0 & 0 \\
Former Soviet Union & 0 & 0 & 0 & 0 & 0 & 0 \\
India & 0 & 0 & 0 & 0 & 0 & 423 \\
Japan & 0 & 0 & 0 & 0 & 0 & 0 \\
Middle East & 0 & 0 & 0 & 0 & 0 & 0 \\
Mexico & 0 & 0 & 170 & 0 & 378 & 493 \\
Other Asia & 0 & 0 & 0 & 0 & 0 & 0 \\
South Korea & 0 & 0 & -222 & 0 & -335 & 0 \\
United States & 0 & 0 & -523 & -43 & $-1,223$ & $-4,279$ \\
Western Europe & 0 & 0 & -172 & $-1,576$ & $-1,895$ & 0 \\
\hline Total & $\mathbf{0}$ & $\mathbf{0}$ & $\mathbf{0}$ & $\mathbf{0}$ & $\mathbf{0}$ & $\mathbf{0}$ \\
\hline \hline
\end{tabular}

Table A-355: Delayed technology reference case with high oil price biomass-toliquids production [Million gallons ethanol equivalent]

\begin{tabular}{lrrrrrr} 
& $\mathbf{2 0 0 5}$ & $\mathbf{2 0 1 0}$ & $\mathbf{2 0 1 5}$ & $\mathbf{2 0 2 0}$ & $\mathbf{2 0 2 5}$ & $\mathbf{2 0 3 0}$ \\
Africa & 0 & 0 & 0 & 0 & 0 & 0 \\
Argentina & 0 & 0 & 21 & 110 & 121 & 125 \\
Australia & 0 & 0 & 0 & 0 & 0 & 3 \\
Brazil & 0 & 0 & 106 & 320 & 554 & 556 \\
Canada & 0 & 0 & 93 & 280 & 329 & 329 \\
Caribbean basin & 0 & 0 & 0 & 0 & 0 & 0 \\
China & 0 & 0 & 80 & 240 & 563 & 915 \\
Colombia & 0 & 0 & 61 & 184 & 431 & 681 \\
Eastern Europe & 0 & 0 & 0 & 0 & 125 & 326 \\
Former Soviet Union & 0 & 0 & 0 & 0 & 0 & 0 \\
India & 0 & 0 & 77 & 232 & 544 & 853 \\
Japan & 0 & 0 & 0 & 0 & 0 & 0 \\
Middle East & 0 & 0 & 0 & 0 & 0 & 0 \\
Mexico & 0 & 0 & 72 & 216 & 506 & 556 \\
Other Asia & 0 & 0 & 74 & 336 & 863 & 1,578 \\
South Korea & 0 & 0 & 36 & 51 & 51 & 51 \\
United States & 0 & 0 & 750 & 2,267 & 3,143 & 4,058 \\
Western Europe & 0 & 0 & 0 & 0 & 0 & 0 \\
\hline Total & $\mathbf{0}$ & $\mathbf{0}$ & $\mathbf{1 , 3 6 9}$ & $\mathbf{4 , 2 3 7}$ & $\mathbf{7 , 2 3 1}$ & $\mathbf{1 0 , 0 3 1}$ \\
\hline \hline
\end{tabular}


Table A-356: Delayed technology reference case with high oil price biomass-toliquids consumption [Million gallons ethanol equivalent]

\begin{tabular}{lrrrrrr} 
& $\mathbf{2 0 0 5}$ & $\mathbf{2 0 1 0}$ & $\mathbf{2 0 1 5}$ & $\mathbf{2 0 2 0}$ & $\mathbf{2 0 2 5}$ & $\mathbf{2 0 3 0}$ \\
Africa & 0 & 0 & 0 & 0 & 0 & 0 \\
Australia & 0 & 0 & 0 & 0 & 0 & 3 \\
Canada & 0 & 0 & 0 & 0 & 0 & 0 \\
China & 0 & 0 & 0 & 0 & 0 & 0 \\
Central and South America & 0 & 0 & 0 & 0 & 0 & 0 \\
Eastern Europe & 0 & 0 & 0 & 0 & 125 & 326 \\
Former Soviet Union & 0 & 0 & 0 & 0 & 0 & 0 \\
India & 0 & 0 & 0 & 0 & 0 & 0 \\
Japan & 0 & 0 & 0 & 0 & 0 & 0 \\
Middle East & 0 & 0 & 0 & 0 & 0 & 0 \\
Mexico & 0 & 0 & 0 & 0 & 0 & 0 \\
Other Asia & 0 & 0 & 74 & 336 & 863 & 1,578 \\
South Korea & 0 & 0 & 0 & 0 & 0 & 0 \\
United States & 0 & 0 & 975 & 3,572 & 6,242 & 8,124 \\
Western Europe & 0 & 0 & 321 & 329 & 0 & 0 \\
\hline Total & $\mathbf{0}$ & $\mathbf{0}$ & $\mathbf{1 , 3 6 9}$ & $\mathbf{4 , 2 3 7}$ & $\mathbf{7 , 2 3 1}$ & $\mathbf{1 0 , 0 3 1}$ \\
\hline \hline
\end{tabular}

Table A-357: Delayed technology reference case with high oil price biomass-toliquids net trade [Million gallons ethanol equivalent]

\begin{tabular}{lrrrrrr} 
& $\mathbf{2 0 0 5}$ & $\mathbf{2 0 1 0}$ & $\mathbf{2 0 1 5}$ & $\mathbf{2 0 2 0}$ & $\mathbf{2 0 2 5}$ & $\mathbf{2 0 3 0}$ \\
Africa & 0 & 0 & 0 & 0 & 0 & 0 \\
Australia & 0 & 0 & 0 & 0 & 0 & 0 \\
Canada & 0 & 0 & 93 & 280 & 329 & 329 \\
China & 0 & 0 & 80 & 240 & 563 & 915 \\
Central and South America & 0 & 0 & 189 & 614 & 1,106 & 1,362 \\
Eastern Europe & 0 & 0 & 0 & 0 & 0 & 0 \\
Former Soviet Union & 0 & 0 & 0 & 0 & 0 & 0 \\
India & 0 & 0 & 77 & 232 & 544 & 853 \\
Japan & 0 & 0 & 0 & 0 & 0 & 0 \\
Middle East & 0 & 0 & 0 & 0 & 0 & 0 \\
Mexico & 0 & 0 & 72 & 216 & 506 & 556 \\
Other Asia & 0 & 0 & 0 & 0 & 0 & 0 \\
South Korea & 0 & 0 & 36 & 51 & 51 & 51 \\
United States & 0 & 0 & -225 & $-1,305$ & $-3,099$ & $-4,066$ \\
Western Europe & 0 & 0 & -321 & -329 & 0 & 0 \\
\hline Total & $\mathbf{0}$ & $\mathbf{0}$ & $\mathbf{0}$ & $\mathbf{0}$ & $\mathbf{0}$ & $\mathbf{0}$ \\
\hline \hline
\end{tabular}


Table A-358: Delayed technology reference case with high oil price biodiesel production [Million gallons ethanol equivalent]

\begin{tabular}{lrrrrrr} 
& $\mathbf{2 0 0 5}$ & $\mathbf{2 0 1 0}$ & $\mathbf{2 0 1 5}$ & $\mathbf{2 0 2 0}$ & $\mathbf{2 0 2 5}$ & $\mathbf{2 0 3 0}$ \\
Africa & 0 & 0 & 0 & 0 & 0 & 0 \\
Argentina & 0 & 333 & 776 & 819 & 827 & 827 \\
Australia & 0 & 0 & 0 & 0 & 0 & 0 \\
Brazil & 0 & 333 & 835 & 1,537 & 1,768 & 1,357 \\
Canada & 0 & 0 & 0 & 0 & 0 & 0 \\
Caribbean basin & 0 & 95 & 310 & 675 & 802 & 898 \\
China & 0 & 0 & 0 & 0 & 0 & 0 \\
Colombia & 0 & 119 & 298 & 474 & 650 & 782 \\
Eastern Europe & 0 & 0 & 0 & 0 & 0 & 0 \\
Former Soviet Union & 0 & 0 & 0 & 0 & 0 & 0 \\
India & 0 & 0 & 0 & 0 & 0 & 0 \\
Japan & 0 & 0 & 0 & 0 & 0 & 0 \\
Middle East & 0 & 0 & 0 & 0 & 0 & 0 \\
Mexico & 0 & 0 & 0 & 0 & 0 & 0 \\
Other Asia & 0 & 0 & 0 & 0 & 0 & 0 \\
South Korea & 0 & 0 & 0 & 0 & 0 & 0 \\
United States & 0 & 1,238 & 1,640 & 1,747 & 1,907 & 2,128 \\
Western Europe & 998 & 3,445 & 4,172 & 5,110 & 5,532 & 5,997 \\
\hline Total & $\mathbf{9 9 8}$ & $\mathbf{5 , 5 6 1}$ & $\mathbf{8 , 0 3 0}$ & $\mathbf{1 0 , 3 6 2}$ & $\mathbf{1 1 , 4 8 5}$ & $\mathbf{1 1 , 9 8 9}$ \\
\hline \hline
\end{tabular}

Table A-359: Delayed technology reference case with high oil price biodiesel consumption [Million gallons ethanol equivalent]

\begin{tabular}{lrrrrrr} 
& $\mathbf{2 0 0 5}$ & $\mathbf{2 0 1 0}$ & $\mathbf{2 0 1 5}$ & $\mathbf{2 0 2 0}$ & $\mathbf{2 0 2 5}$ & $\mathbf{2 0 3 0}$ \\
Africa & 0 & 0 & 0 & 0 & 0 & 0 \\
Australia & 0 & 0 & 0 & 0 & 0 & 0 \\
Canada & 0 & 0 & 0 & 0 & 0 & 0 \\
China & 0 & 0 & 0 & 0 & 0 & 0 \\
Central and South America & 0 & 158 & 458 & 969 & 1,300 & 1,486 \\
Eastern Europe & 0 & 0 & 0 & 0 & 0 & 0 \\
Former Soviet Union & 0 & 0 & 0 & 0 & 0 & 0 \\
India & 0 & 0 & 0 & 0 & 0 & 0 \\
Japan & 0 & 0 & 0 & 0 & 0 & 0 \\
Middle East & 0 & 0 & 0 & 0 & 0 & 0 \\
Mexico & 0 & 0 & 0 & 0 & 0 & 0 \\
Other Asia & 0 & 0 & 0 & 0 & 0 & 0 \\
South Korea & 0 & 0 & 0 & 0 & 0 & 0 \\
United States & 0 & 1,238 & 1,713 & 1,747 & 1,907 & 3,517 \\
Western Europe & 998 & 4,165 & 5,860 & 7,646 & 8,278 & 6,986 \\
\hline Total & $\mathbf{9 9 8}$ & $\mathbf{5 , 5 6 1}$ & $\mathbf{8 , 0 3 0}$ & $\mathbf{1 0 , 3 6 2}$ & $\mathbf{1 1 , 4 8 5}$ & $\mathbf{1 1 , 9 8 9}$ \\
\hline \hline
\end{tabular}


Table A-360: Delayed technology reference case with high oil price biodiesel net trade [Million gallons ethanol equivalent]

\begin{tabular}{lrrrrrr} 
& $\mathbf{2 0 0 5}$ & $\mathbf{2 0 1 0}$ & $\mathbf{2 0 1 5}$ & $\mathbf{2 0 2 0}$ & $\mathbf{2 0 2 5}$ & $\mathbf{2 0 3 0}$ \\
Africa & 0 & 0 & 0 & 0 & 0 & 0 \\
Australia & 0 & 0 & 0 & 0 & 0 & 0 \\
Canada & 0 & 0 & 0 & 0 & 0 & 0 \\
China & 0 & 0 & 0 & 0 & 0 & 0 \\
Central and South America & 0 & 720 & 1,761 & 2,535 & 2,747 & 2,378 \\
Eastern Europe & 0 & 0 & 0 & 0 & 0 & 0 \\
Former Soviet Union & 0 & 0 & 0 & 0 & 0 & 0 \\
India & 0 & 0 & 0 & 0 & 0 & 0 \\
Japan & 0 & 0 & 0 & 0 & 0 & 0 \\
Middle East & 0 & 0 & 0 & 0 & 0 & 0 \\
Mexico & 0 & 0 & 0 & 0 & 0 & 0 \\
Other Asia & 0 & 0 & 0 & 0 & 0 & 0 \\
South Korea & 0 & 0 & 0 & 0 & 0 & 0 \\
United States & 0 & 0 & -73 & 0 & 0 & $-1,389$ \\
Western Europe & 0 & -720 & $-1,688$ & $-2,535$ & $-2,747$ & -989 \\
\hline Total & $\mathbf{0}$ & $\mathbf{0}$ & $\mathbf{0}$ & $\mathbf{0}$ & $\mathbf{0}$ & $\mathbf{0}$ \\
\hline \hline
\end{tabular}




\section{A-21 Delayed Technology Reference Case with Low Oil Price}

\section{Table A-361: Delayed technology reference case with low oil price ethanol production [Million gallons]}

\begin{tabular}{lrrrrrr} 
& $\mathbf{2 0 0 5}$ & $\mathbf{2 0 1 0}$ & $\mathbf{2 0 1 5}$ & $\mathbf{2 0 2 0}$ & $\mathbf{2 0 2 5}$ & $\mathbf{2 0 3 0}$ \\
Africa & 300 & 330 & 364 & 402 & 455 & 490 \\
Argentina & 0 & 178 & 664 & 973 & 1,003 & 1,015 \\
Australia & 0 & 0 & 66 & 217 & 585 & 1,172 \\
Brazil & 4,866 & 5,977 & 7,624 & 9,752 & 11,947 & 13,338 \\
Canada & 0 & 0 & 259 & 650 & 650 & 650 \\
Caribbean basin & 210 & 328 & 573 & 683 & 683 & 683 \\
China & 344 & 384 & 614 & 993 & 1,228 & 1,228 \\
Colombia & 196 & 215 & 411 & 782 & 1,501 & 2,227 \\
Eastern Europe & 110 & 226 & 434 & 780 & 1,037 & 1,149 \\
Former Soviet Union & 0 & 0 & 0 & 0 & 0 & 0 \\
India & 313 & 275 & 534 & 974 & 1,850 & 2,527 \\
Japan & 0 & 0 & 95 & 348 & 612 & 644 \\
Middle East & 0 & 0 & 0 & 0 & 0 & 0 \\
Mexico & 0 & 0 & 199 & 601 & 1,408 & 1,546 \\
Other Asia & 0 & 0 & 0 & 0 & 0 & 0 \\
South Korea & 0 & 0 & 30 & 30 & 30 & 30 \\
United States & 3,850 & 11,612 & 15,732 & 16,156 & 17,521 & 21,444 \\
Western Europe & 600 & 2,549 & 2,755 & 3,398 & 5,291 & 8,013 \\
\hline Total & $\mathbf{1 0 , 7 8 9}$ & $\mathbf{2 2 , 0 7 4}$ & $\mathbf{3 0 , 3 5 3}$ & $\mathbf{3 6 , 7 3 9}$ & $\mathbf{4 5 , 8 0 2}$ & $\mathbf{5 6 , 1 5 7}$ \\
\hline \hline
\end{tabular}


Table A-362: Delayed technology reference case with low oil price ethanol consumption [Million gallons]

\begin{tabular}{lrrrrrr} 
& $\mathbf{2 0 0 5}$ & $\mathbf{2 0 1 0}$ & $\mathbf{2 0 1 5}$ & $\mathbf{2 0 2 0}$ & $\mathbf{2 0 2 5}$ & $\mathbf{2 0 3 0}$ \\
Africa & 300 & 330 & 364 & 402 & 455 & 490 \\
Australia & 0 & 0 & 66 & 217 & 585 & 1,172 \\
Canada & 145 & 0 & 233 & 585 & 650 & 650 \\
China & 297 & 0 & 0 & 259 & 450 & 430 \\
Central and South America & 3,788 & 4,853 & 6,134 & 7,161 & 7,959 & 8,860 \\
Eastern Europe & 699 & 226 & 872 & 953 & 1,037 & 1,149 \\
Former Soviet Union & 0 & 0 & 0 & 0 & 0 & 0 \\
India & 313 & 275 & 534 & 925 & 1,850 & 2,399 \\
Japan & 263 & 250 & 339 & 698 & 1,008 & 1,060 \\
Middle East & 0 & 0 & 0 & 0 & 0 & 0 \\
Mexico & 0 & 0 & 30 & 78 & 183 & 201 \\
Other Asia & 0 & 0 & 0 & 0 & 0 & 0 \\
South Korea & 264 & 335 & 400 & 414 & 413 & 413 \\
United States & 3,984 & 11,612 & 17,037 & 19,797 & 23,562 & 28,685 \\
Western Europe & 736 & 4,193 & 4,344 & 5,249 & 7,649 & 10,649 \\
\hline Total & $\mathbf{1 0 , 7 8 9}$ & $\mathbf{2 2 , 0 7 4}$ & $\mathbf{3 0 , 3 5 3}$ & $\mathbf{3 6 , 7 3 9}$ & $\mathbf{4 5 , 8 0 2}$ & $\mathbf{5 6 , 1 5 7}$ \\
\hline \hline
\end{tabular}

Table A-363: Delayed technology reference case with low oil price ethanol net trade [Million gallons]

\begin{tabular}{lrrrrrr} 
Africa & 0 & 0 & 0 & 0 & 0 & 0 \\
Australia & 0 & 0 & 0 & 0 & 0 & 0 \\
Canada & -145 & 0 & 26 & 65 & 0 & 0 \\
China & 47 & 384 & 614 & 734 & 778 & 798 \\
Central and South America & 1,484 & 1,845 & 3,137 & 5,029 & 7,175 & 8,403 \\
Eastern Europe & -589 & 0 & -438 & -173 & 0 & 0 \\
Former Soviet Union & 0 & 0 & 0 & 0 & 0 & 0 \\
India & 0 & 0 & 0 & 49 & 0 & 128 \\
Japan & -263 & -250 & -244 & -350 & -395 & -415 \\
Middle East & 0 & 0 & 0 & 0 & 0 & 0 \\
Mexico & 0 & 0 & 170 & 523 & 1,225 & 1,345 \\
Other Asia & 0 & 0 & 0 & 0 & 0 & 0 \\
South Korea & -264 & -335 & -370 & -384 & -383 & -382 \\
United States & -134 & 0 & $-1,305$ & $-3,641$ & $-6,042$ & $-7,240$ \\
Western Europe & -136 & $-1,644$ & $-1,589$ & $-1,851$ & $-2,358$ & $-2,636$ \\
\hline Total & $\mathbf{0}$ & $\mathbf{0}$ & $\mathbf{0}$ & $\mathbf{0}$ & $\mathbf{0}$ & $\mathbf{0}$ \\
\hline \hline
\end{tabular}


Table A-364: Delayed technology reference case with low oil price grain ethanol production [Million gallons]

\begin{tabular}{lrrrrrr} 
& $\mathbf{2 0 0 5}$ & $\mathbf{2 0 1 0}$ & $\mathbf{2 0 1 5}$ & $\mathbf{2 0 2 0}$ & $\mathbf{2 0 2 5}$ & $\mathbf{2 0 3 0}$ \\
Africa & 0 & 0 & 0 & 0 & 0 & 0 \\
Argentina & 0 & 178 & 604 & 667 & 667 & 668 \\
Australia & 0 & 0 & 0 & 0 & 0 & 0 \\
Brazil & 0 & 0 & 0 & 0 & 0 & 0 \\
Canada & 0 & 0 & 0 & 0 & 0 & 0 \\
Caribbean basin & 0 & 0 & 0 & 0 & 0 & 0 \\
China & 344 & 344 & 344 & 325 & 0 & 0 \\
Colombia & 0 & 0 & 0 & 0 & 0 & 0 \\
Eastern Europe & 110 & 226 & 348 & 477 & 367 & 251 \\
Former Soviet Union & 0 & 0 & 0 & 0 & 0 & 0 \\
India & 0 & 0 & 0 & 0 & 0 & 0 \\
Japan & 0 & 0 & 0 & 0 & 0 & 0 \\
Middle East & 0 & 0 & 0 & 0 & 0 & 0 \\
Mexico & 0 & 0 & 0 & 0 & 0 & 0 \\
Other Asia & 0 & 0 & 0 & 0 & 0 & 0 \\
South Korea & 0 & 0 & 0 & 0 & 0 & 0 \\
United States & 3,850 & 11,612 & 15,000 & 15,000 & 15,000 & 14,477 \\
Western Europe & 600 & 2,549 & 2,589 & 2,705 & 3,178 & 3,604 \\
\hline Total & $\mathbf{4 , 9 0 4}$ & $\mathbf{1 4 , 9 0 9}$ & $\mathbf{1 8 , 8 8 5}$ & $\mathbf{1 9 , 1 7 5}$ & $\mathbf{1 9 , 2 1 2}$ & $\mathbf{1 9 , 0 0 0}$ \\
\hline \hline
\end{tabular}

Table A-365: Delayed technology reference case with low oil price sugar ethanol production [Million gallons]

\begin{tabular}{lrrrrrr} 
& $\mathbf{2 0 0 5}$ & $\mathbf{2 0 1 0}$ & $\mathbf{2 0 1 5}$ & $\mathbf{2 0 2 0}$ & $\mathbf{2 0 2 5}$ & $\mathbf{2 0 3 0}$ \\
Africa & 300 & 330 & 364 & 402 & 444 & 490 \\
Argentina & 0 & 0 & 0 & 0 & 0 & 0 \\
Australia & 0 & 0 & 0 & 0 & 0 & 0 \\
Brazil & 4,866 & 5,977 & 7,329 & 8,862 & 10,406 & 11,793 \\
Canada & 0 & 0 & 0 & 0 & 0 & 0 \\
Caribbean basin & 210 & 328 & 573 & 683 & 683 & 683 \\
China & 0 & 40 & 48 & 0 & 0 & 0 \\
Colombia & 196 & 215 & 241 & 270 & 302 & 334 \\
Eastern Europe & 0 & 0 & 0 & 0 & 0 & 0 \\
Former Soviet Union & 0 & 0 & 0 & 0 & 0 & 0 \\
India & 313 & 275 & 320 & 329 & 338 & 332 \\
Japan & 0 & 0 & 0 & 0 & 0 & 0 \\
Middle East & 0 & 0 & 0 & 0 & 0 & 0 \\
Mexico & 0 & 0 & 0 & 0 & 0 & 0 \\
Other Asia & 0 & 0 & 0 & 0 & 0 & 0 \\
South Korea & 0 & 0 & 0 & 0 & 0 & 0 \\
United States & 0 & 0 & 0 & 0 & 0 & 0 \\
Western Europe & 0 & 0 & 0 & 0 & 0 & 0 \\
\hline Total & $\mathbf{5 , 8 8 4}$ & $\mathbf{7 , 1 6 5}$ & $\mathbf{8 , 8 7 4}$ & $\mathbf{1 0 , 5 4 6}$ & $\mathbf{1 2 , 1 7 4}$ & $\mathbf{1 3 , 6 3 2}$ \\
\hline \hline
\end{tabular}


Table A-366: Delayed technology reference case with low oil price cellulosic ethanol production [Million gallons]

\begin{tabular}{lrrrrrr} 
& $\mathbf{2 0 0 5}$ & $\mathbf{2 0 1 0}$ & $\mathbf{2 0 1 5}$ & $\mathbf{2 0 2 0}$ & $\mathbf{2 0 2 5}$ & $\mathbf{2 0 3 0}$ \\
Africa & 0 & 0 & 0 & 0 & 11 & 0 \\
Argentina & 0 & 0 & 59 & 306 & 335 & 347 \\
Australia & 0 & 0 & 66 & 217 & 585 & 1,172 \\
Brazil & 0 & 0 & 296 & 890 & 1,541 & 1,546 \\
Canada & 0 & 0 & 259 & 650 & 650 & 650 \\
Caribbean basin & 0 & 0 & 0 & 0 & 0 & 0 \\
China & 0 & 0 & 222 & 668 & 1,228 & 1,228 \\
Colombia & 0 & 0 & 170 & 512 & 1,199 & 1,893 \\
Eastern Europe & 0 & 0 & 85 & 303 & 670 & 897 \\
Former Soviet Union & 0 & 0 & 0 & 0 & 0 & 0 \\
India & 0 & 0 & 214 & 645 & 1,512 & 2,196 \\
Japan & 0 & 0 & 95 & 348 & 612 & 644 \\
Middle East & 0 & 0 & 0 & 0 & 0 & 0 \\
Mexico & 0 & 0 & 199 & 601 & 1,408 & 1,546 \\
Other Asia & 0 & 0 & 0 & 0 & 0 & 0 \\
South Korea & 0 & 0 & 30 & 30 & 30 & 30 \\
United States & 0 & 0 & 732 & 1,156 & 2,521 & 6,967 \\
Western Europe & 0 & 0 & 166 & 693 & 2,113 & 4,410 \\
\hline Total & $\mathbf{0}$ & $\mathbf{0}$ & $\mathbf{2 , 5 9 3}$ & $\mathbf{7 , 0 1 8}$ & $\mathbf{1 4 , 4 1 6}$ & $\mathbf{2 3 , 5 2 5}$ \\
\hline \hline
\end{tabular}

Table A-367: Delayed technology reference case with low oil price grain ethanol consumption [Million gallons]

\begin{tabular}{lrrrrrr} 
& $\mathbf{2 0 0 5}$ & $\mathbf{2 0 1 0}$ & $\mathbf{2 0 1 5}$ & $\mathbf{2 0 2 0}$ & $\mathbf{2 0 2 5}$ & $\mathbf{2 0 3 0}$ \\
Africa & 0 & 0 & 0 & 0 & 0 & 0 \\
Australia & 0 & 0 & 0 & 0 & 0 & 0 \\
Canada & 0 & 0 & 0 & 0 & 0 & 0 \\
China & 297 & 0 & 0 & 259 & 0 & 0 \\
Central and South America & 0 & 36 & 100 & 127 & 100 & 102 \\
Eastern Europe & 110 & 226 & 348 & 477 & 367 & 251 \\
Former Soviet Union & 0 & 0 & 0 & 0 & 0 & 0 \\
India & 0 & 0 & 0 & 0 & 0 & 0 \\
Japan & 0 & 192 & 196 & 67 & 0 & 0 \\
Middle East & 0 & 0 & 0 & 0 & 0 & 0 \\
Mexico & 0 & 0 & 0 & 0 & 0 & 0 \\
Other Asia & 0 & 0 & 0 & 0 & 0 & 0 \\
South Korea & 47 & 295 & 148 & 0 & 0 & 0 \\
United States & 3,850 & 11,612 & 15,000 & 15,000 & 15,000 & 14,477 \\
Western Europe & 600 & 2,549 & 3,094 & 3,246 & 3,745 & 4,170 \\
\hline Total & $\mathbf{4 , 9 0 4}$ & $\mathbf{1 4 , 9 0 9}$ & $\mathbf{1 8 , 8 8 5}$ & $\mathbf{1 9 , 1 7 5}$ & $\mathbf{1 9 , 2 1 2}$ & $\mathbf{1 9 , 0 0 0}$ \\
\hline \hline
\end{tabular}


Table A-368: Delayed technology reference case with low oil price sugar ethanol consumption [Million gallons]

\begin{tabular}{lrrrrrr} 
& $\mathbf{2 0 0 5}$ & $\mathbf{2 0 1 0}$ & $\mathbf{2 0 1 5}$ & $\mathbf{2 0 2 0}$ & $\mathbf{2 0 2 5}$ & $\mathbf{2 0 3 0}$ \\
Africa & 300 & 330 & 364 & 402 & 444 & 490 \\
Australia & 0 & 0 & 0 & 0 & 0 & 0 \\
Canada & 145 & 0 & 0 & 0 & 0 & 0 \\
China & 0 & 0 & 0 & 0 & 0 & 0 \\
Central and South America & 3,788 & 4,818 & 6,035 & 6,909 & 7,859 & 8,759 \\
Eastern Europe & 589 & 0 & 412 & 108 & 0 & 0 \\
Former Soviet Union & 0 & 0 & 0 & 0 & 0 & 0 \\
India & 313 & 275 & 320 & 329 & 338 & 332 \\
Japan & 263 & 58 & 48 & 0 & 0 & 0 \\
Middle East & 0 & 0 & 0 & 0 & 0 & 0 \\
Mexico & 0 & 0 & 0 & 0 & 0 & 0 \\
Other Asia & 0 & 0 & 0 & 0 & 0 & 0 \\
South Korea & 217 & 40 & 0 & 0 & 0 & 0 \\
United States & 134 & 0 & 788 & 2,753 & 2,687 & 2,110 \\
Western Europe & 136 & 1,644 & 908 & 44 & 846 & 1,941 \\
\hline Total & $\mathbf{5 , 8 8 4}$ & $\mathbf{7 , 1 6 5}$ & $\mathbf{8 , 8 7 4}$ & $\mathbf{1 0 , 5 4 6}$ & $\mathbf{1 2 , 1 7 4}$ & $\mathbf{1 3 , 6 3 2}$ \\
\hline \hline
\end{tabular}

Table A-369: Delayed technology reference case with low oil price cellulosic ethanol consumption [Million gallons]

\begin{tabular}{lrrrrrr} 
& $\mathbf{2 0 0 5}$ & $\mathbf{2 0 1 0}$ & $\mathbf{2 0 1 5}$ & $\mathbf{2 0 2 0}$ & $\mathbf{2 0 2 5}$ & $\mathbf{2 0 3 0}$ \\
Africa & 0 & 0 & 0 & 0 & 11 & 0 \\
Australia & 0 & 0 & 66 & 217 & 585 & 1,172 \\
Canada & 0 & 0 & 233 & 585 & 650 & 650 \\
China & 0 & 0 & 0 & 0 & 450 & 430 \\
Central and South America & 0 & 0 & 0 & 125 & 0 & 0 \\
Eastern Europe & 0 & 0 & 111 & 368 & 670 & 897 \\
Former Soviet Union & 0 & 0 & 0 & 0 & 0 & 0 \\
India & 0 & 0 & 214 & 597 & 1,512 & 2,067 \\
Japan & 0 & 0 & 95 & 632 & 1,008 & 1,060 \\
Middle East & 0 & 0 & 0 & 0 & 0 & 0 \\
Mexico & 0 & 0 & 30 & 78 & 183 & 201 \\
Other Asia & 0 & 0 & 0 & 0 & 0 & 0 \\
South Korea & 0 & 0 & 252 & 414 & 413 & 413 \\
United States & 0 & 0 & 1,249 & 2,044 & 5,875 & 12,098 \\
Western Europe & 0 & 0 & 343 & 1,958 & 3,059 & 4,538 \\
\hline Total & $\mathbf{0}$ & $\mathbf{0}$ & $\mathbf{2 , 5 9 3}$ & $\mathbf{7 , 0 1 8}$ & $\mathbf{1 4 , 4 1 6}$ & $\mathbf{2 3 , 5 2 5}$ \\
\hline \hline
\end{tabular}


Table A-370: Delayed technology reference case with low oil price grain ethanol net trade [Million gallons]

\begin{tabular}{lrrrrrr} 
& $\mathbf{2 0 0 5}$ & $\mathbf{2 0 1 0}$ & $\mathbf{2 0 1 5}$ & $\mathbf{2 0 2 0}$ & $\mathbf{2 0 2 5}$ & $\mathbf{2 0 3 0}$ \\
Africa & 0 & 0 & 0 & 0 & 0 & 0 \\
Australia & 0 & 0 & 0 & 0 & 0 & 0 \\
Canada & 0 & 0 & 0 & 0 & 0 & 0 \\
China & 47 & 344 & 344 & 67 & 0 & 0 \\
Central and South America & 0 & 143 & 505 & 541 & 567 & 566 \\
Eastern Europe & 0 & 0 & 0 & 0 & 0 & 0 \\
Former Soviet Union & 0 & 0 & 0 & 0 & 0 & 0 \\
India & 0 & 0 & 0 & 0 & 0 & 0 \\
Japan & 0 & -192 & -196 & -67 & 0 & 0 \\
Middle East & 0 & 0 & 0 & 0 & 0 & 0 \\
Mexico & 0 & 0 & 0 & 0 & 0 & 0 \\
Other Asia & 0 & 0 & 0 & 0 & 0 & 0 \\
South Korea & -47 & -295 & -148 & 0 & 0 & 0 \\
United States & 0 & 0 & 0 & 0 & 0 & 0 \\
Western Europe & 0 & 0 & -505 & -541 & -567 & -566 \\
\hline Total & $\mathbf{0}$ & $\mathbf{0}$ & $\mathbf{0}$ & $\mathbf{0}$ & $\mathbf{0}$ & $\mathbf{0}$ \\
\hline \hline
\end{tabular}

Table A-371: Delayed technology reference case with low oil price sugar ethanol net trade [Million gallons]

\begin{tabular}{lrrrrrr} 
& $\mathbf{2 0 0 5}$ & $\mathbf{2 0 1 0}$ & $\mathbf{2 0 1 5}$ & $\mathbf{2 0 2 0}$ & $\mathbf{2 0 2 5}$ & $\mathbf{2 0 3 0}$ \\
Africa & 0 & 0 & 0 & 0 & 0 & 0 \\
Australia & 0 & 0 & 0 & 0 & 0 & 0 \\
Canada & -145 & 0 & 0 & 0 & 0 & 0 \\
China & 0 & 40 & 48 & 0 & 0 & 0 \\
Central and South America & 1,484 & 1,702 & 2,108 & 2,906 & 3,533 & 4,051 \\
Eastern Europe & -589 & 0 & -412 & -108 & 0 & 0 \\
Former Soviet Union & 0 & 0 & 0 & 0 & 0 & 0 \\
India & 0 & 0 & 0 & 0 & 0 & 0 \\
Japan & -263 & -58 & -48 & 0 & 0 & 0 \\
Middle East & 0 & 0 & 0 & 0 & 0 & 0 \\
Mexico & 0 & 0 & 0 & 0 & 0 & 0 \\
Other Asia & 0 & 0 & 0 & 0 & 0 & 0 \\
South Korea & -217 & -40 & 0 & 0 & 0 & 0 \\
United States & -134 & 0 & -788 & $-2,753$ & $-2,687$ & $-2,110$ \\
Western Europe & -136 & $-1,644$ & -908 & -44 & -846 & $-1,941$ \\
\hline Total & $\mathbf{0}$ & $\mathbf{0}$ & $\mathbf{0}$ & $\mathbf{0}$ & $\mathbf{0}$ & $\mathbf{0}$ \\
\hline \hline
\end{tabular}


Table A-372: Delayed technology reference case with low oil price cellulosic ethanol net trade [Million gallons]

\begin{tabular}{lrrrrrr} 
& $\mathbf{2 0 0 5}$ & $\mathbf{2 0 1 0}$ & $\mathbf{2 0 1 5}$ & $\mathbf{2 0 2 0}$ & $\mathbf{2 0 2 5}$ & $\mathbf{2 0 3 0}$ \\
Africa & 0 & 0 & 0 & 0 & 0 & 0 \\
Australia & 0 & 0 & 0 & 0 & 0 & 0 \\
Canada & 0 & 0 & 26 & 65 & 0 & 0 \\
China & 0 & 0 & 222 & 668 & 778 & 798 \\
Central and South America & 0 & 0 & 525 & 1,582 & 3,076 & 3,786 \\
Eastern Europe & 0 & 0 & -26 & -65 & 0 & 0 \\
Former Soviet Union & 0 & 0 & 0 & 0 & 0 & 0 \\
India & 0 & 0 & 0 & 49 & 0 & 128 \\
Japan & 0 & 0 & 0 & -284 & -395 & -415 \\
Middle East & 0 & 0 & 0 & 0 & 0 & 0 \\
Mexico & 0 & 0 & 170 & 523 & 1,225 & 1,345 \\
Other Asia & 0 & 0 & 0 & 0 & 0 & 0 \\
South Korea & 0 & 0 & -222 & -384 & -383 & -382 \\
United States & 0 & 0 & -518 & -888 & $-3,355$ & $-5,130$ \\
Western Europe & 0 & 0 & -177 & $-1,266$ & -946 & -128 \\
\hline Total & $\mathbf{0}$ & $\mathbf{0}$ & $\mathbf{0}$ & $\mathbf{0}$ & $\mathbf{0}$ & $\mathbf{0}$ \\
\hline \hline
\end{tabular}

Table A-373: Delayed technology reference case with low oil price biomass-toliquids production [Million gallons ethanol equivalent]

\begin{tabular}{lrrrrrr} 
& $\mathbf{2 0 0 5}$ & $\mathbf{2 0 1 0}$ & $\mathbf{2 0 1 5}$ & $\mathbf{2 0 2 0}$ & $\mathbf{2 0 2 5}$ & $\mathbf{2 0 3 0}$ \\
Africa & 0 & 0 & 0 & 0 & 0 & 0 \\
Argentina & 0 & 0 & 21 & 110 & 121 & 125 \\
Australia & 0 & 0 & 0 & 0 & 0 & 0 \\
Brazil & 0 & 0 & 106 & 320 & 554 & 556 \\
Canada & 0 & 0 & 93 & 234 & 234 & 234 \\
Caribbean basin & 0 & 0 & 0 & 0 & 0 & 0 \\
China & 0 & 0 & 80 & 240 & 442 & 442 \\
Colombia & 0 & 0 & 61 & 184 & 431 & 681 \\
Eastern Europe & 0 & 0 & 0 & 0 & 0 & 0 \\
Former Soviet Union & 0 & 0 & 0 & 0 & 0 & 0 \\
India & 0 & 0 & 77 & 232 & 544 & 790 \\
Japan & 0 & 0 & 0 & 0 & 0 & 0 \\
Middle East & 0 & 0 & 0 & 0 & 0 & 0 \\
Mexico & 0 & 0 & 72 & 216 & 506 & 556 \\
Other Asia & 0 & 0 & 0 & 0 & 0 & 0 \\
South Korea & 0 & 0 & 31 & 65 & 65 & 65 \\
United States & 0 & 0 & 750 & 2,467 & 3,398 & 4,383 \\
Western Europe & 0 & 0 & 0 & 0 & 0 & 0 \\
\hline Total & $\mathbf{0}$ & $\mathbf{0}$ & $\mathbf{1 , 2 9 1}$ & $\mathbf{4 , 0 6 7}$ & $\mathbf{6 , 2 9 5}$ & $\mathbf{7 , 8 3 0}$ \\
\hline \hline
\end{tabular}


Table A-374: Delayed technology reference case with low oil price biomass-toliquids consumption [Million gallons ethanol equivalent]

\begin{tabular}{lrrrrrr} 
& $\mathbf{2 0 0 5}$ & $\mathbf{2 0 1 0}$ & $\mathbf{2 0 1 5}$ & $\mathbf{2 0 2 0}$ & $\mathbf{2 0 2 5}$ & $\mathbf{2 0 3 0}$ \\
Africa & 0 & 0 & 0 & 0 & 0 & 0 \\
Australia & 0 & 0 & 0 & 0 & 0 & 0 \\
Canada & 0 & 0 & 0 & 0 & 0 & 0 \\
China & 0 & 0 & 0 & 0 & 0 & 0 \\
Central and South America & 0 & 0 & 0 & 0 & 0 & 0 \\
Eastern Europe & 0 & 0 & 0 & 0 & 0 & 0 \\
Former Soviet Union & 0 & 0 & 0 & 0 & 0 & 0 \\
India & 0 & 0 & 0 & 0 & 0 & 0 \\
Japan & 0 & 0 & 0 & 0 & 0 & 0 \\
Middle East & 0 & 0 & 0 & 0 & 0 & 0 \\
Mexico & 0 & 0 & 0 & 0 & 0 & 0 \\
Other Asia & 0 & 0 & 0 & 0 & 0 & 0 \\
South Korea & 0 & 0 & 0 & 0 & 0 & 0 \\
United States & 0 & 0 & 1,107 & 3,972 & 6,295 & 7,831 \\
Western Europe & 0 & 0 & 184 & 96 & 0 & 0 \\
\hline Total & $\mathbf{0}$ & $\mathbf{0}$ & $\mathbf{1 , 2 9 1}$ & $\mathbf{4 , 0 6 8}$ & $\mathbf{6 , 2 9 5}$ & $\mathbf{7 , 8 3 1}$ \\
\hline \hline
\end{tabular}

Table A-375: Delayed technology reference case with low oil price biomass-toliquids net trade [Million gallons ethanol equivalent]

\begin{tabular}{lrrrrrr} 
& $\mathbf{2 0 0 5}$ & $\mathbf{2 0 1 0}$ & $\mathbf{2 0 1 5}$ & $\mathbf{2 0 2 0}$ & $\mathbf{2 0 2 5}$ & $\mathbf{2 0 3 0}$ \\
Africa & 0 & 0 & 0 & 0 & 0 & 0 \\
Australia & 0 & 0 & 0 & 0 & 0 & 0 \\
Canada & 0 & 0 & 93 & 234 & 234 & 234 \\
China & 0 & 0 & 80 & 240 & 442 & 442 \\
Central and South America & 0 & 0 & 189 & 614 & 1,106 & 1,362 \\
Eastern Europe & 0 & 0 & 0 & 0 & 0 & 0 \\
Former Soviet Union & 0 & 0 & 0 & 0 & 0 & 0 \\
India & 0 & 0 & 77 & 232 & 544 & 790 \\
Japan & 0 & 0 & 0 & 0 & 0 & 0 \\
Middle East & 0 & 0 & 0 & 0 & 0 & 0 \\
Mexico & 0 & 0 & 72 & 216 & 506 & 556 \\
Other Asia & 0 & 0 & 0 & 0 & 0 & 0 \\
South Korea & 0 & 0 & 31 & 65 & 65 & 65 \\
United States & 0 & 0 & -357 & $-1,505$ & $-2,897$ & $-3,448$ \\
Western Europe & 0 & 0 & -184 & -96 & 0 & 0 \\
\hline Total & $\mathbf{0}$ & $\mathbf{0}$ & $\mathbf{0}$ & $\mathbf{0}$ & $\mathbf{0}$ & $\mathbf{0}$ \\
\hline \hline
\end{tabular}


Table A-376: Delayed technology reference case with low oil price biodiesel production [Million gallons ethanol equivalent]

\begin{tabular}{lrrrrrr} 
& $\mathbf{2 0 0 5}$ & $\mathbf{2 0 1 0}$ & $\mathbf{2 0 1 5}$ & $\mathbf{2 0 2 0}$ & $\mathbf{2 0 2 5}$ & $\mathbf{2 0 3 0}$ \\
Africa & 0 & 0 & 0 & 0 & 0 & 0 \\
Argentina & 0 & 333 & 776 & 819 & 827 & 239 \\
Australia & 0 & 0 & 0 & 0 & 0 & 0 \\
Brazil & 0 & 333 & 877 & 1,642 & 2,353 & 1,359 \\
Canada & 0 & 0 & 0 & 0 & 0 & 0 \\
Caribbean basin & 0 & 95 & 310 & 675 & 802 & 898 \\
China & 0 & 0 & 0 & 0 & 0 & 0 \\
Colombia & 0 & 119 & 298 & 474 & 650 & 782 \\
Eastern Europe & 0 & 0 & 0 & 0 & 0 & 0 \\
Former Soviet Union & 0 & 0 & 0 & 0 & 0 & 0 \\
India & 0 & 0 & 0 & 0 & 0 & 0 \\
Japan & 0 & 0 & 0 & 0 & 0 & 0 \\
Middle East & 0 & 0 & 0 & 0 & 0 & 0 \\
Mexico & 0 & 0 & 0 & 0 & 0 & 0 \\
Other Asia & 0 & 0 & 0 & 0 & 0 & 0 \\
South Korea & 0 & 0 & 0 & 0 & 0 & 0 \\
United States & 0 & 1,238 & 1,640 & 1,747 & 1,907 & 2,072 \\
Western Europe & 998 & 2,643 & 3,281 & 3,307 & 3,760 & 4,114 \\
\hline Total & $\mathbf{9 9 8}$ & $\mathbf{4 , 7 6 0}$ & $\mathbf{7 , 1 8 2}$ & $\mathbf{8 , 6 6 4}$ & $\mathbf{1 0 , 2 9 9}$ & $\mathbf{9 , 4 6 5}$ \\
\hline \hline
\end{tabular}

Table A-377: Delayed technology reference case with low oil price biodiesel consumption [Million gallons ethanol equivalent]

\begin{tabular}{lrrrrrr} 
& $\mathbf{2 0 0 5}$ & $\mathbf{2 0 1 0}$ & $\mathbf{2 0 1 5}$ & $\mathbf{2 0 2 0}$ & $\mathbf{2 0 2 5}$ & $\mathbf{2 0 3 0}$ \\
Africa & 0 & 0 & 0 & 0 & 0 & 0 \\
Australia & 0 & 0 & 0 & 0 & 0 & 0 \\
Canada & 0 & 0 & 0 & 0 & 0 & 0 \\
China & 0 & 0 & 0 & 0 & 0 & 0 \\
Central and South America & 0 & 158 & 477 & 985 & 1,309 & 1,494 \\
Eastern Europe & 0 & 0 & 0 & 0 & 0 & 0 \\
Former Soviet Union & 0 & 0 & 0 & 0 & 0 & 0 \\
India & 0 & 0 & 0 & 0 & 0 & 0 \\
Japan & 0 & 0 & 0 & 0 & 0 & 0 \\
Middle East & 0 & 0 & 0 & 0 & 0 & 0 \\
Mexico & 0 & 0 & 0 & 0 & 0 & 0 \\
Other Asia & 0 & 0 & 0 & 0 & 0 & 0 \\
South Korea & 0 & 0 & 0 & 0 & 0 & 0 \\
United States & 0 & 1,238 & 1,713 & 1,747 & 2,313 & 3,413 \\
Western Europe & 998 & 3,364 & 4,992 & 5,932 & 6,677 & 4,557 \\
\hline Total & $\mathbf{9 9 8}$ & $\mathbf{4 , 7 6 0}$ & $\mathbf{7 , 1 8 2}$ & $\mathbf{8 , 6 6 4}$ & $\mathbf{1 0 , 2 9 9}$ & $\mathbf{9 , 4 6 5}$ \\
\hline \hline
\end{tabular}


Table A-378: Delayed technology reference case with low oil price biodiesel net trade [Million gallons ethanol equivalent]

\begin{tabular}{lrrrrrr} 
& $\mathbf{2 0 0 5}$ & $\mathbf{2 0 1 0}$ & $\mathbf{2 0 1 5}$ & $\mathbf{2 0 2 0}$ & $\mathbf{2 0 2 5}$ & $\mathbf{2 0 3 0}$ \\
Africa & 0 & 0 & 0 & 0 & 0 & 0 \\
Australia & 0 & 0 & 0 & 0 & 0 & 0 \\
Canada & 0 & 0 & 0 & 0 & 0 & 0 \\
China & 0 & 0 & 0 & 0 & 0 & 0 \\
Central and South America & 0 & 720 & 1,784 & 2,625 & 3,324 & 1,784 \\
Eastern Europe & 0 & 0 & 0 & 0 & 0 & 0 \\
Former Soviet Union & 0 & 0 & 0 & 0 & 0 & 0 \\
India & 0 & 0 & 0 & 0 & 0 & 0 \\
Japan & 0 & 0 & 0 & 0 & 0 & 0 \\
Middle East & 0 & 0 & 0 & 0 & 0 & 0 \\
Mexico & 0 & 0 & 0 & 0 & 0 & 0 \\
Other Asia & 0 & 0 & 0 & 0 & 0 & 0 \\
South Korea & 0 & 0 & 0 & 0 & 0 & 0 \\
United States & 0 & 0 & -73 & 0 & -406 & $-1,341$ \\
Western Europe & 0 & -720 & $-1,711$ & $-2,625$ & $-2,917$ & -443 \\
\hline Total & $\mathbf{0}$ & $\mathbf{0}$ & $\mathbf{0}$ & $\mathbf{0}$ & $\mathbf{0}$ & $\mathbf{0}$ \\
\hline \hline
\end{tabular}




\section{A-22 Delayed Technology Reference Case with High Oil Price and High Feedstock Availability}

Table A-379: Delayed technology reference case with high oil price and high feedstock availability ethanol production [Million gallons]

\begin{tabular}{lrrrrrr} 
& $\mathbf{2 0 0 5}$ & $\mathbf{2 0 1 0}$ & $\mathbf{2 0 1 5}$ & $\mathbf{2 0 2 0}$ & $\mathbf{2 0 2 5}$ & $\mathbf{2 0 3 0}$ \\
Africa & 300 & 330 & 364 & 402 & 455 & 502 \\
Argentina & 0 & 59 & 125 & 504 & 581 & 607 \\
Australia & 0 & 0 & 71 & 267 & 709 & 1,174 \\
Brazil & 5,225 & 8,034 & 12,103 & 16,859 & 20,867 & 26,166 \\
Canada & 0 & 0 & 259 & 779 & 950 & 950 \\
Caribbean basin & 164 & 282 & 687 & 920 & 942 & 942 \\
China & 344 & 119 & 579 & 1,602 & 2,173 & 3,860 \\
Colombia & 0 & 0 & 170 & 512 & 887 & 887 \\
Eastern Europe & 110 & 226 & 428 & 721 & 1,037 & 1,055 \\
Former Soviet Union & 0 & 0 & 6 & 19 & 57 & 175 \\
India & 313 & 842 & 1,300 & 1,728 & 2,200 & 2,822 \\
Japan & 0 & 0 & 95 & 369 & 608 & 648 \\
Middle East & 0 & 0 & 0 & 0 & 0 & 0 \\
Mexico & 0 & 0 & 199 & 601 & 665 & 665 \\
Other Asia & 0 & 0 & 0 & 0 & 0 & 0 \\
South Korea & 0 & 0 & 27 & 27 & 27 & 27 \\
United States & 3,850 & 11,413 & 14,769 & 15,865 & 16,465 & 17,006 \\
Western Europe & 600 & 2,465 & 2,755 & 3,235 & 5,205 & 7,923 \\
\hline Total & $\mathbf{1 0 , 9 0 5}$ & $\mathbf{2 3 , 7 7 1}$ & $\mathbf{3 3 , 9 3 7}$ & $\mathbf{4 4 , 4 0 8}$ & $\mathbf{5 3 , 8 2 8}$ & $\mathbf{6 5 , 4 0 9}$ \\
\hline \hline
\end{tabular}


Table A-380: Delayed technology reference case with high oil price and high feedstock availability ethanol consumption [Million gallons]

\begin{tabular}{lrrrrrr} 
& $\mathbf{2 0 0 5}$ & $\mathbf{2 0 1 0}$ & $\mathbf{2 0 1 5}$ & $\mathbf{2 0 2 0}$ & $\mathbf{2 0 2 5}$ & $\mathbf{2 0 3 0}$ \\
Africa & 300 & 330 & 364 & 402 & 455 & 502 \\
Australia & 0 & 0 & 71 & 353 & 929 & 1,174 \\
Canada & 103 & 261 & 850 & 895 & 948 & 950 \\
China & 442 & 202 & 2,039 & 3,397 & 4,132 & 5,063 \\
Central and South America & 3,764 & 4,783 & 6,025 & 7,072 & 7,838 & 8,632 \\
Eastern Europe & 699 & 783 & 872 & 953 & 1,037 & 1,055 \\
Former Soviet Union & 0 & 0 & 6 & 19 & 57 & 175 \\
India & 313 & 842 & 1,300 & 1,642 & 1,980 & 2,399 \\
Japan & 300 & 325 & 448 & 757 & 1,041 & 1,139 \\
Middle East & 0 & 0 & 0 & 0 & 0 & 0 \\
Mexico & 0 & 0 & 30 & 998 & 1,030 & 1,052 \\
Other Asia & 0 & 0 & 0 & 2,637 & 3,004 & 3,472 \\
South Korea & 264 & 335 & 400 & 414 & 413 & 413 \\
United States & 3,984 & 11,668 & 17,188 & 19,784 & 23,400 & 28,677 \\
Western Europe & 736 & 4,241 & 4,344 & 5,086 & 7,563 & 10,707 \\
\hline Total & $\mathbf{1 0 , 9 0 5}$ & $\mathbf{2 3 , 7 7 1}$ & $\mathbf{3 3 , 9 3 7}$ & $\mathbf{4 4 , 4 0 8}$ & $\mathbf{5 3 , 8 2 8}$ & $\mathbf{6 5 , 4 0 9}$ \\
\hline \hline
\end{tabular}

Table A-381: Delayed technology reference case with high oil price and high feedstock availability ethanol net trade [Million gallons]

Africa

\begin{tabular}{rrrrrr}
$\mathbf{2 0 0 5}$ & $\mathbf{2 0 1 0}$ & $\mathbf{2 0 1 5}$ & $\mathbf{2 0 2 0}$ & $\mathbf{2 0 2 5}$ & $\mathbf{2 0 3 0}$ \\
0 & 0 & 0 & 0 & 0 & 0 \\
0 & 0 & 0 & -86 & -220 & 0 \\
-103 & -261 & -592 & -116 & 2 & 0 \\
-98 & -83 & $-1,460$ & $-1,795$ & $-1,959$ & $-1,204$ \\
1,624 & 3,593 & 7,060 & 11,723 & 15,439 & 19,970 \\
-589 & -557 & -444 & -233 & 0 & 0 \\
0 & 0 & 0 & 0 & 0 & 0 \\
0 & 0 & 0 & 86 & 220 & 423 \\
-300 & -325 & -353 & -388 & -433 & -490 \\
0 & 0 & 0 & 0 & 0 & 0 \\
0 & 0 & 170 & -398 & -365 & -387 \\
0 & 0 & 0 & $-2,637$ & $-3,004$ & $-3,472$ \\
-264 & -335 & -373 & -387 & -386 & -386 \\
-134 & -256 & $-2,419$ & $-3,919$ & $-6,935$ & $-11,671$ \\
-136 & $-1,776$ & $-1,589$ & $-1,851$ & $-2,358$ & $-2,783$ \\
\hline $\mathbf{0}$ & $\mathbf{0}$ & $\mathbf{0}$ & $\mathbf{0}$ & $\mathbf{0}$ & $\mathbf{0}$ \\
\hline
\end{tabular}


Table A-382: Delayed technology reference case with high oil price and high feedstock availability grain ethanol production [Million gallons]

\begin{tabular}{lrrrrrr} 
& $\mathbf{2 0 0 5}$ & $\mathbf{2 0 1 0}$ & $\mathbf{2 0 1 5}$ & $\mathbf{2 0 2 0}$ & $\mathbf{2 0 2 5}$ & $\mathbf{2 0 3 0}$ \\
Africa & 0 & 0 & 0 & 0 & 0 & 0 \\
Argentina & 0 & 59 & 66 & 66 & 66 & 61 \\
Australia & 0 & 0 & 0 & 0 & 0 & 0 \\
Brazil & 0 & 0 & 0 & 0 & 0 & 0 \\
Canada & 0 & 0 & 0 & 0 & 0 & 0 \\
Caribbean basin & 0 & 0 & 0 & 0 & 0 & 0 \\
China & 344 & 0 & 0 & 325 & 0 & 0 \\
Colombia & 0 & 0 & 0 & 0 & 0 & 0 \\
Eastern Europe & 110 & 226 & 348 & 477 & 474 & 358 \\
Former Soviet Union & 0 & 0 & 0 & 0 & 0 & 0 \\
India & 0 & 0 & 0 & 0 & 0 & 0 \\
Japan & 0 & 0 & 0 & 0 & 0 & 0 \\
Middle East & 0 & 0 & 0 & 0 & 0 & 0 \\
Mexico & 0 & 0 & 0 & 0 & 0 & 0 \\
Other Asia & 0 & 0 & 0 & 0 & 0 & 0 \\
South Korea & 0 & 0 & 0 & 0 & 0 & 0 \\
United States & 3,850 & 11,413 & 14,038 & 14,815 & 12,984 & 10,846 \\
Western Europe & 600 & 2,465 & 2,589 & 2,542 & 3,178 & 3,604 \\
\hline Total & $\mathbf{4 , 9 0 4}$ & $\mathbf{1 4 , 1 6 3}$ & $\mathbf{1 7 , 0 4 0}$ & $\mathbf{1 8 , 2 2 5}$ & $\mathbf{1 6 , 7 0 1}$ & $\mathbf{1 4 , 8 6 8}$ \\
\hline \hline
\end{tabular}

Table A-383: Delayed technology reference case with high oil price and high feedstock availability sugar ethanol production [Million gallons]

\begin{tabular}{lrrrrrr} 
& $\mathbf{2 0 0 5}$ & $\mathbf{2 0 1 0}$ & $\mathbf{2 0 1 5}$ & $\mathbf{2 0 2 0}$ & $\mathbf{2 0 2 5}$ & $\mathbf{2 0 3 0}$ \\
Africa & 300 & 330 & 364 & 402 & 444 & 490 \\
Argentina & 0 & 0 & 0 & 0 & 0 & 0 \\
Australia & 0 & 0 & 0 & 0 & 0 & 0 \\
Brazil & 5,225 & 8,034 & 11,807 & 15,969 & 18,782 & 22,055 \\
Canada & 0 & 0 & 0 & 0 & 0 & 0 \\
Caribbean basin & 164 & 282 & 687 & 920 & 942 & 942 \\
China & 0 & 119 & 358 & 609 & 609 & 609 \\
Colombia & 0 & 0 & 0 & 0 & 0 & 0 \\
Eastern Europe & 0 & 0 & 0 & 0 & 0 & 0 \\
Former Soviet Union & 0 & 0 & 0 & 0 & 0 & 0 \\
India & 313 & 842 & 1,086 & 1,086 & 1,086 & 1,086 \\
Japan & 0 & 0 & 0 & 0 & 0 & 0 \\
Middle East & 0 & 0 & 0 & 0 & 0 & 0 \\
Mexico & 0 & 0 & 0 & 0 & 0 & 0 \\
Other Asia & 0 & 0 & 0 & 0 & 0 & 0 \\
South Korea & 0 & 0 & 0 & 0 & 0 & 0 \\
United States & 0 & 0 & 0 & 0 & 0 & 0 \\
Western Europe & 0 & 0 & 0 & 0 & 0 & 0 \\
\hline Total & $\mathbf{6 , 0 0 1}$ & $\mathbf{9 , 6 0 8}$ & $\mathbf{1 4 , 3 0 2}$ & $\mathbf{1 8 , 9 8 7}$ & $\mathbf{2 1 , 8 6 3}$ & $\mathbf{2 5 , 1 8 3}$ \\
\hline \hline
\end{tabular}


Table A-384: Delayed technology reference case with high oil price and high feedstock availability cellulosic ethanol production [Million gallons]

\begin{tabular}{lrrrrrr} 
& $\mathbf{2 0 0 5}$ & $\mathbf{2 0 1 0}$ & $\mathbf{2 0 1 5}$ & $\mathbf{2 0 2 0}$ & $\mathbf{2 0 2 5}$ & $\mathbf{2 0 3 0}$ \\
Africa & 0 & 0 & 0 & 0 & 11 & 11 \\
Argentina & 0 & 0 & 59 & 438 & 516 & 547 \\
Australia & 0 & 0 & 71 & 267 & 709 & 1,174 \\
Brazil & 0 & 0 & 296 & 890 & 2,085 & 4,111 \\
Canada & 0 & 0 & 259 & 779 & 950 & 950 \\
Caribbean basin & 0 & 0 & 0 & 0 & 0 & 0 \\
China & 0 & 0 & 222 & 667 & 1,564 & 3,251 \\
Colombia & 0 & 0 & 170 & 512 & 887 & 887 \\
Eastern Europe & 0 & 0 & 80 & 243 & 563 & 697 \\
Former Soviet Union & 0 & 0 & 6 & 19 & 57 & 175 \\
India & 0 & 0 & 214 & 642 & 1,114 & 1,736 \\
Japan & 0 & 0 & 95 & 369 & 608 & 648 \\
Middle East & 0 & 0 & 0 & 0 & 0 & 0 \\
Mexico & 0 & 0 & 199 & 601 & 665 & 665 \\
Other Asia & 0 & 0 & 0 & 0 & 0 & 0 \\
South Korea & 0 & 0 & 27 & 27 & 27 & 27 \\
United States & 0 & 0 & 732 & 1,050 & 3,481 & 6,161 \\
Western Europe & 0 & 0 & 166 & 693 & 2,027 & 4,320 \\
\hline Total & $\mathbf{0}$ & $\mathbf{0}$ & $\mathbf{2 , 5 9 5}$ & $\mathbf{7 , 1 9 6}$ & $\mathbf{1 5 , 2 6 4}$ & $\mathbf{2 5 , 3 5 8}$ \\
\hline \hline
\end{tabular}

Table A-385: Delayed technology reference case with high oil price and high feedstock availability grain ethanol consumption [Million gallons]

\begin{tabular}{lrrrrrr} 
& $\mathbf{2 0 0 5}$ & $\mathbf{2 0 1 0}$ & $\mathbf{2 0 1 5}$ & $\mathbf{2 0 2 0}$ & $\mathbf{2 0 2 5}$ & $\mathbf{2 0 3 0}$ \\
Africa & 0 & 0 & 0 & 0 & 0 & 0 \\
Australia & 0 & 0 & 0 & 0 & 0 & 0 \\
Canada & 0 & 0 & 0 & 0 & 0 & 0 \\
China & 344 & 0 & 0 & 325 & 0 & 0 \\
Central and South America & 0 & 12 & 19 & 66 & 58 & 61 \\
Eastern Europe & 110 & 226 & 348 & 477 & 474 & 358 \\
Former Soviet Union & 0 & 0 & 0 & 0 & 0 & 0 \\
India & 0 & 0 & 0 & 0 & 0 & 0 \\
Japan & 0 & 0 & 0 & 0 & 0 & 0 \\
Middle East & 0 & 0 & 0 & 0 & 0 & 0 \\
Mexico & 0 & 0 & 0 & 0 & 0 & 0 \\
Other Asia & 0 & 0 & 0 & 0 & 0 & 0 \\
South Korea & 0 & 48 & 47 & 0 & 7 & 0 \\
United States & 3,850 & 11,413 & 14,038 & 14,815 & 12,984 & 10,846 \\
Western Europe & 600 & 2,465 & 2,589 & 2,542 & 3,178 & 3,604 \\
\hline Total & $\mathbf{4 , 9 0 4}$ & $\mathbf{1 4 , 1 6 3}$ & $\mathbf{1 7 , 0 4 0}$ & $\mathbf{1 8 , 2 2 5}$ & $\mathbf{1 6 , 7 0 1}$ & $\mathbf{1 4 , 8 6 8}$ \\
\hline \hline
\end{tabular}


Table A-386: Delayed technology reference case with high oil price and high feedstock availability sugar ethanol consumption [Million gallons]

\begin{tabular}{lrrrrrr} 
& $\mathbf{2 0 0 5}$ & $\mathbf{2 0 1 0}$ & $\mathbf{2 0 1 5}$ & $\mathbf{2 0 2 0}$ & $\mathbf{2 0 2 5}$ & $\mathbf{2 0 3 0}$ \\
Africa & 300 & 330 & 364 & 402 & 444 & 490 \\
Australia & 0 & 0 & 0 & 0 & 0 & 0 \\
Canada & 103 & 261 & 592 & 116 & 0 & 0 \\
China & 98 & 202 & 1,818 & 2,404 & 2,568 & 1,389 \\
Central and South America & 3,764 & 4,771 & 5,980 & 6,929 & 7,647 & 8,438 \\
Eastern Europe & 589 & 557 & 444 & 233 & 0 & 0 \\
Former Soviet Union & 0 & 0 & 0 & 0 & 0 & 0 \\
India & 313 & 842 & 1,086 & 1,086 & 1,086 & 1,086 \\
Japan & 300 & 325 & 353 & 388 & 433 & 490 \\
Middle East & 0 & 0 & 0 & 0 & 0 & 0 \\
Mexico & 0 & 0 & 0 & 398 & 941 & 895 \\
Other Asia & 0 & 0 & 0 & 2,637 & 3,004 & 3,472 \\
South Korea & 264 & 288 & 327 & 387 & 379 & 386 \\
United States & 134 & 256 & 1,750 & 2,156 & 3,002 & 5,752 \\
Western Europe & 136 & 1,776 & 1,589 & 1,851 & 2,358 & 2,783 \\
\hline Total & $\mathbf{6 , 0 0 1}$ & $\mathbf{9 , 6 0 8}$ & $\mathbf{1 4 , 3 0 2}$ & $\mathbf{1 8 , 9 8 7}$ & $\mathbf{2 1 , 8 6 3}$ & $\mathbf{2 5 , 1 8 3}$ \\
\hline \hline
\end{tabular}

Table A-387: Delayed technology reference case with high oil price and high feedstock availability cellulosic ethanol consumption [Million gallons]

\begin{tabular}{lrrrrrr} 
& $\mathbf{2 0 0 5}$ & $\mathbf{2 0 1 0}$ & $\mathbf{2 0 1 5}$ & $\mathbf{2 0 2 0}$ & $\mathbf{2 0 2 5}$ & $\mathbf{2 0 3 0}$ \\
Africa & 0 & 0 & 0 & 0 & 11 & 11 \\
Australia & 0 & 0 & 71 & 353 & 929 & 1,174 \\
Canada & 0 & 0 & 259 & 779 & 948 & 950 \\
China & 0 & 0 & 222 & 667 & 1,564 & 3,674 \\
Central and South America & 0 & 0 & 26 & 77 & 133 & 133 \\
Eastern Europe & 0 & 0 & 80 & 243 & 563 & 697 \\
Former Soviet Union & 0 & 0 & 6 & 19 & 57 & 175 \\
India & 0 & 0 & 214 & 556 & 894 & 1,313 \\
Japan & 0 & 0 & 95 & 369 & 608 & 648 \\
Middle East & 0 & 0 & 0 & 0 & 0 & 0 \\
Mexico & 0 & 0 & 30 & 601 & 89 & 157 \\
Other Asia & 0 & 0 & 0 & 0 & 0 & 0 \\
South Korea & 0 & 0 & 27 & 27 & 27 & 27 \\
United States & 0 & 0 & 1,400 & 2,813 & 7,414 & 12,079 \\
Western Europe & 0 & 0 & 166 & 693 & 2,027 & 4,320 \\
\hline Total & $\mathbf{0}$ & $\mathbf{0}$ & $\mathbf{2 , 5 9 5}$ & $\mathbf{7 , 1 9 6}$ & $\mathbf{1 5 , 2 6 4}$ & $\mathbf{2 5 , 3 5 8}$ \\
\hline \hline
\end{tabular}


Table A-388: Delayed technology reference case with high oil price and high feedstock availability grain ethanol net trade [Million gallons]

\begin{tabular}{lrrrrrr} 
& $\mathbf{2 0 0 5}$ & $\mathbf{2 0 1 0}$ & $\mathbf{2 0 1 5}$ & $\mathbf{2 0 2 0}$ & $\mathbf{2 0 2 5}$ & $\mathbf{2 0 3 0}$ \\
Africa & 0 & 0 & 0 & 0 & 0 & 0 \\
Australia & 0 & 0 & 0 & 0 & 0 & 0 \\
Canada & 0 & 0 & 0 & 0 & 0 & 0 \\
China & 0 & 0 & 0 & 0 & 0 & 0 \\
Central and South America & 0 & 48 & 47 & 0 & 7 & 0 \\
Eastern Europe & 0 & 0 & 0 & 0 & 0 & 0 \\
Former Soviet Union & 0 & 0 & 0 & 0 & 0 & 0 \\
India & 0 & 0 & 0 & 0 & 0 & 0 \\
Japan & 0 & 0 & 0 & 0 & 0 & 0 \\
Middle East & 0 & 0 & 0 & 0 & 0 & 0 \\
Mexico & 0 & 0 & 0 & 0 & 0 & 0 \\
Other Asia & 0 & 0 & 0 & 0 & 0 & 0 \\
South Korea & 0 & -48 & -47 & 0 & -7 & 0 \\
United States & 0 & 0 & 0 & 0 & 0 & 0 \\
Western Europe & 0 & 0 & 0 & 0 & 0 & 0 \\
\hline Total & $\mathbf{0}$ & $\mathbf{0}$ & $\mathbf{0}$ & $\mathbf{0}$ & $\mathbf{0}$ & $\mathbf{0}$ \\
\hline \hline
\end{tabular}

Table A-389: Delayed technology reference case with high oil price and high feedstock availability sugar ethanol net trade [Million gallons]

\begin{tabular}{lrrrrrr} 
Africa & 0 & 0 & 0 & 0 & 0 & 0 \\
Australia & 0 & 0 & 0 & 0 & 0 & 0 \\
Canada & -103 & -261 & -592 & -116 & 0 & 0 \\
China & -98 & -83 & $-1,460$ & $-1,795$ & $-1,959$ & -780 \\
Central and South America & 1,624 & 3,545 & 6,514 & 9,960 & 12,077 & 14,559 \\
Eastern Europe & -589 & -557 & -444 & -233 & 0 & 0 \\
Former Soviet Union & 0 & 0 & 0 & 0 & 0 & 0 \\
India & 0 & 0 & 0 & 0 & 0 & 0 \\
Japan & -300 & -325 & -353 & -388 & -433 & -490 \\
Middle East & 0 & 0 & 0 & 0 & 0 & 0 \\
Mexico & 0 & 0 & 0 & -398 & -941 & -895 \\
Other Asia & 0 & 0 & 0 & $-2,637$ & $-3,004$ & $-3,472$ \\
South Korea & -264 & -288 & -327 & -387 & -379 & -386 \\
United States & -134 & -256 & $-1,750$ & $-2,156$ & $-3,002$ & $-5,752$ \\
Western Europe & -136 & $-1,776$ & $-1,589$ & $-1,851$ & $-2,358$ & $-2,783$ \\
\hline Total & $\mathbf{0}$ & $\mathbf{0}$ & $\mathbf{0}$ & $\mathbf{0}$ & $\mathbf{0}$ & $\mathbf{0}$ \\
\hline \hline
\end{tabular}


Table A-390: Delayed technology reference case with high oil price and high feedstock availability cellulosic ethanol net trade [Million gallons]

\begin{tabular}{lrrrrrr} 
& $\mathbf{2 0 0 5}$ & $\mathbf{2 0 1 0}$ & $\mathbf{2 0 1 5}$ & $\mathbf{2 0 2 0}$ & $\mathbf{2 0 2 5}$ & $\mathbf{2 0 3 0}$ \\
Africa & 0 & 0 & 0 & 0 & 0 & 0 \\
Australia & 0 & 0 & 0 & -86 & -220 & 0 \\
Canada & 0 & 0 & 0 & 0 & 2 & 0 \\
China & 0 & 0 & 0 & 0 & 0 & -423 \\
Central and South America & 0 & 0 & 499 & 1,763 & 3,355 & 5,411 \\
Eastern Europe & 0 & 0 & 0 & 0 & 0 & 0 \\
Former Soviet Union & 0 & 0 & 0 & 0 & 0 & 0 \\
India & 0 & 0 & 0 & 86 & 220 & 423 \\
Japan & 0 & 0 & 0 & 0 & 0 & 0 \\
Middle East & 0 & 0 & 0 & 0 & 0 & 0 \\
Mexico & 0 & 0 & 170 & 0 & 576 & 508 \\
Other Asia & 0 & 0 & 0 & 0 & 0 & 0 \\
South Korea & 0 & 0 & 0 & 0 & 0 & 0 \\
United States & 0 & 0 & -669 & $-1,763$ & $-3,933$ & $-5,918$ \\
Western Europe & 0 & 0 & 0 & 0 & 0 & 0 \\
\hline Total & $\mathbf{0}$ & $\mathbf{0}$ & $\mathbf{0}$ & $\mathbf{0}$ & $\mathbf{0}$ & $\mathbf{0}$ \\
\hline \hline
\end{tabular}

Table A-391: Delayed technology reference case with high oil price and high feedstock availability biomass-to-liquids production [Million gallons ethanol equivalent]

\begin{tabular}{lllrrrr} 
Africa & 0 & 0 & 0 & 0 & 0 & 0 \\
Argentina & 0 & 0 & 21 & 158 & 186 & 197 \\
Australia & 0 & 0 & 0 & 0 & 0 & 0 \\
Brazil & 0 & 0 & 106 & 320 & 750 & 1,333 \\
Canada & 0 & 0 & 93 & 280 & 342 & 342 \\
Caribbean basin & 0 & 0 & 0 & 0 & 0 & 0 \\
China & 0 & 0 & 80 & 240 & 563 & 1,031 \\
Colombia & 0 & 0 & 61 & 184 & 319 & 319 \\
Eastern Europe & 0 & 0 & 0 & 23 & 170 & 399 \\
Former Soviet Union & 0 & 0 & 0 & 0 & 0 & 0 \\
India & 0 & 0 & 77 & 231 & 401 & 625 \\
Japan & 0 & 0 & 0 & 0 & 0 & 0 \\
Middle East & 0 & 0 & 0 & 0 & 0 & 0 \\
Mexico & 0 & 0 & 72 & 216 & 239 & 239 \\
Other Asia & 0 & 0 & 74 & 336 & 863 & 1,578 \\
South Korea & 0 & 0 & 31 & 57 & 57 & 57 \\
United States & 0 & 0 & 750 & 2,240 & 3,109 & 4,014 \\
Western Europe & 0 & 0 & 0 & 0 & 0 & 0 \\
\hline Total & $\mathbf{0}$ & $\mathbf{0}$ & $\mathbf{1 , 3 6 5}$ & $\mathbf{4 , 2 8 5}$ & $\mathbf{6 , 9 9 8}$ & $\mathbf{1 0 , 1 3 3}$ \\
\hline \hline
\end{tabular}


Table A-392: Delayed technology reference case with high oil price and high feedstock availability biomass-to-liquids consumption [Million gallons ethanol equivalent]

\begin{tabular}{lrrrrrr} 
& $\mathbf{2 0 0 5}$ & $\mathbf{2 0 1 0}$ & $\mathbf{2 0 1 5}$ & $\mathbf{2 0 2 0}$ & $\mathbf{2 0 2 5}$ & $\mathbf{2 0 3 0}$ \\
Africa & 0 & 0 & 0 & 0 & 0 & 0 \\
Australia & 0 & 0 & 0 & 0 & 0 & 0 \\
Canada & 0 & 0 & 0 & 0 & 0 & 0 \\
China & 0 & 0 & 0 & 0 & 0 & 0 \\
Central and South America & 0 & 0 & 0 & 0 & 0 & 0 \\
Eastern Europe & 0 & 0 & 0 & 23 & 171 & 400 \\
Former Soviet Union & 0 & 0 & 0 & 0 & 0 & 0 \\
India & 0 & 0 & 0 & 0 & 0 & 0 \\
Japan & 0 & 0 & 0 & 0 & 0 & 0 \\
Middle East & 0 & 0 & 0 & 0 & 0 & 0 \\
Mexico & 0 & 0 & 0 & 0 & 0 & 0 \\
Other Asia & 0 & 0 & 74 & 336 & 863 & 1,578 \\
South Korea & 0 & 0 & 0 & 0 & 0 & 0 \\
United States & 0 & 0 & 1,291 & 3,926 & 5,965 & 8,156 \\
Western Europe & 0 & 0 & 0 & 0 & 0 & 0 \\
\hline Total & $\mathbf{0}$ & $\mathbf{0}$ & $\mathbf{1 , 3 6 5}$ & $\mathbf{4 , 2 8 5}$ & $\mathbf{6 , 9 9 8}$ & $\mathbf{1 0 , 1 3 4}$ \\
\hline \hline
\end{tabular}

Table A-393: Delayed technology reference case with high oil price and high feedstock availability biomass-to-liquids net trade [Million gallons ethanol equivalent]

\begin{tabular}{lrrrrrr} 
& $\mathbf{2 0 0 5}$ & $\mathbf{2 0 1 0}$ & $\mathbf{2 0 1 5}$ & $\mathbf{2 0 2 0}$ & $\mathbf{2 0 2 5}$ & $\mathbf{2 0 3 0}$ \\
Africa & 0 & 0 & 0 & 0 & 0 & 0 \\
Australia & 0 & 0 & 0 & 0 & 0 & 0 \\
Canada & 0 & 0 & 93 & 280 & 342 & 342 \\
China & 0 & 0 & 80 & 240 & 563 & 1,031 \\
Central and South America & 0 & 0 & 189 & 662 & 1,255 & 1,849 \\
Eastern Europe & 0 & 0 & 0 & 0 & 0 & 0 \\
Former Soviet Union & 0 & 0 & 0 & 0 & 0 & 0 \\
India & 0 & 0 & 77 & 231 & 401 & 625 \\
Japan & 0 & 0 & 0 & 0 & 0 & 0 \\
Middle East & 0 & 0 & 0 & 0 & 0 & 0 \\
Mexico & 0 & 0 & 72 & 216 & 239 & 239 \\
Other Asia & 0 & 0 & 0 & 0 & 0 & 0 \\
South Korea & 0 & 0 & 31 & 57 & 57 & 57 \\
United States & 0 & 0 & -542 & $-1,686$ & $-2,856$ & $-4,142$ \\
Western Europe & 0 & 0 & 0 & 0 & 0 & 0 \\
\hline Total & $\mathbf{0}$ & $\mathbf{0}$ & $\mathbf{0}$ & $\mathbf{0}$ & $\mathbf{0}$ & $\mathbf{0}$ \\
\hline \hline
\end{tabular}


Table A-394: Delayed technology reference case with high oil price and high feedstock availability biodiesel production [Million gallons ethanol equivalent]

\begin{tabular}{lrrrrrr} 
& $\mathbf{2 0 0 5}$ & $\mathbf{2 0 1 0}$ & $\mathbf{2 0 1 5}$ & $\mathbf{2 0 2 0}$ & $\mathbf{2 0 2 5}$ & $\mathbf{2 0 3 0}$ \\
Africa & 0 & 0 & 0 & 0 & 0 & 0 \\
Argentina & 0 & 333 & 877 & 1,642 & 1,642 & 198 \\
Australia & 0 & 0 & 0 & 0 & 0 & 0 \\
Brazil & 0 & 333 & 876 & 1,399 & 1,399 & 1,103 \\
Canada & 0 & 0 & 0 & 0 & 0 & 0 \\
Caribbean basin & 0 & 95 & 310 & 742 & 1,611 & 3,359 \\
China & 0 & 0 & 0 & 0 & 0 & 0 \\
Colombia & 0 & 119 & 358 & 838 & 1,648 & 2,074 \\
Eastern Europe & 0 & 0 & 0 & 0 & 0 & 0 \\
Former Soviet Union & 0 & 0 & 0 & 0 & 0 & 0 \\
India & 0 & 0 & 0 & 0 & 0 & 0 \\
Japan & 0 & 0 & 0 & 0 & 0 & 0 \\
Middle East & 0 & 0 & 0 & 0 & 0 & 0 \\
Mexico & 0 & 0 & 0 & 0 & 0 & 0 \\
Other Asia & 0 & 0 & 0 & 0 & 0 & 0 \\
South Korea & 0 & 0 & 0 & 0 & 0 & 0 \\
United States & 0 & 1,182 & 1,640 & 1,747 & 1,850 & 2,072 \\
Western Europe & 998 & 3,445 & 4,172 & 5,110 & 5,532 & 5,997 \\
\hline Total & $\mathbf{9 9 8}$ & $\mathbf{5 , 5 0 5}$ & $\mathbf{8 , 2 3 2}$ & $\mathbf{1 1 , 4 7 8}$ & $\mathbf{1 3 , 6 8 2}$ & $\mathbf{1 4 , 8 0 2}$ \\
\hline \hline
\end{tabular}

Table A-395: Delayed technology reference case with high oil price and high feedstock availability biodiesel consumption [Million gallons ethanol equivalent]

\begin{tabular}{|c|c|c|c|c|c|c|}
\hline Africa & 0 & 0 & 0 & 0 & 0 & 0 \\
\hline Australia & 0 & 0 & 0 & 0 & 0 & 0 \\
\hline Canada & 0 & 0 & 0 & 0 & 0 & 0 \\
\hline China & 0 & 0 & 0 & 0 & 0 & 0 \\
\hline Central and South America & 0 & 158 & 464 & 975 & 1,303 & 1,488 \\
\hline Eastern Europe & 0 & 0 & 0 & 0 & 0 & 0 \\
\hline Former Soviet Union & 0 & 0 & 0 & 0 & 0 & 0 \\
\hline India & 0 & 0 & 0 & 0 & 0 & 0 \\
\hline Japan & 0 & 0 & 0 & 0 & 0 & 0 \\
\hline Middle East & 0 & 0 & 0 & 0 & 0 & 0 \\
\hline Mexico & 0 & 0 & 0 & 0 & 0 & 0 \\
\hline Other Asia & 0 & 0 & 0 & 0 & 0 & 0 \\
\hline South Korea & 0 & 0 & 0 & 0 & 0 & 0 \\
\hline United States & 0 & 1,182 & 1,713 & 2,529 & 4,014 & 3,402 \\
\hline Western Europe & 998 & 4,165 & 6,055 & 7,974 & 8,365 & 9,912 \\
\hline Total & 998 & 5,505 & 8,232 & 11,478 & 13,682 & 14,802 \\
\hline
\end{tabular}


Table A-396: Delayed technology reference case with high oil price and high feedstock availability biodiesel net trade [Million gallons ethanol equivalent]

\begin{tabular}{lrrrrrr} 
& $\mathbf{2 0 0 5}$ & $\mathbf{2 0 1 0}$ & $\mathbf{2 0 1 5}$ & $\mathbf{2 0 2 0}$ & $\mathbf{2 0 2 5}$ & $\mathbf{2 0 3 0}$ \\
Africa & 0 & 0 & 0 & 0 & 0 & 0 \\
Australia & 0 & 0 & 0 & 0 & 0 & 0 \\
Canada & 0 & 0 & 0 & 0 & 0 & 0 \\
China & 0 & 0 & 0 & 0 & 0 & 0 \\
Central and South America & 0 & 720 & 1,956 & 3,646 & 4,997 & 5,245 \\
Eastern Europe & 0 & 0 & 0 & 0 & 0 & 0 \\
Former Soviet Union & 0 & 0 & 0 & 0 & 0 & 0 \\
India & 0 & 0 & 0 & 0 & 0 & 0 \\
Japan & 0 & 0 & 0 & 0 & 0 & 0 \\
Middle East & 0 & 0 & 0 & 0 & 0 & 0 \\
Mexico & 0 & 0 & 0 & 0 & 0 & 0 \\
Other Asia & 0 & 0 & 0 & 0 & 0 & 0 \\
South Korea & 0 & 0 & 0 & 0 & 0 & 0 \\
United States & 0 & 0 & -73 & -782 & $-2,164$ & $-1,330$ \\
Western Europe & 0 & -720 & $-1,883$ & $-2,864$ & $-2,833$ & $-3,915$ \\
\hline Total & $\mathbf{0}$ & $\mathbf{0}$ & $\mathbf{0}$ & $\mathbf{0}$ & $\mathbf{0}$ & $\mathbf{0}$ \\
\hline \hline
\end{tabular}




\section{A-23 Delayed Technology Reference Case with Low Oil Price and Low Feedstock Availability}

Table A-397: Delayed technology reference case with low oil price and low feedstock availability ethanol production [Million gallons]

\begin{tabular}{lrrrrrr} 
& $\mathbf{2 0 0 5}$ & $\mathbf{2 0 1 0}$ & $\mathbf{2 0 1 5}$ & $\mathbf{2 0 2 0}$ & $\mathbf{2 0 2 5}$ & $\mathbf{2 0 3 0}$ \\
Africa & 300 & 330 & 364 & 402 & 455 & 490 \\
Argentina & 0 & 178 & 735 & 1,108 & 1,114 & 1,114 \\
Australia & 0 & 0 & 66 & 217 & 585 & 1,172 \\
Brazil & 4,866 & 4,894 & 6,282 & 7,363 & 8,942 & 11,845 \\
Canada & 0 & 143 & 401 & 851 & 1,167 & 1,167 \\
Caribbean basin & 162 & 80 & 34 & 15 & 0 & 0 \\
China & 344 & 366 & 588 & 1,033 & 1,564 & 1,564 \\
Colombia & 0 & 196 & 366 & 369 & 369 & 369 \\
Eastern Europe & 110 & 226 & 434 & 780 & 1,037 & 1,149 \\
Former Soviet Union & 0 & 0 & 0 & 0 & 0 & 0 \\
India & 313 & 0 & 214 & 645 & 1,512 & 2,807 \\
Japan & 0 & 0 & 95 & 348 & 612 & 644 \\
Middle East & 0 & 0 & 0 & 0 & 0 & 0 \\
Mexico & 0 & 0 & 0 & 0 & 0 & 0 \\
Other Asia & 0 & 0 & 0 & 0 & 0 & 0 \\
South Korea & 0 & 0 & 35 & 35 & 35 & 26 \\
United States & 3,850 & 11,612 & 15,767 & 18,450 & 21,743 & 25,031 \\
Western Europe & 600 & 2,570 & 2,755 & 3,398 & 5,291 & 8,392 \\
\hline Total & $\mathbf{1 0 , 5 4 4}$ & $\mathbf{2 0 , 5 9 4}$ & $\mathbf{2 8 , 1 3 5}$ & $\mathbf{3 5 , 0 1 3}$ & $\mathbf{4 4 , 4 2 6}$ & $\mathbf{5 5 , 7 7 0}$ \\
\hline
\end{tabular}


Table A-398: Delayed technology reference case with low oil price and low feedstock availability ethanol consumption [Million gallons]

\begin{tabular}{lrrrrrr} 
& $\mathbf{2 0 0 5}$ & $\mathbf{2 0 1 0}$ & $\mathbf{2 0 1 5}$ & $\mathbf{2 0 2 0}$ & $\mathbf{2 0 2 5}$ & $\mathbf{2 0 3 0}$ \\
Africa & 300 & 330 & 364 & 402 & 455 & 490 \\
Australia & 0 & 0 & 66 & 217 & 585 & 1,172 \\
Canada & 101 & 128 & 361 & 766 & 1,050 & 1,050 \\
China & 121 & 0 & 0 & 0 & 0 & 444 \\
Central and South America & 3,764 & 4,831 & 6,105 & 7,083 & 7,767 & 8,557 \\
Eastern Europe & 699 & 226 & 434 & 780 & 1,037 & 1,149 \\
Former Soviet Union & 0 & 0 & 0 & 0 & 0 & 0 \\
India & 313 & 0 & 214 & 613 & 1,361 & 2,386 \\
Japan & 263 & 132 & 216 & 615 & 997 & 1,083 \\
Middle East & 0 & 0 & 0 & 0 & 0 & 0 \\
Mexico & 0 & 0 & 0 & 0 & 0 & 0 \\
Other Asia & 0 & 0 & 0 & 0 & 0 & 0 \\
South Korea & 264 & 0 & 400 & 414 & 413 & 413 \\
United States & 3,984 & 11,612 & 15,767 & 18,874 & 23,112 & 28,564 \\
Western Europe & 736 & 3,335 & 4,208 & 5,249 & 7,649 & 10,461 \\
\hline Total & $\mathbf{1 0 , 5 4 4}$ & $\mathbf{2 0 , 5 9 4}$ & $\mathbf{2 8 , 1 3 5}$ & $\mathbf{3 5 , 0 1 3}$ & $\mathbf{4 4 , 4 2 6}$ & $\mathbf{5 5 , 7 7 0}$ \\
\hline \hline
\end{tabular}

Table A-399: Delayed technology reference case with low oil price and low feedstock availability ethanol net trade [Million gallons]

\begin{tabular}{lrrrrrr} 
& $\mathbf{2 0 0 5}$ & $\mathbf{2 0 1 0}$ & $\mathbf{2 0 1 5}$ & $\mathbf{2 0 2 0}$ & $\mathbf{2 0 2 5}$ & $\mathbf{2 0 3 0}$ \\
Africa & 0 & 0 & 0 & 0 & 0 & 0 \\
Australia & 0 & 0 & 0 & 0 & 0 & 0 \\
Canada & -101 & 14 & 40 & 85 & 117 & 117 \\
China & 224 & 366 & 588 & 1,033 & 1,564 & 1,120 \\
Central and South America & 1,264 & 517 & 1,312 & 1,771 & 2,659 & 4,770 \\
Eastern Europe & -589 & 0 & 0 & 0 & 0 & 0 \\
Former Soviet Union & 0 & 0 & 0 & 0 & 0 & 0 \\
India & 0 & 0 & 0 & 32 & 151 & 421 \\
Japan & -263 & -132 & -121 & -267 & -385 & -439 \\
Middle East & 0 & 0 & 0 & 0 & 0 & 0 \\
Mexico & 0 & 0 & 0 & 0 & 0 & 0 \\
Other Asia & 0 & 0 & 0 & 0 & 0 & 0 \\
South Korea & -264 & 0 & -365 & -379 & -378 & -387 \\
United States & -134 & 0 & 0 & -424 & $-1,369$ & $-3,533$ \\
Western Europe & -136 & -765 & $-1,453$ & $-1,851$ & $-2,358$ & $-2,069$ \\
\hline Total & $\mathbf{0}$ & $\mathbf{0}$ & $\mathbf{0}$ & $\mathbf{0}$ & $\mathbf{0}$ & $\mathbf{0}$ \\
\hline \hline
\end{tabular}


Table A-400: Delayed technology reference case with low oil price and low feedstock availability grain ethanol production [Million gallons]

\begin{tabular}{lrrrrrr} 
& $\mathbf{2 0 0 5}$ & $\mathbf{2 0 1 0}$ & $\mathbf{2 0 1 5}$ & $\mathbf{2 0 2 0}$ & $\mathbf{2 0 2 5}$ & $\mathbf{2 0 3 0}$ \\
Africa & 0 & 0 & 0 & 0 & 0 & 0 \\
Argentina & 0 & 178 & 675 & 833 & 839 & 839 \\
Australia & 0 & 0 & 0 & 0 & 0 & 0 \\
Brazil & 0 & 0 & 0 & 0 & 0 & 0 \\
Canada & 0 & 143 & 143 & 72 & 0 & 0 \\
Caribbean basin & 0 & 0 & 0 & 0 & 0 & 0 \\
China & 344 & 366 & 366 & 366 & 0 & 0 \\
Colombia & 0 & 0 & 0 & 0 & 0 & 0 \\
Eastern Europe & 110 & 226 & 348 & 477 & 367 & 251 \\
Former Soviet Union & 0 & 0 & 0 & 0 & 0 & 0 \\
India & 0 & 0 & 0 & 0 & 0 & 0 \\
Japan & 0 & 0 & 0 & 0 & 0 & 0 \\
Middle East & 0 & 0 & 0 & 0 & 0 & 0 \\
Mexico & 0 & 0 & 0 & 0 & 0 & 0 \\
Other Asia & 0 & 0 & 0 & 0 & 0 & 0 \\
South Korea & 0 & 0 & 0 & 0 & 0 & 0 \\
United States & 3,850 & 11,612 & 15,000 & 15,000 & 15,000 & 15,000 \\
Western Europe & 600 & 2,570 & 2,589 & 2,705 & 3,178 & 3,813 \\
\hline Total & $\mathbf{4 , 9 0 4}$ & $\mathbf{1 5 , 0 9 4}$ & $\mathbf{1 9 , 1 2 1}$ & $\mathbf{1 9 , 4 5 3}$ & $\mathbf{1 9 , 3 8 4}$ & $\mathbf{1 9 , 9 0 4}$ \\
\hline \hline
\end{tabular}

Table A-401: Delayed technology reference case with low oil price and low feedstock availability sugar ethanol production [Million gallons]

\begin{tabular}{lrrrrrr} 
& $\mathbf{2 0 0 5}$ & $\mathbf{2 0 1 0}$ & $\mathbf{2 0 1 5}$ & $\mathbf{2 0 2 0}$ & $\mathbf{2 0 2 5}$ & $\mathbf{2 0 3 0}$ \\
Africa & 300 & 330 & 364 & 402 & 444 & 490 \\
Argentina & 0 & 0 & 0 & 0 & 0 & 0 \\
Australia & 0 & 0 & 0 & 0 & 0 & 0 \\
Brazil & 4,866 & 4,894 & 5,978 & 6,473 & 6,857 & 7,093 \\
Canada & 0 & 0 & 0 & 0 & 0 & 0 \\
Caribbean basin & 162 & 80 & 34 & 15 & 0 & 0 \\
China & 0 & 0 & 0 & 0 & 0 & 0 \\
Colombia & 0 & 196 & 196 & 0 & 0 & 0 \\
Eastern Europe & 0 & 0 & 0 & 0 & 0 & 0 \\
Former Soviet Union & 0 & 0 & 0 & 0 & 0 & 0 \\
India & 313 & 0 & 0 & 0 & 0 & 0 \\
Japan & 0 & 0 & 0 & 0 & 0 & 0 \\
Middle East & 0 & 0 & 0 & 0 & 0 & 0 \\
Mexico & 0 & 0 & 0 & 0 & 0 & 0 \\
Other Asia & 0 & 0 & 0 & 0 & 0 & 0 \\
South Korea & 0 & 0 & 0 & 0 & 0 & 0 \\
United States & 0 & 0 & 0 & 0 & 0 & 0 \\
Western Europe & 0 & 0 & 0 & 0 & 0 & 0 \\
\hline Total & $\mathbf{5 , 6 4 0}$ & $\mathbf{5 , 5 0 0}$ & $\mathbf{6 , 5 7 2}$ & $\mathbf{6 , 8 9 0}$ & $\mathbf{7 , 3 0 1}$ & $\mathbf{7 , 5 8 4}$ \\
\hline \hline
\end{tabular}


Table A-402: Delayed technology reference case with low oil price and low feedstock availability cellulosic ethanol production [Million gallons]

\begin{tabular}{lrrrrrr} 
& $\mathbf{2 0 0 5}$ & $\mathbf{2 0 1 0}$ & $\mathbf{2 0 1 5}$ & $\mathbf{2 0 2 0}$ & $\mathbf{2 0 2 5}$ & $\mathbf{2 0 3 0}$ \\
Africa & 0 & 0 & 0 & 0 & 11 & 0 \\
Argentina & 0 & 0 & 59 & 275 & 275 & 275 \\
Australia & 0 & 0 & 66 & 217 & 585 & 1,172 \\
Brazil & 0 & 0 & 304 & 890 & 2,085 & 4,751 \\
Canada & 0 & 0 & 259 & 779 & 1,167 & 1,167 \\
Caribbean basin & 0 & 0 & 0 & 0 & 0 & 0 \\
China & 0 & 0 & 222 & 667 & 1,564 & 1,564 \\
Colombia & 0 & 0 & 170 & 369 & 369 & 369 \\
Eastern Europe & 0 & 0 & 85 & 303 & 670 & 897 \\
Former Soviet Union & 0 & 0 & 0 & 0 & 0 & 0 \\
India & 0 & 0 & 214 & 645 & 1,512 & 2,807 \\
Japan & 0 & 0 & 95 & 348 & 612 & 644 \\
Middle East & 0 & 0 & 0 & 0 & 0 & 0 \\
Mexico & 0 & 0 & 0 & 0 & 0 & 0 \\
Other Asia & 0 & 0 & 0 & 0 & 0 & 0 \\
South Korea & 0 & 0 & 35 & 35 & 35 & 26 \\
United States & 0 & 0 & 767 & 3,450 & 6,743 & 10,031 \\
Western Europe & 0 & 0 & 166 & 693 & 2,113 & 4,579 \\
\hline Total & $\mathbf{0}$ & $\mathbf{0}$ & $\mathbf{2 , 4 4 2}$ & $\mathbf{8 , 6 7 0}$ & $\mathbf{1 7 , 7 4 2}$ & $\mathbf{2 8 , 2 8 2}$ \\
\hline \hline
\end{tabular}

Table A-403: Delayed technology reference case with low oil price and low feedstock availability grain ethanol consumption [Million gallons]

\begin{tabular}{lrrrrrr} 
& $\mathbf{2 0 0 5}$ & $\mathbf{2 0 1 0}$ & $\mathbf{2 0 1 5}$ & $\mathbf{2 0 2 0}$ & $\mathbf{2 0 2 5}$ & $\mathbf{2 0 3 0}$ \\
Africa & 0 & 0 & 0 & 0 & 0 & 0 \\
Australia & 0 & 0 & 0 & 0 & 0 & 0 \\
Canada & 0 & 128 & 143 & 72 & 0 & 0 \\
China & 121 & 0 & 0 & 0 & 0 & 0 \\
Central and South America & 0 & 36 & 110 & 144 & 111 & 111 \\
Eastern Europe & 110 & 226 & 348 & 477 & 367 & 251 \\
Former Soviet Union & 0 & 0 & 0 & 0 & 0 & 0 \\
India & 0 & 0 & 0 & 0 & 0 & 0 \\
Japan & 0 & 132 & 121 & 267 & 0 & 0 \\
Middle East & 0 & 0 & 0 & 0 & 0 & 0 \\
Mexico & 0 & 0 & 0 & 0 & 0 & 0 \\
Other Asia & 0 & 0 & 0 & 0 & 0 & 0 \\
South Korea & 224 & 0 & 245 & 99 & 0 & 0 \\
United States & 3,850 & 11,612 & 15,000 & 15,000 & 15,000 & 15,000 \\
Western Europe & 600 & 2,960 & 3,154 & 3,394 & 3,905 & 4,541 \\
\hline Total & $\mathbf{4 , 9 0 4}$ & $\mathbf{1 5 , 0 9 4}$ & $\mathbf{1 9 , 1 2 1}$ & $\mathbf{1 9 , 4 5 3}$ & $\mathbf{1 9 , 3 8 4}$ & $\mathbf{1 9 , 9 0 4}$ \\
\hline \hline
\end{tabular}


Table A-404: Delayed technology reference case with low oil price and low feedstock availability sugar ethanol consumption [Million gallons]

\begin{tabular}{lrrrrrr} 
& $\mathbf{2 0 0 5}$ & $\mathbf{2 0 1 0}$ & $\mathbf{2 0 1 5}$ & $\mathbf{2 0 2 0}$ & $\mathbf{2 0 2 5}$ & $\mathbf{2 0 3 0}$ \\
Africa & 300 & 330 & 364 & 402 & 444 & 490 \\
Australia & 0 & 0 & 0 & 0 & 0 & 0 \\
Canada & 101 & 0 & 0 & 0 & 0 & 0 \\
China & 0 & 0 & 0 & 0 & 0 & 0 \\
Central and South America & 3,764 & 4,795 & 5,995 & 6,063 & 5,514 & 7,093 \\
Eastern Europe & 589 & 0 & 0 & 0 & 0 & 0 \\
Former Soviet Union & 0 & 0 & 0 & 0 & 0 & 0 \\
India & 313 & 0 & 0 & 0 & 0 & 0 \\
Japan & 263 & 0 & 0 & 0 & 0 & 0 \\
Middle East & 0 & 0 & 0 & 0 & 0 & 0 \\
Mexico & 0 & 0 & 0 & 0 & 0 & 0 \\
Other Asia & 0 & 0 & 0 & 0 & 0 & 0 \\
South Korea & 41 & 0 & 0 & 0 & 0 & 0 \\
United States & 134 & 0 & 0 & 424 & 1,342 & 0 \\
Western Europe & 136 & 375 & 213 & 0 & 0 & 0 \\
\hline Total & $\mathbf{5 , 6 4 0}$ & $\mathbf{5 , 5 0 0}$ & $\mathbf{6 , 5 7 2}$ & $\mathbf{6 , 8 9 0}$ & $\mathbf{7 , 3 0 1}$ & $\mathbf{7 , 5 8 4}$ \\
\hline \hline
\end{tabular}

Table A-405: Delayed technology reference case with low oil price and low feedstock availability cellulosic ethanol consumption [Million gallons]

\begin{tabular}{lllrrrr} 
Africa & 0 & 0 & 0 & 0 & 11 & 0 \\
Australia & 0 & 0 & 66 & 217 & 585 & 1,172 \\
Canada & 0 & 0 & 219 & 694 & 1,050 & 1,050 \\
China & 0 & 0 & 0 & 0 & 0 & 444 \\
Central and South America & 0 & 0 & 0 & 876 & 2,141 & 1,353 \\
Eastern Europe & 0 & 0 & 85 & 303 & 670 & 897 \\
Former Soviet Union & 0 & 0 & 0 & 0 & 0 & 0 \\
India & 0 & 0 & 214 & 613 & 1,361 & 2,386 \\
Japan & 0 & 0 & 95 & 348 & 997 & 1,083 \\
Middle East & 0 & 0 & 0 & 0 & 0 & 0 \\
Mexico & 0 & 0 & 0 & 0 & 0 & 0 \\
Other Asia & 0 & 0 & 0 & 0 & 0 & 0 \\
South Korea & 0 & 0 & 155 & 315 & 413 & 413 \\
United States & 0 & 0 & 767 & 3,450 & 6,770 & 13,564 \\
Western Europe & 0 & 0 & 841 & 1,855 & 3,744 & 5,920 \\
\hline Total & $\mathbf{0}$ & $\mathbf{0}$ & $\mathbf{2 , 4 4 2}$ & $\mathbf{8 , 6 7 0}$ & $\mathbf{1 7 , 7 4 2}$ & $\mathbf{2 8 , 2 8 2}$ \\
\hline \hline
\end{tabular}


Table A-406: Delayed technology reference case with low oil price and low feedstock availability grain ethanol net trade [Million gallons]

\begin{tabular}{lrrrrrr} 
& $\mathbf{2 0 0 5}$ & $\mathbf{2 0 1 0}$ & $\mathbf{2 0 1 5}$ & $\mathbf{2 0 2 0}$ & $\mathbf{2 0 2 5}$ & $\mathbf{2 0 3 0}$ \\
Africa & 0 & 0 & 0 & 0 & 0 & 0 \\
Australia & 0 & 0 & 0 & 0 & 0 & 0 \\
Canada & 0 & 14 & 0 & 0 & 0 & 0 \\
China & 224 & 366 & 366 & 366 & 0 & 0 \\
Central and South America & 0 & 143 & 565 & 689 & 728 & 728 \\
Eastern Europe & 0 & 0 & 0 & 0 & 0 & 0 \\
Former Soviet Union & 0 & 0 & 0 & 0 & 0 & 0 \\
India & 0 & 0 & 0 & 0 & 0 & 0 \\
Japan & 0 & -132 & -121 & -267 & 0 & 0 \\
Middle East & 0 & 0 & 0 & 0 & 0 & 0 \\
Mexico & 0 & 0 & 0 & 0 & 0 & 0 \\
Other Asia & 0 & 0 & 0 & 0 & 0 & 0 \\
South Korea & -224 & 0 & -245 & -99 & 0 & 0 \\
United States & 0 & 0 & 0 & 0 & 0 & 0 \\
Western Europe & 0 & -390 & -565 & -689 & -728 & -728 \\
\hline Total & $\mathbf{0}$ & $\mathbf{0}$ & $\mathbf{0}$ & $\mathbf{0}$ & $\mathbf{0}$ & $\mathbf{0}$ \\
\hline \hline
\end{tabular}

Table A-407: Delayed technology reference case with low oil price and low feedstock availability sugar ethanol net trade [Million gallons]

\begin{tabular}{|c|c|c|c|c|c|c|}
\hline Africa & 0 & 0 & 0 & 0 & 0 & 0 \\
\hline Australia & 0 & 0 & 0 & 0 & 0 & 0 \\
\hline Canada & -101 & 0 & 0 & 0 & 0 & 0 \\
\hline China & 0 & 0 & 0 & 0 & 0 & 0 \\
\hline Central and South America & 1,264 & 375 & 213 & 424 & 1,342 & 0 \\
\hline Eastern Europe & -589 & 0 & 0 & 0 & 0 & 0 \\
\hline Former Soviet Union & 0 & 0 & 0 & 0 & 0 & 0 \\
\hline India & 0 & 0 & 0 & 0 & 0 & 0 \\
\hline Japan & -263 & 0 & 0 & 0 & 0 & 0 \\
\hline Middle East & 0 & 0 & 0 & 0 & 0 & 0 \\
\hline Mexico & 0 & 0 & 0 & 0 & 0 & 0 \\
\hline Other Asia & 0 & 0 & 0 & 0 & 0 & 0 \\
\hline South Korea & -41 & 0 & 0 & 0 & 0 & 0 \\
\hline United States & -134 & 0 & 0 & -424 & $-1,342$ & 0 \\
\hline Western Europe & -136 & -375 & -213 & 0 & 0 & 0 \\
\hline Total & $\mathbf{0}$ & $\mathbf{0}$ & $\mathbf{0}$ & $\mathbf{0}$ & $\mathbf{0}$ & $\mathbf{0}$ \\
\hline
\end{tabular}


Table A-408: Delayed technology reference case with low oil price and low feedstock availability cellulosic ethanol net trade [Million gallons]

\begin{tabular}{lrrrrrr} 
& $\mathbf{2 0 0 5}$ & $\mathbf{2 0 1 0}$ & $\mathbf{2 0 1 5}$ & $\mathbf{2 0 2 0}$ & $\mathbf{2 0 2 5}$ & $\mathbf{2 0 3 0}$ \\
Africa & 0 & 0 & 0 & 0 & 0 & 0 \\
Australia & 0 & 0 & 0 & 0 & 0 & 0 \\
Canada & 0 & 0 & 40 & 85 & 117 & 117 \\
China & 0 & 0 & 222 & 667 & 1,564 & 1,120 \\
Central and South America & 0 & 0 & 533 & 658 & 589 & 4,043 \\
Eastern Europe & 0 & 0 & 0 & 0 & 0 & 0 \\
Former Soviet Union & 0 & 0 & 0 & 0 & 0 & 0 \\
India & 0 & 0 & 0 & 32 & 151 & 421 \\
Japan & 0 & 0 & 0 & 0 & -385 & -439 \\
Middle East & 0 & 0 & 0 & 0 & 0 & 0 \\
Mexico & 0 & 0 & 0 & 0 & 0 & 0 \\
Other Asia & 0 & 0 & 0 & 0 & 0 & 0 \\
South Korea & 0 & 0 & -121 & -280 & -378 & -387 \\
United States & 0 & 0 & 0 & 0 & -27 & $-3,533$ \\
Western Europe & 0 & 0 & -675 & $-1,163$ & $-1,631$ & $-1,341$ \\
\hline Total & $\mathbf{0}$ & $\mathbf{0}$ & $\mathbf{0}$ & $\mathbf{0}$ & $\mathbf{0}$ & $\mathbf{0}$ \\
\hline \hline
\end{tabular}

Table A-409: Delayed technology reference case with low oil price and low feedstock availability biomass-to-liquids production [Million gallons ethanol equivalent]

\begin{tabular}{lllrrrr} 
Africa & 0 & 0 & 0 & 0 & 0 & 0 \\
Argentina & 0 & 0 & 21 & 99 & 99 & 99 \\
Australia & 0 & 0 & 0 & 0 & 0 & 0 \\
Brazil & 0 & 0 & 97 & 320 & 750 & 1,333 \\
Canada & 0 & 0 & 93 & 280 & 420 & 420 \\
Caribbean basin & 0 & 0 & 0 & 0 & 0 & 0 \\
China & 0 & 0 & 79 & 240 & 563 & 563 \\
Colombia & 0 & 0 & 61 & 133 & 133 & 133 \\
Eastern Europe & 0 & 0 & 0 & 0 & 0 & 0 \\
Former Soviet Union & 0 & 0 & 0 & 0 & 0 & 0 \\
India & 0 & 0 & 77 & 232 & 544 & 1,010 \\
Japan & 0 & 0 & 0 & 0 & 0 & 0 \\
Middle East & 0 & 0 & 0 & 0 & 0 & 0 \\
Mexico & 0 & 0 & 0 & 0 & 0 & 0 \\
Other Asia & 0 & 0 & 0 & 0 & 0 & 0 \\
South Korea & 0 & 0 & 36 & 66 & 66 & 66 \\
United States & 0 & 0 & 786 & 1,253 & 1,849 & 2,406 \\
Western Europe & 0 & 0 & 0 & 0 & 0 & 0 \\
\hline Total & $\mathbf{0}$ & $\mathbf{0}$ & $\mathbf{1 , 2 5 0}$ & $\mathbf{2 , 6 2 3}$ & $\mathbf{4 , 4 2 3}$ & $\mathbf{6 , 0 2 9}$ \\
\hline \hline
\end{tabular}


Table A-410: Delayed technology reference case with low oil price and low feedstock availability biomass-to-liquids consumption [Million gallons ethanol equivalent]

\begin{tabular}{lrrrrrr} 
& $\mathbf{2 0 0 5}$ & $\mathbf{2 0 1 0}$ & $\mathbf{2 0 1 5}$ & $\mathbf{2 0 2 0}$ & $\mathbf{2 0 2 5}$ & $\mathbf{2 0 3 0}$ \\
Africa & 0 & 0 & 0 & 0 & 0 & 0 \\
Australia & 0 & 0 & 0 & 0 & 0 & 0 \\
Canada & 0 & 0 & 0 & 0 & 0 & 0 \\
China & 0 & 0 & 0 & 0 & 0 & 0 \\
Central and South America & 0 & 0 & 0 & 0 & 0 & 0 \\
Eastern Europe & 0 & 0 & 0 & 0 & 0 & 0 \\
Former Soviet Union & 0 & 0 & 0 & 0 & 0 & 0 \\
India & 0 & 0 & 0 & 0 & 0 & 0 \\
Japan & 0 & 0 & 0 & 0 & 0 & 0 \\
Middle East & 0 & 0 & 0 & 0 & 0 & 0 \\
Mexico & 0 & 0 & 0 & 0 & 0 & 0 \\
Other Asia & 0 & 0 & 0 & 0 & 0 & 0 \\
South Korea & 0 & 0 & 0 & 0 & 0 & 0 \\
United States & 0 & 0 & 774 & 1,253 & 3,821 & 6,029 \\
Western Europe & 0 & 0 & 477 & 1,370 & 602 & 0 \\
\hline Total & $\mathbf{0}$ & $\mathbf{0}$ & $\mathbf{1 , 2 5 0}$ & $\mathbf{2 , 6 2 3}$ & $\mathbf{4 , 4 2 3}$ & $\mathbf{6 , 0 2 9}$ \\
\hline \hline
\end{tabular}

Table A-411: Delayed technology reference case with low oil price and low feedstock availability biomass-to-liquids net trade [Million gallons ethanol equivalent]

\begin{tabular}{lrrrrrr} 
& $\mathbf{2 0 0 5}$ & $\mathbf{2 0 1 0}$ & $\mathbf{2 0 1 5}$ & $\mathbf{2 0 2 0}$ & $\mathbf{2 0 2 5}$ & $\mathbf{2 0 3 0}$ \\
Africa & 0 & 0 & 0 & 0 & 0 & 0 \\
Australia & 0 & 0 & 0 & 0 & 0 & 0 \\
Canada & 0 & 0 & 93 & 280 & 420 & 420 \\
China & 0 & 0 & 79 & 240 & 563 & 563 \\
Central and South America & 0 & 0 & 180 & 552 & 982 & 1,565 \\
Eastern Europe & 0 & 0 & 0 & 0 & 0 & 0 \\
Former Soviet Union & 0 & 0 & 0 & 0 & 0 & 0 \\
India & 0 & 0 & 77 & 232 & 544 & 1,010 \\
Japan & 0 & 0 & 0 & 0 & 0 & 0 \\
Middle East & 0 & 0 & 0 & 0 & 0 & 0 \\
Mexico & 0 & 0 & 0 & 0 & 0 & 0 \\
Other Asia & 0 & 0 & 0 & 0 & 0 & 0 \\
South Korea & 0 & 0 & 36 & 66 & 66 & 66 \\
United States & 0 & 0 & 12 & 0 & $-1,972$ & $-3,623$ \\
Western Europe & 0 & 0 & -477 & $-1,370$ & -602 & 0 \\
\hline Total & $\mathbf{0}$ & $\mathbf{0}$ & $\mathbf{0}$ & $\mathbf{0}$ & $\mathbf{0}$ & $\mathbf{0}$ \\
\hline \hline
\end{tabular}


Table A-412: Delayed technology reference case with low oil price and low feedstock availability biodiesel production [Million gallons ethanol equivalent]

\begin{tabular}{lrrrrrr} 
& $\mathbf{2 0 0 5}$ & $\mathbf{2 0 1 0}$ & $\mathbf{2 0 1 5}$ & $\mathbf{2 0 2 0}$ & $\mathbf{2 0 2 5}$ & $\mathbf{2 0 3 0}$ \\
Africa & 0 & 0 & 0 & 0 & 0 & 0 \\
Argentina & 0 & 333 & 877 & 1,491 & 1,472 & 1,458 \\
Australia & 0 & 0 & 0 & 0 & 0 & 0 \\
Brazil & 0 & 333 & 835 & 1,537 & 1,748 & 1,647 \\
Canada & 0 & 0 & 0 & 0 & 0 & 0 \\
Caribbean basin & 0 & 95 & 310 & 742 & 1,145 & 1,220 \\
China & 0 & 0 & 0 & 0 & 0 & 0 \\
Colombia & 0 & 0 & 0 & 0 & 0 & 0 \\
Eastern Europe & 0 & 0 & 0 & 0 & 0 & 0 \\
Former Soviet Union & 0 & 0 & 0 & 0 & 0 & 0 \\
India & 0 & 0 & 0 & 0 & 0 & 0 \\
Japan & 0 & 0 & 0 & 0 & 0 & 0 \\
Middle East & 0 & 0 & 0 & 0 & 0 & 0 \\
Mexico & 0 & 0 & 0 & 0 & 0 & 0 \\
Other Asia & 0 & 0 & 0 & 0 & 0 & 0 \\
South Korea & 0 & 0 & 0 & 0 & 0 & 0 \\
United States & 0 & 1,238 & 1,644 & 1,803 & 1,907 & 2,128 \\
Western Europe & 998 & 2,643 & 2,984 & 3,240 & 3,684 & 4,114 \\
\hline Total & $\mathbf{9 9 8}$ & $\mathbf{4 , 6 4 1}$ & $\mathbf{6 , 6 5 0}$ & $\mathbf{8 , 8 1 3}$ & $\mathbf{9 , 9 5 6}$ & $\mathbf{1 0 , 5 6 7}$ \\
\hline \hline
\end{tabular}

Table A-413: Delayed technology reference case with low oil price and low feedstock availability biodiesel consumption [Million gallons ethanol equivalent]

\begin{tabular}{lrrrrrr} 
& $\mathbf{2 0 0 5}$ & $\mathbf{2 0 1 0}$ & $\mathbf{2 0 1 5}$ & $\mathbf{2 0 2 0}$ & $\mathbf{2 0 2 5}$ & $\mathbf{2 0 3 0}$ \\
Africa & 0 & 0 & 0 & 0 & 0 & 0 \\
Australia & 0 & 0 & 0 & 0 & 0 & 0 \\
Canada & 0 & 0 & 0 & 0 & 0 & 0 \\
China & 0 & 0 & 0 & 0 & 0 & 0 \\
Central and South America & 0 & 126 & 443 & 937 & 1,265 & 1,453 \\
Eastern Europe & 0 & 0 & 0 & 0 & 0 & 0 \\
Former Soviet Union & 0 & 0 & 0 & 0 & 0 & 0 \\
India & 0 & 0 & 0 & 0 & 0 & 0 \\
Japan & 0 & 0 & 0 & 0 & 0 & 0 \\
Middle East & 0 & 0 & 0 & 0 & 0 & 0 \\
Mexico & 0 & 0 & 0 & 0 & 0 & 0 \\
Other Asia & 0 & 0 & 0 & 0 & 0 & 0 \\
South Korea & 0 & 0 & 0 & 0 & 0 & 0 \\
United States & 0 & 1,238 & 2,494 & 4,055 & 3,623 & 5,000 \\
Western Europe & 998 & 3,277 & 3,713 & 3,822 & 5,068 & 4,114 \\
\hline Total & $\mathbf{9 9 8}$ & $\mathbf{4 , 6 4 1}$ & $\mathbf{6 , 6 5 0}$ & $\mathbf{8 , 8 1 3}$ & $\mathbf{9 , 9 5 6}$ & $\mathbf{1 0 , 5 6 7}$ \\
\hline \hline
\end{tabular}


Table A-414: Delayed technology reference case with low oil price and low feedstock availability biodiesel net trade [Million gallons ethanol equivalent]

\begin{tabular}{lrrrrrr} 
& $\mathbf{2 0 0 5}$ & $\mathbf{2 0 1 0}$ & $\mathbf{2 0 1 5}$ & $\mathbf{2 0 2 0}$ & $\mathbf{2 0 2 5}$ & $\mathbf{2 0 3 0}$ \\
Africa & 0 & 0 & 0 & 0 & 0 & 0 \\
Australia & 0 & 0 & 0 & 0 & 0 & 0 \\
Canada & 0 & 0 & 0 & 0 & 0 & 0 \\
China & 0 & 0 & 0 & 0 & 0 & 0 \\
Central and South America & 0 & 634 & 1,579 & 2,833 & 3,101 & 2,872 \\
Eastern Europe & 0 & 0 & 0 & 0 & 0 & 0 \\
Former Soviet Union & 0 & 0 & 0 & 0 & 0 & 0 \\
India & 0 & 0 & 0 & 0 & 0 & 0 \\
Japan & 0 & 0 & 0 & 0 & 0 & 0 \\
Middle East & 0 & 0 & 0 & 0 & 0 & 0 \\
Mexico & 0 & 0 & 0 & 0 & 0 & 0 \\
Other Asia & 0 & 0 & 0 & 0 & 0 & 0 \\
South Korea & 0 & 0 & 0 & 0 & 0 & 0 \\
United States & 0 & 0 & -850 & $-2,251$ & $-1,716$ & $-2,872$ \\
Western Europe & 0 & -634 & -729 & -582 & $-1,385$ & 0 \\
Total & $\mathbf{0}$ & $\mathbf{0}$ & $\mathbf{0}$ & $\mathbf{0}$ & $\mathbf{0}$ & $\mathbf{0}$ \\
\hline \hline
\end{tabular}




\section{A-24 Delayed Technology Reference Case with E20}

\section{Table A-415: Delayed technology reference case with E20 ethanol production} [Million gallons]

\begin{tabular}{lrrrrrr} 
& $\mathbf{2 0 0 5}$ & $\mathbf{2 0 1 0}$ & $\mathbf{2 0 1 5}$ & $\mathbf{2 0 2 0}$ & $\mathbf{2 0 2 5}$ & $\mathbf{2 0 3 0}$ \\
Africa & 300 & 330 & 364 & 402 & 455 & 502 \\
Argentina & 0 & 178 & 699 & 1,607 & 1,665 & 1,309 \\
Australia & 0 & 0 & 66 & 247 & 630 & 1,172 \\
Brazil & 4,866 & 5,977 & 7,624 & 9,926 & 12,492 & 13,634 \\
Canada & 0 & 0 & 259 & 779 & 1,020 & 1,020 \\
Caribbean basin & 210 & 328 & 703 & 779 & 779 & 779 \\
China & 344 & 384 & 614 & 1,041 & 1,319 & 1,319 \\
Colombia & 196 & 215 & 411 & 782 & 1,501 & 1,574 \\
Eastern Europe & 110 & 226 & 434 & 780 & 1,037 & 1,149 \\
Former Soviet Union & 0 & 0 & 6 & 19 & 57 & 175 \\
India & 313 & 279 & 541 & 992 & 1,893 & 2,543 \\
Japan & 0 & 0 & 95 & 348 & 612 & 647 \\
Middle East & 0 & 0 & 0 & 0 & 0 & 0 \\
Mexico & 0 & 0 & 199 & 601 & 1,408 & 1,546 \\
Other Asia & 0 & 0 & 0 & 0 & 0 & 0 \\
South Korea & 0 & 0 & 35 & 35 & 35 & 35 \\
United States & 3,850 & 11,612 & 15,767 & 19,509 & 22,849 & 24,199 \\
Western Europe & 600 & 2,568 & 2,755 & 3,398 & 5,291 & 8,013 \\
\hline Total & $\mathbf{1 0 , 7 8 9}$ & $\mathbf{2 2 , 0 9 7}$ & $\mathbf{3 0 , 5 7 1}$ & $\mathbf{4 1 , 2 4 3}$ & $\mathbf{5 3 , 0 4 3}$ & $\mathbf{5 9 , 6 1 4}$ \\
\hline \hline
\end{tabular}


Table A-416: Delayed technology reference case with E20 ethanol consumption [Million gallons]

\begin{tabular}{lrrrrrr} 
& $\mathbf{2 0 0 5}$ & $\mathbf{2 0 1 0}$ & $\mathbf{2 0 1 5}$ & $\mathbf{2 0 2 0}$ & $\mathbf{2 0 2 5}$ & $\mathbf{2 0 3 0}$ \\
Africa & 300 & 330 & 364 & 402 & 455 & 502 \\
Australia & 0 & 0 & 66 & 247 & 630 & 1,172 \\
Canada & 145 & 0 & 233 & 701 & 918 & 1,020 \\
China & 297 & 0 & 0 & 0 & 545 & 1,463 \\
Central and South America & 3,788 & 4,853 & 6,148 & 7,248 & 8,030 & 8,797 \\
Eastern Europe & 699 & 226 & 872 & 780 & 1,037 & 1,149 \\
Former Soviet Union & 0 & 0 & 6 & 19 & 57 & 175 \\
India & 313 & 279 & 541 & 942 & 1,704 & 2,399 \\
Japan & 263 & 288 & 373 & 698 & 1,008 & 1,100 \\
Middle East & 0 & 0 & 0 & 0 & 0 & 0 \\
Mexico & 0 & 0 & 30 & 78 & 183 & 201 \\
Other Asia & 0 & 0 & 0 & 0 & 0 & 0 \\
South Korea & 264 & 335 & 400 & 414 & 413 & 413 \\
United States & 3,984 & 11,612 & 17,249 & 24,464 & 30,414 & 32,293 \\
Western Europe & 736 & 4,174 & 4,290 & 5,249 & 7,649 & 8,934 \\
\hline Total & $\mathbf{1 0 , 7 8 9}$ & $\mathbf{2 2 , 0 9 7}$ & $\mathbf{3 0 , 5 7 1}$ & $\mathbf{4 1 , 2 4 3}$ & $\mathbf{5 3 , 0 4 3}$ & $\mathbf{5 9 , 6 1 4}$ \\
\hline \hline
\end{tabular}

Table A-417: Delayed technology reference case with E20 ethanol net trade [Million gallons]

\begin{tabular}{lrrrrrr} 
Africa & 0 & 0 & 0 & 0 & 0 & 0 \\
Australia & 0 & 0 & 0 & 0 & 0 & 0 \\
Canada & -145 & 0 & 26 & 78 & 102 & 0 \\
China & 47 & 384 & 614 & 1,041 & 774 & -144 \\
Central and South America & 1,484 & 1,845 & 3,289 & 5,845 & 8,407 & 8,500 \\
Eastern Europe & -589 & 0 & -438 & 0 & 0 & 0 \\
Former Soviet Union & 0 & 0 & 0 & 0 & 0 & 0 \\
India & 0 & 0 & 0 & 50 & 189 & 144 \\
Japan & -263 & -288 & -278 & -350 & -395 & -453 \\
Middle East & 0 & 0 & 0 & 0 & 0 & 0 \\
Mexico & 0 & 0 & 170 & 523 & 1,225 & 1,345 \\
Other Asia & 0 & 0 & 0 & 0 & 0 & 0 \\
South Korea & -264 & -335 & -365 & -379 & -378 & -378 \\
United States & -134 & 0 & $-1,482$ & $-4,955$ & $-7,565$ & $-8,094$ \\
Western Europe & -136 & $-1,607$ & $-1,535$ & $-1,851$ & $-2,358$ & -920 \\
\hline Total & $\mathbf{0}$ & $\mathbf{0}$ & $\mathbf{0}$ & $\mathbf{0}$ & $\mathbf{0}$ & $\mathbf{0}$ \\
\hline \hline
\end{tabular}


Table A-418: Delayed technology reference case with E20 grain ethanol production [Million gallons]

\begin{tabular}{lrrrrrr} 
& $\mathbf{2 0 0 5}$ & $\mathbf{2 0 1 0}$ & $\mathbf{2 0 1 5}$ & $\mathbf{2 0 2 0}$ & $\mathbf{2 0 2 5}$ & $\mathbf{2 0 3 0}$ \\
Africa & 0 & 0 & 0 & 0 & 0 & 0 \\
Argentina & 0 & 178 & 640 & 1,260 & 1,317 & 962 \\
Australia & 0 & 0 & 0 & 0 & 0 & 0 \\
Brazil & 0 & 0 & 0 & 0 & 0 & 0 \\
Canada & 0 & 0 & 0 & 0 & 0 & 0 \\
Caribbean basin & 0 & 0 & 0 & 0 & 0 & 0 \\
China & 344 & 344 & 344 & 325 & 0 & 0 \\
Colombia & 0 & 0 & 0 & 0 & 0 & 0 \\
Eastern Europe & 110 & 226 & 348 & 477 & 367 & 251 \\
Former Soviet Union & 0 & 0 & 0 & 0 & 0 & 0 \\
India & 0 & 0 & 0 & 0 & 0 & 0 \\
Japan & 0 & 0 & 0 & 0 & 0 & 0 \\
Middle East & 0 & 0 & 0 & 0 & 0 & 0 \\
Mexico & 0 & 0 & 0 & 0 & 0 & 0 \\
Other Asia & 0 & 0 & 0 & 0 & 0 & 0 \\
South Korea & 0 & 0 & 0 & 0 & 0 & 0 \\
United States & 3,850 & 11,612 & 15,000 & 15,000 & 15,000 & 14,640 \\
Western Europe & 600 & 2,568 & 2,589 & 2,705 & 3,178 & 3,604 \\
\hline Total & $\mathbf{4 , 9 0 4}$ & $\mathbf{1 4 , 9 2 8}$ & $\mathbf{1 8 , 9 2 1}$ & $\mathbf{1 9 , 7 6 7}$ & $\mathbf{1 9 , 8 6 2}$ & $\mathbf{1 9 , 4 5 7}$ \\
\hline \hline
\end{tabular}

Table A-419: Delayed technology reference case with E20 sugar ethanol production [Million gallons]

\begin{tabular}{lrrrrrr} 
& $\mathbf{2 0 0 5}$ & $\mathbf{2 0 1 0}$ & $\mathbf{2 0 1 5}$ & $\mathbf{2 0 2 0}$ & $\mathbf{2 0 2 5}$ & $\mathbf{2 0 3 0}$ \\
Africa & 300 & 330 & 364 & 402 & 444 & 490 \\
Argentina & 0 & 0 & 0 & 0 & 0 & 0 \\
Australia & 0 & 0 & 0 & 0 & 0 & 0 \\
Brazil & 4,866 & 5,977 & 7,329 & 9,036 & 10,651 & 11,793 \\
Canada & 0 & 0 & 0 & 0 & 0 & 0 \\
Caribbean basin & 210 & 328 & 703 & 779 & 779 & 779 \\
China & 0 & 40 & 48 & 48 & 48 & 48 \\
Colombia & 196 & 215 & 241 & 270 & 302 & 302 \\
Eastern Europe & 0 & 0 & 0 & 0 & 0 & 0 \\
Former Soviet Union & 0 & 0 & 0 & 0 & 0 & 0 \\
India & 313 & 279 & 327 & 347 & 381 & 348 \\
Japan & 0 & 0 & 0 & 0 & 0 & 0 \\
Middle East & 0 & 0 & 0 & 0 & 0 & 0 \\
Mexico & 0 & 0 & 0 & 0 & 0 & 0 \\
Other Asia & 0 & 0 & 0 & 0 & 0 & 0 \\
South Korea & 0 & 0 & 0 & 0 & 0 & 0 \\
United States & 0 & 0 & 0 & 0 & 0 & 0 \\
Western Europe & 0 & 0 & 0 & 0 & 0 & 0 \\
\hline Total & $\mathbf{5 , 8 8 4}$ & $\mathbf{7 , 1 6 9}$ & $\mathbf{9 , 0 1 1}$ & $\mathbf{1 0 , 8 8 2}$ & $\mathbf{1 2 , 6 0 6}$ & $\mathbf{1 3 , 7 6 0}$ \\
\hline \hline
\end{tabular}


Table A-420: Delayed technology reference case with E20 cellulosic ethanol production [Million gallons]

\begin{tabular}{lrrrrrr} 
& $\mathbf{2 0 0 5}$ & $\mathbf{2 0 1 0}$ & $\mathbf{2 0 1 5}$ & $\mathbf{2 0 2 0}$ & $\mathbf{2 0 2 5}$ & $\mathbf{2 0 3 0}$ \\
Africa & 0 & 0 & 0 & 0 & 11 & 11 \\
Argentina & 0 & 0 & 59 & 347 & 347 & 347 \\
Australia & 0 & 0 & 66 & 247 & 630 & 1,172 \\
Brazil & 0 & 0 & 296 & 890 & 1,841 & 1,841 \\
Canada & 0 & 0 & 259 & 779 & 1,020 & 1,020 \\
Caribbean basin & 0 & 0 & 0 & 0 & 0 & 0 \\
China & 0 & 0 & 222 & 668 & 1,271 & 1,271 \\
Colombia & 0 & 0 & 170 & 512 & 1,199 & 1,272 \\
Eastern Europe & 0 & 0 & 85 & 303 & 670 & 897 \\
Former Soviet Union & 0 & 0 & 6 & 19 & 57 & 175 \\
India & 0 & 0 & 214 & 645 & 1,512 & 2,196 \\
Japan & 0 & 0 & 95 & 348 & 612 & 647 \\
Middle East & 0 & 0 & 0 & 0 & 0 & 0 \\
Mexico & 0 & 0 & 199 & 601 & 1,408 & 1,546 \\
Other Asia & 0 & 0 & 0 & 0 & 0 & 0 \\
South Korea & 0 & 0 & 35 & 35 & 35 & 35 \\
United States & 0 & 0 & 767 & 4,509 & 7,849 & 9,560 \\
Western Europe & 0 & 0 & 166 & 693 & 2,113 & 4,410 \\
\hline Total & $\mathbf{0}$ & $\mathbf{0}$ & $\mathbf{2 , 6 3 9}$ & $\mathbf{1 0 , 5 9 4}$ & $\mathbf{2 0 , 5 7 4}$ & $\mathbf{2 6 , 3 9 7}$ \\
\hline \hline
\end{tabular}

Table A-421: Delayed technology reference case with E20 grain ethanol consumption [Million gallons]

\begin{tabular}{lrrrrrr} 
& $\mathbf{2 0 0 5}$ & $\mathbf{2 0 1 0}$ & $\mathbf{2 0 1 5}$ & $\mathbf{2 0 2 0}$ & $\mathbf{2 0 2 5}$ & $\mathbf{2 0 3 0}$ \\
Africa & 0 & 0 & 0 & 0 & 0 & 0 \\
Australia & 0 & 0 & 0 & 0 & 0 & 0 \\
Canada & 0 & 0 & 0 & 0 & 0 & 0 \\
China & 297 & 0 & 0 & 0 & 0 & 0 \\
Central and South America & 0 & 36 & 105 & 209 & 167 & 131 \\
Eastern Europe & 110 & 226 & 348 & 477 & 367 & 251 \\
Former Soviet Union & 0 & 0 & 0 & 0 & 0 & 0 \\
India & 0 & 0 & 0 & 0 & 0 & 0 \\
Japan & 0 & 192 & 230 & 303 & 0 & 453 \\
Middle East & 0 & 0 & 0 & 0 & 0 & 0 \\
Mexico & 0 & 0 & 0 & 0 & 0 & 0 \\
Other Asia & 0 & 0 & 0 & 0 & 0 & 0 \\
South Korea & 47 & 295 & 144 & 23 & 0 & 378 \\
United States & 3,850 & 11,612 & 15,000 & 15,000 & 15,000 & 14,640 \\
Western Europe & 600 & 2,568 & 3,094 & 3,756 & 4,329 & 3,604 \\
\hline Total & $\mathbf{4 , 9 0 4}$ & $\mathbf{1 4 , 9 2 8}$ & $\mathbf{1 8 , 9 2 1}$ & $\mathbf{1 9 , 7 6 7}$ & $\mathbf{1 9 , 8 6 2}$ & $\mathbf{1 9 , 4 5 7}$ \\
\hline \hline
\end{tabular}


Table A-422: Delayed technology reference case with E20 sugar ethanol consumption [Million gallons]

\begin{tabular}{lrrrrrr} 
& $\mathbf{2 0 0 5}$ & $\mathbf{2 0 1 0}$ & $\mathbf{2 0 1 5}$ & $\mathbf{2 0 2 0}$ & $\mathbf{2 0 2 5}$ & $\mathbf{2 0 3 0}$ \\
Africa & 300 & 330 & 364 & 402 & 444 & 490 \\
Australia & 0 & 0 & 0 & 0 & 0 & 0 \\
Canada & 145 & 0 & 0 & 0 & 0 & 0 \\
China & 0 & 0 & 0 & 0 & 48 & 48 \\
Central and South America & 3,788 & 4,818 & 6,043 & 7,040 & 7,864 & 8,666 \\
Eastern Europe & 589 & 0 & 412 & 0 & 0 & 0 \\
Former Soviet Union & 0 & 0 & 0 & 0 & 0 & 0 \\
India & 313 & 279 & 327 & 347 & 381 & 348 \\
Japan & 263 & 96 & 48 & 48 & 0 & 0 \\
Middle East & 0 & 0 & 0 & 0 & 0 & 0 \\
Mexico & 0 & 0 & 0 & 0 & 0 & 0 \\
Other Asia & 0 & 0 & 0 & 0 & 0 & 0 \\
South Korea & 217 & 40 & 0 & 0 & 0 & 0 \\
United States & 134 & 0 & 788 & 2,753 & 3,094 & 3,289 \\
Western Europe & 136 & 1,607 & 1,029 & 293 & 776 & 920 \\
\hline Total & $\mathbf{5 , 8 8 4}$ & $\mathbf{7 , 1 6 9}$ & $\mathbf{9 , 0 1 1}$ & $\mathbf{1 0 , 8 8 2}$ & $\mathbf{1 2 , 6 0 6}$ & $\mathbf{1 3 , 7 6 0}$ \\
\hline \hline
\end{tabular}

Table A-423: Delayed technology reference case with E20 cellulosic ethanol consumption [Million gallons]

\begin{tabular}{lrrrrrr} 
& $\mathbf{2 0 0 5}$ & $\mathbf{2 0 1 0}$ & $\mathbf{2 0 1 5}$ & $\mathbf{2 0 2 0}$ & $\mathbf{2 0 2 5}$ & $\mathbf{2 0 3 0}$ \\
Africa & 0 & 0 & 0 & 0 & 11 & 11 \\
Australia & 0 & 0 & 66 & 247 & 630 & 1,172 \\
Canada & 0 & 0 & 233 & 701 & 918 & 1,020 \\
China & 0 & 0 & 0 & 0 & 497 & 1,415 \\
Central and South America & 0 & 0 & 0 & 0 & 0 & 0 \\
Eastern Europe & 0 & 0 & 111 & 303 & 670 & 897 \\
Former Soviet Union & 0 & 0 & 6 & 19 & 57 & 175 \\
India & 0 & 0 & 214 & 596 & 1,323 & 2,051 \\
Japan & 0 & 0 & 95 & 348 & 1,008 & 647 \\
Middle East & 0 & 0 & 0 & 0 & 0 & 0 \\
Mexico & 0 & 0 & 30 & 78 & 183 & 201 \\
Other Asia & 0 & 0 & 0 & 0 & 0 & 0 \\
South Korea & 0 & 0 & 256 & 392 & 413 & 35 \\
United States & 0 & 0 & 1,461 & 6,711 & 12,320 & 14,365 \\
Western Europe & 0 & 0 & 166 & 1,200 & 2,545 & 4,410 \\
\hline Total & $\mathbf{0}$ & $\mathbf{0}$ & $\mathbf{2 , 6 3 9}$ & $\mathbf{1 0 , 5 9 4}$ & $\mathbf{2 0 , 5 7 4}$ & $\mathbf{2 6 , 3 9 7}$ \\
\hline \hline
\end{tabular}


Table A-424: Delayed technology reference case with E20 grain ethanol net trade [Million gallons]

\begin{tabular}{lrrrrrr} 
& $\mathbf{2 0 0 5}$ & $\mathbf{2 0 1 0}$ & $\mathbf{2 0 1 5}$ & $\mathbf{2 0 2 0}$ & $\mathbf{2 0 2 5}$ & $\mathbf{2 0 3 0}$ \\
Africa & 0 & 0 & 0 & 0 & 0 & 0 \\
Australia & 0 & 0 & 0 & 0 & 0 & 0 \\
Canada & 0 & 0 & 0 & 0 & 0 & 0 \\
China & 47 & 344 & 344 & 325 & 0 & 0 \\
Central and South America & 0 & 143 & 535 & 1,051 & 1,151 & 831 \\
Eastern Europe & 0 & 0 & 0 & 0 & 0 & 0 \\
Former Soviet Union & 0 & 0 & 0 & 0 & 0 & 0 \\
India & 0 & 0 & 0 & 0 & 0 & 0 \\
Japan & 0 & -192 & -230 & -303 & 0 & -453 \\
Middle East & 0 & 0 & 0 & 0 & 0 & 0 \\
Mexico & 0 & 0 & 0 & 0 & 0 & 0 \\
Other Asia & 0 & 0 & 0 & 0 & 0 & 0 \\
South Korea & -47 & -295 & -144 & -23 & 0 & -378 \\
United States & 0 & 0 & 0 & 0 & 0 & 0 \\
Western Europe & 0 & 0 & -506 & $-1,051$ & $-1,151$ & 0 \\
\hline Total & $\mathbf{0}$ & $\mathbf{0}$ & $\mathbf{0}$ & $\mathbf{0}$ & $\mathbf{0}$ & $\mathbf{0}$ \\
\hline \hline
\end{tabular}

Table A-425: Delayed technology reference case with E20 sugar ethanol net trade [Million gallons]

\begin{tabular}{lrrrrrr} 
Africa & 0 & 0 & 0 & 0 & 0 & 0 \\
Australia & 0 & 0 & 0 & 0 & 0 & 0 \\
Canada & -145 & 0 & 0 & 0 & 0 & 0 \\
China & 0 & 40 & 48 & 48 & 0 & 0 \\
Central and South America & 1,484 & 1,702 & 2,229 & 3,045 & 3,869 & 4,209 \\
Eastern Europe & -589 & 0 & -412 & 0 & 0 & 0 \\
Former Soviet Union & 0 & 0 & 0 & 0 & 0 & 0 \\
India & 0 & 0 & 0 & 0 & 0 & 0 \\
Japan & -263 & -96 & -48 & -48 & 0 & 0 \\
Middle East & 0 & 0 & 0 & 0 & 0 & 0 \\
Mexico & 0 & 0 & 0 & 0 & 0 & 0 \\
Other Asia & 0 & 0 & 0 & 0 & 0 & 0 \\
South Korea & -217 & -40 & 0 & 0 & 0 & 0 \\
United States & -134 & 0 & -788 & $-2,753$ & $-3,094$ & $-3,289$ \\
Western Europe & -136 & $-1,607$ & $-1,029$ & -293 & -776 & -920 \\
\hline Total & $\mathbf{0}$ & $\mathbf{0}$ & $\mathbf{0}$ & $\mathbf{0}$ & $\mathbf{0}$ & $\mathbf{0}$ \\
\hline \hline
\end{tabular}


Table A-426: Delayed technology reference case with E20 cellulosic ethanol net trade [Million gallons]

\begin{tabular}{lrrrrrr} 
& $\mathbf{2 0 0 5}$ & $\mathbf{2 0 1 0}$ & $\mathbf{2 0 1 5}$ & $\mathbf{2 0 2 0}$ & $\mathbf{2 0 2 5}$ & $\mathbf{2 0 3 0}$ \\
Africa & 0 & 0 & 0 & 0 & 0 & 0 \\
Australia & 0 & 0 & 0 & 0 & 0 & 0 \\
Canada & 0 & 0 & 26 & 78 & 102 & 0 \\
China & 0 & 0 & 222 & 668 & 774 & -144 \\
Central and South America & 0 & 0 & 525 & 1,749 & 3,387 & 3,460 \\
Eastern Europe & 0 & 0 & -26 & 0 & 0 & 0 \\
Former Soviet Union & 0 & 0 & 0 & 0 & 0 & 0 \\
India & 0 & 0 & 0 & 50 & 189 & 144 \\
Japan & 0 & 0 & 0 & 0 & -395 & 0 \\
Middle East & 0 & 0 & 0 & 0 & 0 & 0 \\
Mexico & 0 & 0 & 170 & 523 & 1,225 & 1,345 \\
Other Asia & 0 & 0 & 0 & 0 & 0 & 0 \\
South Korea & 0 & 0 & -222 & -357 & -378 & 0 \\
United States & 0 & 0 & -694 & $-2,202$ & $-4,471$ & $-4,805$ \\
Western Europe & 0 & 0 & 0 & -508 & -432 & 0 \\
\hline Total & $\mathbf{0}$ & $\mathbf{0}$ & $\mathbf{0}$ & $\mathbf{0}$ & $\mathbf{0}$ & $\mathbf{0}$ \\
\hline \hline
\end{tabular}

Table A-427: Delayed technology reference case with E20 biomass-to-liquids production [Million gallons ethanol equivalent]

\begin{tabular}{lrrrrrr} 
& $\mathbf{2 0 0 5}$ & $\mathbf{2 0 1 0}$ & $\mathbf{2 0 1 5}$ & $\mathbf{2 0 2 0}$ & $\mathbf{2 0 2 5}$ & $\mathbf{2 0 3 0}$ \\
Africa & 0 & 0 & 0 & 0 & 0 & 0 \\
Argentina & 0 & 0 & 21 & 125 & 125 & 125 \\
Australia & 0 & 0 & 0 & 0 & 0 & 0 \\
Brazil & 0 & 0 & 106 & 320 & 662 & 662 \\
Canada & 0 & 0 & 93 & 280 & 367 & 367 \\
Caribbean basin & 0 & 0 & 0 & 0 & 0 & 0 \\
China & 0 & 0 & 80 & 240 & 457 & 457 \\
Colombia & 0 & 0 & 61 & 184 & 431 & 458 \\
Eastern Europe & 0 & 0 & 0 & 0 & 0 & 0 \\
Former Soviet Union & 0 & 0 & 0 & 0 & 0 & 0 \\
India & 0 & 0 & 77 & 232 & 544 & 790 \\
Japan & 0 & 0 & 0 & 0 & 0 & 0 \\
Middle East & 0 & 0 & 0 & 0 & 0 & 0 \\
Mexico & 0 & 0 & 72 & 216 & 506 & 556 \\
Other Asia & 0 & 0 & 0 & 0 & 0 & 0 \\
South Korea & 0 & 0 & 36 & 50 & 50 & 50 \\
United States & 0 & 0 & 786 & 1,253 & 1,849 & 2,406 \\
Western Europe & 0 & 0 & 0 & 0 & 0 & 0 \\
\hline Total & $\mathbf{0}$ & $\mathbf{0}$ & $\mathbf{1 , 3 3 2}$ & $\mathbf{2 , 9 0 0}$ & $\mathbf{4 , 9 9 2}$ & $\mathbf{5 , 8 7 1}$ \\
\hline \hline
\end{tabular}


Table A-428: Delayed technology reference case with E20 biomass-to-liquids consumption [Million gallons ethanol equivalent]

\begin{tabular}{lrrrrrr} 
& $\mathbf{2 0 0 5}$ & $\mathbf{2 0 1 0}$ & $\mathbf{2 0 1 5}$ & $\mathbf{2 0 2 0}$ & $\mathbf{2 0 2 5}$ & $\mathbf{2 0 3 0}$ \\
Africa & 0 & 0 & 0 & 0 & 0 & 0 \\
Australia & 0 & 0 & 0 & 0 & 0 & 0 \\
Canada & 0 & 0 & 0 & 0 & 0 & 0 \\
China & 0 & 0 & 0 & 0 & 0 & 0 \\
Central and South America & 0 & 0 & 0 & 0 & 0 & 0 \\
Eastern Europe & 0 & 0 & 0 & 0 & 0 & 0 \\
Former Soviet Union & 0 & 0 & 0 & 0 & 0 & 0 \\
India & 0 & 0 & 0 & 0 & 0 & 0 \\
Japan & 0 & 0 & 0 & 0 & 0 & 0 \\
Middle East & 0 & 0 & 0 & 0 & 0 & 0 \\
Mexico & 0 & 0 & 0 & 0 & 0 & 0 \\
Other Asia & 0 & 0 & 0 & 0 & 0 & 0 \\
South Korea & 0 & 0 & 0 & 0 & 0 & 0 \\
United States & 0 & 0 & 1,059 & 2,812 & 4,992 & 5,871 \\
Western Europe & 0 & 0 & 273 & 88 & 0 & 0 \\
\hline Total & $\mathbf{0}$ & $\mathbf{0}$ & $\mathbf{1 , 3 3 2}$ & $\mathbf{2 , 9 0 1}$ & $\mathbf{4 , 9 9 2}$ & $\mathbf{5 , 8 7 1}$ \\
\hline \hline
\end{tabular}

Table A-429: Delayed technology reference case with E20 biomass-to-liquids net trade [Million gallons ethanol equivalent]

\begin{tabular}{lrrrrrr} 
& $\mathbf{2 0 0 5}$ & $\mathbf{2 0 1 0}$ & $\mathbf{2 0 1 5}$ & $\mathbf{2 0 2 0}$ & $\mathbf{2 0 2 5}$ & $\mathbf{2 0 3 0}$ \\
Africa & 0 & 0 & 0 & 0 & 0 & 0 \\
Australia & 0 & 0 & 0 & 0 & 0 & 0 \\
Canada & 0 & 0 & 93 & 280 & 367 & 367 \\
China & 0 & 0 & 80 & 240 & 457 & 457 \\
Central and South America & 0 & 0 & 189 & 629 & 1,218 & 1,245 \\
Eastern Europe & 0 & 0 & 0 & 0 & 0 & 0 \\
Former Soviet Union & 0 & 0 & 0 & 0 & 0 & 0 \\
India & 0 & 0 & 77 & 232 & 544 & 790 \\
Japan & 0 & 0 & 0 & 0 & 0 & 0 \\
Middle East & 0 & 0 & 0 & 0 & 0 & 0 \\
Mexico & 0 & 0 & 72 & 216 & 506 & 556 \\
Other Asia & 0 & 0 & 0 & 0 & 0 & 0 \\
South Korea & 0 & 0 & 36 & 50 & 50 & 50 \\
United States & 0 & 0 & -273 & $-1,559$ & $-3,143$ & $-3,465$ \\
Western Europe & 0 & 0 & -273 & -88 & 0 & 0 \\
\hline Total & $\mathbf{0}$ & $\mathbf{0}$ & $\mathbf{0}$ & $\mathbf{0}$ & $\mathbf{0}$ & $\mathbf{0}$ \\
\hline \hline
\end{tabular}


Table A-430: Delayed technology reference case with E20 biodiesel production [Million gallons ethanol equivalent]

\begin{tabular}{lrrrrrr} 
& $\mathbf{2 0 0 5}$ & $\mathbf{2 0 1 0}$ & $\mathbf{2 0 1 5}$ & $\mathbf{2 0 2 0}$ & $\mathbf{2 0 2 5}$ & $\mathbf{2 0 3 0}$ \\
Africa & 0 & 0 & 0 & 0 & 0 & 0 \\
Argentina & 0 & 333 & 776 & 819 & 827 & 239 \\
Australia & 0 & 0 & 0 & 0 & 0 & 0 \\
Brazil & 0 & 333 & 877 & 1,642 & 1,793 & 1,355 \\
Canada & 0 & 0 & 0 & 0 & 0 & 0 \\
Caribbean basin & 0 & 95 & 310 & 675 & 802 & 898 \\
China & 0 & 0 & 0 & 0 & 0 & 0 \\
Colombia & 0 & 119 & 298 & 474 & 650 & 782 \\
Eastern Europe & 0 & 0 & 0 & 0 & 0 & 0 \\
Former Soviet Union & 0 & 0 & 0 & 0 & 0 & 0 \\
India & 0 & 0 & 0 & 0 & 0 & 0 \\
Japan & 0 & 0 & 0 & 0 & 0 & 0 \\
Middle East & 0 & 0 & 0 & 0 & 0 & 0 \\
Mexico & 0 & 0 & 0 & 0 & 0 & 0 \\
Other Asia & 0 & 0 & 0 & 0 & 0 & 0 \\
South Korea & 0 & 0 & 0 & 0 & 0 & 0 \\
United States & 0 & 1,238 & 1,640 & 1,747 & 1,907 & 2,072 \\
Western Europe & 998 & 3,178 & 3,578 & 3,930 & 4,468 & 4,788 \\
\hline Total & $\mathbf{9 9 8}$ & $\mathbf{5 , 2 9 4}$ & $\mathbf{7 , 4 7 9}$ & $\mathbf{9 , 2 8 7}$ & $\mathbf{1 0 , 4 4 8}$ & $\mathbf{1 0 , 1 3 4}$ \\
\hline \hline
\end{tabular}

Table A-431: Delayed technology reference case with E20 biodiesel consumption [Million gallons ethanol equivalent]

\begin{tabular}{lrrrrrr} 
& $\mathbf{2 0 0 5}$ & $\mathbf{2 0 1 0}$ & $\mathbf{2 0 1 5}$ & $\mathbf{2 0 2 0}$ & $\mathbf{2 0 2 5}$ & $\mathbf{2 0 3 0}$ \\
Africa & 0 & 0 & 0 & 0 & 0 & 0 \\
Australia & 0 & 0 & 0 & 0 & 0 & 0 \\
Canada & 0 & 0 & 0 & 0 & 0 & 0 \\
China & 0 & 0 & 0 & 0 & 0 & 0 \\
Central and South America & 0 & 158 & 472 & 984 & 1,306 & 1,490 \\
Eastern Europe & 0 & 0 & 0 & 0 & 0 & 0 \\
Former Soviet Union & 0 & 0 & 0 & 0 & 0 & 0 \\
India & 0 & 0 & 0 & 0 & 0 & 0 \\
Japan & 0 & 0 & 0 & 0 & 0 & 0 \\
Middle East & 0 & 0 & 0 & 0 & 0 & 0 \\
Mexico & 0 & 0 & 0 & 0 & 0 & 0 \\
Other Asia & 0 & 0 & 0 & 0 & 0 & 0 \\
South Korea & 0 & 0 & 0 & 0 & 0 & 0 \\
United States & 0 & 1,238 & 1,713 & 1,747 & 1,907 & 2,072 \\
Western Europe & 998 & 3,898 & 5,294 & 6,556 & 7,235 & 6,572 \\
\hline Total & $\mathbf{9 9 8}$ & $\mathbf{5 , 2 9 4}$ & $\mathbf{7 , 4 7 9}$ & $\mathbf{9 , 2 8 7}$ & $\mathbf{1 0 , 4 4 8}$ & $\mathbf{1 0 , 1 3 4}$ \\
\hline \hline
\end{tabular}


Table A-432: Delayed technology reference case with E20 biodiesel net trade [Million gallons ethanol equivalent]

\begin{tabular}{lrrrrrr} 
& $\mathbf{2 0 0 5}$ & $\mathbf{2 0 1 0}$ & $\mathbf{2 0 1 5}$ & $\mathbf{2 0 2 0}$ & $\mathbf{2 0 2 5}$ & $\mathbf{2 0 3 0}$ \\
Africa & 0 & 0 & 0 & 0 & 0 & 0 \\
Australia & 0 & 0 & 0 & 0 & 0 & 0 \\
Canada & 0 & 0 & 0 & 0 & 0 & 0 \\
China & 0 & 0 & 0 & 0 & 0 & 0 \\
Central and South America & 0 & 720 & 1,788 & 2,626 & 2,767 & 1,784 \\
Eastern Europe & 0 & 0 & 0 & 0 & 0 & 0 \\
Former Soviet Union & 0 & 0 & 0 & 0 & 0 & 0 \\
India & 0 & 0 & 0 & 0 & 0 & 0 \\
Japan & 0 & 0 & 0 & 0 & 0 & 0 \\
Middle East & 0 & 0 & 0 & 0 & 0 & 0 \\
Mexico & 0 & 0 & 0 & 0 & 0 & 0 \\
Other Asia & 0 & 0 & 0 & 0 & 0 & 0 \\
South Korea & 0 & 0 & 0 & 0 & 0 & 0 \\
United States & 0 & 0 & -73 & 0 & 0 & 0 \\
Western Europe & 0 & -720 & $-1,716$ & $-2,626$ & $-2,767$ & $-1,784$ \\
\hline Total & $\mathbf{0}$ & $\mathbf{0}$ & $\mathbf{0}$ & $\mathbf{0}$ & $\mathbf{0}$ & $\mathbf{0}$ \\
\hline \hline
\end{tabular}




\section{A-25 Delayed Technology Reference Case with $\$ 50$ per Tonne $\mathrm{CO}_{2}$}

\section{Table A-433: Delayed technology reference case with $\$ 50$ per tonne $\mathrm{CO}_{2}$ ethanol production [Million gallons]}

\begin{tabular}{lrrrrrr} 
& $\mathbf{2 0 0 5}$ & $\mathbf{2 0 1 0}$ & $\mathbf{2 0 1 5}$ & $\mathbf{2 0 2 0}$ & $\mathbf{2 0 2 5}$ & $\mathbf{2 0 3 0}$ \\
Africa & 300 & 330 & 73 & 83 & 91 & 98 \\
Argentina & 0 & 178 & 664 & 910 & 940 & 952 \\
Australia & 0 & 0 & 66 & 262 & 704 & 1,174 \\
Brazil & 4,866 & 5,977 & 7,624 & 9,752 & 12,283 & 14,455 \\
Canada & 0 & 0 & 259 & 779 & 1,014 & 1,014 \\
Caribbean basin & 210 & 328 & 683 & 683 & 779 & 890 \\
China & 344 & 384 & 614 & 1,045 & 1,622 & 2,607 \\
Colombia & 196 & 215 & 411 & 782 & 1,501 & 2,231 \\
Eastern Europe & 110 & 226 & 428 & 738 & 1,037 & 1,149 \\
Former Soviet Union & 0 & 0 & 0 & 0 & 0 & 0 \\
India & 313 & 279 & 541 & 992 & 1,893 & 2,822 \\
Japan & 0 & 0 & 95 & 348 & 579 & 579 \\
Middle East & 0 & 0 & 0 & 0 & 0 & 0 \\
Mexico & 0 & 0 & 199 & 601 & 1,408 & 1,546 \\
Other Asia & 0 & 0 & 0 & 0 & 0 & 0 \\
South Korea & 0 & 0 & 30 & 30 & 30 & 32 \\
United States & 3,850 & 11,612 & 15,767 & 16,455 & 18,676 & 19,862 \\
Western Europe & 600 & 2,570 & 2,755 & 3,235 & 5,291 & 8,183 \\
\hline Total & $\mathbf{1 0 , 7 8 9}$ & $\mathbf{2 2 , 0 9 9}$ & $\mathbf{3 0 , 2 0 9}$ & $\mathbf{3 6 , 6 9 4}$ & $\mathbf{4 7 , 8 4 7}$ & $\mathbf{5 7 , 5 9 2}$ \\
\hline \hline
\end{tabular}


Table A-434: Delayed technology reference case with $\$ 50$ per tonne $\mathrm{CO}_{2}$ ethanol consumption [Million gallons]

\begin{tabular}{lrrrrrr} 
& $\mathbf{2 0 0 5}$ & $\mathbf{2 0 1 0}$ & $\mathbf{2 0 1 5}$ & $\mathbf{2 0 2 0}$ & $\mathbf{2 0 2 5}$ & $\mathbf{2 0 3 0}$ \\
Africa & 300 & 330 & 73 & 83 & 91 & 98 \\
Australia & 0 & 0 & 66 & 262 & 704 & 1,174 \\
Canada & 145 & 0 & 233 & 779 & 912 & 924 \\
China & 294 & 0 & 0 & 399 & 1,622 & 3,030 \\
Central and South America & 3,788 & 4,853 & 6,141 & 7,153 & 7,958 & 8,865 \\
Eastern Europe & 699 & 226 & 872 & 953 & 1,037 & 1,149 \\
Former Soviet Union & 0 & 0 & 0 & 0 & 0 & 0 \\
India & 313 & 279 & 541 & 992 & 1,893 & 2,399 \\
Japan & 265 & 291 & 373 & 698 & 974 & 1,031 \\
Middle East & 0 & 0 & 0 & 0 & 0 & 0 \\
Mexico & 0 & 0 & 30 & 78 & 1,031 & 1,053 \\
Other Asia & 0 & 0 & 0 & 0 & 0 & 0 \\
South Korea & 264 & 335 & 400 & 414 & 413 & 413 \\
United States & 3,984 & 11,612 & 17,166 & 19,797 & 23,562 & 28,385 \\
Western Europe & 736 & 4,173 & 4,313 & 5,086 & 7,650 & 9,071 \\
\hline Total & $\mathbf{1 0 , 7 8 9}$ & $\mathbf{2 2 , 0 9 9}$ & $\mathbf{3 0 , 2 0 9}$ & $\mathbf{3 6 , 6 9 4}$ & $\mathbf{4 7 , 8 4 7}$ & $\mathbf{5 7 , 5 9 2}$ \\
\hline \hline
\end{tabular}

Table A-435: Delayed technology reference case with $\$ 50$ per tonne $\mathrm{CO}_{2}$ ethanol net trade [Million gallons]

\begin{tabular}{lrrrrrr} 
Africa & 0 & 0 & 0 & 0 & 0 & 0 \\
Australia & 0 & 0 & 0 & 0 & 0 & 0 \\
Canada & -145 & 0 & 26 & 0 & 101 & 90 \\
China & 50 & 384 & 614 & 646 & 0 & -423 \\
Central and South America & 1,484 & 1,845 & 3,241 & 4,974 & 7,545 & 9,663 \\
Eastern Europe & -589 & 0 & -444 & -215 & 0 & 0 \\
Former Soviet Union & 0 & 0 & 0 & 0 & 0 & 0 \\
India & 0 & 0 & 0 & 0 & 0 & 423 \\
Japan & -265 & -291 & -278 & -350 & -395 & -453 \\
Middle East & 0 & 0 & 0 & 0 & 0 & 0 \\
Mexico & 0 & 0 & 170 & 523 & 377 & 493 \\
Other Asia & 0 & 0 & 0 & 0 & 0 & 0 \\
South Korea & -264 & -335 & -370 & -384 & -383 & -381 \\
United States & -134 & 0 & $-1,400$ & $-3,342$ & $-4,886$ & $-8,522$ \\
Western Europe & -136 & $-1,604$ & $-1,559$ & $-1,851$ & $-2,359$ & -889 \\
\hline Total & $\mathbf{0}$ & $\mathbf{0}$ & $\mathbf{0}$ & $\mathbf{0}$ & $\mathbf{0}$ & $\mathbf{0}$ \\
\hline \hline
\end{tabular}


Table A-436: Delayed technology reference case with $\$ 50$ per tonne $\mathrm{CO}_{2}$ grain ethanol production [Million gallons]

\begin{tabular}{lrrrrrr} 
& $\mathbf{2 0 0 5}$ & $\mathbf{2 0 1 0}$ & $\mathbf{2 0 1 5}$ & $\mathbf{2 0 2 0}$ & $\mathbf{2 0 2 5}$ & $\mathbf{2 0 3 0}$ \\
Africa & 0 & 0 & 0 & 0 & 0 & 0 \\
Argentina & 0 & 178 & 604 & 604 & 604 & 604 \\
Australia & 0 & 0 & 0 & 0 & 0 & 0 \\
Brazil & 0 & 0 & 0 & 0 & 0 & 0 \\
Canada & 0 & 0 & 0 & 0 & 0 & 0 \\
Caribbean basin & 0 & 0 & 0 & 0 & 0 & 0 \\
China & 344 & 344 & 344 & 325 & 0 & 0 \\
Colombia & 0 & 0 & 0 & 0 & 0 & 0 \\
Eastern Europe & 110 & 226 & 348 & 477 & 367 & 251 \\
Former Soviet Union & 0 & 0 & 0 & 0 & 0 & 0 \\
India & 0 & 0 & 0 & 0 & 0 & 0 \\
Japan & 0 & 0 & 0 & 0 & 0 & 0 \\
Middle East & 0 & 0 & 0 & 0 & 0 & 0 \\
Mexico & 0 & 0 & 0 & 0 & 0 & 0 \\
Other Asia & 0 & 0 & 0 & 0 & 0 & 0 \\
South Korea & 0 & 0 & 0 & 0 & 0 & 0 \\
United States & 3,850 & 11,612 & 15,000 & 15,000 & 14,132 & 11,111 \\
Western Europe & 600 & 2,570 & 2,589 & 2,542 & 3,178 & 3,604 \\
\hline Total & $\mathbf{4 , 9 0 4}$ & $\mathbf{1 4 , 9 3 0}$ & $\mathbf{1 8 , 8 8 6}$ & $\mathbf{1 8 , 9 4 9}$ & $\mathbf{1 8 , 2 8 2}$ & $\mathbf{1 5 , 5 7 1}$ \\
\hline \hline
\end{tabular}

Table A-437: Delayed technology reference case with $\$ 50$ per tonne $\mathrm{CO}_{2}$ sugar ethanol production [Million gallons]

\begin{tabular}{lrrrrrr} 
& $\mathbf{2 0 0 5}$ & $\mathbf{2 0 1 0}$ & $\mathbf{2 0 1 5}$ & $\mathbf{2 0 2 0}$ & $\mathbf{2 0 2 5}$ & $\mathbf{2 0 3 0}$ \\
Africa & 300 & 330 & 73 & 83 & 91 & 98 \\
Argentina & 0 & 0 & 0 & 0 & 0 & 0 \\
Australia & 0 & 0 & 0 & 0 & 0 & 0 \\
Brazil & 4,866 & 5,977 & 7,329 & 8,862 & 10,406 & 12,578 \\
Canada & 0 & 0 & 0 & 0 & 0 & 0 \\
Caribbean basin & 210 & 328 & 683 & 683 & 779 & 890 \\
China & 0 & 40 & 48 & 53 & 58 & 64 \\
Colombia & 196 & 215 & 241 & 270 & 302 & 339 \\
Eastern Europe & 0 & 0 & 0 & 0 & 0 & 0 \\
Former Soviet Union & 0 & 0 & 0 & 0 & 0 & 0 \\
India & 313 & 279 & 327 & 347 & 381 & 452 \\
Japan & 0 & 0 & 0 & 0 & 0 & 0 \\
Middle East & 0 & 0 & 0 & 0 & 0 & 0 \\
Mexico & 0 & 0 & 0 & 0 & 0 & 0 \\
Other Asia & 0 & 0 & 0 & 0 & 0 & 0 \\
South Korea & 0 & 0 & 0 & 0 & 0 & 0 \\
United States & 0 & 0 & 0 & 0 & 0 & 0 \\
Western Europe & 0 & 0 & 0 & 0 & 0 & 0 \\
\hline Total & $\mathbf{5 , 8 8 4}$ & $\mathbf{7 , 1 7 0}$ & $\mathbf{8 , 7 0 1}$ & $\mathbf{1 0 , 2 9 7}$ & $\mathbf{1 2 , 0 1 8}$ & $\mathbf{1 4 , 4 2 0}$ \\
\hline \hline
\end{tabular}


Table A-438: Delayed technology reference case with $\$ 50$ per tonne $\mathrm{CO}_{2}$ cellulosic ethanol production [Million gallons]

\begin{tabular}{lrrrrrr} 
& $\mathbf{2 0 0 5}$ & $\mathbf{2 0 1 0}$ & $\mathbf{2 0 1 5}$ & $\mathbf{2 0 2 0}$ & $\mathbf{2 0 2 5}$ & $\mathbf{2 0 3 0}$ \\
Africa & 0 & 0 & 0 & 0 & 0 & 0 \\
Argentina & 0 & 0 & 59 & 306 & 335 & 347 \\
Australia & 0 & 0 & 66 & 262 & 704 & 1,174 \\
Brazil & 0 & 0 & 296 & 890 & 1,877 & 1,877 \\
Canada & 0 & 0 & 259 & 779 & 1,014 & 1,014 \\
Caribbean basin & 0 & 0 & 0 & 0 & 0 & 0 \\
China & 0 & 0 & 222 & 667 & 1,564 & 2,543 \\
Colombia & 0 & 0 & 170 & 512 & 1,199 & 1,893 \\
Eastern Europe & 0 & 0 & 80 & 261 & 670 & 897 \\
Former Soviet Union & 0 & 0 & 0 & 0 & 0 & 0 \\
India & 0 & 0 & 214 & 645 & 1,512 & 2,371 \\
Japan & 0 & 0 & 95 & 348 & 579 & 579 \\
Middle East & 0 & 0 & 0 & 0 & 0 & 0 \\
Mexico & 0 & 0 & 199 & 601 & 1,408 & 1,546 \\
Other Asia & 0 & 0 & 0 & 0 & 0 & 0 \\
South Korea & 0 & 0 & 30 & 30 & 30 & 32 \\
United States & 0 & 0 & 767 & 1,455 & 4,544 & 8,751 \\
Western Europe & 0 & 0 & 166 & 693 & 2,113 & 4,579 \\
\hline Total & $\mathbf{0}$ & $\mathbf{0}$ & $\mathbf{2 , 6 2 3}$ & $\mathbf{7 , 4 4 8}$ & $\mathbf{1 7 , 5 4 8}$ & $\mathbf{2 7 , 6 0 1}$ \\
\hline \hline
\end{tabular}

Table A-439: Delayed technology reference case with $\$ 50$ per tonne $\mathrm{CO}_{2}$ grain ethanol consumption [Million gallons]

\begin{tabular}{lrrrrrr} 
& $\mathbf{2 0 0 5}$ & $\mathbf{2 0 1 0}$ & $\mathbf{2 0 1 5}$ & $\mathbf{2 0 2 0}$ & $\mathbf{2 0 2 5}$ & $\mathbf{2 0 3 0}$ \\
Africa & 0 & 0 & 0 & 0 & 0 & 0 \\
Australia & 0 & 0 & 0 & 0 & 0 & 0 \\
Canada & 0 & 0 & 0 & 0 & 0 & 0 \\
China & 294 & 0 & 0 & 325 & 0 & 0 \\
Central and South America & 0 & 36 & 100 & 118 & 94 & 95 \\
Eastern Europe & 110 & 226 & 348 & 477 & 367 & 251 \\
Former Soviet Union & 0 & 0 & 0 & 0 & 0 & 0 \\
India & 0 & 0 & 0 & 0 & 0 & 0 \\
Japan & 0 & 192 & 230 & 0 & 294 & 363 \\
Middle East & 0 & 0 & 0 & 0 & 0 & 0 \\
Mexico & 0 & 0 & 0 & 0 & 0 & 0 \\
Other Asia & 0 & 0 & 0 & 0 & 0 & 0 \\
South Korea & 50 & 295 & 148 & 88 & 216 & 34 \\
United States & 3,850 & 11,612 & 15,000 & 15,000 & 14,132 & 11,223 \\
Western Europe & 600 & 2,570 & 3,060 & 2,940 & 3,178 & 3,604 \\
\hline Total & $\mathbf{4 , 9 0 4}$ & $\mathbf{1 4 , 9 3 0}$ & $\mathbf{1 8 , 8 8 6}$ & $\mathbf{1 8 , 9 4 9}$ & $\mathbf{1 8 , 2 8 2}$ & $\mathbf{1 5 , 5 7 1}$ \\
\hline \hline
\end{tabular}


Table A-440: Delayed technology reference case with $\$ 50$ per tonne $\mathrm{CO}_{2}$ sugar ethanol consumption [Million gallons]

\begin{tabular}{lrrrrrr} 
& $\mathbf{2 0 0 5}$ & $\mathbf{2 0 1 0}$ & $\mathbf{2 0 1 5}$ & $\mathbf{2 0 2 0}$ & $\mathbf{2 0 2 5}$ & $\mathbf{2 0 3 0}$ \\
Africa & 300 & 330 & 73 & 83 & 91 & 98 \\
Australia & 0 & 0 & 0 & 0 & 0 & 0 \\
Canada & 145 & 0 & 0 & 0 & 0 & 0 \\
China & 0 & 0 & 0 & 53 & 58 & 64 \\
Central and South America & 3,788 & 4,818 & 6,042 & 6,909 & 7,449 & 7,045 \\
Eastern Europe & 589 & 0 & 418 & 153 & 0 & 0 \\
Former Soviet Union & 0 & 0 & 0 & 0 & 0 & 0 \\
India & 313 & 279 & 327 & 347 & 381 & 452 \\
Japan & 265 & 99 & 48 & 0 & 0 & 0 \\
Middle East & 0 & 0 & 0 & 0 & 0 & 0 \\
Mexico & 0 & 0 & 0 & 0 & 0 & 0 \\
Other Asia & 0 & 0 & 0 & 0 & 0 & 0 \\
South Korea & 215 & 40 & 0 & 0 & 0 & 0 \\
United States & 134 & 0 & 788 & 2,753 & 3,961 & 6,761 \\
Western Europe & 136 & 1,604 & 1,005 & 0 & 77 & 0 \\
\hline Total & $\mathbf{5 , 8 8 4}$ & $\mathbf{7 , 1 7 0}$ & $\mathbf{8 , 7 0 1}$ & $\mathbf{1 0 , 2 9 7}$ & $\mathbf{1 2 , 0 1 8}$ & $\mathbf{1 4 , 4 2 0}$ \\
\hline \hline
\end{tabular}

Table A-441: Delayed technology reference case with $\$ 50$ per tonne $\mathrm{CO}_{2}$ cellulosic ethanol consumption [Million gallons]

\begin{tabular}{lllrrrr} 
Africa & 0 & 0 & 0 & 0 & 0 & 0 \\
Australia & 0 & 0 & 66 & 262 & 704 & 1,174 \\
Canada & 0 & 0 & 233 & 779 & 912 & 924 \\
China & 0 & 0 & 0 & 21 & 1,564 & 2,966 \\
Central and South America & 0 & 0 & 0 & 125 & 415 & 1,724 \\
Eastern Europe & 0 & 0 & 106 & 323 & 670 & 897 \\
Former Soviet Union & 0 & 0 & 0 & 0 & 0 & 0 \\
India & 0 & 0 & 214 & 645 & 1,512 & 1,947 \\
Japan & 0 & 0 & 95 & 698 & 680 & 668 \\
Middle East & 0 & 0 & 0 & 0 & 0 & 0 \\
Mexico & 0 & 0 & 30 & 78 & 1,031 & 1,053 \\
Other Asia & 0 & 0 & 0 & 0 & 0 & 0 \\
South Korea & 0 & 0 & 252 & 326 & 197 & 379 \\
United States & 0 & 0 & 1,379 & 2,044 & 5,469 & 10,400 \\
Western Europe & 0 & 0 & 248 & 2,146 & 4,396 & 5,467 \\
\hline Total & $\mathbf{0}$ & $\mathbf{0}$ & $\mathbf{2 , 6 2 3}$ & $\mathbf{7 , 4 4 8}$ & $\mathbf{1 7 , 5 4 8}$ & $\mathbf{2 7 , 6 0 1}$ \\
\hline \hline
\end{tabular}


Table A-442: Delayed technology reference case with $\$ 50$ per tonne $\mathrm{CO}_{2}$ grain ethanol net trade [Million gallons]

\begin{tabular}{lrrrrrr} 
& $\mathbf{2 0 0 5}$ & $\mathbf{2 0 1 0}$ & $\mathbf{2 0 1 5}$ & $\mathbf{2 0 2 0}$ & $\mathbf{2 0 2 5}$ & $\mathbf{2 0 3 0}$ \\
Africa & 0 & 0 & 0 & 0 & 0 & 0 \\
Australia & 0 & 0 & 0 & 0 & 0 & 0 \\
Canada & 0 & 0 & 0 & 0 & 0 & 0 \\
China & 50 & 344 & 344 & 0 & 0 & 0 \\
Central and South America & 0 & 143 & 505 & 486 & 510 & 509 \\
Eastern Europe & 0 & 0 & 0 & 0 & 0 & 0 \\
Former Soviet Union & 0 & 0 & 0 & 0 & 0 & 0 \\
India & 0 & 0 & 0 & 0 & 0 & 0 \\
Japan & 0 & -192 & -230 & 0 & -294 & -363 \\
Middle East & 0 & 0 & 0 & 0 & 0 & 0 \\
Mexico & 0 & 0 & 0 & 0 & 0 & 0 \\
Other Asia & 0 & 0 & 0 & 0 & 0 & 0 \\
South Korea & -50 & -295 & -148 & -88 & -216 & -34 \\
United States & 0 & 0 & 0 & 0 & 0 & -112 \\
Western Europe & 0 & 0 & -471 & -398 & 0 & 0 \\
\hline Total & $\mathbf{0}$ & $\mathbf{0}$ & $\mathbf{0}$ & $\mathbf{0}$ & $\mathbf{0}$ & $\mathbf{0}$ \\
\hline \hline
\end{tabular}

Table A-443: Delayed technology reference case with $\$ 50$ per tonne $\mathrm{CO}_{2}$ sugar ethanol net trade [Million gallons]

\begin{tabular}{lrrrrrr} 
Africa & 0 & 0 & 0 & 0 & 0 & 0 \\
Australia & 0 & 0 & 0 & 0 & 0 & 0 \\
Canada & -145 & 0 & 0 & 0 & 0 & 0 \\
China & 0 & 40 & 48 & 0 & 0 & 0 \\
Central and South America & 1,484 & 1,702 & 2,211 & 2,906 & 4,038 & 6,761 \\
Eastern Europe & -589 & 0 & -418 & -153 & 0 & 0 \\
Former Soviet Union & 0 & 0 & 0 & 0 & 0 & 0 \\
India & 0 & 0 & 0 & 0 & 0 & 0 \\
Japan & -265 & -99 & -48 & 0 & 0 & 0 \\
Middle East & 0 & 0 & 0 & 0 & 0 & 0 \\
Mexico & 0 & 0 & 0 & 0 & 0 & 0 \\
Other Asia & 0 & 0 & 0 & 0 & 0 & 0 \\
South Korea & -215 & -40 & 0 & 0 & 0 & 0 \\
United States & -134 & 0 & -788 & $-2,753$ & $-3,961$ & $-6,761$ \\
Western Europe & -136 & $-1,604$ & $-1,005$ & 0 & -77 & 0 \\
\hline Total & $\mathbf{0}$ & $\mathbf{0}$ & $\mathbf{0}$ & $\mathbf{0}$ & $\mathbf{0}$ & $\mathbf{0}$ \\
\hline \hline
\end{tabular}


Table A-444: Delayed technology reference case with $\$ 50$ per tonne $\mathrm{CO}_{2}$ cellulosic ethanol net trade [Million gallons]

\begin{tabular}{lrrrrrr} 
& $\mathbf{2 0 0 5}$ & $\mathbf{2 0 1 0}$ & $\mathbf{2 0 1 5}$ & $\mathbf{2 0 2 0}$ & $\mathbf{2 0 2 5}$ & $\mathbf{2 0 3 0}$ \\
Africa & 0 & 0 & 0 & 0 & 0 & 0 \\
Australia & 0 & 0 & 0 & 0 & 0 & 0 \\
Canada & 0 & 0 & 26 & 0 & 101 & 90 \\
China & 0 & 0 & 222 & 646 & 0 & -423 \\
Central and South America & 0 & 0 & 525 & 1,582 & 2,997 & 2,392 \\
Eastern Europe & 0 & 0 & -26 & -62 & 0 & 0 \\
Former Soviet Union & 0 & 0 & 0 & 0 & 0 & 0 \\
India & 0 & 0 & 0 & 0 & 0 & 423 \\
Japan & 0 & 0 & 0 & -350 & -101 & -90 \\
Middle East & 0 & 0 & 0 & 0 & 0 & 0 \\
Mexico & 0 & 0 & 170 & 523 & 377 & 493 \\
Other Asia & 0 & 0 & 0 & 0 & 0 & 0 \\
South Korea & 0 & 0 & -222 & -296 & -166 & -347 \\
United States & 0 & 0 & -612 & -589 & -925 & $-1,649$ \\
Western Europe & 0 & 0 & -82 & $-1,453$ & $-2,282$ & -889 \\
\hline Total & $\mathbf{0}$ & $\mathbf{0}$ & $\mathbf{0}$ & $\mathbf{0}$ & $\mathbf{0}$ & $\mathbf{0}$ \\
\hline \hline
\end{tabular}

Table A-445: Delayed technology reference case with $\$ 50$ per tonne $\mathrm{CO}_{2}$ biomass-toliquids production [Million gallons ethanol equivalent]

\begin{tabular}{lrrrrrr} 
& $\mathbf{2 0 0 5}$ & $\mathbf{2 0 1 0}$ & $\mathbf{2 0 1 5}$ & $\mathbf{2 0 2 0}$ & $\mathbf{2 0 2 5}$ & $\mathbf{2 0 3 0}$ \\
Africa & 0 & 0 & 0 & 0 & 0 & 0 \\
Argentina & 0 & 0 & 21 & 110 & 121 & 125 \\
Australia & 0 & 0 & 0 & 0 & 0 & 0 \\
Brazil & 0 & 0 & 106 & 320 & 675 & 675 \\
Canada & 0 & 0 & 93 & 280 & 365 & 365 \\
Caribbean basin & 0 & 0 & 0 & 0 & 0 & 0 \\
China & 0 & 0 & 80 & 240 & 563 & 915 \\
Colombia & 0 & 0 & 61 & 184 & 431 & 681 \\
Eastern Europe & 0 & 0 & 0 & 0 & 0 & 0 \\
Former Soviet Union & 0 & 0 & 0 & 0 & 0 & 0 \\
India & 0 & 0 & 77 & 232 & 544 & 853 \\
Japan & 0 & 0 & 0 & 0 & 0 & 0 \\
Middle East & 0 & 0 & 0 & 0 & 0 & 0 \\
Mexico & 0 & 0 & 72 & 216 & 506 & 556 \\
Other Asia & 0 & 0 & 0 & 0 & 0 & 0 \\
South Korea & 0 & 0 & 31 & 41 & 42 & 42 \\
United States & 0 & 0 & 786 & 3,104 & 4,211 & 5,624 \\
Western Europe & 0 & 0 & 0 & 0 & 0 & 0 \\
\hline Total & $\mathbf{0}$ & $\mathbf{0}$ & $\mathbf{1 , 3 2 7}$ & $\mathbf{4 , 7 2 7}$ & $\mathbf{7 , 4 5 8}$ & $\mathbf{9 , 8 3 5}$ \\
\hline \hline
\end{tabular}


Table A-446: Delayed technology reference case with $\$ 50$ per tonne $\mathrm{CO}_{2}$ biomass-toliquids consumption [Million gallons ethanol equivalent]

\begin{tabular}{lrrrrrr} 
& $\mathbf{2 0 0 5}$ & $\mathbf{2 0 1 0}$ & $\mathbf{2 0 1 5}$ & $\mathbf{2 0 2 0}$ & $\mathbf{2 0 2 5}$ & $\mathbf{2 0 3 0}$ \\
Africa & 0 & 0 & 0 & 0 & 0 & 0 \\
Australia & 0 & 0 & 0 & 0 & 0 & 0 \\
Canada & 0 & 0 & 0 & 0 & 0 & 0 \\
China & 0 & 0 & 0 & 0 & 0 & 0 \\
Central and South America & 0 & 0 & 0 & 0 & 0 & 0 \\
Eastern Europe & 0 & 0 & 0 & 0 & 0 & 0 \\
Former Soviet Union & 0 & 0 & 0 & 0 & 0 & 0 \\
India & 0 & 0 & 0 & 0 & 0 & 0 \\
Japan & 0 & 0 & 0 & 0 & 0 & 0 \\
Middle East & 0 & 0 & 0 & 0 & 0 & 0 \\
Mexico & 0 & 0 & 0 & 0 & 0 & 0 \\
Other Asia & 0 & 0 & 0 & 0 & 0 & 0 \\
South Korea & 0 & 0 & 0 & 0 & 0 & 0 \\
United States & 0 & 0 & 1,057 & 4,370 & 7,457 & 9,835 \\
Western Europe & 0 & 0 & 271 & 356 & 0 & 0 \\
\hline Total & $\mathbf{0}$ & $\mathbf{0}$ & $\mathbf{1 , 3 2 7}$ & $\mathbf{4 , 7 2 7}$ & $\mathbf{7 , 4 5 7}$ & $\mathbf{9 , 8 3 5}$ \\
\hline \hline
\end{tabular}

Table A-447: Delayed technology reference case with $\$ 50$ per tonne $\mathrm{CO}_{2}$ biomass-toliquids net trade [Million gallons ethanol equivalent]

\begin{tabular}{lrrrrrr} 
& $\mathbf{2 0 0 5}$ & $\mathbf{2 0 1 0}$ & $\mathbf{2 0 1 5}$ & $\mathbf{2 0 2 0}$ & $\mathbf{2 0 2 5}$ & $\mathbf{2 0 3 0}$ \\
Africa & 0 & 0 & 0 & 0 & 0 & 0 \\
Australia & 0 & 0 & 0 & 0 & 0 & 0 \\
Canada & 0 & 0 & 93 & 280 & 365 & 365 \\
China & 0 & 0 & 80 & 240 & 563 & 915 \\
Central and South America & 0 & 0 & 189 & 614 & 1,227 & 1,481 \\
Eastern Europe & 0 & 0 & 0 & 0 & 0 & 0 \\
Former Soviet Union & 0 & 0 & 0 & 0 & 0 & 0 \\
India & 0 & 0 & 77 & 232 & 544 & 853 \\
Japan & 0 & 0 & 0 & 0 & 0 & 0 \\
Middle East & 0 & 0 & 0 & 0 & 0 & 0 \\
Mexico & 0 & 0 & 72 & 216 & 506 & 556 \\
Other Asia & 0 & 0 & 0 & 0 & 0 & 0 \\
South Korea & 0 & 0 & 31 & 41 & 42 & 42 \\
United States & 0 & 0 & -271 & $-1,267$ & $-3,246$ & $-4,211$ \\
Western Europe & 0 & 0 & -271 & -356 & 0 & 0 \\
\hline Total & $\mathbf{0}$ & $\mathbf{0}$ & $\mathbf{0}$ & $\mathbf{0}$ & $\mathbf{0}$ & $\mathbf{0}$ \\
\hline \hline
\end{tabular}


Table A-448: Delayed technology reference case with $\$ 50$ per tonne $\mathrm{CO}_{2}$ biodiesel production [Million gallons ethanol equivalent]

\begin{tabular}{lrrrrrr} 
& $\mathbf{2 0 0 5}$ & $\mathbf{2 0 1 0}$ & $\mathbf{2 0 1 5}$ & $\mathbf{2 0 2 0}$ & $\mathbf{2 0 2 5}$ & $\mathbf{2 0 3 0}$ \\
Africa & 0 & 0 & 0 & 0 & 0 & 0 \\
Argentina & 0 & 333 & 776 & 819 & 827 & 94 \\
Australia & 0 & 0 & 0 & 0 & 0 & 0 \\
Brazil & 0 & 333 & 835 & 1,537 & 1,792 & 1,362 \\
Canada & 0 & 0 & 0 & 0 & 0 & 0 \\
Caribbean basin & 0 & 95 & 310 & 675 & 802 & 898 \\
China & 0 & 0 & 0 & 0 & 0 & 0 \\
Colombia & 0 & 119 & 298 & 474 & 650 & 782 \\
Eastern Europe & 0 & 0 & 0 & 0 & 0 & 0 \\
Former Soviet Union & 0 & 0 & 0 & 0 & 0 & 0 \\
India & 0 & 0 & 0 & 0 & 0 & 0 \\
Japan & 0 & 0 & 0 & 0 & 0 & 0 \\
Middle East & 0 & 0 & 0 & 0 & 0 & 0 \\
Mexico & 0 & 0 & 0 & 0 & 0 & 0 \\
Other Asia & 0 & 0 & 0 & 0 & 0 & 0 \\
South Korea & 0 & 0 & 0 & 0 & 0 & 0 \\
United States & 0 & 1,238 & 1,640 & 1,747 & 1,907 & 2,016 \\
Western Europe & 998 & 3,178 & 3,578 & 4,175 & 4,566 & 5,191 \\
\hline Total & $\mathbf{9 9 8}$ & $\mathbf{5 , 2 9 4}$ & $\mathbf{7 , 4 3 7}$ & $\mathbf{9 , 4 2 6}$ & $\mathbf{1 0 , 5 4 4}$ & $\mathbf{1 0 , 3 4 3}$ \\
\hline \hline
\end{tabular}

Table A-449: Delayed technology reference case with $\$ 50$ per tonne $\mathrm{CO}_{2}$ biodiesel consumption [Million gallons ethanol equivalent]

\begin{tabular}{lrrrrrr} 
& $\mathbf{2 0 0 5}$ & $\mathbf{2 0 1 0}$ & $\mathbf{2 0 1 5}$ & $\mathbf{2 0 2 0}$ & $\mathbf{2 0 2 5}$ & $\mathbf{2 0 3 0}$ \\
Africa & 0 & 0 & 0 & 0 & 0 & 0 \\
Australia & 0 & 0 & 0 & 0 & 0 & 0 \\
Canada & 0 & 0 & 0 & 0 & 0 & 0 \\
China & 0 & 0 & 0 & 0 & 0 & 0 \\
Central and South America & 0 & 158 & 472 & 984 & 1,306 & 1,490 \\
Eastern Europe & 0 & 0 & 0 & 0 & 0 & 0 \\
Former Soviet Union & 0 & 0 & 0 & 0 & 0 & 0 \\
India & 0 & 0 & 0 & 0 & 0 & 0 \\
Japan & 0 & 0 & 0 & 0 & 0 & 0 \\
Middle East & 0 & 0 & 0 & 0 & 0 & 0 \\
Mexico & 0 & 0 & 0 & 0 & 0 & 0 \\
Other Asia & 0 & 0 & 0 & 0 & 0 & 0 \\
South Korea & 0 & 0 & 0 & 0 & 0 & 0 \\
United States & 0 & 1,238 & 1,713 & 1,747 & 1,907 & 2,016 \\
Western Europe & 998 & 3,898 & 5,252 & 6,696 & 7,331 & 6,837 \\
\hline Total & $\mathbf{9 9 8}$ & $\mathbf{5 , 2 9 4}$ & $\mathbf{7 , 4 3 7}$ & $\mathbf{9 , 4 2 6}$ & $\mathbf{1 0 , 5 4 4}$ & $\mathbf{1 0 , 3 4 3}$ \\
\hline \hline
\end{tabular}


Table A-450: Delayed technology reference case with $\$ 50$ per tonne $\mathrm{CO}_{2}$ biodiesel net trade [Million gallons ethanol equivalent]

\begin{tabular}{lrrrrrr} 
& $\mathbf{2 0 0 5}$ & $\mathbf{2 0 1 0}$ & $\mathbf{2 0 1 5}$ & $\mathbf{2 0 2 0}$ & $\mathbf{2 0 2 5}$ & $\mathbf{2 0 3 0}$ \\
Africa & 0 & 0 & 0 & 0 & 0 & 0 \\
Australia & 0 & 0 & 0 & 0 & 0 & 0 \\
Canada & 0 & 0 & 0 & 0 & 0 & 0 \\
China & 0 & 0 & 0 & 0 & 0 & 0 \\
Central and South America & 0 & 720 & 1,746 & 2,520 & 2,766 & 1,646 \\
Eastern Europe & 0 & 0 & 0 & 0 & 0 & 0 \\
Former Soviet Union & 0 & 0 & 0 & 0 & 0 & 0 \\
India & 0 & 0 & 0 & 0 & 0 & 0 \\
Japan & 0 & 0 & 0 & 0 & 0 & 0 \\
Middle East & 0 & 0 & 0 & 0 & 0 & 0 \\
Mexico & 0 & 0 & 0 & 0 & 0 & 0 \\
Other Asia & 0 & 0 & 0 & 0 & 0 & 0 \\
South Korea & 0 & 0 & 0 & 0 & 0 & 0 \\
United States & 0 & 0 & -73 & 0 & 0 & 0 \\
Western Europe & 0 & -720 & $-1,674$ & $-2,520$ & $-2,766$ & $-1,646$ \\
\hline Total & $\mathbf{0}$ & $\mathbf{0}$ & $\mathbf{0}$ & $\mathbf{0}$ & $\mathbf{0}$ & $\mathbf{0}$ \\
\hline \hline
\end{tabular}




\section{A-26 Delayed Technology Reference Case with \$20 per Tonne Growers' Payment}

\section{Table A-451: Delayed technology reference case with \$20 per tonne growers’} payment ethanol production [Million gallons]

\begin{tabular}{lrrrrrr} 
& $\mathbf{2 0 0 5}$ & $\mathbf{2 0 1 0}$ & $\mathbf{2 0 1 5}$ & $\mathbf{2 0 2 0}$ & $\mathbf{2 0 2 5}$ & $\mathbf{2 0 3 0}$ \\
Africa & 300 & 330 & 364 & 402 & 455 & 502 \\
Argentina & 0 & 178 & 664 & 910 & 940 & 952 \\
Australia & 0 & 0 & 66 & 247 & 625 & 1,172 \\
Brazil & 4,866 & 5,977 & 7,624 & 9,752 & 11,947 & 13,338 \\
Canada & 0 & 0 & 259 & 779 & 1,004 & 1,004 \\
Caribbean basin & 210 & 328 & 573 & 683 & 775 & 775 \\
China & 344 & 384 & 614 & 1,041 & 1,374 & 1,374 \\
Colombia & 196 & 215 & 411 & 782 & 1,501 & 2,227 \\
Eastern Europe & 110 & 226 & 428 & 738 & 1,037 & 1,149 \\
Former Soviet Union & 0 & 0 & 6 & 19 & 57 & 174 \\
India & 313 & 279 & 541 & 992 & 1,893 & 2,543 \\
Japan & 0 & 0 & 95 & 348 & 612 & 647 \\
Middle East & 0 & 0 & 0 & 0 & 0 & 0 \\
Mexico & 0 & 0 & 199 & 601 & 1,408 & 1,546 \\
Other Asia & 0 & 0 & 0 & 0 & 0 & 0 \\
South Korea & 0 & 0 & 35 & 35 & 35 & 35 \\
United States & 3,850 & 11,612 & 15,767 & 16,400 & 17,491 & 21,182 \\
Western Europe & 600 & 2,568 & 2,755 & 3,398 & 5,291 & 8,013 \\
\hline Total & $\mathbf{1 0 , 7 8 9}$ & $\mathbf{2 2 , 0 9 7}$ & $\mathbf{3 0 , 4 0 0}$ & $\mathbf{3 7 , 1 2 5}$ & $\mathbf{4 6 , 4 4 4}$ & $\mathbf{5 6 , 6 3 0}$ \\
\hline \hline
\end{tabular}


Table A-452: Delayed technology reference case with \$20 per tonne growers’ payment ethanol consumption [Million gallons]

\begin{tabular}{lrrrrrr} 
& $\mathbf{2 0 0 5}$ & $\mathbf{2 0 1 0}$ & $\mathbf{2 0 1 5}$ & $\mathbf{2 0 2 0}$ & $\mathbf{2 0 2 5}$ & $\mathbf{2 0 3 0}$ \\
Africa & 300 & 330 & 364 & 402 & 455 & 502 \\
Australia & 0 & 0 & 66 & 247 & 625 & 1,172 \\
Canada & 145 & 0 & 233 & 779 & 1,004 & 1,020 \\
China & 297 & 0 & 0 & 344 & 600 & 1,374 \\
Central and South America & 3,788 & 4,853 & 6,134 & 7,153 & 7,958 & 8,859 \\
Eastern Europe & 699 & 226 & 872 & 953 & 1,037 & 1,149 \\
Former Soviet Union & 0 & 0 & 6 & 19 & 57 & 174 \\
India & 313 & 279 & 541 & 992 & 1,893 & 2,399 \\
Japan & 263 & 288 & 373 & 699 & 1,008 & 1,100 \\
Middle East & 0 & 0 & 0 & 0 & 0 & 0 \\
Mexico & 0 & 0 & 30 & 78 & 183 & 201 \\
Other Asia & 0 & 0 & 0 & 0 & 0 & 0 \\
South Korea & 264 & 335 & 400 & 414 & 413 & 413 \\
United States & 3,984 & 11,612 & 17,091 & 19,797 & 23,562 & 28,684 \\
Western Europe & 736 & 4,174 & 4,289 & 5,249 & 7,650 & 9,586 \\
\hline Total & $\mathbf{1 0 , 7 8 9}$ & $\mathbf{2 2 , 0 9 7}$ & $\mathbf{3 0 , 4 0 0}$ & $\mathbf{3 7 , 1 2 5}$ & $\mathbf{4 6 , 4 4 4}$ & $\mathbf{5 6 , 6 3 0}$ \\
\hline \hline
\end{tabular}

Table A-453: Delayed technology reference case with $\$ 20$ per tonne growers' payment ethanol net trade [Million gallons]

\begin{tabular}{lrrrrrr} 
Africa & 0 & 0 & 0 & 0 & 0 & 0 \\
Australia & 0 & 0 & 0 & 0 & 0 & 0 \\
Canada & -145 & 0 & 26 & 0 & 0 & -16 \\
China & 47 & 384 & 614 & 697 & 774 & 0 \\
Central and South America & 1,484 & 1,845 & 3,137 & 4,974 & 7,205 & 8,433 \\
Eastern Europe & -589 & 0 & -444 & -215 & 0 & 0 \\
Former Soviet Union & 0 & 0 & 0 & 0 & 0 & 0 \\
India & 0 & 0 & 0 & 0 & 0 & 144 \\
Japan & -263 & -288 & -278 & -351 & -395 & -453 \\
Middle East & 0 & 0 & 0 & 0 & 0 & 0 \\
Mexico & 0 & 0 & 170 & 523 & 1,225 & 1,345 \\
Other Asia & 0 & 0 & 0 & 0 & 0 & 0 \\
South Korea & -264 & -335 & -365 & -379 & -378 & -378 \\
United States & -134 & 0 & $-1,324$ & $-3,397$ & $-6,071$ & $-7,503$ \\
Western Europe & -136 & $-1,607$ & $-1,535$ & $-1,851$ & $-2,359$ & $-1,572$ \\
\hline Total & $\mathbf{0}$ & $\mathbf{0}$ & $\mathbf{0}$ & $\mathbf{0}$ & $\mathbf{0}$ & $\mathbf{0}$ \\
\hline \hline
\end{tabular}


Table A-454: Delayed technology reference case with \$20 per tonne growers' payment grain ethanol production [Million gallons]

\begin{tabular}{lrrrrrr} 
& $\mathbf{2 0 0 5}$ & $\mathbf{2 0 1 0}$ & $\mathbf{2 0 1 5}$ & $\mathbf{2 0 2 0}$ & $\mathbf{2 0 2 5}$ & $\mathbf{2 0 3 0}$ \\
Africa & 0 & 0 & 0 & 0 & 0 & 0 \\
Argentina & 0 & 178 & 604 & 604 & 604 & 604 \\
Australia & 0 & 0 & 0 & 0 & 0 & 0 \\
Brazil & 0 & 0 & 0 & 0 & 0 & 0 \\
Canada & 0 & 0 & 0 & 0 & 0 & 0 \\
Caribbean basin & 0 & 0 & 0 & 0 & 0 & 0 \\
China & 344 & 344 & 344 & 325 & 0 & 0 \\
Colombia & 0 & 0 & 0 & 0 & 0 & 0 \\
Eastern Europe & 110 & 226 & 348 & 477 & 367 & 251 \\
Former Soviet Union & 0 & 0 & 0 & 0 & 0 & 0 \\
India & 0 & 0 & 0 & 0 & 0 & 0 \\
Japan & 0 & 0 & 0 & 0 & 0 & 0 \\
Middle East & 0 & 0 & 0 & 0 & 0 & 0 \\
Mexico & 0 & 0 & 0 & 0 & 0 & 0 \\
Other Asia & 0 & 0 & 0 & 0 & 0 & 0 \\
South Korea & 0 & 0 & 0 & 0 & 0 & 0 \\
United States & 3,850 & 11,612 & 15,000 & 15,000 & 15,000 & 14,903 \\
Western Europe & 600 & 2,568 & 2,589 & 2,705 & 3,178 & 3,604 \\
\hline Total & $\mathbf{4 , 9 0 4}$ & $\mathbf{1 4 , 9 2 8}$ & $\mathbf{1 8 , 8 8 5}$ & $\mathbf{1 9 , 1 1 2}$ & $\mathbf{1 9 , 1 4 9}$ & $\mathbf{1 9 , 3 6 3}$ \\
\hline \hline
\end{tabular}

Table A-455: Delayed technology reference case with \$20 per tonne growers' payment sugar ethanol production [Million gallons]

\begin{tabular}{lrrrrrr} 
& $\mathbf{2 0 0 5}$ & $\mathbf{2 0 1 0}$ & $\mathbf{2 0 1 5}$ & $\mathbf{2 0 2 0}$ & $\mathbf{2 0 2 5}$ & $\mathbf{2 0 3 0}$ \\
Africa & 300 & 330 & 364 & 402 & 444 & 490 \\
Argentina & 0 & 0 & 0 & 0 & 0 & 0 \\
Australia & 0 & 0 & 0 & 0 & 0 & 0 \\
Brazil & 4,866 & 5,977 & 7,329 & 8,862 & 10,406 & 11,793 \\
Canada & 0 & 0 & 0 & 0 & 0 & 0 \\
Caribbean basin & 210 & 328 & 573 & 683 & 775 & 775 \\
China & 0 & 40 & 48 & 48 & 48 & 48 \\
Colombia & 196 & 215 & 241 & 270 & 302 & 334 \\
Eastern Europe & 0 & 0 & 0 & 0 & 0 & 0 \\
Former Soviet Union & 0 & 0 & 0 & 0 & 0 & 0 \\
India & 313 & 279 & 327 & 347 & 381 & 348 \\
Japan & 0 & 0 & 0 & 0 & 0 & 0 \\
Middle East & 0 & 0 & 0 & 0 & 0 & 0 \\
Mexico & 0 & 0 & 0 & 0 & 0 & 0 \\
Other Asia & 0 & 0 & 0 & 0 & 0 & 0 \\
South Korea & 0 & 0 & 0 & 0 & 0 & 0 \\
United States & 0 & 0 & 0 & 0 & 0 & 0 \\
Western Europe & 0 & 0 & 0 & 0 & 0 & 0 \\
\hline Total & $\mathbf{5 , 8 8 4}$ & $\mathbf{7 , 1 6 9}$ & $\mathbf{8 , 8 8 2}$ & $\mathbf{1 0 , 6 1 2}$ & $\mathbf{1 2 , 3 5 6}$ & $\mathbf{1 3 , 7 8 7}$ \\
\hline \hline
\end{tabular}


Table A-456: Delayed technology reference case with \$20 per tonne growers' payment cellulosic ethanol production [Million gallons]

\begin{tabular}{lrrrrrr} 
& $\mathbf{2 0 0 5}$ & $\mathbf{2 0 1 0}$ & $\mathbf{2 0 1 5}$ & $\mathbf{2 0 2 0}$ & $\mathbf{2 0 2 5}$ & $\mathbf{2 0 3 0}$ \\
Africa & 0 & 0 & 0 & 0 & 11 & 11 \\
Argentina & 0 & 0 & 59 & 306 & 336 & 347 \\
Australia & 0 & 0 & 66 & 247 & 625 & 1,172 \\
Brazil & 0 & 0 & 296 & 890 & 1,541 & 1,546 \\
Canada & 0 & 0 & 259 & 779 & 1,004 & 1,004 \\
Caribbean basin & 0 & 0 & 0 & 0 & 0 & 0 \\
China & 0 & 0 & 222 & 668 & 1,326 & 1,326 \\
Colombia & 0 & 0 & 170 & 512 & 1,199 & 1,893 \\
Eastern Europe & 0 & 0 & 80 & 261 & 670 & 897 \\
Former Soviet Union & 0 & 0 & 6 & 19 & 57 & 174 \\
India & 0 & 0 & 214 & 645 & 1,512 & 2,196 \\
Japan & 0 & 0 & 95 & 348 & 612 & 647 \\
Middle East & 0 & 0 & 0 & 0 & 0 & 0 \\
Mexico & 0 & 0 & 199 & 601 & 1,408 & 1,546 \\
Other Asia & 0 & 0 & 0 & 0 & 0 & 0 \\
South Korea & 0 & 0 & 35 & 35 & 35 & 35 \\
United States & 0 & 0 & 767 & 1,400 & 2,491 & 6,279 \\
Western Europe & 0 & 0 & 166 & 693 & 2,113 & 4,410 \\
\hline Total & $\mathbf{0}$ & $\mathbf{0}$ & $\mathbf{2 , 6 3 3}$ & $\mathbf{7 , 4 0 1}$ & $\mathbf{1 4 , 9 3 9}$ & $\mathbf{2 3 , 4 8 0}$ \\
\hline \hline
\end{tabular}

Table A-457: Delayed technology reference case with \$20 per tonne growers’ payment grain ethanol consumption [Million gallons]

\begin{tabular}{lrrrrrr} 
& $\mathbf{2 0 0 5}$ & $\mathbf{2 0 1 0}$ & $\mathbf{2 0 1 5}$ & $\mathbf{2 0 2 0}$ & $\mathbf{2 0 2 5}$ & $\mathbf{2 0 3 0}$ \\
Africa & 0 & 0 & 0 & 0 & 0 & 0 \\
Australia & 0 & 0 & 0 & 0 & 0 & 0 \\
Canada & 0 & 0 & 0 & 0 & 0 & 0 \\
China & 297 & 0 & 0 & 296 & 0 & 0 \\
Central and South America & 0 & 36 & 100 & 118 & 94 & 95 \\
Eastern Europe & 110 & 226 & 348 & 477 & 367 & 251 \\
Former Soviet Union & 0 & 0 & 0 & 0 & 0 & 0 \\
India & 0 & 0 & 0 & 0 & 0 & 0 \\
Japan & 0 & 192 & 230 & 29 & 0 & 131 \\
Middle East & 0 & 0 & 0 & 0 & 0 & 0 \\
Mexico & 0 & 0 & 0 & 0 & 0 & 0 \\
Other Asia & 0 & 0 & 0 & 0 & 0 & 0 \\
South Korea & 47 & 295 & 144 & 33 & 0 & 378 \\
United States & 3,850 & 11,612 & 15,000 & 15,000 & 15,000 & 14,903 \\
Western Europe & 600 & 2,568 & 3,064 & 3,158 & 3,688 & 3,604 \\
\hline Total & $\mathbf{4 , 9 0 4}$ & $\mathbf{1 4 , 9 2 8}$ & $\mathbf{1 8 , 8 8 5}$ & $\mathbf{1 9 , 1 1 2}$ & $\mathbf{1 9 , 1 4 9}$ & $\mathbf{1 9 , 3 6 3}$ \\
\hline \hline
\end{tabular}


Table A-458: Delayed technology reference case with \$20 per tonne growers’ payment sugar ethanol consumption [Million gallons]

\begin{tabular}{lrrrrrr} 
& $\mathbf{2 0 0 5}$ & $\mathbf{2 0 1 0}$ & $\mathbf{2 0 1 5}$ & $\mathbf{2 0 2 0}$ & $\mathbf{2 0 2 5}$ & $\mathbf{2 0 3 0}$ \\
Africa & 300 & 330 & 364 & 402 & 444 & 490 \\
Australia & 0 & 0 & 0 & 0 & 0 & 0 \\
Canada & 145 & 0 & 0 & 0 & 0 & 16 \\
China & 0 & 0 & 0 & 48 & 48 & 48 \\
Central and South America & 3,788 & 4,818 & 6,035 & 6,909 & 7,864 & 8,763 \\
Eastern Europe & 589 & 0 & 418 & 153 & 0 & 0 \\
Former Soviet Union & 0 & 0 & 0 & 0 & 0 & 0 \\
India & 313 & 279 & 327 & 347 & 381 & 348 \\
Japan & 263 & 96 & 48 & 0 & 0 & 178 \\
Middle East & 0 & 0 & 0 & 0 & 0 & 0 \\
Mexico & 0 & 0 & 0 & 0 & 0 & 0 \\
Other Asia & 0 & 0 & 0 & 0 & 0 & 0 \\
South Korea & 217 & 40 & 0 & 0 & 0 & 0 \\
United States & 134 & 0 & 788 & 2,753 & 3,093 & 2,372 \\
Western Europe & 136 & 1,607 & 902 & 0 & 526 & 1,572 \\
\hline Total & $\mathbf{5 , 8 8 4}$ & $\mathbf{7 , 1 6 9}$ & $\mathbf{8 , 8 8 2}$ & $\mathbf{1 0 , 6 1 2}$ & $\mathbf{1 2 , 3 5 6}$ & $\mathbf{1 3 , 7 8 7}$ \\
\hline \hline
\end{tabular}

Table A-459: Delayed technology reference case with $\$ 20$ per tonne growers' payment cellulosic ethanol consumption [Million gallons]

\begin{tabular}{lrrrrrr} 
& $\mathbf{2 0 0 5}$ & $\mathbf{2 0 1 0}$ & $\mathbf{2 0 1 5}$ & $\mathbf{2 0 2 0}$ & $\mathbf{2 0 2 5}$ & $\mathbf{2 0 3 0}$ \\
Africa & 0 & 0 & 0 & 0 & 11 & 11 \\
Australia & 0 & 0 & 66 & 247 & 625 & 1,172 \\
Canada & 0 & 0 & 233 & 779 & 1,004 & 1,004 \\
China & 0 & 0 & 0 & 0 & 552 & 1,326 \\
Central and South America & 0 & 0 & 0 & 125 & 0 & 0 \\
Eastern Europe & 0 & 0 & 106 & 323 & 670 & 897 \\
Former Soviet Union & 0 & 0 & 6 & 19 & 57 & 174 \\
India & 0 & 0 & 214 & 645 & 1,512 & 2,051 \\
Japan & 0 & 0 & 95 & 670 & 1,008 & 791 \\
Middle East & 0 & 0 & 0 & 0 & 0 & 0 \\
Mexico & 0 & 0 & 30 & 78 & 183 & 201 \\
Other Asia & 0 & 0 & 0 & 0 & 0 & 0 \\
South Korea & 0 & 0 & 256 & 381 & 413 & 35 \\
United States & 0 & 0 & 1,304 & 2,044 & 5,469 & 11,409 \\
Western Europe & 0 & 0 & 324 & 2,091 & 3,435 & 4,410 \\
\hline Total & $\mathbf{0}$ & $\mathbf{0}$ & $\mathbf{2 , 6 3 3}$ & $\mathbf{7 , 4 0 1}$ & $\mathbf{1 4 , 9 3 9}$ & $\mathbf{2 3 , 4 8 0}$ \\
\hline \hline
\end{tabular}


Table A-460: Delayed technology reference case with \$20 per tonne growers' payment grain ethanol net trade [Million gallons]

\begin{tabular}{lrrrrrr} 
& $\mathbf{2 0 0 5}$ & $\mathbf{2 0 1 0}$ & $\mathbf{2 0 1 5}$ & $\mathbf{2 0 2 0}$ & $\mathbf{2 0 2 5}$ & $\mathbf{2 0 3 0}$ \\
Africa & 0 & 0 & 0 & 0 & 0 & 0 \\
Australia & 0 & 0 & 0 & 0 & 0 & 0 \\
Canada & 0 & 0 & 0 & 0 & 0 & 0 \\
China & 47 & 344 & 344 & 29 & 0 & 0 \\
Central and South America & 0 & 143 & 505 & 486 & 510 & 509 \\
Eastern Europe & 0 & 0 & 0 & 0 & 0 & 0 \\
Former Soviet Union & 0 & 0 & 0 & 0 & 0 & 0 \\
India & 0 & 0 & 0 & 0 & 0 & 0 \\
Japan & 0 & -192 & -230 & -29 & 0 & -131 \\
Middle East & 0 & 0 & 0 & 0 & 0 & 0 \\
Mexico & 0 & 0 & 0 & 0 & 0 & 0 \\
Other Asia & 0 & 0 & 0 & 0 & 0 & 0 \\
South Korea & -47 & -295 & -144 & -33 & 0 & -378 \\
United States & 0 & 0 & 0 & 0 & 0 & 0 \\
Western Europe & 0 & 0 & -475 & -453 & -510 & 0 \\
\hline Total & $\mathbf{0}$ & $\mathbf{0}$ & $\mathbf{0}$ & $\mathbf{0}$ & $\mathbf{0}$ & $\mathbf{0}$ \\
\hline \hline
\end{tabular}

Table A-461: Delayed technology reference case with $\$ 20$ per tonne growers' payment sugar ethanol net trade [Million gallons]

\begin{tabular}{lrrrrrr} 
& $\mathbf{2 0 0 5}$ & $\mathbf{2 0 1 0}$ & $\mathbf{2 0 1 5}$ & $\mathbf{2 0 2 0}$ & $\mathbf{2 0 2 5}$ & $\mathbf{2 0 3 0}$ \\
Africa & 0 & 0 & 0 & 0 & 0 & 0 \\
Australia & 0 & 0 & 0 & 0 & 0 & 0 \\
Canada & -145 & 0 & 0 & 0 & 0 & -16 \\
China & 0 & 40 & 48 & 0 & 0 & 0 \\
Central and South America & 1,484 & 1,702 & 2,107 & 2,906 & 3,619 & 4,138 \\
Eastern Europe & -589 & 0 & -418 & -153 & 0 & 0 \\
Former Soviet Union & 0 & 0 & 0 & 0 & 0 & 0 \\
India & 0 & 0 & 0 & 0 & 0 & 0 \\
Japan & -263 & -96 & -48 & 0 & 0 & -178 \\
Middle East & 0 & 0 & 0 & 0 & 0 & 0 \\
Mexico & 0 & 0 & 0 & 0 & 0 & 0 \\
Other Asia & 0 & 0 & 0 & 0 & 0 & 0 \\
South Korea & -217 & -40 & 0 & 0 & 0 & 0 \\
United States & -134 & 0 & -788 & $-2,753$ & $-3,093$ & $-2,372$ \\
Western Europe & -136 & $-1,607$ & -902 & 0 & -526 & $-1,572$ \\
\hline Total & $\mathbf{0}$ & $\mathbf{0}$ & $\mathbf{0}$ & $\mathbf{0}$ & $\mathbf{0}$ & $\mathbf{0}$ \\
\hline \hline
\end{tabular}


Table A-462: Delayed technology reference case with \$20 per tonne growers' payment cellulosic ethanol net trade [Million gallons]

\begin{tabular}{lrrrrrr} 
& $\mathbf{2 0 0 5}$ & $\mathbf{2 0 1 0}$ & $\mathbf{2 0 1 5}$ & $\mathbf{2 0 2 0}$ & $\mathbf{2 0 2 5}$ & $\mathbf{2 0 3 0}$ \\
Africa & 0 & 0 & 0 & 0 & 0 & 0 \\
Australia & 0 & 0 & 0 & 0 & 0 & 0 \\
Canada & 0 & 0 & 26 & 0 & 0 & 0 \\
China & 0 & 0 & 222 & 668 & 774 & 0 \\
Central and South America & 0 & 0 & 525 & 1,582 & 3,076 & 3,786 \\
Eastern Europe & 0 & 0 & -26 & -62 & 0 & 0 \\
Former Soviet Union & 0 & 0 & 0 & 0 & 0 & 0 \\
India & 0 & 0 & 0 & 0 & 0 & 144 \\
Japan & 0 & 0 & 0 & -322 & -395 & -144 \\
Middle East & 0 & 0 & 0 & 0 & 0 & 0 \\
Mexico & 0 & 0 & 170 & 523 & 1,225 & 1,345 \\
Other Asia & 0 & 0 & 0 & 0 & 0 & 0 \\
South Korea & 0 & 0 & -222 & -346 & -378 & 0 \\
United States & 0 & 0 & -537 & -644 & $-2,978$ & $-5,130$ \\
Western Europe & 0 & 0 & -158 & $-1,398$ & $-1,322$ & 0 \\
\hline Total & $\mathbf{0}$ & $\mathbf{0}$ & $\mathbf{0}$ & $\mathbf{0}$ & $\mathbf{0}$ & $\mathbf{0}$ \\
\hline \hline
\end{tabular}

Table A-463: Delayed technology reference case with \$20 per tonne growers’ payment biomass-to-liquids production [Million gallons ethanol equivalent]

\begin{tabular}{lrrrrrr} 
& $\mathbf{2 0 0 5}$ & $\mathbf{2 0 1 0}$ & $\mathbf{2 0 1 5}$ & $\mathbf{2 0 2 0}$ & $\mathbf{2 0 2 5}$ & $\mathbf{2 0 3 0}$ \\
Africa & 0 & 0 & 0 & 0 & 0 & 0 \\
Argentina & 0 & 0 & 21 & 110 & 121 & 125 \\
Australia & 0 & 0 & 0 & 0 & 0 & 0 \\
Brazil & 0 & 0 & 106 & 320 & 554 & 556 \\
Canada & 0 & 0 & 93 & 280 & 361 & 361 \\
Caribbean basin & 0 & 0 & 0 & 0 & 0 & 0 \\
China & 0 & 0 & 80 & 240 & 477 & 477 \\
Colombia & 0 & 0 & 61 & 184 & 431 & 681 \\
Eastern Europe & 0 & 0 & 0 & 0 & 0 & 0 \\
Former Soviet Union & 0 & 0 & 0 & 0 & 0 & 0 \\
India & 0 & 0 & 77 & 232 & 544 & 790 \\
Japan & 0 & 0 & 0 & 0 & 0 & 0 \\
Middle East & 0 & 0 & 0 & 0 & 0 & 0 \\
Mexico & 0 & 0 & 72 & 216 & 506 & 556 \\
Other Asia & 0 & 0 & 0 & 0 & 0 & 0 \\
South Korea & 0 & 0 & 36 & 52 & 52 & 52 \\
United States & 0 & 0 & 786 & 2,986 & 4,061 & 5,230 \\
Western Europe & 0 & 0 & 0 & 0 & 0 & 0 \\
\hline Total & $\mathbf{0}$ & $\mathbf{0}$ & $\mathbf{1 , 3 3 2}$ & $\mathbf{4 , 6 2 0}$ & $\mathbf{7 , 1 0 7}$ & $\mathbf{8 , 8 2 6}$ \\
\hline \hline
\end{tabular}


Table A-464: Delayed technology reference case with \$20 per tonne growers’ payment biomass-to-liquids consumption [Million gallons ethanol equivalent]

\begin{tabular}{lrrrrrr} 
& $\mathbf{2 0 0 5}$ & $\mathbf{2 0 1 0}$ & $\mathbf{2 0 1 5}$ & $\mathbf{2 0 2 0}$ & $\mathbf{2 0 2 5}$ & $\mathbf{2 0 3 0}$ \\
Africa & 0 & 0 & 0 & 0 & 0 & 0 \\
Australia & 0 & 0 & 0 & 0 & 0 & 0 \\
Canada & 0 & 0 & 0 & 0 & 0 & 0 \\
China & 0 & 0 & 0 & 0 & 0 & 0 \\
Central and South America & 0 & 0 & 0 & 0 & 0 & 0 \\
Eastern Europe & 0 & 0 & 0 & 0 & 0 & 0 \\
Former Soviet Union & 0 & 0 & 0 & 0 & 0 & 0 \\
India & 0 & 0 & 0 & 0 & 0 & 0 \\
Japan & 0 & 0 & 0 & 0 & 0 & 0 \\
Middle East & 0 & 0 & 0 & 0 & 0 & 0 \\
Mexico & 0 & 0 & 0 & 0 & 0 & 0 \\
Other Asia & 0 & 0 & 0 & 0 & 0 & 0 \\
South Korea & 0 & 0 & 0 & 0 & 0 & 0 \\
United States & 0 & 0 & 1,059 & 4,532 & 7,107 & 8,827 \\
Western Europe & 0 & 0 & 273 & 88 & 0 & 0 \\
\hline Total & $\mathbf{0}$ & $\mathbf{0}$ & $\mathbf{1 , 3 3 2}$ & $\mathbf{4 , 6 2 0}$ & $\mathbf{7 , 1 0 7}$ & $\mathbf{8 , 8 2 7}$ \\
\hline \hline
\end{tabular}

Table A-465: Delayed technology reference case with \$20 per tonne growers’ payment biomass-to-liquids net trade [Million gallons ethanol equivalent]

\begin{tabular}{lrrrrrr} 
& $\mathbf{2 0 0 5}$ & $\mathbf{2 0 1 0}$ & $\mathbf{2 0 1 5}$ & $\mathbf{2 0 2 0}$ & $\mathbf{2 0 2 5}$ & $\mathbf{2 0 3 0}$ \\
Africa & 0 & 0 & 0 & 0 & 0 & 0 \\
Australia & 0 & 0 & 0 & 0 & 0 & 0 \\
Canada & 0 & 0 & 93 & 280 & 361 & 361 \\
China & 0 & 0 & 80 & 240 & 477 & 477 \\
Central and South America & 0 & 0 & 189 & 614 & 1,106 & 1,362 \\
Eastern Europe & 0 & 0 & 0 & 0 & 0 & 0 \\
Former Soviet Union & 0 & 0 & 0 & 0 & 0 & 0 \\
India & 0 & 0 & 77 & 232 & 544 & 790 \\
Japan & 0 & 0 & 0 & 0 & 0 & 0 \\
Middle East & 0 & 0 & 0 & 0 & 0 & 0 \\
Mexico & 0 & 0 & 72 & 216 & 506 & 556 \\
Other Asia & 0 & 0 & 0 & 0 & 0 & 0 \\
South Korea & 0 & 0 & 36 & 52 & 52 & 52 \\
United States & 0 & 0 & -273 & $-1,546$ & $-3,046$ & $-3,597$ \\
Western Europe & 0 & 0 & -273 & -88 & 0 & 0 \\
\hline Total & $\mathbf{0}$ & $\mathbf{0}$ & $\mathbf{0}$ & $\mathbf{0}$ & $\mathbf{0}$ & $\mathbf{0}$ \\
\hline \hline
\end{tabular}


Table A-466: Delayed technology reference case with \$20 per tonne growers' payment biodiesel production [Million gallons ethanol equivalent]

\begin{tabular}{lrrrrrr} 
& $\mathbf{2 0 0 5}$ & $\mathbf{2 0 1 0}$ & $\mathbf{2 0 1 5}$ & $\mathbf{2 0 2 0}$ & $\mathbf{2 0 2 5}$ & $\mathbf{2 0 3 0}$ \\
Africa & 0 & 0 & 0 & 0 & 0 & 0 \\
Argentina & 0 & 333 & 776 & 819 & 827 & 239 \\
Australia & 0 & 0 & 0 & 0 & 0 & 0 \\
Brazil & 0 & 333 & 877 & 1,642 & 1,793 & 1,355 \\
Canada & 0 & 0 & 0 & 0 & 0 & 0 \\
Caribbean basin & 0 & 95 & 310 & 675 & 802 & 898 \\
China & 0 & 0 & 0 & 0 & 0 & 0 \\
Colombia & 0 & 119 & 298 & 474 & 650 & 782 \\
Eastern Europe & 0 & 0 & 0 & 0 & 0 & 0 \\
Former Soviet Union & 0 & 0 & 0 & 0 & 0 & 0 \\
India & 0 & 0 & 0 & 0 & 0 & 0 \\
Japan & 0 & 0 & 0 & 0 & 0 & 0 \\
Middle East & 0 & 0 & 0 & 0 & 0 & 0 \\
Mexico & 0 & 0 & 0 & 0 & 0 & 0 \\
Other Asia & 0 & 0 & 0 & 0 & 0 & 0 \\
South Korea & 0 & 0 & 0 & 0 & 0 & 0 \\
United States & 0 & 1,238 & 1,640 & 1,747 & 1,907 & 2,072 \\
Western Europe & 998 & 3,178 & 3,578 & 3,930 & 4,468 & 4,788 \\
\hline Total & $\mathbf{9 9 8}$ & $\mathbf{5 , 2 9 4}$ & $\mathbf{7 , 4 7 9}$ & $\mathbf{9 , 2 8 7}$ & $\mathbf{1 0 , 4 4 8}$ & $\mathbf{1 0 , 1 3 4}$ \\
\hline \hline
\end{tabular}

Table A-467: Delayed technology reference case with \$20 per tonne growers’ payment biodiesel consumption [Million gallons ethanol equivalent]

\begin{tabular}{lrrrrrr} 
& $\mathbf{2 0 0 5}$ & $\mathbf{2 0 1 0}$ & $\mathbf{2 0 1 5}$ & $\mathbf{2 0 2 0}$ & $\mathbf{2 0 2 5}$ & $\mathbf{2 0 3 0}$ \\
Africa & 0 & 0 & 0 & 0 & 0 & 0 \\
Australia & 0 & 0 & 0 & 0 & 0 & 0 \\
Canada & 0 & 0 & 0 & 0 & 0 & 0 \\
China & 0 & 0 & 0 & 0 & 0 & 0 \\
Central and South America & 0 & 158 & 472 & 984 & 1,306 & 1,490 \\
Eastern Europe & 0 & 0 & 0 & 0 & 0 & 0 \\
Former Soviet Union & 0 & 0 & 0 & 0 & 0 & 0 \\
India & 0 & 0 & 0 & 0 & 0 & 0 \\
Japan & 0 & 0 & 0 & 0 & 0 & 0 \\
Middle East & 0 & 0 & 0 & 0 & 0 & 0 \\
Mexico & 0 & 0 & 0 & 0 & 0 & 0 \\
Other Asia & 0 & 0 & 0 & 0 & 0 & 0 \\
South Korea & 0 & 0 & 0 & 0 & 0 & 0 \\
United States & 0 & 1,238 & 1,713 & 1,747 & 1,907 & 2,724 \\
Western Europe & 998 & 3,898 & 5,294 & 6,556 & 7,235 & 5,920 \\
\hline Total & $\mathbf{9 9 8}$ & $\mathbf{5 , 2 9 4}$ & $\mathbf{7 , 4 7 9}$ & $\mathbf{9 , 2 8 7}$ & $\mathbf{1 0 , 4 4 8}$ & $\mathbf{1 0 , 1 3 4}$ \\
\hline \hline
\end{tabular}


Table A-468: Delayed technology reference case with \$20 per tonne growers’ payment biodiesel production [Million gallons ethanol equivalent]

\begin{tabular}{lrrrrrr} 
& $\mathbf{2 0 0 5}$ & $\mathbf{2 0 1 0}$ & $\mathbf{2 0 1 5}$ & $\mathbf{2 0 2 0}$ & $\mathbf{2 0 2 5}$ & $\mathbf{2 0 3 0}$ \\
Africa & 0 & 0 & 0 & 0 & 0 & 0 \\
Australia & 0 & 0 & 0 & 0 & 0 & 0 \\
Canada & 0 & 0 & 0 & 0 & 0 & 0 \\
China & 0 & 0 & 0 & 0 & 0 & 0 \\
Central and South America & 0 & 720 & 1,788 & 2,626 & 2,767 & 1,784 \\
Eastern Europe & 0 & 0 & 0 & 0 & 0 & 0 \\
Former Soviet Union & 0 & 0 & 0 & 0 & 0 & 0 \\
India & 0 & 0 & 0 & 0 & 0 & 0 \\
Japan & 0 & 0 & 0 & 0 & 0 & 0 \\
Middle East & 0 & 0 & 0 & 0 & 0 & 0 \\
Mexico & 0 & 0 & 0 & 0 & 0 & 0 \\
Other Asia & 0 & 0 & 0 & 0 & 0 & 0 \\
South Korea & 0 & 0 & 0 & 0 & 0 & 0 \\
United States & 0 & 0 & -73 & 0 & 0 & -652 \\
Western Europe & 0 & -720 & $-1,716$ & $-2,626$ & $-2,767$ & $-1,132$ \\
\hline Total & $\mathbf{0}$ & $\mathbf{0}$ & $\mathbf{0}$ & $\mathbf{0}$ & $\mathbf{0}$ & $\mathbf{0}$ \\
\hline \hline
\end{tabular}

